\title{
Petroleum Supply Monthly May 1995 With Data for March 1995
}

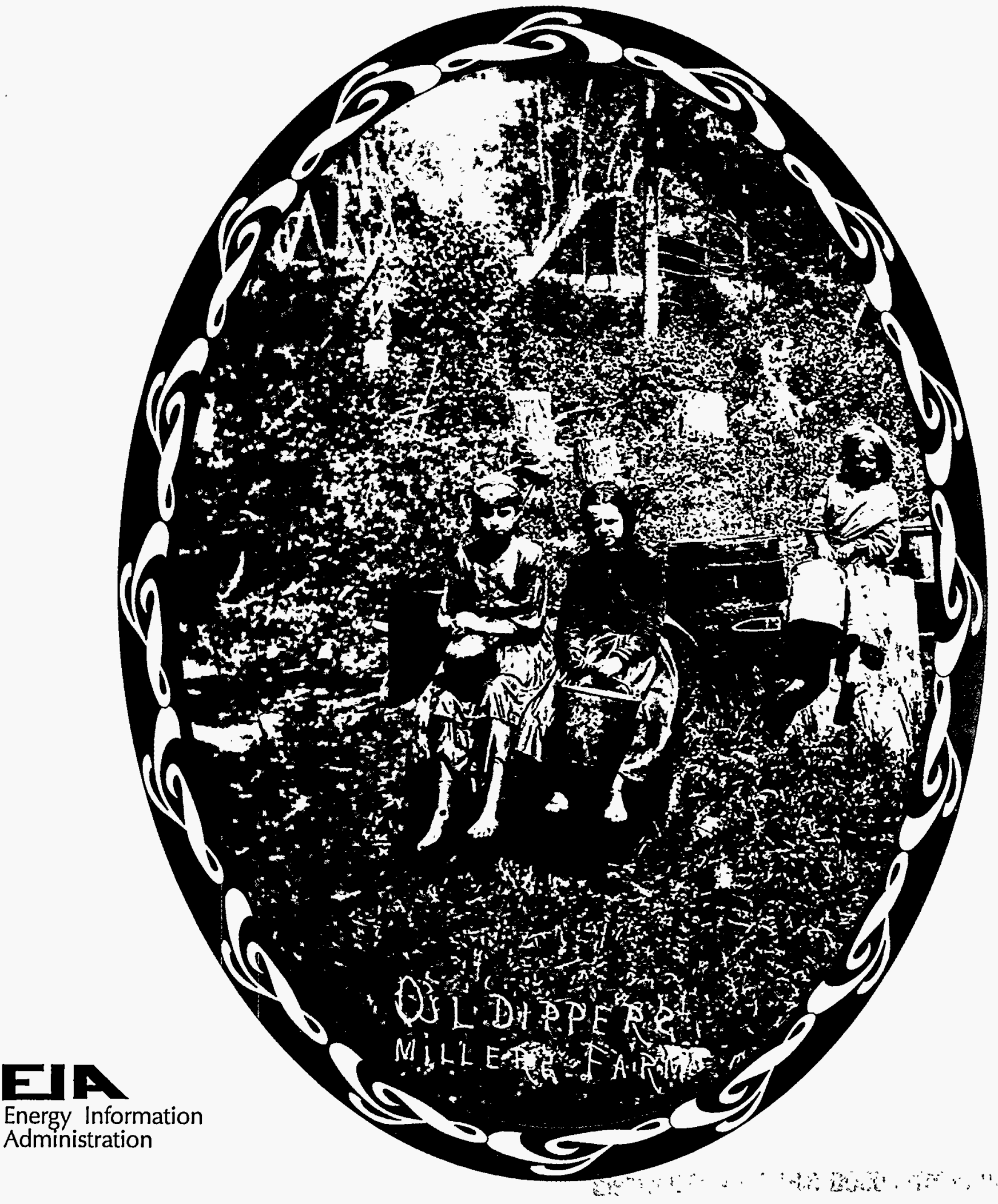


This publication and other Energy Information Administration (EIA) publications may be purchased from the Superintendent of Documents, U.S. Government Printing Office.

All telephone orders should be directed to:

Superintendent of Documents

U.S. Government Printing Office

Main Order Desk

(202) 512-1800

FAX (202) 512-2250

8:00 a.m. to 4:30 p.m., eastern time, M-F

All mail orders should be directed to:

U.S. Government Printing Office

P.O. Box 371954

Pittsburgh, PA $15250-7954$

Complimentary subscriptions and single issues are available to certain groups of subscribers, such as public and academic libraries, Federal, State, local, and foreign governments, and the media. For further information, and for answers to questions on energy statistics, please contact EIA's National Energy Information Center. Address, telephone numbers, and hours appear below.

\author{
National Energy Information Center, EI-231 \\ Energy Information Administration \\ Forrestal Building, Room 1F-048 \\ Washington, DC 20585 \\ (202) $586-8800$
}

TTY: For people who are deaf

or hard of hearing: (202) 586-1181

9:00 a.m. to 5:00 p.m., eastern time, M-F

We thank the following for the use of their photographs and illustrations in this report.

Cities Service Co., page xxvii (courtesy of the American Petroleum Institute).

Standard Oil Co., page 1 (courtesy of the American Petroleum Institute).

Phillips 66 Co., page 33 (courtesy of Phillips 66 Company).

Texaco Inc., page 109 (courtesy of Texaco Inc.).

Standard Oil Co., page 113 (courtesy of the American Petroleum Institute).

Texaco Inc., page 127 (courtesy of the American Petroleum Institute).

American Petroleum Institute, page 133 (courtesy of the American Petroleum Institute).

Atlantic Richfield Co., page 139 (courtesy of the American Petroleum Institute).

Released for printing: May 25, 1995

The Petroleum Supply Monthly (ISSN 0733-0553) is published monthly by the Energy Information Administration, 1000 Independence Avenue, SW., Washington, DC 20585 , and sells for $\$ 83.00$ per year (price is subject to change without advance notice). Second-class postage paid at Washington, DC 20066-9998, and at additional mailing offices. POSTMASTER: Send address changes to Petroleum Supply Monthly, Energy Information Administration, EI-231, 1000 Independence Avenue, SW, Washington, DC 20585.

On The Cover: Starting with the March issue of the Petroleum Supply Monthly, the Energy Information Administration began a 12-month series of historic photographs depicting the early days (1859-1920's) of the petroleum industry. The cover depicts Oil Dippers at Miller Farm, 1863. So much oil ran to waste down Oil Creek because of flowing wells or leaky bulk boats that people along the Creek would stand on the banks with buckets and skim oil off the top of the water, then pour it into barrels. Many a man got started in the oil business in this way.

Photo courtesy of the Pennsylvania Historical and Museum Commission, Drake Well Museum. 


\title{
Petroleum Supply Monthly
}

\author{
May 1995
}

\section{With Data for March 1995}

\author{
Energy Information Administration \\ Office of Oil and Gas \\ U.S. Department of Energy \\ Washington, DC 20585
}

This report was prepared by the Energy Information Administration, the independent statistical and analytical agency within the Department of Energy. The information contained herein should not be construed as advocating or reflecting any policy position of the Department of Energy or any other organization. 


\section{Data Available Electronically}

Data from the Weekly Petroleum Status Report, Winter Fuels Report, and the Petroleum Supply Monthly publications as well as data from other sources are available electronically on the Energy Information Administration's Electronic Publication Bulletin (EPUB) Board, and the Comprehensive Oil and Gas Information Source (COGIS). The schedule for data release is as follows:

\begin{tabular}{l|l|l|}
\hline Publications/Sources & Platform & Information \\
\hline
\end{tabular}

\section{Weekly Petroleum Status Report}

Wednesday 9:00 a.m. (weekly)

Wednesday 5:00 p.m. 6th-12th (monthly)

Thursday by Noon (weekly)

Thursday by Noon 7th-13th (monthly)
EPUB Table 1 (U.S. Balance Sheet) and Data Log (Table 14 plus 4-week averages)

EPUB Table H1 (Petroleum Supply Summary)

COGIS Table 1 (U.S. Balance Sheet) and

Table 14 (Most recent 5-weeks)

COGIS Table H1 (Petroleum Supply Summary)

Winter Fuels Report (October through March)

Wednesday 5:00 p.m. (weekly)

EPUB

Thursday 5:00 p.m. (weekly)

EPUB

Friday by Noon (weekly)

COGIS

Propane Data (April through September)

Second Wednesday of the

month (9:00 a.m.)

\section{Petroleum Supply Monthly}

23rd-26th (monthly)

EPUB

23rd-26th (monthly)

COGIS

EPUB Table D1 U.S. Summary

report month

\section{Oxygenate Data}

15 working days after the

\section{Imports Data}

7th-10th (preliminary)

EPUB

23rd-26th (final)
Propane highlights

All tables and highlights

All tables and highlights

Propane Stocks

Table H1 (Petroleum Supply Summary) and all Summary Statistics and Detailed Statistics Tables

Table H1 (Petroleum Supply Summary), and all Summary Statistics and Detailed Statistics Tables

Table D2 (Fuel Ethanol Production/Stocks) and

Table D3 (MTBE Production/Stocks)

Import data by company from the Form EIA-814, "Monthly Imports Report" 


\section{DISCLAIMER}

This report was prepared as an account of work sponsored by an agency of the United States Government. Neither the United States Government nor any agency thereof, nor any of their employees, make any warranty, express or implied, or assumes any legal liability or responsibility for the accuracy, completeness, or usefulness of any information, apparatus, product, or process disclosed, or represents that its use would not infringe privately owned rights. Reference herein to any specific commercial product, process, or service by trade name, trademark, manufacturer, or otherwise does not necessarily constitute or imply its endorsement, recommendation, or favoring by the United States Government or any agency thereof. The views and opinions of authors expressed herein do not necessarily state or reflect those of the United States Government or any agency thereof. 


\section{DISCLAIMER}

Portions of this document may be illegible in electronic image products. Images are produced from the best available original document. 


\section{Electronic Publishing System (EPUB)}

\section{User Instructions}

EPUB is an electronic publishing system maintained by the Energy Information Administration of the U.S. Department of Energy. EPUB allows the general public to electronically access selected energy data from many of EIA's statistical reports. The system is a menu-driven, bulletin board type system with extensive online help capabilities that can be accessed free of charge 24 hours a day by using a terminal or PC with an asynchronous modem. (EPUB will be taken down briefly at midnight for backup.)

\section{CONFIGURING YOUR PC SOFTWARE}

PC users must provide the following information to their communications software in order to successfully access the EPUB system. Consult your communications software documentation for information on how to correctly configure your software.

Communication Parameters:

BAUD RATE: Up to 28,800 bps

DATA BITS: 8

STOP BITS: 1

PARITY: NONE

DUPLEX: FULL

TERMINAL TYPE: examples: ANSI, ANSI-BBS, VT100

\section{ACCESS PHONE NUMBER}

Once your communications software and/or hardware has been configured, you can access EPUB by dialing (202) 586-2557.

\section{USING EPUB}

When a connection to the system has been made, some users may find that the menu-driven instructions and the online help capabilities will provide enough information to effectively use EPUB. If needed, more extensive information may be found in the EPUB Users Guide, which is available online from the EPUB system or from:

National Energy Information Center, EI-231

Energy Information Administration

Forrestal Building, Room 1F-048

Washington, DC 20585

(202) 586-8800

InternetE-MAIL: infoctr@eia.doe.gov

Hours: 9:00 a.m. to 5:00 p.m. Eastern Time, Monday through Friday

Telecommunications device for the hearing-impaired only:

(202) 586-1181. Hours: 9:00 a.m. to 5:00 p.m. Eastern Time, Monday through Friday

\section{EPUB ASSISTANCE}

For communications or technical assistance, call (202) 586-8959, 8:00 a.m. to 5:00 p.m. Eastern Time, Monday through Friday.

For questions about the content of EPUB reports, call (202) 586-8800, 9:00 a.m. to 5:00 p.m. Eastern Time, Monday through Friday.

\section{EPUB PROVIDES STATISTICAL INFORMATION, AS WELL AS DATA FROM THE FOLLOWING EIA PUBLICATIONS:}

Heating fuel data, (April through September) updated the 2nd week of the month

Oxygenate data, updated approximately 15 working days after the end of the report month

Weekly Petroleum Status Report, updated on Wednesdays (Thursday in event of a holiday) at 9:00 a.m.

Petroleum Supply Monthly, updated between the 23rd and 26th of the month

Petroleum Marketing Monthly, updated on the 20th of the month

Winter Fuels Report, propane inventory data updated Wednesdays at 5:00 p.m. All other data updated on Thursdays (Friday in event of a holiday) at 5:00 p.m. (October through March)

Natural Gas Monthly, updated on the 20th of the month

Weekly Coal Production, updated on Fridays at noon

Quarterly Coal Report, updated 40 days after the end of the quarter

Electric Power Monthly, updated on the 1st of the month

Monthly Energy Review, updated the last week of the month

Short Term Energy Outlook, updated 60 days after the end of the quarter 


\section{Comprehensive Oil and Gas Information Source}

The Comprehensive Oil and Gas Information Source (COGIS) is a project recently developed by the Energy Information Administration (EIA), in cooperation with the U.S. Department of Commerce in an effort to provide more timely information to its customers. COGIS offers the latest oil and gas data published by the EIA. Selected data series from the Petroleum Supply Monthly, the Petroleum Marketing Monthly, the Natural Gas Monthly, the Monthly Energy Review, the Weekly Petroleum Status Report, the Short Term Energy Outlook, and the Winter Fuels Report are available. In addition, COGIS offers timely analysis of major oil and gas trends, and weekly and monthly highlights of oil and gas activity.

Anyone with a workstation connected to an Internet node, or with a personal computer and modem, can have immediate access to oil and gas industry information.

For information, call EIA's National Energy Information Center, (202) 586-8800. To open an account, call the U.S. Department of Commerce, Office of Business Analysis, (202) 482-1986.

Current fee schedule is listed below.

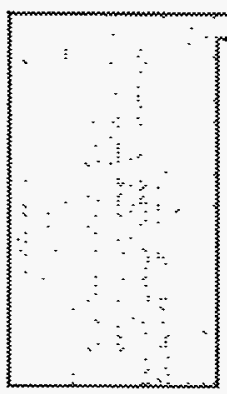

\section{Charge}

Annual Fee

Connect Charge Credit

Connect Charges (per minute based on eastern time)

Weekdays: 8:00 a.m. - noon

Noon - 6:00 p.m.

6:00 p.m. - 8:00 a.m.

(Also weekends and holidays)

Annual Flat Fee Option (cannot use account between 8:00 a.m. and noon)

Maximum 1 hour per day

Maximum 4 hours per day
Means Used to Access the EBB

$\begin{array}{ccc}\text { Up to } 2400 \text { Baud } & 9600 \text { Baud } & \text { Internet (telnet only) } \\ \$ 45.00 & \$ 45.00 & \$ 45.00 \\ \$ 20.00 & \$ 20.00 & \$ 20.00\end{array}$

$\$ 0.20$

$\$ 0.40$

$\$ 0.40$

$\$ 0.15$

$\$ 0.25$

$\$ 0.25$

$\$ 0.05$

$\$ 0.10$

$\$ 0.10$

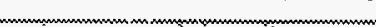




\section{Contacts}

The Petroleum Supply Monthly is prepared by the Petroleum Supply Division of the Office of Oil and Gas, Energy Information Administration, under the direction of Charles C. Heath (202) 586-6860.

Questions and comments concerning the contents of the Petroleum Supply Monthly may be referred to Ronald W. O'Neill (202) 586-9884, Chief of the Industry Analysis Branch, or the following specialists:

\begin{tabular}{|c|c|}
\hline 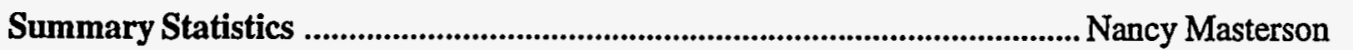 & (202) 586-8393 \\
\hline Supply and Disposition ... & (202) 586-8393 \\
\hline Crude Oil Production ... & (202) 586-2990 \\
\hline Natural Gas Processing . & (202) $586-2990$ \\
\hline Refinery Operations ...... & (202) 586-5786 \\
\hline Imports & (202) 586-9649 \\
\hline 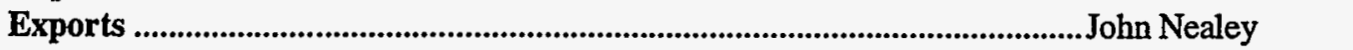 & (202) 586-9670 \\
\hline 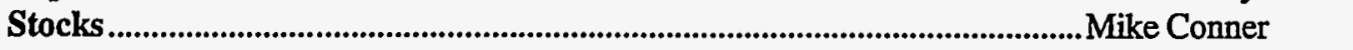 & (202) 586-1795 \\
\hline Mike Conner & (202) 586-1795 \\
\hline Oxygenate Data & (202) 586-9594 \\
\hline
\end{tabular}

Additional information on all energy statistics available from the Energy Information Administration may be obtained from the National Energy Information Center (202) 586-8800. 


\section{Preface}

The Petroleum Supply Monthly (PSM) is one of a family of four publications produced by the Petroleum Supply Division within the Energy Information Administration (EIA) reflecting different levels of data timeliness and completeness. The other publications are the Weekly Petroleum Status Report (WPSR), the Winter Fuels Report, and the Petroleum Supply Annual (PSA).

Data presented in the PSM describe the supply and disposition of petroleum products in the United States and major U.S. geographic regions. The data series describe production, imports and exports, inter-Petroleum Administration for Defense (PAD) District movements, and inventories by the primary suppliers of petroleum products in the United States (50 States and the District of Columbia). The reporting universe includes those petroleum sectors in primary supply. Included are: petroleum refiners, motor gasoline blenders, operators of natural gas processing plants and fractionators, inter-PAD transporters, importers, and major inventory holders of petroleum products and crude oil. When aggregated, the data reported by these sectors approximately represent the consumption of petroleum products in the United States.

Data presented in the PSM are divided into two sections: Summary Statistics and Detailed Statistics.

\section{Summary Statistics}

The tables and figures in the Summary Statistics section of the PSM present a time series of selected petroleum data on a U.S. level. Most time series include preliminary estimates for one month based on the Weekly Petroleum Supply Reporting System; statistics based on the most recent data from the Monthly Petroleum Supply Reporting System (MPSRS); and statistics published in prior issues of the PSM and PSA.

\section{Detailed Statistics}

The Detailed Statistics tables of the PSM present statistics for the most current month available as well as year-to-date. In most cases, the statistics are presented for several geographic areas - - the United States (50 States and the District of Columbia), five PAD Districts, and 12 Refining Districts. At the U.S. and PAD District level, the total volume and the daily rate of activities are presented. The statistics are developed from monthly survey forms submitted by respondents to the EIA and from data provided from other sources.

\section{Appendices}

Four appendices are provided to assist in understanding and interpreting the data presented in this publication:

- Appendix A (District Descriptions and Maps) - Geographic aggregations of the 50 States and the District of Columbia into Refining Districts which make up the PAD Districts.

- Appendix B (Detailed Statistics Explanatory Notes) - Information describing data collection, sources, estimation methodology, data quality control procedures, modifications to reporting requirements and interpretation of tables.

- Appendix C (Impact of Resubmissions) - Information on revisions to published statistics caused by resubmission of respondent survey forms.

- Appendix D (EIA-819M, Monthly Oxygenate Telephone Report) - Preliminary information on production and stocks of fuel ethanol and methyl tertiary butyl ether (MTBE) by PAD District. Data are collected from a sample of respondents reporting on the MPSRS surveys. Data are also published in the WPSR and are available electronically approximately 15 working days after the end of the month.

Industry terminology and product definitions are listed alphabetically in the Glossary. Final statistics for the data series published in the PSM, as well as additional data from the annual refinery and oxygenate capacity surveys are published in the PSA. The PSA is published approximately five months after the end of the report year. 

Article.

Highlights

Tables

\section{Summary Statistics}

S1. Crude Oil and Petroleum Products Overview, 1981-Present ...................................................................................... 2

S2. Crude Oil Supply and Disposition, 1981-Present.................................................................................................................. 6

S3. Crude Oil and Petroleum Product Imports, 1981-Present .................................................................................................... 8

S4. Finished Motor Gasoline Supply and Disposition, 1981-Present ................................................................................. 17

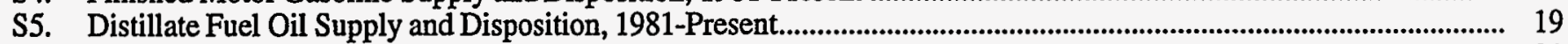

S6. Residual Fuel Oil Supply and Disposition, 1981-Present ............................................................................................. 21

S7. Jet Fuel Supply and Disposition, 1981-Present ............................................................................................................ 23

S8. Propane/Propylene Supply and Disposition, 1981-Present .............................................................................................. 25

S9. Liquefied Petroleum Gases Supply and Disposition, 1981-Present..................................................................................... 27

S10. Other Petroleum Products Supply and Disposition, 1981-Present..................................................................................... 28

Summary Statistics Table and Figure Sources ............................................................................................................... 29

Summary Statistics Explanatory Notes................................................................................................................................. 30

\section{Detailed Statistics}

National Statistics

1. U.S. Petroleum Balance

2. U.S. Supply, Disposition, and Ending Stocks of Crude Oil and Petroleum Products ................................................ 36

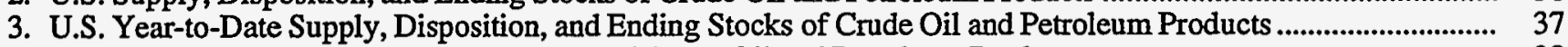

4. U.S. Daily Average Supply and Disposition of Crude Oil and Petroleum Products ......................................................... 38

5. U.S. Year-to-Date Daily Average Supply and Disposition of Crude Oil and Petroleum Products

Supply and Disposition of Crude Oil and Petroleum Products

6. PAD District I

7. Year-to-Date PAD District I

8. Daily Average PAD District I.

9. Year-to-Date Daily Average PAD District I .

10. PAD District II .

11. Year-to-Date PAD District II.

12. Daily Average PAD District II .

13. Year-to-Date Daily Average PAD District II

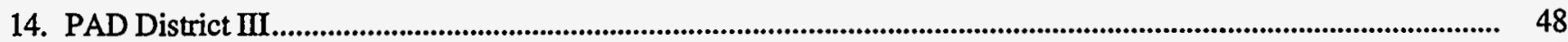

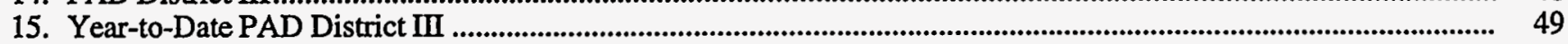

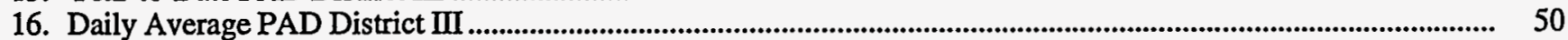

17. Year-to-Date Daily Average PAD District III................................................................................................................ 51

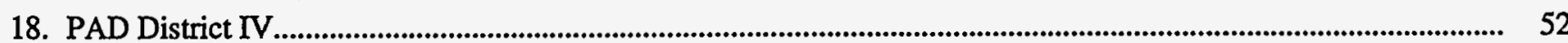

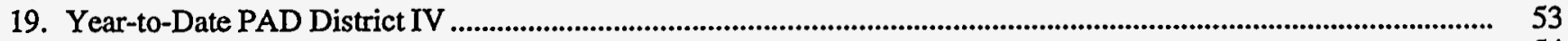

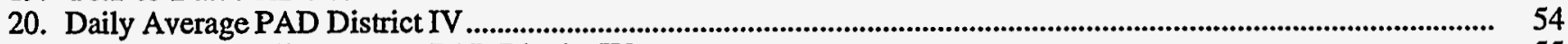

21. Year-to-Date Daily Average PAD District IV ….......................................................................................... 55

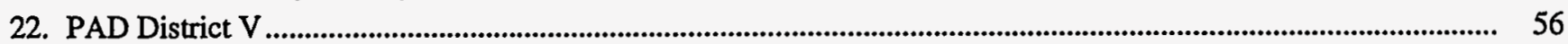

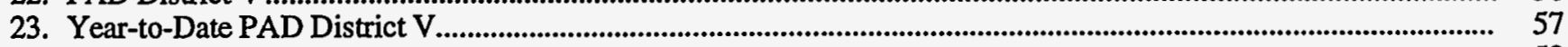

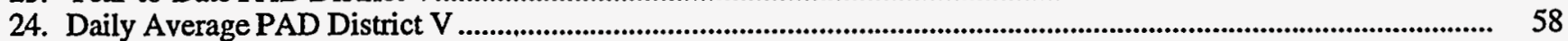

25. Year-to-Date Daily Average PAD District V ................................................................................................................ 59

Production of Crude Oil

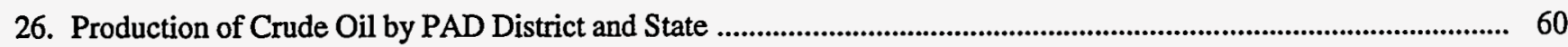

Natural Gas Processing

27. Natural Gas Plant Net Production and Stocks of Petroleum Products by PAD and Refining Districts

Refinery Operations

28. Refinery Input of Crude Oil and Petroleum Products by PAD and Refining Districts .............................................. 62

29. Refinery Net Production of Finished Petroleum Products by PAD and Refining Districts .......................................... 64

30. Refinery Stocks of Crude Oil and Petroleum Products by PAD and Refining Districts ............................................... 66

31. Percent Refinery Yield of Petroleum Products by PAD and Refining Districts........................................................... 68 
Imports of Crude Oil and Petroleum Products

State of Entry

32. Imports of Residual Fuel Oil by Sulfur Content

PAD District

33. Imports of Crude Oil and Petroleum Products

34. Year-to-Date Imports of Crude Oil and Petroleum Products

\section{Country of Origin}

35. United States.

36. PAD District I.

37. PAD District II

38. PAD District III

39. PAD Districts IV and V

40. Year-to-Date United States

41. Year-to-Date PAD District I

42. Year-to-Date PAD District II

43. Year-to-Date PAD District III

44. Year-to-Date PAD Districts IV and V

Exports of Crude Oil and Petroleum Products

45. Exports of Crude Oil and Petroleum Products by PAD District.

46. Year-to-Date Exports of Crude Oil and Petroleum Products by PAD District

47. Exports of Crude Oil and Petroleum Products by Destination

48. Year-to-Date Exports of Crude Oil and Petroleum Products by Destination

Net Imports

49. Net Imports of Crude Oil and Petroleum Products into the United States by Country.

50. Year-to-Date Net Imports of Crude Oil and Petroleum Products into the United States by Country

\section{Stocks}

51. Stocks of Crude Oil and Petroleum Products by PAD District

52. Refinery, Bulk Terminal, and Natural Gas Plant Stocks of Selected Petroleum Products

by PAD District and State

\section{Movements of Crude Oil and Petroleum Products}

53. Movements of Crude Oil and Petroleum Products by Pipeline, Tanker, and Barge Between PAD Districts

54. Movements of Crude Oil and Petroleum Products by Pipeline Between PAD Districts

55. Movements of Crude Oil and Petroleum Products by Tanker and Barge Between PAD Districts

56. Net Movements of Crude Oil and Petroleum Products by Pipeline, Tanker, and Barge Between PAD Districts

\section{Illustrations}

S1. Petroleum Overview, March 1994-Present

S2. Petroleum Products Supplied, March 1994-Present

S3. Crude Oil Supply and Disposition, March 1994-Present .

S4. Crude Oil Ending Stocks, March 1994-Present.

S5. Finished Motor Gasoline Supply and Disposition, March 1994-Present

S6. Motor Gasoline Ending Stocks, March 1994-Present

S7. Distillate Fuel Oil Supply and Disposition, March 1994-Present

S8. Distillate Fuel Oil Ending Stocks, March 1994-Present.

S9. Residual Fuel Oil Supply and Disposition, March 1994-Present.

S10. Residual Fuel Oil Ending Stocks, March 1994-Present

S11. Jet Fuel Supply and Disposition, March 1994-Present

S12. Jet Fuel Ending Stocks, March 1994-Present

S13. Propane/Propylene Supply and Disposition, February 1994-Present.

S14. Propane/Propylene Ending Stocks, February 1994-Present

S15. Liquefied Petroleum Gases Supply and Disposition, February 1994-Present

S16. Liquefied Petroleum Gases Ending Stocks, February 1994-Present.

\section{Appendices}

A. District Descriptions and Maps

B. Detailed Statistics Explanatory Notes

C. Impact of Resubmissions on Major Series, 1994

D. EIA-819M, Monthly Oxygenate Telephone Report

\section{Glossary}

Definitions of Petroleum Products and Other Terms 


\section{Articles}

Feature articles on energy-related subjects are frequently included in this publication. The following articles have appeared in previous issues.

Trends in Petroleum Product Consumption

January 1986

Western Countries Lead U.S. Petroleum Import Sources.

January 1986

U.S. Petroleum Exports Show Slight Upturn

January 1986

Motor Gasoline Trends

February 1986

Oil Imports from Saudi Arabia.

February 1986

Refinery Capacity Trends and Outlook

March 1986

Timeliness and Accuracy of Petroleum Supply Data

April 1986

Midyear Petroleum Review

May 1986

Winter 1986-1987 Distillate Fuel Outlook

July 1986

Recent Trends for Middle Distillates

July 1986

Comparison of Independent Statistics on Petroleum Supply

U.S. Petroleum Developments: 1986

September 1986

U.S. Petroleum Imports, 1986 Regional Highlights

Leading Petroleum Importers, 1986

U.S. Exports of Petroleum Products Reach Record High

Trends in Petroleum Product Consumption

Refinery Capacity: 1987

Motor Gasoline Outlook for Summer 1987.

Motor Gasoline Trends Through 1986.

Timeliness and Accuracy of Petroleum Supply Data .

Midyear Petroleum Review

November 1986

Petroleum Heating Fuels

Distillate Fuel Oil Outlook for Winter 1987/1988

Petroleum Exports

December 1986

December 1986

December 1986

EIA Releases Annual Reserves Summary.

Comparisons of Independent Statistics on Petroleum Supply

The Northeast-Distillate Fuel Oil Supply

U.S. Petroleum Developments: 1987

U.S. Petroleum Import/Export Trends Through 1987.

Motor Gasoline Trends Through 1987

Distillate Fuel Oil Outlook: Winter 1988/1989.

February 1987

March 1987

March 1987

March 1987

April 1987

May 1987

July 1987

July 1987

August 1987

August 1987

September 1987

November 1987

December 1987

January 1988

February 1988

July 1988

Comparison of Independent Statistics on Petroleum Supply

U.S. Petroleum Developments: 1988

September 1988

U.S. Petroleum Trade Trends: 1988

December 1988

Timeliness and Accuracy of Petroleum Supply Data

January 1989

July 1989

July 1989

Distillate Fuel Oil Outlook: Winter 1989/1990

September 1989

Comparisons of Independent Statistics on Petroleum Supply

U.S. Petroleum Developments: 1989.

December 1989

U.S. Petroleum Trade Trends: 1989

January 1990

Motor Gasoline Outlook: 1990

Timeliness and Accuracy of Petroleum Supply Data

February 1990

Heating Fuel Outlook: Winter 1990-91

Comparisons of Independent Statistics on Petroleum Supply

U.S. Petroleum Developments: 1990

April 1990

July 1990

September 1990

U.S. Petroleum Trade 1990.

February 1991

Effects of the Clean Air Act's Highway Diesel Fuel Oil Provisions

March 1991

June 1991

June 1991

Timeliness and Accuracy of Petroleum Supply Data

August 1991

Regulation of Underground Petro

October 1991

U.S. Petroleum Developments: 1991

February 1992

Comparisons of Independent Statistics on Petroleum Supply

March 1992 


\section{Articles (Continued)}

U.S. Petroleum Trade, 1991

April 1992

Timeliness and Accuracy of Petroleum Supply Data

September 1992

Three Dimensional Seismology-A New Perspective

December 1992

Summer 1993 Motor Gasoline Outlook

April 1993

Comparisons of Independent Statistics on Petroleum Supply

May 1993

Drilling Sideways.

June 1993

The Economics of the Clean Air Act Amendments of 1990.

July 1993

Accuracy of Petroleum Supply Data

August 1993

Distillate Fuel Oil Outlook for Winter 1993-1994

October 1993

Propane Outlook for Winter 1993-1994

October 1993

Strategic Shipping Lanes

January 1994

Summer 1994 Motor Gasoline Outlook

April 1994

Accuracy of Petroleum Supply Data

October 1994

Distillate Fuel Oil Assessment for Winter 1994-1995

October 1994

Propane Assessment for Winter 1994-1995

October 1994

Comparisons of Independent Statistics on Petroleum Supply

April 1995 


\section{Feature Article}

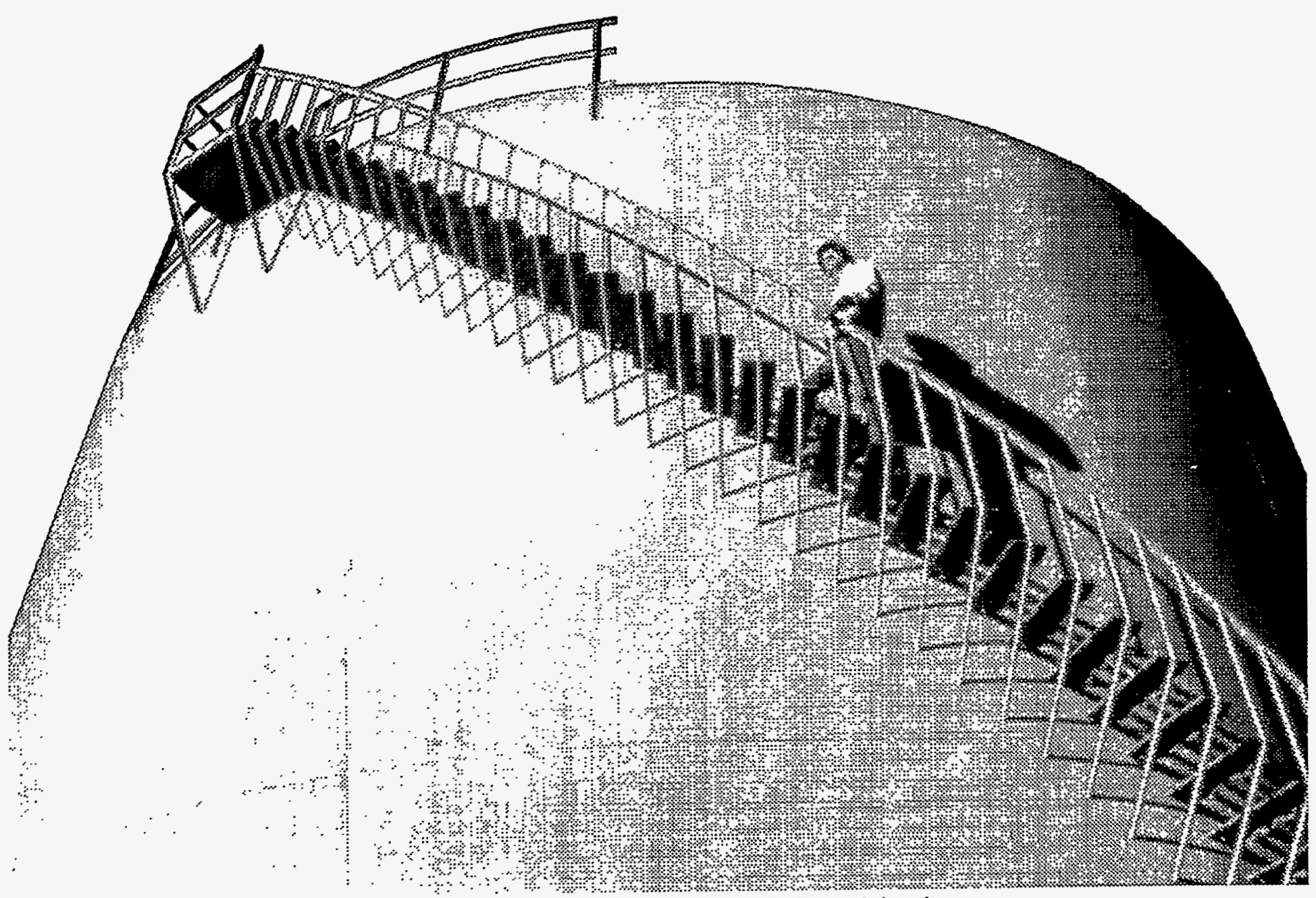





\title{
Summer 1995 Gasoline Assessment
}

\author{
by Aileen Bohn, Charles Dale, and John Zyren ${ }^{1}$
}

\section{Summary}

Fundamental changes to U.S. gasoline markets in recent years add complexity and uncertainty to the summer assessment. Growing demand, without corresponding increases in production capacity, has increased both domestic refinery capacity utilization and dependence on imports. At the same time, expanding product slates, driven by clean air concerns, have reduced flexibility in refinery, transportation, and storage operations. Additionally, regulatory uncertainties affecting demand and specifications for cleaner fuels have complicated suppliers' planning for both shortand long-term product availability.

Summer ${ }^{2}$ gasoline demand is forecast to reach record high levels in 1995, driven by continuing economic growth. In the Mid World Oil Price Case of the Energy Information Administration's (EIA) Short Term Energy Outlook (STEO), summer demand is expected to average 7.89 million barrels per day (MMBD), 1.8 percent higher than summer demand last year (Table FE1). Refinery production to serve this demand is forecast to increase 1.9 percent to 7.36 MMBD. Non-refinery gasoline blending is expected to add another 150 thousand barrels per day (MBD). Net imports are estimated to remain at about the same level as last year, which was slightly higher than the average for 1989-1993. Estimated stock levels begin slightly lower, but end higher than last year's levels.

This will be the first driving season in which reformulated gasoline (RFG) is used. RFG is expected to account for about 30 percent of total demand this summer, peaking at 2.4 MMBD in July and August. Stocks of RFG have been running lower than conventional gasoline on a days supply basis, and there is no reason to expect this pattern to change over the summer under the Mid Case forecast. This would imply that RFG stocks will not provide a significant source of incremental supplies, and RFG imports will have to make up the difference between production and demand. If RFG production does not go higher than 2.2 MMBD, imports of 200 to $300 \mathrm{MBD}$ of RFG may occur; however, much uncertainty exists around the mix of production and imports that will actually occur. Supplies of oxygenate for RFG are expected to be adequate.

The Mid Case world oil price is roughly constant at $\$ 16.75$ per barrel, $\$ 0.50$ per barrel (about 1.2 cents per gallon) higher than last year. Wholesale gasoline spreads over crude price average over 3 cents per gallon higher than last year's average wholesale spread, due to increased costs of producing RFG and to refiners and wholesalers recovering slightly from the low spreads in 1994. This would imply at least a 4-cent per gallon increase in costs to retailers, which is reflected in the wholesale prices shown in Table FE1. The retail price is forecast to increase about 5 cents per gallon over 1994, to average $\$ 1.24$ for the 1995 summer driving season.

If the market returns to a situation similar to that of 1994 , with lower wholesale price spreads over crude, retail prices will likely be 1 to 2 cents per gallon lower than the forecast. However, if world markets experience strong gasoline demand growth and lower incentive to export to the United States, import levels may be lower than forecast, potentially driving stocks lower than forecast. This would tend to push prices higher.

The Mid World Oil Price Case is the basis for the summer driving season forecast described in this article. The STEO case was developed using the Short-Term Integrated Forecasting System, driven principally by three sets of inputs pertaining to key macroeconomic variables, world oil prices, and the weather. Table FE1 compares the major STEO demand drivers (Gross Domestic Product Growth Rate, Disposable Personal Income, Vehicle Miles Traveled, and Vehicle Fuel Efficiency Index), price drivers (the Price of Imported Crude Oil and the Federal Tax Rate), and the model results for demand, supply components, and prices.

\section{Introduction}

As the summer 1995 driving season approaches, gasoline supply and market conditions are somewhat different from past years. Expected record demands this summer, if realized, will push refinery utilization to very high levels. In addition, this is the first summer driving season using RFG. The RFG program, which started in December 1994 at the wholesale level and in January 1995 at retail, impacts the overall gasoline market in a variety of ways.

Gasoline today encompasses many different formulations, grades and volatility classes. Prior to the Clean Air Act Amendments of 1990 (CAAA), gasolines were mainly distinguished by grade (octane rating) and by Reid Vapor Pressure (RVP). The CAAA

\footnotetext{
${ }^{1}$ Michael Burdette, Joanne Shore, and John Hackworth, industry analysts on contract to EIA, also contributed to this article.

2 "Summer" and "driving season" are used throughout this article to refer to the period including the second and third quarters of the calendar year, i.e., April through September.
}

Unless otherwise referenced, data in this article are taken from the following: Weekly Petroleum Status Report, DOE/EIA-0208 April 21 (95-17) and predecessor reports; Petroleum Supply Monthily, April 1995, DOE/EIA-0109(95/04); Petroleum Supply Annual 1993, DOE/EIA-0340, Volume 1 and predecessor reports; Petroleum Marketing Monthly, March 1995, DOE/EIA-0380(95/03); Short-Term Energy Outlook, DOE/EIA-0202(94/2Q) and predecessor reports. All data through 1993 are considered final and are not subject to further revision. 


\begin{tabular}{|c|c|c|c|c|}
\hline \multirow[b]{2}{*}{ Factor } & \multicolumn{3}{|c|}{ History } & \multirow{2}{*}{$\begin{array}{c}\text { STEO } \\
\text { Mid Case } \\
\text { Summer } 1995\end{array}$} \\
\hline & Summer 1992 & Summer 1993 & Summer 1994 & \\
\hline 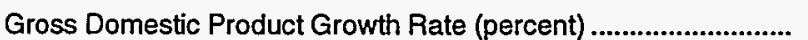 & 2.3 & 3.1 & 4.2 & 2.9 \\
\hline 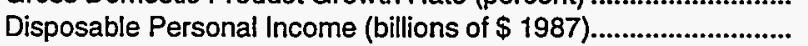 & $\$ 3,630$ & $\$ 3,705$ & $\$ 3,826$ & $\$ 3,963$ \\
\hline 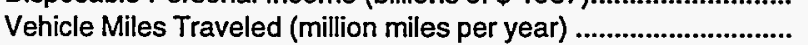 & 6437 & 6603 & 6756 & 6947 \\
\hline 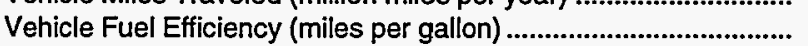 & 20.71 & 20.54 & 20.78 & 20.97 \\
\hline 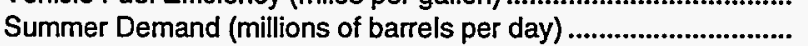 & 7.51 & 7.66 & 7.75 & 7.89 \\
\hline 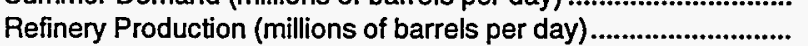 & 7.05 & 7.30 & 7.22 & 7.36 \\
\hline 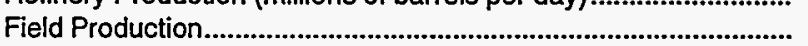 & 0.12 & 0.07 & 0.13 & 0.15 \\
\hline Net imports (millions of barrels per day) & 0.27 & 0.19 & 0.37 & 0.37 \\
\hline 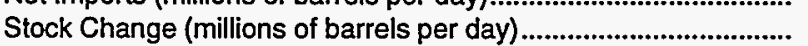 & -0.07 & -0.11 & -0.05 & -0.02 \\
\hline 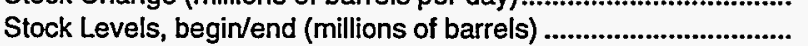 & $220 / 206$ & $230 / 208$ & $214 / 205$ & $212 / 209$ \\
\hline 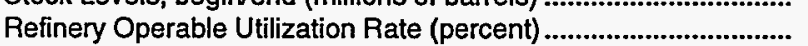 & 89.8 & 93.3 & 95.0 & 94.3 \\
\hline 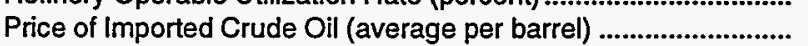 & $\$ 19.04$ & $\$ 16.63$ & $\$ 16.25$ & $\$ 16.75$ \\
\hline 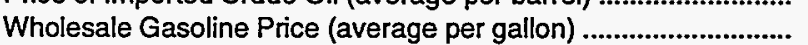 & $\$ 0.71$ & $\$ 0.65$ & $\$ 0.62$ & $\$ 0.66$ \\
\hline Federal Tax Rate (average per gallon) & $\$ 0.14$ & $\$ 0.14$ & $\$ 0.18$ & $\$ 0.18$ \\
\hline 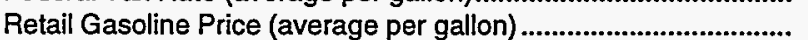 & $\$ 1.21$ & $\$ 1.18$ & $\$ 1.19$ & $\$ 1.24$ \\
\hline
\end{tabular}

Source: Short-Term Energy Outlook, Petroleum Supply Monthly/Annual, and Petroleum Marketing Monthly/Annual.

added oxygenated gasoline for carbon monoxide control, and most recently RFG, designed to reduce ground-level ozone pollution. Products vary by season and by geographic location. For example, oxygenated fuels (both oxygenated gasoline and oxygenated reformulated gasoline) are only required during the winter months and in limited geographic areas. The RFG program was the most complex new product program implemented by the industry to date, requiring major refinery changes. In addition, RFG must be kept segregated from other gasolines and tracked from refinery to outlet. The addition of RFG to the gasoline pool increases the demand for oxygenates during this summer over previous summers, and presents a new set of uncertainties for refiners and marketers.

This article provides an overview of the gasoline market as it stands facing the 1995 driving season, followed by a discussion of the assessment for gasoline supply, demand, and prices this summer.

\section{Overview of the Motor Gasoline Market}

The 1995 summer driving season is expected to create record high demands for gasoline. Preliminary estimates show first quarter 1995 demand as high as 4.1 percent over first quarter 1994. Total production and net imports were 7.4 MMBD, or 5.0 percent higher in the first quarter 1995 than first quarter 1994. Primary inventories (stocks) ended March at 212 million barrels (MMB), which is quite low, but not the lowest March ever. Finished gasoline stocks ended the quarter at about 22 days supply (based on the demand forecast for April), much lower than the 5-year average of 25 days. Prices are significantly above last year's pre-season levels, partly due to higher crude oil costs, and have begun their usual seasonal increase.

The addition of RFG to the gasoline pool this year has significantly affected gasoline markets (see box on "Introduction of Reformulated Gasoline"). The low stock levels experienced through the first quarter were in large part due to low RFG stocks. Uncertainties surrounding opt-outs, i.e., the unexpected withdrawal of voluntarily participating areas, and weak RFG prices relative to conventional fuels, constrained RFG production and imports, thereby keeping stocks from growing much beyond their initial levels when the program began last December. After strengthening somewhat through late January, the price differential narrowed in February and March, as oxygenate costs eased and conventional gasoline prices began their seasonal climb.

\section{Demand}

Motor gasoline demand has shown strong growth for the past several years. Gasoline demand was influenced by a recession that began in the last half of 1990 and lasted through the first half of 1992. With the rebound in the economy, the need for motor gasoline increased in 1992 and 1993 (Table FE2). Gasoline

Table FE2. Finished Gasoline Demand (Thousands of Barrels Per Day)

\begin{tabular}{|c|c|c|c|c|c|c|}
\hline Years & Quarter 1 & Quarter 2 & Quarter 3 & Quarter 4 & Annual & Annual Growth \\
\hline 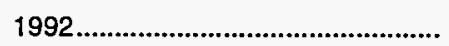 & 7,072 & 7,436 & 7,583 & 7,409 & 7,376 & $1.1 \% 1$ \\
\hline 1993 & 7,044 & 7,573 & 7,754 & 7,525 & 7,476 & $1.4 \%$ \\
\hline 1994 & 7,186 & 7,682 & 7,826 & 7,644 & 7,587 & $1.5 \%$ \\
\hline $1995 \ldots$ & 7,484 & & & & & \\
\hline
\end{tabular}

\footnotetext{
${ }^{1}$ This percentage was calculated from 1991 to 1992 using 1992 data not adjusted for blending components, since only unadjusted 1991 data is available.
} 


\section{Introduction of Reformulated Gasoline (RFG)}

Throughout 1994, the petroleum industry prepared for the introduction of reformulated gasoline (RFG), required year-round in almost a third of the U.S. gasoline market by the Clean Air Act Amendments of 1990 (CAAA). As the latest in a series of regulatory initiatives designed to improve the environmental quality of motor fuels, the CAAA necessitated a proliferation of reformulated and other clean products in the gasoline market by the end of 1994. Transition to the RFG program progressed without significant supply shortfalls or price runups, in contrast to the start-up of the low sulfur diesel program when a combination of infrastructure problems and low stocks led to price spikes in some regions.

Prior to the start of the program, RFG use was expected in 9 mandated areas and various others that had "opted-in", which combined constituted about 35 percent of U.S. gasoline consumption. The original forecasts for RFG demand of about 2.5 MMBD in December 1994 and January $1995^{3}$ never materialized, mainly because some areas elected to opt out of the program. These areas, which combined represented about $200 \mathrm{MBD}$ of RFG demand, were among the most distant from supply facilities. Their exit reduced the risk of localized outages.

RFG production began in September, then accelerated as refiners built stocks before the program startup. RFG production was expected to reach peak levels earlier than it did. One of the reasons for the delay was the Colonial Pipeline break in October, which, by raising conventional gasoline prices relative to RFG, discouraged an early switch to RFG production. Once the break was repaired and market concern over conventional supplies eased, the differential returned, and RFG production surged.

The peak production level of 2.2 MMBD was reached in the first 3 weeks of December, prior to the realization of downward, opt-out related pressures. Although production never reached forecast levels, refiners began to cut output in late December, in response to lower demand, reduced financial incentives, and increased uncertainty as to what gasolines would eventually be required in many areas.

RFG stocks built fairly quickly once production began, and hit $40 \mathrm{MMB}$ by the end of November, when RFG supply from terminals was required. Stocks peaked at about $44 \mathrm{MMB}$ in mid-December, or about 23 days of supply at estimated January demand levels. After that, RFG stocks gradually declined, as falling demand was more than offset by production cuts. RFG stocks in late February stabilized around $40 \mathrm{MMB}$, or 21 days supply, low by historical standards, and have since fluctuated between 40 and $45 \mathrm{MMB}$.

Despite the relatively smooth startup of the RFG program, and minimal price impacts on consumers, several issues emerged which cloud the outlook. Following the opting-out of various counties in Pennsylvania, New York, and Maine, and an early adjustment to lower oxygen content in northern New Jersey, the State of Wisconsin requested the suspension of RFG requirements for the remainder of the winter. The request, ultimately denied by EPA, was based on consumer complaints of high cost, health hazards (nausea and dizziness), and engine damage, all attributed to RFG. Particular complaints were lodged against MTBE as the source of noxious vapors, especially during cold weather. A unique aspect of the State's request was that the Milwaukee area, unlike the earlier opt-out areas in the East, was one of the nine severe ozone non-attainment areas mandated to participate in the program. Wisconsin has since rescinded its late opt-in of three other counties, and its state legislature has continued to seek legal means to exit the program, or at least to outlaw the use of MTBE in the State. Other jurisdictions are reported to be following the Wisconsin situation with great interest.

${ }^{3}$ EIA, The Energy Information Administration's Assessment of Reformulated Gasoline (October 1994) and The Energy Information Administration's Assessment of Reformulated Gasoline: An Update (December 1994).

demand continued a strong growth pattern in 1994 as the economy continued to improve. Real disposable personal income increased at a rate of 3.5 percent, compared with 1.5 percent in 1993. Gross Domestic Product rose by a robust 4.0 percent (in constant dollars), contrasted to 3.1 percent in $1993 .{ }^{4}$ This led to continued growth of the vehicle fleet and an increase in the average number of miles driven per vehicle, both of which boost gasoline consumption. Since the late 1970's, improvements in fuel efficiency, measured by miles per gallon, tended to slow demand growth; however, fuel efficiency in 1992 and 1993 began to fall slightly, removing the moderating effect it had on consumption historically.

In 1994, gasoline demand, as measured by product supplied, set a record of almost 7.6 MMBD. This represented a rise of 1.5 percent from 1993's record of 7.5 MMBD. Concurrently, distillate and jet fuels were also in high demand.

Overall, gasoline demand has continued its relatively strong upward trend in 1995 to date (Figure FE1), averaging 7.5 MMBD

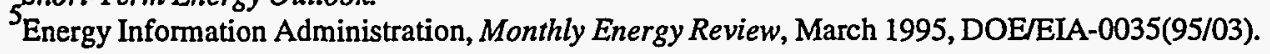




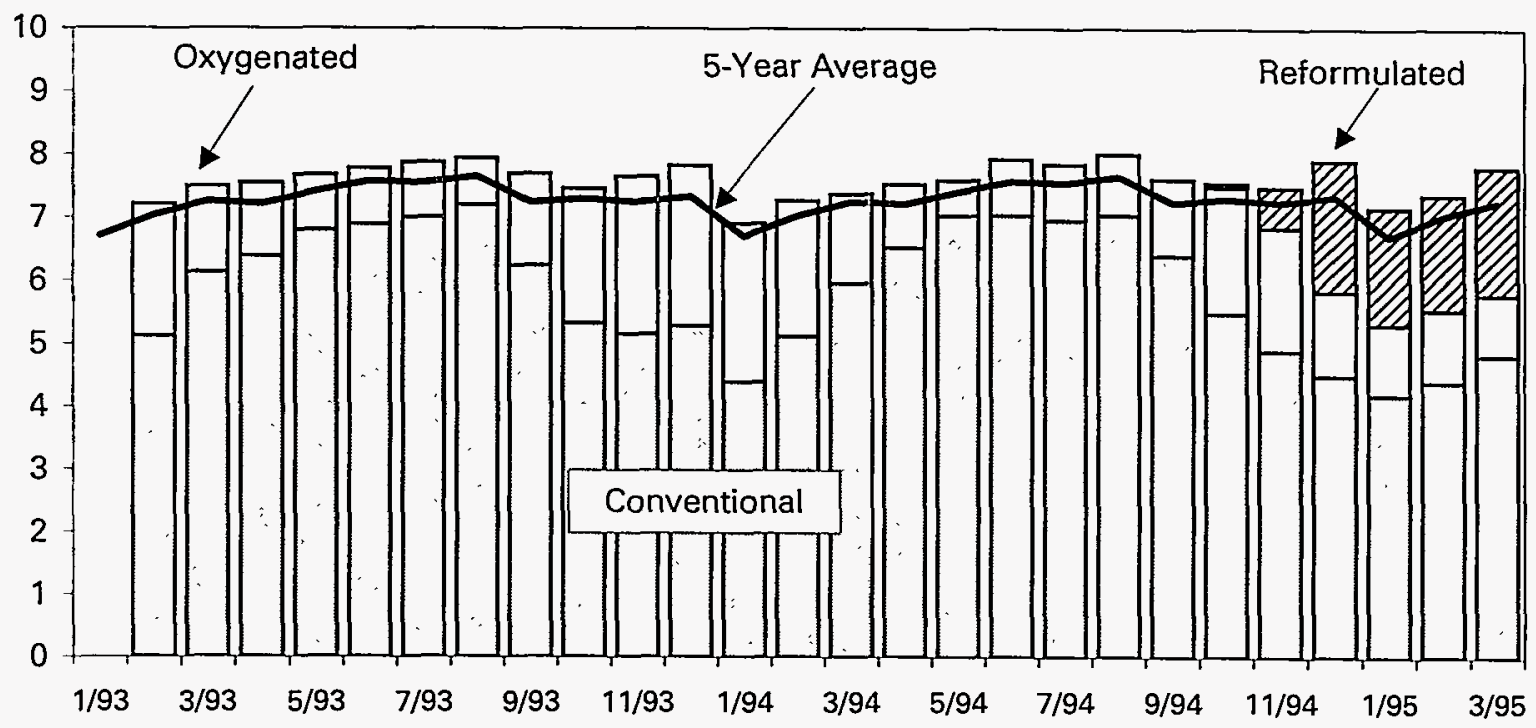

Source: Petroleum Supply Monthly/Annual.

through the first quarter compared to $7.2 \mathrm{MMBD}$ for the first quarter 1994. RFG demand averaged 1.9 MMBD for the first quarter for 1995 or 26 percent of gasoline demand. However, RFG demand has not reached the levels anticipated by the Energy Information Administration (EIA) last fall, ${ }^{6}$ mainly because some areas elected to opt out of the program. These areas represented about 200,000 barrels per day, roughly 10 percent of first quarter average RFG demand.

\section{Production}

To meet higher demand, gasoline production increased in 1992 and 1993, but dropped slightly in 1994, as imports returned to more typical levels in satisfying incremental requirements (Table FE3). Total gasoline production for the first quarter 1995 was 7.3 MMBD, up 5.9 percent over the 1994 first quarter production of 6.9 MMBD. RFG represented 25 percent of the first quarter's total gasoline production. The typical dip in spring production for refinery turnarounds did not occur in 1995 , possibly due to the recent refinery upgrades completed in preparation for RFG production. Last year, for example, total gasoline production averaged 6.7 MMBD in March, but this year, March production averaged 7.3 MMBD.

A stronger economy also generated demand growth in other petroleum products. As a result, total refinery utilization, measured as gross inputs divided by operable distillation capacity, grew from 91.4 percent in 1993 to 92.6 percent in 1994. (Operable capacity remained essentially the same during these two years.) As the 1995 gasoline season is about to begin, utilization was 89.4 percent in March, higher than last year's March utilization of 87.4 percent, and high utilizations are expected this summer to meet strong demand projections.

As a result of the RFG program, oxygenates will play a more important role this summer than previously. Prior to the RFG program, increased oxygenates were only required during the winter. RFG contains increased oxygenates throughout the year,

6Energy Information Administration, The Energy Information Administration's Assessment of Reformulated Gasoline, Volumes 1\&2, SR/OOG/94-02, Washington, DC, October 1994, and The Energy Information Administration's Assessment of Reformulated Gasoline: An Update, SR/OOG/94-03, Washington, DC, December 1994.

Table FE3. Total Gasoline Production

(Thousands of Barrels Per Day)

\begin{tabular}{|c|c|c|c|c|c|c|}
\hline Years & Quarter 1 & Quarter 2 & Quarter 3 & Quarter 4 & Annual & Annual Growth \\
\hline 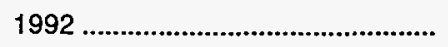 & 6,809 & 7,081 & 7,027 & 7,311 & 7,058 & $1.2 \%$ \\
\hline 1993 & 7,091 & 7,340 & 7,413 & 7,590 & 7,360 & $4.3 \%^{1}$ \\
\hline 1994 & 6,876 & 7,300 & 7,347 & 7,617 & 7,300 & $-0.8 \%$ \\
\hline 1995 & 7,279 & & & & & \\
\hline
\end{tabular}

\footnotetext{
${ }^{1}$ Field blended gasoline was not available until the end of 1992. Thus, the growth shown from 1992 to 1993 is slightly over-stated; however, refinery production alone grew 3.5 percent between 1992 and 1993.

Source: Petroleum Supply Monthly/Annual.
} 
so oxygenate supply is of interest this summer. Methyl tertiary butyl ether (MTBE), one of the primary oxygenates in RFG, is used in conventional gasoline, as well as RFG, to enhance octane levels. MTBE demand peaked during the winter when both oxygenated gasoline and RFG were required (see box on "Oxygenates"). The industry increased capacity and production in anticipation of the RFG program startup. However, methanol ${ }^{7}$ production plant problems caused concerns this past winter over adequacy of MTBE supplies, driving MTBE prices to record highs. Prices receded during the winter due to opt-outs, the end of the oxygenated gasoline season and resolution of methanol plant problems, but have recently strengthened. MTBE production levels are expected to be adequate to meet demand.

\section{Imports and Exports}

In 1994, high domestic demand and sufficient price differentials with Europe stimulated imports, which represented 5 percent of total gasoline supplied during the year, the highest level since 1990 (Figure FE2). However, as expected, in November and December, imports fell off as the RFG program began. During 1994, over 40 percent of U.S. gasoline imports came from the Caribbean region, while Canada provided 13 percent. Brazil and the United Kingdom provided less than 7 percent each. For the first quarter of 1995 , total gasoline imports have been modest, averaging $263 \mathrm{MBD}$ compared to $302 \mathrm{MBD}$ for the first quarter 1994. Through February, RFG represented 42 percent of the imports of which 31 percent came from Canada, and 34 percent each from both the Virgin Islands and from Venezuela. The first RFG imports from Europe were registered in February 1995.

Average total gasoline exports for 1994 were $103 \mathrm{MBD}$, about the same level as in 1993 and 1992 . Over 60 percent of the exports went to Mexico. It is possible that the RFG program may result

Figure FE2. U.S. Total Motor Gasoline Imports and Exports (Cents per Gallon)

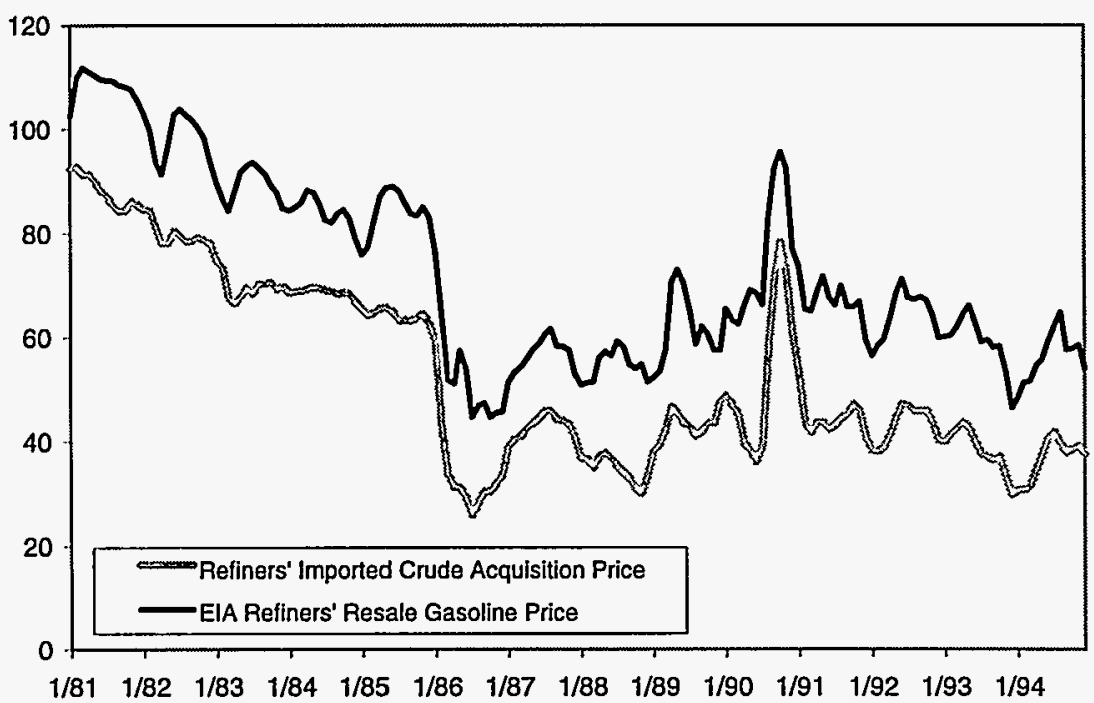

Source: Petroleum Supply Monthly/Annual. in increased exports as companies seek new markets for gasoline that does not comply with U.S. specifications. As the RFG program began in December 1994, exports jumped to 248 MBD compared to 171 MBD in December 1993. This marked the highest single month for gasoline exports since 1964, when export statistics were initiated. However, they retreated to an average of $103 \mathrm{MBD}$ in the first quarter of 1995 . It is still too early to tell if exports will increase substantially in 1995.

In spite of 1995's high gasoline demand, net imports for the first quarter declined from last year, averaging $160 \mathrm{MBD}$, compared to $208 \mathrm{MBD}$ in 1994 and $113 \mathrm{MBD}$ in 1993.

\section{Stocks}

Gasoline stocks at the primary level remained lower than average from March through October 1994 (see "Expectations for the Summer Driving Season," Figure FE7). In October, conventional stocks dropped as tanks were drawn down to make room for oxygenated and reformulated gasolines. By the end of November, finished gasoline stocks had recovered to average levels of 24 days of supply, while, as expected, RFG inventories covered only 19 days of supply. ${ }^{8}$ During December, oxygenated stocks dropped, while conventional and RFG increased slightly, but not enough to keep total stocks from returning to below average levels.

Normally, total gasoline stocks build in January as gasoline demand falls off and refinery production stays strong to produce seasonally higher distillate volumes for heating fuel. The typical seasonal build did not occur in early 1995 . Oxygenated gasoline stocks began to fall as expected, since the oxygenate season was nearing its end in many regions in February. Conventional stocks increased somewhat, but RFG stocks fell, as growing uncertainty over actual requirements in the face of mounting opt-out pressure coupled with weakening relative prices discouraged production.

Stock levels did not change much in February, but fell seasonally in March. The quarter ended with U.S. finished gasoline stocks at about 22 days of supply, compared to the 5-year average of 25 days of supply.

\section{Prices}

Motor gasoline prices in the spring of 1995 have averaged significantly higher than those a year ago, partly because of higher crude oil prices. As of April 21, spot prices for conventional unleaded regular gasoline at New York Harbor stood at 63.0 cents per gallon, 14.4 cents above the same date in

\footnotetext{
${ }^{7}$ Methanol is a key input to MTBE production. ${ }^{8}$ RFG stocks were expected to begin low, which is typical of new product introduction; however, once equilibrium was reached, RFG stock levels (on a days supply basis) were expected to be more typical of conventional stock levels.
} 


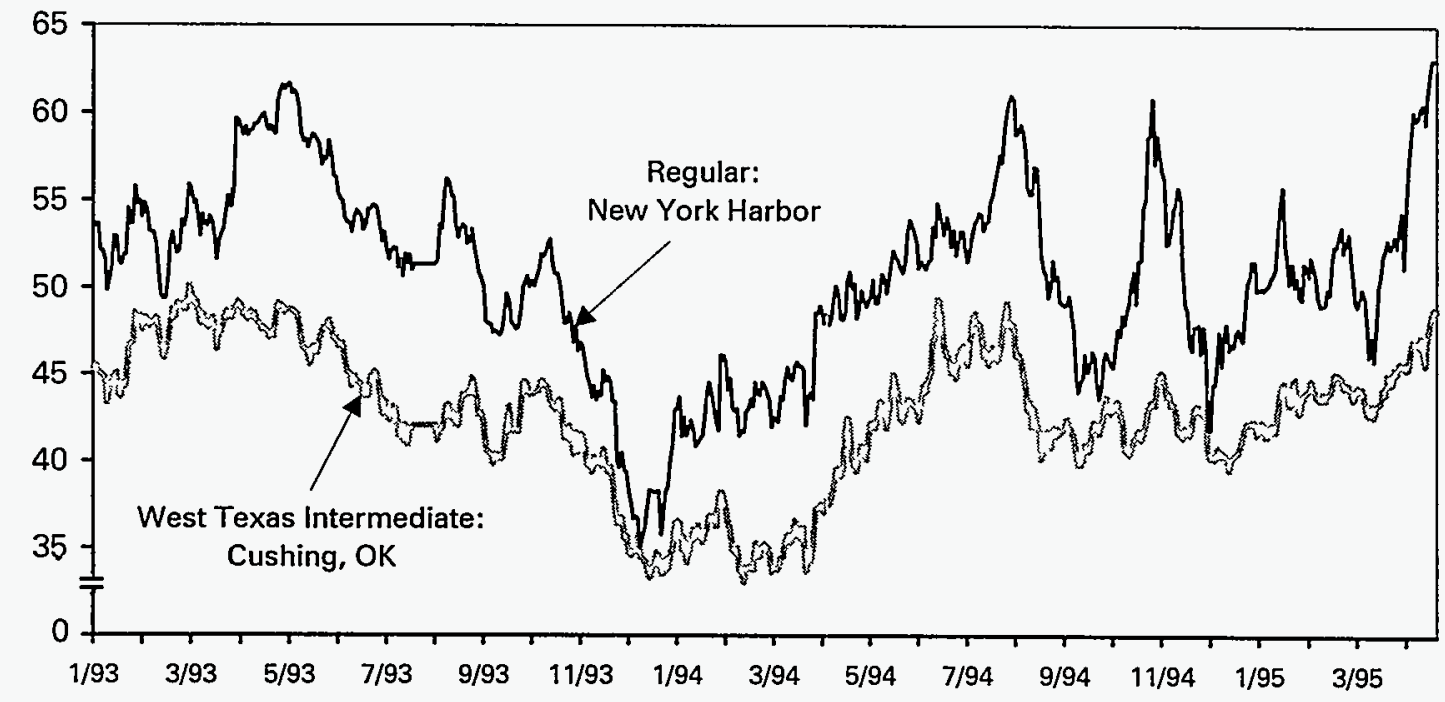

Source: Reuters Information Services.

1994 (Figure FE3). West Texas Intermediate (WTI) crude oil prices were up $\$ 2.90$ per barrel, or 6.9 cents per gallon, in the same period. U.S. average retail prices stood 8.4 cents above the same point in 1994.

On a year-to-year basis, except during unusual market situations, crude oil prices tend to be the most significant determinant of gasoline price levels. Pre-season (spring) gasoline prices were significantly lower in 1994 than the previous year, due to a sustained decline in crude oil prices during most of 1993. However, rising world oil demand and relatively stable production levels brought on a crude oil price recovery in the first half of 1994, only partially offset by a more moderate decline in late summer. Crude oil prices stabilized in the $\$ 17$ to $\$ 19$-per-barrel (WTI) range in the fall of 1994, and remained there until recently, when tightening spring gasoline markets pulled WTI prices above $\$ 20$ per barrel. Conventional gasoline prices generally followed those for crude oil in the fall and winter, although with seasonal influences and significant volatility due to unusual situations, including the introduction of RFG.

After a sharp decline in August and September 1994, wholesale gasoline prices were briefly but dramatically affected by the Colonial Pipeline rupture near Houston on October 20. Because the Colonial is the major supply route to the East Coast, New York Harbor prices jumped 9 cents in a week, only to fall back nearly as quickly once temporary repairs were accomplished. The introduction of RFG then reduced demand for conventional grades, briefly pulling down prices to near-parity with crude oil. Since December, conventional gasoline prices have maintained a more typical seasonal relationship to those for crude oil, with some fluctuation caused by demand uncertainties related to potential and actual opt-outs from the RFG and oxygenated gasoline programs.

While conventional gasoline prices are often measured against crude oil prices, those for oxygenated gasoline and, more recently,
RFG, are measured by their differential to conventional gasoline. Oxygenated gasoline, recently completing its third season, normally trades at a differential to conventional gasoline almost solely determined by the cost of the oxygenate needed. Higher oxygenate demand due to RFG and methanol supply problems (see "Oxygenates" sidebar) resulted in somewhat higher differentials during the past winter than in prior years. While RFG costs entail a number of other components relative to conventional gasoline cost, oxygenate cost is the most significant, similarly inflating RFG prices during its introduction. Since its introduction, the RFG price differential over conventional gasoline has been similar to that for oxygenated gasoline. As a result, after peaking in late January, RFG price differentials have declined during the spring along with oxygenate prices, to current levels of only 0 to 2 cents per gallon.

Retail gasoline price changes typically lag somewhat those on wholesale markets, resulting in less volatility and occasional differences in direction. After declining seasonally throughout the fall, U.S. average cash self-serve regular gasoline prices ended 1994 at 107.8 cents per gallon. Prices rose slightly around the introduction of RFG at the retail level in early January, then declined to an 8-month low of 106.7 cents per gallon in late February. Recently, prices have begun a seasonal rise toward the peak driving season, standing at 111.7 cents per gallon as of April 17, about 8 cents above the same point in 1994.

\section{Expectations for the Summer Driving Season}

As of the end of March, the United States had 22 days worth of finished gasoline stocks, compared to 23 days supply at the same time last year. As the summer driving season begins, demand is expected to surge, reaching 8.1 MMBD at the season's peak. However, supplies through the driving season appear adequate, based on stock levels, production capability and 


\section{Oxygenates}

Oxygenate additives are a key component in reformulated gasoline costs. MTBE, one of the primary oxygenates in RFG, is used in conventional gasoline, as well, to improve the octane rating. Therefore, MTBE demand was expected to reach new peaks in late 1994 due to RFG and oxygenated gasoline sales this winter. Spot MTBE prices had been climbing to over $\$ 1$ per gallon, double what they were the previous year, as shown in Figure FE4. Ethanol prices were also on the increase in the fall.

\section{Figure FE4. Oxygenate Prices} (Cents per Gallon)

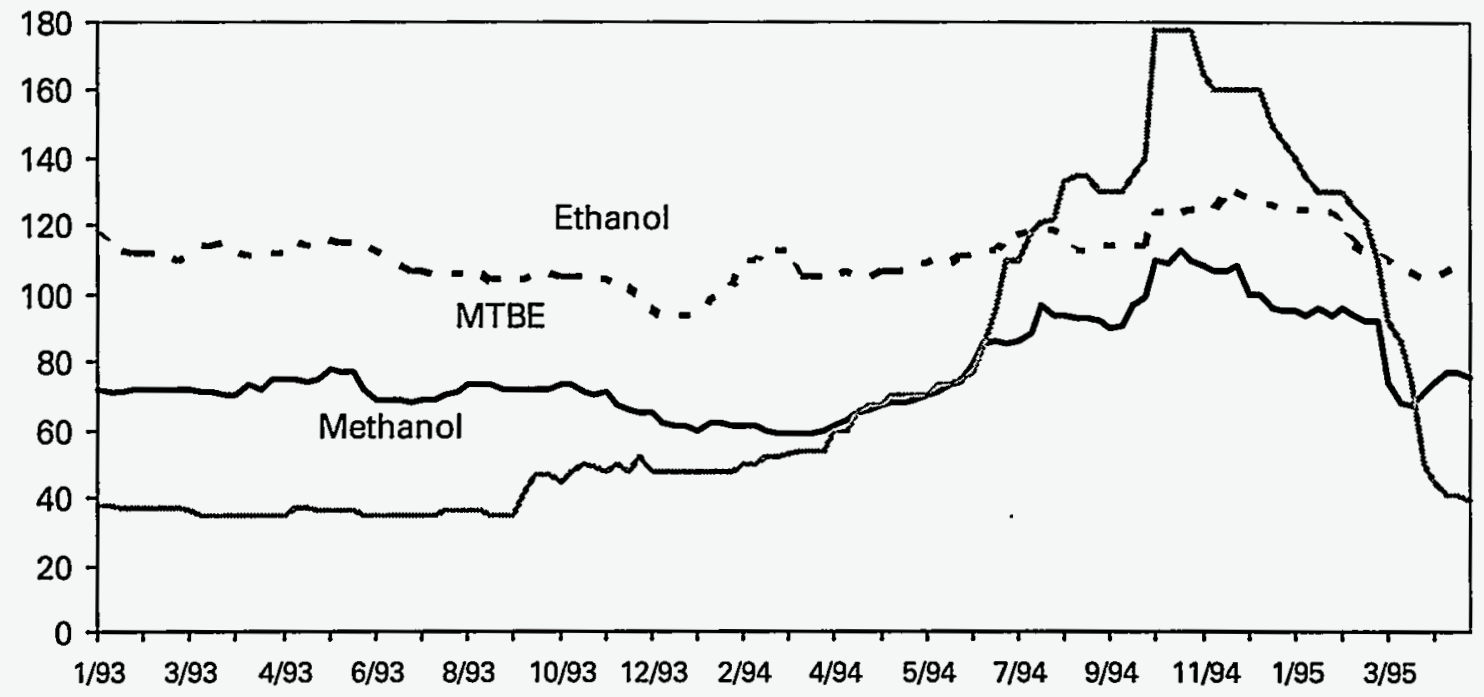

Source: Octane Week, various issues.

Methanol, a major feedstock for MTBE production, was in tight supply last summer and was driving the increases in MTBE prices. Methanol prices increased sharply in June, rising from about 75 cents per gallon to about $\$ 1: 20$ by the beginning of August, triple what they were the previous year. When the Channelview, Texas plant closed for maintenance in August, prices rose further to $\$ 1,35$. Prices then declined slightly; but subsequently shot up to $\$ 1.78$ following a fire in October at an Enron facility in Pasadena, Texas, accounting for about 8 percent of U.S. methanol supply. Prices began to ease in November, then plummeted starting in late December in reaction to falling MTBE demand due to opt-outs from the RFG program. MTBE followed the rise and fall of methanol prices, although less dramatically, but began to recover in late March as its blending value increased with rising gasoline prices.

The annual demand for MTBE-equivalent oxygenates was 320 MBD in 1993 and $327 \mathrm{MBD}$ in 1994, and will be an estimated 440 MBD in 1995. Ethanol provided about half the MTBE-equivalent oxygenate volume consumed in 1993 and 1994, but that percentage will fall to about 40 percent in 1995 (a possible exception is adoption of a renewable oxygenate standard - see next page).

The demand for MTBE alone is projected to rise from an annual average of $158 \mathrm{MBD}$ in 1994 to $255 \mathrm{MBD}$ in 1995 , the difference being attributable to RFG demand. Net imports of MTBE are projected to average $45 \mathrm{MBD}$ in 1995 , much higher than the $24 \mathrm{MBD}$ level of 1994.

Oxygenate stocking patterns are shown in Figure FE5. MTBE exhibits the strong seasonal pattern that has occurred since the first oxygenated gasoline season in the winter of 1992-1993. Stocks of MTBE were generally lower the next winter, as increased production capacity came on stream. MTBE stocks this past winter did not reach theír peaks of 2 years before, when uncertainties about potential demand and potential supply shortages abounded. MTBE stocks fell to 11 MMB at the end of March 1995, which combined with the $3 \mathrm{MMB}$ of ethanol stock assured an adequate supply of oxygenates for the foreseeable future. 
Figure FE5. U.S. Oxygenate Stocks

(Millions of Barrels)

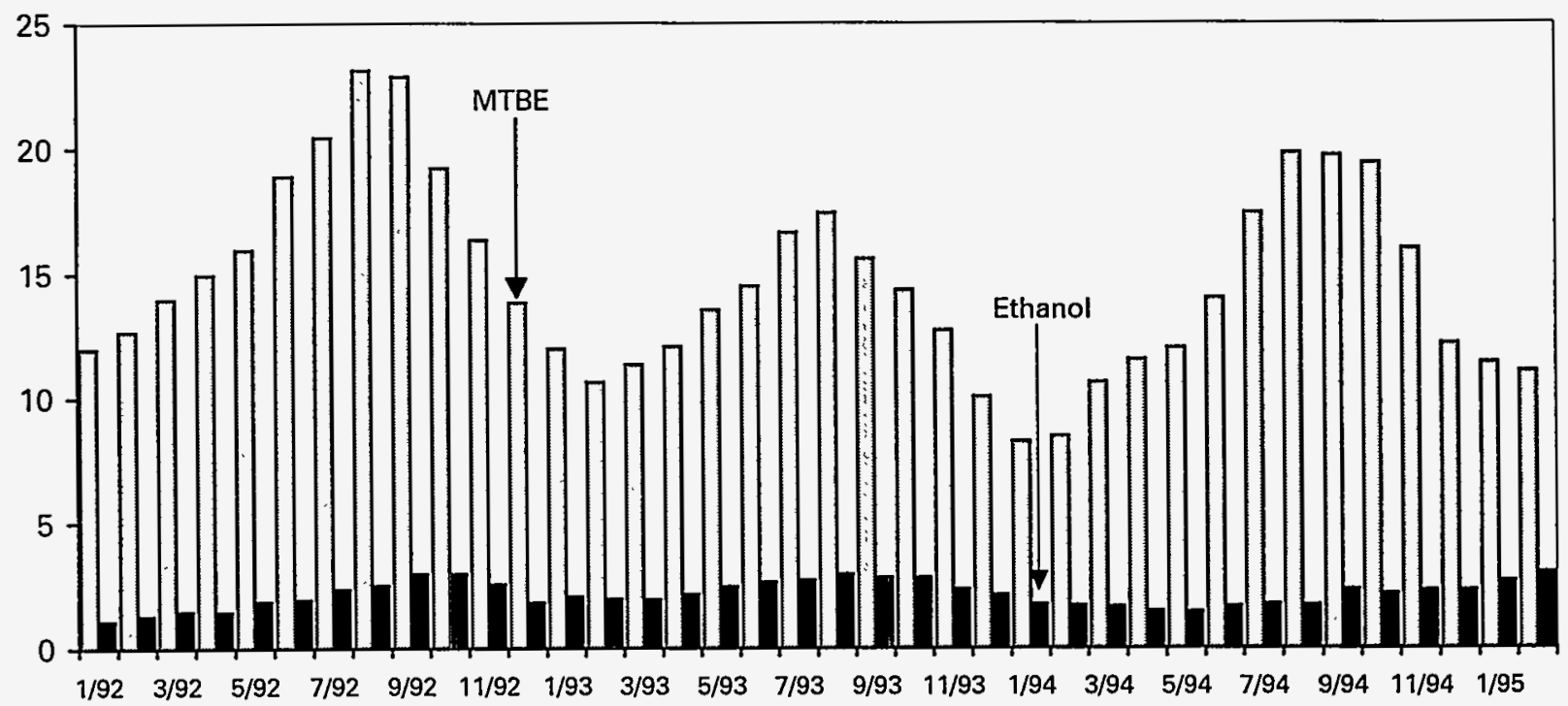

Source: Petroleum Supply Monthly/Annual.

\section{The Renewable Oxygenate Standard}

On June 30,1994, EPA issued the final rule requiring the use of renewable oxygenates in reformulated gasoline. The rule was to be implemented in two phases. The first phase was effective for all of 1995 and required that at least 15 percent of the oxygen content of reformulated gasoline come from renewable sources. The second phase required that, beginning in 1996, 30 percent of the oxygen in reformulated gasoline come from renewable sources. At present, ethanol and its derivatives are essentially the only. renewable oxygenates that are commercially available.

On July 13, 1994, a legal challenge to the renewable oxygenate standard (ROS) for reformulated gasoline was filed in the U.S, Court of Appeals for the District of Columbia. The challenge was filed jointly by the American Petroleum Institute and the National Petroleum Refiners Association. The groups argued that EPA exceeded its authority in issuing the ROS. Later, the American Methanol Institute and the Oxygenated Fuels. Association also joined the legal challenge.

On September 13, 1994, the U.S. Court of Appeals issued a delay in the implementation of ROS until the case was decided. On February 16, 1995, oral arguments were presented before the court's three-judge panel. The court ruled on April 28, 1995, that EPA lacked the authority to mandate the use of renewable oxygenates in motor gasoline.

prospective imports. According to the STEO forecasts, much of the increase in summer demand is to be met by growth in refinery production, an increase in oxygenate blending, and an uptick in net imports. Stock withdrawals during the summer driving season, averaging $20 \mathrm{MBD}$, will be less than half last year's value.

Overall gasoline prices are expected to reflect the typical summer pattern, rising on average by about 8 cents per gallon. RFG prices are anticipated to follow the same pattern.

\section{Demand}

Demand for 1995 is projected to continue the upward growth pattern that has been experienced for the past several years. This summer, demand is expected to be 1.8 percent higher than in summer 1994 (Figure FE6). Continuing economic growth is predicted to result in a 2.8 percent increase in total miles traveled, driving the increase in gasoline consumption. Total miles traveled is influenced by both number of vehicles and miles traveled per vehicle. In a growing economy, both of these factors frequently increase. Fuel efficiency over the summer months, as measured in miles per gallon, is expected to grow slightly compared to the summer of 1994. This increase moderates demand growth; but nevertheless, strong overall growth is anticipated.

Gasoline demand typically peaks in July or August. This year's forecast shows the peak in July, at 8.1 MMBD. Last year's peak month was August, at 8.0 MMBD.

RFG demand is projected to follow total gasoline demand patterns (Table FE4). For the summer season, RFG is forecast to represent 30 percent of total U.S. gasoline demand, 44 percent of demand in PADD I (East Coast) and 38 percent in PADD V (West Coast). 
Figure FE6. Gasoline Production and Demand

(Thousand Barrels per Day)

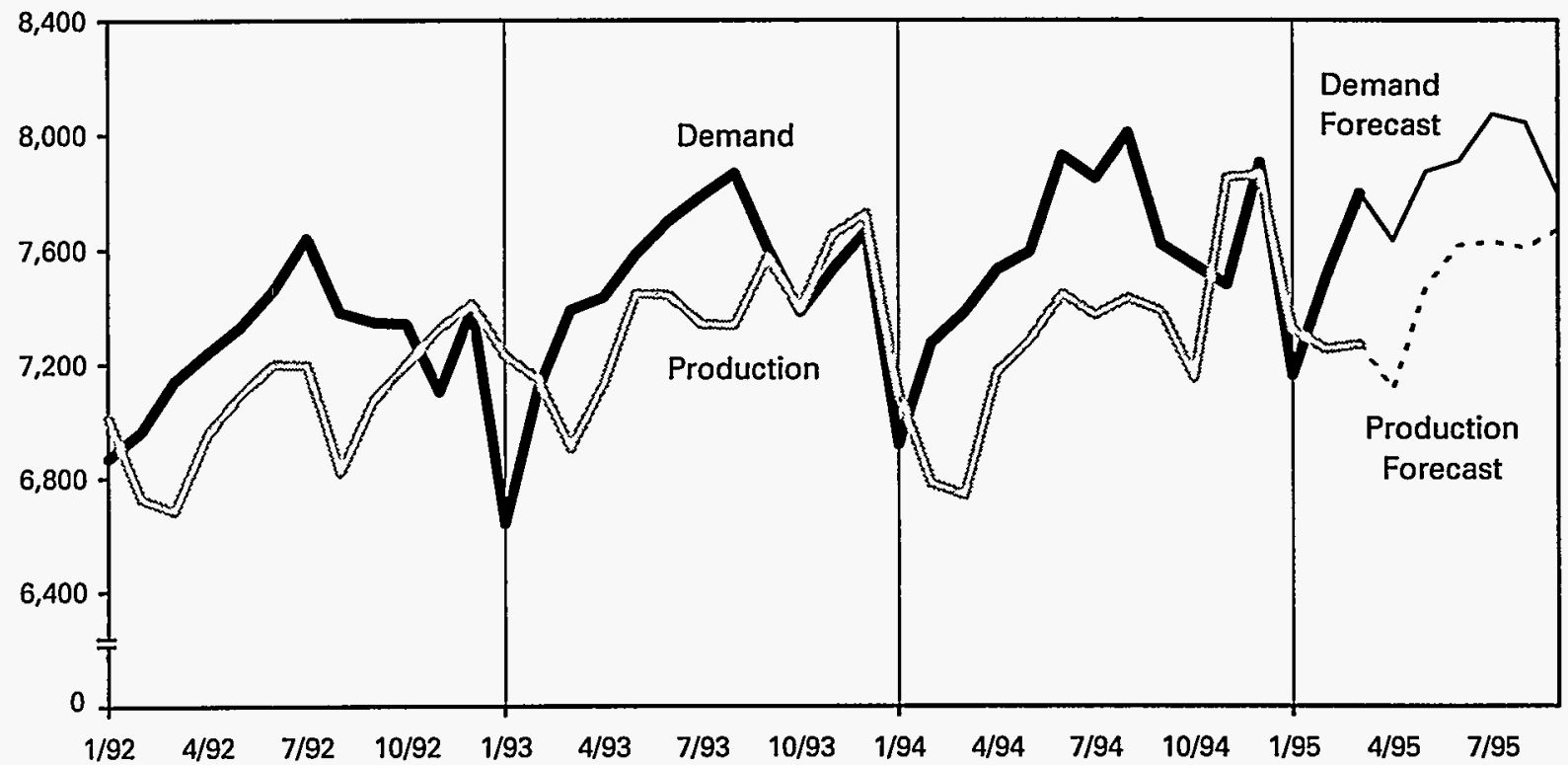

Source: Petroleum Supply Monthly, Petroleum Supply Annual, and Short-Term Energy Outlook.

At its peak, RFG demand is forecast to rise as high as $2.4 \mathrm{MMBD}$, about $200 \mathrm{MBD}$ higher than initial levels seen in mid-December, with higher production and import levels expected to satisfy the bulk of incremental requirements.

\section{Production}

Throughout the driving season, refinery gasoline production is expected to average 1.9 percent higher than last year (Figure FE6). Utilization of operable refinery capacity is expected to average
94.3 percent, slightly lower than last summer's utilization due to capacity additions and lower distillate production. As previously noted, the continued high production rates through March indicate few refinery turn-arounds through the first part of the spring, possibly due to modifications in advance of the start of the RFG program.

The RFG program mandates a minimum oxygen content during the summer for the first time, thereby making oxygenates an important supply source. The additional oxygenates decrease the

Table FE4. PAD District Total Demand

(Millions of Barrels per Day)

\begin{tabular}{|c|c|c|c|c|c|c|c|c|}
\hline Area & Type & April & May & June & July & August & September & $\begin{array}{l}\text { Driving } \\
\text { Season } \\
\text { Average }\end{array}$ \\
\hline $\begin{array}{l}\text { PAD District } 1 \\
\text { PAD District Total }\end{array}$ & $\begin{array}{l}\text { RFG } \\
\text { CONV. }\end{array}$ & $\begin{array}{l}1.189 \\
1.642 \\
2.732\end{array}$ & $\begin{array}{l}1.237 \\
1.552 \\
2.789\end{array}$ & $\begin{array}{l}1.255 \\
1.568 \\
2.822\end{array}$ & $\begin{array}{l}1.266 \\
1.589 \\
2.855\end{array}$ & $\begin{array}{l}1.271 \\
1.574 \\
2.845\end{array}$ & $\begin{array}{l}1.249 \\
1.562 \\
2.812\end{array}$ & $\begin{array}{l}1.245 \\
1.565 \\
2.809\end{array}$ \\
\hline $\begin{array}{l}\text { PAD District } 2 \\
\text { PAD District Total }\end{array}$ & $\begin{array}{l}\text { RFG } \\
\text { CONV. }\end{array}$ & $\begin{array}{l}0.347 \\
1.880 \\
2.228\end{array}$ & $\begin{array}{l}0.368 \\
1.990 \\
2.357\end{array}$ & $\begin{array}{l}0.367 \\
1.986 \\
2.353\end{array}$ & $\begin{array}{l}0.375 \\
2.032 \\
2.407\end{array}$ & $\begin{array}{l}0.374 \\
2.030 \\
2.404\end{array}$ & $\begin{array}{l}0.356 \\
1.924 \\
2.280\end{array}$ & $\begin{array}{l}0.385 \\
1.974 \\
2.339\end{array}$ \\
\hline $\begin{array}{l}\text { PAD District } 3 \\
\text { PAD District Total }\end{array}$ & $\begin{array}{l}\text { RFG } \\
\text { CONV. }\end{array}$ & $\begin{array}{l}0.267 \\
0.847 \\
1.114\end{array}$ & $\begin{array}{l}0.272 \\
0.865 \\
1.137\end{array}$ & $\begin{array}{l}0.266 \\
0.846 \\
1.112\end{array}$ & $\begin{array}{l}0.276 \\
0.876 \\
1.152\end{array}$ & $\begin{array}{l}0.266 \\
0.847 \\
1.113\end{array}$ & $\begin{array}{l}0.259 \\
0.824 \\
1.083\end{array}$ & $\begin{array}{l}0.268 \\
0.851 \\
1.119\end{array}$ \\
\hline $\begin{array}{l}\text { PAD District } 4 \\
\text { PAD District Total }\end{array}$ & $\begin{array}{l}\text { RFG } \\
\text { CONV. }\end{array}$ & $\begin{array}{l}0.000 \\
0.228 \\
0.228\end{array}$ & $\begin{array}{l}0.000 \\
0.244 \\
0.244\end{array}$ & $\begin{array}{l}0.000 \\
0.261 \\
0.261\end{array}$ & $\begin{array}{l}0.000 \\
0.272 \\
0.272\end{array}$ & $\begin{array}{l}0.000 \\
0.276 \\
0.276\end{array}$ & $\begin{array}{l}0.000 \\
0.257 \\
0.257\end{array}$ & $\begin{array}{l}0.000 \\
0.256 \\
0.256\end{array}$ \\
\hline $\begin{array}{l}\text { PAD District } 5 \\
\text { PAD District Total }\end{array}$ & $\begin{array}{l}\text { RFG } \\
\text { CONV. }\end{array}$ & $\begin{array}{l}0.521 \\
0.809 \\
1.331\end{array}$ & $\begin{array}{l}0.516 \\
0.825 \\
1.342\end{array}$ & $\begin{array}{l}0.523 \\
0.837 \\
1.360\end{array}$ & $\begin{array}{l}0.529 \\
0.857 \\
1.386\end{array}$ & $\begin{array}{l}0.537 \\
0.866 \\
1.403\end{array}$ & $\begin{array}{l}0.520 \\
0.839 \\
1.359\end{array}$ & $\begin{array}{l}0.524 \\
0.839 \\
1.363\end{array}$ \\
\hline US Total & $\begin{array}{l}\text { RFG } \\
\text { CONV. }\end{array}$ & $\begin{array}{l}2.325 \\
5.307 \\
7.632\end{array}$ & $\begin{array}{l}2.392 \\
5.477 \\
7.869\end{array}$ & $\begin{array}{l}2.410 \\
5.496 \\
7.906\end{array}$ & $\begin{array}{l}2.447 \\
5.625 \\
8.072\end{array}$ & $\begin{array}{l}2.449 \\
5.593 \\
8.042\end{array}$ & $\begin{array}{l}2.383 \\
5.407 \\
7.790\end{array}$ & $\begin{array}{l}2.401 \\
5.486 \\
7.887\end{array}$ \\
\hline
\end{tabular}

$\because$ Source: Short-Term Energy Outlook. 
need for crude oil inputs to refineries to meet gasoline demand. The reduction in crude input, in turn, can reduce refinery utilization (as measured at the distillation unit) below that which would occur producing only conventional gasoline. Countering this effect is the lower efficiency of RFG (which means more gallons of RFG are needed to travel the same number of miles), and the need to reduce butane content in gasoline in order for RFG to meet its low RVP specifications. The increased volume of oxygenates alone is estimated to reduce crude input by about 2 percent. However, the countering effects of energy efficiency and lower butane each increase the need for crude by about 0.5 percent. The net impact on crude runs this summer due to RFG might be to lower the utilization close to 1 percent from that which would have been required producing conventional gasoline alone.

\section{Imports and Exports}

Strong domestic gasoline demand is anticipated to result in net import levels similar to last summer's, about 370 MBD. Imports from the Virgin Islands, Canada, and Venezuela are expected to dominate. Because imports of gasoline flow mainly to the Northeast, a major RFG area, much of the summer imports will be in the form of RFG or reformulated blendstocks for oxygenate blending (RBOB). Based on the assumption that low RFG stock levels will prevent drawing down RFG stocks significantly, the gap between demand and production of RFG will have to be filled with imports. With RFG demand forecast to average $2.4 \mathrm{MMBD}$, and production running no higher than 2.2 MMBD (the highest weekly average seen to date), imports of RFG might run 200-300 MBD.

Gasoline exports are estimated to continue through the summer at an average level of $127 \mathrm{MBD}$, compared to $74 \mathrm{MBD}$ on average during the 1994 driving season. Refiners are apt to sell conventional gasoline that does not meet U.S. standards, but is valuable in international markets.

\section{Stocks}

Total gasoline stocks (not including oxygenates) are beginning the 1995 summer driving season lower than last year at $212 \mathrm{MMB}$ versus $214 \mathrm{MMB}$ in 1994 (Figure FE7). Although production is forecast to be higher than in 1994 and net imports are expected to be about the same, strong demand will keep stock levels lower than last year for the first half of the summer. In 1995, stock levels are not forecast to decline as fast as last year's stocks during the summer due to better wholesale margins in 1995 keeping production up. Thus, 1995 stocks eventually exceed 1994 stocks in the second half of the summer. Throughout the summer, though, stocks hover around the lower bound of the historical average range. The lower stocks in 1995 are partially due to the RFG stocks, which are expected to remain about $45 \mathrm{MMB}$ throughout the summer, consistent with their pattern to date. RFG stocks are not forecast separately, but if they average about 20 days of supply, which is about where RFG is starting the summer, stocks might approach $50 \mathrm{MMB}$ prior to RFG's peak demand of 2.4 MMBD. However, stocks generally don't increase during the summer months. Thus, we might expect RFG stocks to fluctuate around $45 \mathrm{MMB}$ this summer.

Finished gasoline stocks begin the season at 22 days of supply in March, drop to 21 days during July, but recover to 22 days in August as demand falls off and stocks begin rebuilding. Last year, finished gasoline stocks averaged about 1 day supply higher than the 1995 forecast.

\section{Prices}

Excluding unusual changes in crude oil prices or gasoline supply disruptions, gasoline prices normally exhibit a demand-driven seasonal pattern, rising in the spring to a peak in the June-August period, and declining through the fall to a low between December and February. Crude oil prices seldom change this overall pattern,

\section{Figure FE7. Total Gasoline Stocks (Million Barrels)}

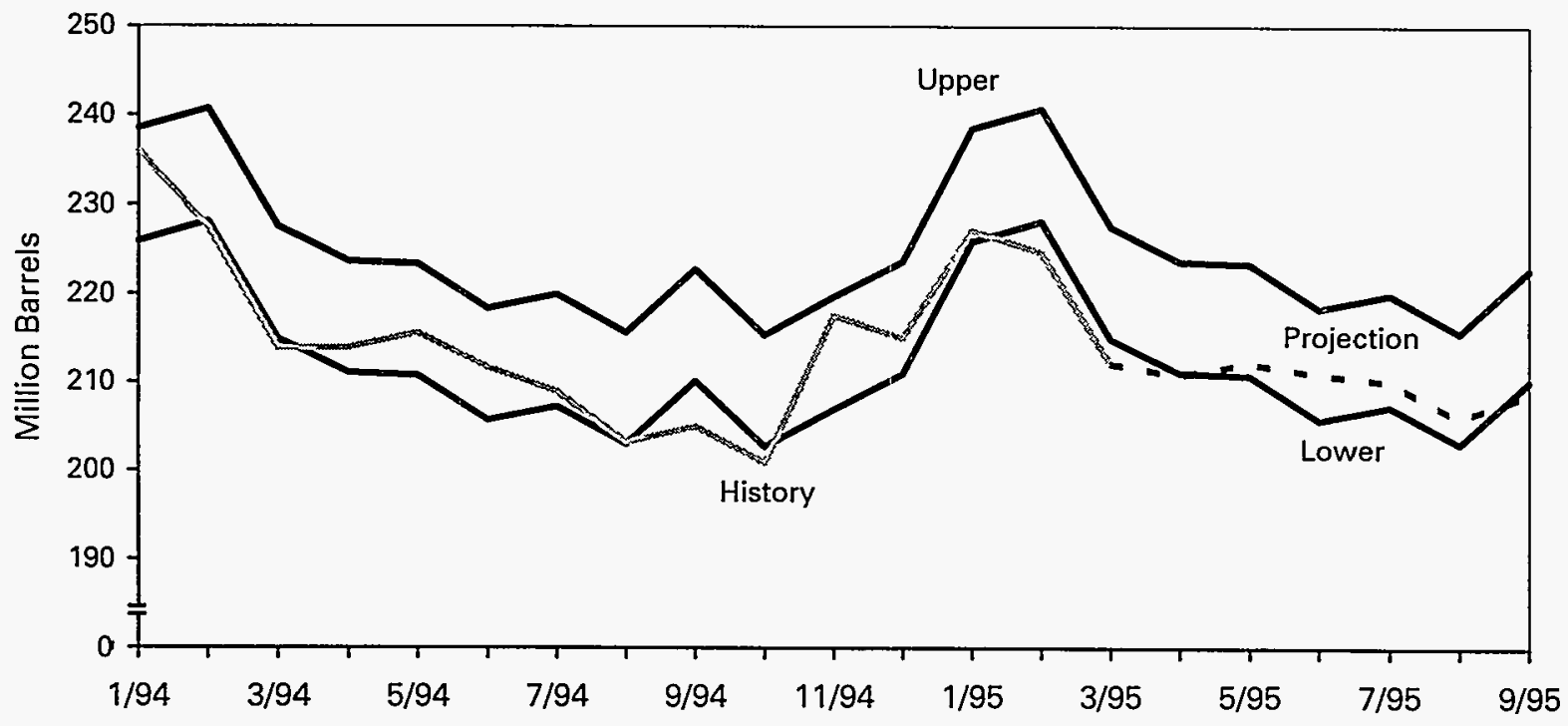

Source: Petroleum Supply Monthly and Short-Term Energy Outlook. 
but often impact the magnitude of seasonal change. STEO estimates that the average world crude oil price will remain relatively flat through the second and third quarters of 1995, at about $\$ 16.75$ per barrel, rising slightly in the fourth quarter to $\$ 17.00$. This forecast assumes stable to slightly rising world crude oil production offsetting similar increases in demand, as OPEC and other sources compensate for declining output in the United States and the former Soviet Union. Therefore, gasoline prices are expected to be primarily dependent on product-specific factors, i.e. the gasoline supply/demand balance, throughout the summer driving season. This forecast, of course, does not attempt to anticipate crude oil or petroleum product market disruptions, international political developments, or any of the other unforeseeable events that often are significant price determinants in petroleum markets.

Based on expectations for world crude oil prices and the various supply and demand factors outlined above, STEO forecasts gasoline prices to follow a fairly typical seasonal pattern this summer. Unlike 1994 , which saw an unusually sharp price rise due to tightening of world crude oil markets, wholesale gasoline prices are expected to rise about 8 cents on average over the 3-month period ending June 1995. Retail prices, exhibiting a typical lag relationship to wholesale, are expected to increase more gradually, but over a longer period, gaining about 8 cents on average from their winter low through a late-summer peak. Prices at both levels are then expected to gradually decline through the remainder of the year.

\section{Uncertainties and Sensitivities}

The main concerns for this summer are centered around high demand, high refinery utilization, low stocks and RFG's first summer driving season. The high utilization and low stocks affect marginal costs to provide more gasoline, and thus influence the mix of production and imports. RFG adds to uncertainties surrounding imports and forces oxygenates into the picture as an important supply component for the summer months. In addition, high utilization, low stocks, and delivery of the new RFG reduce the flexibility of the system. Prices will be influenced by how these factors come together this summer. The Mid World Oil Price Case (Mid Case) assumes gasoline spreads (gasoline price over crude price) are typical of spreads seen in 1992 and 1993. These spreads (and therefore price) may vary from this assumption due to variation in the factors discussed below.

In the STEO Mid Case described above, demand is high, requiring high refinery utilizations. As demand increases over the summer season, three sources of supply are available to meet this increase: domestic production, withdrawals from stocks, and imports. The mix of these sources of supply affects prices. In the past, when utilizations were lower, increases in demand over the summer season were met mainly by U.S. refiners increasing their throughput and by stockdraws. This year's high utilizations entering the summer season present a different economic situation for refiners. At high utilizations, it is more expensive to produce additional gasoline than at lower utilizations. Thus, even if gasoline prices are rising (with crude price fixed), costs to produce additional gasoline will also be rising. As a result, refiners who increase production may not gain much increase in their margins in spite of the price increase. This situation tends to put upward pressure on gasoline prices.

In addition, refiners co-produce distillate with gasoline. During the summer, distillate prices are usually weak, and if too many refiners produce more distillate than is needed, prices will weaken even more. Thus, refiners must consider the tradeoffs among increasing gasoline prices, rising marginal costs to produce gasoline, and potentially having to "dump" distillate co-produced with gasoline in the face of deteriorating distillate margins.

At the same time U.S. refiners are making their decisions, foreign refiners are watching U.S. prices. Foreign refiners may be operating at lower utilizations and thus have lower marginal costs than U.S. refiners, and they may have better markets close at hand for their distillate. (Europe uses a much larger percent of distillate than does the United States.) Depending on gasoline prices in their own markets relative to the United States, foreign refiners may find it attractive to bring product to the United States. Under these circumstances, prices would tend to hold steady or experience downward pressure. That is, at some point, the supply-demand balance and price situation can become more attractive for importers than for domestic refiners to provide the additional gasoline to the United States. The relationship between imports and domestic production is dynamic, being driven by marginal costs and price differentials between world markets, which are not static. A large degree of uncertainty exists around the ultimate mix that will occur between imports and domestic production.

On the other hand, if foreign markets are tight this summer and foreign gasoline markets are more attractive to foreign producers than the U.S. market, U.S. gasoline stocks may move lower than those shown in the base case, driving prices higher before additional domestic production or imports pick up the slack.

While the uncertainties discussed above would tend to push prices higher over the summer, depressed light-heavy crude price differences are keeping prices down. For the past few years, the Atlantic basin has experienced a glut of light crudes (crudes that produce high gasoline yields). Mainly as a result of the light-crude surplus, the price difference between the light and heavy crudes is smaller than normal. This results in refiners being able to produce increases in gasoline at lower cost than when premium crude prices are high. Since all refiners can benefit in varying degrees from the low light crude prices, the low differential tends to keep all gasoline prices down.

In the event that production and imports are not as high as indicated in the Mid Case, stocks will be drawn down to lower levels than those shown in Figure FE7, putting upward pressure on prices. This might occur if prices are not attractive enough initially to draw imports at the forecast levels. For example, if production plus imports fall $200 \mathrm{MBD}$ lower in May than predicted, stocks will also fall, pushing prices about 1 to 2 cents 
per gallon higher than the Mid Case on average over the summer driving season. However, if these low stocks drop below local working levels, prices will go even higher. Note that higher prices, in turn, provide incentive for increases in both production and imports.

If, instead, gasoline spreads over crude are similar to those experienced in 1994, prices will be lower than in the STEO forecast. In 1994, the average spread of gasoline price over crude during the summer was about 3 cents per gallon lower than forecast in the Mid Case. Assuming some of this difference in spread is due to increased costs to produce RFG in 1995, retail prices under this scenario may only average $\$ 1.22$ per gallon this summer.

RFG complicates this picture even more. As described earlier, a large portion of gasoline imports are expected to be RFG. The highest monthly imports of RFG received to date were 125 MBD in December. The import balances shown in the Mid Case imply RFG imports might go as high as 200-300 MBD. Where the additional imports are likely to come from and the price needed to attract them is not known at this time.

Finally, refiners and marketers are dealing with regulatory uncertainties on top of market uncertainties this summer. The largest uncertainty is for RFG demand. Will other areas opt out and when? Several factors are affecting areas' decisions to opt out. RFG is more expensive than conventional fuel, and has slightly lower fuel efficiency. In addition, health concerns over MTBE have been raised. The uncertainty in RFG demand will tend to keep RFG stocks low, since no company wants to have this expensive product caught in an area that opts out, potentially having to sell it at a loss as prices move down to conventional gasoline levels following the opt-out.

While no problems are foreseen this summer, the gasoline markets are fraught with uncertainty. While prices to consumers will be higher than last year due to higher costs to produce gasoline, margins for refiners may not be much better than in 1994. 


\section{Highlights}

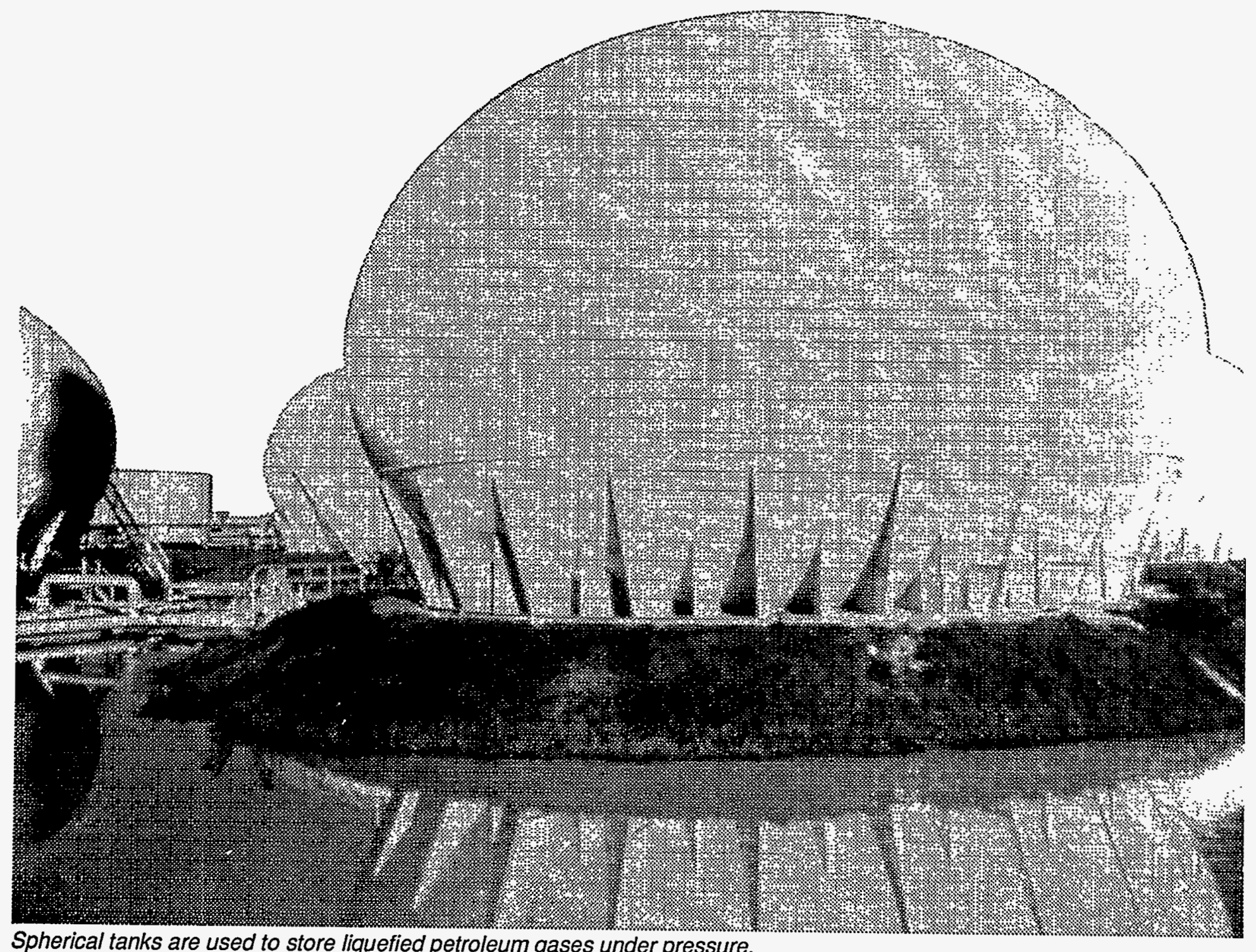

Spherical tanks are used to store liquefied petroleum gases under pressure.

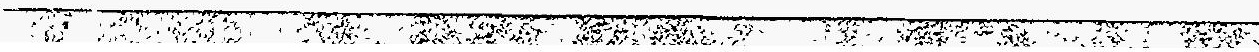





\section{Highlights}

Despite the economy's deceleration, total demand for refined petroleum products (measured as products supplied) for April $1995^{1}$ averaged a healthy 17.4 million barrels per day (Table H1), slightly lower than the April 1994 level but 3 percent above the April 1993 level. This demand level was sustained by record-high April finished motor gasoline and distillate demand volumes. Slowing economic growth ${ }^{2}$ and cooler-than-normal spring weather had little effect on demand levels this month. April 1995 temperatures in the U.S. were 8 percent cooler than normal and 24 percent cooler than last year.

Other April 1995 highlights include:

- The United States Environmental Protection Agency (EPA) withdrew three Wisconsin counties--Kewaunee, Manitowoc, and Sheboygan--from the federal reformulated gasoline (RFG) program citing the receipt of adverse public comments to their proposed rulemaking and a letter from Governor Tommy G. Thompson. Prior to the withdrawal, these counties were expected to require year-round RFG beginning June $1,1995^{3}$

- On April 28, a three-judge appellate panel for the District of Columbia decreed that the EPA did not have the authority to mandate the use of renewable oxygenates in RFG manufacturing. Ethanol, a renewable oxygenate, would have benefitted most if the renewable oxygenate for RFG standard were upheld. Despite the ruling, ethanol marketers remained optimistic about future market share growth. ${ }^{4}$

- Retail prices of finished motor gasoline averaged 6.3 cents per gallon more in April 1995 than in April 1994. Higher crude oil prices, more stringent fuel formulation regulations, unplanned refinery outages, and reduced imports have helped to increase motor gasoline prices. 5

- Crude oil imports climbed to a record high for April; domestic crude oil production continued to decline.

- Refiners in the Midwest are hopeful that proposed crude oil pipeline capacity additions and flow-direction reversals will alleviate the supply problems experienced in past summers.

\section{Motor Gasoline}

Finished motor gasoline demand averaged 7.7 million barrels per day, an all-time April high. Production of finished motor gasoline averaged 7.5 million barrels per day, the highest April level ever. At 0.3 million barrels per day, imports of finished motor gasoline were below the normal range for this time of year. Stock levels dropped to 167 million barrels, well below the normal seasonal range and an indication that inventory management at refineries and bulk terminals is critical. Reformulated gasoline (RFG) production picked up in April, averaging 1.9 million barrels per day. RFG stocks remained relatively flat at 41 million barrels, approximately 21 days supply.

As mentioned above, retail prices of finished motor gasoline have increased steadily in recent months. Industry analysts point to several factors affecting the motor gasoline market: increased crude oil prices, refiners' difficulties meeting the increasing, ever-changing types of gasoline specifications; planned refinery maintenance and unplanned refinery outages; increased consumer demand; and reduced ability to meet incremental demand increases with imports due to the stricter product specifications of the Clean Air Act Amendments of 1990.

Refiners are required to produce several different grades and types of motor gasolines to meet a variety of seasonal and regional fuel specifications, making it difficult to manage output volumes and inventory levels. For example, the RFG oxygen content used in the New York metropolitan area is different within the area. New Jersey opted to end its winter oxygenated fuel season on February 28 , reverting to the 2.0 weight percent oxygen content in RFG; the rest of the area must use oxygenated program RFG with 2.7 weight percent oxygen through April 30. In addition, May 1 marks the start of the low-RVP season at the wholesale level. ${ }^{6}$ A detailed description of the motor gasoline varieties being marketed today and a map displaying where and when they are required is at the end of this section.

\section{Distillate Fuel Oil}

Distillate fuel oil demand reached 3.3 million barrels per day, the highest April level ever. Production of distillate fuel oil was strong as well, averaging 3.2 million barrels per day. Distillate fuel oil imports of 0.1 million barrels per day were slightly below the normal range for this time of year. At 114 million barrels, inventories of distillate fuel oil were the highest April level since 1981. Low-sulfur distillate fuel oil stocks totaled 61 million barrels, nearly 54 percent of the total.

\footnotetext{
'April 1995 data are monthly-from-weekly estimates based on the Energy Information Administration's Weekly Petroleum Supply Reporting System.

"Economy's Pace Slowing Sharply," The Washington Post, April 19, 1995, pp. A1 and A20.

$3^{3}$ "Regulation of Fuels and Fuel Additives: Standards for Reformulated and Conventional Gasoline Withdrawal of Reformulated Gasoline Program Extension in Wisconsin," Federal Register, May 3, 1995, pp. 21724-21725.

4 "Court Shoots Down Ethanol Mandate, Says EPA Overstepped Its Authority," The Oil Daily, May 1, 1995, pp. 1 and 6.

"Summer Gasoline, Refinery Outages Send Prices Soaring," Octane Week, April 17, 1995, pp. 1 and 10.

"Market Chaos Tightens Gasoline Supplies, Sends Prices Up 3 Times Faster Than Crude," The Oil Daily, April 21, 1995, pp. 1 and 7.
} 


\begin{tabular}{|c|c|c|c|c|c|c|}
\hline \multirow{2}{*}{ Category } & \multicolumn{3}{|c|}{1995} & \multirow{2}{*}{1994} & \multicolumn{2}{|c|}{ January - April } \\
\hline & $\begin{array}{l}\text { Estimated } \\
\text { April }\end{array}$ & March & Difference ${ }^{a}$ & & 1995 & 1994 \\
\hline 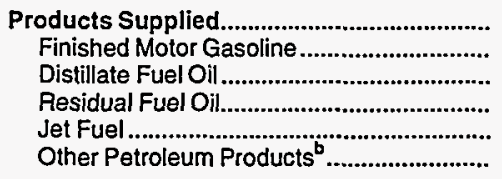 & $\begin{array}{r}17.4 \\
7.7 \\
3.3 \\
0.8 \\
1.4 \\
4.2\end{array}$ & $\begin{array}{r}17.4 \\
7.8 \\
3.3 \\
0.8 \\
1.5 \\
4.0\end{array}$ & $\begin{array}{r}(s) \\
(s) \\
(s) \\
(s) \\
-0.1 \\
0.2\end{array}$ & $\begin{array}{r}17.4 \\
7.5 \\
3.1 \\
1.1 \\
1.5 \\
4.2\end{array}$ & $\begin{array}{r}17.6 \\
7.5 \\
3.4 \\
0.9 \\
1.5 \\
4.3\end{array}$ & $\begin{array}{r}17.7 \\
7.3 \\
3.4 \\
1.2 \\
1.5 \\
4.3\end{array}$ \\
\hline Crude Oil Inputs........................................... & 13.9 & 13.5 & 0.5 & 13.8 & 13.6 & 13.3 \\
\hline Operating Utilization Rate (\%)...................... & 93.4 & 89.0 & 4.4 & 97.5 & 90.9 & 92.6 \\
\hline 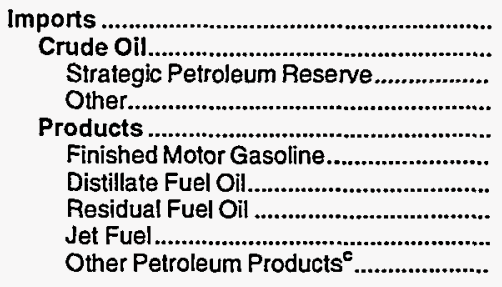 & $\begin{array}{l}8.5 \\
7.2 \\
0.0 \\
7.2 \\
1.3 \\
0.3 \\
0.1 \\
0.1 \\
0.1 \\
0.7\end{array}$ & $\begin{array}{l}9.0 \\
7.4 \\
0.0 \\
7.4 \\
1.6 \\
0.3 \\
0.2 \\
0.2 \\
0.1 \\
0.8\end{array}$ & $\begin{array}{r}-0.6 \\
-0.2 \\
0.0 \\
-0.2 \\
-0.3 \\
-0.1 \\
-0.1 \\
-0.1 \\
(s) \\
-0.1\end{array}$ & $\begin{array}{l}8.9 \\
6.9 \\
(s) \\
6.9 \\
2.0 \\
0.5 \\
0.2 \\
0.3 \\
0.1 \\
0.9\end{array}$ & $\begin{array}{l}8.4 \\
6.9 \\
0.0 \\
6.9 \\
1.5 \\
0.2 \\
0.2 \\
0.2 \\
0.1 \\
0.8\end{array}$ & $\begin{array}{l}8.5 \\
6.4 \\
\text { (s) } \\
6.4 \\
2.1 \\
0.3 \\
0.2 \\
0.4 \\
0.1 \\
0.9\end{array}$ \\
\hline 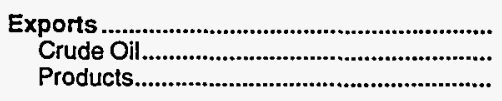 & $\begin{array}{l}1.0 \\
0.1 \\
0.9\end{array}$ & $\begin{array}{l}0.9 \\
0.1 \\
0.9\end{array}$ & $\begin{array}{l}\text { (s) } \\
(s) \\
(s)\end{array}$ & $\begin{array}{l}0.9 \\
0.1 \\
0.7\end{array}$ & $\begin{array}{l}1.0 \\
0.1 \\
0.9\end{array}$ & $\begin{array}{l}0.9 \\
0.1 \\
0.8\end{array}$ \\
\hline 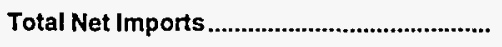 & 7.5 & 8.1 & -0.6 & 8.1 & 7.5 & 7.6 \\
\hline 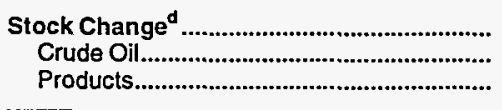 & $\begin{array}{l}-0.3 \\
-0.1 \\
-0.2\end{array}$ & $\begin{array}{r}-0.1 \\
0.3 \\
-0.5\end{array}$ & $\begin{array}{r}-0.1 \\
-0.4 \\
0.3\end{array}$ & $\begin{array}{r}0.3 \\
-0.1 \\
0.3\end{array}$ & $\begin{array}{r}-0.5 \\
(5) \\
-0.5\end{array}$ & $\begin{array}{r}-0.5 \\
(s) \\
-0.5\end{array}$ \\
\hline 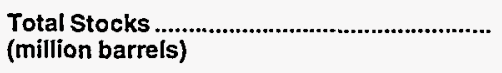 & 1,598 & 1,599 & -1 & 1,585 & - & - \\
\hline 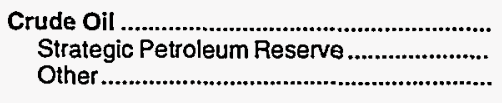 & $\begin{array}{l}926 \\
592 \\
335\end{array}$ & $\begin{array}{l}929 \\
592 \\
338\end{array}$ & $\begin{array}{r}-3 \\
0 \\
-3\end{array}$ & $\begin{array}{l}926 \\
591 \\
335\end{array}$ & $\overline{-}$ & - \\
\hline 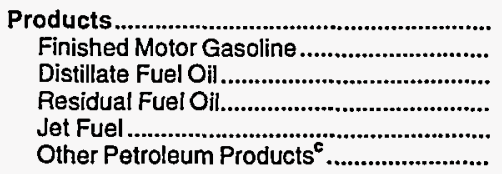 & $\begin{array}{r}672 \\
167 \\
114 \\
36 \\
38 \\
317\end{array}$ & $\begin{array}{r}669 \\
168 \\
115 \\
38 \\
39 \\
309\end{array}$ & $\begin{array}{r}2 \\
-1 \\
-2 \\
-2 \\
-1 \\
7\end{array}$ & $\begin{array}{r}659 \\
177 \\
103 \\
39 \\
38 \\
302\end{array}$ & $\begin{array}{l}- \\
\overline{-} \\
\overline{-} \\
-\end{array}$ & 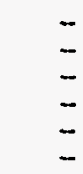 \\
\hline
\end{tabular}

Difference is equal to volume for current month minus volume for previous month.

b Includes crude oil product supplied, natural gas liquids, liquefied refinery gases (LRG's), other liquids, and all finished petroleum products except finished motor gasoline, distillate fuel oil, residual fuel oil, and jet fuel.

c Includes natural gas liquids, liquefied refinery gases (LRG's), other liquids, and all finished petroleum products except motor gasoline, jet fuel, distillate fuel oil, and residual fuel oil.

A negative number indicates a decrease in stocks and a positive number indicates an increase.

$(s)=$ Less than 0.05 million barrels per day, or less than 0.05 percent, or less than 0.5 million barrels.

E=Estimated.

Note: Totals may not equal sum of components due to independent rounding.

Source: Energy Information Administration (EIA), 1993, Petroleum Supply Annual, Volume II; appropriate issues of the Petroleum Supply Monthly and the Weekly Petroleum Status Report

Data for the current month are preliminary estimates, based on weekly submissions. For an explanation of estimation methodology and accuracy, see Appendix A of Weekly Petroleum Status Report and the article, "Accuracy of Petroleum Supply Data", published in the October 1994, Petroleum Supply Monthly. 
Table H2. U.S. Refinery Inputs, Capacities and Utilization Rates: 1993-1994

(Thousand Barrels per Day, Except Where Noted)

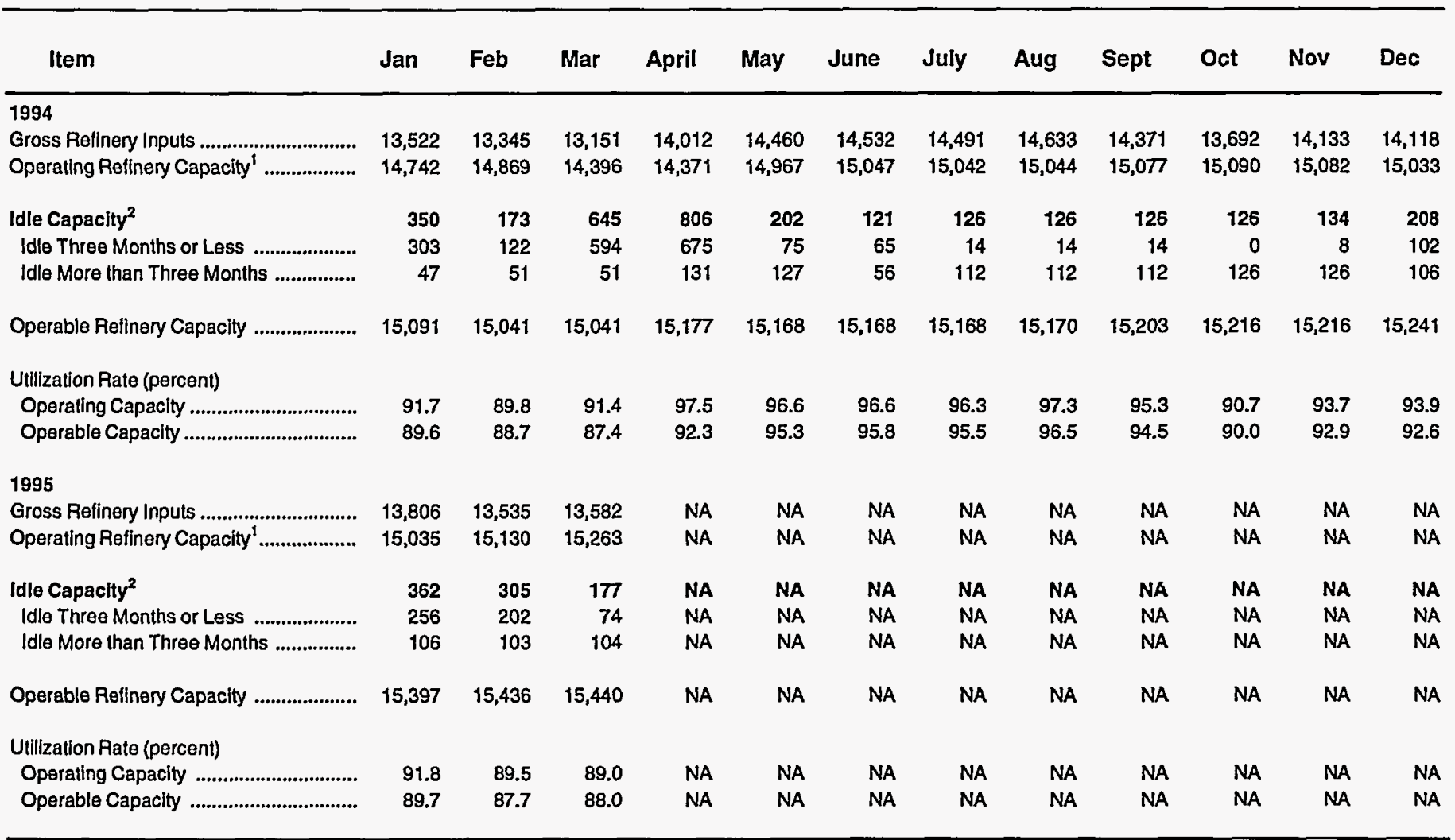

\footnotetext{
'Operating capacity equals the operable capacity less the total idle capacity.

2 Idle capacity is the component of operable capacity that is not in operation and not under active repair, but is capable of being placed in operation within 30 days; and capacity not in operation but is under active repair that can be completed within 90 days.

NA $=$ Not Available

Note: Totals may not equal sum of components due to independent rounding.

Sources: Energy Information Administration (EIA), 1993, Petroleum Supply Annual, Volume II, Table 16; ElA, Petroleum Supply Monthly, 1994 data issue, Table 28.
}

The expected price differential between sulfur grades of distillate fuel oil is finally beginning to appear as low-sulfur prices have increased, allowing refiners to realize returns on their significant capital investments in process improvements and unit upgrades. Demand for low-sulfur fuel oil has put upward pressure on prices and supply has tightened. These actions are significant because U.S. refiners have the capacity to make 70 percent of their output low-sulfur, while only $45-50$ percent is needed to satisfy federal requirements. However, some industry analysts expect this trend to last only for the spring, stating that the price differential will narrow again soon. ${ }^{7}$

\section{Residual Fuel Oil}

As petroleum products continued to be displaced by low-priced competing fuels at electric power plants, demand for residual fuel oil dropped to 0.8 million barrels per day, the lowest April level in decades. Production of residual fuel oil averaged 0.8 million barrels per day, the lowest April level since 1972. Stock levels settled at 36 million barrels, the lowest April level since 1987.

\section{Kerosene-type Jet Fuel}

Buoyed by increased international air cargo traffic ${ }^{8}$, demand for kerosene-type jet fuel averaged 1.4 million barrels per day, within the normal range for April. Kerosene-type jet fuel production also fell within the normal seasonal range at 1.3 million barrels per day. Month-ending stock levels were slightly higher than last year, but were within the normal range.

\section{Propane}

Stockholders added nearly 5.3 million barrels of propane to primary storage during April 1995, a level that is roughly comparable with April stockbuilds in recent years. However, at an estimated 30.4 million barrels, U.S. inventories were at their second lowest April level in a quarter century. Regionally, East Coast inventories were relatively unchanged at 2.2 million barrels, while Midwest and Gulf Coast inventories increased by 1.9 million barrels and 3.3 million barrels, respectively. Inventories remain at the normal level for the Gulf Coust and Midwest regions while in the East Coast stocks were slightly below their normal level for this time of year.

\footnotetext{
7"Demand Helps Low-Sulfur No. 2 Diesel Price Outstrip Sagging High-Sulfur Heating Oil," The Oil Daily, April 26, 1995 , pp. 1 and 4.

"Strong Exports Drive Air Cargo Growth," Journal of Commerce, April 12, 1995, pp. 1 A and 8A.
} 


\section{Crude Oil}

Domestic crude oil production continued to decline, sinking to 6.6 million barrels per day, the lowest level since April 1958. As a result of the declining domestic production and relatively favorable prices, crude oil imports reached an April-record high of 7.2 million barrels per day. Stock levels of crude oil (excluding the Strategic Petroleum Reserve) were slightly below the normal seasonal range at 335 million barrels.

Stock levels in Midwest are being watched closely this year after the crude oil supply problems experienced in recent summers. Several companies have taken steps to ease the burden on crude pipelines serving Petroleum Administration for Defense District II. Mobil Oil converted and reversed a products pipeline that now has the capacity to carry more than 200 thousand barrels per day from Nederland, Texas to Patoka, Illinois. ${ }^{9}$ Imports of crude oil from Canada into the central states will be shipped on the newly expanded Interprovincial Pipeline. Carrying capacity on this pipeline was increased by 175 thousand barrels per day in December, with about 83 percent of the increase allocated for refineries in the Chicago, Illinois area. ${ }^{10}$

\section{Refinery Operations}

As refiners returned selected units from turnaround and maintenance activities, crude oil inputs remained strong, averaging 13.9 million barrels per day. The estimated refinery operable utilization rate, gross inputs divided by the total refining capacity with idle units included, averaged 91.3 percent.

\section{News Highlight}

\section{Motor Gasoline Varieties and Areas of Consumption}

\section{Introduction}

The Clean Air Act Amendments of 1990 (CAA) mandate various requirements for achieving the National Ambient Air Quality Standards for regulated air pollutants. As a consequence of the CAA, various areas in the United States must use specific compositions of gasoline at specific times of the year. These varieties are known as conventional, oxygenated conventional, reformulated and oxygenated program reformulated gasolines. In addition to the CAA regulations, other rules also exist specifically addressing the Reid Vapor Pressure (RVP) of gasoline.

The interaction of the regulations is critical, and can cause areas to be under simultaneous mandates in some cases, and under different revolving mandates depending on the time of the year. For example, the regulations for reformulated gasoline are in effect year-round, but may be modified, but not preempted, by oxygenated gasoline requirements during the winter months.
This sidebar will describe the regulations, illustrate their interactions and relate them to the impacted areas.

\section{The Federal Regulations}

\section{Reid Vapor Pressure}

During the late 1980s and early 1990s, EPA issued regulations to limit, in the summer, the volatility of motor gasoline used in the lower 48 States. The purpose of these regulations is to control evaporative emissions of volatile organic compounds (VOCs) that contribute to ground-level ozone formation. The regulations were enacted in two phases. Phase I RVP regulations became effective on June 1, 1989 and limited RVP to one of three possible values $(10.5,9.5$, or 9.0 pounds per square inch RVP) based on summer climatic conditions. Phase II regulations were implemented in the summer of 1992. Phase II standards were more stringent than Phase I, with possible values of $9.0,7.8$, or 7.0 pounds per square inch RVP.

The 9.0 psi standard is required for all states in the country in the month of May. From June 1 to September 15, the 9.0 psi standard is required for states that fall into the Northern Region and for areas in the Southern Region that are ozone attainment areas. From June 1 to September 15, the 7.8 psi standard is required for ozone nonattainment areas in the Southern Region. To implement the even stricter RVP standard of $7.0 \mathrm{psi}$, a special request is needed from the Governor of the requesting State to the Administrator of EPA. This request must establish the need for $7.0 \mathrm{psi}$ RVP gasoline to meet ozone standards.

\section{Oxygenated Gasoline}

The CAA contains major provisions on oxygenated gasoline. The purpose of these provisions is to reduce the incidence of unacceptable carbon monoxide levels during the winter months in 39 of 41 carbon monoxide non-attainment areas.

The Act requires that gasoline sold during specified winter months (at least four months) contain at least 2.7 weight percent oxygen in carbon monoxide non-attainment areas. This program went into effect on November 1,1992 . The oxygenate used to achieve the minimum oxygen requirement is not specified, and neither is the actual method or location of blending.

Oxygenated fuel is to be used in the entire Metropolitan Statistical Area (MSA) that contains the non-attainment area. The MSA often encompasses a much larger area than the non-attainment area itself.

\section{Reformulated Gasoline}

The CAA also mandates that areas that are in ozone non-attainment must take action to achieve attainment by specified dates. The nine worst ozone non-attainment areas are required to use reformulated gasoline (RFG). The other ozone non-attainment areas can "opt in" to the RFG program, or they can

\footnotetext{
9."Mobil to Begin Shipments," The Oil Daily, April 24, 1995, p. 5.

10"U.S. Crude Oil Pipeline Expansions Should Keep WTI Prices in Check," Bloomberg Oil Buyers' Guide, May 8, 1995 , pp. 1 and 3.
} 
Figure H1. Clean Motor Gasoline Used in 1995 in Response to CAAA 90 and Applicable RVP Requirements by Area

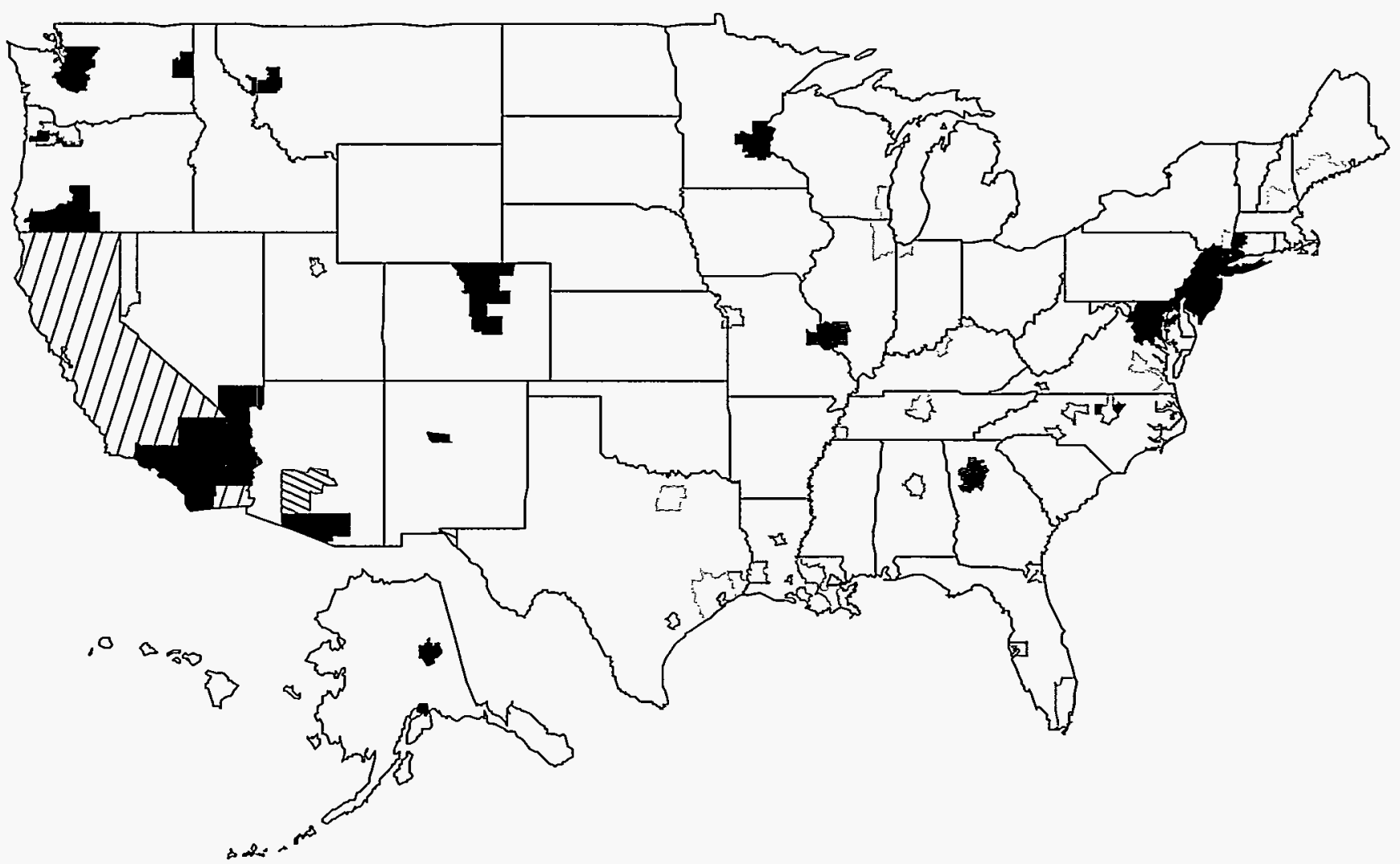

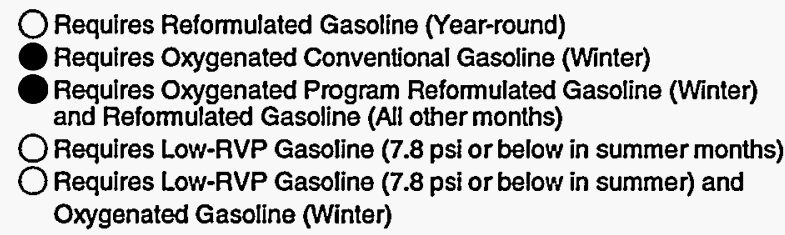

Oxygenated Gasoline (Winter)
(1) Requires Low-RVP Gasoline (7.8 psi or below in summer) and Califomia Oxygenated Gasoline (Winter)

QRequires Ultra-Low-RVP Gasoline (7.0 psi or below in summer) and Oxygenated Gasoline (Winter)

Requires Ultra-Low-RVP Gasoline (7.0 psi or below in summer months except in the St. Louis Metropolitan Area of Illinois which requires 7.2 psi or below in summer months)

ORequires 9.0 psi or below RVP Gasoline (Summer), except Alaska and Hawaii

Sources: Derived from: "Reformulated Gasoline and Anti-Dumping Questions and Answers," EPA, July 1, 1994; "A Summarized Version of the Applicable Volatility Standards for Conventional Gasoline Areas," unpublished EPA document, January 1995; telephone conversations with regional and national EPA offices; and telephone conversations with state environmental offices.

use alternative strategies, such as use of low-RVP gasoline, to achieve attainment. El Paso, Texas; Atlanta, Georgia; St. Louis, Missouri; and Phoenix, Arizona are examples of ozone non-attainment areas that are currently taking the low-RVP approach to achieve attainment. The nine RFG-mandated areas and all "opt-ins" are required to use reformulated gasoline. Areas that are in ozone attainment, or that have not opted-in to the reformulated gasoline program, cannot be required to use RFG.

\section{California Reformulated and Oxygenated Gasolines}

The State of California has air pollution problems that are unique. The Clean Air Act Amendments of 1990 and resultant EPA regulations contain numerous special provisions for California. Two metropolitan areas in California are included in the nine areas required to participate in the year round Federal reformulated gasoline program beginning January 1,1995. These areas are the Los Angeles Consolidated Metropolitan Statistical Area and San Diego County. California has developed its own statewide reformulated gasoline program that supplants the Federal program beginning March 1, 1996. California's oxygenated fuel regulations also vary from Federal standards by accepting the lower average oxygen content of 2.0 weight percent, rather than 2.7 weight percent.

\section{Linking Regulations and Geographic Areas}

Figure $\mathrm{H} 1$ depicts areas of the United States under various mandates. A description of each unique area follows below:

Requires Reformulated Gasoline - The area requires reformulated gasoline throughout the year to alleviate ground-level ozone pollution.

Requires Oxygenated Conventional Gasoline - The area requires the winter-only use of oxygenated gasoline ( 2.7 minimum weight percent oxygen) for at least four winter months. The precise months covered by the winter requirement vary between September and April, inclusive. These areas are combating 
carbon monoxide pollution. These areas are required to use 9.0 psi conventional gasoline from June 1 through September 15.

Requires Oxygenated Program Reformulated Gasoline (OPRG) - The area requires reformulated gasoline throughout the year. During the winter control period (at least four months long), the RFG must also meet a 2.7 minimum weight percent oxygen content standard. The oxygen standard for the RFG used outside the winter control period is 2.0 minimum weight percent oxygen. The OPRG areas are combating both ozone and carbon monoxide pollution.

Requires Low-RVP Gasoline (7.8 psi) - Summer-only federal program that requires conventional gasoline with an RVP $7.8 \mathrm{psi}$ at the retail level June 1 (May 1 at the terminal level) through at least September 15 each year. Some areas (under state authority) have extended the retail requirement to include all of May or all of September. Areas that are designated as nonattainment for ozone and are in states in the "southern-tier" are required to enforce the $7.8 \mathrm{psi}$ RVP restriction. The intent of the program is to lessen ground-level ozone pollution. There are no environmental volatility requirements outside the summer controls.

Requires Low RVP Gasoline (Summer) and Oxygenated Gasoline (Winter) - Summer-only federal program that requires conventional gasoline with an RVP 7.8 psi at the retail level June 1 (May 1 at the terminal level) through at least September 15 each year. Some areas (under state authority) have extended the retail requirement to include all of May or all of September. Areas that are designated as nonattainment for ozone and are in states in the "southern-tier" are required to enforce the 7.8 psi RVP restriction. The intent of the program is to lessen ground-level ozone pollution. There are no environmental volatility requirements outside the summer controls. These areas also require oxygenated gasoline containing 2.7 minimum weight percent oxygen during the winter. The RVP and oxygenate requirements are exclusive of each other (i.e., they effect different seasons and are not simultaneously required). These areas are combating both carbon monoxide and ozone pollution.

Requires Low-RVP Gasoline (Summer) and California Oxygenated Gasoline (Winter) - The state of California requires the statewide summertime use of $7.8 \mathrm{psi}$ RVP gasoline (regardless of ozone attainment status) in areas that are not under the federal mandate to use RFG. The summertime period varies within the state between April 1 and October 31. The purpose of this program is to reduce ground-level ozone pollution. The state requires that these same non-RFG areas use California-specification oxygenated gasoline (oxygen content of 1.8-2.2 weight percent) in the winter to reduce carbon monoxide pollution. The winter control period varies within the state between September and February. The RVP and oxygenate requirements are exclusive of each other (i.e., they effect different seasons and are not simultaneously required).

Requires Ultra-Low RVP Gasoline (Summer) and Oxygenated Gasoline (Winter) - The area is under a state-imposed requirement to use gasoline with an RVP below the federal limit of $7.8 \mathrm{psi}$ RVP (to date only southern areas have selected the restricted RVP strategy). Reduced RVP fuels are an alternative to the use of RFG and are required in the summer only. These areas also require oxygenated gasoline containing 2.7 minimum weight percent oxygen during the winter. The RVP and oxygenate requirements are exclusive of each other (i.e., they effect different seasons and are not simultaneously required). El Paso, Texas and Phoenix, Arizona are the two areas with both oxygenated fuels and ultra-low RVP ( $7.0 \mathrm{psi}$ ) requirements. These areas are combating both carbon monoxide and ozone pollution.

Requires Ultra-Low RVP Gasoline - The area is under a state-imposed requirement to use gasoline with an RVP below the federal limit of 7.8 psi RVP (to date only southern areas have selected the restricted RVP strategy). Reduced RVP fuels are an alternative to the use of RFG and are required in the summer only. Atlanta, Georgia and the portion of the St. Louis, Missouri metropolitan area that is in Missouri require gasoline with a summer RVP of 7.0 psi. The portion of the St. Louis metropolitan area that is in Illinois requires a maximum RVP of 7.2 psi or the use of RFG (with an RVP limit of 8.1 psi for Illinois, VOC-control region 2). Areas using ultra-low RVP strategies are combating ground-level ozone pollution.

Requires 9.0 psi or below RVP Gasoline (Summer) - All areas that are not otherwise identified (excluding Hawaii and Alaska) are required to use 9.0 psi conventional gasoline from June 1 through September 15. 


\section{Summary Statistics}

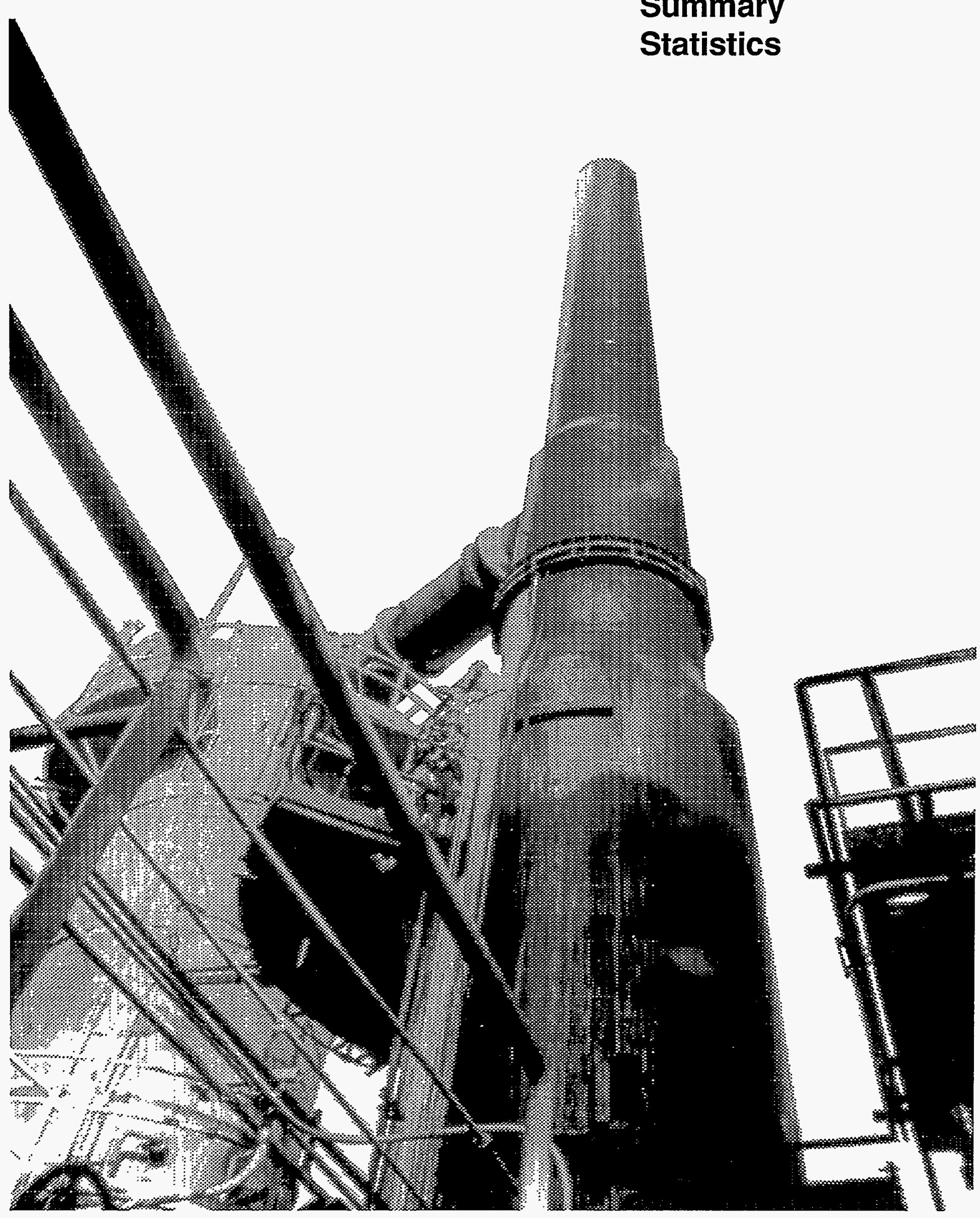

Incinerators such as this one at a chemical installation turn toxic chemicals into water vapor and other harmless elements. 
Table S1. Crude Oil and Petroleum Products Overview, 1981 - Present (Thousand Barrels per Day, Except Where Noted)

\begin{tabular}{|c|c|c|c|c|c|c|c|c|}
\hline & \multirow[b]{2}{*}{ Year/Month } & \multicolumn{3}{|c|}{ Field Production } & \multicolumn{2}{|c|}{ Stock Change ${ }^{a}$} & \multirow[b]{2}{*}{$\begin{array}{l}\text { Petroleum } \\
\text { Products } \\
\text { Supplied }\end{array}$} & \multirow{2}{*}{\begin{tabular}{|c|}
$\begin{array}{c}\text { Ending } \\
\text { Stocks }\end{array}$ \\
(Million Barrels) \\
$\begin{array}{c}\text { Crude Oild and } \\
\text { Petroleum } \\
\text { Products }\end{array}$ \\
\end{tabular}} \\
\hline & & $\begin{array}{c}\text { Total } \\
\text { Domestic }\end{array}$ & $\begin{array}{c}\text { Crude } \\
\text { Oil }\end{array}$ & $\begin{array}{l}\text { Natural } \\
\text { Gas Plant } \\
\text { Liquids }\end{array}$ & $\begin{array}{c}\text { Crugie } \\
\text { Oil }^{d}\end{array}$ & $\begin{array}{l}\text { Petroleum } \\
\text { Products }\end{array}$ & & \\
\hline $\begin{array}{l}1981 \\
1982 \\
1983 \\
1984 \\
1985 \\
1986 \\
1987 \\
1988 \\
1989 \\
1990 \\
1991 \\
1992\end{array}$ & 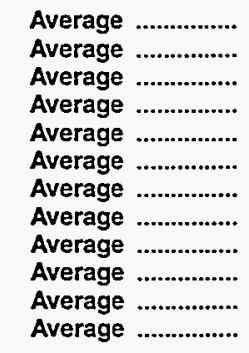 & $\begin{array}{r}10,230 \\
10,252 \\
10,299 \\
10,554 \\
10,636 \\
10,289 \\
10,008 \\
9,818 \\
9,219 \\
8,994 \\
9,168 \\
8,996\end{array}$ & $\begin{array}{l}8,572 \\
8,649 \\
8,688 \\
8,879 \\
8,971 \\
8,680 \\
8,349 \\
8,140 \\
7,613 \\
7,355 \\
7,417 \\
7,171\end{array}$ & $\begin{array}{l}1,609 \\
1,550 \\
1,559 \\
1,630 \\
1,609 \\
1,551 \\
1,595 \\
1,625 \\
1,546 \\
1,559 \\
1,659 \\
1,697\end{array}$ & $\begin{array}{r}g_{290} \\
136 \\
g_{214} \\
199 \\
50 \\
78 \\
128 \\
1 \\
86 \\
-35 \\
-42 \\
-1\end{array}$ & $\begin{array}{r}g-130 \\
-283 \\
g-234 \\
81 \\
-153 \\
124 \\
-87 \\
-29 \\
-129 \\
142 \\
32 \\
-68\end{array}$ & $\begin{array}{l}16,058 \\
15,296 \\
15,231 \\
15,726 \\
15,726 \\
16,281 \\
16,665 \\
17,283 \\
17,325 \\
16,988 \\
16,714 \\
17,033\end{array}$ & $\begin{array}{r}1,484 \\
91,430 \\
1,454 \\
1,556 \\
1,519 \\
1,593 \\
1,607 \\
1,597 \\
1,581 \\
1,621 \\
1,617 \\
91,592\end{array}$ \\
\hline \multicolumn{2}{|c|}{ 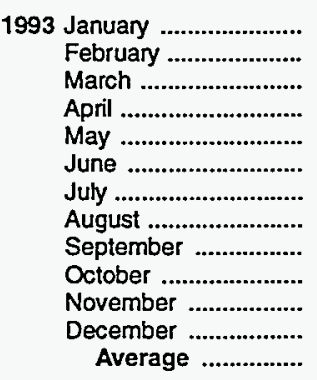 } & $\begin{array}{l}9,254 \\
8,907 \\
8,987 \\
8,897 \\
8,800 \\
8,747 \\
8,657 \\
8,720 \\
8,652 \\
8,893 \\
8,847 \\
8,668 \\
8,836\end{array}$ & $\begin{array}{l}6,961 \\
6,943 \\
6,974 \\
6,881 \\
6,847 \\
6,795 \\
6,688 \\
6,758 \\
6,712 \\
6,839 \\
6,912 \\
6,858 \\
6,847\end{array}$ & $\begin{array}{l}1,737 \\
1,777 \\
1,793 \\
1,802 \\
1,732 \\
1,753 \\
1,741 \\
1,747 \\
1,732 \\
1,768 \\
1,670 \\
1,579 \\
1,736\end{array}$ & $\begin{array}{r}295 \\
219 \\
212 \\
523 \\
147 \\
2 \\
6 \\
-505 \\
-439 \\
328 \\
251 \\
-53 \\
81\end{array}$ & $\begin{array}{r}9560 \\
-796 \\
-602 \\
356 \\
915 \\
573 \\
497 \\
299 \\
86 \\
403 \\
-320 \\
-1,198 \\
70\end{array}$ & $\begin{array}{l}16,173 \\
17,334 \\
17,575 \\
16,781 \\
16,508 \\
17,096 \\
17,357 \\
17,332 \\
17,650 \\
17,323 \\
17,780 \\
17,953 \\
17,237\end{array}$ & $\begin{array}{r}91,618 \\
1,602 \\
1,590 \\
1,617 \\
1,650 \\
1,667 \\
1,682 \\
1,676 \\
1,665 \\
1,688 \\
1,686 \\
1,647 \\
-\end{array}$ \\
\hline \multicolumn{2}{|c|}{ 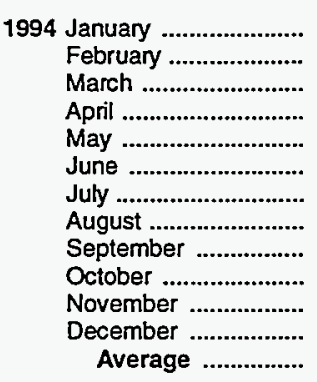 } & $\begin{array}{l}E_{8,674} \\
E_{8,586} \\
E_{8,688} \\
E_{8,528} \\
E_{8,546} \\
E_{8,546} \\
E_{8,580} \\
E_{8,537} \\
E_{8,613} \\
E_{8,600} \\
E_{8,649} \\
E_{8,764} \\
E_{8,610}\end{array}$ & $\begin{array}{l}E_{6,777} \\
E_{6,745} \\
E_{6,719} \\
E_{6,634} \\
E_{6,658} \\
E_{6,567} \\
E_{6,528} \\
E_{6,547} \\
E_{6,551} \\
E_{6,578} \\
E_{6,542} \\
E_{6,686} \\
E_{6,627}\end{array}$ & $\begin{array}{l}1,619 \\
1,642 \\
1,676 \\
1,687 \\
1,715 \\
1,736 \\
1,756 \\
1,766 \\
1,793 \\
1,747 \\
1,796 \\
1,799 \\
1,728\end{array}$ & $\begin{array}{r}-16 \\
-164 \\
339 \\
-58 \\
-213 \\
-204 \\
187 \\
-43 \\
112 \\
294 \\
106 \\
-155 \\
17\end{array}$ & $\begin{array}{r}-831 \\
-1,225 \\
-438 \\
311 \\
977 \\
457 \\
855 \\
291 \\
580 \\
-546 \\
329 \\
-776 \\
4\end{array}$ & $\begin{array}{l}17,924 \\
18,302 \\
17,289 \\
17,428 \\
17,094 \\
17,830 \\
17,474 \\
18,107 \\
17,469 \\
17,656 \\
17,340 \\
18,280 \\
17,679\end{array}$ & $\begin{array}{r}1,620 \\
1,581 \\
1,578 \\
1,585 \\
1,609 \\
1,616 \\
1,649 \\
1,656 \\
1,677 \\
1,669 \\
1,682 \\
1,654 \\
-\end{array}$ \\
\hline \multicolumn{2}{|c|}{$\begin{array}{l}1995 \text { January ....................... } \\
\text { February ....................... } \\
\text { March ....................... } \\
\text { April* ....................... } \\
\text { 4-Mo. Average ........... }\end{array}$} & $\begin{array}{r}E_{8,664} \\
E_{8,832} \\
\operatorname{RE}_{8,625} \\
E_{8,665} \\
E_{8,693}\end{array}$ & $\begin{array}{r}E_{6,596} \\
E_{6,703} \\
\text { RE }_{6,606} \\
\text { PE } 6,577 \\
\text { PE } 6,619\end{array}$ & $\begin{array}{r}1,773 \\
1,774 \\
R_{1,773} \\
E_{1,774} \\
E_{1,774}\end{array}$ & $\begin{array}{r}-279 \\
-48 \\
R_{344} \\
E_{-105} \\
E_{-20}\end{array}$ & $\begin{array}{r}-117 \\
-1,315 \\
R-484 \\
E_{-}-160 \\
E_{-502}\end{array}$ & $\begin{array}{r}17,167 \\
18,355 \\
R_{17,403} \\
E_{17,353} \\
E_{17,552}\end{array}$ & $\begin{array}{r}1,641 \\
1,603 \\
R 1,599 \\
E_{1,598} \\
-\end{array}$ \\
\hline \multicolumn{2}{|c|}{$\begin{array}{l}1994 \text { 4-Mo. Average ........... } \\
1993 \text { 4-Mo. Average ........... }\end{array}$} & $\begin{array}{r}E_{8,621} \\
9,015\end{array}$ & $\begin{array}{r}E_{6,719} \\
6,940\end{array}$ & $\begin{array}{l}1,656 \\
1,777\end{array}$ & $\begin{array}{r}31 \\
313\end{array}$ & $\begin{array}{l}-536 \\
-108\end{array}$ & $\begin{array}{l}17,724 \\
16,958\end{array}$ & - \\
\hline
\end{tabular}

\footnotetext{
a negative number indicates a decrease in stocks and a positive number indicates an increase.

b Stocks are totals as of end of period.

c Includes crude oil, natural gas plant liquids, and other liquids. Beginning in 1993, fuel ethanol blended into finished motor gasoline and oxygenate production from merchant MTBE plants are also included.

d Includes stocks located in the Strategic Petroleum Reserve.

e Includes crude oil for storage in the Strategic Petroleum Reserve.

i Net Imports equal Imports minus Exports.

9 In January 1981 and 1983 , numerous respondents were added to surveys affecting stocks reported and stock change calculations. Stock changes are calculated using new basis stock levels. Bulk terminal and pipeline stocks of oxygenates were added beginning in January 1993. See Summary Statistics Explanatory Note 4.

Footnotes continued on following page.
} 
Table S1. Crude Oil and Petroleum Products Overview, 1981 - Present (Continued) (Thousand Barrels per Day, Except Where Noted)

\begin{tabular}{|c|c|c|c|c|c|c|c|c|}
\hline \multirow{2}{*}{\multicolumn{2}{|c|}{ YearMonth }} & \multicolumn{3}{|c|}{ Imports } & \multicolumn{3}{|c|}{ Exports } & \multirow[b]{2}{*}{$\underset{\text { Imports }}{\text { Net }}$} \\
\hline & & Total & $\underset{\text { Oill }^{\mathrm{e}}}{\text { Crude }}$ & $\begin{array}{l}\text { Petroleum } \\
\text { Products }\end{array}$ & Total & $\begin{array}{l}\text { Crude } \\
\text { Oil }\end{array}$ & $\begin{array}{l}\text { Petroleum } \\
\text { Products }\end{array}$ & \\
\hline $\begin{array}{l}1981 \\
1982 \\
1983 \\
1984 \\
1985 \\
1986 \\
1987 \\
1988 \\
1989 \\
1990 \\
1991 \\
1992\end{array}$ & 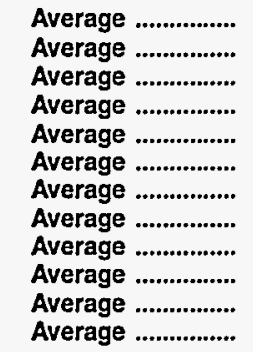 & $\begin{array}{l}5,996 \\
5,113 \\
5,051 \\
5,437 \\
5,067 \\
6,224 \\
6,678 \\
7,402 \\
8,061 \\
8,018 \\
7,627 \\
7,888\end{array}$ & $\begin{array}{l}4,396 \\
3,488 \\
3,329 \\
3,426 \\
3,201 \\
4,178 \\
4,674 \\
5,107 \\
5,843 \\
5,894 \\
5,782 \\
6,083\end{array}$ & $\begin{array}{l}1,599 \\
1,625 \\
1,722 \\
2,011 \\
1,866 \\
2,045 \\
2,004 \\
2,295 \\
2,217 \\
2,123 \\
1,844 \\
1,805\end{array}$ & $\begin{array}{r}595 \\
815 \\
739 \\
722 \\
781 \\
785 \\
764 \\
815 \\
859 \\
857 \\
1,001 \\
950\end{array}$ & $\begin{array}{l}228 \\
236 \\
164 \\
181 \\
204 \\
154 \\
151 \\
155 \\
142 \\
109 \\
116 \\
89\end{array}$ & $\begin{array}{l}367 \\
579 \\
575 \\
541 \\
577 \\
631 \\
613 \\
661 \\
717 \\
748 \\
885 \\
861\end{array}$ & $\begin{array}{l}5,401 \\
4,298 \\
4,312 \\
4,715 \\
4,286 \\
5,439 \\
5,914 \\
6,587 \\
7,202 \\
7,161 \\
6,626 \\
6,938\end{array}$ \\
\hline \multicolumn{2}{|c|}{ 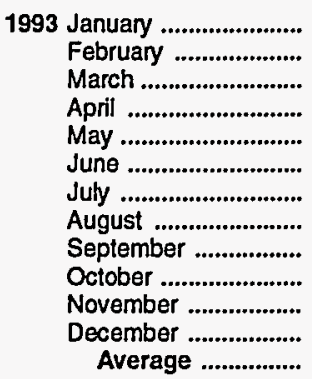 } & $\begin{array}{l}8,004 \\
7,948 \\
8,285 \\
8,768 \\
8,663 \\
8,805 \\
9,219 \\
8,429 \\
8,531 \\
9,197 \\
8,903 \\
8,645 \\
8,620\end{array}$ & $\begin{array}{l}6,292 \\
6,156 \\
6,488 \\
6,928 \\
6,809 \\
7,201 \\
7,289 \\
6,641 \\
6,581 \\
7,181 \\
6,997 \\
6,838 \\
6,787\end{array}$ & $\begin{array}{l}1,712 \\
1,792 \\
1,797 \\
1,840 \\
1,854 \\
1,604 \\
1,930 \\
1,789 \\
1,950 \\
2,015 \\
1,906 \\
1,807 \\
1,833\end{array}$ & $\begin{array}{r}1,135 \\
1,033 \\
970 \\
1,067 \\
1,082 \\
900 \\
1,001 \\
829 \\
902 \\
881 \\
980 \\
1,250 \\
1,003\end{array}$ & $\begin{array}{r}129 \\
166 \\
139 \\
73 \\
112 \\
150 \\
62 \\
55 \\
107 \\
62 \\
67 \\
63 \\
98\end{array}$ & $\begin{array}{r}1,006 \\
867 \\
831 \\
994 \\
970 \\
750 \\
938 \\
774 \\
795 \\
819 \\
913 \\
1,188 \\
904\end{array}$ & $\begin{array}{l}6,869 \\
6,915 \\
7,315 \\
7,701 \\
7,581 \\
7,905 \\
8,218 \\
7,600 \\
7,629 \\
8,316 \\
7,923 \\
7,394 \\
7,618\end{array}$ \\
\hline \multicolumn{2}{|c|}{ 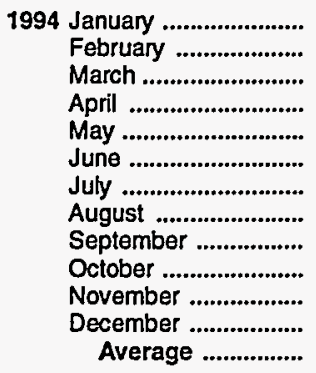 } & $\begin{array}{l}7,914 \\
8,501 \\
8,500 \\
8,927 \\
9,155 \\
9,263 \\
9,778 \\
9,523 \\
9,526 \\
8,642 \\
8,527 \\
8,861 \\
8,929\end{array}$ & $\begin{array}{l}5,961 \\
6,313 \\
6,377 \\
6,937 \\
7,163 \\
7,358 \\
7,867 \\
7,528 \\
7,722 \\
6,993 \\
6,863 \\
7,193 \\
7,027\end{array}$ & $\begin{array}{l}1,953 \\
2,187 \\
2,123 \\
1,990 \\
1,993 \\
1,906 \\
1,911 \\
1,996 \\
1,804 \\
1,649 \\
1,663 \\
1,668 \\
1,902\end{array}$ & $\begin{array}{r}927 \\
882 \\
936 \\
868 \\
929 \\
867 \\
877 \\
913 \\
891 \\
997 \\
1,000 \\
1,208 \\
942\end{array}$ & $\begin{array}{r}110 \\
116 \\
40 \\
120 \\
118 \\
107 \\
84 \\
72 \\
61 \\
138 \\
102 \\
118 \\
99\end{array}$ & $\begin{array}{r}817 \\
766 \\
896 \\
749 \\
812 \\
760 \\
793 \\
841 \\
830 \\
859 \\
898 \\
1,090 \\
843\end{array}$ & $\begin{array}{l}6,987 \\
7,619 \\
7,564 \\
8,059 \\
8,226 \\
8,396 \\
8,901 \\
8,611 \\
8,635 \\
7,646 \\
7,527 \\
7,653 \\
7,986\end{array}$ \\
\hline \multicolumn{2}{|c|}{ 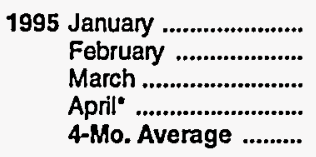 } & $\begin{array}{r}7,955 \\
8,358 \\
R_{9,020} \\
E_{8,454} \\
E_{8,449}\end{array}$ & $\begin{array}{r}6,503 \\
6,565 \\
R_{7,409} \\
E_{6,181}^{7,921}\end{array}$ & $\begin{array}{r}1,452 \\
1,793 \\
R_{1,612} \\
E_{1,272} \\
E_{1,528}\end{array}$ & $\begin{array}{r}978 \\
1,062 \\
R_{948}^{\prime} \\
E_{970} \\
988\end{array}$ & $\begin{array}{r}113 \\
95 \\
\mathrm{R}_{68} \\
\mathrm{E}_{99} \\
\mathrm{E}_{93}\end{array}$ & $\begin{array}{r}865 \\
967 \\
R_{880} \\
E_{871} \\
E_{894}\end{array}$ & $\begin{array}{r}6,977 \\
7,296 \\
R_{8,073} \\
E_{7,484} \\
E_{7,461}\end{array}$ \\
\hline \multicolumn{2}{|c|}{$\begin{array}{l}1994 \text { 4-Mo. Average ......... } \\
1993 \text { 4-Mo. Average ........ }\end{array}$} & $\begin{array}{l}8,456 \\
8,255\end{array}$ & $\begin{array}{l}6,395 \\
6,470\end{array}$ & $\begin{array}{l}2,061 \\
1,785\end{array}$ & $\begin{array}{r}904 \\
1,051\end{array}$ & $\begin{array}{r}96 \\
126\end{array}$ & $\begin{array}{l}808 \\
925\end{array}$ & $\begin{array}{l}7,551 \\
7,203\end{array}$ \\
\hline
\end{tabular}

Footnotes continued.

$\mathrm{R}=$ Revised data. $\mathrm{E}=$ Estimated. $\mathrm{PE}=$ Preliminary estimate. $\mathrm{RE}=$ Revised estimate.

- See Summary Statistics Explanatory Note 1.

Notes: - Crude oil includes lease condensate. - Italics denote estimates based upon preliminary data. - Geographic coverage is the 50 States and the District of Columbia. - Totals may not equal sum of components due to independent rounding.

Source: See Summary Statistics Table and Figure Sources. 
Figure S1. Petroleum Overview, March 1994 - Present

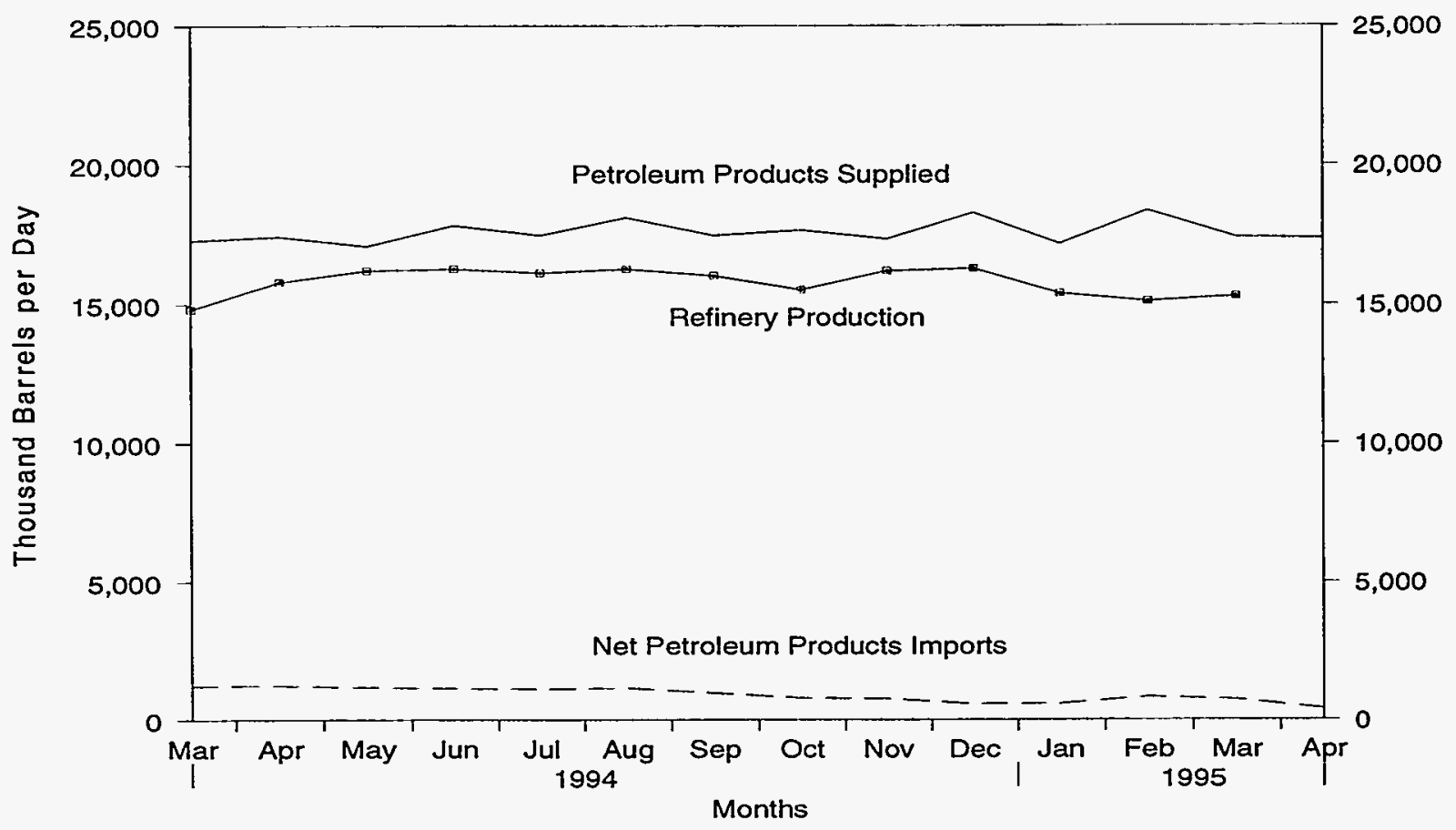

Source: Energy Information Administration, Petroleum Supply Monthly, Table S1. See Summary Statistics Table and Figure Sources.

Figure S2. Petroleum Products Supplied, March 1994 - Present

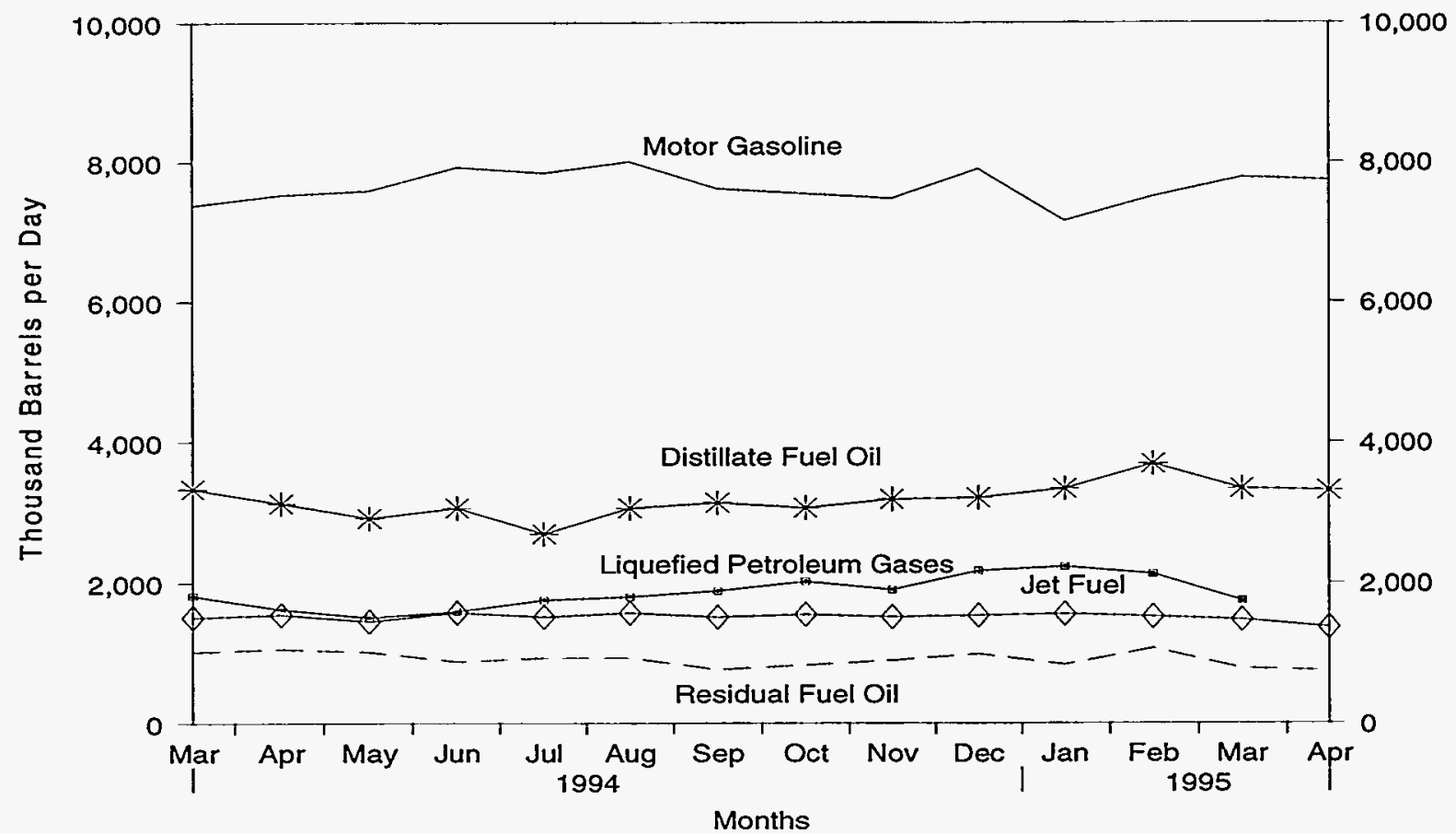

Source: Energy Information Administration, Petroleum Supply Monthly, Tables S4-S7, and S9. See Summary Statistics Table and Figure Sources. 
Figure S3. Crude Oil Supply and Disposition, March 1994 - Present

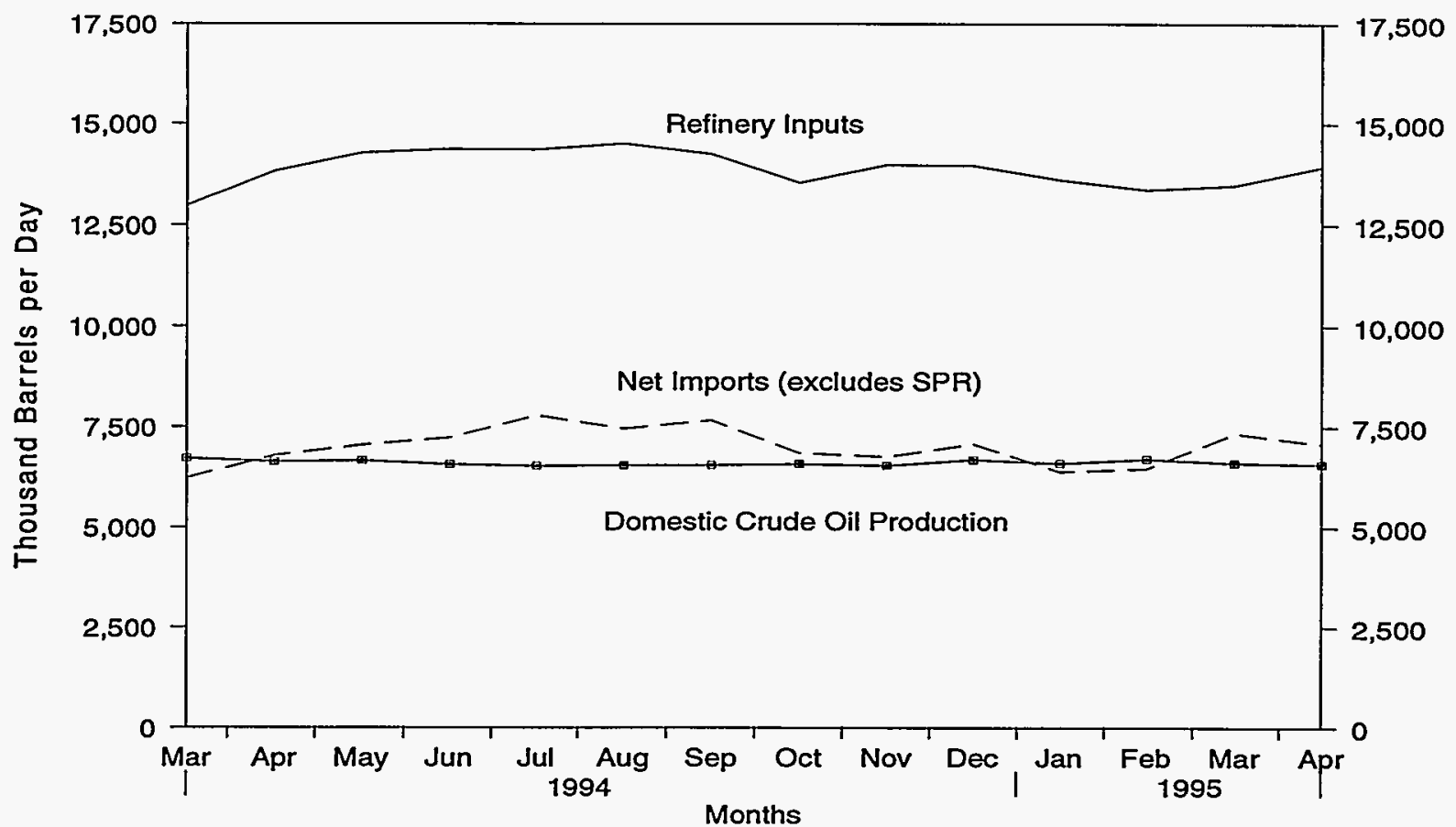

Source: Energy Information Administration, Petroleum Supply Monthly, Table S2. See Summary Statistics Table and Figure Sources.

\section{Figure S4. Crude Oil Ending Stocks, ${ }^{1}$ March 1994 - Present}

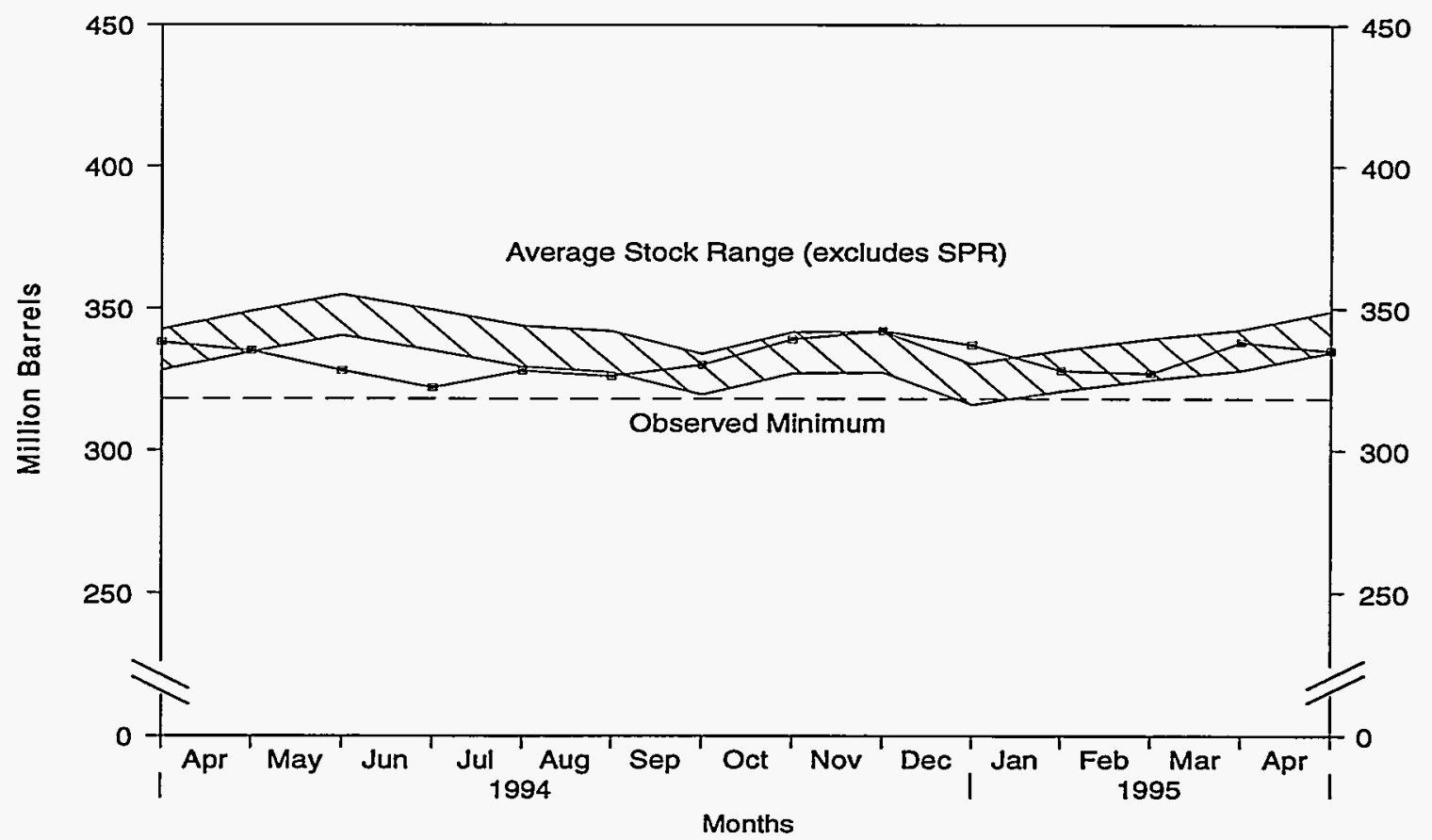

'Excludes stocks held in the Strategic Petroleum Reserve (SPR).

Note: The Observed Minimum for crude oil stocks in the last 36-month period was 318.1 million barrels, occurring in December 1992. Source: Energy Information Administration, Petroleum Supply Monthly, Table S2. See Summary Statistics Table and Figure Sources. 
Table S2. Crude Oil Supply and Disposition, 1981 - Present (Thousand Barrels per Day, Except Where Noted)

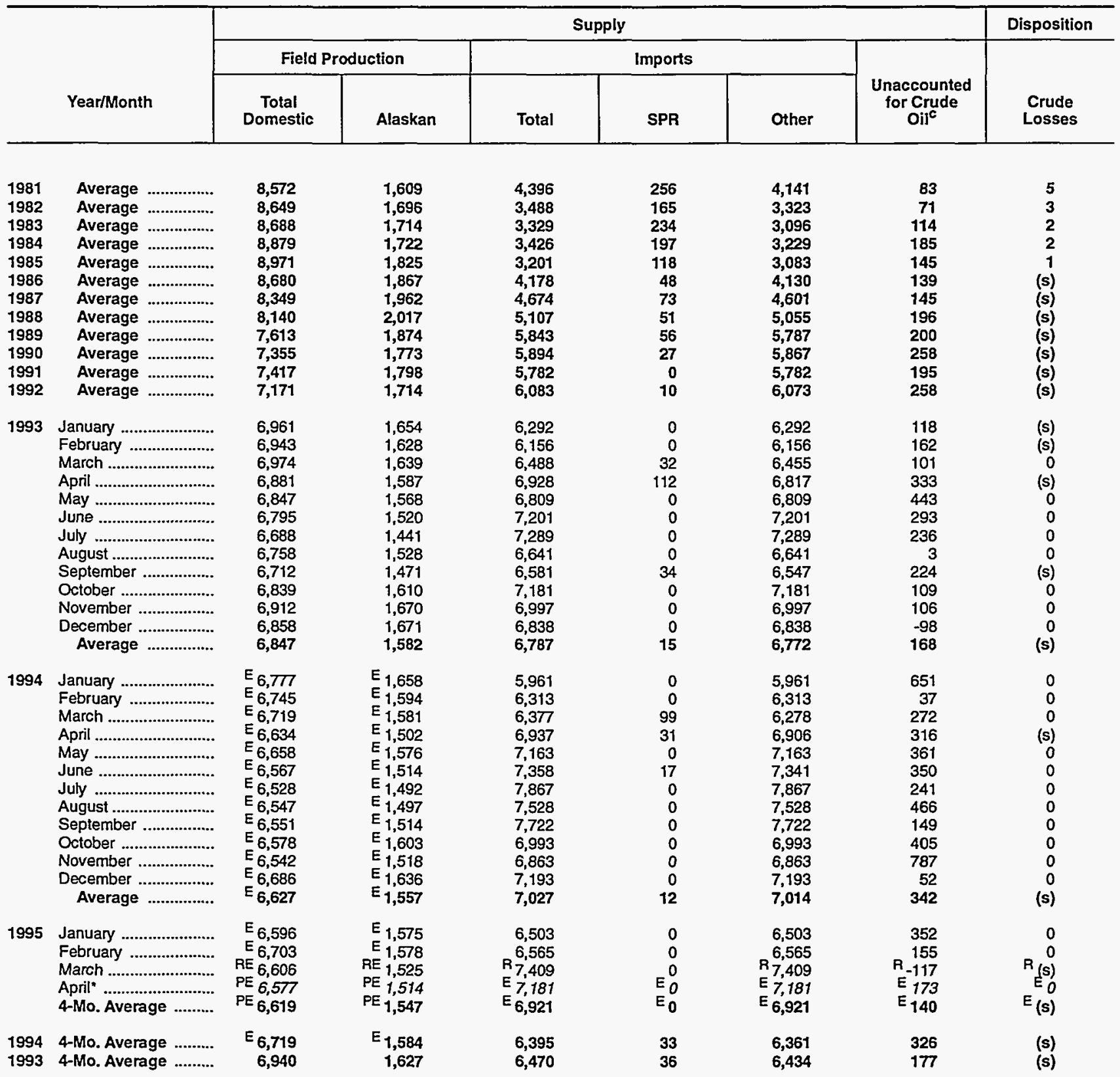

Stocks are totals as of end of period.

b A negative number indicates a decrease in stocks and a positive number indicates an increase

c Unaccounted for crude oil represents the difference between the supply and disposition of crude oil. Preliminary estimates of crude oil imports at the National level have historically understated final values by approximately 50 thousand barrels per day. This causes the preliminary values of unaccounted for crude oil to overstate the final values by the same amount.

$d$ Previously published as crude used directiy.

e Stock changes are calculated using new basis stock levels. See Summary Statistics Explanatory Note 4.

Footnotes continued on following page. 
Table S2. Crude Oil Supply and Disposition, 1981 - Present (Continued) (Thousand Barrels per Day, Except Where Noted)

\begin{tabular}{|c|c|c|c|c|c|c|c|c|c|}
\hline & \multirow[b]{3}{*}{ Year/Month } & \multicolumn{5}{|c|}{ Disposition } & \multicolumn{3}{|c|}{ Ending Stocks ${ }^{\mathbf{a}}$ (Million Barrels) } \\
\hline & & \multicolumn{2}{|c|}{ Stock Change ${ }^{b}$} & \multirow[b]{2}{*}{$\begin{array}{l}\text { Refinery } \\
\text { Inputs }\end{array}$} & \multirow[b]{2}{*}{ Exports } & \multirow[b]{2}{*}{$\begin{array}{l}\text { Product } \\
\text { Supplied }\end{array}$} & \multirow[b]{2}{*}{ Total } & \multirow[b]{2}{*}{ SPR } & \multirow[b]{2}{*}{$\begin{array}{l}\text { Other } \\
\text { Primary }\end{array}$} \\
\hline & & SPR & Other & & & & & & \\
\hline $\begin{array}{l}1981 \\
1982 \\
1983 \\
1984 \\
1985 \\
1986 \\
1987 \\
1988 \\
1989 \\
1990 \\
1991 \\
1992\end{array}$ & 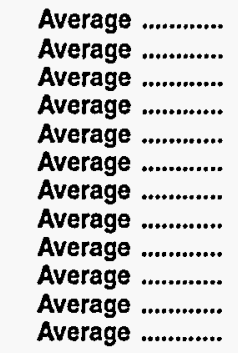 & $\begin{array}{r}336 \\
174 \\
234 \\
195 \\
117 \\
50 \\
80 \\
52 \\
56 \\
16 \\
-47 \\
17\end{array}$ & $\begin{array}{r}\theta-46 \\
-38 \\
\theta^{-20} \\
4 \\
-67 \\
28 \\
49 \\
-51 \\
30 \\
-51 \\
5 \\
-18\end{array}$ & $\begin{array}{l}12,470 \\
11,774 \\
11,685 \\
12,044 \\
12,002 \\
12,716 \\
12,854 \\
13,246 \\
13,401 \\
13,409 \\
13,301 \\
13,411\end{array}$ & $\begin{array}{l}228 \\
236 \\
164 \\
181 \\
204 \\
154 \\
151 \\
155 \\
142 \\
109 \\
116 \\
89\end{array}$ & $\begin{array}{r}d 58 \\
d 59 \\
66 \\
64 \\
60 \\
49 \\
34 \\
40 \\
28 \\
24 \\
18 \\
13\end{array}$ & $\begin{array}{r}594 \\
{ }^{\circ} 644 \\
723 \\
796 \\
814 \\
843 \\
890 \\
890 \\
921 \\
908 \\
893 \\
893\end{array}$ & $\begin{array}{l}230 \\
294 \\
379 \\
451 \\
493 \\
512 \\
541 \\
560 \\
580 \\
586 \\
569 \\
\mathbf{5 7 5}\end{array}$ & $\begin{array}{r}363 \\
e 350 \\
344 \\
345 \\
321 \\
331 \\
349 \\
330 \\
341 \\
323 \\
325 \\
318\end{array}$ \\
\hline 1993 & 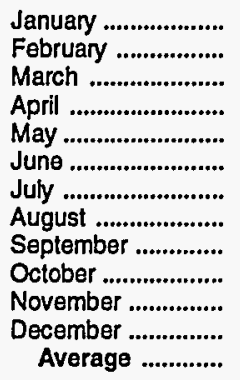 & $\begin{array}{r}19 \\
18 \\
58 \\
136 \\
13 \\
21 \\
19 \\
24 \\
52 \\
19 \\
18 \\
9 \\
34\end{array}$ & $\begin{array}{r}276 \\
201 \\
154 \\
387 \\
134 \\
-20 \\
-13 \\
-529 \\
-491 \\
309 \\
233 \\
-62 \\
47\end{array}$ & $\begin{array}{l}12,938 \\
12,865 \\
13,200 \\
13,538 \\
13,829 \\
14,129 \\
14,136 \\
13,844 \\
13,841 \\
13,729 \\
13,686 \\
13,571 \\
13,613\end{array}$ & $\begin{array}{r}129 \\
166 \\
139 \\
73 \\
112 \\
150 \\
62 \\
55 \\
107 \\
62 \\
67 \\
63 \\
98\end{array}$ & $\begin{array}{r}10 \\
10 \\
11 \\
9 \\
10 \\
8 \\
9 \\
8 \\
8 \\
10 \\
10 \\
16 \\
10\end{array}$ & $\begin{array}{r}902 \\
908 \\
915 \\
930 \\
935 \\
935 \\
935 \\
920 \\
906 \\
917 \\
924 \\
922 \\
-\end{array}$ & $\begin{array}{r}575 \\
576 \\
578 \\
582 \\
582 \\
583 \\
583 \\
584 \\
586 \\
586 \\
587 \\
587 \\
-\end{array}$ & $\begin{array}{r}327 \\
332 \\
337 \\
349 \\
353 \\
352 \\
352 \\
335 \\
321 \\
330 \\
337 \\
335 \\
-\end{array}$ \\
\hline 1994 & 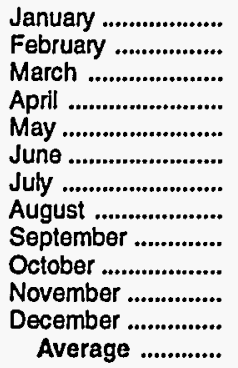 & $\begin{array}{r}4 \\
(s) \\
99 \\
31 \\
(s) \\
16 \\
(s) \\
(s) \\
0 \\
0 \\
(s) \\
(s) \\
13\end{array}$ & $\begin{array}{r}-19 \\
-164 \\
241 \\
-89 \\
-213 \\
-220 \\
187 \\
-43 \\
112 \\
294 \\
106 \\
-155 \\
5\end{array}$ & $\begin{array}{l}13,285 \\
13,132 \\
12,978 \\
13,817 \\
14,269 \\
14,364 \\
14,356 \\
14,505 \\
14,240 \\
13,537 \\
13,978 \\
13,958 \\
13,872\end{array}$ & $\begin{array}{r}110 \\
116 \\
40 \\
120 \\
118 \\
107 \\
84 \\
72 \\
61 \\
138 \\
102 \\
118 \\
99\end{array}$ & $\begin{array}{r}10 \\
12 \\
10 \\
9 \\
9 \\
7 \\
8 \\
7 \\
9 \\
8 \\
7 \\
10 \\
9\end{array}$ & $\begin{array}{r}922 \\
917 \\
928 \\
926 \\
920 \\
913 \\
919 \\
918 \\
921 \\
930 \\
934 \\
929 \\
-\end{array}$ & $\begin{array}{r}587 \\
587 \\
590 \\
591 \\
591 \\
592 \\
592 \\
592 \\
592 \\
592 \\
592 \\
592 \\
-\end{array}$ & $\begin{array}{r}335 \\
330 \\
338 \\
335 \\
328 \\
322 \\
328 \\
326 \\
330 \\
339 \\
342 \\
337 \\
-\end{array}$ \\
\hline 1995 & $\begin{array}{l}\text { January .................... } \\
\text { February ................. } \\
\text { March ..................... } \\
\text { April" ...................... } \\
\text { 4-Mo. Average ....... }\end{array}$ & $\begin{array}{r}(s) \\
(s) \\
E_{(s)}^{(s)} \\
E_{(s)}\end{array}$ & $\begin{array}{r}-279 \\
-48 \\
R_{344} \\
E_{-105} \\
E_{-20}\end{array}$ & $\begin{array}{r}13,610 \\
13,367 \\
R_{13,478} \\
E_{13,930} \\
E_{13,599}\end{array}$ & $\begin{array}{r}113 \\
95 \\
\mathrm{R}_{68} \\
\mathrm{E}_{99} \\
\mathrm{E}_{93}\end{array}$ & $\begin{array}{r}7 \\
8 \\
R_{7} \\
E_{7} \\
E_{8}\end{array}$ & $\begin{array}{r}920 \\
919 \\
929 \\
\text { E } 926 \\
-\end{array}$ & $\begin{array}{r}592 \\
592 \\
592 \\
592 \\
-\end{array}$ & $\begin{array}{r}328 \\
327 \\
338 \\
335 \\
-\end{array}$ \\
\hline $\begin{array}{l}1994 \\
1993\end{array}$ & $\begin{array}{l}\text { 4-Mo. Average ...... } \\
\text { 4-Mo. Average ...... }\end{array}$ & $\begin{array}{l}34 \\
58\end{array}$ & $\begin{array}{r}-3 \\
255\end{array}$ & $\begin{array}{l}13,303 \\
13,138\end{array}$ & $\begin{array}{r}96 \\
126\end{array}$ & $\begin{array}{l}10 \\
10\end{array}$ & $\overline{-}$ & $\overline{-}$ & 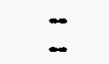 \\
\hline
\end{tabular}

Footnotes continued.

$R=$ Revised data. $(s)=$ Less than 500 barrels per day. $E=$ Estimated. $P E=$ Preliminary estimate. $R E=R e v i s e d$ estimate.

SPR = Strategic Petroleum Reserve.

See Summary Statistics Explanatory Note 1

Notes: - Crude oll includes lease condensate. - Italics denote estimates based upon preliminary data. $\bullet$ Geographic coverage is the 50 States and the District of Columbia. - Totals may not equal sum of components due to independent rounding.

Source: See Summary Statistics Table and Figure Sources. 
Table S3. Crude Oil and Petroleum Product Imports, 1981 - Present (Thousand Barrels per Day)

\begin{tabular}{|c|c|c|c|c|c|c|c|c|c|}
\hline & \multirow{3}{*}{ Year/Month } & \multicolumn{8}{|c|}{ Imports from Arab-OPEC Sources } \\
\hline & & \multicolumn{2}{|c|}{ Algeria } & \multicolumn{2}{|c|}{ Iraq } & \multicolumn{2}{|c|}{ Kuwait $^{b}$} & \multicolumn{2}{|c|}{ Libya } \\
\hline & & Total & Crude Oil & Total & Crude Oil & Total & Crude Oil & Total & Crude OIl \\
\hline $\begin{array}{l}1981 \\
1982 \\
1983 \\
1984 \\
1985 \\
1986 \\
1987 \\
1988 \\
1989 \\
1990 \\
1991 \\
1992\end{array}$ & 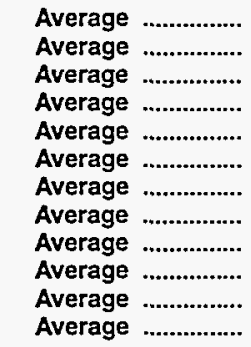 & $\begin{array}{l}311 \\
170 \\
240 \\
323 \\
187 \\
271 \\
295 \\
300 \\
269 \\
280 \\
253 \\
196\end{array}$ & $\begin{array}{r}261 \\
90 \\
176 \\
194 \\
84 \\
78 \\
115 \\
58 \\
60 \\
63 \\
44 \\
24\end{array}$ & $\begin{array}{r}(s) \\
3 \\
10 \\
12 \\
46 \\
81 \\
83 \\
345 \\
449 \\
518 \\
0 \\
0\end{array}$ & $\begin{array}{r}0 \\
3 \\
10 \\
12 \\
46 \\
81 \\
82 \\
343 \\
441 \\
514 \\
0 \\
0\end{array}$ & $\begin{array}{r}0 \\
5 \\
14 \\
36 \\
21 \\
68 \\
84 \\
92 \\
157 \\
86 \\
6 \\
51\end{array}$ & $\begin{array}{r}0 \\
2 \\
7 \\
24 \\
4 \\
28 \\
70 \\
80 \\
155 \\
79 \\
6 \\
39\end{array}$ & $\begin{array}{r}319 \\
26 \\
0 \\
1 \\
4 \\
0 \\
0 \\
0 \\
0 \\
0 \\
0 \\
0\end{array}$ & $\begin{array}{r}317 \\
23 \\
0 \\
0 \\
0 \\
0 \\
0 \\
0 \\
0 \\
0 \\
0 \\
0\end{array}$ \\
\hline 1993 & 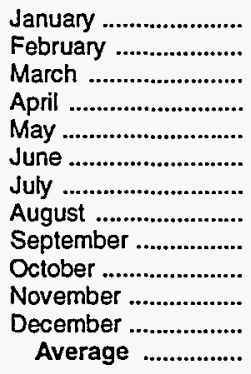 & $\begin{array}{l}153 \\
256 \\
185 \\
258 \\
228 \\
169 \\
246 \\
241 \\
192 \\
317 \\
222 \\
169 \\
220\end{array}$ & $\begin{array}{r}28 \\
0 \\
7 \\
26 \\
3 \\
32 \\
6 \\
28 \\
0 \\
80 \\
52 \\
25 \\
24\end{array}$ & $\begin{array}{l}0 \\
0 \\
0 \\
0 \\
0 \\
0 \\
0 \\
0 \\
0 \\
0 \\
0 \\
0 \\
0\end{array}$ & $\begin{array}{l}0 \\
0 \\
0 \\
0 \\
0 \\
0 \\
0 \\
0 \\
0 \\
0 \\
0 \\
0 \\
0\end{array}$ & $\begin{array}{l}144 \\
251 \\
316 \\
279 \\
222 \\
235 \\
368 \\
467 \\
445 \\
530 \\
486 \\
484 \\
353\end{array}$ & $\begin{array}{l}129 \\
229 \\
300 \\
279 \\
222 \\
235 \\
362 \\
451 \\
431 \\
526 \\
470 \\
484 \\
344\end{array}$ & $\begin{array}{l}0 \\
0 \\
0 \\
0 \\
0 \\
0 \\
0 \\
0 \\
0 \\
0 \\
0 \\
0 \\
0\end{array}$ & $\begin{array}{l}0 \\
0 \\
0 \\
0 \\
0 \\
0 \\
0 \\
0 \\
0 \\
0 \\
0 \\
0 \\
0\end{array}$ \\
\hline 1994 & 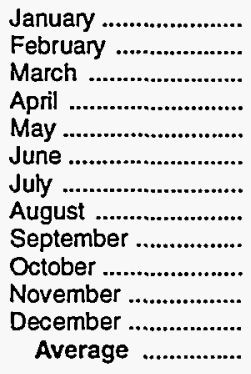 & $\begin{array}{l}233 \\
226 \\
278 \\
245 \\
261 \\
178 \\
301 \\
282 \\
237 \\
217 \\
203 \\
259 \\
244\end{array}$ & $\begin{array}{r}35 \\
20 \\
22 \\
30 \\
0 \\
2 \\
38 \\
39 \\
20 \\
38 \\
20 \\
39 \\
25\end{array}$ & $\begin{array}{l}0 \\
0 \\
0 \\
0 \\
0 \\
0 \\
0 \\
0 \\
0 \\
0 \\
0 \\
0 \\
0\end{array}$ & $\begin{array}{l}0 \\
0 \\
0 \\
0 \\
0 \\
0 \\
0 \\
0 \\
0 \\
0 \\
0 \\
0 \\
0\end{array}$ & $\begin{array}{l}309 \\
423 \\
476 \\
261 \\
362 \\
255 \\
345 \\
306 \\
361 \\
165 \\
249 \\
240 \\
312\end{array}$ & $\begin{array}{l}309 \\
423 \\
476 \\
238 \\
362 \\
255 \\
345 \\
306 \\
361 \\
148 \\
240 \\
227 \\
307\end{array}$ & $\begin{array}{l}0 \\
0 \\
0 \\
0 \\
0 \\
0 \\
0 \\
0 \\
0 \\
0 \\
0 \\
0 \\
0\end{array}$ & $\begin{array}{l}0 \\
0 \\
0 \\
0 \\
0 \\
0 \\
0 \\
0 \\
0 \\
0 \\
0 \\
0 \\
0\end{array}$ \\
\hline 1995 & 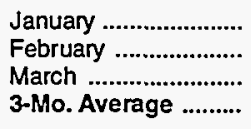 & $\begin{array}{l}168 \\
358 \\
196 \\
237\end{array}$ & $\begin{array}{r}0 \\
64 \\
19 \\
27\end{array}$ & $\begin{array}{l}0 \\
0 \\
0 \\
0\end{array}$ & $\begin{array}{l}0 \\
0 \\
0 \\
0\end{array}$ & $\begin{array}{l}130 \\
346 \\
252 \\
239\end{array}$ & $\begin{array}{l}120 \\
324 \\
252 \\
229\end{array}$ & $\begin{array}{l}0 \\
0 \\
0 \\
0\end{array}$ & $\begin{array}{l}0 \\
0 \\
0 \\
0\end{array}$ \\
\hline $\begin{array}{l}1994 \\
1993\end{array}$ & $\begin{array}{l}\text { 3-Mo. Average ......... } \\
\text { 3-Mo. Average ......... }\end{array}$ & $\begin{array}{l}246 \\
196\end{array}$ & $\begin{array}{l}26 \\
12\end{array}$ & $\begin{array}{l}0 \\
0\end{array}$ & $\begin{array}{l}0 \\
0\end{array}$ & $\begin{array}{l}402 \\
237\end{array}$ & $\begin{array}{l}402 \\
219\end{array}$ & $\begin{array}{l}0 \\
0\end{array}$ & $\begin{array}{l}0 \\
0\end{array}$ \\
\hline
\end{tabular}

See footnotes at end of table. 
Table S3. Crude Oil and Petroleum Product Imports, 1981 - Present (Continued) (Thousand Barrels per Day)

\begin{tabular}{|c|c|c|c|c|c|c|c|c|c|}
\hline & \multirow{3}{*}{ Year/Month } & \multicolumn{8}{|c|}{ Imports from Arab-OPEC Sources } \\
\hline & & \multicolumn{2}{|c|}{ Qatar } & \multicolumn{2}{|c|}{$\begin{array}{c}\text { Saudi } \\
\text { Arabiab }^{b}\end{array}$} & \multicolumn{2}{|c|}{$\begin{array}{c}\text { United } \\
\text { Arab } \\
\text { Emirates }\end{array}$} & \multicolumn{2}{|c|}{$\begin{array}{l}\text { Total } \\
\text { Arab } \\
\text { OPEC }\end{array}$} \\
\hline & & Total & Crude Oll & Total & Crude Oil & Total & Crude Oil & Total & Crude Oil \\
\hline $\begin{array}{l}1981 \\
1982 \\
1983 \\
1984 \\
1985 \\
1986 \\
1987 \\
1988 \\
1989 \\
1990 \\
1991 \\
1992\end{array}$ & $\begin{array}{l}\text { Average } \\
\text { Average }\end{array}$................. & $\begin{array}{r}7 \\
7 \\
(s) \\
5 \\
(s) \\
13 \\
0 \\
0 \\
2 \\
4 \\
0 \\
1\end{array}$ & $\begin{array}{r}7 \\
7 \\
0 \\
4 \\
0 \\
12 \\
0 \\
0 \\
2 \\
4 \\
0 \\
0\end{array}$ & $\begin{array}{r}1,129 \\
552 \\
337 \\
325 \\
168 \\
685 \\
751 \\
1,073 \\
1,224 \\
1,339 \\
1,802 \\
1,720\end{array}$ & $\begin{array}{r}1,112 \\
530 \\
321 \\
309 \\
132 \\
618 \\
642 \\
911 \\
1,116 \\
1,195 \\
1,703 \\
1,597\end{array}$ & $\begin{array}{r}81 \\
92 \\
30 \\
117 \\
45 \\
44 \\
61 \\
29 \\
28 \\
17 \\
3 \\
6\end{array}$ & $\begin{array}{r}77 \\
81 \\
18 \\
90 \\
35 \\
38 \\
56 \\
23 \\
21 \\
9 \\
2 \\
0\end{array}$ & $\begin{array}{r}1,848 \\
854 \\
632 \\
819 \\
472 \\
1,162 \\
1,274 \\
1,839 \\
2,130 \\
2,244 \\
2,064 \\
1,974 \\
\end{array}$ & $\begin{array}{r}1,774 \\
736 \\
533 \\
634 \\
300 \\
854 \\
965 \\
1,415 \\
1,794 \\
1,864 \\
1,754 \\
1,660\end{array}$ \\
\hline 1993 & 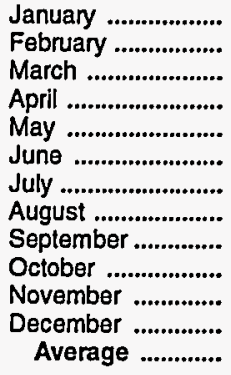 & $\begin{array}{l}0 \\
0 \\
6 \\
0 \\
0 \\
0 \\
0 \\
0 \\
0 \\
0 \\
0 \\
0 \\
1\end{array}$ & $\begin{array}{l}0 \\
0 \\
0 \\
0 \\
0 \\
0 \\
0 \\
0 \\
0 \\
0 \\
0 \\
0 \\
0\end{array}$ & $\begin{array}{l}1,688 \\
1,626 \\
1,479 \\
1,644 \\
1,524 \\
1,540 \\
1,283 \\
1,151 \\
1,329 \\
1,115 \\
1,281 \\
1,330 \\
1,414\end{array}$ & $\begin{array}{r}1,571 \\
1,480 \\
1,349 \\
1,515 \\
1,361 \\
1,413 \\
1,171 \\
1,036 \\
1,181 \\
969 \\
1,152 \\
1,205 \\
1,282\end{array}$ & $\begin{array}{r}0 \\
0 \\
0 \\
17 \\
59 \\
66 \\
19 \\
0 \\
0 \\
0 \\
1 \\
0 \\
14\end{array}$ & $\begin{array}{r}0 \\
0 \\
0 \\
17 \\
59 \\
66 \\
0 \\
0 \\
0 \\
0 \\
0 \\
0 \\
12\end{array}$ & $\begin{array}{l}1,984 \\
2,133 \\
1,987 \\
2,198 \\
2,034 \\
2,010 \\
1,917 \\
1,859 \\
1,966 \\
1,961 \\
1,989 \\
1,983 \\
2,000\end{array}$ & $\begin{array}{l}1,728 \\
1,709 \\
1,655 \\
1,837 \\
1,646 \\
1,746 \\
1,538 \\
1,515 \\
1,612 \\
1,574 \\
1,673 \\
1,713 \\
1,661\end{array}$ \\
\hline 1994 & 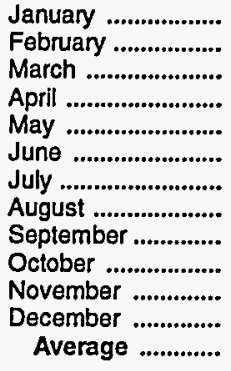 & $\begin{array}{l}0 \\
0 \\
0 \\
0 \\
0 \\
0 \\
0 \\
0 \\
0 \\
0 \\
0 \\
0 \\
0\end{array}$ & $\begin{array}{l}0 \\
0 \\
0 \\
0 \\
0 \\
0 \\
0 \\
0 \\
0 \\
0 \\
0 \\
0 \\
0\end{array}$ & $\begin{array}{l}1,320 \\
1,071 \\
1,128 \\
1,586 \\
1,438 \\
1,395 \\
1,414 \\
1,360 \\
1,486 \\
1,601 \\
1,477 \\
1,526 \\
1,402\end{array}$ & $\begin{array}{l}1,175 \\
1,023 \\
1,055 \\
1,428 \\
1,394 \\
1,277 \\
1,310 \\
1,271 \\
1,364 \\
1,500 \\
1,357 \\
1,388 \\
1,297\end{array}$ & $\begin{array}{r}0 \\
0 \\
0 \\
4 \\
0 \\
0 \\
53 \\
0 \\
40 \\
38 \\
0 \\
15 \\
13\end{array}$ & $\begin{array}{r}0 \\
0 \\
0 \\
0 \\
0 \\
0 \\
53 \\
0 \\
40 \\
23 \\
0 \\
15 \\
11\end{array}$ & $\begin{array}{l}1,863 \\
1,719 \\
1,883 \\
2,097 \\
2,062 \\
1,829 \\
2,113 \\
1,948 \\
2,125 \\
2,020 \\
1,929 \\
2,040 \\
1,971\end{array}$ & $\begin{array}{l}1,520 \\
1,467 \\
1,553 \\
1,696 \\
1,757 \\
1,535 \\
1,745 \\
1,615 \\
1,786 \\
1,709 \\
1,617 \\
1,669 \\
1,640\end{array}$ \\
\hline 1995 & $\begin{array}{l}\text { January .................. } \\
\text { February ................. } \\
\text { March .................. } \\
\text { 3-Mo. Average ....... }\end{array}$ & $\begin{array}{l}0 \\
0 \\
0 \\
0\end{array}$ & $\begin{array}{l}0 \\
0 \\
0 \\
0\end{array}$ & $\begin{array}{l}1,309 \\
1,181 \\
1,535 \\
1,347\end{array}$ & $\begin{array}{l}1,251 \\
1,134 \\
1,410 \\
1,269\end{array}$ & $\begin{array}{r}20 \\
13 \\
0 \\
11\end{array}$ & $\begin{array}{r}20 \\
13 \\
0 \\
11\end{array}$ & $\begin{array}{l}1,628 \\
1,897 \\
1,983 \\
1,834\end{array}$ & $\begin{array}{l}1,391 \\
1,535 \\
1,681 \\
1,536\end{array}$ \\
\hline $\begin{array}{l}1994 \\
1993\end{array}$ & $\begin{array}{l}\text { 3-Mo. Average ...... } \\
\text { 3-Mo. Average ...... }\end{array}$ & $\begin{array}{l}0 \\
2\end{array}$ & $\begin{array}{l}0 \\
0\end{array}$ & $\begin{array}{l}1,176 \\
1,597\end{array}$ & $\begin{array}{l}1,086 \\
1,466\end{array}$ & $\begin{array}{l}0 \\
0\end{array}$ & $\begin{array}{l}0 \\
0\end{array}$ & $\begin{array}{l}1,825 \\
2,032\end{array}$ & $\begin{array}{l}1,515 \\
1,697\end{array}$ \\
\hline
\end{tabular}

See footnotes at end of table. 
Table S3. Crude Oil and Petroleum Product Imports, 1981 - Present (Continued) (Thousand Barrels per Day)

\begin{tabular}{|c|c|c|c|c|c|c|c|c|c|}
\hline & \multirow{3}{*}{ Year/Month } & \multicolumn{8}{|c|}{ Imports from Other-OPEC Sources } \\
\hline & & \multicolumn{2}{|c|}{ Ecuador } & \multicolumn{2}{|c|}{ Gabon } & \multicolumn{2}{|c|}{ Indonesia } & \multicolumn{2}{|c|}{ Iran } \\
\hline & & Total & Crude Oil & Total & Crude Oll & Total & Crude Oll & Total & Crude OII \\
\hline $\begin{array}{l}1981 \\
1982 \\
1983 \\
1984 \\
1985 \\
1986 \\
1987 \\
1988 \\
1989 \\
1990 \\
1991 \\
1992\end{array}$ & $\begin{array}{l}\text { Average } \\
\text { Average }\end{array}$ & $\begin{array}{l}48 \\
42 \\
61 \\
55 \\
67 \\
77 \\
29 \\
47 \\
89 \\
49 \\
63 \\
65\end{array}$ & $\begin{array}{l}38 \\
32 \\
56 \\
47 \\
56 \\
64 \\
23 \\
33 \\
80 \\
38 \\
53 \\
62\end{array}$ & $\begin{array}{r}35 \\
40 \\
59 \\
58 \\
52 \\
26 \\
35 \\
16 \\
50 \\
64 \\
84 \\
124\end{array}$ & $\begin{array}{r}35 \\
40 \\
59 \\
57 \\
51 \\
25 \\
35 \\
15 \\
49 \\
64 \\
84 \\
123\end{array}$ & $\begin{array}{r}366 \\
248 \\
338 \\
343 \\
314 \\
318 \\
285 \\
205 \\
183 \\
114 \\
111 \\
78\end{array}$ & $\begin{array}{r}318 \\
226 \\
315 \\
304 \\
292 \\
297 \\
262 \\
186 \\
158 \\
98 \\
102 \\
70\end{array}$ & $\begin{array}{r}0 \\
35 \\
48 \\
10 \\
27 \\
19 \\
98 \\
\text { f } \\
\text { (s) } \\
0 \\
0 \\
32 \\
0\end{array}$ & $\begin{array}{r}0 \\
35 \\
48 \\
10 \\
27 \\
19 \\
98 \\
1(8) \\
0 \\
0 \\
32 \\
0\end{array}$ \\
\hline 1993 & 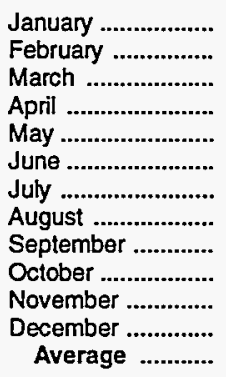 & $\begin{array}{r}76 \\
14 \\
59 \\
74 \\
56 \\
75 \\
96 \\
121 \\
49 \\
146 \\
115 \\
84 \\
81\end{array}$ & $\begin{array}{r}70 \\
14 \\
59 \\
62 \\
56 \\
75 \\
96 \\
121 \\
49 \\
135 \\
106 \\
84 \\
78\end{array}$ & $\begin{array}{r}90 \\
88 \\
126 \\
127 \\
169 \\
107 \\
168 \\
152 \\
211 \\
242 \\
143 \\
191 \\
152\end{array}$ & $\begin{array}{r}89 \\
88 \\
123 \\
127 \\
169 \\
107 \\
166 \\
152 \\
211 \\
242 \\
136 \\
191 \\
151\end{array}$ & $\begin{array}{r}37 \\
52 \\
67 \\
76 \\
82 \\
97 \\
55 \\
95 \\
51 \\
131 \\
74 \\
156 \\
81\end{array}$ & $\begin{array}{r}37 \\
51 \\
64 \\
76 \\
82 \\
67 \\
55 \\
80 \\
40 \\
82 \\
34 \\
114 \\
65\end{array}$ & $\begin{array}{l}0 \\
0 \\
0 \\
0 \\
0 \\
0 \\
0 \\
0 \\
0 \\
0 \\
0 \\
0 \\
0\end{array}$ & $\begin{array}{l}0 \\
0 \\
0 \\
0 \\
0 \\
0 \\
0 \\
0 \\
0 \\
0 \\
0 \\
0 \\
0\end{array}$ \\
\hline 1994 & 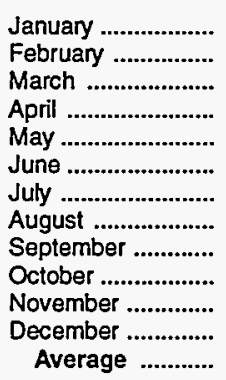 & $\begin{array}{l}\text { (c) } \\
\text { (c) } \\
\text { (c) } \\
(c) \\
\text { (c) } \\
(c) \\
(c) \\
(c) \\
(c) \\
(c) \\
(c) \\
\text { (c) }\end{array}$ & $\begin{array}{l}\text { (c) } \\
(c) \\
(c) \\
(c) \\
(c) \\
(c) \\
(c) \\
(c) \\
(c) \\
(c) \\
(c) \\
(c) \\
(c)\end{array}$ & $\begin{array}{r}144 \\
212 \\
91 \\
288 \\
187 \\
223 \\
216 \\
142 \\
194 \\
235 \\
254 \\
154 \\
194\end{array}$ & $\begin{array}{r}144 \\
208 \\
91 \\
288 \\
187 \\
223 \\
216 \\
142 \\
194 \\
235 \\
254 \\
154 \\
194\end{array}$ & $\begin{array}{r}140 \\
103 \\
112 \\
88 \\
94 \\
155 \\
196 \\
119 \\
61 \\
96 \\
71 \\
113 \\
113\end{array}$ & $\begin{array}{r}81 \\
59 \\
50 \\
88 \\
76 \\
155 \\
196 \\
112 \\
61 \\
89 \\
56 \\
95 \\
93\end{array}$ & $\begin{array}{l}0 \\
0 \\
0 \\
0 \\
0 \\
0 \\
0 \\
0 \\
0 \\
0 \\
0 \\
0 \\
0\end{array}$ & $\begin{array}{l}0 \\
0 \\
0 \\
0 \\
0 \\
0 \\
0 \\
0 \\
0 \\
0 \\
0 \\
0 \\
0\end{array}$ \\
\hline 1995 & $\begin{array}{l}\text { January .............. } \\
\text { February ............... } \\
\text { March ................. } \\
\text { 3-Mo. Average ...... }\end{array}$ & $\begin{array}{l}(c) \\
(c) \\
(c) \\
(c)\end{array}$ & $\begin{array}{l}\text { (c) } \\
(c) \\
\text { (c) } \\
\text { (c) }\end{array}$ & $\begin{array}{l}224 \\
186 \\
159 \\
190\end{array}$ & $\begin{array}{l}224 \\
186 \\
159 \\
190\end{array}$ & $\begin{array}{r}38 \\
129 \\
51 \\
71\end{array}$ & $\begin{array}{l}38 \\
87 \\
29 \\
50\end{array}$ & $\begin{array}{l}0 \\
0 \\
0 \\
0\end{array}$ & $\begin{array}{l}0 \\
0 \\
0 \\
0\end{array}$ \\
\hline $\begin{array}{l}1994 \\
1993\end{array}$ & $\begin{array}{l}\text { 3-Mo. Average ..... } \\
\text { 3-Mo. Average ..... }\end{array}$ & $\begin{array}{l}\text { (c) } \\
51\end{array}$ & $\begin{array}{l}\text { (c) } \\
49\end{array}$ & $\begin{array}{l}147 \\
102\end{array}$ & $\begin{array}{l}146 \\
100\end{array}$ & $\begin{array}{r}119 \\
52\end{array}$ & $\begin{array}{l}63 \\
51\end{array}$ & $\begin{array}{l}0 \\
0\end{array}$ & $\begin{array}{l}\mathbf{0} \\
\mathbf{0}\end{array}$ \\
\hline
\end{tabular}

See footnotes at end of table. 
Table S3. Crude Oil and Petroleum Product Imports, 1981 - Present (Continued)

(Thousand Barrels per Day)

\begin{tabular}{|c|c|c|c|c|c|c|c|c|c|}
\hline & \multirow{3}{*}{ Year/Month } & \multicolumn{6}{|c|}{ Imports from Other-OPEC Sources } & \multirow{2}{*}{\multicolumn{2}{|c|}{$\begin{array}{c}\text { Total } \\
\text { OPEC } \\
\text { OP,d }\end{array}$}} \\
\hline & & \multicolumn{2}{|c|}{ Nigeria } & \multicolumn{2}{|c|}{ Venezuela } & \multicolumn{2}{|c|}{$\begin{array}{l}\text { Total } \\
\text { Other } \\
\text { OPEC }\end{array}$} & & \\
\hline & & Total & Crude Oil & Total & Crude Oil & Total & Crude Oil & Total & Crude Oil \\
\hline $\begin{array}{l}1981 \\
1982 \\
1983 \\
1984 \\
1985 \\
1986 \\
1987 \\
1988 \\
1989 \\
1990 \\
1991 \\
1992\end{array}$ & 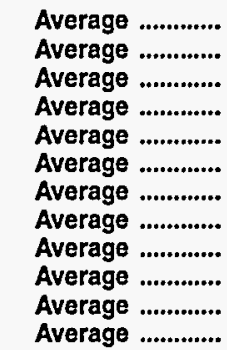 & $\begin{array}{l}620 \\
514 \\
302 \\
216 \\
293 \\
440 \\
535 \\
618 \\
815 \\
800 \\
703 \\
681\end{array}$ & $\begin{array}{l}611 \\
510 \\
301 \\
207 \\
280 \\
437 \\
529 \\
607 \\
800 \\
784 \\
683 \\
665\end{array}$ & $\begin{array}{r}406 \\
412 \\
422 \\
548 \\
605 \\
793 \\
804 \\
794 \\
873 \\
1,025 \\
1,035 \\
1,170\end{array}$ & $\begin{array}{l}147 \\
155 \\
164 \\
253 \\
306 \\
416 \\
488 \\
439 \\
495 \\
666 \\
668 \\
826\end{array}$ & $\begin{array}{l}1,476 \\
1,291 \\
1,231 \\
1,230 \\
1,358 \\
1,674 \\
1,787 \\
1,681 \\
2,010 \\
2,052 \\
2,028 \\
2,117\end{array}$ & $\begin{array}{r}1,149 \\
998 \\
944 \\
878 \\
1,012 \\
1,259 \\
1,435 \\
1,281 \\
1,582 \\
1,650 \\
1,622 \\
1,746\end{array}$ & $\begin{array}{l}3,323 \\
2,146 \\
1,862 \\
2,049 \\
1,830 \\
2,837 \\
3,060 \\
3,520 \\
4,140 \\
4,296 \\
4,092 \\
4,092\end{array}$ & $\begin{array}{l}2,922 \\
1,734 \\
1,477 \\
1,512 \\
1,312 \\
2,113 \\
2,400 \\
2,696 \\
3,376 \\
3,514 \\
3,377 \\
3,406\end{array}$ \\
\hline 1993 & 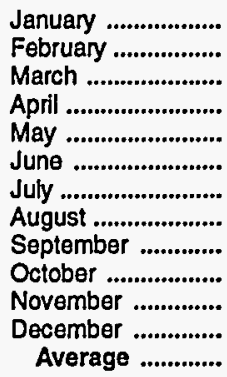 & $\begin{array}{l}729 \\
927 \\
928 \\
892 \\
760 \\
848 \\
893 \\
562 \\
514 \\
603 \\
636 \\
598 \\
740\end{array}$ & $\begin{array}{l}729 \\
913 \\
892 \\
871 \\
741 \\
827 \\
888 \\
549 \\
496 \\
593 \\
612 \\
569 \\
722\end{array}$ & $\begin{array}{l}1,397 \\
1,296 \\
1,173 \\
1,314 \\
1,264 \\
1,292 \\
1,384 \\
1,383 \\
1,273 \\
1,276 \\
1,322 \\
1,230 \\
1,300\end{array}$ & $\begin{array}{r}1,038 \\
925 \\
835 \\
1,023 \\
992 \\
999 \\
1,068 \\
1,135 \\
1,050 \\
993 \\
1,108 \\
952 \\
1,010\end{array}$ & $\begin{array}{l}2,330 \\
2,377 \\
2,354 \\
2,483 \\
2,331 \\
2,418 \\
2,596 \\
2,313 \\
2,097 \\
2,398 \\
2,290 \\
2,260 \\
2,354\end{array}$ & $\begin{array}{l}1,962 \\
1,990 \\
1,973 \\
2,158 \\
2,040 \\
2,075 \\
2,273 \\
2,037 \\
1,845 \\
2,045 \\
1,997 \\
1,910 \\
2,026\end{array}$ & $\begin{array}{l}4,314 \\
4,510 \\
4,341 \\
4,682 \\
4,365 \\
4,428 \\
4,513 \\
4,172 \\
4,063 \\
4,359 \\
4,279 \\
4,242 \\
4,354\end{array}$ & $\begin{array}{l}3,690 \\
3,699 \\
3,628 \\
3,995 \\
3,686 \\
3,821 \\
3,811 \\
3,552 \\
3,457 \\
3,619 \\
3,670 \\
3,624 \\
3,687\end{array}$ \\
\hline 1994 & 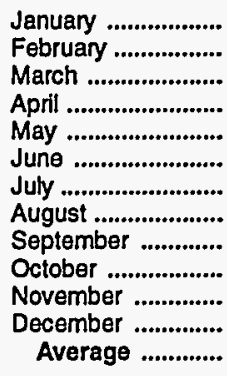 & $\begin{array}{r}310 \\
576 \\
441 \\
631 \\
732 \\
842 \\
703 \\
1,037 \\
578 \\
569 \\
485 \\
739 \\
637\end{array}$ & $\begin{array}{r}274 \\
557 \\
402 \\
621 \\
730 \\
837 \\
694 \\
1,010 \\
578 \\
559 \\
478 \\
739 \\
624\end{array}$ & $\begin{array}{l}1,185 \\
1,204 \\
1,219 \\
1,272 \\
1,297 \\
1,449 \\
1,298 \\
1,241 \\
1,410 \\
1,385 \\
1,433 \\
1,405 \\
1,317\end{array}$ & $\begin{array}{r}901 \\
946 \\
915 \\
1,016 \\
1,004 \\
1,088 \\
1,030 \\
992 \\
1,106 \\
1,101 \\
1,085 \\
1,183 \\
1,031\end{array}$ & $\begin{array}{l}1,780 \\
2,094 \\
1,862 \\
2,280 \\
2,309 \\
2,669 \\
2,413 \\
2,539 \\
2,243 \\
2,284 \\
2,243 \\
2,411 \\
2,261\end{array}$ & $\begin{array}{l}1,400 \\
1,770 \\
1,457 \\
2,014 \\
1,996 \\
2,303 \\
2,136 \\
2,255 \\
1,939 \\
1,984 \\
1,873 \\
2,171 \\
1,942\end{array}$ & $\begin{array}{l}3,643 \\
3,814 \\
3,745 \\
4,377 \\
4,371 \\
4,498 \\
4,525 \\
4,487 \\
4,368 \\
4,304 \\
4,172 \\
4,451 \\
4,232\end{array}$ & $\begin{array}{l}2,920 \\
3,237 \\
3,010 \\
3,710 \\
3,753 \\
3,838 \\
3,881 \\
3,870 \\
3,725 \\
3,693 \\
3,490 \\
3,840 \\
3,582\end{array}$ \\
\hline 1995 & $\begin{array}{l}\text { January .................. } \\
\text { February .................... } \\
\text { March ................... } \\
\text { 3-Mo. Average ...... }\end{array}$ & $\begin{array}{l}583 \\
463 \\
687 \\
581\end{array}$ & $\begin{array}{l}575 \\
463 \\
676 \\
575\end{array}$ & $\begin{array}{l}1,355 \\
1,439 \\
1,499 \\
1,431\end{array}$ & $\begin{array}{l}1,059 \\
1,083 \\
1,209 \\
1,118\end{array}$ & $\begin{array}{l}2,201 \\
2,217 \\
2,396 \\
2,273\end{array}$ & $\begin{array}{l}1,897 \\
1,819 \\
2,073 \\
1,933\end{array}$ & $\begin{array}{l}3,828 \\
4,114 \\
4,379 \\
4,107\end{array}$ & $\begin{array}{l}3,288 \\
3,354 \\
3,754 \\
3,469\end{array}$ \\
\hline $\begin{array}{l}1994 \\
1993\end{array}$ & $\begin{array}{l}\text { 3-Mo. Average ...... } \\
\text { 3-Mo. Average ...... }\end{array}$ & $\begin{array}{l}438 \\
859\end{array}$ & $\begin{array}{l}406 \\
842\end{array}$ & $\begin{array}{l}1,203 \\
1,289\end{array}$ & $\begin{array}{l}920 \\
933\end{array}$ & $\begin{array}{l}1,906 \\
2,353\end{array}$ & $\begin{array}{l}1,535 \\
1,974\end{array}$ & $\begin{array}{l}3,731 \\
4,384\end{array}$ & $\begin{array}{l}3,050 \\
3,672\end{array}$ \\
\hline
\end{tabular}

See footnotes at end of table. 
Table S3. Crude Oil and Petroleum Product Imports, 1981 - Present (Continued) (Thousand Barrels per Day)

\begin{tabular}{|c|c|c|c|c|c|c|c|c|c|c|c|c|c|}
\hline & \multirow{3}{*}{ Year/Month } & \multicolumn{12}{|c|}{ Imports from Non-OPEC Sources ${ }^{a}$} \\
\hline & & \multicolumn{2}{|c|}{ Angola } & \multicolumn{2}{|c|}{ Australia } & \multicolumn{2}{|c|}{$\begin{array}{l}\text { Bahama } \\
\text { Islands }\end{array}$} & \multicolumn{2}{|c|}{ Brazil } & \multicolumn{2}{|c|}{ Canada } & \multicolumn{2}{|c|}{$\begin{array}{c}\text { China, } \\
\text { People's } \\
\text { Republic of }\end{array}$} \\
\hline & & Total & Crude Oil & Total & Crude Oil & Total & Crude Oil & Total & Crude Oil & Total & Crude Oll & Total & Crude Oil \\
\hline $\begin{array}{l}1981 \\
1982 \\
1983 \\
1984 \\
1985 \\
1986 \\
1987 \\
1988 \\
1989 \\
1990 \\
1991 \\
1992\end{array}$ & $\begin{array}{l}\text { Average } \\
\text { Average } \\
\text { Average }\end{array}$................... & $\begin{array}{r}49 \\
44 \\
78 \\
90 \\
110 \\
112 \\
192 \\
212 \\
284 \\
237 \\
254 \\
336\end{array}$ & $\begin{array}{r}45 \\
42 \\
71 \\
85 \\
104 \\
102 \\
180 \\
203 \\
279 \\
236 \\
254 \\
336\end{array}$ & $\begin{array}{r}5 \\
5 \\
4 \\
38 \\
37 \\
41 \\
58 \\
64 \\
36 \\
53 \\
26 \\
19\end{array}$ & $\begin{array}{r}0 \\
(s) \\
0 \\
25 \\
21 \\
30 \\
49 \\
59 \\
31 \\
47 \\
21 \\
17\end{array}$ & $\begin{array}{r}74 \\
65 \\
125 \\
88 \\
40 \\
37 \\
37 \\
32 \\
34 \\
37 \\
35 \\
36\end{array}$ & $\begin{array}{l}0 \\
0 \\
0 \\
0 \\
0 \\
0 \\
0 \\
0 \\
0 \\
0 \\
0 \\
0\end{array}$ & $\begin{array}{l}23 \\
47 \\
41 \\
60 \\
61 \\
50 \\
84 \\
98 \\
82 \\
49 \\
22 \\
20\end{array}$ & $\begin{array}{r}14 \\
19 \\
2 \\
(5) \\
0 \\
0 \\
0 \\
0 \\
0 \\
0 \\
0 \\
0\end{array}$ & $\begin{array}{r}447 \\
482 \\
547 \\
630 \\
770 \\
807 \\
848 \\
999 \\
931 \\
934 \\
1,033 \\
1,069\end{array}$ & $\begin{array}{l}164 \\
214 \\
274 \\
341 \\
468 \\
570 \\
608 \\
681 \\
630 \\
643 \\
743 \\
797\end{array}$ & $\begin{array}{l}18 \\
40 \\
34 \\
46 \\
59 \\
90 \\
82 \\
88 \\
80 \\
80 \\
91 \\
90\end{array}$ & $\begin{array}{r}0 \\
8 \\
6 \\
15 \\
36 \\
68 \\
63 \\
82 \\
76 \\
77 \\
87 \\
84\end{array}$ \\
\hline 1993 & 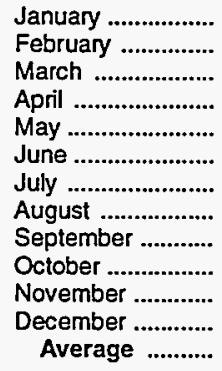 & $\begin{array}{l}354 \\
348 \\
408 \\
344 \\
299 \\
209 \\
402 \\
258 \\
282 \\
440 \\
307 \\
379 \\
336\end{array}$ & $\begin{array}{l}354 \\
348 \\
408 \\
344 \\
299 \\
209 \\
402 \\
258 \\
282 \\
440 \\
307 \\
379 \\
336\end{array}$ & $\begin{array}{r}(s) \\
0 \\
0 \\
0 \\
13 \\
34 \\
40 \\
33 \\
0 \\
53 \\
0 \\
53 \\
19\end{array}$ & $\begin{array}{r}0 \\
0 \\
0 \\
0 \\
13 \\
34 \\
40 \\
27 \\
0 \\
47 \\
0 \\
53 \\
18\end{array}$ & $\begin{array}{r}18 \\
26 \\
38 \\
16 \\
8 \\
7 \\
31 \\
41 \\
37 \\
53 \\
29 \\
30 \\
28\end{array}$ & $\begin{array}{l}0 \\
0 \\
0 \\
0 \\
0 \\
0 \\
0 \\
0 \\
0 \\
0 \\
0 \\
0 \\
0\end{array}$ & $\begin{array}{r}3 \\
22 \\
27 \\
56 \\
41 \\
19 \\
48 \\
32 \\
59 \\
15 \\
61 \\
10 \\
33\end{array}$ & $\begin{array}{l}0 \\
0 \\
0 \\
0 \\
0 \\
0 \\
0 \\
0 \\
0 \\
0 \\
0 \\
0 \\
0\end{array}$ & $\begin{array}{l}1,052 \\
1,095 \\
1,033 \\
1,052 \\
1,128 \\
1,117 \\
1,264 \\
1,247 \\
1,319 \\
1,370 \\
1,236 \\
1,255 \\
1,181\end{array}$ & $\begin{array}{r}778 \\
782 \\
770 \\
783 \\
874 \\
911 \\
991 \\
966 \\
1,023 \\
1,030 \\
917 \\
964 \\
900\end{array}$ & $\begin{array}{r}60 \\
44 \\
79 \\
0 \\
40 \\
48 \\
24 \\
38 \\
91 \\
61 \\
68 \\
61 \\
51\end{array}$ & $\begin{array}{r}60 \\
44 \\
73 \\
0 \\
40 \\
46 \\
24 \\
38 \\
89 \\
61 \\
68 \\
61 \\
50\end{array}$ \\
\hline 1994 & 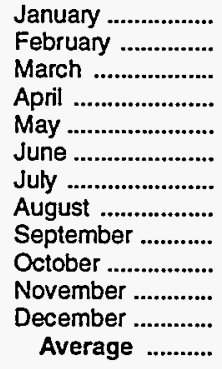 & $\begin{array}{l}338 \\
295 \\
291 \\
284 \\
354 \\
278 \\
304 \\
358 \\
455 \\
286 \\
328 \\
402 \\
331\end{array}$ & $\begin{array}{l}338 \\
282 \\
265 \\
284 \\
331 \\
278 \\
299 \\
347 \\
448 \\
286 \\
328 \\
380 \\
322 \\
\end{array}$ & $\begin{array}{r}12 \\
0 \\
11 \\
0 \\
32 \\
11 \\
44 \\
13 \\
35 \\
22 \\
22 \\
0 \\
17\end{array}$ & $\begin{array}{r}0 \\
0 \\
11 \\
0 \\
32 \\
11 \\
44 \\
13 \\
35 \\
22 \\
22 \\
0 \\
16\end{array}$ & $\begin{array}{r}28 \\
79 \\
52 \\
39 \\
58 \\
14 \\
18 \\
20 \\
17 \\
15 \\
8 \\
6 \\
29\end{array}$ & $\begin{array}{l}0 \\
0 \\
0 \\
0 \\
0 \\
0 \\
0 \\
0 \\
0 \\
0 \\
0 \\
0 \\
0\end{array}$ & $\begin{array}{r}11 \\
12 \\
10 \\
42 \\
96 \\
62 \\
53 \\
38 \\
21 \\
18 \\
0 \\
8 \\
31\end{array}$ & $\begin{array}{l}0 \\
0 \\
0 \\
0 \\
0 \\
0 \\
0 \\
0 \\
0 \\
0 \\
0 \\
8 \\
1\end{array}$ & $\begin{array}{l}1,234 \\
1,364 \\
1,328 \\
1,191 \\
1,157 \\
1,202 \\
1,224 \\
1,350 \\
1,151 \\
1,092 \\
1,096 \\
1,386 \\
1,231\end{array}$ & $\begin{array}{r}905 \\
994 \\
987 \\
930 \\
905 \\
973 \\
984 \\
1,056 \\
886 \\
839 \\
844 \\
1,054 \\
946\end{array}$ & $\begin{array}{r}81 \\
44 \\
107 \\
70 \\
80 \\
37 \\
92 \\
64 \\
63 \\
18 \\
79 \\
40 \\
65\end{array}$ & $\begin{array}{r}78 \\
44 \\
104 \\
67 \\
80 \\
36 \\
92 \\
64 \\
63 \\
18 \\
79 \\
40 \\
64\end{array}$ \\
\hline 1995 & $\begin{array}{l}\text { January ............... } \\
\text { February ............... } \\
\text { March ................. } \\
\text { 3-Mo. Average .... }\end{array}$ & $\begin{array}{l}273 \\
348 \\
427 \\
349\end{array}$ & $\begin{array}{l}262 \\
335 \\
416 \\
338\end{array}$ & $\begin{array}{r}21 \\
22 \\
0 \\
14\end{array}$ & $\begin{array}{r}21 \\
22 \\
0 \\
14\end{array}$ & $\begin{array}{l}6 \\
8 \\
7 \\
7\end{array}$ & $\begin{array}{l}0 \\
0 \\
0 \\
0\end{array}$ & $\begin{array}{l}0 \\
0 \\
0 \\
0\end{array}$ & $\begin{array}{l}0 \\
0 \\
0 \\
0\end{array}$ & $\begin{array}{l}1,349 \\
1,310 \\
1,206 \\
1,288\end{array}$ & $\begin{array}{r}1,009 \\
965 \\
891 \\
955\end{array}$ & $\begin{array}{l}64 \\
21 \\
54 \\
47\end{array}$ & $\begin{array}{l}62 \\
21 \\
54 \\
46\end{array}$ \\
\hline $\begin{array}{l}1994 \\
1993\end{array}$ & $\begin{array}{l}\text { 3-Mo. Average .... } \\
\text { 3-Mo. Average .... }\end{array}$ & $\begin{array}{l}308 \\
371\end{array}$ & $\begin{array}{l}296 \\
371\end{array}$ & $\begin{array}{r}8 \\
(s)\end{array}$ & $\begin{array}{l}4 \\
0\end{array}$ & $\begin{array}{l}52 \\
27\end{array}$ & $\begin{array}{l}0 \\
0\end{array}$ & $\begin{array}{l}11 \\
17\end{array}$ & $\begin{array}{l}0 \\
0\end{array}$ & $\begin{array}{l}1,307 \\
1,059\end{array}$ & $\begin{array}{l}961 \\
777\end{array}$ & $\begin{array}{l}79 \\
62\end{array}$ & $\begin{array}{l}77 \\
60\end{array}$ \\
\hline
\end{tabular}


Table S3. Crude Oil and Petroleum Product Imports, 1981 - Present (Continued) (Thousand Barrels per Day)

\begin{tabular}{|c|c|c|c|c|c|c|c|c|c|c|c|c|c|}
\hline & \multirow{3}{*}{ Year/Month } & \multicolumn{12}{|c|}{ Imports from Non-OPEC Sources ${ }^{a}$} \\
\hline & & \multicolumn{2}{|c|}{ Colombia } & \multicolumn{2}{|c|}{ Ecuadore } & \multicolumn{2}{|c|}{ Italy } & \multicolumn{2}{|c|}{ Malaysia } & \multicolumn{2}{|c|}{ Mexico } & \multicolumn{2}{|c|}{ Netherlands } \\
\hline & & Total & Crude Oil & Total & Crude Oil & Total & Crude Oil & Total & Crude Oil & Total & Crude Oil & Total & Crude Oil \\
\hline $\begin{array}{l}1981 \\
1982 \\
1983 \\
1984 \\
1985 \\
1986 \\
1987 \\
1988 \\
1989 \\
1990 \\
1991 \\
1992\end{array}$ & 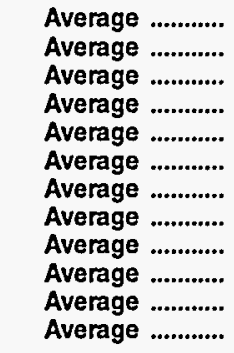 & $\begin{array}{r}1 \\
5 \\
10 \\
8 \\
23 \\
87 \\
148 \\
134 \\
172 \\
182 \\
163 \\
126\end{array}$ & $\begin{array}{r}0 \\
0 \\
0 \\
0 \\
0 \\
57 \\
115 \\
106 \\
136 \\
140 \\
123 \\
102\end{array}$ & $\begin{array}{l}- \\
\overline{-} \\
\overline{-} \\
\overline{-} \\
\overline{-} \\
\overline{-} \\
\overline{-}\end{array}$ & $\begin{array}{l}- \\
- \\
- \\
- \\
- \\
\overline{-} \\
\overline{-} \\
-\end{array}$ & $\begin{array}{l}11 \\
18 \\
18 \\
45 \\
60 \\
76 \\
54 \\
65 \\
34 \\
58 \\
47 \\
55\end{array}$ & $\begin{array}{r}0 \\
\text { (s) } \\
\text { (s) } \\
(\mathrm{s}) \\
(\mathrm{s}) \\
0 \\
1 \\
5 \\
3 \\
2 \\
3 \\
0\end{array}$ & $\begin{array}{r}36 \\
20 \\
4 \\
1 \\
3 \\
12 \\
13 \\
19 \\
39 \\
41 \\
24 \\
10\end{array}$ & $\begin{array}{r}33 \\
18 \\
3 \\
0 \\
1 \\
11 \\
12 \\
19 \\
39 \\
40 \\
24 \\
10\end{array}$ & $\begin{array}{l}522 \\
685 \\
826 \\
748 \\
816 \\
699 \\
655 \\
747 \\
767 \\
755 \\
807 \\
830\end{array}$ & $\begin{array}{l}469 \\
645 \\
766 \\
659 \\
715 \\
621 \\
602 \\
674 \\
716 \\
689 \\
759 \\
787\end{array}$ & $\begin{array}{l}30 \\
35 \\
65 \\
65 \\
58 \\
54 \\
60 \\
61 \\
49 \\
55 \\
29 \\
26\end{array}$ & $\begin{array}{r}\text { (s) } \\
\text { (s) } \\
3 \\
3 \\
0 \\
0 \\
0 \\
0 \\
0 \\
0 \\
0 \\
0\end{array}$ \\
\hline 1993 & $\begin{array}{l}\text { January .................. } \\
\text { February ................ } \\
\text { March .................... } \\
\text { April ..................... } \\
\text { May ........................ } \\
\text { June ..................... } \\
\text { July ..................... } \\
\text { August ................... } \\
\text { September ............ } \\
\text { October ................. } \\
\text { November ............. } \\
\text { December .............. } \\
\text { Average ........... }\end{array}$ & $\begin{array}{l}188 \\
148 \\
161 \\
178 \\
147 \\
176 \\
204 \\
131 \\
224 \\
192 \\
164 \\
134 \\
171\end{array}$ & $\begin{array}{r}167 \\
137 \\
129 \\
165 \\
90 \\
143 \\
184 \\
101 \\
170 \\
182 \\
143 \\
85 \\
141\end{array}$ & $\begin{array}{l}- \\
\overline{-} \\
\overline{-} \\
\overline{-} \\
\overline{-} \\
\overline{-} \\
-\end{array}$ & $\begin{array}{l}- \\
\overline{-} \\
\overline{-} \\
\overline{-} \\
\overline{-} \\
\overline{-} \\
\overline{-} \\
-\end{array}$ & $\begin{array}{r}56 \\
34 \\
43 \\
14 \\
26 \\
25 \\
25 \\
50 \\
32 \\
40 \\
30 \\
0 \\
31\end{array}$ & $\begin{array}{l}0 \\
0 \\
0 \\
0 \\
0 \\
0 \\
0 \\
0 \\
0 \\
0 \\
0 \\
0 \\
0\end{array}$ & $\begin{array}{r}0 \\
0 \\
11 \\
8 \\
21 \\
0 \\
11 \\
14 \\
28 \\
14 \\
0 \\
28 \\
11\end{array}$ & $\begin{array}{r}0 \\
0 \\
10 \\
8 \\
10 \\
0 \\
11 \\
14 \\
28 \\
10 \\
0 \\
28 \\
10\end{array}$ & $\begin{array}{r}858 \\
807 \\
844 \\
832 \\
917 \\
987 \\
943 \\
862 \\
929 \\
1,013 \\
1,116 \\
909 \\
919\end{array}$ & $\begin{array}{r}820 \\
748 \\
798 \\
796 \\
846 \\
959 \\
878 \\
809 \\
867 \\
951 \\
1,041 \\
837 \\
863\end{array}$ & $\begin{array}{r}11 \\
18 \\
10 \\
0 \\
10 \\
10 \\
21 \\
17 \\
22 \\
0 \\
(s) \\
6 \\
10\end{array}$ & $\begin{array}{l}0 \\
0 \\
0 \\
0 \\
0 \\
0 \\
0 \\
0 \\
0 \\
0 \\
0 \\
0 \\
0\end{array}$ \\
\hline 1994 & 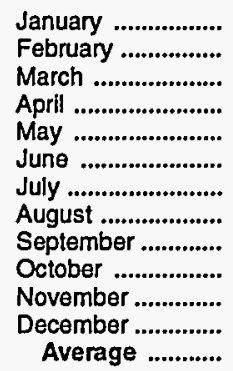 & $\begin{array}{l}182 \\
184 \\
188 \\
241 \\
105 \\
112 \\
127 \\
181 \\
144 \\
215 \\
118 \\
124 \\
160\end{array}$ & $\begin{array}{r}149 \\
131 \\
167 \\
197 \\
75 \\
101 \\
127 \\
181 \\
144 \\
215 \\
118 \\
124 \\
144\end{array}$ & $\begin{array}{r}128 \\
96 \\
37 \\
52 \\
85 \\
72 \\
144 \\
115 \\
63 \\
110 \\
85 \\
96 \\
90\end{array}$ & $\begin{array}{r}128 \\
96 \\
37 \\
52 \\
85 \\
72 \\
144 \\
115 \\
63 \\
110 \\
85 \\
96 \\
90\end{array}$ & $\begin{array}{r}8 \\
35 \\
16 \\
13 \\
19 \\
12 \\
35 \\
52 \\
34 \\
21 \\
17 \\
9 \\
22\end{array}$ & $\begin{array}{l}0 \\
0 \\
0 \\
0 \\
0 \\
0 \\
0 \\
0 \\
0 \\
0 \\
0 \\
0 \\
0\end{array}$ & $\begin{array}{r}11 \\
19 \\
13 \\
3 \\
0 \\
10 \\
36 \\
13 \\
9 \\
0 \\
0 \\
6 \\
10\end{array}$ & $\begin{array}{r}0 \\
15 \\
0 \\
0 \\
0 \\
10 \\
36 \\
7 \\
0 \\
0 \\
0 \\
0 \\
6\end{array}$ & $\begin{array}{r}971 \\
967 \\
1,067 \\
987 \\
957 \\
1,040 \\
926 \\
928 \\
1,043 \\
940 \\
1,037 \\
963 \\
985\end{array}$ & $\begin{array}{r}945 \\
926 \\
1,014 \\
963 \\
917 \\
974 \\
889 \\
885 \\
963 \\
881 \\
981 \\
944 \\
940\end{array}$ & $\begin{array}{r}35 \\
43 \\
33 \\
23 \\
79 \\
38 \\
35 \\
33 \\
34 \\
18 \\
1 \\
4 \\
31\end{array}$ & $\begin{array}{l}0 \\
0 \\
0 \\
0 \\
0 \\
0 \\
0 \\
0 \\
0 \\
0 \\
0 \\
0 \\
0\end{array}$ \\
\hline 1995 & $\begin{array}{l}\text { January ................. } \\
\text { February ................ } \\
\text { March .................. } \\
\text { 3-Mo. Average ..... }\end{array}$ & $\begin{array}{l}191 \\
158 \\
257 \\
203\end{array}$ & $\begin{array}{l}181 \\
148 \\
238 \\
191\end{array}$ & $\begin{array}{l}130 \\
107 \\
104 \\
114\end{array}$ & $\begin{array}{l}130 \\
107 \\
104 \\
114\end{array}$ & $\begin{array}{l}4 \\
1 \\
8 \\
5\end{array}$ & $\begin{array}{l}0 \\
0 \\
0 \\
0\end{array}$ & $\begin{array}{r}21 \\
0 \\
0 \\
7\end{array}$ & $\begin{array}{r}21 \\
0 \\
0 \\
7\end{array}$ & $\begin{array}{r}942 \\
919 \\
1,006 \\
957\end{array}$ & $\begin{array}{l}909 \\
888 \\
961 \\
920\end{array}$ & $\begin{array}{r}0 \\
17 \\
29 \\
15\end{array}$ & $\begin{array}{l}0 \\
0 \\
0 \\
0\end{array}$ \\
\hline $\begin{array}{l}1994 \\
1993\end{array}$ & $\begin{array}{l}\text { 3-Mo. Average ..... } \\
\text { 3-Mo. Average ..... }\end{array}$ & $\begin{array}{l}185 \\
166\end{array}$ & $\begin{array}{l}150 \\
145\end{array}$ & $\begin{array}{r}87 \\
-\end{array}$ & $\begin{array}{r}87 \\
-\end{array}$ & $\begin{array}{l}19 \\
45\end{array}$ & $\begin{array}{l}0 \\
0\end{array}$ & $\begin{array}{r}14 \\
4\end{array}$ & $\begin{array}{l}5 \\
4\end{array}$ & $\begin{array}{r}1,003 \\
837\end{array}$ & $\begin{array}{l}963 \\
790\end{array}$ & $\begin{array}{l}37 \\
13\end{array}$ & $\begin{array}{l}0 \\
0\end{array}$ \\
\hline
\end{tabular}

See footnotes at end of table. 
Table S3. Crude Oil and Petroleum Product Imports, 1981 - Present (Continued) (Thousand Barrels per Day)

\begin{tabular}{|c|c|c|c|c|c|c|c|c|c|c|c|c|c|}
\hline & \multirow{3}{*}{ Year/Month } & \multicolumn{12}{|c|}{ Imports from Non-OPEC Sources ${ }^{a}$} \\
\hline & & \multicolumn{2}{|c|}{$\begin{array}{l}\text { Netherlands } \\
\text { Antilles }\end{array}$} & \multicolumn{2}{|c|}{ Norway } & \multicolumn{2}{|c|}{$\begin{array}{c}\text { Puerto } \\
\text { Rico }\end{array}$} & \multicolumn{2}{|c|}{ Russia $^{\circ}$} & \multicolumn{2}{|c|}{ Spain } & \multicolumn{2}{|c|}{$\begin{array}{l}\text { Trinidad } \\
\text { and } \\
\text { Topage }\end{array}$} \\
\hline & & Total & Crude Oil & Total & Crude Oil & Total & Crude Oil & Total & Crude OII & Total & Crude Oil & Total & Crude Oll \\
\hline $\begin{array}{l}1981 \\
1982 \\
1983 \\
1984 \\
1985 \\
1986 \\
1987 \\
1988 \\
1989 \\
1990 \\
1991 \\
1992\end{array}$ & 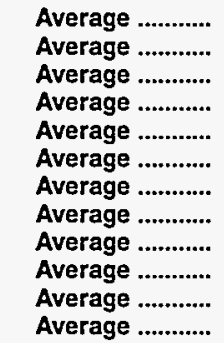 & $\begin{array}{r}197 \\
175 \\
189 \\
188 \\
40 \\
25 \\
29 \\
36 \\
42 \\
31 \\
81 \\
65\end{array}$ & $\begin{array}{l}0 \\
0 \\
0 \\
0 \\
0 \\
0 \\
0 \\
0 \\
0 \\
0 \\
0 \\
0\end{array}$ & $\begin{array}{r}119 \\
102 \\
66 \\
114 \\
32 \\
60 \\
80 \\
67 \\
138 \\
102 \\
82 \\
127\end{array}$ & $\begin{array}{r}114 \\
102 \\
65 \\
112 \\
31 \\
53 \\
50 \\
70 \\
62 \\
127 \\
96 \\
74 \\
119\end{array}$ & $\begin{array}{l}62 \\
50 \\
40 \\
42 \\
28 \\
21 \\
21 \\
22 \\
32 \\
32 \\
27 \\
26\end{array}$ & $\begin{array}{l}0 \\
0 \\
0 \\
0 \\
0 \\
0 \\
0 \\
0 \\
0 \\
0 \\
0 \\
0\end{array}$ & $\begin{array}{r}5 \\
1 \\
1 \\
13 \\
8 \\
18 \\
11 \\
29 \\
48 \\
45 \\
29 \\
18\end{array}$ & $\begin{array}{r}\text { (s) } \\
0 \\
(s) \\
(s) \\
(s) \\
(s) \\
0 \\
0 \\
0 \\
1 \\
1 \\
5\end{array}$ & $\begin{array}{r}1 \\
3 \\
2 \\
11 \\
29 \\
53 \\
55 \\
68 \\
67 \\
47 \\
33 \\
32\end{array}$ & $\begin{array}{l}(s) \\
(s) \\
(s) \\
0 \\
1 \\
0 \\
0 \\
0 \\
0 \\
0 \\
0 \\
0\end{array}$ & $\begin{array}{r}133 \\
112 \\
96 \\
94 \\
113 \\
125 \\
106 \\
97 \\
94 \\
96 \\
88 \\
95\end{array}$ & $\begin{array}{r}102 \\
92 \\
83 \\
87 \\
98 \\
93 \\
75 \\
71 \\
73 \\
76 \\
72 \\
70\end{array}$ \\
\hline 1993 & 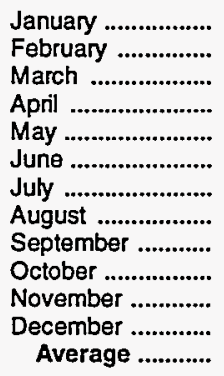 & $\begin{array}{r}73 \\
80 \\
61 \\
97 \\
81 \\
55 \\
52 \\
56 \\
101 \\
122 \\
90 \\
118 \\
82\end{array}$ & $\begin{array}{l}0 \\
0 \\
0 \\
0 \\
0 \\
0 \\
0 \\
0 \\
0 \\
0 \\
0 \\
0 \\
0\end{array}$ & $\begin{array}{r}70 \\
62 \\
122 \\
170 \\
222 \\
160 \\
215 \\
180 \\
113 \\
115 \\
162 \\
108 \\
142\end{array}$ & $\begin{array}{r}70 \\
61 \\
115 \\
170 \\
222 \\
160 \\
215 \\
161 \\
113 \\
93 \\
155 \\
101 \\
137\end{array}$ & $\begin{array}{l}37 \\
21 \\
26 \\
18 \\
38 \\
29 \\
49 \\
30 \\
28 \\
30 \\
23 \\
14 \\
29\end{array}$ & $\begin{array}{l}0 \\
0 \\
0 \\
0 \\
0 \\
0 \\
0 \\
0 \\
0 \\
0 \\
0 \\
0 \\
0\end{array}$ & $\begin{array}{l}0 \\
0 \\
0 \\
0 \\
0 \\
0 \\
0 \\
0 \\
0 \\
0 \\
0 \\
0 \\
0\end{array}$ & $\begin{array}{l}0 \\
0 \\
0 \\
0 \\
0 \\
0 \\
0 \\
0 \\
0 \\
0 \\
0 \\
0 \\
0\end{array}$ & $\begin{array}{l}44 \\
19 \\
21 \\
61 \\
42 \\
20 \\
41 \\
37 \\
54 \\
33 \\
30 \\
42 \\
37\end{array}$ & $\begin{array}{l}0 \\
0 \\
0 \\
0 \\
0 \\
0 \\
0 \\
0 \\
0 \\
0 \\
0 \\
0 \\
0\end{array}$ & $\begin{array}{r}59 \\
72 \\
92 \\
78 \\
68 \\
77 \\
82 \\
50 \\
70 \\
69 \\
66 \\
103 \\
74\end{array}$ & $\begin{array}{l}48 \\
58 \\
71 \\
55 \\
51 \\
55 \\
53 \\
37 \\
55 \\
54 \\
55 \\
71 \\
55\end{array}$ \\
\hline 1994 & 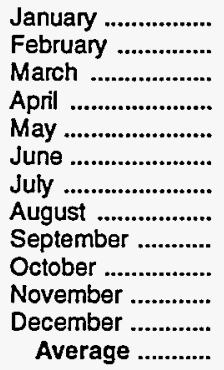 & $\begin{array}{r}162 \\
119 \\
102 \\
73 \\
70 \\
69 \\
121 \\
114 \\
95 \\
77 \\
96 \\
43 \\
95\end{array}$ & $\begin{array}{l}0 \\
0 \\
0 \\
0 \\
0 \\
0 \\
0 \\
0 \\
0 \\
0 \\
0 \\
0 \\
0\end{array}$ & $\begin{array}{l}101 \\
199 \\
108 \\
205 \\
159 \\
176 \\
276 \\
206 \\
347 \\
310 \\
214 \\
125 \\
202\end{array}$ & $\begin{array}{r}96 \\
166 \\
108 \\
184 \\
159 \\
158 \\
257 \\
198 \\
336 \\
300 \\
195 \\
123 \\
190\end{array}$ & $\begin{array}{r}20 \\
11 \\
14 \\
17 \\
21 \\
42 \\
43 \\
23 \\
17 \\
20 \\
6 \\
10 \\
20\end{array}$ & $\begin{array}{l}0 \\
0 \\
0 \\
0 \\
0 \\
0 \\
0 \\
0 \\
0 \\
0 \\
0 \\
0 \\
0\end{array}$ & $\begin{array}{r}11 \\
14 \\
34 \\
0 \\
32 \\
133 \\
82 \\
21 \\
6 \\
30 \\
0 \\
0 \\
30\end{array}$ & $\begin{array}{r}0 \\
0 \\
34 \\
0 \\
32 \\
133 \\
82 \\
15 \\
0 \\
30 \\
0 \\
0 \\
27\end{array}$ & $\begin{array}{l}26 \\
31 \\
37 \\
45 \\
53 \\
50 \\
25 \\
38 \\
56 \\
35 \\
22 \\
26 \\
37\end{array}$ & $\begin{array}{l}0 \\
0 \\
0 \\
0 \\
0 \\
0 \\
0 \\
0 \\
0 \\
0 \\
0 \\
0 \\
0\end{array}$ & $\begin{array}{r}79 \\
92 \\
68 \\
76 \\
68 \\
106 \\
63 \\
92 \\
64 \\
79 \\
59 \\
74 \\
77\end{array}$ & $\begin{array}{l}60 \\
80 \\
54 \\
56 \\
58 \\
79 \\
55 \\
55 \\
56 \\
65 \\
55 \\
74 \\
62\end{array}$ \\
\hline 1995 & $\begin{array}{l}\text { January ................. } \\
\text { February ............... } \\
\text { March ................ } \\
\text { 3-Mo. Average .... }\end{array}$ & $\begin{array}{l}75 \\
58 \\
68 \\
67\end{array}$ & $\begin{array}{l}0 \\
0 \\
0 \\
0\end{array}$ & $\begin{array}{l}200 \\
194 \\
241 \\
212\end{array}$ & $\begin{array}{l}170 \\
164 \\
209 \\
181\end{array}$ & $\begin{array}{r}6 \\
7 \\
13 \\
9\end{array}$ & $\begin{array}{l}0 \\
0 \\
0 \\
0\end{array}$ & $\begin{array}{l}0 \\
0 \\
0 \\
0\end{array}$ & $\begin{array}{l}0 \\
0 \\
0 \\
0\end{array}$ & $\begin{array}{r}7 \\
9 \\
16 \\
11\end{array}$ & $\begin{array}{l}0 \\
0 \\
0 \\
0\end{array}$ & $\begin{array}{l}91 \\
60 \\
70 \\
74\end{array}$ & $\begin{array}{l}91 \\
60 \\
70 \\
74\end{array}$ \\
\hline $\begin{array}{l}1994 \\
1993\end{array}$ & $\begin{array}{l}\text { 3-Mo. Average .... } \\
\text { 3-Mo. Average .... }\end{array}$ & $\begin{array}{r}128 \\
71\end{array}$ & $\begin{array}{l}0 \\
0\end{array}$ & $\begin{array}{r}134 \\
85\end{array}$ & $\begin{array}{r}122 \\
83\end{array}$ & $\begin{array}{l}15 \\
28\end{array}$ & $\begin{array}{l}0 \\
0\end{array}$ & $\begin{array}{r}20 \\
0\end{array}$ & $\begin{array}{r}12 \\
0\end{array}$ & $\begin{array}{l}31 \\
28\end{array}$ & $\begin{array}{l}0 \\
0\end{array}$ & $\begin{array}{l}79 \\
75\end{array}$ & $\begin{array}{l}64 \\
59\end{array}$ \\
\hline
\end{tabular}

See footnotes at end of table. 
Table S3. Crude Oil and Petroleum Product Imports, 1981 - Present (Continued) (Thousand Barrels per Day)

\begin{tabular}{|c|c|c|c|c|c|c|c|c|c|c|c|}
\hline & \multirow{3}{*}{ Year/Month } & \multicolumn{8}{|c|}{ Imports from Non-OPEC Sources ${ }^{a}$} & & \\
\hline & & \multicolumn{2}{|c|}{$\begin{array}{l}\text { United } \\
\text { Kingdom }\end{array}$} & \multicolumn{2}{|c|}{$\begin{array}{l}\text { Virgin } \\
\text { Islands }\end{array}$} & \multicolumn{2}{|c|}{$\begin{array}{l}\text { Other } \\
\text { Non- } \\
\text { QPEC }\end{array}$} & \multicolumn{2}{|c|}{$\begin{array}{l}\text { Total } \\
\text { Non- } \\
\text { OPEC }\end{array}$} & \multicolumn{2}{|c|}{$\begin{array}{c}\text { Total } \\
\text { Imports }\end{array}$} \\
\hline & & Total & Crude OII & Total & Crude Oil & Total & Crude Oil & Total & Crude Oil & Total & Crude OII \\
\hline $\begin{array}{l}1981 \\
1982 \\
1983 \\
1984 \\
1985 \\
1986 \\
1987 \\
1988 \\
1989 \\
1990 \\
1991 \\
1992\end{array}$ & 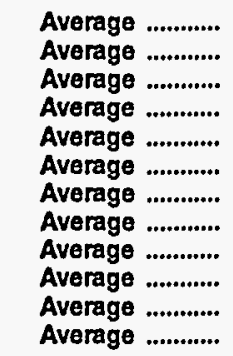 & $\begin{array}{l}375 \\
456 \\
382 \\
402 \\
310 \\
350 \\
352 \\
315 \\
215 \\
189 \\
138 \\
230\end{array}$ & $\begin{array}{l}369 \\
441 \\
365 \\
378 \\
278 \\
317 \\
304 \\
254 \\
160 \\
155 \\
106 \\
200\end{array}$ & $\begin{array}{l}327 \\
316 \\
282 \\
294 \\
247 \\
244 \\
272 \\
242 \\
321 \\
282 \\
243 \\
249\end{array}$ & $\begin{array}{l}0 \\
0 \\
0 \\
0 \\
0 \\
0 \\
0 \\
0 \\
0 \\
0 \\
0 \\
0\end{array}$ & $\begin{array}{l}236 \\
306 \\
378 \\
411 \\
394 \\
426 \\
459 \\
487 \\
457 \\
417 \\
282 \\
335\end{array}$ & $\begin{array}{l}163 \\
174 \\
215 \\
210 \\
137 \\
144 \\
196 \\
196 \\
197 \\
180 \\
137 \\
149\end{array}$ & $\begin{array}{l}2,672 \\
2,968 \\
3,189 \\
3,388 \\
3,237 \\
3,387 \\
3,617 \\
3,882 \\
3,921 \\
3,721 \\
3,535 \\
3,796\end{array}$ & $\begin{array}{l}1,474 \\
1,754 \\
1,853 \\
1,914 \\
1,888 \\
2,065 \\
2,274 \\
2,411 \\
2,467 \\
2,381 \\
2,405 \\
2,676\end{array}$ & $\begin{array}{l}5,996 \\
5,113 \\
5,051 \\
5,437 \\
5,067 \\
6,224 \\
6,678 \\
7,402 \\
8,061 \\
8,018 \\
7,627 \\
7,888\end{array}$ & $\begin{array}{l}4,396 \\
3,488 \\
3,329 \\
3,426 \\
3,201 \\
4,178 \\
4,674 \\
5,107 \\
5,843 \\
5,894 \\
5,782 \\
6,083\end{array}$ \\
\hline 1993 & 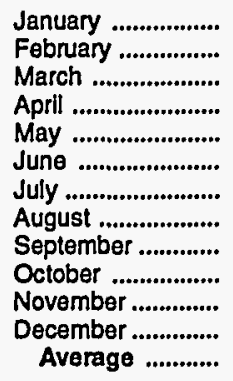 & $\begin{array}{l}229 \\
173 \\
332 \\
413 \\
522 \\
458 \\
292 \\
343 \\
286 \\
353 \\
351 \\
432 \\
350\end{array}$ & $\begin{array}{l}201 \\
127 \\
298 \\
337 \\
495 \\
408 \\
247 \\
323 \\
217 \\
338 \\
340 \\
403 \\
312\end{array}$ & $\begin{array}{l}252 \\
244 \\
244 \\
245 \\
279 \\
290 \\
202 \\
256 \\
184 \\
236 \\
330 \\
288 \\
254\end{array}$ & $\begin{array}{l}0 \\
0 \\
0 \\
0 \\
0 \\
0 \\
0 \\
0 \\
0 \\
0 \\
0 \\
0 \\
0\end{array}$ & $\begin{array}{l}325 \\
223 \\
393 \\
472 \\
363 \\
581 \\
600 \\
556 \\
552 \\
453 \\
503 \\
394 \\
452\end{array}$ & $\begin{array}{l}104 \\
151 \\
186 \\
243 \\
152 \\
405 \\
299 \\
356 \\
251 \\
233 \\
270 \\
231 \\
240\end{array}$ & $\begin{array}{l}3,690 \\
3,438 \\
3,944 \\
4,087 \\
4,298 \\
4,377 \\
4,705 \\
4,257 \\
4,468 \\
4,838 \\
4,624 \\
4,402 \\
4,266\end{array}$ & $\begin{array}{l}2,602 \\
2,457 \\
2,859 \\
2,933 \\
3,123 \\
3,380 \\
3,477 \\
3,088 \\
3,124 \\
3,562 \\
3,327 \\
3,214 \\
3,100\end{array}$ & $\begin{array}{l}8,004 \\
7,948 \\
8,285 \\
8,768 \\
8,663 \\
8,805 \\
9,219 \\
8,429 \\
8,531 \\
9,197 \\
8,903 \\
8,645 \\
8,620\end{array}$ & $\begin{array}{l}6,292 \\
6,156 \\
6,488 \\
6,928 \\
6,809 \\
7,201 \\
7,289 \\
6,641 \\
6,581 \\
7,181 \\
6,997 \\
6,838 \\
6,787\end{array}$ \\
\hline 1994 & $\begin{array}{l}\text { January ................. } \\
\text { February ................. } \\
\text { March .................... } \\
\text { April ........................ } \\
\text { May ......................... } \\
\text { June ..................... } \\
\text { July ....................... } \\
\text { August .................... } \\
\text { September ............. } \\
\text { October .................. } \\
\text { November .............. } \\
\text { December ............. } \\
\text { Average ............ }\end{array}$ & $\begin{array}{l}205 \\
290 \\
459 \\
377 \\
404 \\
537 \\
678 \\
509 \\
736 \\
370 \\
618 \\
305 \\
458\end{array}$ & $\begin{array}{l}161 \\
232 \\
394 \\
282 \\
345 \\
485 \\
578 \\
473 \\
717 \\
323 \\
507 \\
255 \\
396\end{array}$ & $\begin{array}{l}276 \\
351 \\
325 \\
325 \\
312 \\
361 \\
294 \\
356 \\
360 \\
313 \\
292 \\
369 \\
328\end{array}$ & $\begin{array}{l}0 \\
0 \\
0 \\
0 \\
0 \\
0 \\
0 \\
0 \\
0 \\
0 \\
0 \\
0 \\
0\end{array}$ & $\begin{array}{l}353 \\
441 \\
454 \\
488 \\
643 \\
405 \\
634 \\
513 \\
409 \\
350 \\
257 \\
414 \\
447\end{array}$ & $\begin{array}{l}181 \\
111 \\
191 \\
212 \\
390 \\
209 \\
400 \\
249 \\
287 \\
212 \\
159 \\
254 \\
239\end{array}$ & $\begin{array}{l}4,271 \\
4,687 \\
4,755 \\
4,550 \\
4,784 \\
4,766 \\
5,253 \\
5,036 \\
5,159 \\
4,338 \\
4,355 \\
4,410 \\
4,697\end{array}$ & $\begin{array}{l}3,041 \\
3,077 \\
3,366 \\
3,227 \\
3,409 \\
3,520 \\
3,986 \\
3,658 \\
3,997 \\
3,300 \\
3,374 \\
3,352 \\
3,444\end{array}$ & $\begin{array}{l}7,914 \\
8,501 \\
8,500 \\
8,927 \\
9,155 \\
9,263 \\
9,778 \\
9,523 \\
9,526 \\
8,642 \\
8,527 \\
8,861 \\
8,929\end{array}$ & $\begin{array}{l}5,961 \\
6,313 \\
6,377 \\
6,937 \\
7,163 \\
7,358 \\
7,867 \\
7,528 \\
7,722 \\
6,993 \\
6,863 \\
7,193 \\
7,027\end{array}$ \\
\hline 1995 & $\begin{array}{l}\text { January .................. } \\
\text { February ................ } \\
\text { March ................... } \\
\text { 3-Mo. Average ...... }\end{array}$ & $\begin{array}{l}256 \\
382 \\
663 \\
435\end{array}$ & $\begin{array}{l}228 \\
359 \\
621 \\
404\end{array}$ & $\begin{array}{l}283 \\
322 \\
298 \\
300\end{array}$ & $\begin{array}{l}0 \\
0 \\
0 \\
0\end{array}$ & $\begin{array}{l}209 \\
300 \\
174 \\
225\end{array}$ & $\begin{array}{r}131 \\
143 \\
91 \\
121\end{array}$ & $\begin{array}{l}4,126 \\
4,244 \\
4,641 \\
4,340\end{array}$ & $\begin{array}{l}3,215 \\
3,211 \\
3,655 \\
3,365\end{array}$ & $\begin{array}{l}7,955 \\
8,358 \\
9,020 \\
8,447\end{array}$ & $\begin{array}{l}6,503 \\
6,565 \\
7,409 \\
6,834\end{array}$ \\
\hline $\begin{array}{l}1994 \\
1993\end{array}$ & $\begin{array}{l}\text { 3-Mo. Average ..... } \\
\text { 3-Mo. Average ..... }\end{array}$ & $\begin{array}{l}319 \\
247\end{array}$ & $\begin{array}{l}263 \\
211\end{array}$ & $\begin{array}{l}316 \\
247\end{array}$ & $\begin{array}{l}0 \\
0\end{array}$ & $\begin{array}{l}415 \\
317\end{array}$ & $\begin{array}{l}162 \\
146\end{array}$ & $\begin{array}{l}4,567 \\
3,699\end{array}$ & $\begin{array}{l}3,164 \\
2,645\end{array}$ & $\begin{array}{l}8,298 \\
8,083\end{array}$ & $\begin{array}{l}6,214 \\
6,317\end{array}$ \\
\hline
\end{tabular}

${ }^{\text {a }}$ Includes petroleum imported into the United States indirectly from members of the Organization of Petroleum Exporting Countries (OPEC) primarily from Caribbean and West European areas as petroleum products that were refined from crude oil produced by OPEC.

Imports from the Neutral Zone between Kuwait and Saudi Arabia are included in imports from Saudi Arabia.

c On December 31, 1992, Ecuador withdrew as a member of OPEC. As of January 1, 1994, imports of petroleum from Ecuador appear under imports from Non-OPEC Sources.

Excludes petroleum imported into the United States indirectly from members of the Organization of Petroleum Exporting Countries (OPEC), primarily from Caribbean and West European areas, as petroleum products that were refined from crude oil produced by OPEC.

Imports from other States in the former U.S.S.R. may be included in imports from Russia for the years 1981 through 1992.

A small amount of Iranian crude oil entered the United States in January 1988 from the Virgin Islands. This oil originated in Iran and was exported to the Virgin Islands prior to the signing of Executive Order 12613 on October 29, 1987.

(s) = Less than 500 barrels per day.

Notes: - Geographic coverage is the 50 States and the District of Columbia. - Totals may not equal sum of components due to independent rounding.

Source: See Summary Statistics Table and Figure Sources. 
Figure S5. Finished Motor Gasoline Supply and Disposition, March 1994 - Present

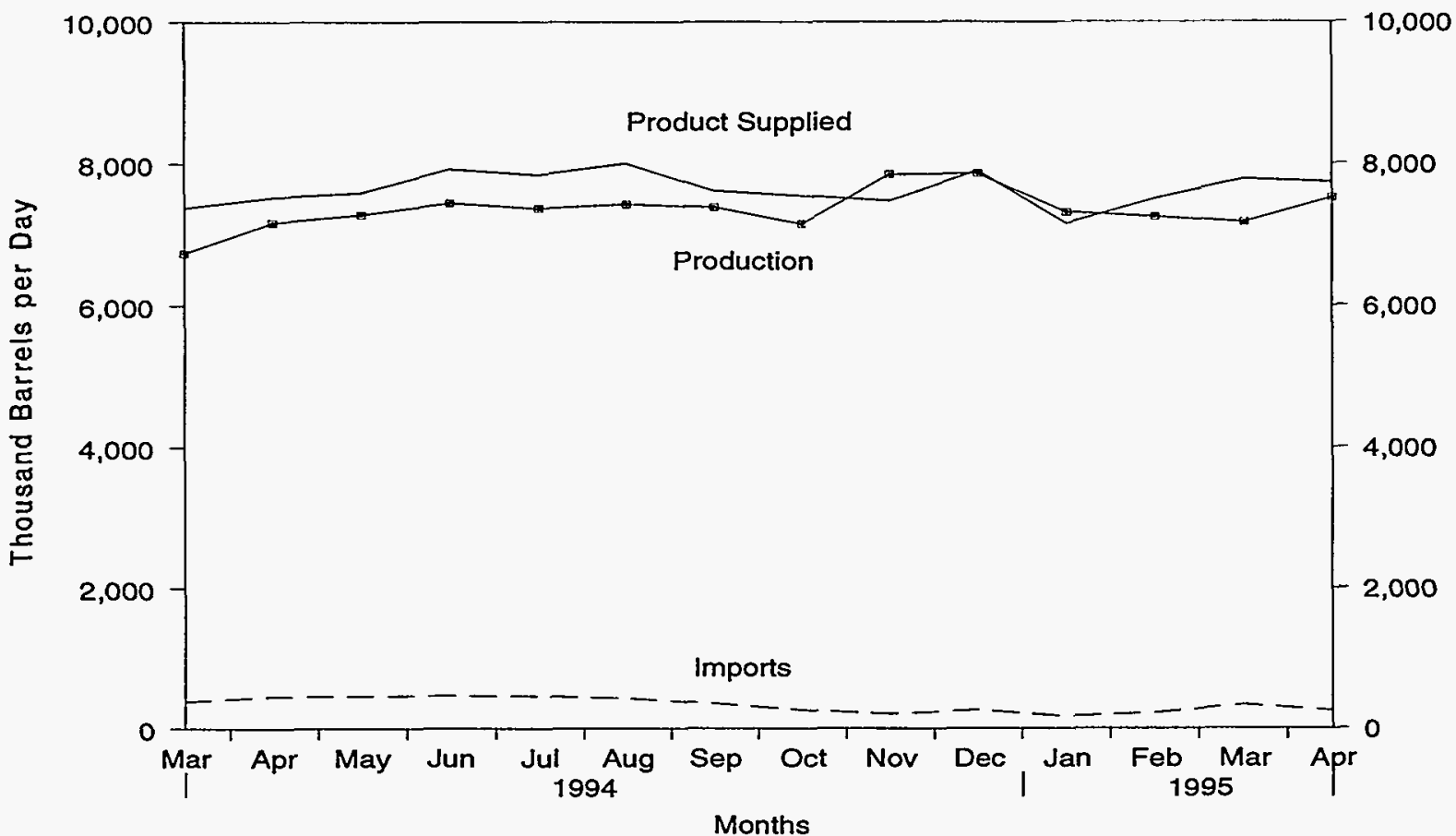

Source: Energy Information Administration, Petroleum Supply Monthly, Table S4. See Summary Statistics Table and Figure Sources.

Figure S6. Motor Gasoline Ending Stocks, March 1994 - Present

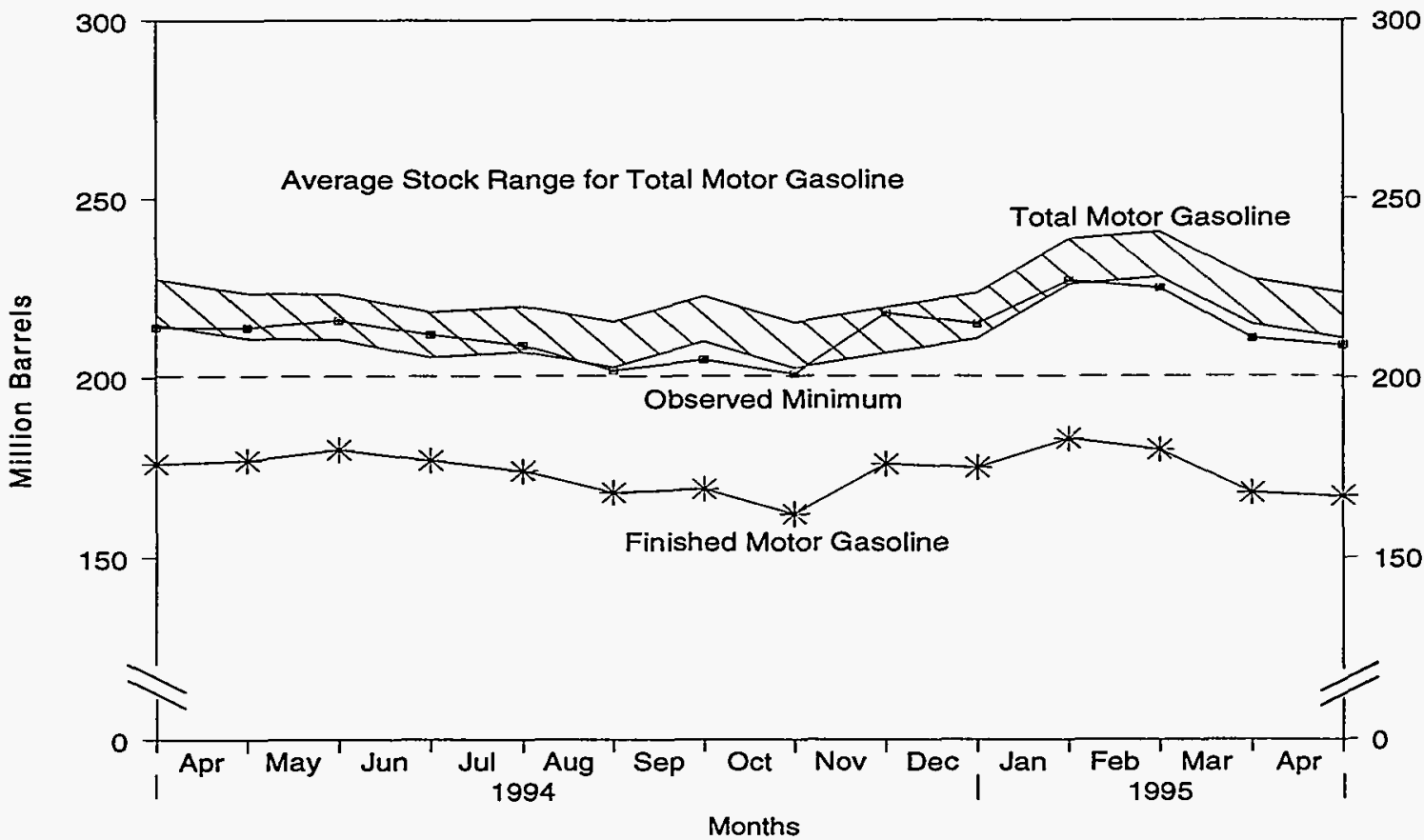

Note: - Total motor gasoline includes motor gasoline blending components and finished motor gasoline. $\bullet$ The Observed Minimum for total motor gasoline stocks in the last 36-month period was 200.9 million barrels, occurring in October 1994.

Source: Energy Information Administration, Petroleum Supply Monthly, Table S4. See Summary Statistics Table and Figure Sources. 
Table S4. Finished Motor Gasoline Supply and Disposition, 1981 - Present (Thousand Barrels per Day, Except Where Noted)

\begin{tabular}{|c|c|c|c|c|c|c|c|c|c|}
\hline & \multirow{3}{*}{ Year/Month } & \multicolumn{2}{|c|}{ Supply } & \multicolumn{3}{|c|}{ Disposition } & \multirow{2}{*}{\multicolumn{2}{|c|}{$\begin{array}{l}\begin{array}{l}\text { Ending Stocks } \\
\text { (Million Barrels) }\end{array} \\
\text { Motor Gasoline }\end{array}$}} & \multirow{3}{*}{$\begin{array}{c}\begin{array}{c}\text { Ending Stocks } \\
\text { (Million Barrels) }\end{array} \\
\text { Oxygenates }\end{array}$} \\
\hline & & \multirow{2}{*}{$\begin{array}{c}\text { Total } \\
\text { Production }\end{array}$} & \multirow[b]{2}{*}{ Importsc } & \multirow{2}{*}{$\begin{array}{c}\text { Stock } \\
\text { Change }\end{array}$} & \multirow[b]{2}{*}{ Exports } & \multirow{2}{*}{$\begin{array}{l}\text { Product } \\
\text { Supplied }\end{array}$} & & & \\
\hline & & & & & & & Total $^{\ominus}$ & Finished & \\
\hline $\begin{array}{l}1981 \\
1982 \\
1983 \\
1984 \\
1985 \\
1986 \\
1987 \\
1988 \\
1989 \\
1990 \\
1991 \\
1992\end{array}$ & 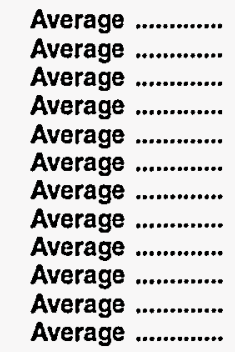 & $\begin{array}{l}6,405 \\
6,338 \\
6,340 \\
6,453 \\
6,419 \\
6,752 \\
6,841 \\
6,956 \\
6,963 \\
6,959 \\
6,975 \\
7,058\end{array}$ & $\begin{array}{l}157 \\
197 \\
247 \\
299 \\
381 \\
326 \\
384 \\
405 \\
369 \\
342 \\
297 \\
294\end{array}$ & $\begin{array}{r}f-28 \\
-25 \\
f-45 \\
54 \\
-41 \\
11 \\
-15 \\
3 \\
-35 \\
10 \\
3 \\
-11\end{array}$ & $\begin{array}{r}2 \\
20 \\
10 \\
6 \\
10 \\
33 \\
35 \\
22 \\
39 \\
55 \\
82 \\
96\end{array}$ & $\begin{array}{l}6,588 \\
6,539 \\
6,622 \\
6,693 \\
6,831 \\
7,034 \\
7,206 \\
7,336 \\
7,328 \\
7,235 \\
7,188 \\
7,268\end{array}$ & $\begin{array}{r}\mathbf{2 5 3} \\
\mathbf{f} 235 \\
222 \\
243 \\
223 \\
233 \\
226 \\
228 \\
213 \\
220 \\
219 \\
216\end{array}$ & $\begin{array}{l}203 \\
1194 \\
186 \\
205 \\
190 \\
194 \\
189 \\
190 \\
177 \\
181 \\
182 \\
178\end{array}$ & $\begin{array}{l}- \\
- \\
- \\
- \\
- \\
- \\
- \\
-\end{array}$ \\
\hline 1993 & 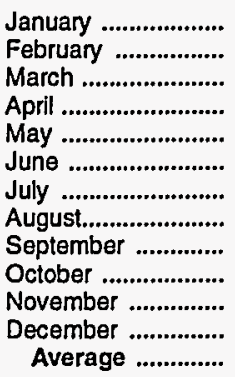 & $\begin{array}{l}7,228 \\
7,144 \\
6,904 \\
7,126 \\
7,446 \\
7,442 \\
7,337 \\
7,335 \\
7,573 \\
7,394 \\
7,652 \\
7,725 \\
7,360\end{array}$ & $\begin{array}{l}204 \\
216 \\
177 \\
253 \\
323 \\
251 \\
300 \\
283 \\
267 \\
210 \\
252 \\
231 \\
247\end{array}$ & $\begin{array}{r}652 \\
149 \\
-417 \\
-168 \\
93 \\
-88 \\
-240 \\
-323 \\
148 \\
142 \\
245 \\
132 \\
26\end{array}$ & $\begin{array}{r}142 \\
99 \\
109 \\
111 \\
90 \\
81 \\
92 \\
77 \\
85 \\
80 \\
126 \\
162 \\
105\end{array}$ & $\begin{array}{l}6,639 \\
7,112 \\
7,389 \\
7,435 \\
7,585 \\
7,700 \\
7,785 \\
7,864 \\
7,607 \\
7,382 \\
7,533 \\
7,661 \\
7,476\end{array}$ & $\begin{array}{r}240 \\
245 \\
230 \\
225 \\
225 \\
221 \\
215 \\
202 \\
208 \\
212 \\
222 \\
226 \\
-\end{array}$ & $\begin{array}{r}198 \\
202 \\
189 \\
184 \\
187 \\
184 \\
177 \\
167 \\
171 \\
176 \\
183 \\
187 \\
-\end{array}$ & $\begin{array}{l}15 \\
14 \\
15 \\
15 \\
17 \\
18 \\
20 \\
21 \\
19 \\
18 \\
16 \\
13 \\
-\end{array}$ \\
\hline 1994 & 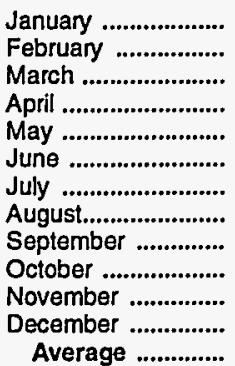 & $\begin{array}{l}7,098 \\
6,780 \\
6,740 \\
7,171 \\
7,282 \\
7,448 \\
7,372 \\
7,432 \\
7,387 \\
7,149 \\
7,849 \\
7,860 \\
7,300\end{array}$ & $\begin{array}{l}206 \\
281 \\
387 \\
460 \\
464 \\
473 \\
464 \\
434 \\
360 \\
263 \\
209 \\
265 \\
356\end{array}$ & $\begin{array}{r}291 \\
-288 \\
-340 \\
28 \\
90 \\
-93 \\
-88 \\
-211 \\
53 \\
-245 \\
470 \\
-8 \\
-28\end{array}$ & $\begin{array}{r}97 \\
77 \\
88 \\
73 \\
64 \\
88 \\
78 \\
70 \\
74 \\
110 \\
108 \\
231 \\
97\end{array}$ & $\begin{array}{l}6,916 \\
7,272 \\
7,379 \\
7,530 \\
7,592 \\
7,926 \\
7,846 \\
8,007 \\
7,619 \\
7,547 \\
7,479 \\
7,902 \\
7,587\end{array}$ & $\begin{array}{r}236 \\
227 \\
214 \\
214 \\
216 \\
212 \\
209 \\
202 \\
205 \\
201 \\
218 \\
215 \\
-\end{array}$ & $\begin{array}{r}195 \\
187 \\
176 \\
177 \\
180 \\
177 \\
174 \\
168 \\
169 \\
162 \\
176 \\
175 \\
-\end{array}$ & $\begin{array}{l}11 \\
11 \\
13 \\
15 \\
16 \\
18 \\
22 \\
24 \\
25 \\
23 \\
20 \\
17 \\
-\end{array}$ \\
\hline 1995 & $\begin{array}{l}\text { January ................... } \\
\text { February ................. } \\
\text { March ...................... } \\
\text { April* ...................... } \\
\text { 4-Mo. Average ....... }\end{array}$ & $\begin{array}{r}7,317 \\
7,250 \\
R_{7,171} \\
E_{7,520} \\
E_{7,314}\end{array}$ & $\begin{array}{r}174 \\
223 \\
R_{336} \\
E_{254} \\
E_{247}\end{array}$ & $\begin{array}{r}235 \\
-116 \\
R_{-380}^{-38} \\
E_{-66}\end{array}$ & $\begin{array}{r}100 \\
84 \\
R_{107} \\
E_{101} \\
E_{98}\end{array}$ & $\begin{array}{r}7,157 \\
7,505 \\
R_{7,780} \\
E_{7,739} \\
E_{7,544}\end{array}$ & $\begin{array}{r}227 \\
225 \\
R_{211} \\
E_{209} \\
-\end{array}$ & $\begin{array}{r}183 \\
180 \\
R_{168} \\
E_{167} \\
-\end{array}$ & $\begin{array}{r}16 \\
16 \\
15 \\
\text { NA } \\
-\end{array}$ \\
\hline $\begin{array}{l}1994 \\
1993\end{array}$ & $\begin{array}{l}\text { 4-Mo. Average ...... } \\
\text { 4-Mo. Average ...... }\end{array}$ & $\begin{array}{l}6,949 \\
7,099\end{array}$ & $\begin{array}{l}334 \\
212\end{array}$ & $\begin{array}{r}-73 \\
53\end{array}$ & $\begin{array}{r}84 \\
116\end{array}$ & $\begin{array}{l}7,272 \\
7,142\end{array}$ & $\underline{-}$ & - & - \\
\hline
\end{tabular}

\footnotetext{
a Stocks are totals as of end of period.

b Beginning in 1993, motor gasoline production and product supplied includes blending of fuel ethanol and an adjustment to correct for the imbalance of motor gasoline blending components.

c Beginning in 1981, excludes blending components.

d A negative number indicates a decrease in stocks and a positive number indicates an increase.

- Includes motor gasoline blending components but excludes stocks of oxygenates.

f In January 1981 and 1983 , numerous respondents were added to surveys affecting stocks reported and stock change calculations. Stock changes are calculated using new basis stock levels. See Summary Statistics Explanatory Note 4.

$R=$ Revised data. $E=$ Estimated. NA = Not Available.

- See Summary Statistics Explanatory Note 1.

Notes: - Italics denote estimates based upon preliminary data. • Geographic coverage is the 50 States and the District of Columbia. - Totals may not equal sum of components due to independent rounding.

Source: See Summary Statistics Table and Figure Sources.
} 
Figure S7. Distillate Fuel Oil Supply and Disposition, March 1994 - Present

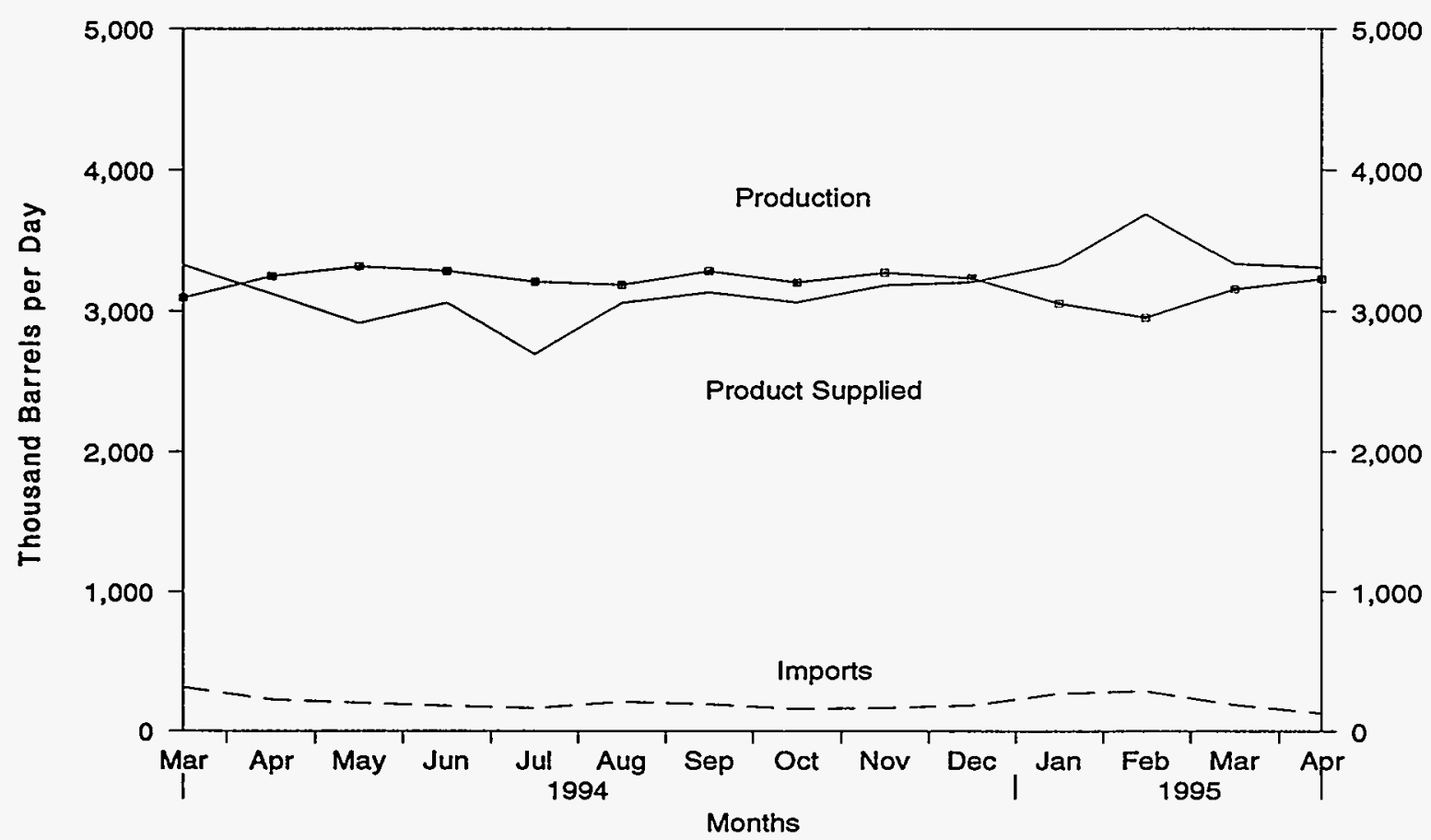

Source: Energy Information Administration, Petroleum Supply Monthly, Table S5. See Summary Statistics Table and Figure Sources.

Figure S8. Distillate Fuel Oil Ending Stocks, March 1994 - Present

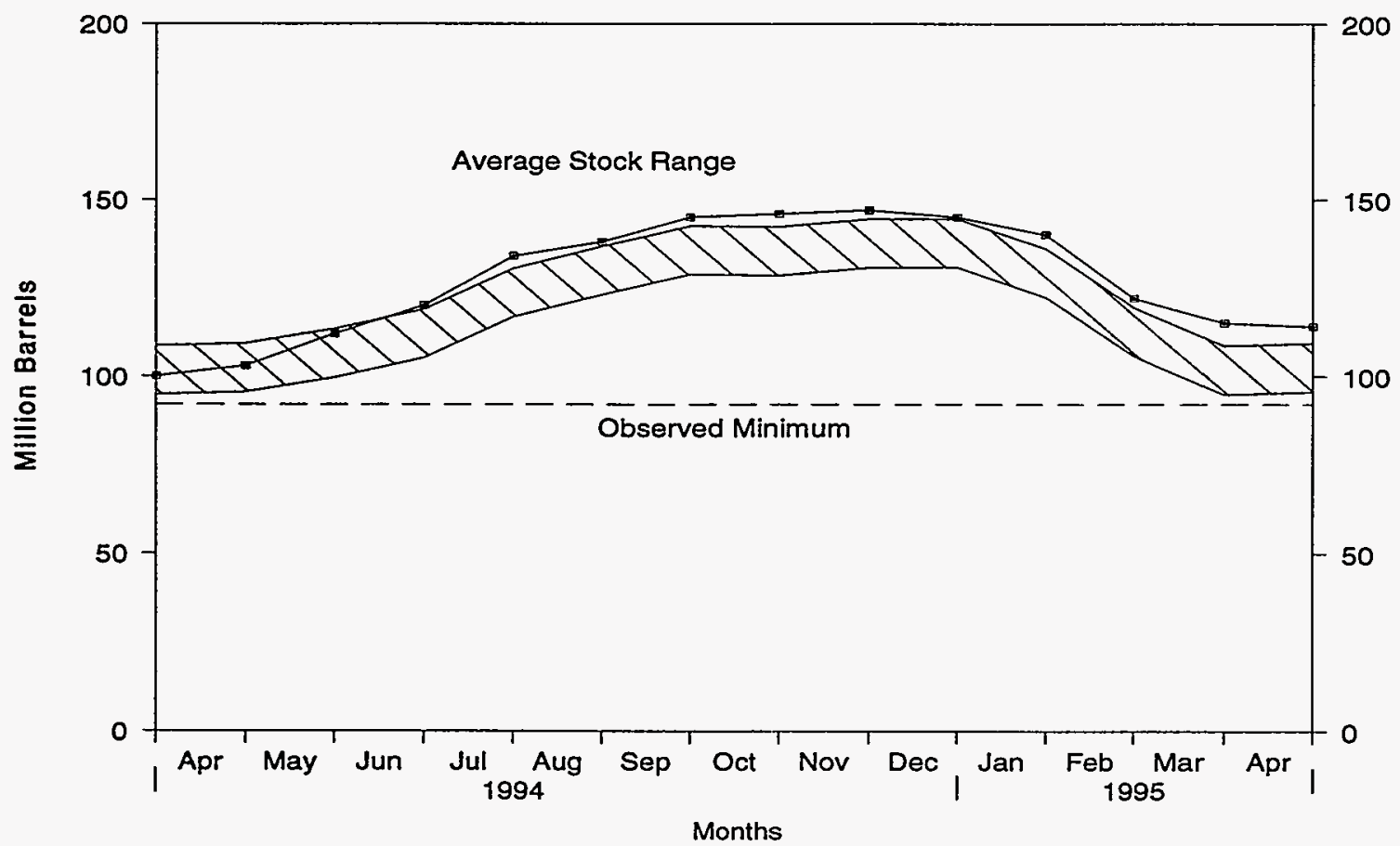

Note: The Observed Minimum for distillate fuel oil stocks in the last 36-month period was 92.1 million barrels, occurring in April 1992.

Source: Energy Information Administration, Petroleum Supply Monthly, Table S5. See Summary Statistics Table and Figure Sources. 
Table S5. Distillate Fuel Oil Supply and Disposition, 1981 - Present (Thousand Barrels per Day, Except Where Noted)

\begin{tabular}{|c|c|c|c|c|c|c|c|c|c|}
\hline & \multirow{3}{*}{ Year/Month } & \multicolumn{2}{|c|}{ Supply" } & \multicolumn{3}{|c|}{ Disposition } & \multirow{2}{*}{\multicolumn{3}{|c|}{$\begin{array}{l}\text { Ending Stocks" } \\
\text { (Million Barrels) }\end{array}$}} \\
\hline & & \multirow[b]{2}{*}{$\begin{array}{c}\text { Total } \\
\text { Production }\end{array}$} & \multirow[b]{2}{*}{ Imports } & \multirow[b]{2}{*}{$\begin{array}{c}\text { Stock } \\
\text { Change }^{c}\end{array}$} & \multirow[b]{2}{*}{ Exports } & \multirow[b]{2}{*}{$\begin{array}{l}\text { Product } \\
\text { Supplied }\end{array}$} & & & \\
\hline & & & & & & & Total & $\begin{array}{l}0.05 \% \text { Sulfur } \\
\text { and Under }\end{array}$ & $\begin{array}{l}\text { Greater than } \\
0.05 \% \text { Sulfur }\end{array}$ \\
\hline $\begin{array}{l}1981 \\
1982 \\
1983 \\
1984 \\
1985 \\
1986 \\
1987 \\
1988 \\
1989 \\
1990 \\
1991 \\
1992\end{array}$ & 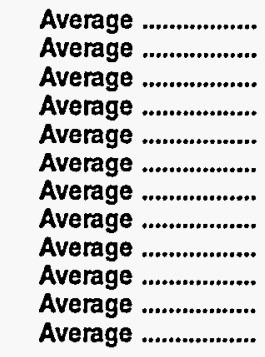 & $\begin{array}{l}2,613 \\
2,606 \\
2,456 \\
2,681 \\
2,687 \\
2,798 \\
2,731 \\
2,859 \\
2,899 \\
2,925 \\
2,962 \\
2,974\end{array}$ & $\begin{array}{r}173 \\
93 \\
174 \\
272 \\
200 \\
247 \\
255 \\
302 \\
306 \\
278 \\
205 \\
216\end{array}$ & $\begin{array}{r}d-38 \\
-35 \\
d-124 \\
57 \\
-48 \\
31 \\
-56 \\
-30 \\
-49 \\
73 \\
31 \\
-8\end{array}$ & $\begin{array}{r}5 \\
74 \\
64 \\
51 \\
67 \\
100 \\
66 \\
69 \\
97 \\
109 \\
215 \\
219\end{array}$ & $\begin{array}{l}2,829 \\
2,671 \\
2,690 \\
2,845 \\
2,868 \\
2,914 \\
2,976 \\
3,122 \\
3,157 \\
3,021 \\
2,921 \\
2,979\end{array}$ & $\begin{array}{r}192 \\
d 179 \\
140 \\
161 \\
144 \\
155 \\
134 \\
124 \\
106 \\
132 \\
144 \\
141\end{array}$ & $\begin{array}{l}- \\
- \\
\overline{-} \\
\overline{-} \\
\overline{-} \\
\overline{-} \\
-\end{array}$ & $\begin{array}{l}\overline{-} \\
\overline{-} \\
\overline{-} \\
\overline{-} \\
\overline{-} \\
\overline{-}\end{array}$ \\
\hline 1993 & 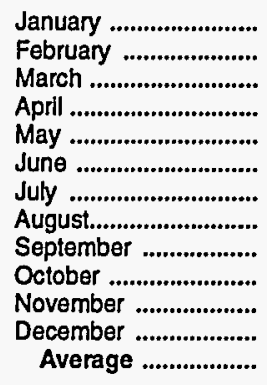 & $\begin{array}{l}2,914 \\
2,815 \\
2,919 \\
3,047 \\
2,994 \\
3,093 \\
3,186 \\
3,100 \\
3,205 \\
3,432 \\
3,474 \\
3,382 \\
3,132 \\
\end{array}$ & $\begin{array}{l}182 \\
224 \\
235 \\
209 \\
153 \\
168 \\
130 \\
159 \\
137 \\
242 \\
214 \\
160 \\
184\end{array}$ & $\begin{array}{r}-318 \\
-727 \\
-420 \\
71 \\
106 \\
241 \\
346 \\
243 \\
102 \\
453 \\
127 \\
-267 \\
1\end{array}$ & $\begin{array}{l}287 \\
301 \\
154 \\
241 \\
355 \\
158 \\
296 \\
196 \\
267 \\
237 \\
342 \\
453 \\
274\end{array}$ & $\begin{array}{l}3,128 \\
3,465 \\
3,420 \\
2,943 \\
2,685 \\
2,863 \\
2,674 \\
2,820 \\
2,973 \\
2,983 \\
3,218 \\
3,357 \\
3,041\end{array}$ & $\begin{array}{r}131 \\
110 \\
97 \\
99 \\
103 \\
110 \\
121 \\
128 \\
131 \\
145 \\
149 \\
141 \\
-\end{array}$ & $\begin{array}{l}15 \\
12 \\
11 \\
12 \\
12 \\
15 \\
21 \\
44 \\
48 \\
55 \\
64 \\
64 \\
-\end{array}$ & $\begin{array}{r}115 \\
99 \\
87 \\
88 \\
91 \\
95 \\
100 \\
84 \\
84 \\
90 \\
85 \\
77 \\
-\end{array}$ \\
\hline 1994 & 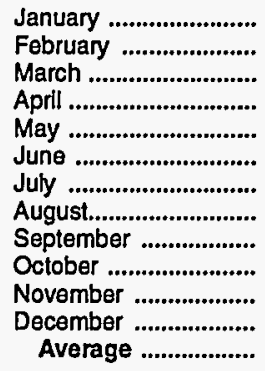 & $\begin{array}{l}3,117 \\
3,019 \\
3,095 \\
3,250 \\
3,319 \\
3,287 \\
3,211 \\
3,189 \\
3,286 \\
3,206 \\
3,274 \\
3,236 \\
3,208\end{array}$ & $\begin{array}{l}160 \\
276 \\
313 \\
226 \\
202 \\
181 \\
164 \\
211 \\
193 \\
159 \\
166 \\
185 \\
202\end{array}$ & $\begin{array}{r}-746 \\
-505 \\
-142 \\
100 \\
317 \\
239 \\
461 \\
147 \\
205 \\
46 \\
44 \\
-70 \\
11\end{array}$ & $\begin{array}{l}332 \\
235 \\
220 \\
252 \\
289 \\
168 \\
220 \\
193 \\
140 \\
256 \\
211 \\
284 \\
234\end{array}$ & $\begin{array}{l}3,692 \\
3,565 \\
3,330 \\
3,124 \\
2,915 \\
3,061 \\
2,694 \\
3,060 \\
3,135 \\
3,063 \\
3,185 \\
3,207 \\
3,166\end{array}$ & $\begin{array}{r}118 \\
104 \\
100 \\
103 \\
112 \\
120 \\
134 \\
138 \\
145 \\
146 \\
147 \\
145 \\
-\end{array}$ & $\begin{array}{l}56 \\
49 \\
50 \\
56 \\
61 \\
61 \\
68 \\
67 \\
66 \\
67 \\
70 \\
72 \\
-\end{array}$ & $\begin{array}{l}62 \\
55 \\
50 \\
46 \\
52 \\
58 \\
65 \\
72 \\
79 \\
79 \\
78 \\
73 \\
-\end{array}$ \\
\hline 1995 & $\begin{array}{l}\text { January ....................... } \\
\text { February ....................... } \\
\text { March ........................... } \\
\text { April" ........................... } \\
\text { 4-Mo. Average ........... }\end{array}$ & $\begin{array}{r}3,055 \\
2,954 \\
R 3,156 \\
E_{3,229} \\
E_{3,101}\end{array}$ & $\begin{array}{r}270 \\
287 \\
R \quad 188 \\
E_{128} \\
E_{217}\end{array}$ & $\begin{array}{r}-152 \\
-660 \\
R-208 \\
E_{-166} \\
E_{-289}\end{array}$ & $\begin{array}{r}141 \\
212 \\
R_{216} \\
E_{212} \\
E_{195}\end{array}$ & $\begin{array}{r}3,335 \\
3,689 \\
R_{3,336} \\
E_{3,310} \\
E_{3,412}\end{array}$ & $\begin{array}{r}140 \\
122 \\
R_{115} \\
E 114 \\
-\end{array}$ & $\begin{array}{r}69 \\
63 \\
R_{59} \\
E_{61} \\
-\end{array}$ & $\begin{array}{r}71 \\
59 \\
\mathrm{R} 56 \\
\mathrm{E} 53 \\
-\end{array}$ \\
\hline $\begin{array}{l}1994 \\
1993\end{array}$ & $\begin{array}{l}\text { 4-Mo. Average .......... } \\
\text { 4-Mo. Average ......... }\end{array}$ & $\begin{array}{l}3,122 \\
2,925\end{array}$ & $\begin{array}{l}243 \\
212\end{array}$ & $\begin{array}{l}-322 \\
-343\end{array}$ & $\begin{array}{l}260 \\
244\end{array}$ & $\begin{array}{l}3,427 \\
3,236\end{array}$ & - & $\overline{-}$ & - \\
\hline
\end{tabular}

Excludes 10,000 barrels per day in 1981 and 1982 previously published as crude used directly.

Stocks are totals as of end of period.

c A negative number indicates a decrease in stocks and a positive number indicates an increase.

d In January 1981 and 1983, numerous respondents were added to surveys affecting stocks reported and stock change calculations. Stock changes are calculated using new stock basis stock levels. See Summary Statistics Explanatory Note 4.

$\mathrm{R}=$ Revised data. $\mathrm{E}=$ Estimated.

- See Summary Statistics Explanatory Note 1.

Notes: - Italics denote estimates based upon preliminary data. • Geographic coverage is the 50 States and the District of Columbia. • Totals may not equal sum of components due to independent rounding.

Source: See Summary Statistics Table and Figure Sources. 
Figure S9. Residual Fuel Oil Supply and Disposition, March 1994 - Present

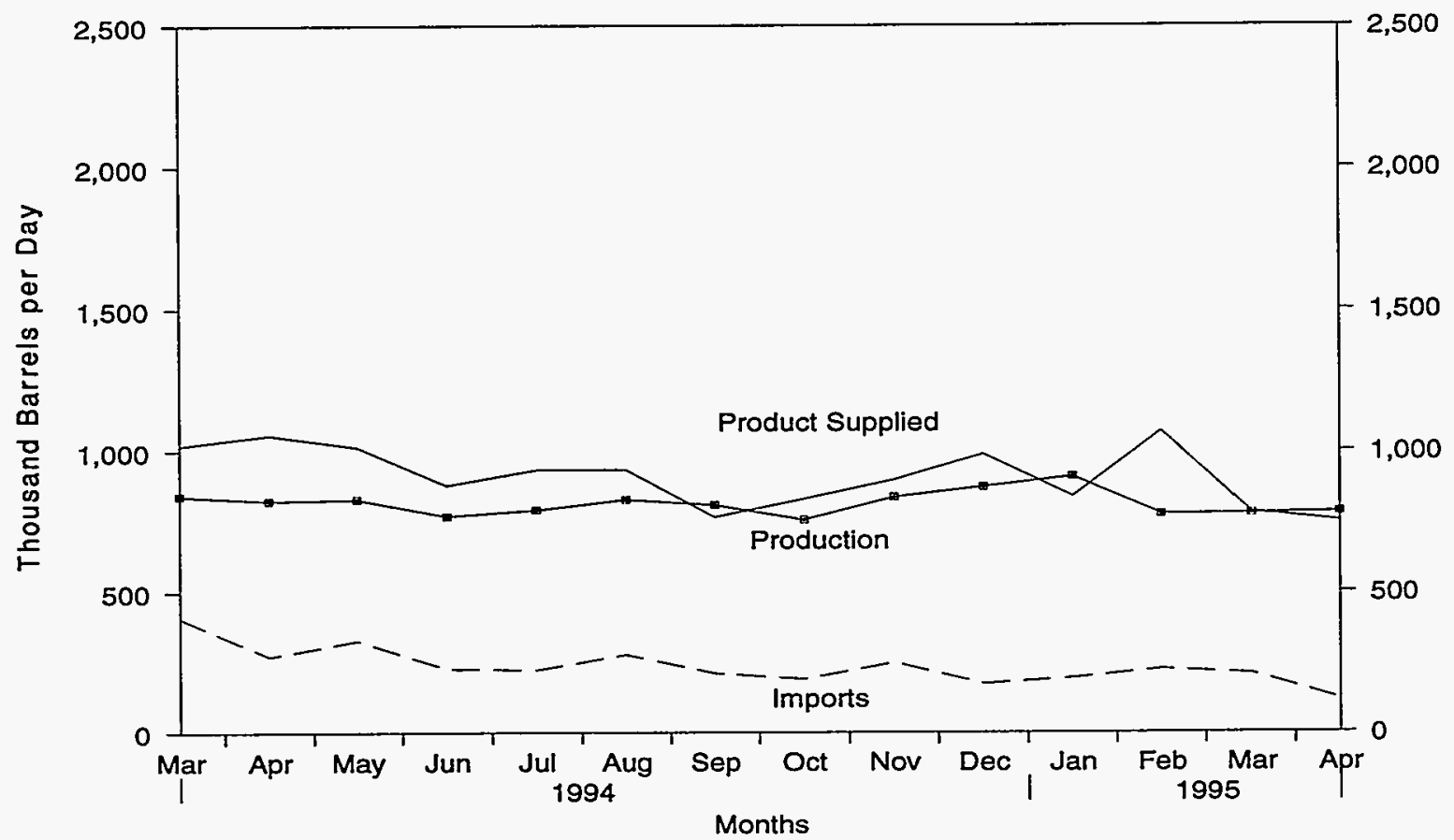

Source: Energy Information Administration, Petroleum Supply Monthly, Table S6. See Summary Statistics Table and Figure Sources.

Figure S10. Residual Fuel Oil Ending Stocks, March 1994 - Present

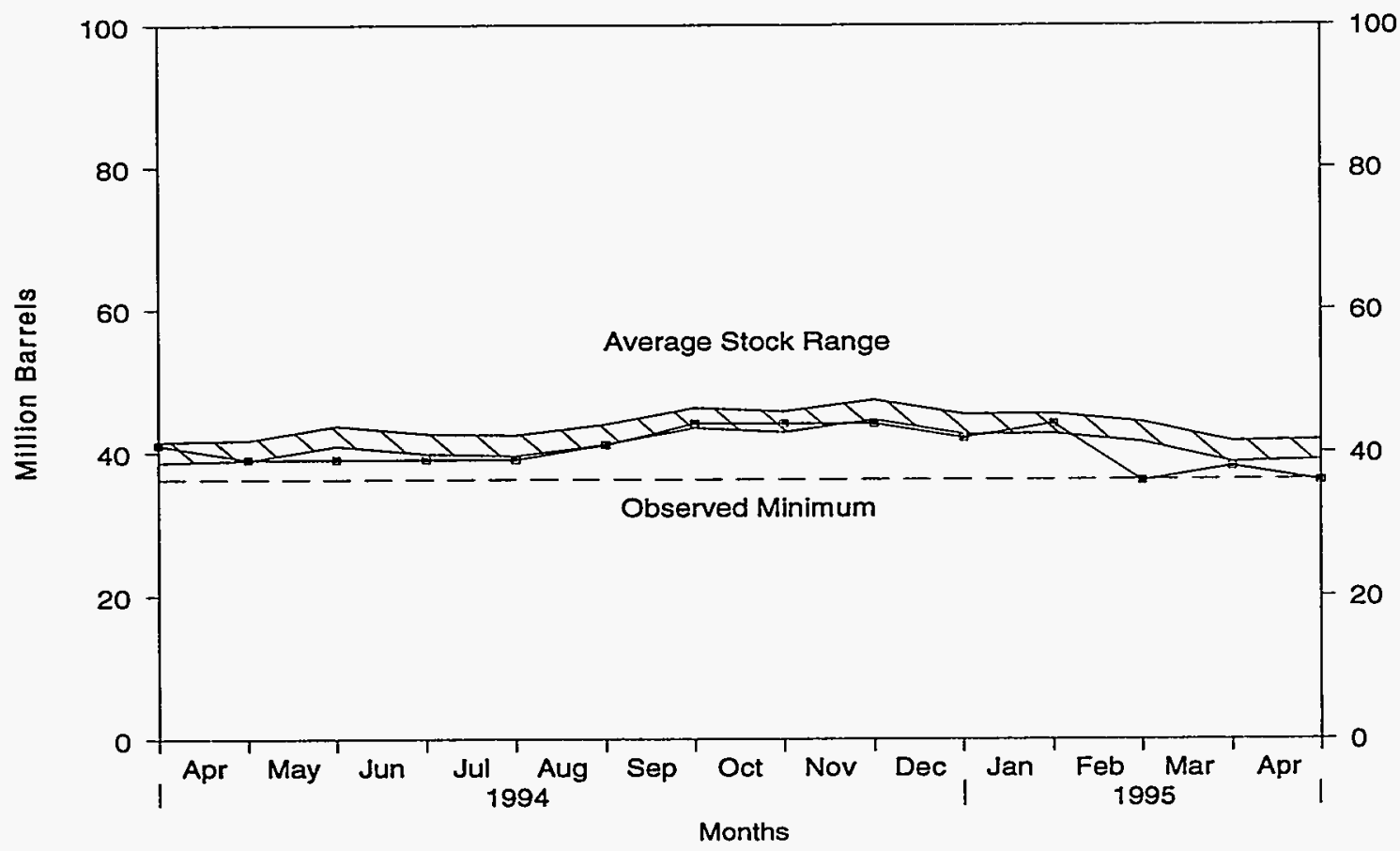

Note: The Observed Minimum for residual fuel oil stocks in the last 36-month period was 36.2 million barrels, occurring in February 1995. Source: Energy Information Administration, Petroleum Supply Monthly, Table S6. See Summary Statistics Table and Figure Sources. 
Table S6. Residual Fuel Oil Supply and Disposition, 1981 - Present (Thousand Barrels per Day, Except Where Noted)

\begin{tabular}{|c|c|c|c|c|c|c|c|}
\hline \multirow{2}{*}{\multicolumn{2}{|c|}{ Year/Month }} & \multicolumn{2}{|c|}{ Supply } & \multicolumn{3}{|c|}{ Disposition } & \multirow[b]{2}{*}{$\begin{array}{c}\text { Ending } \\
\text { Stocks } \\
\text { (Million Barrels) }\end{array}$} \\
\hline & & $\begin{array}{c}\text { Total } \\
\text { Production }\end{array}$ & Imports & Change $_{\text {Stock }}^{\text {bhang }}$ & Exports & $\begin{array}{l}\text { Product } \\
\text { Supplied }^{a}\end{array}$ & \\
\hline $\begin{array}{l}1981 \\
1982 \\
1983 \\
1984 \\
1985 \\
1986 \\
1987 \\
1988 \\
1989 \\
1990 \\
1991 \\
1992\end{array}$ & $\begin{array}{l}\text { Average } \\
\text { Average } \\
\text { Average } \\
\text { Average }\end{array}$ Average & $\begin{array}{r}1,321 \\
1,070 \\
852 \\
891 \\
882 \\
889 \\
885 \\
926 \\
954 \\
950 \\
934 \\
892\end{array}$ & $\begin{array}{l}800 \\
776 \\
699 \\
681 \\
510 \\
669 \\
565 \\
644 \\
629 \\
504 \\
453 \\
375\end{array}$ & $\begin{array}{r}d-37 \\
-32 \\
d-55 \\
12 \\
-7 \\
-8 \\
(s) \\
-8 \\
-2 \\
13 \\
4 \\
-20\end{array}$ & $\begin{array}{l}118 \\
209 \\
185 \\
190 \\
197 \\
147 \\
186 \\
200 \\
215 \\
211 \\
226 \\
193\end{array}$ & $\begin{array}{l}2,088 \\
1,716 \\
1,421 \\
1,369 \\
1,202 \\
1,418 \\
1,264 \\
1,378 \\
1,370 \\
1,229 \\
1,158 \\
1,094\end{array}$ & $\begin{array}{r}78 \\
d 66 \\
49 \\
53 \\
50 \\
47 \\
47 \\
45 \\
44 \\
49 \\
50 \\
43\end{array}$ \\
\hline 1993 & 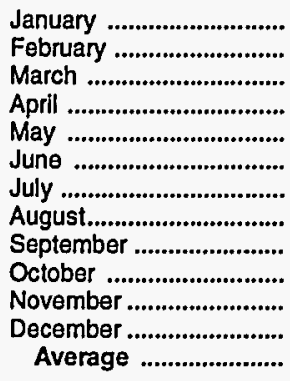 & $\begin{array}{l}820 \\
840 \\
818 \\
896 \\
908 \\
795 \\
762 \\
752 \\
822 \\
841 \\
899 \\
869 \\
835\end{array}$ & $\begin{array}{l}385 \\
332 \\
360 \\
377 \\
316 \\
308 \\
337 \\
387 \\
430 \\
412 \\
361 \\
467 \\
373\end{array}$ & $\begin{array}{r}44 \\
-74 \\
-47 \\
32 \\
54 \\
87 \\
-102 \\
64 \\
-31 \\
103 \\
48 \\
-129 \\
4\end{array}$ & $\begin{array}{r}133 \\
113 \\
152 \\
169 \\
137 \\
147 \\
122 \\
120 \\
110 \\
94 \\
86 \\
98 \\
123\end{array}$ & $\begin{array}{r}1,028 \\
1,132 \\
1,073 \\
1,071 \\
1,033 \\
870 \\
1,079 \\
955 \\
1,173 \\
1,057 \\
1,126 \\
1,367 \\
1,080\end{array}$ & $\begin{array}{l}44 \\
42 \\
40 \\
41 \\
43 \\
46 \\
43 \\
44 \\
44 \\
47 \\
48 \\
44 \\
-\end{array}$ \\
\hline 1994 & 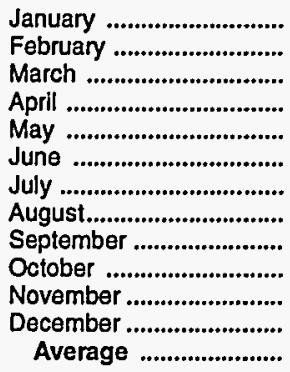 & $\begin{array}{l}813 \\
859 \\
841 \\
825 \\
830 \\
770 \\
791 \\
828 \\
809 \\
756 \\
836 \\
873 \\
819\end{array}$ & $\begin{array}{l}503 \\
586 \\
407 \\
272 \\
328 \\
227 \\
223 \\
277 \\
211 \\
190 \\
248 \\
173 \\
302\end{array}$ & $\begin{array}{r}-16 \\
-152 \\
54 \\
-70 \\
13 \\
-3 \\
-2 \\
52 \\
113 \\
-18 \\
5 \\
-58 \\
-6\end{array}$ & $\begin{array}{r}64 \\
127 \\
175 \\
110 \\
129 \\
122 \\
83 \\
120 \\
141 \\
134 \\
182 \\
115 \\
125\end{array}$ & $\begin{array}{r}1,267 \\
1,470 \\
1,019 \\
1,057 \\
1,015 \\
879 \\
933 \\
934 \\
766 \\
830 \\
897 \\
988 \\
1,002\end{array}$ & $\begin{array}{r}44 \\
39 \\
41 \\
39 \\
39 \\
39 \\
39 \\
41 \\
44 \\
44 \\
44 \\
42 \\
-\end{array}$ \\
\hline 1995 & 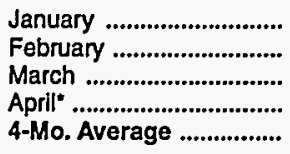 & $\begin{array}{r}909 \\
776 \\
R_{778} \\
E_{783} \\
E_{813}\end{array}$ & $\begin{array}{r}194 \\
225 \\
R_{209} \\
E_{118} \\
E_{186}\end{array}$ & $\begin{array}{r}60 \\
-275 \\
R 50 \\
E_{-13} \\
E_{-39}\end{array}$ & $\begin{array}{r}203 \\
208 \\
R_{154} \\
E_{163} \\
E_{181}\end{array}$ & $\begin{array}{r}839 \\
1,069 \\
R_{783} \\
E_{751} \\
E_{856}\end{array}$ & $\begin{array}{r}44 \\
36 \\
\mathrm{R}_{38} \\
-\end{array}$ \\
\hline $\begin{array}{l}1994 \\
1993\end{array}$ & $\begin{array}{l}\text { 4-Mo. Average ................ } \\
\text { 4-Mo. Average ............... }\end{array}$ & $\begin{array}{l}834 \\
843\end{array}$ & $\begin{array}{l}440 \\
364\end{array}$ & $\begin{array}{l}-43 \\
-10\end{array}$ & $\begin{array}{l}119 \\
142\end{array}$ & $\begin{array}{l}1,198 \\
1,075\end{array}$ & - \\
\hline
\end{tabular}

a Excludes 48,000 barrels per day in 1981 and 1982 previously published as crude used directly.

b A negative number indicates a decrease in stocks and a positive number indicates an increase

c Stocks are totals as of end of period.

d In January 1981 and 1983 , numerous respondents were added to surveys affecting stocks reported and stock change calculations. Stock changes are calculated using new basis stock levels. See Summary Statistics Explanatory Note 4.

$R=$ Revised data. $(s)=$ Less than 500 barrels per day. $E=$ Estimated.

- See Summary Statistics Explanatory Note 1.

Notes: - Italics denote estimates based upon preliminary data. • Geographic coverage is the 50 States and the District of Columbia. $\bullet$ Totals may not equal sum of components due to independent rounding.

Source: See Summary Statistics Table and Figure Sources. 
Figure S11. Jet Fuel Supply and Disposition, March 1994 - Present

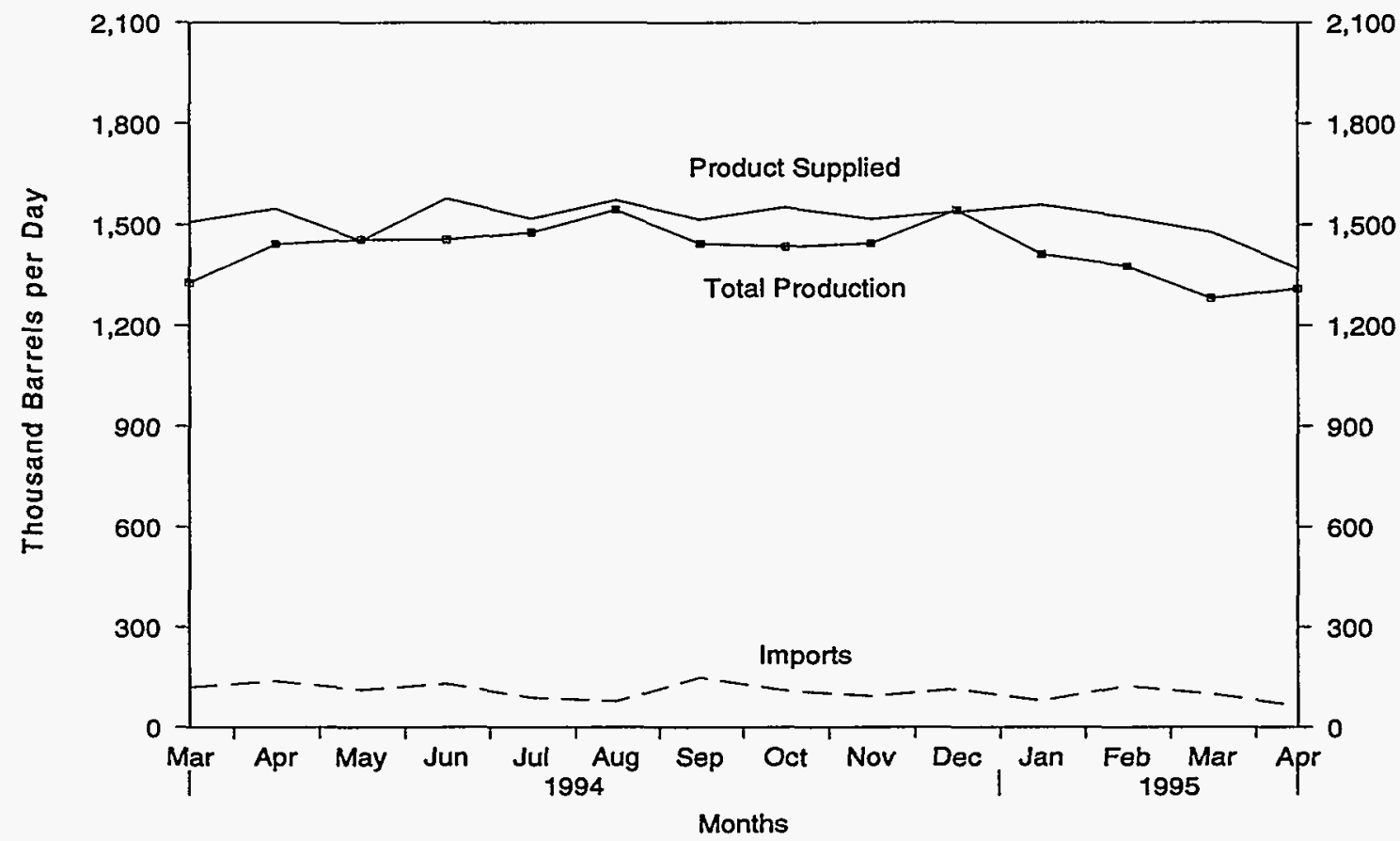

Source: Energy Information Administration, Petroleum Supply Monthly, Table S7. See Summary Statistics Table and Figure Sources.

Figure S12. Jet Fuel Ending Stocks, March 1994 - Present

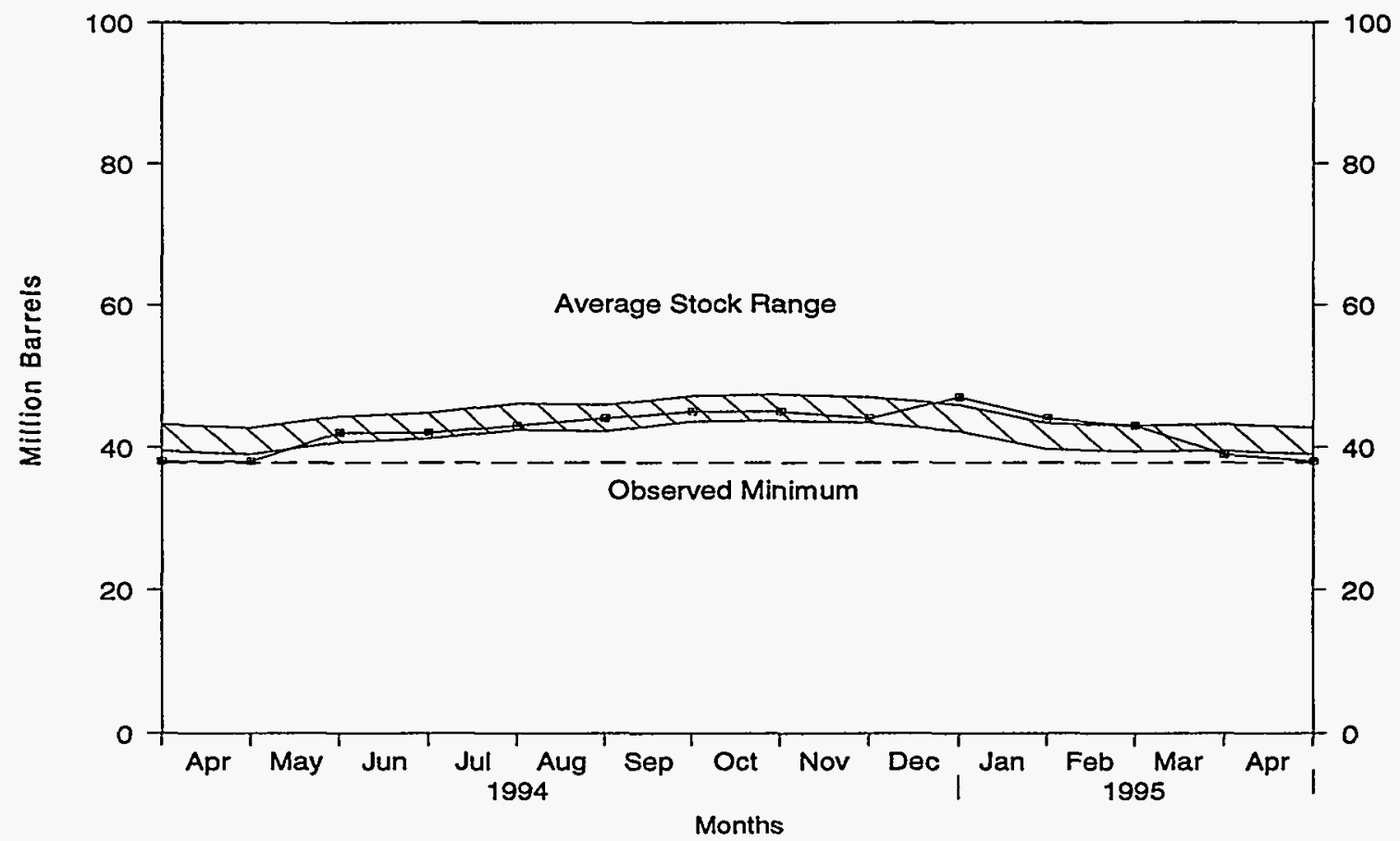

Note: The Observed Minimum for total jet fuel stocks in the last 36-month period was 37.8 million barrels, occurring in March 1994.

Source: Energy Information Administration, Petroleum Supply Monthly, Table S7. See Summary Statistics Table and Figure Sources. 
Table S7. Jet Fuel Supply and Disposition, 1981 - Present

(Thousand Barrels per Day, Except Where Noted)

\begin{tabular}{|c|c|c|c|c|c|c|c|c|c|c|}
\hline & \multirow[b]{3}{*}{ Year/Month } & \multicolumn{3}{|c|}{ Supply } & \multicolumn{4}{|c|}{ Disposition } & \multicolumn{2}{|c|}{$\begin{array}{l}\text { Ending Stocks } \\
\text { (Million Barrels) }\end{array}$} \\
\hline & & \multicolumn{2}{|c|}{ Production } & \multirow[b]{2}{*}{ Imports } & \multirow[b]{2}{*}{$\begin{array}{c}\text { Stock } \\
\text { Change }\end{array}$} & \multirow[b]{2}{*}{ Exports } & \multicolumn{2}{|c|}{ Product Supplied } & \multirow[b]{2}{*}{ Total } & \multirow[b]{2}{*}{$\begin{array}{l}\text { Kerosene- } \\
\text { Type }\end{array}$} \\
\hline & & Total & Kerosene-Type & & & & Total & Kerosene-Type & & \\
\hline $\begin{array}{l}1981 \\
1982 \\
1983 \\
1984 \\
1985 \\
1986 \\
1987 \\
1988 \\
1989 \\
1990 \\
1991 \\
1992\end{array}$ & 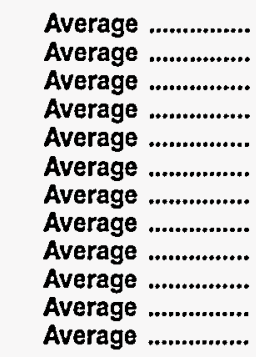 & $\begin{array}{r}968 \\
978 \\
1,022 \\
1,132 \\
1,189 \\
1,293 \\
1,343 \\
1,370 \\
1,403 \\
1,488 \\
1,438 \\
1,399\end{array}$ & $\begin{array}{r}775 \\
778 \\
817 \\
919 \\
983 \\
1,097 \\
1,138 \\
1,164 \\
1,197 \\
1,311 \\
1,274 \\
1,254\end{array}$ & $\begin{array}{r}38 \\
29 \\
29 \\
62 \\
39 \\
57 \\
67 \\
90 \\
106 \\
108 \\
67 \\
82\end{array}$ & $\begin{array}{r}c-4 \\
-12 \\
c(s) \\
9 \\
-4 \\
25 \\
(s) \\
-17 \\
-8 \\
31 \\
-9 \\
-16\end{array}$ & $\begin{array}{r}2 \\
6 \\
6 \\
9 \\
13 \\
18 \\
24 \\
28 \\
27 \\
43 \\
43 \\
43\end{array}$ & $\begin{array}{l}1,007 \\
1,013 \\
1,046 \\
1,175 \\
1,218 \\
1,307 \\
1,385 \\
1,449 \\
1,489 \\
1,522 \\
1,471 \\
1,454\end{array}$ & $\begin{array}{r}809 \\
804 \\
839 \\
953 \\
1,005 \\
1,105 \\
1,181 \\
1,236 \\
1,284 \\
1,340 \\
1,296 \\
1,310\end{array}$ & $\begin{array}{r}41 \\
637 \\
39 \\
42 \\
40 \\
50 \\
50 \\
44 \\
41 \\
52 \\
49 \\
43\end{array}$ & $\begin{array}{r}34 \\
\mathbf{3 1} \\
\mathbf{3 2} \\
\mathbf{3 5} \\
\mathbf{3 4} \\
\mathbf{4 3} \\
42 \\
38 \\
34 \\
46 \\
44 \\
39\end{array}$ \\
\hline 1993 & 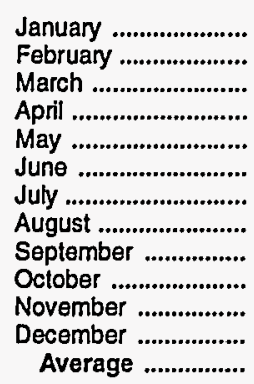 & $\begin{array}{l}1,437 \\
1,440 \\
1,463 \\
1,391 \\
1,427 \\
1,547 \\
1,485 \\
1,358 \\
1,338 \\
1,329 \\
1,386 \\
1,459 \\
1,422\end{array}$ & $\begin{array}{l}1,308 \\
1,316 \\
1,332 \\
1,265 \\
1,302 \\
1,407 \\
1,359 \\
1,257 \\
1,241 \\
1,242 \\
1,301 \\
1,382 \\
1,309\end{array}$ & $\begin{array}{r}89 \\
110 \\
76 \\
88 \\
75 \\
111 \\
94 \\
100 \\
106 \\
143 \\
105 \\
105 \\
100\end{array}$ & $\begin{array}{r}-64 \\
53 \\
-15 \\
-23 \\
42 \\
83 \\
42 \\
-98 \\
-69 \\
-27 \\
8 \\
-13 \\
-7\end{array}$ & $\begin{array}{r}134 \\
17 \\
101 \\
88 \\
60 \\
45 \\
71 \\
42 \\
16 \\
20 \\
29 \\
85 \\
59\end{array}$ & $\begin{array}{l}1,456 \\
1,480 \\
1,453 \\
1,413 \\
1,401 \\
1,530 \\
1,466 \\
1,514 \\
1,497 \\
1,479 \\
1,453 \\
1,493 \\
1,469\end{array}$ & $\begin{array}{l}1,369 \\
1,337 \\
1,335 \\
1,299 \\
1,288 \\
1,362 \\
1,338 \\
1,413 \\
1,357 \\
1,389 \\
1,357 \\
1,441 \\
1,357\end{array}$ & $\begin{array}{r}41 \\
43 \\
42 \\
41 \\
43 \\
45 \\
47 \\
43 \\
41 \\
41 \\
41 \\
40 \\
-\end{array}$ & $\begin{array}{l}36 \\
38 \\
38 \\
37 \\
38 \\
41 \\
43 \\
40 \\
38 \\
37 \\
38 \\
38 \\
-\end{array}$ \\
\hline 1994 & 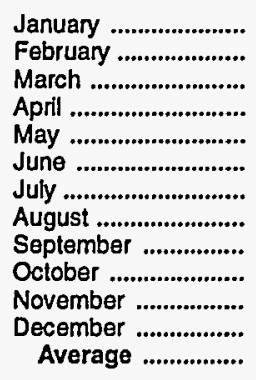 & $\begin{array}{l}1,461 \\
1,379 \\
1,327 \\
1,442 \\
1,456 \\
1,456 \\
1,477 \\
1,544 \\
1,444 \\
1,435 \\
1,444 \\
1,543 \\
1,451\end{array}$ & $\begin{array}{l}1,394 \\
1,331 \\
1,271 \\
1,393 \\
1,402 \\
1,399 \\
1,420 \\
1,498 \\
1,419 \\
1,409 \\
1,433 \\
1,533 \\
1,409\end{array}$ & $\begin{array}{r}116 \\
138 \\
120 \\
138 \\
112 \\
130 \\
88 \\
77 \\
149 \\
110 \\
93 \\
114 \\
115\end{array}$ & $\begin{array}{r}36 \\
-41 \\
-77 \\
20 \\
106 \\
-2 \\
36 \\
38 \\
46 \\
-25 \\
(s) \\
86 \\
19\end{array}$ & $\begin{array}{r}40 \\
35 \\
14 \\
12 \\
9 \\
11 \\
11 \\
10 \\
31 \\
18 \\
19 \\
33 \\
20\end{array}$ & $\begin{array}{l}1,502 \\
1,522 \\
1,509 \\
1,548 \\
1,453 \\
1,578 \\
1,518 \\
1,573 \\
1,516 \\
1,552 \\
1,517 \\
1,538 \\
1,527\end{array}$ & $\begin{array}{l}1,453 \\
1,471 \\
1,440 \\
1,467 \\
1,401 \\
1,516 \\
1,452 \\
1,519 \\
1,461 \\
1,518 \\
1,495 \\
1,526 \\
1,477\end{array}$ & $\begin{array}{l}41 \\
40 \\
38 \\
38 \\
42 \\
42 \\
43 \\
44 \\
45 \\
45 \\
44 \\
47 \\
-\end{array}$ & $\begin{array}{r}39 \\
38 \\
36 \\
36 \\
40 \\
40 \\
41 \\
42 \\
44 \\
43 \\
43 \\
46 \\
-\end{array}$ \\
\hline 1995 & $\begin{array}{l}\text { January .................... } \\
\text { February ..................... } \\
\text { March ...................... } \\
\text { April" .......................... } \\
\text { 4-Mo. Average ......... }\end{array}$ & $\begin{array}{r}1,412 \\
1,376 \\
R_{1,281} \\
E_{1,309} \\
E_{1,344}\end{array}$ & $\begin{array}{r}1,402 \\
1,366 \\
R_{1,272} \\
E_{1,295} \\
E_{1,333}\end{array}$ & $\begin{array}{r}79 \\
123 \\
\mathrm{R}_{99} \\
\mathrm{E}_{63} \\
\mathrm{E}_{90}\end{array}$ & $\begin{array}{r}-101 \\
-44 \\
R-113 \\
E_{-25} \\
E_{-72}\end{array}$ & $\begin{array}{r}33 \\
21 \\
R_{17} \\
E_{27} \\
E_{25}\end{array}$ & $\begin{array}{r}1,559 \\
1,522 \\
R_{1,477} \\
E_{1,369} \\
E_{1,482}\end{array}$ & $\begin{array}{r}1,548 \\
1,516 \\
R_{1,461} \\
E_{1,356}^{1,470}\end{array}$ & $\begin{array}{r}44 \\
43 \\
39 \\
38 \\
-\end{array}$ & $\begin{array}{r}43 \\
42 \\
38 \\
38 \\
-\end{array}$ \\
\hline $\begin{array}{l}1994 \\
1993\end{array}$ & $\begin{array}{l}\text { 4-Mo. Average ......... } \\
\text { 4-Mo. Average ......... }\end{array}$ & $\begin{array}{l}1,402 \\
1,433\end{array}$ & $\begin{array}{l}1,347 \\
1,305\end{array}$ & $\begin{array}{r}128 \\
90\end{array}$ & $\begin{array}{l}-15 \\
-14\end{array}$ & $\begin{array}{l}25 \\
87\end{array}$ & $\begin{array}{l}1,520 \\
1,450\end{array}$ & $\begin{array}{l}1,457 \\
1,335\end{array}$ & - & - \\
\hline
\end{tabular}

Stocks are totals as of end of period.

b A negative number indicates a decrease in stocks and a positive number indicates an increase.

c In January 1981, 1983, and 1984, a new stock basis was established affecting stocks reported and stock change calculations. Stock changes are calculated using new basis stock levels. See Summary Statistics Explanatory Note 4.

$R=$ Revised data ( $s$ ) = Less than 500 barrels per day. $E=$ Estimated.

- See Summary Statistics Explanatory Note 1.

Notes: - Italics denote estimates based upon preliminary data. Geographic coverage is the 50 States and the District of Columbia. $\bullet$ Totals may not equal sum of components due to independent rounding.

Source: See Summary Statistics Table and Figure Sources. 
Figure S13. Propane/Propylene Supply and Disposition, February 1994 - Present

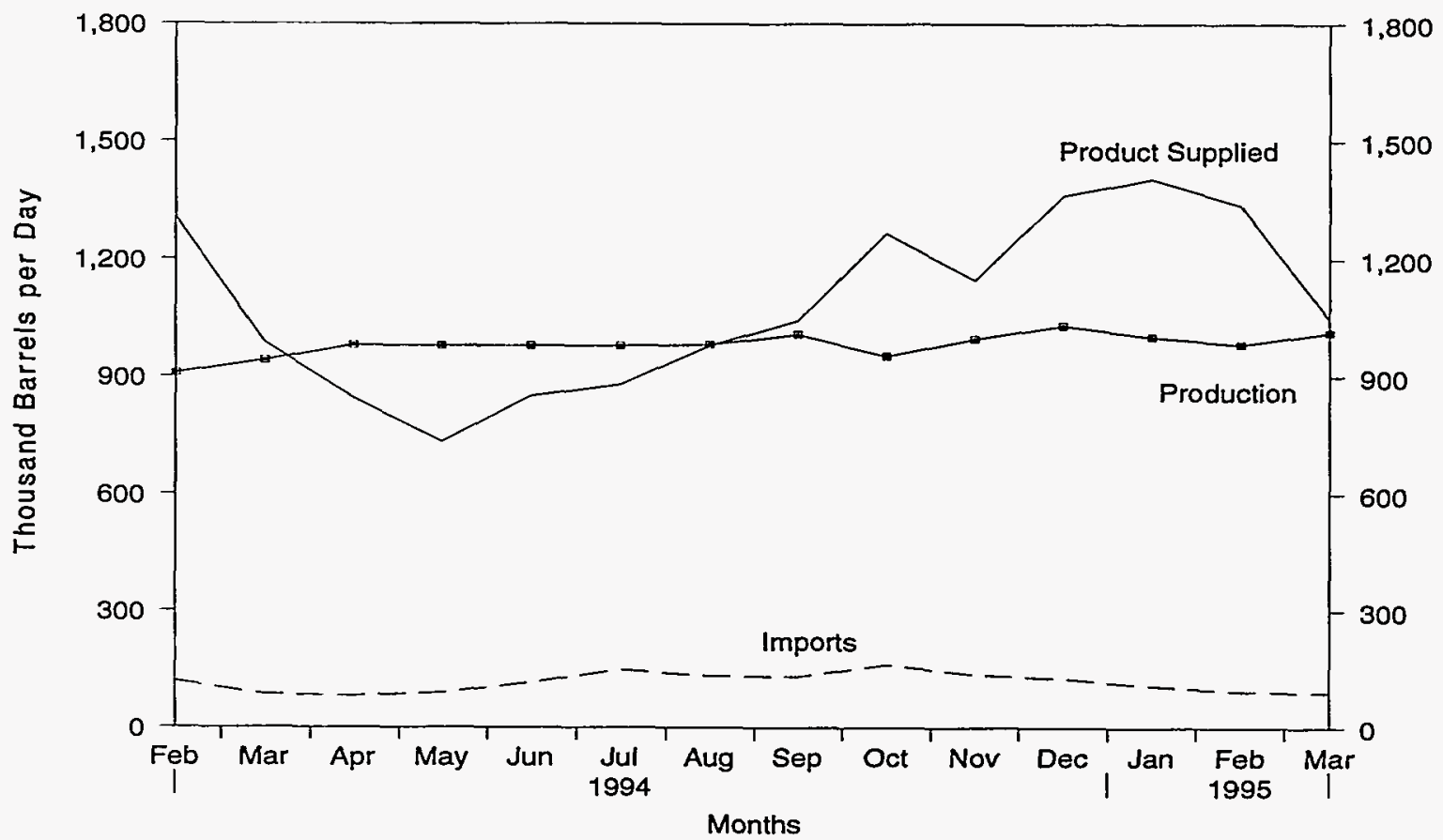

Source: Energy Information Administration, Petroleum Supply Monthly, Table S8. See Summary Statistics Table and Figure Sources.

Figure S14. Propane/Propylene Ending Stocks, February 1994 - Present

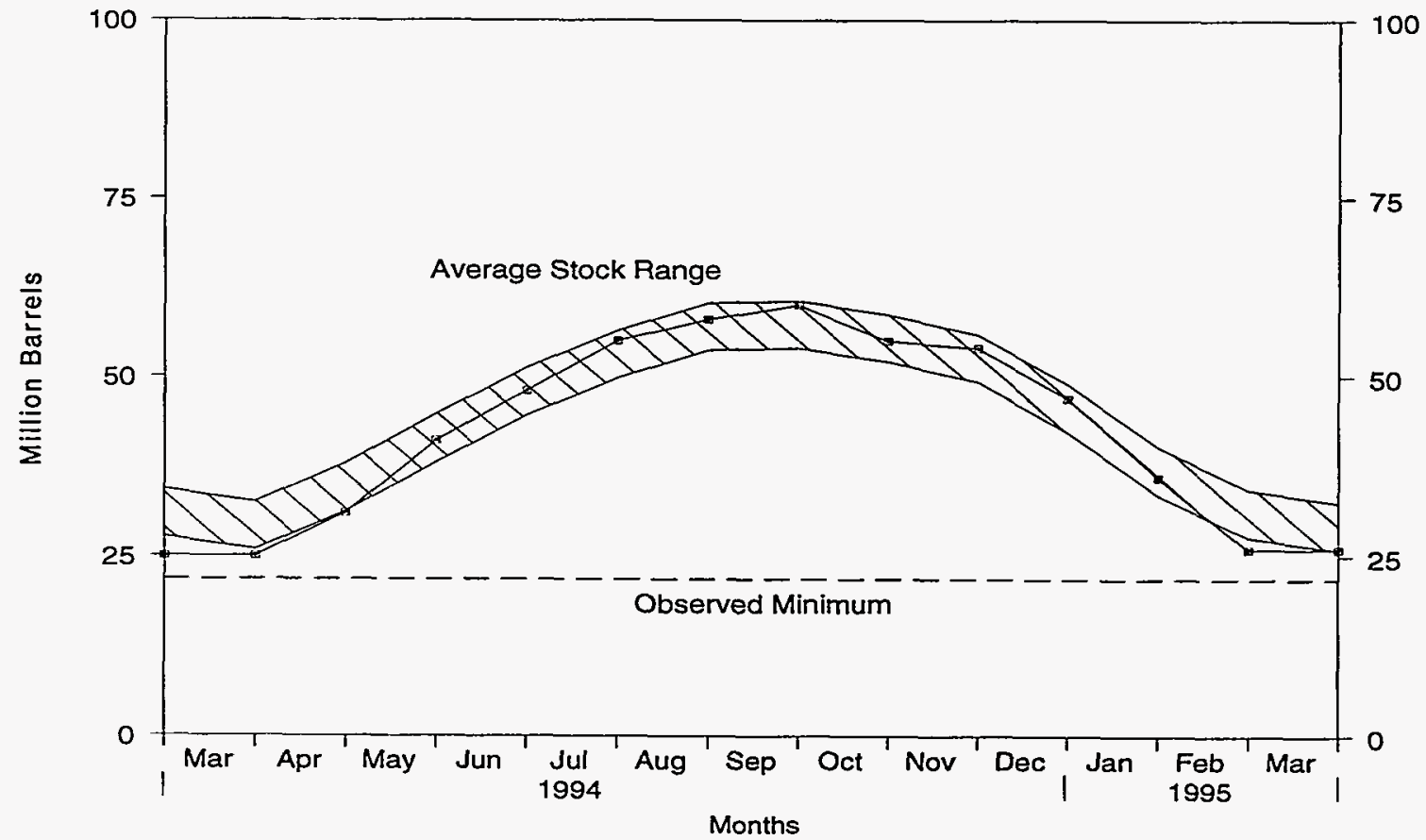

Note: The Observed Minimum for propane stocks in the last 36 month period was 21.8 million barrels, occurring in March 1993.

Source: Energy Information Administration, Petroleum Supply Monthly, Table S8. See Summary Statistics Table and Figure Sources. 
Table S8. Propane/Propylene Supply and Disposition, 1981 - Present (Thousand Barrels per Day, Except Where Noted)

\begin{tabular}{|c|c|c|c|c|c|c|c|c|}
\hline & \multirow[b]{2}{*}{ Year/Month } & \multicolumn{2}{|c|}{ Supply } & \multicolumn{4}{|c|}{ Disposition } & \multirow[b]{2}{*}{$\begin{array}{c}\text { Ending } \\
\text { Stocks } \\
\text { (Million Barrels) }\end{array}$} \\
\hline & & $\begin{array}{c}\text { Total } \\
\text { Production }\end{array}$ & Imports & $\begin{array}{c}\text { Stock } \\
\text { Change }^{a}\end{array}$ & $\begin{array}{l}\text { Refinery } \\
\text { Inputs }\end{array}$ & Exports & $\begin{array}{l}\text { Product } \\
\text { Supplied }\end{array}$ & \\
\hline $\begin{array}{l}1981 \\
1982 \\
1983 \\
1984 \\
1985 \\
1986 \\
1987 \\
1988 \\
1989 \\
1990 \\
1991 \\
1992\end{array}$ & 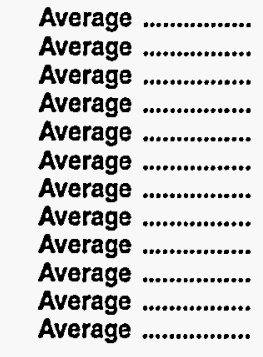 & $\begin{array}{l}745 \\
711 \\
730 \\
806 \\
816 \\
817 \\
828 \\
863 \\
862 \\
878 \\
915 \\
956\end{array}$ & $\begin{array}{r}70 \\
63 \\
44 \\
67 \\
67 \\
110 \\
88 \\
106 \\
111 \\
115 \\
91 \\
85\end{array}$ & $\begin{array}{r}c \\
c 18 \\
-59 \\
c-24 \\
c 7 \\
-50 \\
64 \\
-41 \\
7 \\
-52 \\
48 \\
-3 \\
-24\end{array}$ & $\begin{array}{r}5 \\
4 \\
4 \\
4 \\
3 \\
4 \\
8 \\
8 \\
11 \\
\text { (s) } \\
\text { (s) } \\
\text { (s) }\end{array}$ & $\begin{array}{l}18 \\
31 \\
43 \\
30 \\
48 \\
28 \\
24 \\
31 \\
24 \\
28 \\
28 \\
33\end{array}$ & $\begin{array}{r}773 \\
798 \\
751 \\
833 \\
883 \\
831 \\
924 \\
923 \\
990 \\
917 \\
982 \\
1,032\end{array}$ & $\begin{array}{r}76 \\
c_{54} \\
c^{c} 48 \\
58 \\
39 \\
63 \\
48 \\
50 \\
32 \\
49 \\
48 \\
39\end{array}$ \\
\hline 1993 & 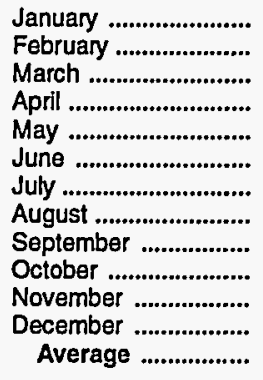 & $\begin{array}{l}968 \\
964 \\
966 \\
980 \\
951 \\
967 \\
963 \\
960 \\
969 \\
954 \\
963 \\
953 \\
963\end{array}$ & $\begin{array}{r}79 \\
82 \\
85 \\
108 \\
96 \\
75 \\
118 \\
116 \\
132 \\
107 \\
138 \\
102 \\
103\end{array}$ & $\begin{array}{r}-212 \\
-255 \\
-109 \\
238 \\
266 \\
265 \\
256 \\
178 \\
92 \\
-11 \\
-126 \\
-195 \\
34\end{array}$ & $\begin{array}{r}1 \\
(s) \\
(s) \\
(s) \\
0 \\
0 \\
0 \\
0 \\
0 \\
0 \\
0 \\
0 \\
(s)\end{array}$ & $\begin{array}{l}31 \\
37 \\
32 \\
40 \\
30 \\
23 \\
26 \\
27 \\
17 \\
13 \\
17 \\
25 \\
26\end{array}$ & $\begin{array}{r}1,227 \\
1,264 \\
1,129 \\
809 \\
750 \\
754 \\
800 \\
871 \\
992 \\
1,059 \\
1,209 \\
1,225 \\
1,006\end{array}$ & $\begin{array}{l}32 \\
25 \\
22 \\
29 \\
37 \\
45 \\
53 \\
59 \\
61 \\
61 \\
57 \\
51 \\
-\end{array}$ \\
\hline 1994 & 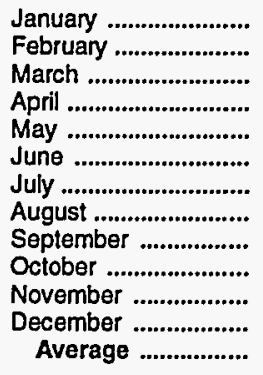 & $\begin{array}{r}892 \\
908 \\
941 \\
980 \\
978 \\
979 \\
979 \\
982 \\
1,008 \\
953 \\
997 \\
1,031 \\
969\end{array}$ & $\begin{array}{r}134 \\
119 \\
85 \\
81 \\
89 \\
115 \\
149 \\
133 \\
131 \\
162 \\
137 \\
127 \\
122\end{array}$ & $\begin{array}{r}-555 \\
-316 \\
11 \\
196 \\
313 \\
224 \\
226 \\
107 \\
77 \\
-176 \\
-40 \\
-233 \\
-13\end{array}$ & $\begin{array}{r}0 \\
6 \\
0 \\
0 \\
0 \\
0 \\
0 \\
0 \\
0 \\
0 \\
0 \\
0 \\
\text { (s) }\end{array}$ & $\begin{array}{l}19 \\
30 \\
29 \\
20 \\
20 \\
20 \\
22 \\
28 \\
20 \\
24 \\
27 \\
29 \\
24\end{array}$ & $\begin{array}{r}1,562 \\
1,308 \\
987 \\
845 \\
733 \\
850 \\
880 \\
980 \\
1,043 \\
1,267 \\
1,147 \\
1,363 \\
1,080\end{array}$ & $\begin{array}{l}34 \\
25 \\
25 \\
31 \\
41 \\
48 \\
55 \\
58 \\
60 \\
55 \\
54 \\
47 \\
-\end{array}$ \\
\hline 1995 & 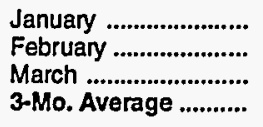 & $\begin{array}{r}1,002 \\
983 \\
1,013 \\
1,000\end{array}$ & $\begin{array}{r}108 \\
94 \\
90 \\
97\end{array}$ & $\begin{array}{r}-350 \\
-361 \\
16 \\
-228\end{array}$ & $\begin{array}{r}0 \\
0 \\
(s) \\
(s)\end{array}$ & $\begin{array}{r}55 \\
100 \\
39 \\
63\end{array}$ & $\begin{array}{l}1,405 \\
1,338 \\
1,048 \\
1,261\end{array}$ & $\begin{array}{l}36 \\
26 \\
26 \\
-\end{array}$ \\
\hline $\begin{array}{l}1994 \\
1993\end{array}$ & $\begin{array}{l}\text { 3-Mo. Average .......... } \\
\text { 3-Mo. Average ......... }\end{array}$ & $\begin{array}{l}914 \\
966\end{array}$ & $\begin{array}{r}113 \\
82\end{array}$ & $\begin{array}{l}-286 \\
-190\end{array}$ & $\begin{array}{r}2 \\
(s)\end{array}$ & $\begin{array}{l}26 \\
33\end{array}$ & $\begin{array}{l}1,285 \\
1,205\end{array}$ & $\ddot{-}$ \\
\hline
\end{tabular}

\footnotetext{
a A negative number indicates a decrease in stocks and a positive number indicates an increase.

b Stocks are totals as of end of period.

c In January 1981, 1983, and 1984, a new stock basis was established affecting stocks reported and stock change calculations. Stock changes are calculated using new basis stock levels. See Summary Statistics Explanatory Note 4.

(s) = Less than 500 barrels per day.

Notes: - Geographic coverage is the 50 States and the District of Columbia. - Totals may not equal sum of components due to independent rounding. Source: See Summary Statistics Table and Figure Sources.
} 
Figure S15. Liquefied Petroleum Gases Supply and Disposition, February 1994 - Present

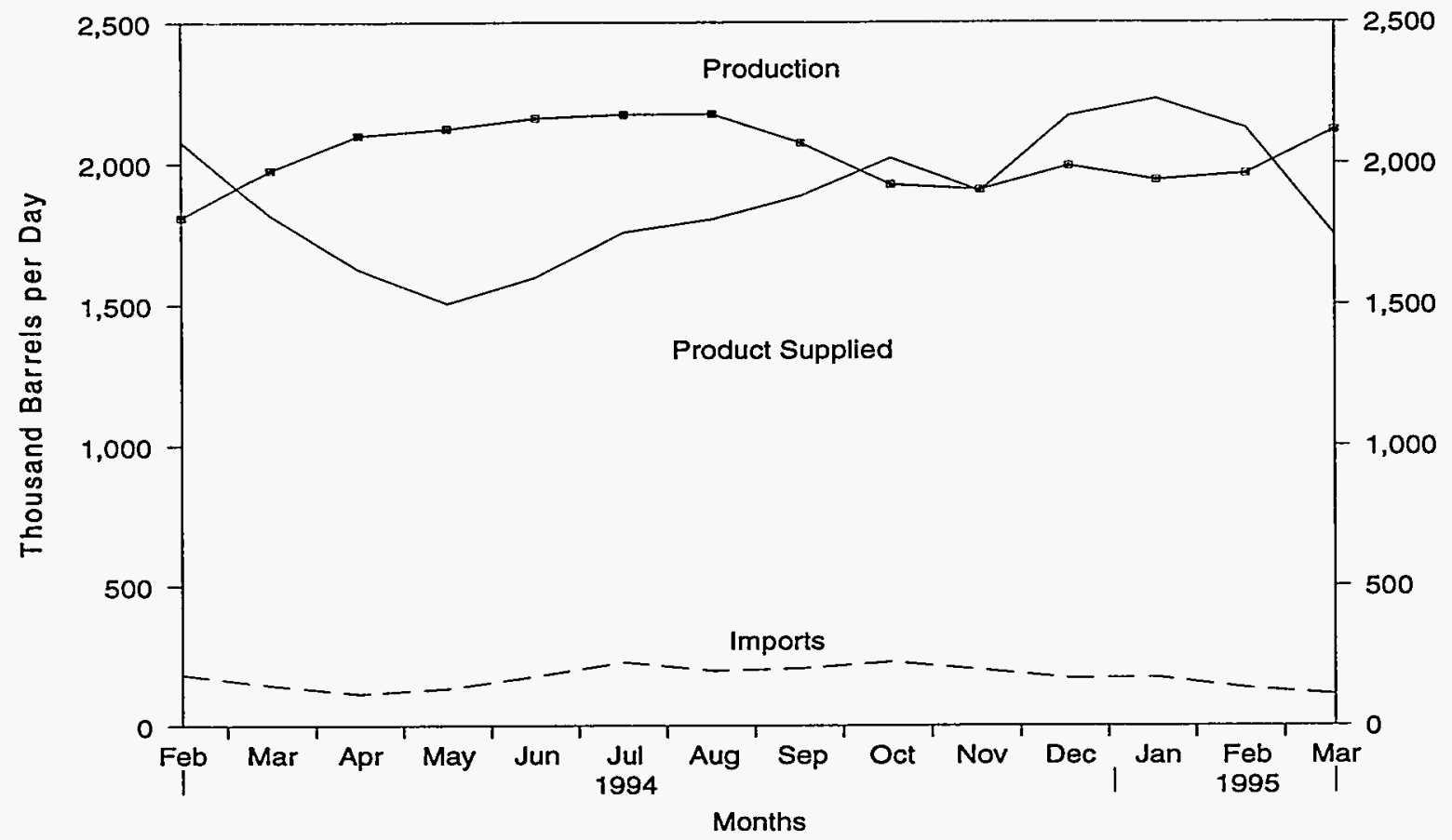

Source: Energy Information Administration, Petroleum Supply Monthly, Table S9. See Summary Statistics Table and Figure Sources.

Figure S16. Liquefied Petroleum Gases Ending Stocks, February 1994 - Present

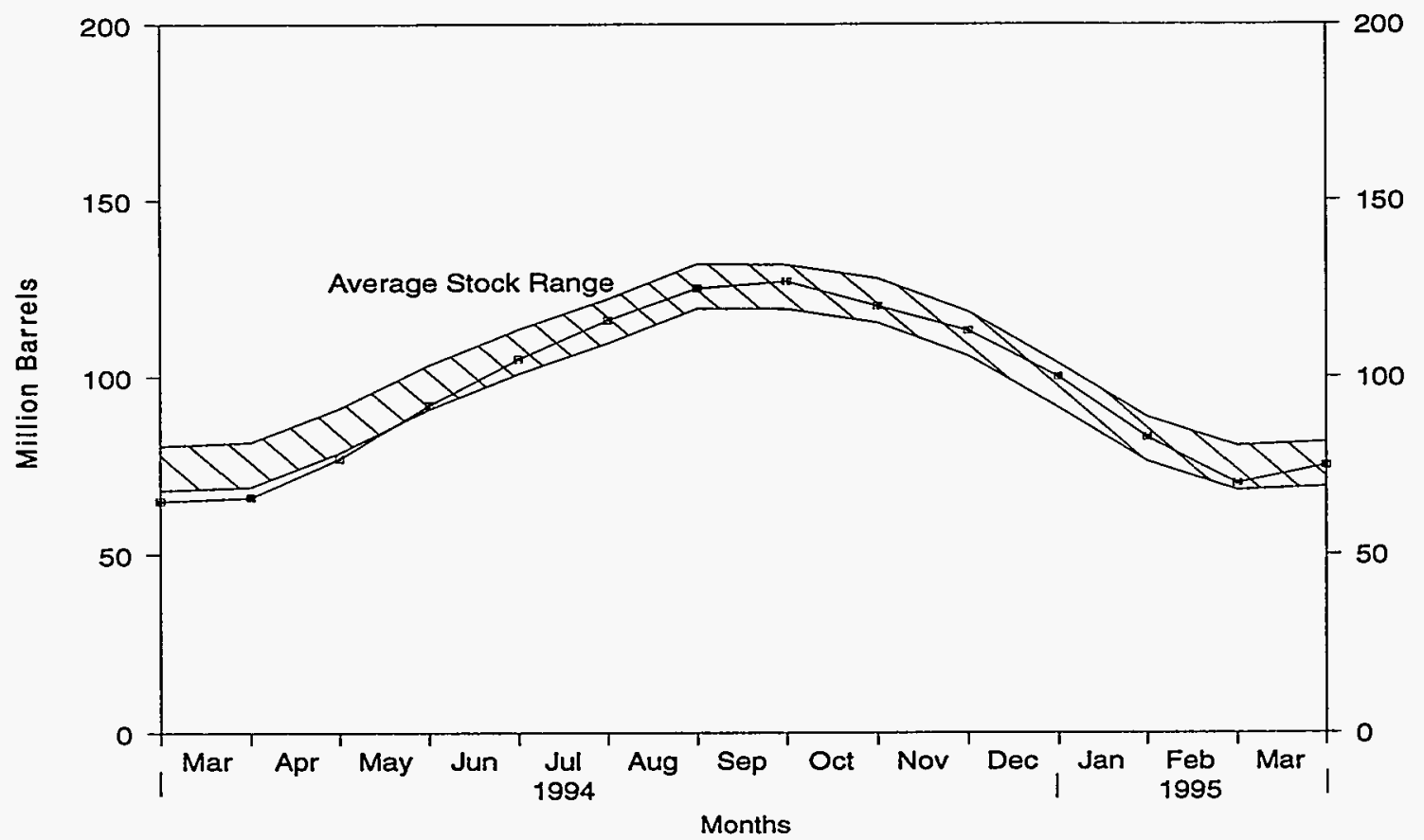

Source: Energy Information Administration, Petroleum Supply Monthly, Table S9. See Summary Statistics Table and Figure Sources. 
Table S9. Liquefied Petroleum Gases Supply and Disposition, 1981 - Present (Thousand Barrels per Day, Except Where Noted)

\begin{tabular}{|c|c|c|c|c|c|c|c|c|}
\hline & \multirow[b]{2}{*}{ Year/Month } & \multicolumn{2}{|c|}{ Supply } & \multicolumn{4}{|c|}{ Disposition } & \multirow[b]{2}{*}{$\begin{array}{c}\text { Ending } \\
\text { Stocks } \\
\text { (Million Barrels) }\end{array}$} \\
\hline & & $\begin{array}{c}\text { Total } \\
\text { Production }\end{array}$ & Imports & $\begin{array}{l}\text { Stock } \\
\text { Change }\end{array}$ & $\begin{array}{l}\text { Refinery } \\
\text { Inputs }\end{array}$ & Exports & $\begin{array}{l}\text { Product } \\
\text { Supplied }\end{array}$ & \\
\hline $\begin{array}{l}1981 \\
1982 \\
1983 \\
1984 \\
1985 \\
1986 \\
1987 \\
1988 \\
1989 \\
1990 \\
1991 \\
1992\end{array}$ & 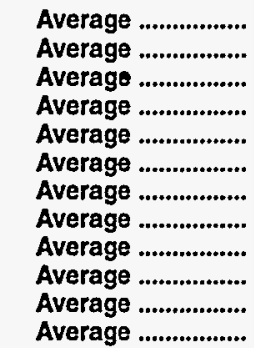 & $\begin{array}{l}1,571 \\
1,528 \\
1,642 \\
1,697 \\
1,704 \\
1,695 \\
1,748 \\
1,817 \\
1,791 \\
1,749 \\
1,871 \\
1,972\end{array}$ & $\begin{array}{l}244 \\
226 \\
190 \\
195 \\
187 \\
242 \\
190 \\
209 \\
181 \\
188 \\
147 \\
131\end{array}$ & $\begin{array}{r}c 18 \\
-111 \\
c-4 \\
c-19 \\
-75 \\
80 \\
-15 \\
1 \\
-47 \\
48 \\
-15 \\
-10\end{array}$ & $\begin{array}{l}289 \\
300 \\
253 \\
291 \\
304 \\
302 \\
304 \\
321 \\
315 \\
293 \\
304 \\
309\end{array}$ & $\begin{array}{l}42 \\
65 \\
73 \\
48 \\
62 \\
42 \\
38 \\
49 \\
35 \\
40 \\
41 \\
49\end{array}$ & $\begin{array}{l}1,466 \\
1,499 \\
1,509 \\
1,572 \\
1,599 \\
1,512 \\
1,612 \\
1,656 \\
1,668 \\
1,556 \\
1,689 \\
1,755\end{array}$ & $\begin{array}{r}135 \\
c 94 \\
{ }^{c} 101 \\
101 \\
74 \\
103 \\
97 \\
97 \\
80 \\
98 \\
92 \\
89\end{array}$ \\
\hline 1993 & 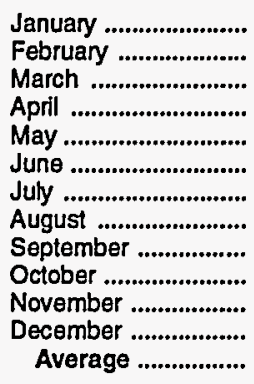 & $\begin{array}{l}1,845 \\
1,929 \\
2,103 \\
2,172 \\
2,116 \\
2,141 \\
2,125 \\
2,105 \\
1,984 \\
1,899 \\
1,789 \\
1,710 \\
1,993\end{array}$ & $\begin{array}{l}126 \\
138 \\
124 \\
161 \\
153 \\
111 \\
175 \\
168 \\
210 \\
200 \\
181 \\
166 \\
160\end{array}$ & $\begin{array}{r}-492 \\
-309 \\
53 \\
472 \\
540 \\
489 \\
391 \\
442 \\
204 \\
-154 \\
-527 \\
-545 \\
49\end{array}$ & $\begin{array}{l}444 \\
363 \\
256 \\
250 \\
254 \\
247 \\
246 \\
269 \\
312 \\
381 \\
469 \\
440 \\
327\end{array}$ & $\begin{array}{l}39 \\
55 \\
47 \\
69 \\
50 \\
41 \\
54 \\
45 \\
35 \\
21 \\
21 \\
40 \\
43\end{array}$ & $\begin{array}{l}1,980 \\
1,958 \\
1,871 \\
1,542 \\
1,425 \\
1,476 \\
1,609 \\
1,517 \\
1,644 \\
1,851 \\
2,007 \\
1,942 \\
1,734\end{array}$ & $\begin{array}{r}73 \\
65 \\
66 \\
81 \\
97 \\
112 \\
124 \\
138 \\
144 \\
139 \\
123 \\
106 \\
-\end{array}$ \\
\hline 1994 & 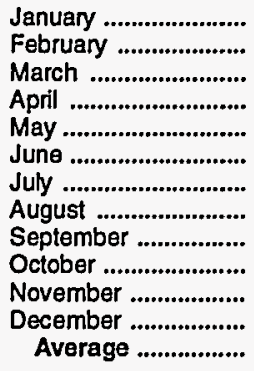 & $\begin{array}{l}1,710 \\
1,809 \\
1,976 \\
2,099 \\
2,123 \\
2,161 \\
2,174 \\
2,175 \\
2,073 \\
1,925 \\
1,907 \\
1,991 \\
2,011\end{array}$ & $\begin{array}{l}187 \\
182 \\
144 \\
114 \\
133 \\
177 \\
227 \\
196 \\
205 \\
228 \\
199 \\
169 \\
180\end{array}$ & $\begin{array}{r}-902 \\
-474 \\
35 \\
341 \\
477 \\
448 \\
358 \\
296 \\
71 \\
-229 \\
-226 \\
-448 \\
-19\end{array}$ & $\begin{array}{l}381 \\
343 \\
232 \\
218 \\
243 \\
251 \\
246 \\
236 \\
264 \\
322 \\
396 \\
399 \\
294\end{array}$ & $\begin{array}{l}28 \\
44 \\
37 \\
29 \\
32 \\
41 \\
40 \\
37 \\
56 \\
40 \\
35 \\
41 \\
38\end{array}$ & $\begin{array}{l}2,390 \\
2,077 \\
1,816 \\
1,625 \\
1,505 \\
1,597 \\
1,757 \\
1,803 \\
1,886 \\
2,019 \\
1,902 \\
2,168 \\
1,878\end{array}$ & $\begin{array}{r}79 \\
65 \\
66 \\
77 \\
92 \\
105 \\
116 \\
125 \\
127 \\
120 \\
113 \\
100 \\
-\end{array}$ \\
\hline 1995 & 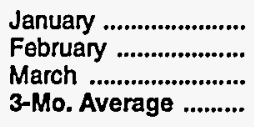 & $\begin{array}{l}1,941 \\
1,964 \\
2,117 \\
2,009\end{array}$ & $\begin{array}{r}172 \\
134 \\
111 \\
139\end{array}$ & $\begin{array}{r}-542 \\
-456 \\
175 \\
-268\end{array}$ & $\begin{array}{l}363 \\
306 \\
248 \\
306\end{array}$ & $\begin{array}{r}64 \\
122 \\
57 \\
80\end{array}$ & $\begin{array}{l}2,228 \\
2,125 \\
1,747 \\
2,031\end{array}$ & $\begin{array}{l}83 \\
70 \\
75 \\
-\end{array}$ \\
\hline $\begin{array}{l}1994 \\
1993\end{array}$ & $\begin{array}{l}\text { 3-Mo. Average ......... } \\
\text { 3-Mo. Average .......... }\end{array}$ & $\begin{array}{l}1,832 \\
1,960\end{array}$ & $\begin{array}{l}171 \\
129\end{array}$ & $\begin{array}{l}-446 \\
-248\end{array}$ & $\begin{array}{l}318 \\
354\end{array}$ & $\begin{array}{l}36 \\
46\end{array}$ & $\begin{array}{l}2,095 \\
1,936\end{array}$ & $\overline{-}$ \\
\hline
\end{tabular}

a negative number indicates a decrease in stocks and a positive number indicates an increase.

b tocks are totals as of end of period.

c In January 1981, 1983, and 1984, a new stock basis was established affecting stocks reported and stock change calculations. Stock changes are calculated using new basis stock levels. See Summary Statistics Explanatory Note 4.

Notes: - Liquefied petroleum gases includes ethane/ethylene, propane/propylene, normal butane/butylene, and isobutane/isobutylene. • Beginning in January 1984, unfractionated stream, is reported by individual product. • Geographic coverage is the 50 States and the District of Columbia. - Totals may not equal sum of components due to independent rounding.

Source: See Summary Statistics Table and Figure Sources. 
Table S10. Other Petroleum Products Supply and Disposition, 1981 - Present (Thousand Barrels per Day, Except Where Noted)

\begin{tabular}{|c|c|c|c|c|c|c|c|c|}
\hline & \multirow[b]{2}{*}{ Year/Month } & \multicolumn{2}{|c|}{ Supply } & \multicolumn{4}{|c|}{ Disposition } & \multirow[b]{2}{*}{$\begin{array}{c}\text { Ending } \\
\text { Stocks } \\
\text { (Million Barrels) }\end{array}$} \\
\hline & & $\begin{array}{c}\text { Total } \\
\text { Production }\end{array}$ & Imports & $\begin{array}{l}\text { Stock } \\
\text { Change }\end{array}$ & $\begin{array}{l}\text { Refinery } \\
\text { Inputs }\end{array}$ & Exports & $\begin{array}{l}\text { Products } \\
\text { Supplied }\end{array}$ & \\
\hline $\begin{array}{l}1981 \\
1982 \\
1983 \\
1984 \\
1985 \\
1986 \\
1987 \\
1988 \\
1989 \\
1990 \\
1991 \\
1992\end{array}$ & 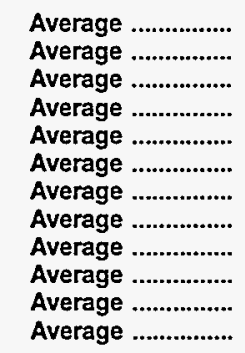 & $\begin{array}{l}2,771 \\
2,475 \\
2,437 \\
2,500 \\
2,532 \\
2,704 \\
2,737 \\
2,773 \\
2,771 \\
2,842 \\
2,826 \\
2,928\end{array}$ & $\begin{array}{l}188 \\
305 \\
382 \\
503 \\
550 \\
504 \\
543 \\
645 \\
627 \\
705 \\
675 \\
707\end{array}$ & $\begin{array}{r}c-42 \\
-68 \\
c^{c}-6 \\
c_{-32} \\
22 \\
-15 \\
-1 \\
22 \\
12 \\
-32 \\
18 \\
-3\end{array}$ & $\begin{array}{l}723 \\
787 \\
712 \\
791 \\
886 \\
888 \\
829 \\
799 \\
797 \\
887 \\
936 \\
906\end{array}$ & $\begin{array}{l}197 \\
205 \\
236 \\
236 \\
227 \\
291 \\
264 \\
294 \\
305 \\
289 \\
277 \\
263\end{array}$ & $\begin{array}{l}2,081 \\
1,856 \\
1,877 \\
2,007 \\
1,947 \\
2,045 \\
2,187 \\
2,303 \\
2,285 \\
2,402 \\
2,269 \\
2,470\end{array}$ & $\begin{array}{r}c_{241} \\
c_{216} \\
217 \\
198 \\
206 \\
201 \\
200 \\
208 \\
213 \\
201 \\
208 \\
207\end{array}$ \\
\hline 1993 & 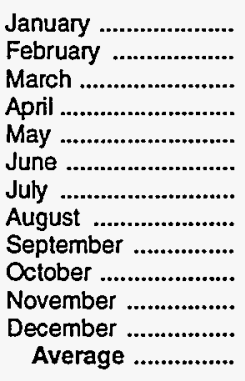 & $\begin{array}{l}3,147 \\
2,853 \\
2,887 \\
2,935 \\
2,941 \\
3,099 \\
3,213 \\
3,167 \\
3,067 \\
3,195 \\
3,080 \\
2,816 \\
3,035\end{array}$ & $\begin{array}{l}726 \\
773 \\
826 \\
753 \\
834 \\
654 \\
894 \\
693 \\
800 \\
810 \\
795 \\
678 \\
770\end{array}$ & $\begin{array}{r}c_{739} \\
111 \\
245 \\
-29 \\
80 \\
-239 \\
61 \\
-28 \\
-268 \\
-114 \\
-222 \\
-376 \\
-2\end{array}$ & $\begin{array}{r}929 \\
1,057 \\
843 \\
1,033 \\
1,048 \\
1,064 \\
1,008 \\
940 \\
1,104 \\
1,189 \\
1,355 \\
1,403 \\
1,081\end{array}$ & $\begin{array}{l}271 \\
282 \\
269 \\
315 \\
278 \\
278 \\
303 \\
294 \\
282 \\
369 \\
309 \\
349 \\
300\end{array}$ & $\begin{array}{l}1,933 \\
2,176 \\
2,356 \\
2,368 \\
2,368 \\
2,650 \\
2,735 \\
2,654 \\
2,749 \\
2,561 \\
2,433 \\
2,117 \\
2,426\end{array}$ & $\begin{array}{r}229 \\
233 \\
240 \\
239 \\
242 \\
235 \\
237 \\
236 \\
228 \\
224 \\
217 \\
206 \\
-\end{array}$ \\
\hline 1994 & 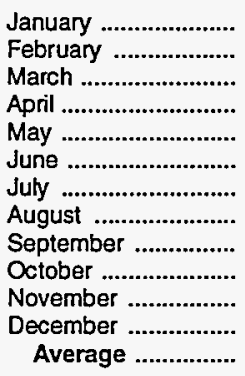 & $\begin{array}{l}2,719 \\
2,779 \\
2,805 \\
2,901 \\
3,088 \\
3,127 \\
3,155 \\
3,087 \\
3,086 \\
3,067 \\
2,996 \\
2,862 \\
2,974\end{array}$ & $\begin{array}{l}780 \\
725 \\
753 \\
780 \\
754 \\
716 \\
745 \\
801 \\
686 \\
700 \\
749 \\
762 \\
746\end{array}$ & $\begin{array}{r}507 \\
236 \\
32 \\
-108 \\
-26 \\
-133 \\
89 \\
-31 \\
92 \\
-75 \\
37 \\
-278 \\
27\end{array}$ & $\begin{array}{r}590 \\
638 \\
939 \\
981 \\
975 \\
865 \\
733 \\
782 \\
754 \\
902 \\
1,013 \\
1,049 \\
853\end{array}$ & $\begin{array}{l}256 \\
248 \\
361 \\
272 \\
288 \\
331 \\
361 \\
411 \\
388 \\
300 \\
344 \\
386 \\
329\end{array}$ & $\begin{array}{l}2,147 \\
2,383 \\
2,226 \\
2,536 \\
2,605 \\
2,781 \\
2,717 \\
2,725 \\
2,538 \\
2,638 \\
2,352 \\
2,467 \\
2,510\end{array}$ & $\begin{array}{r}221 \\
228 \\
229 \\
226 \\
225 \\
221 \\
223 \\
223 \\
225 \\
223 \\
224 \\
215 \\
-\end{array}$ \\
\hline 1995 & 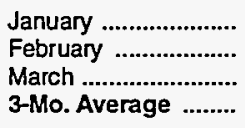 & $\begin{array}{l}2,819 \\
2,914 \\
2,797 \\
2,841\end{array}$ & $\begin{array}{l}563 \\
802 \\
669 \\
674\end{array}$ & $\begin{array}{r}383 \\
236 \\
-8 \\
203\end{array}$ & $\begin{array}{l}634 \\
722 \\
873 \\
744\end{array}$ & $\begin{array}{l}324 \\
320 \\
329 \\
324\end{array}$ & $\begin{array}{l}2,041 \\
2,438 \\
2,273 \\
2,244\end{array}$ & $\begin{array}{r}227 \\
234 \\
234 \\
-\end{array}$ \\
\hline $\begin{array}{l}1994 \\
1993\end{array}$ & $\begin{array}{l}\text { 3-Mo. Average ........ } \\
\text { 3-Mo. Average ......... }\end{array}$ & $\begin{array}{l}2,768 \\
2,966\end{array}$ & $\begin{array}{l}754 \\
775\end{array}$ & $\begin{array}{l}259 \\
373\end{array}$ & $\begin{array}{l}725 \\
939\end{array}$ & $\begin{array}{l}289 \\
274\end{array}$ & $\begin{array}{l}2,248 \\
2,154\end{array}$ & $\overline{-}$ \\
\hline
\end{tabular}

a A negative number indicates a decrease in stocks and a positive number indicates an increase.

b Stocks are totals as of end of period.

c In January 1981, 1983, and 1984, a new stock basis was established affecting stocks reported and stock change calculations. Stock changes are calculated using new basis stock levels. Bulk terminal and pipeline stocks of oxygenates were added beginning in January 1993 . See Summary Statistics Explanatory Note 4.

Notes: - Other petroleum products includes pentanes plus, other hydrocarbons and oxygenates, unfinished oils, gasoline blending components and all finished petroleum products except finished motor gasoline, distillate fuel oil, residual fuel oil, jet fuel, liquefied petroleum gases, and crude oil product supplied. - Geographic coverage is the 50 States and the District of Columbia. - Totals may not equal sum of components due to independent rounding.

Source: See Summary Statistics Table and Figure Sources. 


\section{Summary Statistics Tables and Figures Sources}

Information about petroleum supply and disposition at the National level are presented in the Summary Statistics tables. Industry terminology and product definitions are listed alphabetically in the Glossary.

The data presented in these tables are from several sources and represent different levels of timeliness and data finality.

- U.S. Department of Energy, Energy Information Administration (EIA), Petroleum Supply Annual (1981 through 1993).

- EIA, Petroleum Supply Monthly (January 1994 through March 1995).
- EIA, Weekly Petroleum Supply Reporting System (except domestic crude oil production) (April 1995). A more detailed explanation is provided in Summary Statistics Explanatory Note 1.

- Domestic crude oil production estimate is based on historical statistics from State conservation agencies and the Minerals Management Service of the U.S. Department of the Interior. (January 1994 through April 1995). Refer to Summary Statistics Explanatory Note 2 for a more detailed explanation. 


\section{Summary Statistics Explanatory Notes}

The following explanatory notes are provided to assist in understanding and interpreting the data presented in the Summary Statistics section of this publication.

\section{Note 1. Preliminary Monthly Statistics Derivation}

Data collected from the Weekly Petroleum Supply Reporting System (WPSRS) are used to develop estimates of the most current monthly quantities. The forms that comprise the WPSRS are:

$\begin{array}{ll}\text { Eorm Number } & \text { Name } \\ \text { EIA-800 } & \text { "Weekly Refinery Report" } \\ \text { EIA-801 } & \text { "Weekly Bulk Terminal Report" } \\ \text { EIA-802 } & \text { "Weekly Product Pipeline Report" } \\ \text { EIA-803 } & \text { "Weekly Crude Oil Stocks Report" } \\ \text { EIA-804 } & \text { "Weekly Imports Report" }\end{array}$

A sample of all petroleum companies report weekly data to the Energy Information Administration (EIA) on crude oil and petroleum products stocks, refinery inputs and production, and crude oil and petroleum product imports. The sample of companies that report weekly is selected from the universe of companies that report on the comparable monthly surveys.

The sampling procedure used for the weekly system is the cut-off method. In the cut-off method, companies are ranked from largest to smallest on the basis of the quantities reported during a 12-month period. Companies are chosen for the sample beginning with the largest companies with additional companies added until the total sample coverage represents a minimum of 90 percent of each item by geographic region being measured. All monthly-from-weekly estimates are shown in italics.

In calculating monthly estimates based upon weekly submissions, an interpolation process is used to make the weekly figures comparable to the monthly. The interpolation process is designed to resolve the timing differences between the weekly and the monthly systems -- the time-of-day of reporting periods and the day-of-month of reporting periods. The end of the weekly reporting period (exactly 1 week long) is $7 \mathrm{a} . \mathrm{m}$. Friday. The end of the monthly reporting period (one calendar month long) is 12 midnight on the last day of the month. To resolve the difference in the time-of-day of the weekly and monthly reporting periods, it is assumed that there is no activity during the period 12 midnight Thursday through 7 a.m. Friday. Thus, for the purposes of interpolation, the weekly system reporting period is assumed to end at 12 midnight on Thursday. The resolution of the day-of-month differences depends on whether the series is a cumulative one (such as production and imports) or a value at a fixed point-in-time (i.e., stocks).

For cumulative items (all items except stocks) the following method is used to calculate a monthly-from-weekly figure for a given month. First, a weight is assigned to each week in the month based on the number of days in that week that are in the month. (All intermediate weeks in a month will have a weight of seven; the beginning and ending weeks in the month may have a weight of less than seven, according to the number of days of the week that are in the month.) The weight for each week is then multiplied by the average daily volume for that week. To arrive at the monthly-from-weekly figure, a sum is taken of these weighted weekly volumes. The daily average for the monthly-from-weekly figure is calculated by dividing the total monthly-from-weekly figure by the number of days in the month.

Stock figures are not cumulative but represent inventories as of the last day of the reporting period. When the reporting week does not coincide with the end of a reporting month, an interpolation is necessary to derive a monthly-from-weekly figure for end-of-month stocks.

To derive the monthly-from-weekly stock figures, the two weekly reports that bracket the end of the month are used. Average daily stock change and the number of interpolated days are determined. The average daily stock change is defined as one-seventh of the difference between the stock level at the end of the last full week of the month and the stock level at the end of the week containing the last day of the month. The number of interpolation days is defined as the number of days between the end of the preceding weekly reporting period (midnight Thursday) and the end of the monthly reporting period. The end-of-month stock levels are then estimated as the sum of (a) the stock level reported the last full week of the month, plus (b) the number of interpolation days multiplied by the average daily stock change for the week.

The monthly-from-weekly exports data are derived from the most recent data published in the Weekly Petroleum Status Report. Beginning with statistics for the first week ending in October 1991, weekly estimates of exports are forecast using an autoregressive integrated moving-average (ARIMA) procedure. The ARIMA procedure models a value as a linear combination of its own past values and present and past values of other related time series. The most recent 5 years of 
past data are used to obtain the forecast. In addition, for the major products and crude oil, 5 years of related price data are used. The price data include some U.S. and some foreign series.

\section{Note 2. Domestic Crude Oil Production}

The Energy Information Administration (EIA) collects monthly crude oil production data on an ongoing basis. Data on crude oil production for States are reported to the EIA by State government agencies. Data on crude oil production for Federal offshore areas are reported to the EIA by the Minerals Management Service of the U.S. Department of the Interior and the Conservation Committee of California Oil Producers.

Currently, all except four crude oil producing States (Michigan, New York, Ohio, and Pennsylvania) report production on a monthly basis. These four States report crude oil production on an annual basis. Estimates of monthly crude oil production for these four States are made by the EIA using data reported on Form EIA-182, "Domestic Crude Oil First Purchase Report." After the end of each calendar year, the monthly crude oil production estimates are updated using annual reports from various State agencies, the Minerals Management Service, and the Conservation Committee of California Oil Producers. The final estimate is published in the Petroleum Supply Annual. There is a time lag of approximately 4 months between the end of the production month and the time when most monthly State crude oil production data become available.

In order to present more timely crude oil production estimates, the EIA prepares an original, forecast estimate on the first day of the production month (indicated with a "PE"). Approximately 45 days later, this original estimate of monthly crude oil production is replaced by State-level interim estimates (indicated with an "RE"). The State-level interim estimates are based on: (a) data reported by the States (e.g., production data for Alaska are typically reported to the EIA before the interim estimate is made); (b) first purchase data reported on Form EIA-182, "Domestic Crude Oil First Purchase Report;" (c) exponential or hyperbolic curve fitted projections based on recent State data; or (d) constant level projections based on the average production rate during a recent time period.

\section{Note 3. Figures}

Figures associated with the Summary Statistics tables are provided which depict the balance between supply, disposition, and ending stocks for various commodities.

The national inventory (stocks) graphs (Figures S4, S6, S8, $S 10, S 12, S 14$, and $S 16$ ) for crude oil, finished motor gasoline, distillate fuel oil, residual fuel oil, jet fuel, propane/propylene, and liquefied petroleum gases, in this publication include features to assist in comparing current inventory levels with past inventory levels and observed minimum operating levels. These features are described below.

The graphs displaying inventory levels provide the reader with actual inventory data compared to an average range from the most recent 3-year period running from January through December or from July through June. The ranges are updated every 6 months in April and October. The 3-year period is adjusted by dropping the oldest 6 months and including the most recent 6 months. The ranges also reflect seasonal variation determined from a 7-year period. The seasonal factors, which determine the shape of the upper and lower curves, are updated annually in October, using the most recent year's final monthly data.

The monthly seasonal factors are estimated by means of a seasonal adjustment technique developed at the U.S. Bureau of the Census (Census X-11). The seasonal factors are assumed to be stable (i.e., unchanging from year to year) and additive (i.e., the series is deseasonalized by subtracting the seasonal factor for the appropriate month from the reported inventory levels). The intent of deseasonalization is to remove only variation from the data. Thus, a deseasonalized series would contain the same trends, cyclical components, and irregularities as the original data.

After seasonal factors are derived, data from the most recent 3-year period (January through December or July through June) are deseasonalized. The average of the deseasonalized 36-month series determines the midpoint of the deseasonalized average band. The standard deviation of the deseasonalized 36 months is calculated adjusting for extreme data points. The upper curve of the average range is defined as the average plus the seasonal factors plus the standard deviation. The lower curve is defined as the average plus the seasonal factors minus the standard deviation. Thus, the width of the average range is twice the standard deviation.

The lines labeled "observed minimum" are the lowest inventory level observed during the most recent 36-month period as published in the Petroleum Supply Monthly.

\section{Note 4. Frames Maintenance}

In January 1981 and 1983, numerous respondents were added to bulk terminal and pipeline surveys affecting subsequent stocks reported and stock change calculations. Using the expanded coverage (new basis), the end-of-year stocks, in million barrels, would have been as listed below.

- Crude Oil: 1982- 645 (Total) and 351 (Other Primary). 
- Crude Oil and Petroleum Products: 1980- 1,425; and 1982- 1,461 .

- Motor Gasoline: 1980- 263 (Total) and 214 (Finished); 1982- 244 (Total) and 202 (Finished).

- Distillate Fuel Oil: 1980- 205; and 1982- 186.

- $\quad$ Residual Fuel Oil: 1980- 91; and 1982- 69.

- Jet Fuel: 1980- 42 (Total) and 36 (Kerosene-type); and 1982- 39 (Total) and 32 (Kerosene-type).

- Propane/Propylene: 1980-69; and 1982- 57.

- Liquefied Petroleum Gases: 1980- 128; and 1982- 102.

- Other Petroleum Products: 1980- 207; and 1982- 219.

Stock change calculations beginning in 1981 and 1983 were made using new basis stock levels.

Stocks of Alaskan crude oil in-transit were included for the first time in January 1981. The major impact of this change is on the reporting of stock change calculations. Using the expanded coverage (new basis), 1980 end-of-year crude oil stocks would have been 488 million barrels (Total) and 380 million barrels (Other Primary).

Beginning with January 1984, natural gas liquids supply and disposition data were collected on a component basis rather than a product basis. This change affected stocks reported and stock change calculations. Under the new basis, end-of-year 1983 stocks would have been:

- Propane/Propylene: 1983-55.

- Liquefied Petroleum Gases: 1983- 108.

- Other Petroleum Products: 1983-210.

In response to changes in the Clean Air Act Amendments of 1990 requiring that all gasoline sold in carbon monoxide nonattainment areas have an oxygen content of 2.7 percent (by weight) during winter months, the Energy Information Administration (EIA) conducted a frame identifier survey in 1991 of companies that produce, blend, store, or import oxygenates. The purpose of this survey was to (1) identify all U.S. producers, blenders, storers, and importers of oxygenates; and (2) collect supply and blending data for 1990 and end of 1990 inventory data on those oxygenates blended into motor gasoline. A summary of the results from the identification survey were published in the Weekly Petroleum Status Report dated February 12, 1992 and in the February 1992 issue of the Petroleum Supply Monthly.

In order to continue to provide relevant information about U.S. and regional gasoline supply, the EIA conducted a second frame identifier survey of these companies during 1992. As a result, a number of respondents were added to the monthly surveys effective in January 1993: 19 blenders, 25 stock holders, and 8 importers. This change did not affect stocks reported and therefore did not cause a new basis stock level to be calculated. 


\section{Detailed Statistics}

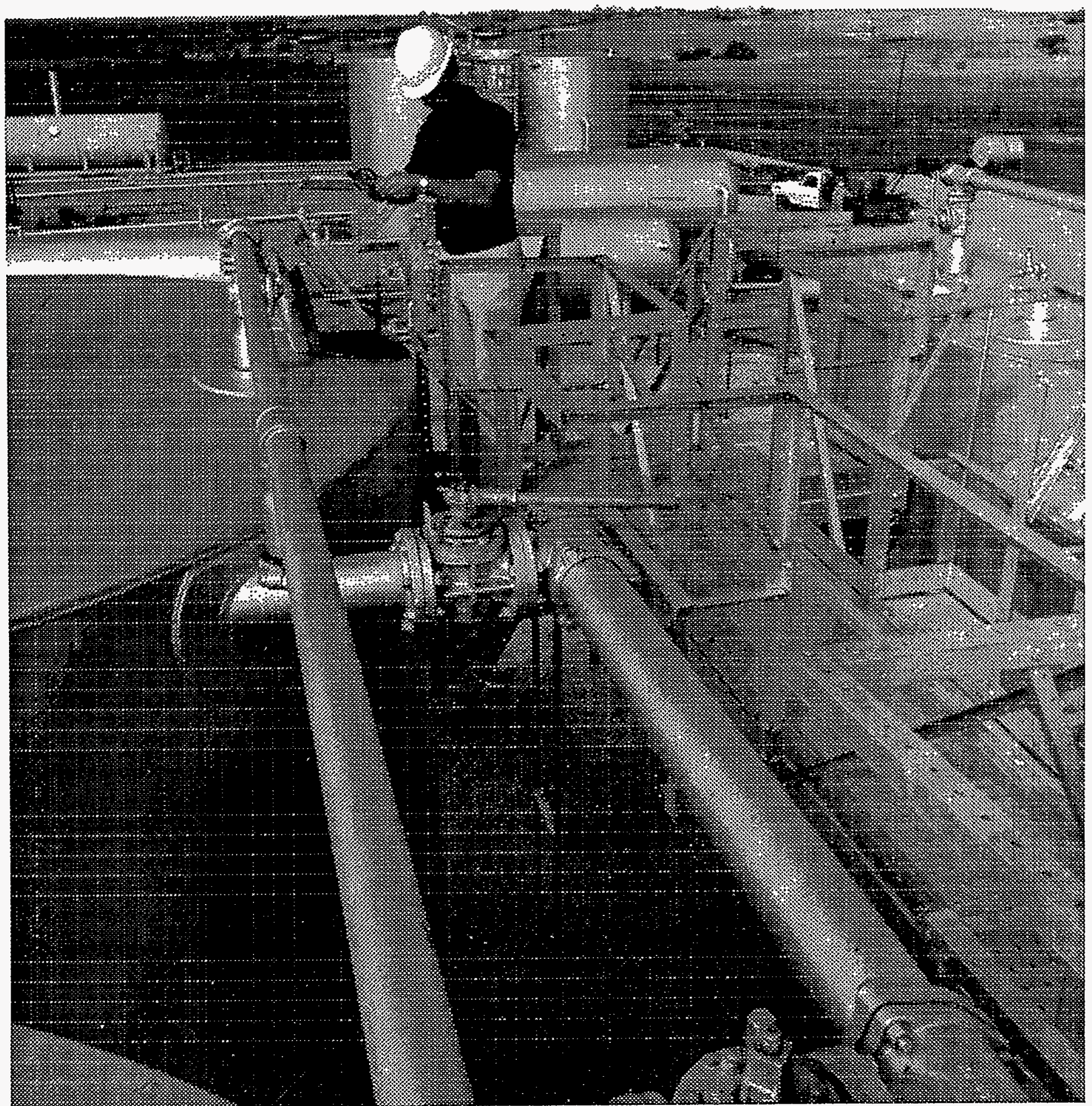

At some locations, oil skimmers and knockout tanks (in background) are used to remove waste water from the crude oil. The crude oil is then put into storage tanks and gauged. 



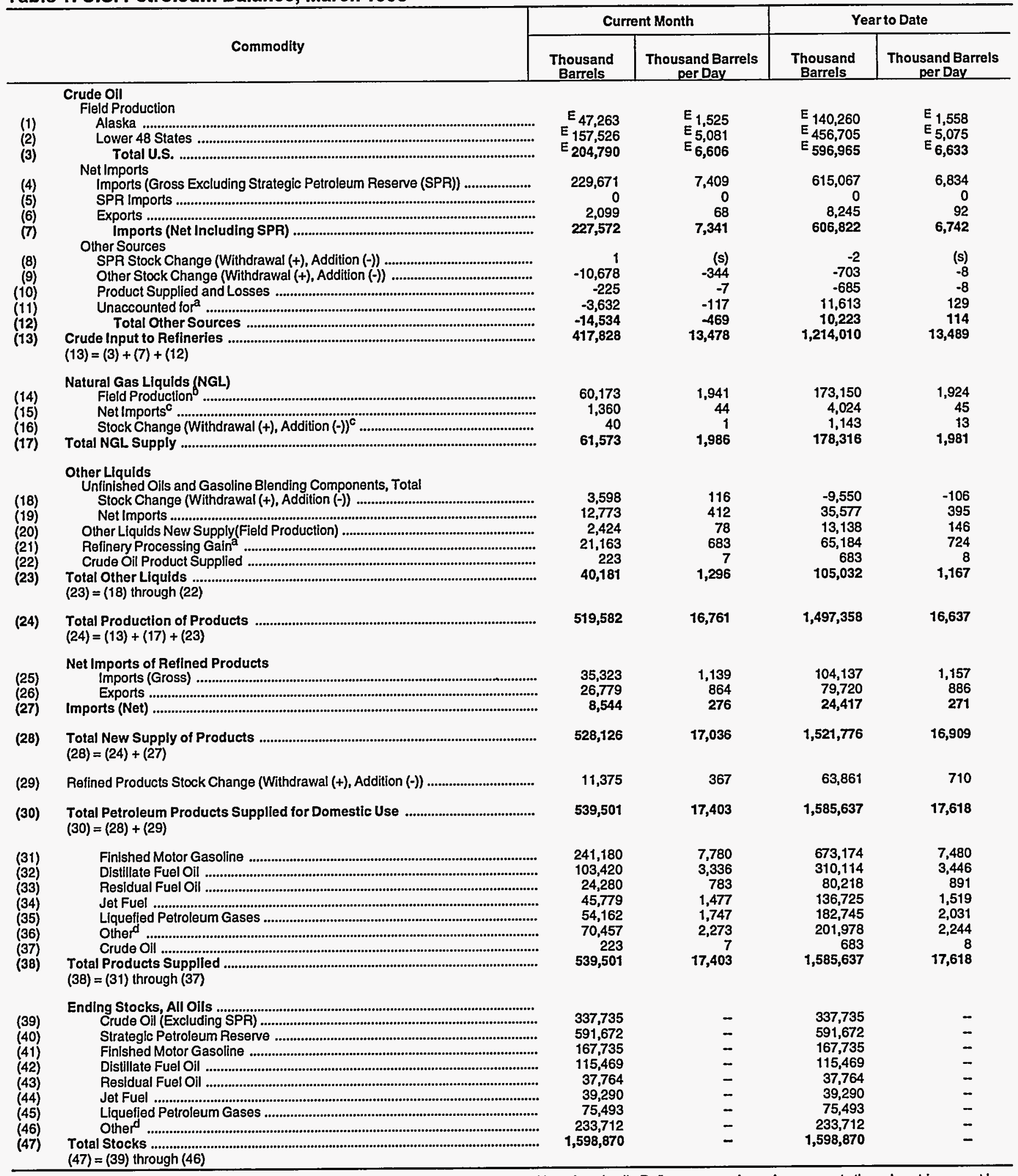

a Unaccounted for crude oil represents the difference between the supply and disposition of crude oil. Refinery processing gain represents the volumetric amount by which total output is greater than input for a given period of time. Preliminary estimates of crude oil imports at the National level have historically understated final values by approximately 50 thousand barrels per day. This causes the preliminary values of unaccounted for crude oil to overstate the final values by the same amount.

Includes fuel ethanol blended into finished motor gasoline.

c Includes products in the pentanes plus category only.

d Includes pentanes plus, other liquids, and all finished petroleum products except finished motor gasoline, distillate fuel oil, residual fuel oil, jet fuel, and liquefied petroleum gases.

$E=$ Estimated.

Note: Totals may not equal sum of components due to independent rounding.

Sources: - Energy Information Administration (EIA), Monthly Petroleum Supply Reporting System. — Domestic crude oil production estimates based on historical statistics from State conservation agencies and the Minerals Management Service of the U.S. Department of the Interior. - Export data from the Bureau of the Census and Form EIA-810, "Monthly Refinery Report."

Energy Information Administration/Petroleum Supply Monthly 
Table 2. U.S. Supply, Disposition, and Ending Stocks of Crude Oil and Petroleum Products, March 1995

(Thousand Barrels)

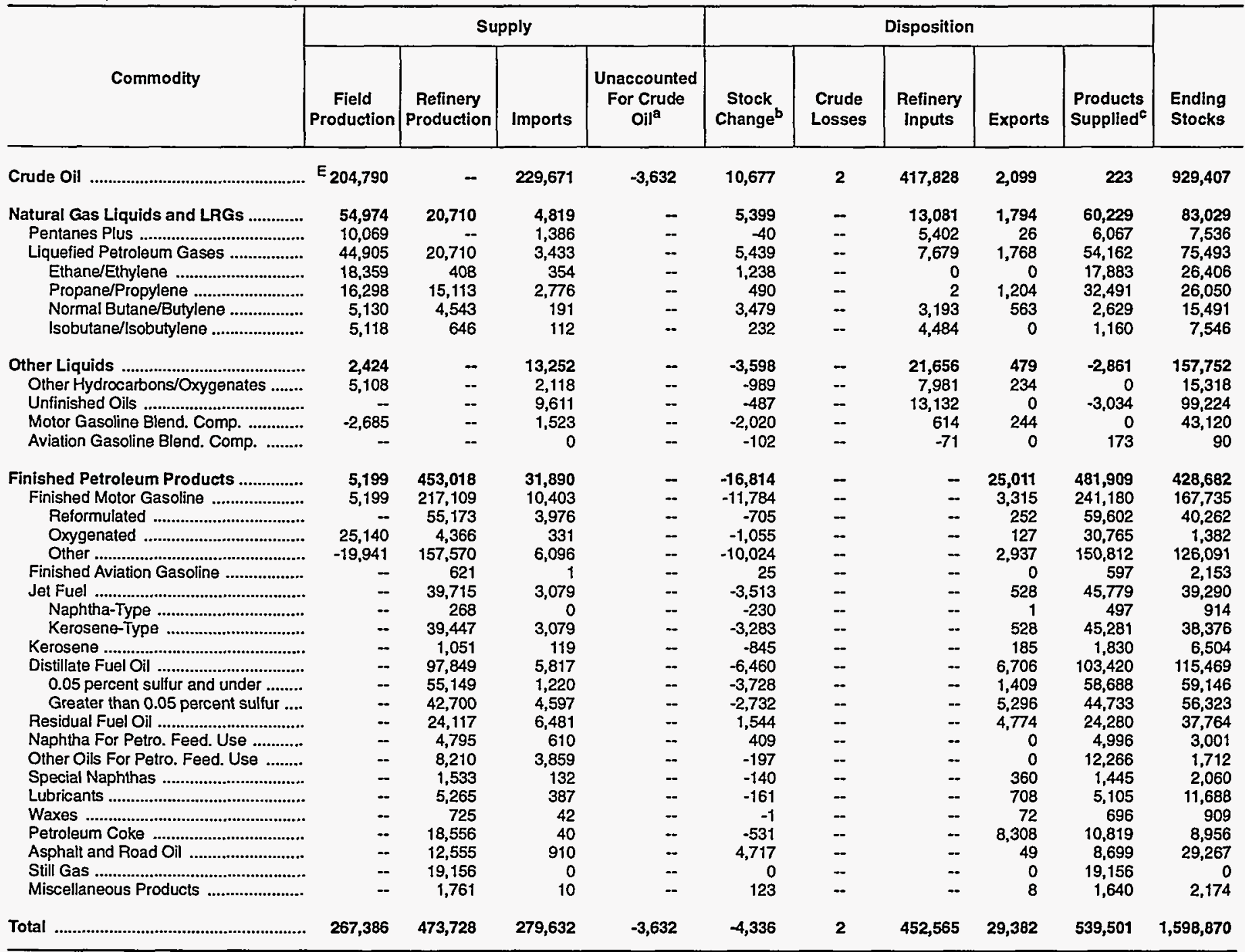

a Unaccounted for crude oil represents the difference between the supply and disposition of crude oil. Preliminary estimates of crude oil imports at the National level have historically understated final values by approximately 50,000 barrels per day. This causes the preliminary values of unaccounted for crude oil to overstate the final values by the same amount

b A negative number indicates a decrease in stocks and a positive number indicates an increase in stocks.

c Products supplied is equal to field production, plus refinery production, plus imports, plus unaccounted for crude oil, minus stock change, minus crude losses, minus refinery inputs, minus exports.

(s) = Less than 500 barrels.

$\mathrm{E}=$ Estimated.

$L R G=$ Liquefied Refinery Gas.

Note: Totals may not equal sum of components due to independent rounding.

Sources: • Energy Information Administration (EIA) Forms EIA-810, "Monthly Refinery Report," EIA-811, "Monthly Bulk Terminal Report," ElA-812, "Monthly Product Pipeline Report," ElA-813, "Monthly Crude Oil Report," EIA-814, "Monthly Imports Report," EIA-816, "Monthly Natural Gas Liquids Report," EIA-817, "Monthly Tanker and Barge Movement Report," and ElA-819M, "Monthly Oxygenate Telephone Report." - Domestic crude oil production estimates based on historical statistics from State conservation agencies and the Minerals Management Service of the U.S. Department of the Interior. $\bullet$ Export data from the Bureau of the Census and Form EIA-810, "Monthly Refinery Report." 
Table 3. U.S. Year-to-Date Supply, Disposition, and Ending Stocks of Crude Oil and Petroleum Products, January-March 1995

(Thousand Barrels)

\begin{tabular}{|c|c|c|c|c|c|c|c|c|c|c|}
\hline \multirow[b]{2}{*}{ Commodity } & \multicolumn{4}{|c|}{ Supply } & \multicolumn{5}{|c|}{ Disposition } & \multirow[b]{2}{*}{$\begin{array}{l}\text { Ending } \\
\text { Stocks }\end{array}$} \\
\hline & $\begin{array}{c}\text { Field } \\
\text { Production }\end{array}$ & $\begin{array}{c}\text { Refinery } \\
\text { Production }\end{array}$ & Imports & $\begin{array}{c}\text { Unaccounted } \\
\text { For Crude } \\
\text { Oil }^{\mathrm{a}}\end{array}$ & $\begin{array}{c}\text { Stock } \\
\text { Change }^{b}\end{array}$ & $\begin{array}{l}\text { Crude } \\
\text { Losses }\end{array}$ & $\begin{array}{l}\text { Refinery } \\
\text { Inputs }\end{array}$ & Exports & $\begin{array}{l}\text { Products } \\
\text { Supplied }^{c}\end{array}$ & \\
\hline 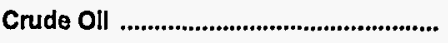 & $E_{596,965}$ & $\cdots$ & 615,067 & 11,613 & 705 & 2 & $1,214,010$ & 8,245 & 683 & 929,407 \\
\hline $\begin{array}{l}\text { Natural Gas Liquids and LRGs ............. } \\
\text { Pentanes Plus ...................................... } \\
\text { Llquefled Petroleum Gases ............... } \\
\text { Ethane/Ethylene ............................... } \\
\text { Propane/Propylene ....................... } \\
\text { Normal Butane/Butylene ................ } \\
\text { Isobutane/lsobutylene ................... }\end{array}$ & $\begin{array}{r}159,627 \\
29,057 \\
130,570 \\
53,870 \\
47,009 \\
14,095 \\
15,596\end{array}$ & $\begin{array}{r}50,209 \\
50,209 \\
1,455 \\
42,986 \\
5,173 \\
595\end{array}$ & $\begin{array}{r}16,615 \\
4,098 \\
12,517 \\
1,647 \\
8,752 \\
1,169 \\
949\end{array}$ & $\begin{array}{l}- \\
- \\
- \\
- \\
-\end{array}$ & $\begin{array}{r}-25,266 \\
-1,143 \\
-24,123 \\
1,117 \\
-20,480 \\
-4,236 \\
-524\end{array}$ & $\begin{array}{l}- \\
- \\
- \\
- \\
-\end{array}$ & $\begin{array}{r}44,057 \\
16,545 \\
27,512 \\
0 \\
2 \\
15,429 \\
12,081\end{array}$ & $\begin{array}{r}7,236 \\
74 \\
7,162 \\
0 \\
5,711 \\
1,451 \\
0\end{array}$ & $\begin{array}{r}200,424 \\
17,679 \\
182,745 \\
55,855 \\
113,514 \\
7,793 \\
5,583\end{array}$ & $\begin{array}{r}83,029 \\
7,536 \\
75,493 \\
26,406 \\
26,050 \\
15,491 \\
7,546\end{array}$ \\
\hline $\begin{array}{l}\text { Other Llquids ........................................ } \\
\text { Other Hydrocarbons/Oxygenates ...... } \\
\text { Unfinished Oils ..................................... } \\
\text { Motor Gasoline Blend. Comp. ............ } \\
\text { Aviation Gasoline Blend. Comp. ......... }\end{array}$ & $\begin{array}{r}13,138 \\
20,077 \\
- \\
-6,939 \\
--\end{array}$ & $\begin{array}{l}- \\
- \\
- \\
-\end{array}$ & $\begin{array}{r}36,954 \\
4,333 \\
29,953 \\
2,668 \\
0\end{array}$ & $\begin{array}{l}- \\
- \\
- \\
-\end{array}$ & $\begin{array}{r}9,550 \\
-1,854 \\
7,845 \\
3,558 \\
1\end{array}$ & $\begin{array}{l}- \\
- \\
- \\
-\end{array}$ & $\begin{array}{r}50,403 \\
25,388 \\
33,706 \\
-8,330 \\
-361\end{array}$ & $\begin{array}{r}1,377 \\
876 \\
0 \\
501 \\
0\end{array}$ & $\begin{array}{r}-11,238 \\
0 \\
-11,598 \\
0 \\
360\end{array}$ & $\begin{array}{r}157,752 \\
15,318 \\
99,224 \\
43,120 \\
90\end{array}$ \\
\hline 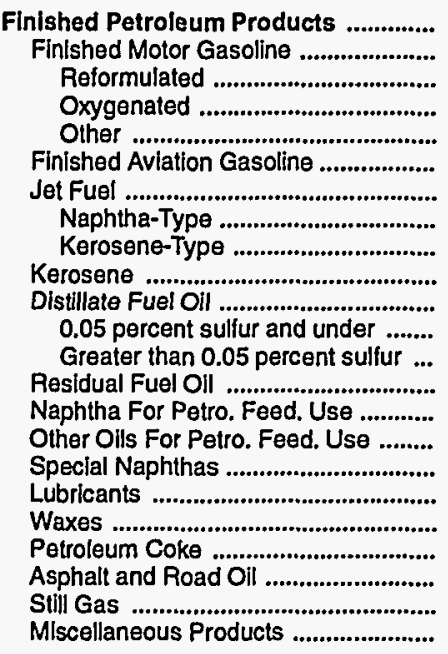 & $\begin{array}{r}13,523 \\
13,523 \\
- \\
65,840 \\
-52,317 \\
-- \\
- \\
- \\
- \\
- \\
- \\
- \\
- \\
- \\
- \\
- \\
- \\
- \\
- \\
-\end{array}$ & $\begin{array}{r}1,323,445 \\
838,622 \\
159,317 \\
21,323 \\
457,982 \\
1,751 \\
122,016 \\
869 \\
121,147 \\
5,846 \\
275,270 \\
151,806 \\
123,464 \\
74,029 \\
15,391 \\
22,917 \\
4,698 \\
15,708 \\
1,989 \\
54,752 \\
30,514 \\
55,532 \\
4,410\end{array}$ & $\begin{array}{r}91,620 \\
22,050 \\
10,274 \\
1,229 \\
10,547 \\
5 \\
8,973 \\
150 \\
8,823 \\
332 \\
22,207 \\
7,039 \\
15,168 \\
18,781 \\
2,350 \\
12,660 \\
538 \\
894 \\
140 \\
281 \\
2,376 \\
0 \\
33\end{array}$ & $\begin{array}{l}- \\
- \\
- \\
- \\
- \\
- \\
- \\
- \\
- \\
- \\
- \\
- \\
- \\
- \\
- \\
- \\
- \\
- \\
-\end{array}$ & $\begin{array}{r}-39,738 \\
-7,757 \\
-2,600 \\
-6,638 \\
1,481 \\
-135 \\
-7,873 \\
-268 \\
-7,605 \\
-1,457 \\
-29,654 \\
-12,677 \\
-16,977 \\
-4,288 \\
746 \\
303 \\
-169 \\
116 \\
-19 \\
-251 \\
10,696 \\
0 \\
4\end{array}$ & $\begin{array}{l}- \\
- \\
- \\
- \\
- \\
- \\
- \\
- \\
- \\
- \\
- \\
- \\
- \\
- \\
-\end{array}$ & $\begin{array}{l}- \\
- \\
- \\
- \\
- \\
- \\
- \\
- \\
- \\
- \\
- \\
- \\
- \\
- \\
- \\
- \\
- \\
- \\
-\end{array}$ & $\begin{array}{r}72,558 \\
8,777 \\
252 \\
487 \\
8,038 \\
0 \\
2,137 \\
298 \\
1,839 \\
335 \\
17,017 \\
3,815 \\
13,202 \\
16,880 \\
0 \\
0 \\
2,065 \\
2,176 \\
189 \\
22,738 \\
219 \\
0 \\
24\end{array}$ & $\begin{array}{r}1,395,768 \\
673,174 \\
171,939 \\
94,543 \\
406,692 \\
1,891 \\
136,725 \\
989 \\
135,736 \\
7,300 \\
310,114 \\
167,707 \\
142,407 \\
80,218 \\
16,995 \\
35,274 \\
3,340 \\
14,310 \\
1,959 \\
32,546 \\
21,975 \\
55,532 \\
4,415\end{array}$ & $\begin{array}{r}428,682 \\
167,735 \\
40,262 \\
1,382 \\
126,091 \\
2,153 \\
39,290 \\
914 \\
38,376 \\
6,504 \\
115,469 \\
59,146 \\
56,323 \\
37,764 \\
3,001 \\
1,712 \\
2,060 \\
11,688 \\
909 \\
8,956 \\
29,267 \\
0 \\
2,174\end{array}$ \\
\hline 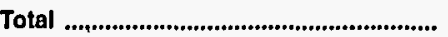 & 783,253 & $1,373,654$ & 760,256 & 11,613 & $-54,749$ & 2 & $1,308,470$ & 89,416 & $1,585,637$ & $1,598,870$ \\
\hline
\end{tabular}

anaccounted for crude oil represents the difference between the supply and disposition of crude oil. Preliminary estimates of crude oil imports at the National level have historically understated final values by approximately 50,000 barrels per day. This causes the preliminary values of unaccounted for crude oil to overstate the

final values by the same amount.

A A negative number indicates a decrease in stocks and a positive number indicates an increase in stocks.

c Products supplied is equal to field production, plus refinery production, plus imports, plus unaccounted for crude oil, minus stock change, minus crude losses, minus refinery inputs, minus exports.

(s) = Less than 500 barrels.

$E=$ Estimated.

LRG = Liquefied Refinery Gas.

Note: Totals may not equal sum of components due to independent rounding.

Sources: - Energy Information Administration (EIA) Forms EIA-810, "Monthly Refinery Report," EIA-811, "Monthly Bulk Terminal Report," EIA-812, "Monthly Product Pipeline Report," EIA-813, "Monthly Crude Oil Report," EIA-814, "Monthly Imports Report," EIA-816, "Monthly Natural Gas Liquids Report," EIA-817, "Monthly Tanker and Barge Movement Report," and EIA-819M, "Monthly Oxygenate Telephone Report" - Domestic crude oil production estimates based on historical statistics from State conservation agencies and the Minerals Management Service of the U.S. Department of the interior. - Export data from the Bureau of the Census and Form EIA-610, "Monthly Refinery Report." 
Table 4. U.S. Daily Average Supply and Disposition of Crude Oil and Petroleum Products, March 1995

(Thousand Barrels per Day)

\begin{tabular}{|c|c|c|c|c|c|c|c|c|c|}
\hline \multirow[b]{2}{*}{ Commodity } & \multicolumn{4}{|c|}{ Supply } & \multicolumn{5}{|c|}{ Disposition } \\
\hline & $\begin{array}{c}\text { Field } \\
\text { Production }\end{array}$ & $\begin{array}{c}\text { Refinery } \\
\text { Production }\end{array}$ & Imports & $\begin{array}{c}\text { Unaccounted } \\
\text { For Crude } \\
\text { Oil }^{\mathrm{a}}\end{array}$ & $\underset{\text { Change }^{b}}{\text { Stock }}$ & $\begin{array}{l}\text { Crude } \\
\text { Losses }\end{array}$ & $\begin{array}{l}\text { Refinery } \\
\text { Inputs }\end{array}$ & Exports & $\begin{array}{l}\text { Products } \\
\text { Supplied }^{\mathrm{C}}\end{array}$ \\
\hline 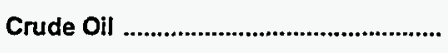 & $E_{6,606}$ & - & 7,409 & -117 & 344 & (s) & 13,478 & 68 & 7 \\
\hline 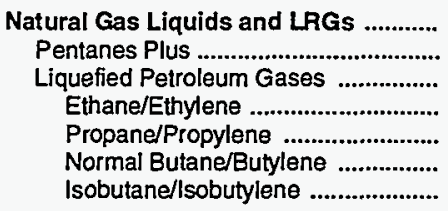 & $\begin{array}{r}1,773 \\
325 \\
1,449 \\
592 \\
526 \\
165 \\
165\end{array}$ & $\begin{array}{r}668 \\
- \\
668 \\
13 \\
488 \\
147 \\
21\end{array}$ & $\begin{array}{r}155 \\
45 \\
111 \\
11 \\
90 \\
6 \\
4\end{array}$ & $\begin{array}{l}- \\
\overline{-} \\
\overline{-} \\
\overline{-} \\
-\end{array}$ & $\begin{array}{r}174 \\
-1 \\
175 \\
40 \\
16 \\
112 \\
7\end{array}$ & $\begin{array}{l}- \\
\overline{-} \\
\overline{-} \\
\overline{-}\end{array}$ & $\begin{array}{r}422 \\
174 \\
248 \\
0 \\
(s) \\
103 \\
145\end{array}$ & $\begin{array}{r}58 \\
1 \\
57 \\
0 \\
39 \\
18 \\
0\end{array}$ & $\begin{array}{r}1,943 \\
196 \\
1,747 \\
577 \\
1,048 \\
85 \\
37\end{array}$ \\
\hline $\begin{array}{l}\text { Other Liquids ............................... } \\
\text { Other Hydrocarons/Oxygenates ...... } \\
\text { Unfinished Oils ............................. } \\
\text { Motor Gasoline Blend. Comp. ............ } \\
\text { Aviation Gasoline Blend. Comp. ........ }\end{array}$ & $\begin{array}{r}78 \\
165 \\
-87 \\
-\end{array}$ & $\begin{array}{l}- \\
- \\
-\end{array}$ & $\begin{array}{r}427 \\
68 \\
310 \\
49 \\
0\end{array}$ & $\begin{array}{l}- \\
- \\
-\end{array}$ & $\begin{array}{r}-116 \\
-32 \\
-16 \\
-65 \\
-3\end{array}$ & $\begin{array}{l}\overline{-} \\
\overline{-} \\
-\end{array}$ & $\begin{array}{r}699 \\
257 \\
424 \\
20 \\
-2\end{array}$ & $\begin{array}{r}15 \\
8 \\
0 \\
8 \\
0\end{array}$ & $\begin{array}{r}-92 \\
0 \\
-98 \\
0 \\
6\end{array}$ \\
\hline 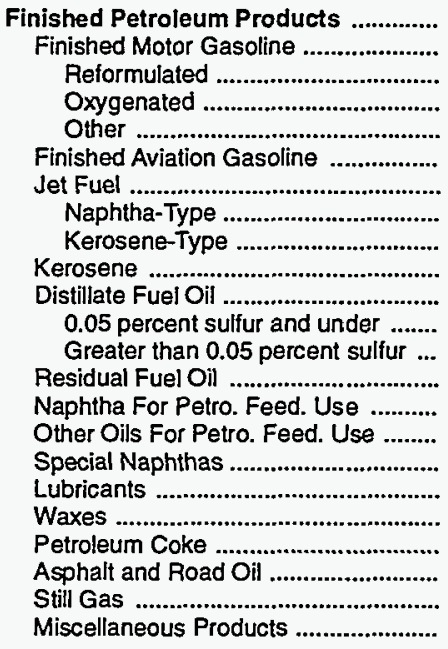 & $\begin{array}{r}168 \\
168 \\
- \\
811 \\
-643 \\
- \\
- \\
- \\
- \\
- \\
- \\
- \\
- \\
- \\
- \\
- \\
- \\
-\end{array}$ & $\begin{array}{r}14,613 \\
7,004 \\
1,780 \\
141 \\
5,083 \\
20 \\
1,281 \\
9 \\
1,272 \\
34 \\
3,156 \\
1,779 \\
1,377 \\
778 \\
155 \\
265 \\
49 \\
170 \\
23 \\
599 \\
405 \\
618 \\
57\end{array}$ & $\begin{array}{r}1,029 \\
336 \\
128 \\
11 \\
197 \\
(s) \\
99 \\
0 \\
99 \\
4 \\
188 \\
39 \\
148 \\
209 \\
20 \\
124 \\
4 \\
12 \\
1 \\
1 \\
29 \\
0 \\
(\mathrm{~s})\end{array}$ & $\begin{array}{l}- \\
- \\
- \\
- \\
- \\
- \\
- \\
- \\
- \\
- \\
- \\
- \\
- \\
- \\
- \\
- \\
- \\
-\end{array}$ & $\begin{array}{r}-542 \\
-380 \\
-23 \\
-34 \\
-323 \\
1 \\
-113 \\
-7 \\
-106 \\
-27 \\
-208 \\
-120 \\
-88 \\
50 \\
13 \\
-6 \\
-5 \\
-5 \\
(s) \\
-17 \\
152 \\
0 \\
4\end{array}$ & $\begin{array}{l}- \\
- \\
- \\
- \\
- \\
- \\
- \\
- \\
- \\
- \\
- \\
- \\
- \\
- \\
- \\
- \\
-\end{array}$ & $\begin{array}{l}- \\
- \\
- \\
- \\
- \\
- \\
- \\
- \\
- \\
- \\
- \\
- \\
- \\
- \\
- \\
- \\
- \\
-\end{array}$ & $\begin{array}{r}807 \\
107 \\
8 \\
4 \\
95 \\
0 \\
17 \\
(s) \\
17 \\
6 \\
216 \\
45 \\
171 \\
154 \\
0 \\
0 \\
12 \\
23 \\
2 \\
268 \\
2 \\
0 \\
(s)\end{array}$ & $\begin{array}{r}15,545 \\
7,780 \\
1,923 \\
992 \\
4,865 \\
19 \\
1,477 \\
16 \\
1,461 \\
59 \\
3,336 \\
1,893 \\
1,443 \\
783 \\
161 \\
396 \\
47 \\
165 \\
22 \\
349 \\
281 \\
618 \\
53\end{array}$ \\
\hline Total & 8,625 & 15,282 & 9,020 & -117 & -140 & (s) & 14,599 & 948 & 17,403 \\
\hline
\end{tabular}

a Unaccounted for crude oil represents the difference between the supply and disposition of crude oil. Preliminary estimates of crude oll imports at the National level have historically understated final values by approximately 50,000 barrels per day. This causes the preliminary values of unaccounted for crude oil to overstate the final values by the same amount.

b A negative number indicates a decrease in stocks and a positive number indicates an increase in stocks.

c Products supplied is equal to field production, plus refinery production, plus imports, plus unaccounted for crude oil, minus stock change, minus crude losses, minus refinery inputs, minus exports.

$(s)=$ Less than 500 barrels per day.

$E=$ Estimated

LRG = Liquefied Refinery Gas.

Note: Totals may not equal sum of components due to independent rounding

Sources: • Energy Information Administration (EIA) Forms EIA-810, "Monthly Refinery Report," EIA-811, "Monthly Bulk Terminal Report," EIA-812, "Monthly Product Pipeline Report," EIA-813, "Monthly Crude Oil Report," EIA-814, "Monthly Imports Report," EIA-816, "Monthly Natural Gas Liquids Report," EIA-817, "Monthly Tanker and Barge Movement Report," and EIA-819M, "Monthly Oxygenate Telephone Report". - Domestic crude oil production estimates based on historical statistics from State conservation agencies and the Minerals Management Service of the U.S. Department of the Interior. - Export data from the Bureau of the Census and Form EIA-810, "Monthly Refinen Report." 
Table 5. U.S. Year-to-Date Daily Average Supply and Disposition of Crude Oil and Petroleum Products, January-March 1995

(Thousand Barrels per Day)

\begin{tabular}{|c|c|c|c|c|c|c|c|c|c|}
\hline \multirow[b]{2}{*}{ Commodity } & \multicolumn{4}{|c|}{ Supply } & \multicolumn{5}{|c|}{ Disposition } \\
\hline & $\begin{array}{c}\text { Field } \\
\text { Production }\end{array}$ & $\begin{array}{c}\text { Refinery } \\
\text { Production }\end{array}$ & Imports & $\begin{array}{c}\text { Unaccounted } \\
\text { For Crude } \\
\text { Oil }^{\mathrm{a}}\end{array}$ & $\begin{array}{c}\text { Stock } \\
\text { Changeb }\end{array}$ & $\begin{array}{l}\text { Crude } \\
\text { Losses }\end{array}$ & $\begin{array}{c}\text { Refinery } \\
\text { Inputs }\end{array}$ & Exports & $\begin{array}{l}\text { Products } \\
\text { Supplied }\end{array}$ \\
\hline 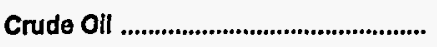 & $E_{6,633}$ & - & 6,834 & 129 & 8 & (s) & 13,489 & 92 & 8 \\
\hline $\begin{array}{l}\text { Natural Gas Lilquids and LRGs .......... } \\
\text { Pentanes Plus ..................................... } \\
\text { Llquefied Petroleum Gases ............... } \\
\text { Ethane/Ethylene ............................. } \\
\text { Propane/Propylene ....................... } \\
\text { Normal Butane/Butylene ................ } \\
\text { Isobutane/lsobutylene .................... }\end{array}$ & $\begin{array}{r}1,774 \\
323 \\
1,451 \\
599 \\
522 \\
157 \\
173\end{array}$ & $\begin{array}{r}558 \\
- \\
558 \\
16 \\
478 \\
57 \\
7\end{array}$ & $\begin{array}{r}185 \\
46 \\
139 \\
18 \\
97 \\
13 \\
11\end{array}$ & $\begin{array}{l}- \\
- \\
- \\
-\end{array}$ & $\begin{array}{r}-281 \\
-13 \\
-268 \\
12 \\
-228 \\
-47 \\
-6\end{array}$ & $\begin{array}{l}- \\
- \\
- \\
-\end{array}$ & $\begin{array}{r}490 \\
184 \\
306 \\
0 \\
(s) \\
171 \\
134\end{array}$ & $\begin{array}{r}80 \\
1 \\
80 \\
0 \\
63 \\
16 \\
0\end{array}$ & $\begin{array}{r}2,227 \\
196 \\
2,031 \\
621 \\
1,261 \\
87 \\
62\end{array}$ \\
\hline $\begin{array}{l}\text { Other Llquids .................................... } \\
\text { Other Hydrocarbons/Oxygenates ..... } \\
\text { Unflnished Oils ................................... } \\
\text { Motor Gasoline Blend. Comp. ............ } \\
\text { Aviation Gasoline Blend. Comp. ........ }\end{array}$ & $\begin{array}{r}146 \\
223 \\
-- \\
-77 \\
--\end{array}$ & $\begin{array}{l}- \\
- \\
-\end{array}$ & $\begin{array}{r}411 \\
48 \\
333 \\
30 \\
0\end{array}$ & $\overline{-}$ & $\begin{array}{r}106 \\
-21 \\
87 \\
40 \\
(s)\end{array}$ & $\overline{-}$ & $\begin{array}{r}560 \\
282 \\
375 \\
-93 \\
-4\end{array}$ & $\begin{array}{r}15 \\
10 \\
0 \\
6 \\
0\end{array}$ & $\begin{array}{r}-125 \\
0 \\
-129 \\
0 \\
4\end{array}$ \\
\hline 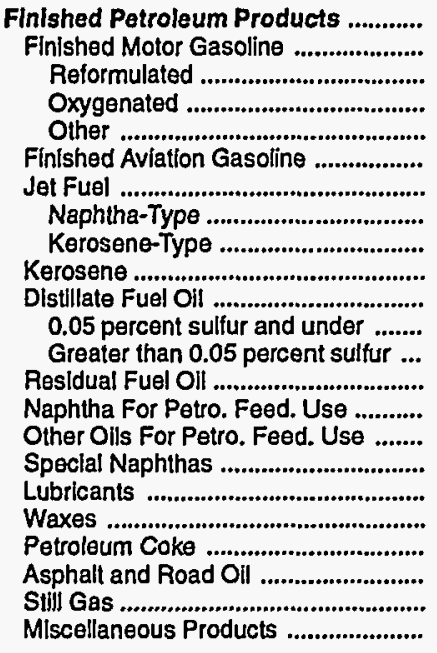 & $\begin{array}{r}150 \\
150 \\
-- \\
732 \\
-581 \\
- \\
- \\
- \\
- \\
- \\
- \\
- \\
- \\
- \\
- \\
- \\
- \\
- \\
- \\
-\end{array}$ & $\begin{array}{r}14,705 \\
7,096 \\
1,770 \\
237 \\
5,089 \\
19 \\
1,356 \\
10 \\
1,346 \\
65 \\
3,059 \\
1,687 \\
1,372 \\
823 \\
171 \\
255 \\
52 \\
175 \\
22 \\
608 \\
339 \\
617 \\
49\end{array}$ & $\begin{array}{r}1,018 \\
245 \\
114 \\
14 \\
117 \\
\langle s) \\
100 \\
2 \\
98 \\
4 \\
247 \\
78 \\
169 \\
209 \\
26 \\
141 \\
6 \\
10 \\
2 \\
3 \\
26 \\
0 \\
(s)\end{array}$ & $\begin{array}{l}- \\
- \\
- \\
- \\
- \\
- \\
- \\
- \\
- \\
- \\
- \\
- \\
- \\
- \\
- \\
- \\
- \\
- \\
- \\
-\end{array}$ & $\begin{array}{r}-442 \\
-86 \\
-29 \\
-74 \\
16 \\
-2 \\
-87 \\
-3 \\
-85 \\
-16 \\
-329 \\
-141 \\
-189 \\
-48 \\
8 \\
3 \\
-2 \\
1 \\
(s) \\
-3 \\
119 \\
0 \\
(s)\end{array}$ & $\begin{array}{l}- \\
- \\
- \\
- \\
- \\
- \\
- \\
- \\
- \\
- \\
- \\
- \\
- \\
- \\
- \\
- \\
-\end{array}$ & $\begin{array}{l}- \\
- \\
- \\
- \\
- \\
- \\
- \\
- \\
- \\
- \\
- \\
- \\
- \\
- \\
- \\
- \\
- \\
-\end{array}$ & $\begin{array}{r}806 \\
98 \\
3 \\
5 \\
89 \\
0 \\
24 \\
3 \\
20 \\
4 \\
189 \\
42 \\
147 \\
188 \\
0 \\
0 \\
23 \\
24 \\
2 \\
253 \\
2 \\
0 \\
(s)\end{array}$ & $\begin{array}{r}15,509 \\
7,480 \\
1,910 \\
1,050 \\
4,519 \\
21 \\
1,519 \\
11 \\
1,508 \\
81 \\
3,446 \\
1,863 \\
1,582 \\
891 \\
189 \\
392 \\
37 \\
159 \\
22 \\
362 \\
244 \\
617 \\
49\end{array}$ \\
\hline 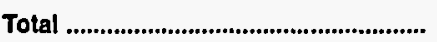 & 8,703 & 15,263 & 8,447 & 129 & -608 & (s) & 14,539 & 994 & 17,618 \\
\hline
\end{tabular}

a Unaccounted for crude oil represents the difference between the supply and disposition of crude oil. Preliminary estimates of crude oil imports at the National lovel have historically understated final values by approximately 50,000 barrels per day. This causes the preliminary values of unaccounted for crude oll to overstate the final values by the same amount.

b A negative number indicates a decrease in stocks and a positive number indicates an increase in stocks.

c Products supplied is equal to field production, plus refinery production, plus imports, plus unaccounted for crude oil, minus stock change, minus crude losses, minus refinery inputs, minus exports.

(s) = Less than 500 barrels per day.

$E=$ Estimated.

LRG = Liquefled Refinery Gas.

Note: Totals may not equal sum of components due to independent rounding.

Sources: - Energy information Administration (EIA) Forms ElA-810, "Monthly Refinery Report," ElA-811, "Monthly Bulk Terminal Report," EIA-812, "Monthly Product Pipeline Report," ElA-813, "Monthly Crude Oil Report," ElA-814, "Monthly Imports Report," ElA-816, "Monthly Natural Gas Liquids Report," EIA-817, "Monthly Tanker and Barge Movement Report," and ElA-819M, "Monthly Oxygenate Telephone Report". • Domestic crude oil production estimates based on historical statistics from State conservation agencies and the Minerals Management Service of the U.S. Department of the Interior. "Export data from the Bureau of the Census and Form EIA-810, "Monthly Refinery Report." 
Table 6. PAD District I-Supply, Disposition, and Ending Stocks of Crude Oil and Petroleum Products, March 1995

(Thousand Barrels)

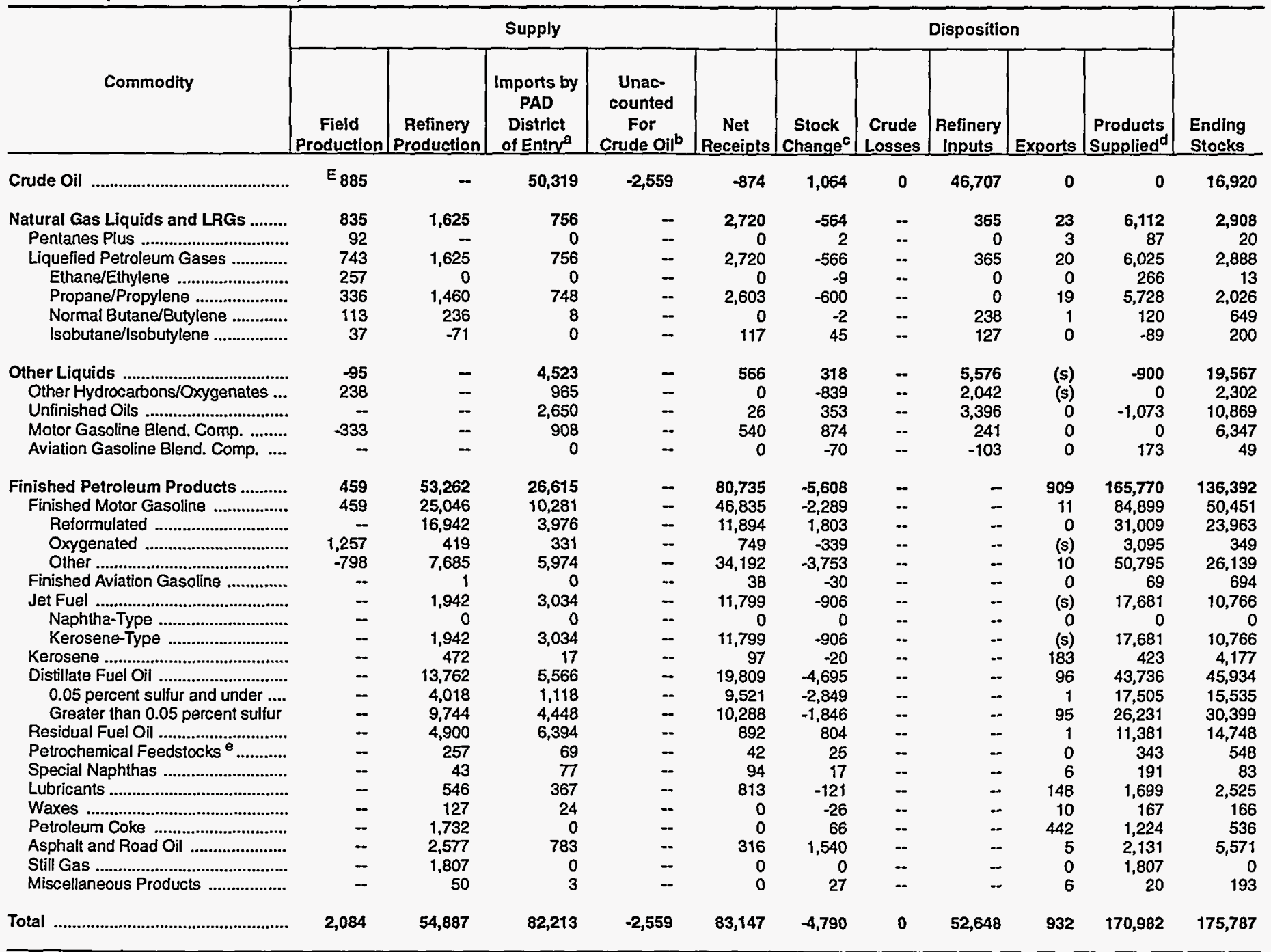

a Represents the PAD District in which the material entered the United States and not necessarily where the crude oil or product is processed and/or consumed.

b Unaccounted for crude oil represents the difference between the supply and disposition of crude oil.

c A negative number indicates a decrease in stocks and a positive number indicates an increase in stocks.

d Products supplied is equal to field production, plus refinery production, plus imports, plus unaccounted for crude oil, plus net receipts, minus stock change, minus crude losses, minus refinery inputs, minus exports.

Includes naphtha less than $401^{\circ} \mathrm{F}$ endpoint and other oils equal to or greater than $401^{\circ} \mathrm{F}$ endpoint.

(s) = Less than 500 barrels.

$\dot{E}=$ Estimated.

LRG $=$ Liquefied Refinery Gas.

Note: Totals may not equal sum of components due to independent rounding.

Sources: - Energy Information Administration (EIA) Forms ElA-810, "Monthly Refinery Report," EIA-811, "Monthly Bulk Terminal Report," EIA-812, "Monthly Product Pipeline Report," ElA-813, "Monthly Crude Oil Report," EIA-814, "Monthly Imports Report," ElA-816, "Monthly Natural Gas Liquids Report," EIA-817, "Monthly Tanker and Barge Movement Report," and ElA-819M, "Monthly Oxygenate Telephone Report". - Domestic crude oil production estimates based on historical statistics from State conservation agencies and the Minerals Management Service of the U.S. Department of the Interior. - Export data from the Bureau of the Census and Form ElA-810, "Monthly Refinery Report." 
Table 7. PAD District 1-Year-to-Date Supply, Disposition, and Ending Stocks of Crude Oil and Petroleum Products, January-March 1995

(Thousand Barrels)

\begin{tabular}{|c|c|c|c|c|c|c|c|c|c|c|c|}
\hline \multirow[b]{2}{*}{ Commodity } & \multicolumn{5}{|c|}{ Supply } & \multicolumn{5}{|c|}{ Disposition } & \multirow[b]{2}{*}{$\begin{array}{l}\text { Ending } \\
\text { Stocks }\end{array}$} \\
\hline & $\begin{array}{c}\text { Field } \\
\text { Production }\end{array}$ & $\begin{array}{c}\text { Pefinery } \\
\text { Production }\end{array}$ & $\begin{array}{c}\text { Imports by } \\
\text { PAD } \\
\text { District } \\
\text { of Entry }\end{array}$ & $\begin{array}{l}\text { Unac- } \\
\text { counted } \\
\text { For } \\
\text { Crude Oil }\end{array}$ & $\begin{array}{c}\text { Net } \\
\text { Receipts }\end{array}$ & $\begin{array}{c}\text { Stock } \\
\text { Change }\end{array}$ & $\begin{array}{c}\text { Crude } \\
\text { Losses }\end{array}$ & $\begin{array}{c}\text { Refinery } \\
\text { Inputs }\end{array}$ & Exports & $\begin{array}{l}\text { Products } \\
\text { Supplied }\end{array}$ & \\
\hline Crude Oll ............................................... & $E_{2,460}$ & - & 120,432 & 10,052 & $-2,366$ & 1,365 & 0 & 129,213 & 0 & 0 & 16,920 \\
\hline 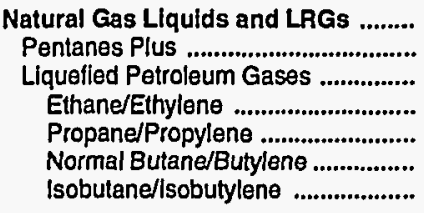 & $\begin{array}{r}2,366 \\
253 \\
2,113 \\
738 \\
951 \\
319 \\
105\end{array}$ & $\begin{array}{r}3,537 \\
3,537 \\
0 \\
4,166 \\
-429 \\
-200\end{array}$ & $\begin{array}{r}2,588 \\
0 \\
2,588 \\
0 \\
2,454 \\
104 \\
30\end{array}$ & $\begin{array}{l}- \\
- \\
- \\
- \\
-\end{array}$ & $\begin{array}{r}11,407 \\
0 \\
11,407 \\
0 \\
10,892 \\
398 \\
117\end{array}$ & $\begin{array}{r}-4,361 \\
-48 \\
-4,313 \\
13 \\
-3,502 \\
-797 \\
-27\end{array}$ & $\begin{array}{l}- \\
- \\
- \\
- \\
-\end{array}$ & $\begin{array}{r}1,140 \\
56 \\
1,084 \\
0 \\
0 \\
745 \\
339\end{array}$ & $\begin{array}{r}92 \\
6 \\
86 \\
0 \\
83 \\
3 \\
0\end{array}$ & $\begin{array}{r}23,027 \\
239 \\
22,788 \\
725 \\
21,882 \\
441 \\
-260\end{array}$ & $\begin{array}{r}2,908 \\
20 \\
2,888 \\
13 \\
2,026 \\
649 \\
200\end{array}$ \\
\hline $\begin{array}{l}\text { Other Llquids ..................................... } \\
\text { Other Hydrocarbons/Oxygenates ...... } \\
\text { Unflnished Oils ................................... } \\
\text { Motor Gasoline Blend. Comp. ........... } \\
\text { Aviation Gasoline Blend. Comp. ........ }\end{array}$ & $\begin{array}{r}1,645 \\
3,022 \\
-1,377 \\
--\end{array}$ & $\begin{array}{l}- \\
- \\
-\end{array}$ & $\begin{array}{r}14,883 \\
1,735 \\
11,096 \\
2,052 \\
0\end{array}$ & $\begin{array}{l}- \\
- \\
-\end{array}$ & $\begin{array}{r}1,556 \\
0 \\
26 \\
1,530 \\
0\end{array}$ & $\begin{array}{r}817 \\
-1,879 \\
1,125 \\
1,596 \\
-25\end{array}$ & $\begin{array}{l}- \\
- \\
-\end{array}$ & $\begin{array}{r}19,637 \\
6,632 \\
12,767 \\
608 \\
-370\end{array}$ & $\begin{array}{l}5 \\
4 \\
0 \\
1 \\
0\end{array}$ & $\begin{array}{r}-2,375 \\
0 \\
-2,770 \\
0 \\
395\end{array}$ & $\begin{array}{r}19,567 \\
2,302 \\
10,869 \\
6,347 \\
49\end{array}$ \\
\hline 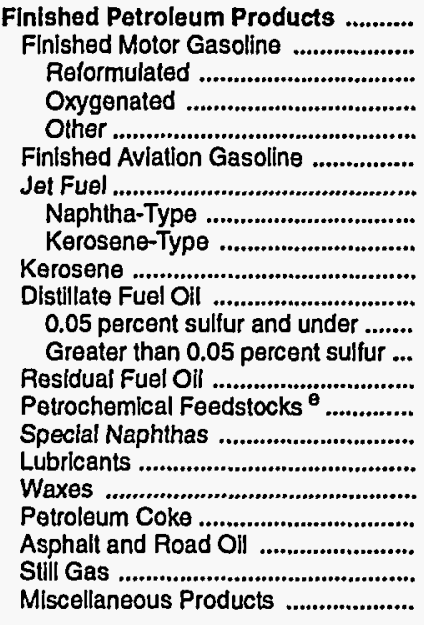 & 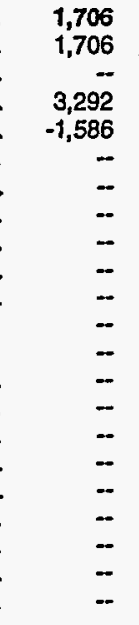 & $\begin{array}{r}152,790 \\
74,569 \\
47,655 \\
1,374 \\
25,540 \\
23 \\
6,116 \\
0 \\
6,116 \\
1,381 \\
38,341 \\
9,523 \\
28,818 \\
14,166 \\
791 \\
198 \\
1,672 \\
418 \\
4,707 \\
4,923 \\
5,305 \\
180\end{array}$ & $\begin{array}{r}72,350 \\
21,701 \\
10,274 \\
1,229 \\
10,198 \\
0 \\
8,147 \\
0 \\
8,147 \\
74 \\
21,316 \\
6,727 \\
14,589 \\
17,697 \\
125 \\
219 \\
772 \\
85 \\
0 \\
2,205 \\
0 \\
9\end{array}$ & $\begin{array}{l}- \\
- \\
- \\
- \\
- \\
- \\
- \\
- \\
- \\
- \\
- \\
- \\
- \\
- \\
- \\
-\end{array}$ & $\begin{array}{r}235,510 \\
132,189 \\
33,071 \\
1,290 \\
97,828 \\
120 \\
37,053 \\
0 \\
37,053 \\
483 \\
59,108 \\
28,479 \\
30,629 \\
3,018 \\
32 \\
320 \\
2,473 \\
0 \\
0 \\
670 \\
0 \\
44\end{array}$ & $\begin{array}{r}-26,715 \\
-3,112 \\
905 \\
-1,712 \\
-2,305 \\
-359 \\
-2,373 \\
-4 \\
-2,369 \\
-236 \\
-20,160 \\
-6,553 \\
-13,607 \\
-3,038 \\
173 \\
-21 \\
-26 \\
-20 \\
-91 \\
2,676 \\
0 \\
-128\end{array}$ & $\begin{array}{l}- \\
- \\
-- \\
- \\
-- \\
- \\
- \\
- \\
- \\
- \\
-- \\
- \\
- \\
- \\
- \\
- \\
- \\
- \\
- \\
-\end{array}$ & $\begin{array}{l}- \\
- \\
- \\
- \\
- \\
- \\
- \\
- \\
- \\
- \\
- \\
- \\
- \\
- \\
- \\
-\end{array}$ & $\begin{array}{r}2,448 \\
38 \\
0 \\
3 \\
35 \\
0 \\
2 \\
1 \\
1 \\
329 \\
218 \\
89 \\
129 \\
682 \\
0 \\
17 \\
394 \\
30 \\
706 \\
11 \\
0 \\
21\end{array}$ & $\begin{array}{r}486,624 \\
233,239 \\
90,095 \\
8,894 \\
134,250 \\
502 \\
53,687 \\
3 \\
53,684 \\
1,845 \\
138,707 \\
51,193 \\
87,514 \\
37,237 \\
775 \\
741 \\
4,549 \\
493 \\
4,092 \\
5,111 \\
5,305 \\
340\end{array}$ & $\begin{array}{r}136,392 \\
50,451 \\
23,963 \\
349 \\
26,139 \\
694 \\
10,766 \\
0 \\
10,766 \\
4,177 \\
45,934 \\
15,535 \\
30,399 \\
14,748 \\
548 \\
83 \\
2,525 \\
166 \\
536 \\
5,571 \\
0 \\
193\end{array}$ \\
\hline 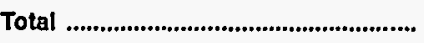 & 8,176 & 156,327 & 210,253 & 10,052 & 246,107 & $-28,894$ & 0 & 149,990 & 2,544 & 507,276 & 175,787 \\
\hline
\end{tabular}

a Represents the PAD District in which the material entered the United States and not necessarily where the crude oil or product is processed and/or consumed.

b Unaccounted for crude oil represents the difference between the supply and disposition of crude oil.

c A negative number indicates a decrease in stocks and a positive number indicates an increase in stocks.

d Products supplied is equal to field production, plus refinery production, plus imports, plus unaccounted for crude oil, plus net receipts, minus stock change, minus crude losses, minus refinery inputs, minus exports.

$\theta$ Includes naphtha less than $401^{\circ} \mathrm{F}$ endpoint and other oils equal to or greater than $401^{\circ} \mathrm{F}$ endpoint.

(s) $=$ Less than 500 barrels.

$\mathrm{E}=$ Estimated.

LRG = Liquefied Refinery Gas.

Note: Totals may not equal sum of components due to independent rounding

Sources: - Energy Information Administration (ElA) Forms EIA-810, "Monthly Refinery Report," EIA-811, "Monthly Bulk Terminal Report" EIA-812, "Monthly Product Pipeline Report," EIA-813, "Monthly Crude Oil Report," EIA-814, "Monthly Imports Report," EIA-816, "Monthly Natural Gas Liquids Report," EIA-817, "Monthly Tanker and Barge Movement Report," and ElA-819M, "Monthly Oxygenate Telephone Report". - Domestic crude oil production estimates based on historical statistics from State conservation agencles and the Minerals Management Service of the U.S. Department of the Interior. • Export data from the Bureau of the Census and Form ElA-810, "Monthly Refinery Report." 
Table 8. PAD District —Daily Average Supply and Disposition of Crude Oil and Petroleum Products, March 1995 (Thousand Barrels per Day)

\begin{tabular}{|c|c|c|c|c|c|c|c|c|c|c|}
\hline \multirow[b]{2}{*}{ Commodity } & \multicolumn{5}{|c|}{ Supply } & \multicolumn{5}{|c|}{ Disposition } \\
\hline & $\begin{array}{c}\text { Field } \\
\text { Production }\end{array}$ & $\begin{array}{c}\text { Refinery } \\
\text { Production }\end{array}$ & $\begin{array}{c}\text { Imports by } \\
\text { PAD } \\
\text { District } \\
\text { of Entry }\end{array}$ & $\begin{array}{l}\text { Unac- } \\
\text { counted } \\
\text { For } \\
\text { Crude Oil }\end{array}$ & $\begin{array}{c}\text { Net } \\
\text { Receipts }\end{array}$ & $\begin{array}{c}\text { Stock } \\
\text { Change }\end{array}$ & $\begin{array}{c}\text { Crude } \\
\text { Losses }\end{array}$ & $\begin{array}{l}\text { Refinery } \\
\text { Inputs }\end{array}$ & Exports & $\begin{array}{l}\text { Products } \\
\text { Supplied }\end{array}$ \\
\hline 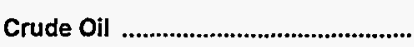 & $E_{29}$ & - & 1,623 & -83 & -28 & 34 & 0 & 1,507 & 0 & 0 \\
\hline $\begin{array}{l}\text { Natural Gas Liquids and LRGs ........ } \\
\text { Pentanes Plus ............................... } \\
\text { Liquefied Petroleum Gases ............... } \\
\text { Ethane/Ethylene ............................ } \\
\text { Propane/Propylene ....................... } \\
\text { Normal Butane/Butylene ................. } \\
\text { Isobutane/lsobutylene ................... }\end{array}$ & $\begin{array}{r}27 \\
3 \\
24 \\
8 \\
11 \\
4 \\
1\end{array}$ & $\begin{array}{r}52 \\
52 \\
0 \\
47 \\
8 \\
-2\end{array}$ & $\begin{array}{r}24 \\
0 \\
24 \\
0 \\
24 \\
(s) \\
0\end{array}$ & $\begin{array}{l}- \\
- \\
- \\
- \\
-\end{array}$ & $\begin{array}{r}88 \\
0 \\
88 \\
0 \\
84 \\
0 \\
4\end{array}$ & $\begin{array}{r}-18 \\
(s) \\
-18 \\
(s) \\
-19 \\
(s) \\
1\end{array}$ & $\begin{array}{l}- \\
- \\
- \\
- \\
-\end{array}$ & $\begin{array}{r}12 \\
0 \\
12 \\
0 \\
0 \\
8 \\
4\end{array}$ & $\begin{array}{r}1 \\
\text { (s) } \\
1 \\
0 \\
1 \\
\text { (s) } \\
0\end{array}$ & $\begin{array}{r}197 \\
3 \\
194 \\
9 \\
185 \\
4 \\
-3\end{array}$ \\
\hline $\begin{array}{l}\text { Other Liquids .................................. } \\
\text { Other Hydrocarbons/Oxygenates ..... } \\
\text { Untinished Oils ................................... } \\
\text { Motor Gasoline Blend. Comp. ........... } \\
\text { Aviation Gasoline Blend. Comp. ........ }\end{array}$ & $\begin{array}{r}-3 \\
8 \\
-11 \\
-\end{array}$ & $\begin{array}{l}- \\
- \\
-\end{array}$ & $\begin{array}{r}146 \\
31 \\
85 \\
29 \\
0\end{array}$ & $\begin{array}{l}- \\
- \\
-\end{array}$ & $\begin{array}{r}18 \\
0 \\
1 \\
17 \\
0\end{array}$ & $\begin{array}{r}10 \\
-27 \\
11 \\
28 \\
-2\end{array}$ & $\begin{array}{l}- \\
- \\
-\end{array}$ & $\begin{array}{r}180 \\
66 \\
110 \\
8 \\
-3\end{array}$ & $\begin{array}{r}\text { (s) } \\
\text { (s) } \\
0 \\
0 \\
0\end{array}$ & $\begin{array}{r}-29 \\
0 \\
-35 \\
0 \\
6\end{array}$ \\
\hline 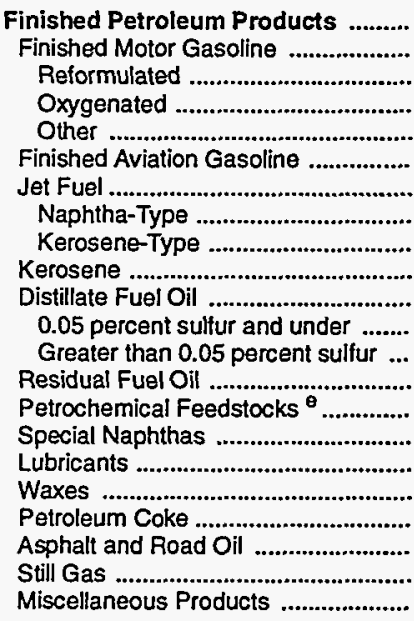 & $\begin{array}{r}15 \\
15 \\
-11 \\
-26 \\
- \\
- \\
- \\
- \\
- \\
- \\
- \\
- \\
- \\
- \\
- \\
-\end{array}$ & $\begin{array}{r}1,718 \\
808 \\
547 \\
14 \\
248 \\
(s) \\
63 \\
0 \\
63 \\
15 \\
444 \\
130 \\
314 \\
158 \\
8 \\
1 \\
18 \\
4 \\
56 \\
83 \\
58 \\
2\end{array}$ & $\begin{array}{r}859 \\
332 \\
128 \\
11 \\
193 \\
0 \\
98 \\
0 \\
98 \\
1 \\
180 \\
36 \\
143 \\
206 \\
2 \\
2 \\
12 \\
1 \\
0 \\
25 \\
0 \\
(\mathrm{~s})\end{array}$ & $\begin{array}{l}- \\
- \\
- \\
- \\
- \\
- \\
- \\
- \\
- \\
- \\
- \\
- \\
- \\
- \\
- \\
- \\
- \\
-\end{array}$ & $\begin{array}{r}2,604 \\
1,511 \\
384 \\
24 \\
1,103 \\
1 \\
381 \\
0 \\
381 \\
3 \\
639 \\
307 \\
332 \\
29 \\
1 \\
3 \\
26 \\
0 \\
0 \\
10 \\
0 \\
0\end{array}$ & $\begin{array}{r}-181 \\
-74 \\
58 \\
-11 \\
-121 \\
-1 \\
-29 \\
0 \\
-29 \\
-1 \\
-151 \\
-92 \\
-60 \\
26 \\
1 \\
1 \\
-4 \\
-1 \\
2 \\
50 \\
0 \\
1\end{array}$ & $\begin{array}{l}- \\
- \\
- \\
- \\
- \\
- \\
- \\
- \\
- \\
- \\
- \\
- \\
- \\
- \\
- \\
- \\
- \\
- \\
-\end{array}$ & $\begin{array}{l}- \\
- \\
- \\
- \\
- \\
- \\
- \\
- \\
- \\
- \\
- \\
- \\
- \\
- \\
- \\
- \\
- \\
-\end{array}$ & $\begin{array}{r}29 \\
(s) \\
0 \\
(s) \\
(s) \\
0 \\
(s) \\
0 \\
(s) \\
6 \\
3 \\
(s) \\
3 \\
(s) \\
0 \\
(s) \\
5 \\
(s) \\
14 \\
(s) \\
0 \\
(s)\end{array}$ & $\begin{array}{r}5,347 \\
2,739 \\
1,000 \\
100 \\
1,639 \\
2 \\
570 \\
0 \\
570 \\
14 \\
1,411 \\
565 \\
846 \\
367 \\
11 \\
6 \\
55 \\
5 \\
39 \\
69 \\
58 \\
1\end{array}$ \\
\hline Total & 67 & 1,771 & 2,652 & -83 & 2,682 & -155 & $\mathbf{0}$ & 1,698 & 30 & 5,516 \\
\hline
\end{tabular}

a Represents the PAD District in which the material entered the United States and not necessarily where the crude oil or product is processed and/or consumed.

b Unaccounted for crude oil represents the diference between the supply and disposition of crude oil.

c A negative number indicates a decrease in stocks and a positive number indicates an increase in stocks.

d Products supplied is equal to field production, plus refinery production, plus imports, plus unaccounted for crude oil, plus net receipts, minus stock change, minus crude losses, minus refinery inputs, minus exports.

- Includes naphtha less than $401^{\circ} \mathrm{F}$ endpoint and other oils equal to or greater than $401^{\circ} \mathrm{F}$ endpoint.

$(s)=$ Less than 500 barrels per day.

$\mathbf{E}=$ Estimated.

$\mathrm{LRG}=$ Liquefied Refinery Gas.

Note: Totals may not equal sum of components due to independent rounding.

Sources: • Energy Information Administration (EIA) Forms EIA-810, "Monthly Refinery Report," EIA-811, "Monthly Bulk Terminal Report," EIA-812, "Monthly Product Pipeline Report," EIA-813, "Monthly Crude Oil Report," EIA-814, "Monthly Imports Report," EIA-816, "Monthly Natural Gas Liquids Report," EIA-817, "Monthly Tanker and Barge Movement Report," and ElA-819M, "Monthly Oxygenate Telephone Report". - Domestic crude oil production estimates based on historical statistics from State conservation agencles and the Minerals Management Service of the U.S. Department of the interior. • Export data from the Bureau of the Census and Form ElA-810, "Monthly Refinery Report." 
Table 9. PAD District 1-Year-to-Date Daily Average Supply and Disposition of Crude Oil and Petroleum Products, January-March 1995

(Thousand Barrels per Day)

\begin{tabular}{|c|c|c|c|c|c|c|c|c|c|c|}
\hline \multirow[b]{2}{*}{ Commodity } & \multicolumn{5}{|c|}{ Supply } & \multicolumn{5}{|c|}{ Disposition } \\
\hline & $\begin{array}{c}\text { Field } \\
\text { Production }\end{array}$ & $\begin{array}{c}\text { Refinery } \\
\text { Production }\end{array}$ & $\begin{array}{l}\text { Imports by } \\
\text { PAD } \\
\text { District } \\
\text { of Entry }\end{array}$ & $\begin{array}{l}\text { Unac- } \\
\text { counted } \\
\text { For } \\
\text { Crude Oilb }\end{array}$ & $\begin{array}{c}\text { Net } \\
\text { Receipts }\end{array}$ & $\begin{array}{c}\text { Stock } \\
\text { Change }^{c}\end{array}$ & $\begin{array}{c}\text { Crude } \\
\text { Losses }\end{array}$ & $\begin{array}{c}\text { Refinery } \\
\text { Inputs }\end{array}$ & Exports & $\begin{array}{l}\text { Products } \\
\text { Supplied }\end{array}$ \\
\hline 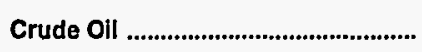 & $E_{27}$ & - & 1,338 & 112 & -26 & 15 & 0 & 1,436 & 0 & 0 \\
\hline 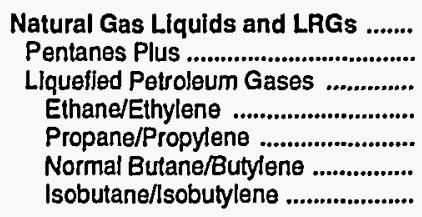 & $\begin{array}{r}26 \\
3 \\
23 \\
8 \\
11 \\
4 \\
1\end{array}$ & $\begin{array}{r}39 \\
- \\
39 \\
0 \\
46 \\
-5 \\
-2\end{array}$ & $\begin{array}{r}29 \\
0 \\
29 \\
0 \\
27 \\
1 \\
1\end{array}$ & $\begin{array}{l}= \\
= \\
=\end{array}$ & $\begin{array}{r}127 \\
0 \\
127 \\
0 \\
121 \\
4 \\
1\end{array}$ & $\begin{array}{r}-48 \\
-1 \\
-48 \\
(s) \\
-39 \\
-9 \\
(s)\end{array}$ & $\begin{array}{l}= \\
\overline{-} \\
\overline{-}\end{array}$ & $\begin{array}{r}13 \\
1 \\
12 \\
0 \\
0 \\
8 \\
4\end{array}$ & $\begin{array}{r}1 \\
\text { (s) } \\
1 \\
0 \\
1 \\
\text { (s) } \\
0\end{array}$ & $\begin{array}{r}256 \\
3 \\
253 \\
8 \\
243 \\
24 \\
5 \\
-3\end{array}$ \\
\hline $\begin{array}{l}\text { Other Liquilds ................................ } \\
\text { Other Hydrocarbons/OXxgenates .... } \\
\text { Unfinished Oils ................................. } \\
\text { Motor Gasoline Blend. Comp. ......... } \\
\text { Aviation Gasoline Blend. Comp. ....... }\end{array}$ & $\begin{array}{r}18 \\
34 \\
-\overline{-15} \\
-\end{array}$ & $\begin{array}{l}- \\
- \\
-\end{array}$ & $\begin{array}{r}165 \\
19 \\
123 \\
23 \\
0\end{array}$ & $\begin{array}{l}= \\
= \\
=\end{array}$ & $\begin{array}{r}17 \\
0 \\
(\mathrm{~s}) \\
17 \\
0\end{array}$ & $\begin{array}{r}9 \\
-21 \\
13 \\
18 \\
(s)\end{array}$ & $\begin{array}{l}\overline{-} \\
\bar{z}\end{array}$ & $\begin{array}{r}218 \\
74 \\
142 \\
7 \\
-4\end{array}$ & $\begin{array}{r}\text { (s) } \\
\text { (s) } \\
0 \\
\text { (s) } \\
0\end{array}$ & $\begin{array}{r}-26 \\
0 \\
-31 \\
0 \\
4\end{array}$ \\
\hline 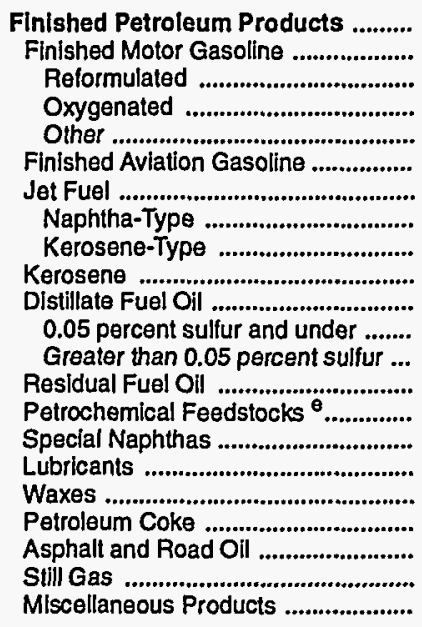 & $\begin{array}{r}19 \\
19 \\
37 \\
-18 \\
= \\
= \\
= \\
= \\
= \\
= \\
= \\
= \\
= \\
= \\
= \\
-\end{array}$ & $\begin{array}{r}1,698 \\
829 \\
530 \\
15 \\
284 \\
(5) \\
68 \\
0 \\
68 \\
15 \\
426 \\
106 \\
320 \\
157 \\
9 \\
2 \\
19 \\
5 \\
52 \\
55 \\
59 \\
2\end{array}$ & $\begin{array}{r}804 \\
241 \\
114 \\
14 \\
113 \\
0 \\
91 \\
0 \\
91 \\
1 \\
1 \\
237 \\
75 \\
162 \\
197 \\
1 \\
2 \\
9 \\
1 \\
0 \\
25 \\
0 \\
\text { (s) }\end{array}$ & $\begin{array}{l}= \\
= \\
= \\
= \\
= \\
= \\
= \\
= \\
= \\
= \\
= \\
= \\
=\end{array}$ & $\begin{array}{r}2,617 \\
1,469 \\
367 \\
14 \\
1,087 \\
1 \\
412 \\
0 \\
012 \\
412 \\
5 \\
657 \\
316 \\
340 \\
34 \\
(\mathrm{~s}) \\
4 \\
27 \\
0 \\
0 \\
7 \\
0 \\
0 \\
\text { (s) }\end{array}$ & $\begin{array}{r}-297 \\
-35 \\
10 \\
-19 \\
-26 \\
-4 \\
-26 \\
(s) \\
-26 \\
-3 \\
-224 \\
-73 \\
-151 \\
-34 \\
2 \\
(s) \\
(s) \\
(s) \\
-1 \\
30 \\
0 \\
-1\end{array}$ & 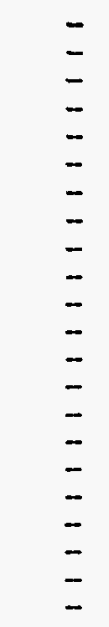 & $\begin{array}{l}= \\
= \\
= \\
= \\
= \\
= \\
= \\
= \\
= \\
= \\
= \\
= \\
= \\
=\end{array}$ & $\begin{array}{r}27 \\
\text { (s) } \\
0 \\
(s) \\
(s) \\
0 \\
(s) \\
(s) \\
(s) \\
4 \\
2 \\
1 \\
1 \\
8 \\
0 \\
(s) \\
4 \\
(s) \\
8 \\
(s) \\
0 \\
(s)\end{array}$ & $\begin{array}{r}5,407 \\
2,592 \\
1,001 \\
99 \\
1,492 \\
6 \\
597 \\
(5) \\
596 \\
20 \\
1,541 \\
569 \\
972 \\
414 \\
9 \\
8 \\
51 \\
5 \\
45 \\
57 \\
59 \\
4\end{array}$ \\
\hline 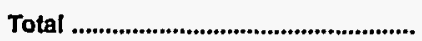 & 91 & 1,737 & 2,336 & 112 & 2,735 & -321 & 0 & 1,667 & 28 & 5,636 \\
\hline
\end{tabular}

a Represents the PAD District in which the material entered the United States and not necessarily where the crude oil or product is processed and/or consumed.

b Unaccounted for crude oil represents the difference between the supply and disposition of crude oil.

c A negative number indicates a decrease in stocks and a positive number indicates an increase in stocks.

d Products supplied is equal to field production, plus refinery production, plus imports, plus unaccounted for crude oil, plus net receipts, minus stock change, minus crude losses, minus refinery inputs, minus exports.

- Includes naphtha less than $401^{\circ} \mathrm{F}$ endpoint and other oils equal to or greater than $401^{\circ} \mathrm{F}$ endpoint.

(s) = Less than 500 barrels per day.

$E=$ Estimated

LRG = Liquefied Refinery Gas.

Note: Totals may not equal sum of components due to independent rounding

Sources: • Energy Information Administration (EIA) Forms ElA-810, "Monthly Refinery Report," ElA-811, "Monthly Bulk Terminal Report," ElA-812, "Monthly Product Plpeline Report," ElA-813, "Monthly Crude Oil Report," ElA-814, "Monthly Imports Report," ElA-816, "Monthly Natural Gas Liquids Report," ElA-817, "Monthly Tanker and Barge Movement Report," and ElA-819M, "Monthly Oxygenate Telephone Report". - Domestic crude oil production estimates based on historical statistles from State conservation agencles and the Minerals Management Service of the U.S. Department of the Interior. • Export data from the Bureau of the Census and Form EIA-B10, "Monthly Relinery Report." 
Table 10. PAD District II-Supply, Disposition, and Ending Stocks of Crude Oil and Petroleum Products, March 1995 (Thousand Barrels)

\begin{tabular}{|c|c|c|c|c|c|c|c|c|c|c|c|}
\hline \multirow[b]{2}{*}{ Commodity } & \multicolumn{5}{|c|}{ Supply } & \multicolumn{5}{|c|}{ Disposition } & \multirow[b]{2}{*}{$\begin{array}{l}\text { Ending } \\
\text { Stocks }\end{array}$} \\
\hline & $\begin{array}{c}\text { Field } \\
\text { Production }\end{array}$ & $\begin{array}{c}\text { Refinery } \\
\text { Production }\end{array}$ & $\begin{array}{c}\text { Imports by } \\
\text { PAD } \\
\text { District } \\
\text { of Entry }\end{array}$ & $\begin{array}{l}\text { Unac- } \\
\text { counted } \\
\text { For } \\
\text { Crude Oil } \\
\end{array}$ & $\begin{array}{c}\text { Net } \\
\text { Receipts }\end{array}$ & $\begin{array}{c}\text { Stock } \\
\text { Change }\end{array}$ & $\begin{array}{c}\text { Crude } \\
\text { Losses }\end{array}$ & $\begin{array}{c}\text { Refinery } \\
\text { Inputs }\end{array}$ & Exports & $\begin{array}{l}\text { Products } \\
\text { Supplied }\end{array}$ & \\
\hline Crude Oil & $E_{17,787}$ & - & 20,381 & 2,555 & 54,501 & 5,185 & 0 & 90,002 & 37 & 0 & 73,030 \\
\hline $\begin{array}{l}\text { Natural Gas Liquids and LRGs .......... } \\
\text { Pentanes Plus ..................................... } \\
\text { Liquefied Petroleum Gases ............... } \\
\text { Ethane/Ethylene ........................... } \\
\text { Propane/Propylene ........................ } \\
\text { Normal Butane/Butylene .............. } \\
\text { Isobutane/lsobutylene ................... }\end{array}$ & $\begin{array}{r}9,515 \\
1,331 \\
8,184 \\
2,912 \\
3,468 \\
1,140 \\
664\end{array}$ & $\begin{array}{r}4,137 \\
- \\
4,137 \\
0 \\
3,410 \\
622 \\
105\end{array}$ & $\begin{array}{r}2,076 \\
33 \\
2,043 \\
354 \\
1,505 \\
134 \\
50\end{array}$ & $\begin{array}{l}-. \\
-. \\
-. \\
. . \\
-. \\
-\end{array}$ & $\begin{array}{r}907 \\
902 \\
5 \\
-986 \\
629 \\
-59 \\
421\end{array}$ & $\begin{array}{r}-464 \\
9 \\
-473 \\
-552 \\
-389 \\
201 \\
267\end{array}$ & $\begin{array}{l}- \\
- \\
- \\
- \\
-\end{array}$ & $\begin{array}{r}2,977 \\
1,100 \\
1,877 \\
0 \\
2 \\
585 \\
1,290\end{array}$ & $\begin{array}{r}165 \\
23 \\
142 \\
0 \\
65 \\
78 \\
0\end{array}$ & $\begin{array}{r}13,957 \\
1,134 \\
12,823 \\
2,832 \\
9,334 \\
973 \\
-317\end{array}$ & $\begin{array}{r}18,057 \\
2,410 \\
15,647 \\
2,411 \\
8,527 \\
2,653 \\
2,056\end{array}$ \\
\hline $\begin{array}{l}\text { Other Liquids ................................... } \\
\text { Other Hydrocarbons/Oxygenates ..... } \\
\text { Unifinished Oils .................................... } \\
\text { Motor Gasoline Blend. Comp. ........... } \\
\text { Aviation Gasoline Blend. Comp. ....... }\end{array}$ & $\begin{array}{r}-1,583 \\
907 \\
-- \\
-2,490 \\
--\end{array}$ & $\begin{array}{l}-- \\
- \\
-- \\
- \\
-\end{array}$ & $\begin{array}{l}8 \\
1 \\
3 \\
4 \\
0\end{array}$ & $\begin{array}{l}- \\
-- \\
- \\
-\end{array}$ & $\begin{array}{r}1,832 \\
0 \\
150 \\
1,682 \\
0\end{array}$ & $\begin{array}{r}-1,572 \\
135 \\
-605 \\
-1,077 \\
-25\end{array}$ & $\begin{array}{l}- \\
- \\
- \\
-\end{array}$ & $\begin{array}{r}2,108 \\
773 \\
1,039 \\
271 \\
25\end{array}$ & $\begin{array}{r}2 \\
(s) \\
0 \\
2 \\
0\end{array}$ & $\begin{array}{r}-281 \\
0 \\
-281 \\
0 \\
0\end{array}$ & $\begin{array}{r}26,976 \\
2,533 \\
14,271 \\
10,157 \\
15\end{array}$ \\
\hline 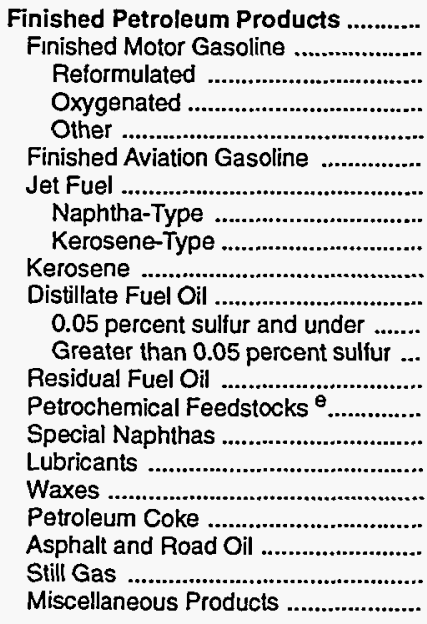 & $\begin{array}{rr}. . & 4,401 \\
. . & 4,401 \\
. . & - \\
. . & 19,106 \\
. . & -14,705 \\
. & -- \\
. . & -. \\
. . & -. \\
. . & -- \\
. . & -- \\
. . & -- \\
. & - \\
. . & -- \\
. . & -- \\
. & -- \\
. . & -- \\
. . & -- \\
. . & -- \\
. . & - \\
. & -\end{array}$ & $\begin{array}{r}95,340 \\
51,293 \\
5,644 \\
2,091 \\
43,558 \\
68 \\
5,266 \\
-1 \\
5,267 \\
142 \\
22,738 \\
13,679 \\
9,059 \\
1,582 \\
1,041 \\
419 \\
672 \\
131 \\
3,488 \\
4,216 \\
3,775 \\
509\end{array}$ & $\begin{array}{r}465 \\
101 \\
0 \\
0 \\
101 \\
1 \\
0 \\
0 \\
0 \\
0 \\
129 \\
86 \\
43 \\
87 \\
34 \\
45 \\
20 \\
11 \\
0 \\
34 \\
0 \\
3\end{array}$ & $\begin{array}{l}- \\
-- \\
-- \\
-- \\
-- \\
-- \\
-- \\
-- \\
-- \\
-- \\
-- \\
-- \\
-- \\
-- \\
-- \\
-- \\
-- \\
-- \\
-- \\
- \\
-- \\
-\end{array}$ & $\begin{array}{r}22,848 \\
12,399 \\
180 \\
-209 \\
12,428 \\
89 \\
2,639 \\
68 \\
2,571 \\
16 \\
7,063 \\
6,082 \\
981 \\
124 \\
0 \\
113 \\
325 \\
0 \\
0 \\
80 \\
0 \\
0\end{array}$ & $\begin{array}{r}-4,012 \\
-3,514 \\
-717 \\
-148 \\
-2,649 \\
4 \\
-789 \\
-75 \\
-714 \\
-459 \\
-699 \\
-555 \\
-144 \\
-164 \\
-88 \\
-39 \\
-7 \\
9 \\
-389 \\
2,132 \\
0 \\
-9\end{array}$ & $\begin{array}{l}- \\
- \\
-. \\
- \\
- \\
- \\
- \\
- \\
- \\
- \\
- \\
- \\
- \\
- \\
- \\
- \\
- \\
- \\
- \\
-\end{array}$ & 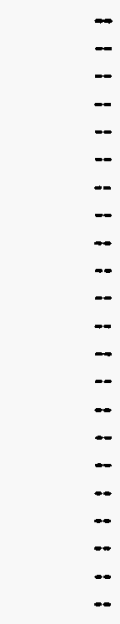 & $\begin{array}{r}355 \\
113 \\
0 \\
4 \\
109 \\
0 \\
0 \\
0 \\
0 \\
(s) \\
31 \\
(s) \\
31 \\
57 \\
0 \\
8 \\
54 \\
6 \\
65 \\
20 \\
0 \\
(s)\end{array}$ & $\begin{array}{r}126,711 \\
71,595 \\
6,541 \\
21,132 \\
43,922 \\
154 \\
8,694 \\
142 \\
8,552 \\
617 \\
30,598 \\
20,402 \\
10,196 \\
1,900 \\
1,163 \\
608 \\
970 \\
127 \\
3,812 \\
2,178 \\
3,775 \\
521\end{array}$ & $\begin{array}{r}107,971 \\
47,103 \\
2,574 \\
418 \\
44,111 \\
377 \\
7,453 \\
257 \\
7,196 \\
1,325 \\
30,480 \\
20,825 \\
9,655 \\
2,179 \\
367 \\
205 \\
1,714 \\
128 \\
2,076 \\
14,332 \\
0 \\
232\end{array}$ \\
\hline Total & 30,120 & 99,477 & 22,930 & 2,555 & 80,088 & -863 & 0 & 95,087 & 559 & 140,387 & 226,034 \\
\hline
\end{tabular}

a Represents the PAD District in which the material entered the United States and not necessarily where the crude oil or product is processed and/or consumed.

b Unaccounted for crude oil represents the difference between the supply and disposition of crude oil.

c A negative number indicates a decrease in stocks and a positive number indicates an increase in stocks.

d Products supplied is equal to field production, plus refinery production, plus imports, plus unaccounted for crude oil, plus net receipts, minus stock change, minus crude losses, minus refinery inputs, minus exports.

includes naphtha less than $401^{\circ} \mathrm{F}$ endpoint and other oils equal to or greater than $401^{\circ} \mathrm{F}$ endpoint.

(s) $=$ Less than 500 barrels.

$\mathrm{E}=$ Estimated.

$\mathrm{LRG}=$ Liquefied Refinery Gas.

Note: Totals may not equal sum of components due to independent rounding.

Sources: - Energy Information Administration (EIA) Forms EIA-810, "Monthly Refinery Report," ElA-811, "Monthly Bulk Terminal Report," EIA-812, "Monthly Product Pipeline Report," EIA-813, "Monthly Crude Oil Report," EIA-814, "Monthly Imports Report," ElA-816, "Monthly Natural Gas Liquids Report," EIA-817, "Monthly Tanker and Barge Movement Report," and EIA-819M, "Monthly Oxygenate Telephone Report". - Domestic crude oil production estimates based on historical statistics from State conservation agencies and the Minerals Management Service of the U.S. Department of the Interior. - Export data from the Bureau of the Census and Form EIA-810, "Monthly Refinery Report." 
Table 11. PAD District II-Year-to-Date Supply, Disposition, and Ending Stocks of Crude Oil and Petroleum Products, January-March 1995

(Thousand Barrels)

\begin{tabular}{|c|c|c|c|c|c|c|c|c|c|c|c|}
\hline \multirow[b]{2}{*}{ Commodity } & \multicolumn{5}{|c|}{ Supply } & \multicolumn{5}{|c|}{ Disposition } & \multirow[b]{2}{*}{$\begin{array}{l}\text { Ending } \\
\text { Stocks }\end{array}$} \\
\hline & $\begin{array}{c}\text { Field } \\
\text { Production }\end{array}$ & $\begin{array}{c}\text { Refinery } \\
\text { Production }\end{array}$ & $\begin{array}{c}\text { Imports by } \\
\text { PAD } \\
\text { District } \\
\text { of Entry }\end{array}$ & $\begin{array}{c}\text { Unac- } \\
\text { counted } \\
\text { For } \\
\text { Crude OH } \\
\end{array}$ & $\begin{array}{c}\text { Net } \\
\text { Receipts }\end{array}$ & $\begin{array}{c}\text { Stock } \\
\text { Change }\end{array}$ & $\begin{array}{c}\text { Crude } \\
\text { Losses }\end{array}$ & $\begin{array}{c}\text { Refinery } \\
\text { Inputs }\end{array}$ & Exports & $\begin{array}{l}\text { Products } \\
\text { Supplied }\end{array}$ & \\
\hline 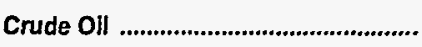 & $E_{51,814}$ & - & 63,563 & 372 & 155,043 & $-3,266$ & $\mathbf{0}$ & 274,020 & 38 & $\mathbf{0}$ & 73,030 \\
\hline $\begin{array}{l}\text { Natural Gas Liquids and LRGs ......... } \\
\text { Pentanes Plus .................................... } \\
\text { Llquefied Petroleum Gases .............. } \\
\text { Ethane/Ethylene ............................ } \\
\text { Propane/Propylene ......................... } \\
\text { Normal Butane/Butylene ............... } \\
\text { Isobutane/lsobutylene ................... }\end{array}$ & $\begin{array}{r}28,543 \\
3,946 \\
24,597 \\
8,942 \\
10,305 \\
3,531 \\
1,819\end{array}$ & $\begin{array}{r}10,428 \\
10,428 \\
1 \\
10,260 \\
111 \\
56\end{array}$ & $\begin{array}{r}7,513 \\
126 \\
7,387 \\
1,025 \\
5,403 \\
633 \\
326\end{array}$ & $\begin{array}{l}- \\
- \\
- \\
- \\
-\end{array}$ & $\begin{array}{r}4,417 \\
2,708 \\
1,709 \\
-3,727 \\
2,870 \\
1,003 \\
1,563\end{array}$ & $\begin{array}{r}-11,897 \\
87 \\
-11,984 \\
-1,052 \\
-9,254 \\
-1,827 \\
149\end{array}$ & $\begin{array}{l}- \\
- \\
- \\
-\end{array}$ & $\begin{array}{r}11,502 \\
3,446 \\
8,056 \\
0 \\
2 \\
4,340 \\
3,714\end{array}$ & $\begin{array}{r}637 \\
68 \\
569 \\
0 \\
263 \\
306 \\
0\end{array}$ & $\begin{array}{r}50,659 \\
3,179 \\
47,480 \\
7,293 \\
37,827 \\
2,459 \\
-99\end{array}$ & $\begin{array}{r}18,057 \\
2,410 \\
15,647 \\
2,411 \\
8,527 \\
2,653 \\
2,056\end{array}$ \\
\hline $\begin{array}{l}\text { Other Liquilds .................................... } \\
\text { Other Hydrocarbons/Oxygenates ..... } \\
\text { Unfinished Oils ................................... } \\
\text { Motor Gasoline Blend. Comp. .......... } \\
\text { Aviatlon Gasoline Blend. Comp. ....... }\end{array}$ & $\begin{array}{r}-2,059 \\
3,041 \\
- \\
-5,099 \\
-\end{array}$ & $\begin{array}{l}- \\
- \\
-\end{array}$ & $\begin{array}{r}14 \\
1 \\
8 \\
5 \\
0\end{array}$ & $\begin{array}{l}- \\
- \\
-\end{array}$ & $\begin{array}{r}4,778 \\
0 \\
105 \\
4,673 \\
0\end{array}$ & $\begin{array}{r}1,205 \\
627 \\
-250 \\
813 \\
15\end{array}$ & $\begin{array}{l}- \\
- \\
-\end{array}$ & $\begin{array}{r}4,005 \\
2,414 \\
2,806 \\
-1,236 \\
21\end{array}$ & $\begin{array}{l}2 \\
1 \\
0 \\
2 \\
0\end{array}$ & $\begin{array}{r}-2,479 \\
0 \\
-2,443 \\
0 \\
-36\end{array}$ & $\begin{array}{r}26,976 \\
2,533 \\
14,271 \\
10,157 \\
15\end{array}$ \\
\hline 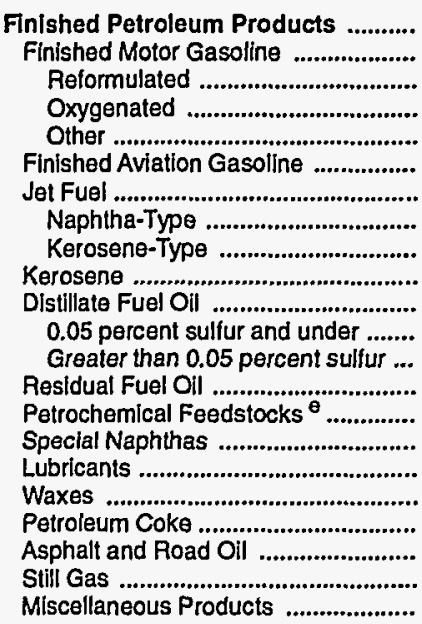 & $\begin{array}{r}10,103 \\
10,103 \\
- \\
50,038 \\
-39,935 \\
- \\
- \\
- \\
- \\
- \\
- \\
- \\
- \\
- \\
- \\
- \\
-\end{array}$ & $\begin{array}{r}293,760 \\
158,314 \\
18,702 \\
6,500 \\
133,112 \\
181 \\
16,819 \\
-1 \\
16,820 \\
2,507 \\
67,089 \\
42,301 \\
24,788 \\
5,760 \\
3,920 \\
1,150 \\
2,104 \\
305 \\
11,620 \\
11,557 \\
11,211 \\
1,223\end{array}$ & $\begin{array}{r}1,279 \\
267 \\
0 \\
0 \\
267 \\
3 \\
0 \\
0 \\
0 \\
0 \\
444 \\
268 \\
176 \\
135 \\
94 \\
217 \\
52 \\
22 \\
0 \\
34 \\
0 \\
11\end{array}$ & $\begin{array}{l}- \\
- \\
- \\
- \\
- \\
- \\
- \\
- \\
- \\
- \\
- \\
- \\
- \\
- \\
- \\
- \\
-\end{array}$ & $\begin{array}{r}55,266 \\
31,207 \\
546 \\
-554 \\
31,215 \\
220 \\
9,337 \\
178 \\
9,159 \\
138 \\
14,041 \\
11,557 \\
2,484 \\
-960 \\
20 \\
303 \\
603 \\
0 \\
0 \\
335 \\
0 \\
22\end{array}$ & $\begin{array}{r}406 \\
-652 \\
-1,262 \\
43 \\
567 \\
-60 \\
-850 \\
3 \\
-853 \\
-346 \\
-4,308 \\
-3,019 \\
-1,289 \\
-70 \\
111 \\
-91 \\
116 \\
33 \\
641 \\
5,853 \\
0 \\
29\end{array}$ & $\begin{array}{l}- \\
- \\
- \\
- \\
- \\
- \\
- \\
- \\
- \\
- \\
- \\
- \\
- \\
- \\
- \\
-\end{array}$ & $\begin{array}{l}- \\
- \\
- \\
- \\
- \\
- \\
- \\
- \\
- \\
- \\
- \\
- \\
- \\
- \\
- \\
- \\
-\end{array}$ & $\begin{array}{r}846 \\
145 \\
0 \\
13 \\
132 \\
0 \\
(s) \\
(s) \\
0 \\
1 \\
35 \\
2 \\
33 \\
181 \\
0 \\
20 \\
155 \\
21 \\
194 \\
93 \\
0 \\
(s)\end{array}$ & $\begin{array}{r}359,156 \\
200,398 \\
20,510 \\
55,929 \\
123,960 \\
464 \\
27,006 \\
174 \\
26,832 \\
2,990 \\
85,847 \\
57,143 \\
28,704 \\
4,824 \\
3,923 \\
1,741 \\
2,488 \\
273 \\
10,785 \\
5,980 \\
11,211 \\
1,227\end{array}$ & $\begin{array}{r}107,971 \\
47,103 \\
2,574 \\
418 \\
44,111 \\
377 \\
7,453 \\
257 \\
7,196 \\
1,325 \\
30,480 \\
20,825 \\
9,655 \\
2,179 \\
367 \\
205 \\
1,714 \\
128 \\
2,076 \\
14,332 \\
0 \\
232\end{array}$ \\
\hline 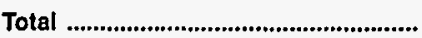 & 88,401 & 304,188 & 72,369 & 372 & 219,504 & $-13,552$ & 0 & 289,527 & 1,523 & 407,336 & 226,034 \\
\hline
\end{tabular}

a Represents the PAD District in which the material entered the United States and not necessarily where the crude oil or product is processed and/or consumed.

Unaccounted for crude oil represents the difference between the supply and disposition of crude oil.

c A negative number indicates a decrease in stocks and a positive number indicates an increase in stocks.

d Products supplied is equal to field production, plus refinery production, plus imports, plus unaccounted for crude oil, plus net receipts, minus stock change, minus crude losses, minus refinery inputs, minus exports.

$\theta$ Includes naphtha less than $401^{\circ} \mathrm{F}$ endpoint and other oils equal to or greater than $401^{\circ} \mathrm{F}$ endpoint.

(s) $=$ Less than 500 barrels.

$E$ = Estimated.

$L R G=$ Llquefied Refinery Gas.

Note: Totals may not equal sum of components due to independent rounding.

Sources: • Energy Information Administration (EIA) Forms EIA-810, "Monthly Refinery Report," EIA-811, "Monthly Bulk Terminal Report," EIA-812, "Monthly Product Pipeline Report," EIA-813, "Monthly Crude Oil Report," ElA-814, "Monthly Imports Report," ElA-816, "Monthly Natural Gas Liquids Report," ElA-817, "Monthly Tanker and Barge Movement Report," and EIA-819M, "Monthly Oxygenate Telephone Report". - Domestic crude oil production estimates based on historical statistics from State conservation agencies and the Minerals Management Service of the U.S. Department of the Interior. - Export data from the Bureau of the Census and Form EIA-B10, "Monthly Refinery Report." 
Table 12. PAD District II-Daily Average Supply and Disposition of Crude Oil and Petroleum Products, March 1995

(Thousand Barrels per Day)

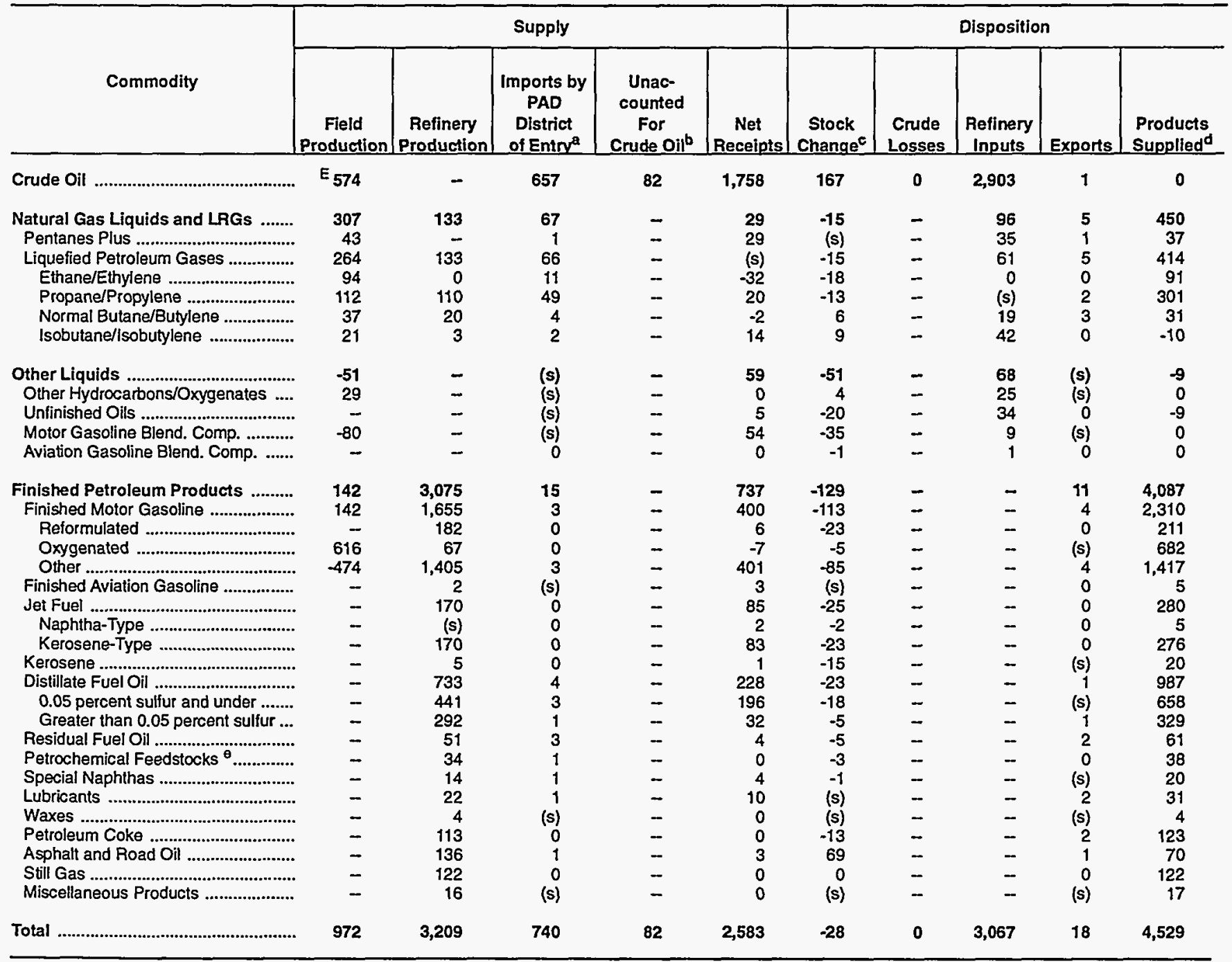

a Represents the PAD District in which the material entered the United States and not necessarily where the crude oil or product is processed and/or consumed.

Unaccounted for crude oil represents the difference between the supply and disposition of crude oil.

c A negative number indicates a decrease in stocks and a positive number indicales an increase in stocks.

Products supplied is equal to field production, plus refinery production, plus imports, plus unaccounted for crude oil, plus net receipts, minus stock change, minus crude losses, minus refinery inputs, minus exports.

Includes naphtha less than $401^{\circ} \mathrm{F}$ endpoint and other oils equal to or greater than $401^{\circ} \mathrm{F}$ endpoint.

(s) = Less than 500 barrels per day.

$E$ = Estimated.

LRG $=$ Liquefied Refinery Gas.

Note: Totals may not equal sum of components due to independent rounding.

Sources: • Energy Information Administration (EIA) Forms EIA-810, "Monthly Refinery Report," EIA-811, "Monthly Bulk Terminal Report," ElA-812, "Monthly Product Pipeline Report," ElA-813, "Monthly Crude Oil Report," EIA-814, "Monthly Imports Report," ElA-816, "Monthly Natural Gas Liquids Report," ElA-817, "Monthly Tanker and Barge Movement Report," and EIA-819M, "Monthly Oxygenate Telephone Report". - Domestic crude oil production estimates based on historical statistics from State conservation agencies and the Minerals Management Service of the U.S. Department of the Interior. - Export data from the Bureau of the Census and Form ElA-810, "Monthly Refinery Report." 
Table 13. PAD District II-Year-to-Date Daily Average Supply and Disposition of Crude Oil and Petroleum Products, January-March 1995

(Thousand Barrels per Day)

\begin{tabular}{|c|c|c|c|c|c|c|c|c|c|c|}
\hline \multirow[b]{2}{*}{ Commodity } & \multicolumn{5}{|c|}{ Supply } & \multicolumn{5}{|c|}{ Disposition } \\
\hline & \begin{tabular}{c|} 
Field \\
Production \\
\end{tabular} & $\begin{array}{c}\text { Refinery } \\
\text { Production }\end{array}$ & $\begin{array}{l}\text { Imports by } \\
\text { PAD } \\
\text { District } \\
\text { of Entry }\end{array}$ & $\begin{array}{l}\text { Unac- } \\
\text { counted } \\
\text { For } \\
\text { Crude Oil } \\
\end{array}$ & $\begin{array}{c}\text { Net } \\
\text { Receipts }\end{array}$ & $\begin{array}{c}\text { Stock } \\
\text { Changec }\end{array}$ & $\begin{array}{l}\text { Crude } \\
\text { Losses }\end{array}$ & $\begin{array}{c}\text { Refinery } \\
\text { Inputs }\end{array}$ & Exports & $\begin{array}{l}\text { Products } \\
\text { Supplied }\end{array}$ \\
\hline 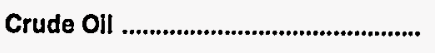 & $E_{576}$ & - & 706 & 4 & 1,723 & -36 & 0 & 3,045 & (s) & 0 \\
\hline $\begin{array}{l}\text { Natural Gas Liquids and LRGs .......... } \\
\text { Pentanes Plus .................................... } \\
\text { Llquefied Petroleum Gases .............. } \\
\text { Ethane/Ethylene .............................. } \\
\text { Propane/Propylene ...................... } \\
\text { Normal Butane/Butylene ................ } \\
\text { Isobutane/lsobutylene ................... }\end{array}$ & $\begin{array}{r}317 \\
44 \\
273 \\
99 \\
115 \\
39 \\
20\end{array}$ & $\begin{array}{r}116 \\
- \\
116 \\
(s) \\
114 \\
1 \\
1\end{array}$ & $\begin{array}{r}83 \\
1 \\
82 \\
11 \\
60 \\
7 \\
4\end{array}$ & $\begin{array}{l}- \\
- \\
- \\
- \\
-\end{array}$ & $\begin{array}{r}49 \\
30 \\
19 \\
-41 \\
32 \\
11 \\
17\end{array}$ & $\begin{array}{r}-132 \\
1 \\
-133 \\
-12 \\
-103 \\
-20 \\
2\end{array}$ & $\begin{array}{l}- \\
- \\
- \\
- \\
-\end{array}$ & $\begin{array}{r}128 \\
38 \\
90 \\
0 \\
(s) \\
48 \\
41\end{array}$ & $\begin{array}{l}7 \\
1 \\
6 \\
0 \\
3 \\
3 \\
0\end{array}$ & $\begin{array}{r}563 \\
35 \\
528 \\
81 \\
420 \\
27 \\
-1\end{array}$ \\
\hline $\begin{array}{l}\text { Other Llquids ..................................... } \\
\text { Other Hydrocarbons/Oxygenates ..... } \\
\text { Unfinished Oils .................................... } \\
\text { Motor Gasoline Blend. Comp. ........... } \\
\text { Avlation Gasoline Blend. Comp. ........ }\end{array}$ & $\begin{array}{r}-23 \\
34 \\
- \\
-57 \\
-\end{array}$ & $\begin{array}{l}- \\
- \\
-\end{array}$ & $\begin{array}{l}\text { (s) } \\
\text { (s) } \\
\text { (s) } \\
\text { (s) } \\
0\end{array}$ & $\begin{array}{l}- \\
\overline{-} \\
-\end{array}$ & $\begin{array}{r}53 \\
0 \\
1 \\
52 \\
0\end{array}$ & $\begin{array}{r}13 \\
7 \\
-3 \\
9 \\
\text { (s) }\end{array}$ & $\begin{array}{l}- \\
- \\
-\end{array}$ & $\begin{array}{r}45 \\
27 \\
31 \\
-14 \\
\text { (s) }\end{array}$ & $\begin{array}{r}\text { (s) } \\
(s) \\
0 \\
(s) \\
0\end{array}$ & $\begin{array}{r}-28 \\
0 \\
-27 \\
0 \\
(s)\end{array}$ \\
\hline 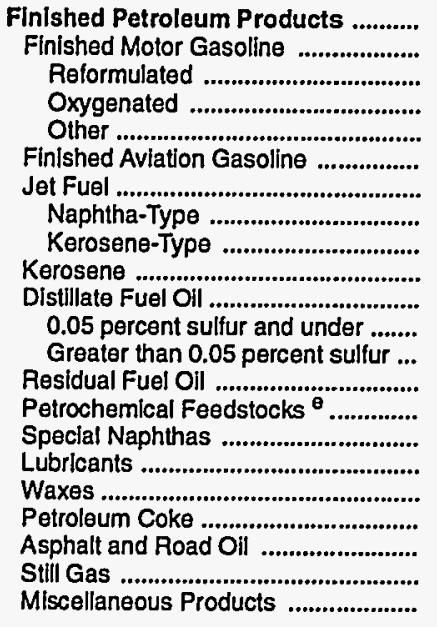 & $\begin{array}{r}112 \\
112 \\
556 \\
-444 \\
- \\
- \\
- \\
- \\
- \\
- \\
- \\
- \\
- \\
- \\
- \\
-\end{array}$ & $\begin{array}{r}3,264 \\
1,759 \\
208 \\
72 \\
1,479 \\
2 \\
187 \\
(s) \\
187 \\
28 \\
745 \\
470 \\
275 \\
64 \\
44 \\
13 \\
23 \\
3 \\
129 \\
128 \\
125 \\
14\end{array}$ & $\begin{array}{r}14 \\
3 \\
0 \\
0 \\
3 \\
(s) \\
0 \\
0 \\
0 \\
0 \\
5 \\
3 \\
2 \\
2 \\
1 \\
2 \\
1 \\
\text { (s) } \\
0 \\
\text { (s) } \\
0 \\
\text { (s) }\end{array}$ & $\begin{array}{l}- \\
- \\
- \\
- \\
- \\
- \\
- \\
- \\
- \\
- \\
- \\
- \\
- \\
- \\
- \\
- \\
-\end{array}$ & $\begin{array}{r}614 \\
347 \\
6 \\
-6 \\
347 \\
2 \\
104 \\
2 \\
102 \\
2 \\
156 \\
128 \\
28 \\
-11 \\
(s) \\
3 \\
7 \\
0 \\
0 \\
4 \\
0 \\
(s)\end{array}$ & $\begin{array}{r}5 \\
-7 \\
-14 \\
(s) \\
6 \\
-1 \\
-9 \\
(s) \\
-9 \\
-4 \\
-48 \\
-34 \\
-14 \\
-1 \\
1 \\
-1 \\
1 \\
(s) \\
7 \\
65 \\
0 \\
(s)\end{array}$ & $\begin{array}{l}- \\
- \\
- \\
- \\
- \\
- \\
- \\
- \\
- \\
- \\
- \\
- \\
- \\
- \\
- \\
- \\
-\end{array}$ & $\begin{array}{l}- \\
- \\
- \\
- \\
- \\
- \\
- \\
- \\
- \\
- \\
- \\
- \\
- \\
- \\
- \\
- \\
-\end{array}$ & $\begin{array}{r}9 \\
2 \\
0 \\
(s) \\
1 \\
0 \\
(s) \\
(s) \\
0 \\
(s) \\
(s) \\
(s) \\
(s) \\
2 \\
0 \\
(s) \\
2 \\
(s) \\
2 \\
1 \\
0 \\
(s)\end{array}$ & $\begin{array}{r}3,991 \\
2,227 \\
228 \\
621 \\
1,377 \\
5 \\
300 \\
2 \\
298 \\
33 \\
954 \\
635 \\
319 \\
54 \\
44 \\
19 \\
28 \\
3 \\
120 \\
66 \\
125 \\
14\end{array}$ \\
\hline Total $\ldots \ldots \ldots \ldots \ldots \ldots$ & 982 & 3,380 & 804 & 4 & 2,439 & -151 & 0 & 3,217 & 17 & 4,526 \\
\hline
\end{tabular}

a Represents the PAD District in which the material entered the United States and not necessarily where the crude oil or product is processed and/or consumed.

b Unaccounted for crude oll represents the difference between the supply and disposition of crude oil.

c A negative number indicates a decrease in stocks and a positive number indicates an increase in stocks.

d Products supplied is equal to field production, plus refinery production, plus imports, plus unaccounted for crude oil, plus net receipts, minus stock change, minus crude losses, minus refinery inpuls, minus exports.

- Includes naphtha less than $401^{\circ} \mathrm{F}$ endpoint and other oils equal to or greater than $401^{\circ} \mathrm{F}$ endpoint.

(s) = Less than 500 barrels per day.

$E=$ Estimated.

LRG = Llquefled Refinery Gas.

Note: Totals may not equal sum of components due to independent rounding.

Sources: • Energy Information Administration (EIA) Forms ElA-810, "Monthly Refinery Report," EIA-811, "Monthly Bulk Terminal Report," ElA-812, "Monthly Product Pipeline Report," EIA-813, "Monthly Crude Oil Report," ElA-814, "Monthly Imports Report," EIA-816, "Monthly Natural Gas Liquids Report," EIA-817, "Monthly Tanker and Barge Movement Report," and EIA-819M, "Monthly Oxygenate Telephone Report". - Domestic crude oil production estimates based on historical statistics from State conservation agencies and the Minerals Management Service of the U.S. Department of the Interior. $\bullet$ Export data from the Bureau of the Census and Form ElA-810, "Monthly Retinery Report." 
Table 14. PAD District III-Supply, Disposition, and Ending Stocks of Crude Oil and Petroleum Products, March 1995

(Thousand Barrels)

\begin{tabular}{|c|c|c|c|c|c|c|c|c|c|c|c|}
\hline \multirow[b]{2}{*}{ Commodity } & \multicolumn{5}{|c|}{ Supply } & \multicolumn{5}{|c|}{ Disposition } & \multirow[b]{2}{*}{$\begin{array}{l}\text { Ending } \\
\text { Stocks }\end{array}$} \\
\hline & $\begin{array}{c}\text { Field } \\
\text { Production }\end{array}$ & $\begin{array}{c}\text { Refinery } \\
\text { Production }\end{array}$ & $\begin{array}{c}\text { Imports by } \\
\text { PAD } \\
\text { District } \\
\text { of Entry }\end{array}$ & $\begin{array}{l}\text { Unac- } \\
\text { counted } \\
\text { For } \\
\text { Crude Oil } \\
\end{array}$ & $\begin{array}{c}\text { Net } \\
\text { Receipts }\end{array}$ & $\begin{array}{c}\text { Stock } \\
\text { Change }\end{array}$ & $\begin{array}{c}\text { Crude } \\
\text { Losses }\end{array}$ & $\begin{array}{c}\text { Refinery } \\
\text { Inputs }\end{array}$ & Exports & $\begin{array}{l}\text { Products } \\
\text { Supplied }\end{array}$ & \\
\hline 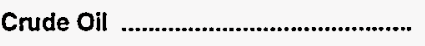 & $E_{96,789}$ & - & 149,048 & $-3,226$ & $-40,566$ & 6,883 & 0 & 195,162 & 0 & 0 & 745,954 \\
\hline $\begin{array}{l}\text { Natural Gas Liquids and LRGs .......... } \\
\text { Pentanes Plus .................................... } \\
\text { Liquefied Petroleum Gases ............... } \\
\text { Ethane/Ethylene ............................ } \\
\text { Propane/Propylene ........................... } \\
\text { Normal Butane/Butylene ................ } \\
\text { Isobutane/lsobutylene ..................... }\end{array}$ & $\begin{array}{r}37,076 \\
5,871 \\
31,205 \\
14,065 \\
10,739 \\
2,717 \\
3,684\end{array}$ & $\begin{array}{r}11,694 \\
\cdots \\
11,694 \\
408 \\
8,593 \\
2,333 \\
360\end{array}$ & $\begin{array}{r}1,208 \\
806 \\
402 \\
0 \\
402 \\
0 \\
0\end{array}$ & $\begin{array}{l}- \\
- \\
- \\
- \\
- \\
-\end{array}$ & $\begin{array}{r}-342 \\
-519 \\
177 \\
2,281 \\
-2,289 \\
478 \\
-293\end{array}$ & $\begin{array}{r}6,284 \\
-46 \\
6,330 \\
1,803 \\
1,649 \\
3,048 \\
-170\end{array}$ & $\begin{array}{l}- \\
- \\
- \\
- \\
- \\
- \\
-\end{array}$ & $\begin{array}{r}6,592 \\
2,714 \\
3,878 \\
0 \\
0 \\
1,397 \\
2,481\end{array}$ & $\begin{array}{r}902 \\
(s) \\
901 \\
0 \\
783 \\
118 \\
0\end{array}$ & $\begin{array}{r}35,858 \\
3,490 \\
32,369 \\
14,951 \\
15,013 \\
965 \\
1,440\end{array}$ & $\begin{array}{r}58,691 \\
4,874 \\
53,817 \\
23,769 \\
14,812 \\
10,659 \\
4,577\end{array}$ \\
\hline $\begin{array}{l}\text { Other Liquids ............................... } \\
\text { Other Hydrocarbons/Oxygenates ..... } \\
\text { Unfinished Oils ................................. } \\
\text { Motor Gasoline Blend. Comp. ........... } \\
\text { Aviation Gasoline Blend. Comp. ....... }\end{array}$ & $\begin{array}{r}3,096 \\
2,951 \\
-- \\
145 \\
\ldots\end{array}$ & $\begin{array}{l}- \\
- \\
- \\
-\end{array}$ & $\begin{array}{r}7,581 \\
12 \\
6,958 \\
611 \\
0\end{array}$ & $\begin{array}{l}- \\
- \\
- \\
--\end{array}$ & $\begin{array}{r}-2,398 \\
0 \\
-176 \\
-2,222 \\
0\end{array}$ & $\begin{array}{r}-418 \\
-233 \\
374 \\
-556 \\
-3\end{array}$ & $\begin{array}{l}- \\
-- \\
-- \\
--\end{array}$ & $\begin{array}{r}10,046 \\
2,963 \\
8,157 \\
-1,077 \\
3\end{array}$ & $\begin{array}{r}400 \\
233 \\
0 \\
167 \\
0\end{array}$ & $\begin{array}{r}-1,749 \\
0 \\
-1,749 \\
0 \\
0\end{array}$ & $\begin{array}{r}68,109 \\
5,941 \\
46,344 \\
15,801 \\
23\end{array}$ \\
\hline 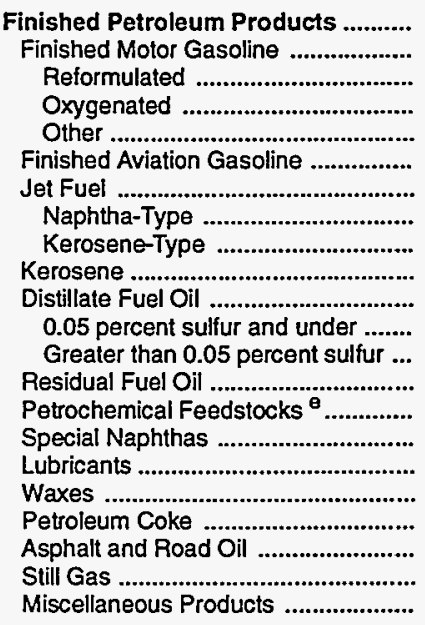 & $\begin{array}{r}56 \\
56 \\
-\overline{2} \\
2,011 \\
-1,955 \\
- \\
- \\
-- \\
- \\
- \\
- \\
- \\
- \\
-- \\
- \\
-- \\
-- \\
- \\
-- \\
-. \\
-. \\
-\end{array}$ & $\begin{array}{r}209,603 \\
96,773 \\
18,587 \\
1,154 \\
77,032 \\
415 \\
19,999 \\
47 \\
19,952 \\
382 \\
43,692 \\
25,289 \\
18,403 \\
10,011 \\
11,336 \\
1,019 \\
3,292 \\
362 \\
8,660 \\
3,722 \\
9,099 \\
841\end{array}$ & $\begin{array}{r}4,537 \\
0 \\
0 \\
0 \\
0 \\
0 \\
20 \\
0 \\
20 \\
102 \\
0 \\
0 \\
0 \\
0 \\
4,307 \\
9 \\
0 \\
2 \\
0 \\
93 \\
0 \\
4\end{array}$ & $\begin{array}{l}- \\
-- \\
-- \\
- \\
- \\
- \\
- \\
- \\
- \\
- \\
- \\
- \\
- \\
- \\
- \\
- \\
- \\
- \\
- \\
-\end{array}$ & $\begin{array}{r}-106,839 \\
-60,720 \\
-12,074 \\
-540 \\
-48,106 \\
-133 \\
-15,711 \\
0 \\
-15,711 \\
-113 \\
-27,363 \\
-15,971 \\
-11,392 \\
-1,016 \\
-42 \\
-207 \\
-1,138 \\
0 \\
0 \\
-396 \\
0 \\
0\end{array}$ & $\begin{array}{r}-2,336 \\
-2,022 \\
-977 \\
-168 \\
-877 \\
-3 \\
-1,245 \\
-132 \\
-1,113 \\
-344 \\
-557 \\
154 \\
-711 \\
982 \\
163 \\
-116 \\
75 \\
-5 \\
122 \\
603 \\
0 \\
11\end{array}$ & $\begin{array}{l}- \\
-- \\
-- \\
-- \\
-- \\
-- \\
-- \\
-- \\
-. \\
-- \\
-- \\
-- \\
-- \\
-- \\
-- \\
-- \\
-- \\
-- \\
-- \\
-- \\
-- \\
--\end{array}$ & $\begin{array}{l}- \\
-- \\
-- \\
-- \\
- \\
-- \\
- \\
- \\
- \\
- \\
- \\
- \\
- \\
- \\
- \\
- \\
- \\
- \\
- \\
- \\
-\end{array}$ & $\begin{array}{r}11,913 \\
2,601 \\
252 \\
111 \\
2,239 \\
0 \\
86 \\
0 \\
86 \\
0 \\
2,695 \\
328 \\
2,368 \\
2,169 \\
0 \\
95 \\
344 \\
43 \\
3,871 \\
8 \\
0 \\
(s)\end{array}$ & $\begin{array}{r}97,781 \\
35,530 \\
7,238 \\
2,682 \\
25,610 \\
285 \\
5,467 \\
179 \\
5,288 \\
715 \\
14,191 \\
8,836 \\
5,354 \\
5,844 \\
15,438 \\
842 \\
1,735 \\
326 \\
4,667 \\
2,808 \\
9,099 \\
834\end{array}$ & $\begin{array}{r}117,392 \\
45,545 \\
7,900 \\
396 \\
37,249 \\
505 \\
12,134 \\
190 \\
11,944 \\
839 \\
25,291 \\
13,039 \\
12,252 \\
13,286 \\
3,468 \\
1,732 \\
5,697 \\
465 \\
2,780 \\
4,268 \\
0 \\
1,382\end{array}$ \\
\hline 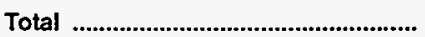 & 137,018 & 221,297 & 162,374 & $-3,226$ & $-150,145$ & 10,413 & 0 & 211,800 & 13,214 & 131,890 & 990,146 \\
\hline
\end{tabular}

a Represents the PAD District in which the material entered the United States and not necessarily where the crude oil or product is processed and/or consumed.

b Unaccounted for crude oil represents the difference between the supply and disposition of crude oil.

c A negative number indicates a decrease in stocks and a positive number indicates an increase in stocks.

d Products supplied is equal to field production, plus refinery production, plus imports, plus unaccounted for crude oil, plus net receipts, minus stock change, minus crude losses, minus refinery inputs, minus exports.

$\theta$ Includes naphtha less than $401^{\circ} \mathrm{F}$ endpoint and other oils equal to or greater than $401^{\circ} \mathrm{F}$ endpoint.

(s) = Less than 500 barrels.

$E=$ Estimated.

LRG $=$ Liquefied Refinery Gas

Note: Totals may not equal sum of components due to independent rounding.

Sources: - Energy Information Administration (EIA) Forms EIA-810, "Monthly Refinery Report," EIA-811, "Monthly Bulk Terminal Report," EIA-812, "Monthly Product

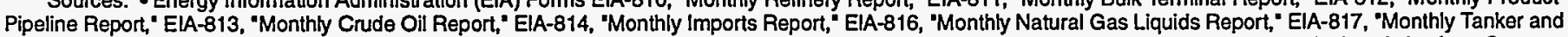
Barge Movement Report," and EIA-819M, "Monthly Oxygenate Telephone Report". - Domestic crude oil production estimates based on historical statistics from State conservation agencies and the Minerals Management Service of the U.S. Department of the Interior. - Export data from the Bureau of the Census and Form ElA-810, "Monthly Refinery Report." 
Table 15. PAD District III-Year-to-Date Supply, Disposition, and Ending Stocks of Crude Oil and Petroleum Products, January-March 1995 (Thousand Barrels)

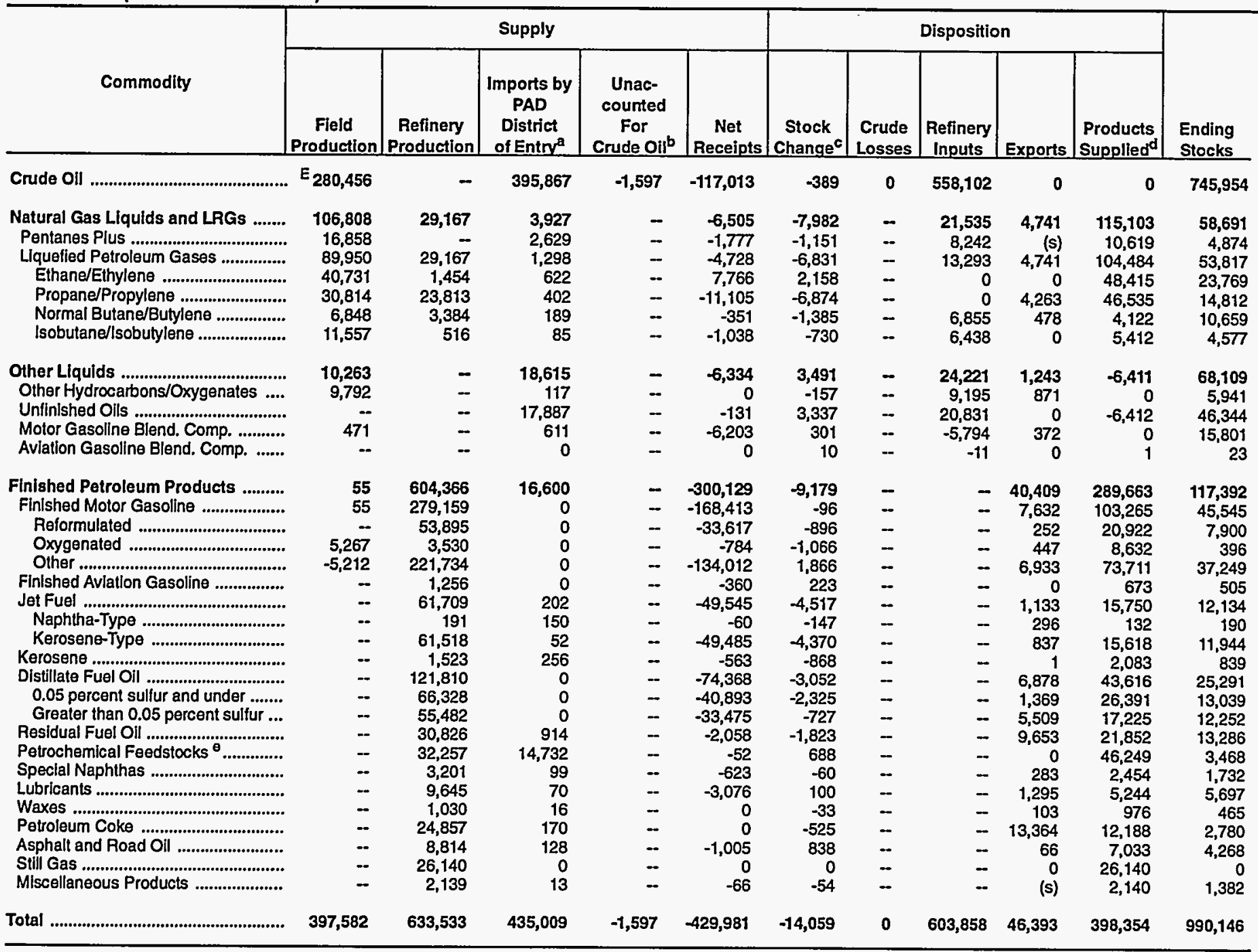

Represents the PAD District in which the material entered the United States and not necessarily where the crude oil or product is processed and/or consumed.

b Unaccounted for crude oil represents the difference between the supply and disposition of crude oil.

c A negative number indicates a decrease in stocks and a positive number indicates an increase in stocks.

Products supplied is equal to field production, plus refinery production, plus imports, plus unaccounted for crude oil, plus net receipts, minus stock change, minus crude losses, minus refinery inputs, minus exports.

- Includes naphtha less than $401^{\circ} \mathrm{F}$ endpoint and other oils equal to or greater than $401^{\circ} \mathrm{F}$ endpoint.

(s) = Less than 500 barrels.

$E=$ Estimated.

LRG = Liquefied Refinery Gas.

Note: Totals may not equal sum of components due to independent rounding.

Sources: - Energy Information Administration (EIA) Forms EIA-810, "Monthly Refinery Report," ElA-811, "Monthly Bulk Terminal Report," ElA-812, "Monthly Product Pipeline Report," EIA-813, "Monthly Crude Oil Report," EIA-814, "Monthly Imports Report," EIA-816, "Monthly Natural Gas Liquids Report," EIA-817, "Monthly Tanker and Barge Movement Report, and EIA-819M, "Monthly Oxygenate Telephone Report". - Domestic crude oil production estimates based on historical statistics from State conservation agencles and the Minerals Management Service of the U.S. Department of the Interior. - Export data from the Bureau of the Census and Form EIA-810, "Monthly Refinery Report." 
Table 16. PAD District III-Daily Average Supply and Disposition of Crude Oil and Petroleum Products, March 1995 (Thousand Barrels per Day)

\begin{tabular}{|c|c|c|c|c|c|c|c|c|c|c|}
\hline \multirow[b]{2}{*}{ Commodity } & \multicolumn{5}{|c|}{ Supply } & \multicolumn{5}{|c|}{ Disposition } \\
\hline & \begin{tabular}{c|} 
Field \\
Production
\end{tabular} & $\begin{array}{c}\text { Refinery } \\
\text { Production }\end{array}$ & $\begin{array}{l}\text { Imports by } \\
\text { PAD } \\
\text { District } \\
\text { of Entry }\end{array}$ & $\begin{array}{l}\text { Unac- } \\
\text { counted } \\
\text { For } \\
\text { Crude Oil' }\end{array}$ & $\begin{array}{c}\text { Net } \\
\text { Receipts }\end{array}$ & $\begin{array}{c}\text { Stock } \\
\text { Change }^{c}\end{array}$ & $\begin{array}{c}\text { Crude } \\
\text { Losses }\end{array}$ & $\begin{array}{c}\text { Refinery } \\
\text { Inputs }\end{array}$ & Exports & $\begin{array}{l}\text { Products } \\
\text { Supplied }^{d}\end{array}$ \\
\hline 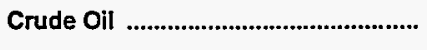 & $E_{3,122}$ & - & 4,808 & -104 & $-1,309$ & 222 & 0 & 6,296 & 0 & 0 \\
\hline $\begin{array}{l}\text { Natural Gas Liquids and LRGs ........ } \\
\text { Pentanes Plus ................................... } \\
\text { Liquefied Petroleum Gases .............. } \\
\text { Ethane/Ethylene ............................. } \\
\text { Propane/Propylene ........................ } \\
\text { Normal Butane/Butylene ................. } \\
\text { Isobutane/lsobutylene ................... }\end{array}$ & $\begin{array}{r}1,196 \\
189 \\
1,007 \\
454 \\
346 \\
88 \\
119\end{array}$ & $\begin{array}{r}377 \\
- \\
377 \\
13 \\
277 \\
75 \\
12\end{array}$ & $\begin{array}{r}39 \\
26 \\
13 \\
0 \\
13 \\
0 \\
0\end{array}$ & $\begin{array}{l}- \\
- \\
- \\
- \\
-\end{array}$ & $\begin{array}{r}-11 \\
-17 \\
6 \\
74 \\
-74 \\
15 \\
-9\end{array}$ & $\begin{array}{r}203 \\
-1 \\
204 \\
58 \\
53 \\
98 \\
-5\end{array}$ & $\begin{array}{l}- \\
- \\
- \\
- \\
-\end{array}$ & $\begin{array}{r}213 \\
88 \\
125 \\
0 \\
0 \\
45 \\
80\end{array}$ & $\begin{array}{r}29 \\
(s) \\
29 \\
0 \\
25 \\
4 \\
0\end{array}$ & $\begin{array}{r}1,157 \\
113 \\
1,044 \\
482 \\
484 \\
31 \\
46\end{array}$ \\
\hline $\begin{array}{l}\text { Other Liquids .................................. } \\
\text { Other Hydrocarbons/Oxygenates .... } \\
\text { Unfinished Oils .................................... } \\
\text { Motor Gasoline Blend. Comp. .......... } \\
\text { Aviation Gasoline Blend. Comp. ....... }\end{array}$ & $\begin{array}{r}100 \\
95 \\
\overline{5} \\
-\end{array}$ & $\begin{array}{l}- \\
- \\
-\end{array}$ & $\begin{array}{r}245 \\
(s) \\
224 \\
20 \\
0\end{array}$ & $\begin{array}{l}\bar{z} \\
\overline{-}\end{array}$ & $\begin{array}{r}-77 \\
0 \\
-6 \\
-72 \\
0\end{array}$ & $\begin{array}{r}-13 \\
-8 \\
12 \\
-18 \\
\text { (s) }\end{array}$ & $\begin{array}{l}- \\
- \\
-\end{array}$ & $\begin{array}{r}324 \\
96 \\
263 \\
-35 \\
(s)\end{array}$ & $\begin{array}{r}13 \\
8 \\
0 \\
5 \\
0\end{array}$ & $\begin{array}{r}-56 \\
0 \\
-56 \\
0 \\
0\end{array}$ \\
\hline 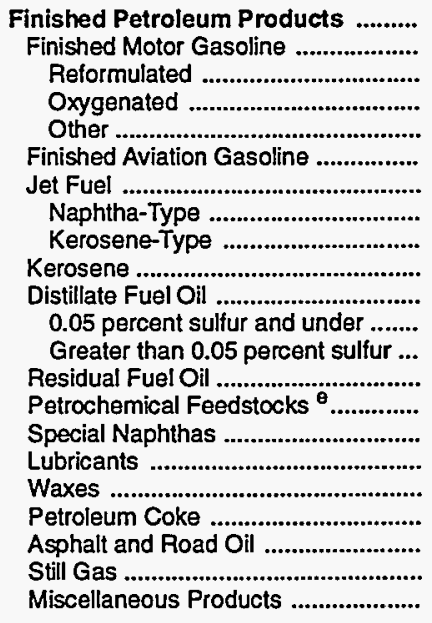 & $\begin{array}{r}2 \\
2 \\
-65 \\
-63 \\
- \\
- \\
- \\
- \\
- \\
- \\
- \\
- \\
- \\
- \\
- \\
- \\
- \\
-\end{array}$ & $\begin{array}{r}6,761 \\
3,122 \\
600 \\
37 \\
2,485 \\
13 \\
645 \\
2 \\
644 \\
12 \\
1,409 \\
816 \\
594 \\
323 \\
366 \\
33 \\
106 \\
12 \\
279 \\
120 \\
294 \\
27\end{array}$ & $\begin{array}{r}146 \\
0 \\
0 \\
0 \\
0 \\
0 \\
1 \\
0 \\
1 \\
3 \\
0 \\
0 \\
0 \\
0 \\
139 \\
\text { (s) } \\
0 \\
\text { (s) } \\
0 \\
3 \\
0 \\
\text { (s) }\end{array}$ & $\begin{array}{l}- \\
- \\
- \\
- \\
- \\
- \\
- \\
- \\
- \\
- \\
- \\
- \\
- \\
- \\
- \\
- \\
-\end{array}$ & $\begin{array}{r}-3,446 \\
-1,959 \\
-389 \\
-17 \\
-1,552 \\
-4 \\
-507 \\
0 \\
-507 \\
-4 \\
-883 \\
-515 \\
-367 \\
-33 \\
-1 \\
-7 \\
-37 \\
0 \\
0 \\
-13 \\
0 \\
0\end{array}$ & $\begin{array}{r}-75 \\
-65 \\
-32 \\
-5 \\
-28 \\
(s) \\
-40 \\
-4 \\
-36 \\
-11 \\
-18 \\
5 \\
-23 \\
32 \\
5 \\
-4 \\
2 \\
(s) \\
4 \\
19 \\
0 \\
(s)\end{array}$ & $\begin{array}{l}- \\
- \\
- \\
- \\
- \\
- \\
- \\
- \\
- \\
- \\
- \\
- \\
- \\
- \\
- \\
- \\
-\end{array}$ & $\begin{array}{l}- \\
- \\
- \\
- \\
- \\
- \\
- \\
- \\
- \\
- \\
- \\
- \\
- \\
- \\
- \\
- \\
- \\
-\end{array}$ & $\begin{array}{r}384 \\
84 \\
8 \\
4 \\
72 \\
0 \\
3 \\
0 \\
3 \\
0 \\
87 \\
11 \\
76 \\
70 \\
0 \\
3 \\
11 \\
1 \\
125 \\
(s) \\
0 \\
(s)\end{array}$ & $\begin{array}{r}3,154 \\
1,146 \\
233 \\
87 \\
826 \\
9 \\
176 \\
6 \\
171 \\
23 \\
458 \\
285 \\
173 \\
189 \\
498 \\
27 \\
56 \\
11 \\
151 \\
91 \\
294 \\
27\end{array}$ \\
\hline Total & 4,420 & 7,139 & 5,238 & -104 & $-4,843$ & 336 & 0 & 6,832 & 426 & 4,255 \\
\hline
\end{tabular}

a Represents the PAD District in which the material entered the United States and not necessarily where the crude oil or product is processed and/or consumed.

b Unaccounted for crude oil represents the difference between the supply and disposition of crude oil.

c A negative number indicates a decrease in stocks and a positive number indicates an increase in stocks.

d Products supplied is equal to field production, plus refinery production, plus imports, plus unaccounted for crude oil, plus net receipts, minus stock change, minus crude losses, minus refinery inputs, minus exports.

${ }^{\circ}$ Includes naphtha less than $401^{\circ} \mathrm{F}$ endpoint and other oils equal to or greater than $401^{\circ} \mathrm{F}$ endpoint.

(s) = Less than 500 barrels per day.

$E=$ Estimated.

LRG = Liquefied Refinery Gas.

Note: Totals may not equal sum of components due to independent rounding.

Sources: • Energy Information Administration (ElA) Forms EIA-810, "Monthly Refinery Report," EIA-811, "Monthly Bulk Terminal Report," EIA-812, "Monthly Product Pipeline Report," ElA-813, "Monthly Crude Oil Report," ElA-814, "Monthly Imports Report," EIA-816, "Monthly Natural Gas Liquids Report," EIA-817, "Monthly Tanker and Barge Movement Report," and EIA-819M, "Monthly Oxygenate Telephone Report". - Domestic crude oil production estimates based on historical statistics from State conservation agencies and the Minerals Management Service of the U.S. Department of the Interior. - Export data from the Bureau of the Census and Form ElA-810, "Monthly Refinery Report. 
Table 17. PAD District II-Year-to-Date Daily Average Supply and Disposition of Crude Oil and Petroleum Products, January-March 1995

(Thousand Barrels per Day)

\begin{tabular}{|c|c|c|c|c|c|c|c|c|c|c|}
\hline \multirow[b]{2}{*}{ Commodity } & \multicolumn{5}{|c|}{ Supply } & \multicolumn{5}{|c|}{ Disposition } \\
\hline & $\begin{array}{c}\text { Fleld } \\
\text { Production }\end{array}$ & $\begin{array}{c}\text { Refinery } \\
\text { Production }\end{array}$ & $\begin{array}{l}\text { Imports by } \\
\text { PAD } \\
\text { District } \\
\text { of Entry }\end{array}$ & $\begin{array}{l}\text { Unac- } \\
\text { counted } \\
\text { For } \\
\text { Crude Oilb }\end{array}$ & $\begin{array}{c}\text { Net } \\
\text { Receipts }\end{array}$ & $\begin{array}{c}\text { Stock } \\
\text { Changec }\end{array}$ & $\begin{array}{c}\text { Crude } \\
\text { Losses }\end{array}$ & $\begin{array}{c}\text { Refinery } \\
\text { Inputs }\end{array}$ & Exports & $\begin{array}{l}\text { Products } \\
\text { Supplled }\end{array}$ \\
\hline 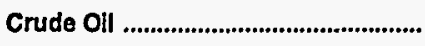 & . $E_{3,116}$ & - & 4,399 & -18 & $-1,300$ & -4 & $\mathbf{0}$ & 6,201 & 0 & 0 \\
\hline $\begin{array}{l}\text { Natural Gas Llquids and LRGs .......... } \\
\text { Pentanes Plus .................................... } \\
\text { Liquefied Petroleum Gases .............. } \\
\text { Ethane/Ethylene ............................. } \\
\text { Propane/Propylene ......................... } \\
\text { Normal Butane/Butylene ................ } \\
\text { Isobutane/lsobutylene ..................... }\end{array}$ & $\begin{array}{r}1,187 \\
187 \\
999 \\
453 \\
342 \\
76 \\
128\end{array}$ & $\begin{array}{r}324 \\
- \\
324 \\
16 \\
265 \\
38 \\
6\end{array}$ & $\begin{array}{r}44 \\
29 \\
14 \\
7 \\
4 \\
2 \\
1\end{array}$ & $\begin{array}{l}- \\
\overline{-} \\
\overline{-} \\
-\end{array}$ & $\begin{array}{r}-72 \\
-20 \\
-53 \\
86 \\
-123 \\
-4 \\
-12\end{array}$ & $\begin{array}{r}-89 \\
-13 \\
-76 \\
24 \\
-76 \\
-15 \\
-8\end{array}$ & $\begin{array}{l}- \\
\overline{-} \\
\overline{-} \\
-\end{array}$ & $\begin{array}{r}239 \\
92 \\
148 \\
0 \\
0 \\
76 \\
72\end{array}$ & $\begin{array}{r}53 \\
(s) \\
53 \\
0 \\
47 \\
5 \\
0\end{array}$ & $\begin{array}{r}1,279 \\
118 \\
1,161 \\
538 \\
517 \\
46 \\
60\end{array}$ \\
\hline $\begin{array}{l}\text { Other Llquids ..................................... } \\
\text { Other Hydrocarbons/Oxygenates ...... } \\
\text { Unfinished Oils ................................... } \\
\text { Motor Gasoline Blend. Comp. ........... } \\
\text { Avlation Gasoline Blend. Comp. ........ }\end{array}$ & $\begin{array}{r}114 \\
109 \\
- \\
5 \\
-\end{array}$ & $\underline{m}=$ & $\begin{array}{r}207 \\
1 \\
199 \\
7 \\
0\end{array}$ & $\begin{array}{l}- \\
\overline{-} \\
-\end{array}$ & $\begin{array}{r}-70 \\
0 \\
-1 \\
-69 \\
0\end{array}$ & $\begin{array}{r}39 \\
-2 \\
37 \\
3 \\
(s)\end{array}$ & $\begin{array}{l}- \\
\overline{-}\end{array}$ & $\begin{array}{r}269 \\
102 \\
231 \\
-64 \\
(s)\end{array}$ & $\begin{array}{r}14 \\
10 \\
0 \\
4 \\
0\end{array}$ & $\begin{array}{r}-71 \\
0 \\
-71 \\
0 \\
(\mathrm{~s})\end{array}$ \\
\hline 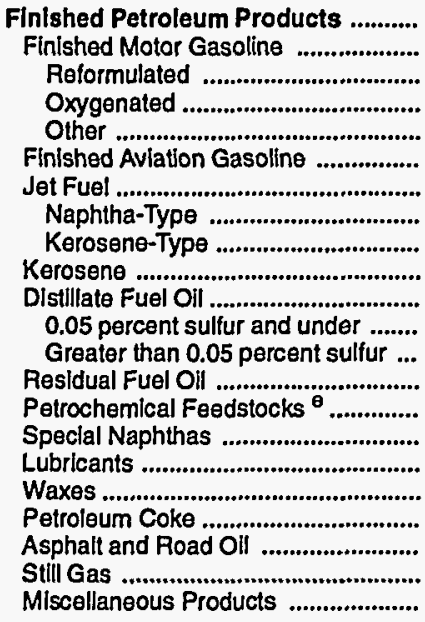 & $\begin{array}{r}1 \\
- \\
59 \\
-58 \\
- \\
- \\
- \\
- \\
- \\
- \\
- \\
- \\
- \\
-\end{array}$ & $\begin{array}{r}6,715 \\
3,102 \\
599 \\
39 \\
2,464 \\
14 \\
686 \\
2 \\
2 \\
684 \\
17 \\
1,353 \\
737 \\
616 \\
343 \\
358 \\
36 \\
107 \\
11 \\
276 \\
98 \\
290 \\
24\end{array}$ & $\begin{array}{r}184 \\
0 \\
0 \\
0 \\
0 \\
0 \\
2 \\
2 \\
1 \\
3 \\
0 \\
0 \\
0 \\
10 \\
164 \\
1 \\
1 \\
\text { (s) } \\
2 \\
1 \\
0 \\
\text { (s) }\end{array}$ & $\begin{array}{l}- \\
- \\
- \\
- \\
- \\
- \\
- \\
- \\
- \\
- \\
- \\
- \\
- \\
- \\
-\end{array}$ & $\begin{array}{r}-3,335 \\
-1,871 \\
-374 \\
-9 \\
-1,489 \\
-4 \\
-551 \\
-1 \\
-550 \\
-6 \\
-826 \\
-454 \\
-372 \\
-23 \\
-1 \\
-7 \\
-34 \\
0 \\
0 \\
-11 \\
0 \\
-1\end{array}$ & $\begin{array}{r}-102 \\
-1 \\
-10 \\
-12 \\
21 \\
2 \\
-50 \\
-2 \\
-49 \\
-10 \\
-34 \\
-26 \\
-8 \\
-20 \\
8 \\
-1 \\
1 \\
(s) \\
-6 \\
9 \\
0 \\
-1\end{array}$ & $\begin{array}{l}- \\
- \\
- \\
- \\
- \\
- \\
- \\
- \\
- \\
- \\
- \\
- \\
- \\
- \\
-\end{array}$ & $\begin{array}{l}- \\
\overline{-} \\
\overline{-} \\
\overline{-} \\
- \\
- \\
- \\
- \\
- \\
- \\
- \\
- \\
- \\
- \\
-\end{array}$ & $\begin{array}{r}449 \\
85 \\
3 \\
5 \\
77 \\
0 \\
13 \\
3 \\
9 \\
(s) \\
76 \\
15 \\
61 \\
107 \\
0 \\
3 \\
14 \\
1 \\
148 \\
1 \\
0 \\
(s)\end{array}$ & $\begin{array}{r}3,218 \\
1,147 \\
232 \\
96 \\
819 \\
7 \\
175 \\
1 \\
174 \\
23 \\
485 \\
293 \\
191 \\
243 \\
514 \\
27 \\
58 \\
11 \\
135 \\
78 \\
290 \\
24\end{array}$ \\
\hline Total ......................................................... & 4,418 & 7,039 & 4,833 & -18 & $-4,778$ & -156 & 0 & 6,710 & 515 & 4,426 \\
\hline
\end{tabular}

a Represents the PAD District in which the material entered the United States and not necessarily where the crude oil or product is processed and/or consumed.

b Unaccounted for crude oil represents the difference between the supply and disposition of crude oil.

c. A negative number indicates a decrease in stocks and a positive number indicates an increase in stocks.

d Products supplied is equal to field production, plus refinery production, plus imports, plus unaccounted for crude oil, plus net receipts, minus stock change, minus crude losses, minus refinery inputs, minus exports.

- Includes naphtha less than $401^{\circ} \mathrm{F}$ endpoint and other oils equal to or greater than $401^{\circ} \mathrm{F}$ endpoint.

(s) $=$ Less than 500 barrels per day.

$E=$ Estimated.

LRG = Llquefied Fefinery Gas.

Note: Totals may not equal sum of components due to independent rounding.

Sources: • Energy Information Administration (ElA) Forms ElA-810, "Monthly Refinery Report," ElA-811, "Monthly Bulk Terminal Report," ElA-812, "Monthly Product Pipeline Report," ElA-813, "Monthly Crude Oil Report," EIA-814, "Monthly Imports Report," ElA-816, "Monthly Natural Gas Liquids Report," ElA-817, "Monthly Tanker and Barge Movement Report," and ElA-819M, "Monthly Oxygenate Telephone Report". - Domestic crude oil production estimates based on historical statistles from State conservation agencies and the Minerals Management Service of the U.S. Department of the Interior. - Export data from the Bureau of the Census and Form EIA-810, "Monthly Refinery Report." 
Table 18. PAD District IV—Supply, Disposition, and Ending Stocks of Crude Oil and Petroleum Products, March 1995

(Thousand Barrels)

\begin{tabular}{|c|c|c|c|c|c|c|c|c|c|c|c|}
\hline \multirow[b]{2}{*}{ Commodity } & \multicolumn{5}{|c|}{ Supply } & \multicolumn{5}{|c|}{ Disposition } & \multirow[b]{2}{*}{$\begin{array}{l}\text { Ending } \\
\text { Stocks }\end{array}$} \\
\hline & \begin{tabular}{|c|} 
Field \\
Production \\
\end{tabular} & $\begin{array}{c}\text { Refinery } \\
\text { Production }\end{array}$ & $\begin{array}{l}\text { Imports by } \\
\text { PAD } \\
\text { District } \\
\text { of Entry }\end{array}$ & $\begin{array}{l}\text { Unac- } \\
\text { counted } \\
\text { For } \\
\text { Crude Oij } \\
\end{array}$ & $\begin{array}{c}\text { Net } \\
\text { Receipts }\end{array}$ & $\begin{array}{c}\text { Stock } \\
\text { Change }\end{array}$ & $\begin{array}{c}\text { Crude } \\
\text { Losses } \\
\end{array}$ & $\begin{array}{c}\text { Refinery } \\
\text { Inputs }\end{array}$ & Exports & $\begin{array}{l}\text { Products } \\
\text { Supplied d }\end{array}$ & \\
\hline 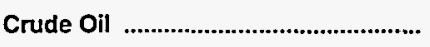 & $E_{12,234}$ & - & 3,971 & 263 & $-2,634$ & -240 & 0 & 14,074 & 0 & $\mathbf{0}$ & 12,844 \\
\hline $\begin{array}{l}\text { Natural Gas Liquids and LRGs ........... } \\
\text { Pentanes Plus ..................................... } \\
\text { Liquefied Petroleum Gases ............... } \\
\text { Ethane/Ethylene ............................ } \\
\text { Propane/Propylene .......................... } \\
\text { Normal Butane/Butylene ............... } \\
\text { Isobutane/lsobutylene .................... }\end{array}$ & $\begin{array}{r}4,281 \\
854 \\
3,427 \\
1,124 \\
1,452 \\
532 \\
319\end{array}$ & $\begin{array}{r}364 \\
- \\
364 \\
0 \\
323 \\
26 \\
15\end{array}$ & $\begin{array}{r}720 \\
547 \\
173 \\
0 \\
120 \\
49 \\
4\end{array}$ & $\begin{array}{l}- \\
- \\
- \\
- \\
-\end{array}$ & $\begin{array}{r}-3,285 \\
-383 \\
-2,902 \\
-1,295 \\
-943 \\
-419 \\
-245\end{array}$ & $\begin{array}{r}65 \\
-10 \\
75 \\
-4 \\
-22 \\
84 \\
17\end{array}$ & $\begin{array}{l}- \\
- \\
- \\
- \\
-\end{array}$ & $\begin{array}{r}352 \\
92 \\
260 \\
0 \\
0 \\
166 \\
94\end{array}$ & $\begin{array}{l}1 \\
0 \\
1 \\
0 \\
0 \\
1 \\
0\end{array}$ & $\begin{array}{r}1,662 \\
936 \\
726 \\
-167 \\
974 \\
-63 \\
-18\end{array}$ & $\begin{array}{r}1,233 \\
181 \\
1,052 \\
213 \\
315 \\
334 \\
190\end{array}$ \\
\hline $\begin{array}{l}\text { Other Liquids .................................... } \\
\text { Other Hydrocarbons/Oxygenates ...... } \\
\text { Unfinished Oils .................................... } \\
\text { Motor Gasoline Blend. Comp. ........... } \\
\text { Aviation Gasoline Blend. Comp. ....... }\end{array}$ & $\begin{array}{r}78 \\
19 \\
-- \\
59 \\
--\end{array}$ & $\begin{array}{l}- \\
- \\
-\end{array}$ & $\begin{array}{l}0 \\
0 \\
0 \\
0 \\
0\end{array}$ & $\begin{array}{l}- \\
- \\
- \\
-\end{array}$ & $\begin{array}{l}0 \\
0 \\
0 \\
0 \\
0\end{array}$ & $\begin{array}{r}234 \\
5 \\
473 \\
-244 \\
0\end{array}$ & $\begin{array}{l}- \\
- \\
-\end{array}$ & $\begin{array}{r}-159 \\
14 \\
-476 \\
303 \\
0\end{array}$ & $\begin{array}{l}0 \\
0 \\
0 \\
0 \\
0\end{array}$ & $\begin{array}{l}3 \\
0 \\
3 \\
0 \\
0\end{array}$ & $\begin{array}{r}5,299 \\
250 \\
2,531 \\
2,518 \\
0\end{array}$ \\
\hline 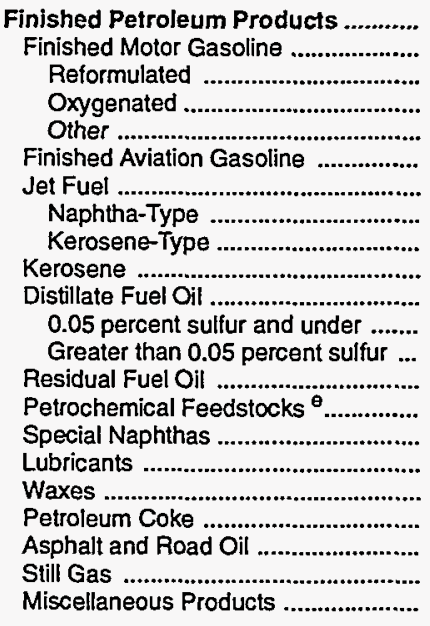 & $\begin{array}{r}-9 \\
-9 \\
-- \\
503 \\
-512 \\
-- \\
-- \\
-- \\
-- \\
-- \\
-- \\
-- \\
-- \\
-- \\
-- \\
-- \\
- \\
- \\
-- \\
-- \\
-- \\
-\end{array}$ & $\begin{array}{r}14,426 \\
7,101 \\
0 \\
-68 \\
7,169 \\
11 \\
783 \\
191 \\
592 \\
26 \\
3,929 \\
2,994 \\
935 \\
451 \\
19 \\
0 \\
0 \\
9 \\
457 \\
1,000 \\
550 \\
90\end{array}$ & $\begin{array}{r}107 \\
12 \\
0 \\
0 \\
12 \\
0 \\
0 \\
0 \\
0 \\
0 \\
95 \\
16 \\
79 \\
0 \\
0 \\
0 \\
0 \\
0 \\
0 \\
0 \\
0 \\
0\end{array}$ & $\begin{array}{l}- \\
- \\
- \\
- \\
- \\
- \\
- \\
- \\
- \\
- \\
- \\
- \\
-- \\
- \\
- \\
-- \\
- \\
- \\
- \\
- \\
--\end{array}$ & $\begin{array}{r}902 \\
-30 \\
0 \\
0 \\
-30 \\
6 \\
907 \\
-147 \\
1,054 \\
0 \\
19 \\
9 \\
10 \\
0 \\
0 \\
0 \\
0 \\
0 \\
0 \\
0 \\
0 \\
0\end{array}$ & $\begin{array}{r}-123 \\
-560 \\
0 \\
-159 \\
-401 \\
-1 \\
-62 \\
-38 \\
-24 \\
20 \\
-4 \\
-130 \\
126 \\
84 \\
0 \\
0 \\
0 \\
1 \\
50 \\
352 \\
0 \\
-3\end{array}$ & $\begin{array}{l}- \\
-- \\
- \\
- \\
-- \\
-- \\
- \\
-- \\
-- \\
-- \\
-- \\
-- \\
-- \\
-- \\
- \\
-\end{array}$ & $\begin{array}{l}- \\
- \\
- \\
- \\
-- \\
- \\
- \\
- \\
- \\
- \\
- \\
- \\
- \\
- \\
- \\
- \\
- \\
-\end{array}$ & $\begin{array}{r}17 \\
10 \\
0 \\
9 \\
1 \\
0 \\
0 \\
0 \\
0 \\
0 \\
0 \\
0 \\
0 \\
0 \\
0 \\
(s) \\
6 \\
0 \\
0 \\
1 \\
0 \\
0\end{array}$ & $\begin{array}{r}15,532 \\
7,624 \\
0 \\
585 \\
7,040 \\
18 \\
1,752 \\
82 \\
1,670 \\
6 \\
4,047 \\
3,149 \\
898 \\
367 \\
19 \\
(s) \\
-6 \\
8 \\
407 \\
647 \\
550 \\
93\end{array}$ & $\begin{array}{r}12,278 \\
4,757 \\
0 \\
92 \\
4,665 \\
45 \\
871 \\
144 \\
727 \\
103 \\
2,748 \\
2,238 \\
510 \\
571 \\
0 \\
1 \\
0 \\
16 \\
243 \\
2,908 \\
0 \\
15\end{array}$ \\
\hline Total & 16,584 & 14,790 & 4,798 & 263 & $-5,017$ & -64 & 0 & 14,267 & 19 & 17,197 & 31,654 \\
\hline
\end{tabular}

a Represents the PAD District in which the material entered the United States and not necessarily where the crude oil or product is processed and/or consumed.

b Unaccounted for crude oil represents the difference between the supply and disposition of crude oil.

c A negative number indicates a decrease in stocks and a positive number indicates an increase in stocks.

d Products supplied is equal to field production, plus refinery production, plus imports, plus unaccounted for crude oil, plus net receipts, minus stock change, minus crude losses, minus refinery inputs, minus exports.

$\theta$ Includes naphtha less than $401^{\circ} \mathrm{F}$ endpoint and other oils equal to or greater than $401^{\circ} \mathrm{F}$ endpoint.

(s) = Less than 500 barrels.

$E=$ Estimated.

LRG = Liquefied Refinery Gas.

Note: Totals may not equal sum of components due to independent rounding.

Sources: • Energy Information Administration (EIA) Forms ElA-810, "Monthly Refinery Report," EIA-811, "Monthly Bulk Terminal Report," EIA-812, "Monthly Product Pipeline Report," EIA-813, "Monthly Crude Oil Report," EIA-814, "Monthly Imports Report," EIA-816, "Monthly Natural Gas Liquids Report," EIA-817, "Monthly Tanker and Barge Movement Report," and EIA-819M, "Monthly Oxygenate Telephone Report". - Domestic crude oil production estimates based on historical statistics from State conservation agencies and the Minerals Management Service of the U.S. Department of the Interior. - Export data from the Bureau of the Census and Form EIA-810, "Monthly Refinery Report." 
Table 19. PAD District IV-Year-to-Date Supply, Disposition, and Ending Stocks of Crude Oil and Petroleum Products, January-March 1995 (Thousand Barrels)

\begin{tabular}{|c|c|c|c|c|c|c|c|c|c|c|c|}
\hline \multirow[b]{2}{*}{ Commodity } & \multicolumn{5}{|c|}{ Supply } & \multicolumn{5}{|c|}{ Disposition } & \multirow[b]{2}{*}{$\begin{array}{l}\text { Ending } \\
\text { Stocks }\end{array}$} \\
\hline & \begin{tabular}{c|} 
Field \\
Production
\end{tabular} & $\begin{array}{c}\text { Refinery } \\
\text { Production }\end{array}$ & $\begin{array}{l}\text { Imports by } \\
\text { PAD } \\
\text { District } \\
\text { of Entry }\end{array}$ & $\begin{array}{l}\text { Unac- } \\
\text { counted } \\
\text { For } \\
\text { Crude Oil }\end{array}$ & $\begin{array}{c}\text { Net } \\
\text { Receipts }\end{array}$ & $\begin{array}{c}\text { Stock } \\
\text { Changec }\end{array}$ & $\begin{array}{c}\text { Crude } \\
\text { Losses }\end{array}$ & $\begin{array}{c}\text { Retinery } \\
\text { Inputs }\end{array}$ & Exports & $\begin{array}{l}\text { Products } \\
\text { Supplied d }\end{array}$ & \\
\hline Crude Oll & $E_{34,426}$ & $\cdots$ & 11,174 & 3,374 & $-7,723$ & 732 & 0 & 40,519 & 0 & 0 & 12,844 \\
\hline $\begin{array}{l}\text { Natural Gas Liquids and LRGs ......... } \\
\text { Pentanes Plus .................................... } \\
\text { Llquefied Petroleum Gases .............. } \\
\text { Ethane/Ethylene ............................ } \\
\text { Propane/Propylene ......................... } \\
\text { Normal Butane/Butylene ................ } \\
\text { Isobutane/lsobutylene ................... }\end{array}$ & $\begin{array}{r}12,280 \\
2,402 \\
9,878 \\
3,455 \\
4,040 \\
1,461 \\
922\end{array}$ & $\begin{array}{r}616 \\
- \\
616 \\
0 \\
889 \\
-264 \\
-9\end{array}$ & $\begin{array}{r}2,087 \\
1,343 \\
744 \\
0 \\
485 \\
243 \\
16\end{array}$ & $\begin{array}{l}- \\
- \\
- \\
- \\
- \\
-\end{array}$ & $\begin{array}{r}-9,319 \\
-931 \\
-8,388 \\
-4,039 \\
-2,657 \\
-1,050 \\
-642\end{array}$ & $\begin{array}{r}-72 \\
-5 \\
-67 \\
-2 \\
-78 \\
-15 \\
28\end{array}$ & $\begin{array}{l}- \\
- \\
- \\
-\end{array}$ & $\begin{array}{r}1,446 \\
431 \\
1.015 \\
0 \\
0 \\
742 \\
273\end{array}$ & $\begin{array}{l}1 \\
0 \\
1 \\
0 \\
0 \\
1 \\
0\end{array}$ & $\begin{array}{r}4,289 \\
2,388 \\
1,901 \\
-582 \\
2,835 \\
-338 \\
-14\end{array}$ & $\begin{array}{r}1,233 \\
181 \\
1,052 \\
213 \\
315 \\
334 \\
190\end{array}$ \\
\hline $\begin{array}{l}\text { Other Liquids ................................... } \\
\text { Other Hydrocarbons/Oxygenates .... } \\
\text { Unfinished Olls .................................. } \\
\text { Motor Gasoline Blend. Comp. .......... } \\
\text { Aviation Gasoline Blend. Comp. ...... }\end{array}$ & $\begin{array}{r}263 \\
237 \\
- \\
26 \\
-\end{array}$ & $\begin{array}{l}- \\
- \\
-\end{array}$ & $\begin{array}{l}0 \\
0 \\
0 \\
0 \\
0\end{array}$ & $\begin{array}{l}- \\
- \\
- \\
-\end{array}$ & $\begin{array}{l}0 \\
0 \\
0 \\
0 \\
0\end{array}$ & $\begin{array}{r}661 \\
-32 \\
532 \\
161 \\
0\end{array}$ & $\begin{array}{l}- \\
- \\
-\end{array}$ & $\begin{array}{r}-432 \\
269 \\
-566 \\
-135 \\
0\end{array}$ & $\begin{array}{l}0 \\
0 \\
0 \\
0 \\
0\end{array}$ & $\begin{array}{r}34 \\
0 \\
34 \\
0 \\
0\end{array}$ & $\begin{array}{r}5,299 \\
250 \\
2,531 \\
2,518 \\
0\end{array}$ \\
\hline 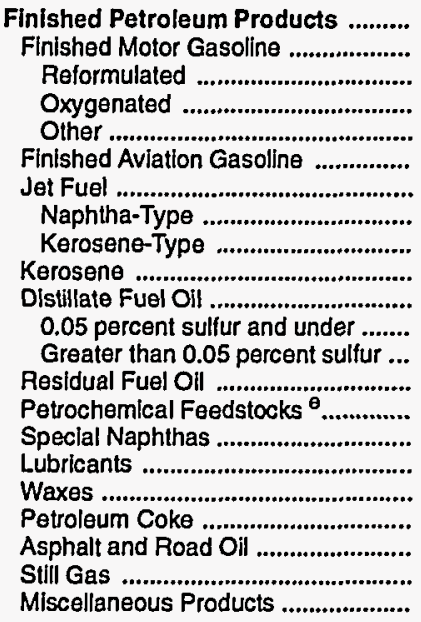 & $\begin{array}{r}106 \\
106 \\
- \\
1,317 \\
-1,211 \\
- \\
- \\
- \\
- \\
- \\
- \\
- \\
- \\
- \\
- \\
- \\
- \\
- \\
- \\
- \\
-\end{array}$ & $\begin{array}{r}42,637 \\
21,143 \\
0 \\
1,676 \\
19,467 \\
37 \\
2,824 \\
618 \\
2,206 \\
208 \\
11,074 \\
8,602 \\
2,472 \\
1,051 \\
63 \\
0 \\
0 \\
16 \\
1,367 \\
2,786 \\
1,797 \\
271\end{array}$ & $\begin{array}{r}383 \\
28 \\
0 \\
0 \\
28 \\
0 \\
0 \\
0 \\
0 \\
0 \\
355 \\
44 \\
311 \\
0 \\
0 \\
0 \\
0 \\
0 \\
0 \\
0 \\
0 \\
0\end{array}$ & $\begin{array}{l}- \\
- \\
-- \\
- \\
- \\
- \\
- \\
- \\
- \\
- \\
- \\
- \\
- \\
- \\
- \\
- \\
- \\
- \\
- \\
- \\
-\end{array}$ & $\begin{array}{r}1,399 \\
-171 \\
0 \\
48 \\
-219 \\
20 \\
1,958 \\
-298 \\
2,256 \\
-58 \\
-350 \\
-272 \\
-78 \\
0 \\
0 \\
0 \\
0 \\
0 \\
0 \\
0 \\
0 \\
0\end{array}$ & $\begin{array}{r}1,018 \\
-3 \\
0 \\
-485 \\
482 \\
3 \\
3 \\
-113 \\
116 \\
12 \\
-407 \\
-50 \\
-357 \\
172 \\
0 \\
0 \\
0 \\
-11 \\
138 \\
1,113 \\
0 \\
-2\end{array}$ & $\begin{array}{l}- \\
- \\
- \\
- \\
- \\
- \\
- \\
- \\
- \\
- \\
- \\
- \\
- \\
- \\
- \\
- \\
-\end{array}$ & $\begin{array}{l}- \\
- \\
- \\
- \\
- \\
- \\
- \\
- \\
- \\
- \\
- \\
- \\
- \\
- \\
- \\
- \\
-\end{array}$ & $\begin{array}{r}33 \\
16 \\
0 \\
15 \\
1 \\
0 \\
0 \\
0 \\
0 \\
0 \\
0 \\
0 \\
0 \\
0 \\
0 \\
1 \\
13 \\
0 \\
0 \\
3 \\
0 \\
\text { (s) }\end{array}$ & $\begin{array}{r}43,474 \\
21,093 \\
0 \\
3,511 \\
17,581 \\
54 \\
4,779 \\
433 \\
4,346 \\
138 \\
11,486 \\
8,424 \\
3,062 \\
879 \\
63 \\
-1 \\
-13 \\
27 \\
1,229 \\
1,670 \\
1,797 \\
273\end{array}$ & $\begin{array}{r}12,278 \\
4,757 \\
0 \\
92 \\
4,665 \\
45 \\
871 \\
144 \\
727 \\
103 \\
2,748 \\
2,238 \\
510 \\
571 \\
0 \\
1 \\
0 \\
16 \\
243 \\
2,908 \\
0 \\
15\end{array}$ \\
\hline 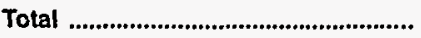 & 47,075 & 43,253 & 13,644 & 3,374 & $-15,643$ & 2,339 & 0 & 41,533 & 34 & 47,796 & 31,654 \\
\hline
\end{tabular}

Represents the PAD District in which the material entered the United States and not necessarily where the crude oil or product is processed and/or consumed.

Unaccounted for crude oil represents the difference between the supply and disposition of crude oil.

c A negative number indicates a decrease in stocks and a positive number indicates an increase in stocks.

d Products supplied is equal to field production, plus refinery production, plus imports, plus unaccounted for crude oil, plus net receipts, minus stock change, minus crude losses, minus refinery inputs, minus exports.

Includes naphtha less than $401^{\circ} \mathrm{F}$ endpoint and other oils equal to or greater than $401^{\circ} \mathrm{F}$ endpoint.

(s) $=$ Less than 500 barrels.

$E=$ Estimated.

LRG $=$ Liquefied Refinery Gas.

Note: Totals may not equal sum of components due to independent rounding.

Sources: - Energy Information Administration (EIA) Forms EIA-810, "Monthly Refinery Report," EIA-811, "Monthly Bulk Terminal Report," EIA-812, "Monthly Product Pipeline Report," EIA-813, "Monthly Crude Oil Report," EIA-814, "Monthly Imports Report," EIA-816, "Monthly Natural Gas Liquids Report," ElA-817, "Monthly Tanker and Barge Movement Report," and EIA-819M, "Monthly Oxygenate Telephone Report". - Domestic crude oil production estimates based on historical statistics from State conservation agencles and the Minerals Management Service of the U.S. Department of the Interior. • Export data from the Bureau of the Census and Form ElA-810, "Monthly Refinery Report." 
Table 20. PAD District IV-Daily Average Supply and Disposition of Crude Oil and Petroleum Products, March 1995

(Thousand Barrels per Day)

\begin{tabular}{|c|c|c|c|c|c|c|c|c|c|c|}
\hline \multirow[b]{2}{*}{ Commodity } & \multicolumn{5}{|c|}{ Supply } & \multicolumn{5}{|c|}{ Disposition } \\
\hline & $\begin{array}{c}\text { Field } \\
\text { Production }\end{array}$ & $\begin{array}{c}\text { Refinery } \\
\text { Production }\end{array}$ & $\begin{array}{c}\text { Imports by } \\
\text { PAD } \\
\text { District } \\
\text { of Entry }\end{array}$ & $\begin{array}{l}\text { Unac- } \\
\text { counted } \\
\text { For } \\
\text { Crude Oil }\end{array}$ & $\begin{array}{c}\text { Net } \\
\text { Receipts }\end{array}$ & $\begin{array}{c}\text { Stock } \\
\text { Change }\end{array}$ & $\begin{array}{c}\text { Crude } \\
\text { Losses } \\
\end{array}$ & $\begin{array}{c}\text { Refinery } \\
\text { Inputs }\end{array}$ & Exports & $\begin{array}{l}\text { Products } \\
\text { Supplled d }^{\text {d }}\end{array}$ \\
\hline 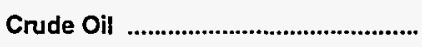 & $E_{395}$ & - & 128 & 8 & -85 & -8 & 0 & 454 & 0 & 0 \\
\hline $\begin{array}{l}\text { Natural Gas Liquids and LRGs ......... } \\
\text { Pentanes Plus ................................... } \\
\text { Liquefied Petroleum Gases .............. } \\
\text { Ethane/Ethylene .......................... } \\
\text { Propane/Propylene ......................... } \\
\text { Normal Butane/Butylene ............... } \\
\text { Isobutane/lsobutylene .................... }\end{array}$ & $\begin{array}{r}138 \\
28 \\
111 \\
36 \\
47 \\
17 \\
10\end{array}$ & $\begin{array}{r}12 \\
\overline{12} \\
0 \\
10 \\
1 \\
(s)\end{array}$ & $\begin{array}{r}23 \\
18 \\
6 \\
0 \\
4 \\
2 \\
\text { (s) }\end{array}$ & $\begin{array}{l}- \\
- \\
- \\
- \\
- \\
-\end{array}$ & $\begin{array}{r}-106 \\
-12 \\
-94 \\
-42 \\
-30 \\
-14 \\
-8\end{array}$ & $\begin{array}{r}2 \\
(s) \\
2 \\
(s) \\
-1 \\
3 \\
1\end{array}$ & $\begin{array}{l}- \\
- \\
\ddot{-} \\
-\end{array}$ & $\begin{array}{r}11 \\
3 \\
8 \\
0 \\
0 \\
5 \\
3\end{array}$ & $\begin{array}{r}\text { (s) } \\
0 \\
\text { (s) } \\
0 \\
0 \\
\text { (s) } \\
0\end{array}$ & $\begin{array}{l}54 \\
30 \\
23 \\
-5 \\
31 \\
-2 \\
-1\end{array}$ \\
\hline $\begin{array}{l}\text { Other Liquids ................................... } \\
\text { Other Hydrocarbons/Oxygenates ..... } \\
\text { Unfinished Oils ................................. } \\
\text { Motor Gasoline Blend. Comp. .......... } \\
\text { Aviation Gasoline Blend. Comp. ....... }\end{array}$ & \begin{tabular}{l}
3 \\
1 \\
\hdashline \\
2 \\
--
\end{tabular} & $\begin{array}{l}- \\
- \\
- \\
-\end{array}$ & $\begin{array}{l}0 \\
0 \\
0 \\
0 \\
0\end{array}$ & $\begin{array}{l}- \\
- \\
-- \\
-\end{array}$ & $\begin{array}{l}0 \\
0 \\
0 \\
0 \\
0\end{array}$ & $\begin{array}{r}8 \\
(s) \\
15 \\
-8 \\
0\end{array}$ & $\begin{array}{l}- \\
\ddot{-} \\
\ddot{-}\end{array}$ & $\begin{array}{r}-5 \\
(s) \\
-15 \\
10 \\
0\end{array}$ & $\begin{array}{l}0 \\
0 \\
0 \\
0 \\
0\end{array}$ & $\begin{array}{r}\text { (s) } \\
0 \\
(s) \\
0 \\
0\end{array}$ \\
\hline 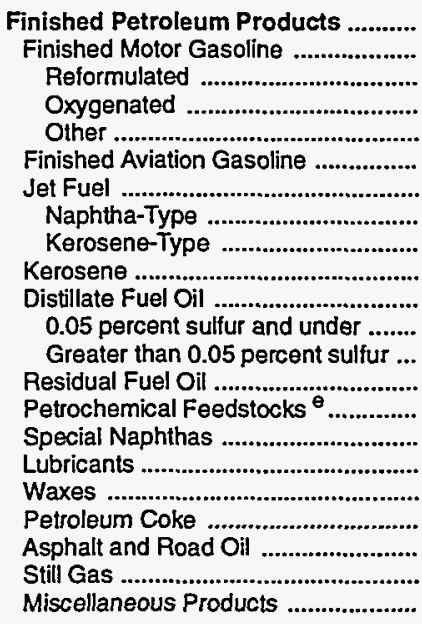 & $\begin{array}{r}(s) \\
(s) \\
-- \\
16 \\
-17 \\
- \\
- \\
-- \\
-- \\
- \\
- \\
- \\
- \\
- \\
- \\
- \\
- \\
- \\
- \\
- \\
-\end{array}$ & $\begin{array}{r}465 \\
229 \\
0 \\
-2 \\
231 \\
(s) \\
25 \\
6 \\
19 \\
1 \\
127 \\
97 \\
30 \\
15 \\
1 \\
0 \\
0 \\
(s) \\
15 \\
32 \\
18 \\
3\end{array}$ & $\begin{array}{r}3 \\
\text { (s) } \\
0 \\
0 \\
(s) \\
0 \\
0 \\
0 \\
0 \\
0 \\
3 \\
1 \\
3 \\
0 \\
0 \\
0 \\
0 \\
0 \\
0 \\
0 \\
0 \\
0\end{array}$ & $\begin{array}{c}- \\
-- \\
- \\
- \\
- \\
- \\
- \\
- \\
- \\
- \\
- \\
- \\
- \\
- \\
- \\
- \\
- \\
- \\
- \\
- \\
-\end{array}$ & $\begin{array}{r}29 \\
-1 \\
0 \\
0 \\
-1 \\
(s) \\
29 \\
-5 \\
34 \\
0 \\
1 \\
(s) \\
(s) \\
0 \\
0 \\
0 \\
0 \\
0 \\
0 \\
0 \\
0 \\
0\end{array}$ & $\begin{array}{r}-4 \\
-18 \\
0 \\
-5 \\
-13 \\
\text { (s) } \\
-2 \\
-1 \\
-1 \\
1 \\
\text { (s) } \\
-4 \\
4 \\
3 \\
0 \\
0 \\
0 \\
\text { (s) } \\
2 \\
11 \\
0 \\
\text { (s) }\end{array}$ & $\begin{array}{l}- \\
- \\
- \\
- \\
- \\
- \\
- \\
- \\
- \\
- \\
- \\
- \\
- \\
- \\
- \\
- \\
-\end{array}$ & $\begin{array}{l}- \\
- \\
- \\
- \\
- \\
- \\
- \\
- \\
- \\
- \\
- \\
- \\
- \\
- \\
- \\
- \\
- \\
- \\
- \\
- \\
-\end{array}$ & $\begin{array}{r}1 \\
\text { (s) } \\
0 \\
(s) \\
(s) \\
0 \\
0 \\
0 \\
0 \\
0 \\
0 \\
0 \\
0 \\
0 \\
0 \\
\text { (s) } \\
\text { (s) } \\
0 \\
0 \\
\text { (s) } \\
0 \\
0\end{array}$ & $\begin{array}{r}501 \\
246 \\
0 \\
19 \\
227 \\
1 \\
57 \\
3 \\
54 \\
(s) \\
131 \\
102 \\
29 \\
12 \\
1 \\
\text { (s) } \\
\text { (s) } \\
\text { (s) } \\
13 \\
21 \\
18 \\
3\end{array}$ \\
\hline Total & 535 & 477 & 155 & 8 & -162 & -2 & 0 & 460 & 1 & 555 \\
\hline
\end{tabular}

a Represents the PAD District in which the material entered the United States and not necessarily where the crude oil or product is processed and/or consumed.

b Unaccounted for crude oil represents the difference botween the supply and disposition of crude oil.

c A negative number indicates a decrease in stocks and a positive number indicates an increase in stocks.

d Products supplied is equal to field production, plus refinery production, plus imports, plus unaccounted for crude oil, plus net receipts, minus stock change, minus crude losses, minus refinery inputs, minus exports.

Includes naphtha less than $401^{\circ} \mathrm{F}$ endpoint and other oils equal to or greater than $401^{\circ} \mathrm{F}$ endpoint.

(s) = Less than 500 barrels per day.

$E=$ Estimated.

LRG = Liquelied Refinery Gas.

Note: Totals may not equal sum of components due to independent rounding.

Sources: - Energy Information Administration (EIA) Forms ElA-810, "Monthly Refinery Report," ElA-811, "Monthly Bulk Terminal Report," EIA-812, "Monthly Product Pipeline Report," ElA-813, "Monthly Crude Oil Report," EIA-814, "Monthly Imports Report," EIA-816, "Monthly Natural Gas Liquids Report," EIA-817, "Monthly Tanker and Barge Movement Report," and ElA-819M, "Monthly Oxygenate Telephone Report'. • Domestic crude oll production estimates based on historical statistics from State conservation agencies and the Minerals Management Service of the U.S. Department of the Interior. - Export data from the Bureau of the Census and Form EIA-810, "Monthly Refinery Report." 
Table 21. PAD District IV-Year-to-Date Daily Average Supply and Disposition of Crude Oil and Petroleum Products, January-March 1995

(Thousand Barrels per Day)

\begin{tabular}{|c|c|c|c|c|c|c|c|c|c|c|}
\hline \multirow[b]{2}{*}{ Commodity } & \multicolumn{5}{|c|}{ Supply } & \multicolumn{5}{|c|}{ Disposition } \\
\hline & $\begin{array}{c}\text { Field } \\
\text { Production }\end{array}$ & $\begin{array}{c}\text { Refinery } \\
\text { Production }\end{array}$ & $\begin{array}{l}\text { Imports by } \\
\text { PAD } \\
\text { District } \\
\text { of Entry }\end{array}$ & $\begin{array}{l}\text { Unac- } \\
\text { counted } \\
\text { For } \\
\text { Crude Oil } \\
\end{array}$ & $\begin{array}{c}\text { Net } \\
\text { Receipts }\end{array}$ & $\begin{array}{c}\text { Stock } \\
\text { Changec }\end{array}$ & $\begin{array}{c}\text { Crude } \\
\text { Losses }\end{array}$ & $\begin{array}{c}\text { Refinery } \\
\text { Inputs }\end{array}$ & Exports & $\begin{array}{l}\text { Products } \\
\text { Supplled }\end{array}$ \\
\hline Crude Oll .................................................. & $E_{383}$ & - & 124 & 37 & -86 & 8 & 0 & 450 & 0 & 0 \\
\hline $\begin{array}{l}\text { Natural Gas Llquids and LRGs ......... } \\
\text { Pentanes Plus ................................... } \\
\text { Liquefled Petroleum Gases .............. } \\
\text { Ethane/Ethylene ............................. } \\
\text { Propane/Propylene ...................... } \\
\text { Normal Butane/Butylene ............... } \\
\text { Isobutane/lsobutylene ................... }\end{array}$ & $\begin{array}{r}136 \\
27 \\
110 \\
38 \\
45 \\
16 \\
10\end{array}$ & $\begin{array}{r}7 \\
\overline{7} \\
0 \\
10 \\
-3 \\
\text { (s) }\end{array}$ & $\begin{array}{r}23 \\
15 \\
8 \\
0 \\
5 \\
3 \\
\text { (s) }\end{array}$ & $\begin{array}{l}- \\
- \\
- \\
-\end{array}$ & $\begin{array}{r}-104 \\
-10 \\
-93 \\
-45 \\
-30 \\
-12 \\
-7\end{array}$ & $\begin{array}{l}-1 \\
(s) \\
-1 \\
(s) \\
-1 \\
(s) \\
(s)\end{array}$ & $\begin{array}{l}- \\
- \\
- \\
-\end{array}$ & $\begin{array}{r}16 \\
5 \\
11 \\
0 \\
0 \\
8 \\
3\end{array}$ & $\begin{array}{r}\text { (s) } \\
0 \\
\text { (s) } \\
0 \\
0 \\
\text { (s) } \\
0\end{array}$ & $\begin{array}{r}48 \\
27 \\
21 \\
-6 \\
32 \\
-4 \\
\text { (s) }\end{array}$ \\
\hline $\begin{array}{l}\text { Other Llquids ..................................... } \\
\text { Other Hydrocarbons/Oxygenates ..... } \\
\text { Unfinished Oils .................................... } \\
\text { Motor Gasoline Blend. Comp. .......... } \\
\text { Aviation Gasoline Blend. Comp. ........ }\end{array}$ & $\begin{array}{r}3 \\
3 \\
- \\
-\end{array}$ & $\begin{array}{l}- \\
\overline{-} \\
-\end{array}$ & $\begin{array}{l}0 \\
0 \\
0 \\
0 \\
0\end{array}$ & $\begin{array}{l}- \\
- \\
-\end{array}$ & $\begin{array}{l}0 \\
0 \\
0 \\
0 \\
0\end{array}$ & $\begin{array}{r}7 \\
(s) \\
6 \\
2 \\
0\end{array}$ & $\begin{array}{l}- \\
- \\
-\end{array}$ & $\begin{array}{r}-5 \\
3 \\
-6 \\
-2 \\
0\end{array}$ & $\begin{array}{l}0 \\
0 \\
0 \\
0 \\
0\end{array}$ & $\begin{array}{r}\text { (s) } \\
0 \\
\text { (s) } \\
0 \\
0\end{array}$ \\
\hline 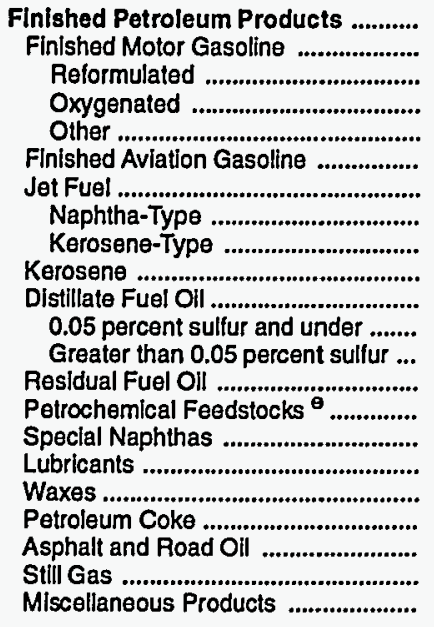 & $\begin{array}{r}1 \\
\overline{1} \\
15 \\
-13 \\
- \\
- \\
- \\
- \\
- \\
- \\
- \\
- \\
- \\
- \\
- \\
-\end{array}$ & $\begin{array}{r}474 \\
235 \\
0 \\
19 \\
216 \\
(s) \\
31 \\
7 \\
25 \\
2 \\
123 \\
96 \\
27 \\
12 \\
1 \\
0 \\
0 \\
\text { (s) } \\
15 \\
31 \\
20 \\
3\end{array}$ & $\begin{array}{r}4 \\
(s) \\
0 \\
0 \\
(s) \\
0 \\
0 \\
0 \\
0 \\
0 \\
4 \\
(s) \\
3 \\
0 \\
0 \\
0 \\
0 \\
0 \\
0 \\
0 \\
0 \\
0\end{array}$ & $\begin{array}{l}- \\
- \\
\overline{-} \\
- \\
\bar{z} \\
\overline{-} \\
- \\
- \\
- \\
- \\
- \\
- \\
- \\
- \\
- \\
- \\
-\end{array}$ & $\begin{array}{r}16 \\
-2 \\
0 \\
1 \\
-2 \\
(s) \\
22 \\
-3 \\
25 \\
-1 \\
-4 \\
-3 \\
-1 \\
0 \\
0 \\
0 \\
0 \\
0 \\
0 \\
0 \\
0 \\
0\end{array}$ & $\begin{array}{r}11 \\
\text { (s) } \\
0 \\
-5 \\
5 \\
(s) \\
(s) \\
-1 \\
1 \\
(s) \\
-5 \\
-1 \\
-4 \\
2 \\
0 \\
0 \\
0 \\
(s) \\
2 \\
12 \\
0 \\
(s)\end{array}$ & $\begin{array}{l}- \\
- \\
- \\
- \\
- \\
- \\
- \\
- \\
- \\
- \\
- \\
- \\
- \\
- \\
- \\
- \\
- \\
- \\
- \\
-\end{array}$ & $\begin{array}{l}- \\
- \\
- \\
- \\
- \\
- \\
- \\
- \\
- \\
- \\
- \\
- \\
- \\
- \\
- \\
- \\
- \\
-\end{array}$ & $\begin{array}{r}(s) \\
(s) \\
0 \\
(s) \\
(s) \\
0 \\
0 \\
0 \\
0 \\
0 \\
0 \\
0 \\
0 \\
0 \\
0 \\
(s) \\
(s) \\
0 \\
0 \\
\text { (s) } \\
0 \\
\text { (s) }\end{array}$ & $\begin{array}{r}483 \\
234 \\
0 \\
39 \\
195 \\
1 \\
53 \\
5 \\
48 \\
2 \\
128 \\
94 \\
34 \\
10 \\
1 \\
(s) \\
(5) \\
(s) \\
14 \\
19 \\
20 \\
3\end{array}$ \\
\hline 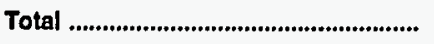 & 523 & 481 & 152 & 37 & -174 & 26 & $\mathbf{0}$ & 461 & (s) & 531 \\
\hline
\end{tabular}

a Represents the PAD District in which the material entered the United States and not necessarily where the crude oil or product is processed and/or consumed.

b Unaccounted for crude oil represents the difference between the supply and disposition of crude oil.

c A negative number indicates a decrease in stocks and a positive number indicates an increase in stocks.

d Products supplied is equal to field production, plus refinery production, plus imports, plus unaccounted for crude oil, plus net receipts, minus stock change, minus crude losses, minus refinery inputs, minus exports.

$\theta$ Includes naphtha less than $401^{\circ} \mathrm{F}$ endpoint and other oils equal to or greater than $401^{\circ} \mathrm{F}$ endpoint.

(s) = Less than 500 barrels per day.

$E=$ Estimated.

$\mathrm{LRG}=$ Liquefied Refinery Gas.

Note: Totals may not equal sum of components due to independent rounding.

Sources: • Energy Information Administration (EIA) Forms ElA-810, "Monthly Refinery Report," ElA-811, "Monthly Bulk Terminal Report," ElA-812, "Monthly Product Pipeline Report," ElA-813, "Monthly Crude Oil Report," ElA-814, "Monthly Imports Report," ElA-816, "Monthly Natural Gas Liquids Report," ElA-817, "Monthly Tanker and Barge Movement Report," and EIA-819M, "Monthly Oxygenate Telephone Report". - Domestic crude oil production estimates based on hlstorical statistics from State conservation agencies and the Minerals Management Service of the U.S. Department of the Interior. $\bullet$ Export data from the Bureau of the Census and Form EIA-810, "Monthly Refinery Report." 
Table 22. PAD District V-Supply, Disposition, and Ending Stocks of Crude Oil and Petroleum Products, March 1995

(Thousand Barrels)

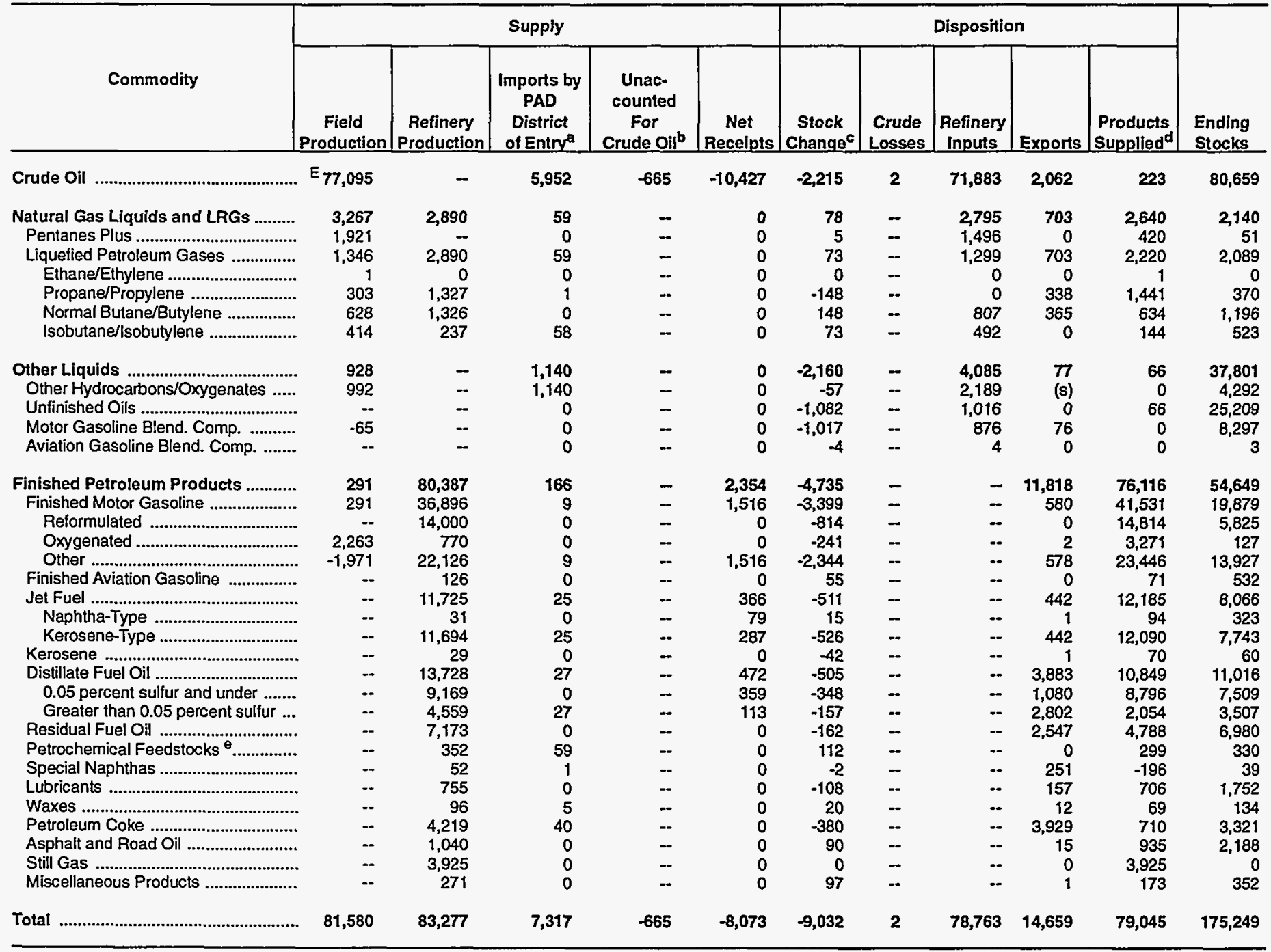

a Represents the PAD District in which the material entered the United States and not necessarily where the crude oil or product is processed and/or consumed.

b Unaccounted for crude oil represents the difference between the supply and disposition of crude oil.

c A negative number indicates a decrease in stocks and a positive number indicates an increase in stocks.

d Products supplied is equal to field production, plus refinery production, plus imports, plus unaccounted for crude oil, plus net receipts, minus stock change, minus crude losses, minus refinery inputs, minus exports.

- Includes naphtha less than $401^{\circ} \mathrm{F}$ endpoint and other oils equal to or greater than $401^{\circ} \mathrm{F}$ endpoint.

(s) $=$ Less than 500 barrels.

$E=$ Estimated.

LRG = Liquefied Refinery Gas.

Note: Totals may not equal sum of components due to independent rounding.

Sources: - Energy Information Administration (EIA) Forms ElA-810, "Monthly Refinery Report," ElA-811, "Monthly Bulk Terminal Report," EIA-812, "Monthly Product Pipeline Report," EIA-813, "Monthly Crude Oil Report," EIA-814, "Monthly Imports Report," ElA-816, "Monthly Natural Gas Liquids Report," ElA-817, "Monthly Tanker and Barge Movement Report," and EIA-819M, "Monthly Oxygenate Telephone Report". - Domestic crude oil production estimates based on historical statistics from State conservation agencies and the Minerals Management Service of the U.S. Department of the Interior. - Export data from the Bureau of the Census and Form ElA-810, "Monthly Refinery Report." 
Table 23. PAD District V-Year-to-Date Supply, Disposition, and Ending Stocks of Crude Oil and Petroleum Products, January-March 1995

(Thousand Barrels)

\begin{tabular}{|c|c|c|c|c|c|c|c|c|c|c|c|}
\hline \multirow[b]{2}{*}{ Commodity } & \multicolumn{5}{|c|}{ Supply } & \multicolumn{5}{|c|}{ Disposition } & \multirow[b]{2}{*}{$\begin{array}{l}\text { Ending } \\
\text { Stocks }\end{array}$} \\
\hline & $\begin{array}{c}\text { Field } \\
\text { Production }\end{array}$ & $\begin{array}{c}\text { Refinery } \\
\text { Production }\end{array}$ & $\begin{array}{c}\text { Imports by } \\
\text { PAD } \\
\text { District } \\
\text { of Entry }\end{array}$ & $\begin{array}{l}\text { Unac- } \\
\text { counted } \\
\text { For } \\
\text { Crude Oilb } \\
\end{array}$ & $\begin{array}{c}\text { Net } \\
\text { Receipts }\end{array}$ & $\begin{array}{c}\text { Stock } \\
\text { Change }^{c}\end{array}$ & $\begin{array}{c}\text { Crude } \\
\text { Losses } \\
\end{array}$ & $\begin{array}{c}\text { Refinery } \\
\text { Inputs }\end{array}$ & Exports & $\begin{array}{l}\text { Products } \\
\text { Supplied }\end{array}$ & \\
\hline 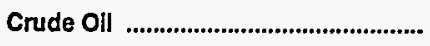 & $E_{227,810}$ & - & 24,031 & -589 & $-27,941$ & 2,263 & 2 & 212,156 & 8,207 & 683 & 80,659 \\
\hline $\begin{array}{l}\text { Natural Gas Liquids and LRGs .......... } \\
\text { Pentanes Plus .................................... } \\
\text { Llquefled Petroleum Gases ................ } \\
\text { Ethane/Ethylene ............................. } \\
\text { Propane/Propylene ......................... } \\
\text { Normal Butane/Butylene ................ } \\
\text { Isobutane/lsobutylene ................... }\end{array}$ & $\begin{array}{r}9,630 \\
5,598 \\
4,032 \\
4 \\
899 \\
1,936 \\
1,193\end{array}$ & $\begin{array}{r}6,461 \\
- \\
6,461 \\
0 \\
3,858 \\
2,371 \\
232\end{array}$ & $\begin{array}{r}500 \\
0 \\
500 \\
0 \\
8 \\
0 \\
492\end{array}$ & $\begin{array}{l}- \\
- \\
- \\
- \\
-\end{array}$ & $\begin{array}{l}0 \\
0 \\
0 \\
0 \\
0 \\
0 \\
0\end{array}$ & $\begin{array}{r}-954 \\
-26 \\
-928 \\
0 \\
-772 \\
-212 \\
56\end{array}$ & $\begin{array}{l}- \\
- \\
- \\
- \\
-\end{array}$ & $\begin{array}{r}8,434 \\
4,370 \\
4,064 \\
0 \\
0 \\
2,747 \\
1,317\end{array}$ & $\begin{array}{r}1,765 \\
(s) \\
1,764 \\
0 \\
1,102 \\
662 \\
0\end{array}$ & $\begin{array}{r}7,346 \\
1,254 \\
6,093 \\
4 \\
4,435 \\
1,110 \\
544\end{array}$ & $\begin{array}{r}2,140 \\
51 \\
2,089 \\
0 \\
370 \\
1,196 \\
523\end{array}$ \\
\hline $\begin{array}{l}\text { Other Liquids ...................................... } \\
\text { Other Hydrocarbons/Oxygenates ...... } \\
\text { Unfinished Olls ................................... } \\
\text { Motor Gasoline Blend. Comp. .......... } \\
\text { Aviation Gasoline Blend. Comp. ........ }\end{array}$ & $\begin{array}{r}3,027 \\
3,986 \\
-960 \\
-\end{array}$ & $\begin{array}{l}- \\
- \\
-\end{array}$ & $\begin{array}{r}3,442 \\
2,480 \\
962 \\
0 \\
0\end{array}$ & $\begin{array}{l}- \\
- \\
-\end{array}$ & $\begin{array}{l}0 \\
0 \\
0 \\
0 \\
0\end{array}$ & $\begin{array}{r}3,376 \\
-413 \\
3,101 \\
687 \\
1\end{array}$ & $\begin{array}{l}- \\
- \\
-\end{array}$ & $\begin{array}{r}2,972 \\
6,878 \\
-2,132 \\
-1,773 \\
-1\end{array}$ & $\begin{array}{r}128 \\
1 \\
0 \\
126 \\
0\end{array}$ & $\begin{array}{r}-7 \\
0 \\
-7 \\
0 \\
0\end{array}$ & $\begin{array}{r}37,801 \\
4,292 \\
25,209 \\
8,297 \\
3\end{array}$ \\
\hline 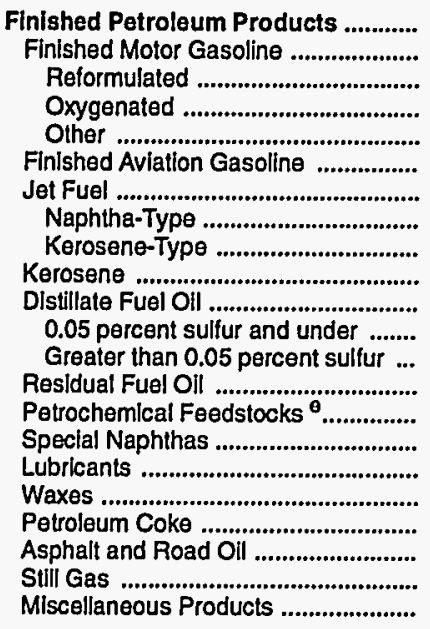 & $\begin{array}{r}1,552 \\
1,552 \\
-- \\
5,926 \\
-4,373 \\
- \\
-- \\
- \\
- \\
- \\
- \\
- \\
- \\
- \\
- \\
- \\
- \\
- \\
- \\
- \\
-\end{array}$ & $\begin{array}{r}229,892 \\
105,437 \\
39,065 \\
8,243 \\
58,129 \\
254 \\
34,548 \\
61 \\
34,487 \\
227 \\
36,956 \\
25,052 \\
11,904 \\
22,226 \\
1,277 \\
149 \\
2,287 \\
220 \\
12,201 \\
2,434 \\
11,079 \\
597\end{array}$ & $\begin{array}{r}1,008 \\
54 \\
0 \\
0 \\
54 \\
2 \\
624 \\
0 \\
624 \\
2 \\
92 \\
0 \\
92 \\
35 \\
59 \\
3 \\
0 \\
17 \\
111 \\
9 \\
0 \\
0\end{array}$ & $\begin{array}{l}- \\
- \\
- \\
- \\
- \\
- \\
- \\
- \\
- \\
- \\
- \\
- \\
- \\
- \\
- \\
- \\
- \\
- \\
- \\
-\end{array}$ & $\begin{array}{r}7,954 \\
5,188 \\
0 \\
0 \\
5,188 \\
0 \\
1,197 \\
180 \\
1,017 \\
0 \\
1,569 \\
1,129 \\
440 \\
0 \\
0 \\
0 \\
0 \\
0 \\
0 \\
0 \\
0 \\
0\end{array}$ & $\begin{array}{r}-5,268 \\
-3,894 \\
-1,347 \\
-3,418 \\
871 \\
58 \\
-136 \\
-7 \\
-129 \\
-19 \\
-1,727 \\
-730 \\
-997 \\
471 \\
77 \\
3 \\
-74 \\
12 \\
-414 \\
216 \\
0 \\
159\end{array}$ & $\begin{array}{l}- \\
- \\
- \\
- \\
- \\
- \\
- \\
- \\
- \\
- \\
- \\
- \\
- \\
- \\
- \\
-\end{array}$ & $\begin{array}{l}- \\
- \\
- \\
- \\
- \\
- \\
- \\
- \\
- \\
- \\
- \\
- \\
- \\
- \\
- \\
- \\
-\end{array}$ & $\begin{array}{r}28,822 \\
947 \\
0 \\
10 \\
937 \\
0 \\
1,002 \\
1 \\
1,001 \\
3 \\
9,887 \\
2,356 \\
7,531 \\
6,364 \\
0 \\
1,745 \\
319 \\
36 \\
8,473 \\
45 \\
0 \\
3\end{array}$ & $\begin{array}{r}216,852 \\
115,179 \\
40,412 \\
17,577 \\
57,190 \\
198 \\
35,503 \\
247 \\
35,256 \\
245 \\
30,457 \\
24,555 \\
5,902 \\
15,426 \\
1,259 \\
-1,596 \\
2,042 \\
189 \\
4,253 \\
2,182 \\
11,079 \\
435\end{array}$ & $\begin{array}{r}54,649 \\
19,879 \\
5,825 \\
127 \\
13,927 \\
532 \\
8,066 \\
323 \\
7,743 \\
60 \\
11,016 \\
7,509 \\
3,507 \\
6,980 \\
330 \\
39 \\
1,752 \\
134 \\
3,321 \\
2,188 \\
0 \\
352\end{array}$ \\
\hline Total & 242,019 & 236,353 & 28,981 & -589 & $-19,987$ & -583 & 2 & 223,562 & 38,922 & 224,874 & 175,249 \\
\hline
\end{tabular}

a Represents the PAD District in which the material entered the United States and not necessarily where the crude oil or product is processed and/or consumed.

b Unaccounted for crude oil represents the difference between the supply and disposition of crude oil.

c A negative number indicates a decrease in stocks and a positive number indicates an increase in stocks.

d Products supplied is equal to field production, plus refinery production, plus imports, plus unaccounted for crude oil, plus net receipts, minus stock change, minus crude losses, minus refinery inputs, minus exports.

${ }^{\circ}$ Includes naphtha less than $401^{\circ} \mathrm{F}$ endpoint and other oils equal to or greater than $401^{\circ} \mathrm{F}$ endpoint.

(s) = Less than 500 barrels.

$E=$ Estimated.

$L R G=$ Liquefied Refinery Gas.

Note: Totals may not equal sum of components due to independent rounding.

Sources: - Energy Information Administration (EIA) Forms EIA-810, "Monthly Refinery Report," EIA-811, "Monthly Bulk Terminal Report," EIA-812, "Monthly Product Pipeline Report," ElA-813, "Monthly Crude Oil Report," ElA-814, "Monthly Imports Report," ElA-816, "Monthly Natural Gas Liquids Report," EIA-817, "Monthly Tanker and Barge Movement Report," and EIA-819M, "Monthly Oxygenate Telephone Report". - Domestic crude oil production estimates based on historical statistics from State conservation agencles and the Minerals Management Service of the U.S. Department of the Interior. • Export data from the Bureau of the Census and Form ElA-810, "Monthly Refinery Report." 
Table 24. PAD District V - Daily Average Supply and Disposition of Crude Oil and Petroleum Products, March 1995

(Thousand Barrels per Day)

\begin{tabular}{|c|c|c|c|c|c|c|c|c|c|c|}
\hline \multirow[b]{2}{*}{ Commodity } & \multicolumn{5}{|c|}{ Supply } & \multicolumn{5}{|c|}{ Disposition } \\
\hline & $\begin{array}{c}\text { Field } \\
\text { Production }\end{array}$ & $\begin{array}{c}\text { Refinery } \\
\text { Production }\end{array}$ & $\begin{array}{l}\text { Imports by } \\
\text { PAD } \\
\text { District } \\
\text { of Entry }\end{array}$ & $\begin{array}{l}\text { Unac- } \\
\text { counted } \\
\text { For } \\
\text { Crude Oil } \\
\end{array}$ & $\begin{array}{c}\text { Net } \\
\text { Receipts }\end{array}$ & $\begin{array}{c}\text { Stock } \\
\text { Change }\end{array}$ & $\begin{array}{c}\text { Crude } \\
\text { Losses }\end{array}$ & $\begin{array}{c}\text { Refinery } \\
\text { Inputs }\end{array}$ & Exports & $\begin{array}{l}\text { Products } \\
\text { Supplied }^{\mathrm{d}}\end{array}$ \\
\hline 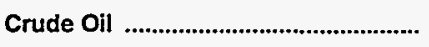 & $E_{2,487}$ & - & 192 & -21 & -336 & -71 & (s) & 2,319 & 67 & 7 \\
\hline $\begin{array}{l}\text { Natural Gas Liquids and LRGs ......... } \\
\text { Pentanes Plus ................................. } \\
\text { Liquefied Petroleum Gases .............. } \\
\text { Ethane/Ethylene ........................... } \\
\text { Propane/Propylene ........................ } \\
\text { Normal Butane/Butylene ................ } \\
\text { Isobutane/Isobutylene ..................... }\end{array}$ & $\begin{array}{r}105 \\
62 \\
43 \\
(s) \\
10 \\
20 \\
13\end{array}$ & $\begin{array}{r}93 \\
\overline{93} \\
0 \\
43 \\
43 \\
8\end{array}$ & $\begin{array}{r}2 \\
0 \\
2 \\
0 \\
\text { (s) } \\
0 \\
2\end{array}$ & $\begin{array}{l}- \\
- \\
- \\
- \\
-\end{array}$ & $\begin{array}{l}0 \\
0 \\
0 \\
0 \\
0 \\
0 \\
0\end{array}$ & $\begin{array}{r}3 \\
(s) \\
2 \\
0 \\
-5 \\
5 \\
2\end{array}$ & $\begin{array}{l}- \\
\overline{-} \\
- \\
- \\
-\end{array}$ & $\begin{array}{r}90 \\
48 \\
42 \\
0 \\
0 \\
26 \\
16\end{array}$ & $\begin{array}{r}23 \\
0 \\
23 \\
0 \\
11 \\
12 \\
0\end{array}$ & $\begin{array}{r}85 \\
14 \\
72 \\
(s) \\
46 \\
20 \\
5\end{array}$ \\
\hline $\begin{array}{l}\text { Other Liquids ................................. } \\
\text { Other Hydrocarbons/Oxygenates ...... } \\
\text { Unfinished Oils ................................ } \\
\text { Motor Gasoline Blend. Comp. ........... } \\
\text { Aviation Gasoline Blend. Comp. ........ }\end{array}$ & $\begin{array}{r}30 \\
32 \\
-2 \\
-\end{array}$ & $\begin{array}{l}- \\
- \\
-\end{array}$ & $\begin{array}{r}37 \\
37 \\
0 \\
0 \\
0\end{array}$ & $\begin{array}{l}- \\
- \\
-\end{array}$ & $\begin{array}{l}0 \\
0 \\
0 \\
0 \\
0\end{array}$ & $\begin{array}{r}-70 \\
-2 \\
-35 \\
-33 \\
(s)\end{array}$ & $\begin{array}{l}- \\
- \\
-\end{array}$ & $\begin{array}{r}132 \\
71 \\
33 \\
28 \\
(s)\end{array}$ & $\begin{array}{r}2 \\
\text { (s) } \\
0 \\
2 \\
0\end{array}$ & $\begin{array}{l}2 \\
0 \\
2 \\
0 \\
0\end{array}$ \\
\hline 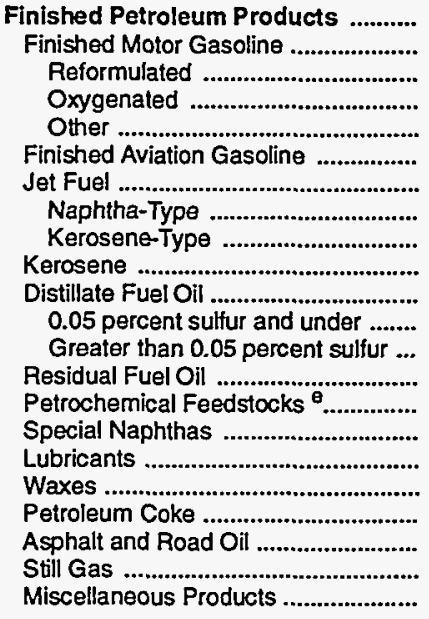 & $\begin{array}{r}9 \\
9 \\
73 \\
-64 \\
- \\
- \\
- \\
- \\
- \\
- \\
- \\
- \\
- \\
- \\
- \\
- \\
-\end{array}$ & $\begin{array}{r}2,593 \\
1,190 \\
452 \\
25 \\
714 \\
4 \\
378 \\
1 \\
377 \\
1 \\
443 \\
296 \\
147 \\
231 \\
11 \\
2 \\
24 \\
3 \\
136 \\
34 \\
127 \\
9\end{array}$ & $\begin{array}{r}5 \\
\text { (s) } \\
0 \\
0 \\
(\mathrm{~s}) \\
0 \\
1 \\
0 \\
1 \\
0 \\
1 \\
0 \\
1 \\
0 \\
2 \\
(\mathrm{~s}) \\
0 \\
\text { (s) } \\
1 \\
0 \\
0 \\
0\end{array}$ & $\begin{array}{l}- \\
- \\
- \\
- \\
- \\
- \\
- \\
- \\
- \\
- \\
- \\
- \\
- \\
- \\
- \\
- \\
- \\
- \\
- \\
-\end{array}$ & $\begin{array}{r}76 \\
49 \\
0 \\
0 \\
49 \\
0 \\
12 \\
3 \\
9 \\
0 \\
15 \\
12 \\
4 \\
0 \\
0 \\
0 \\
0 \\
0 \\
0 \\
0 \\
0 \\
0\end{array}$ & $\begin{array}{r}-153 \\
-110 \\
-26 \\
-8 \\
-76 \\
2 \\
-16 \\
(s) \\
-17 \\
-1 \\
-16 \\
-11 \\
-5 \\
-5 \\
4 \\
(s) \\
-3 \\
1 \\
-12 \\
3 \\
0 \\
3\end{array}$ & $\begin{array}{l}- \\
- \\
- \\
- \\
- \\
- \\
- \\
- \\
- \\
- \\
- \\
- \\
- \\
- \\
- \\
- \\
- \\
- \\
- \\
- \\
-\end{array}$ & $\begin{array}{l}- \\
- \\
- \\
- \\
- \\
- \\
- \\
- \\
- \\
- \\
- \\
- \\
- \\
- \\
- \\
- \\
- \\
- \\
- \\
- \\
-\end{array}$ & $\begin{array}{r}381 \\
19 \\
0 \\
(s) \\
19 \\
0 \\
14 \\
(s) \\
14 \\
(s) \\
125 \\
35 \\
90 \\
82 \\
0 \\
8 \\
5 \\
(s) \\
127 \\
\text { (s) } \\
0 \\
(s)\end{array}$ & $\begin{array}{r}2,455 \\
1,340 \\
478 \\
106 \\
756 \\
2 \\
393 \\
3 \\
390 \\
2 \\
350 \\
284 \\
66 \\
154 \\
10 \\
-6 \\
23 \\
2 \\
23 \\
30 \\
127 \\
6\end{array}$ \\
\hline Total & 2,632 & 2,686 & 236 & -21 & -260 & -291 & (s) & 2,541 & 473 & 2,550 \\
\hline
\end{tabular}

a Represents the PAD District in which the material entered the United States and not necessarily where the crude oil or product is processed and/or consumed.

b Unaccounted for crude oil represents the difference between the supply and disposition of crude oil.

c A negative number indicates a decrease in stocks and a positive number indicates an increase in stocks.

d Products supplied is equal to field production, plus refinery production, plus imports, plus unaccounted for crude oil, plus net receipts, minus stock change, minus crude losses, minus refinery inputs, minus exports.

- Includes naphtha less than $401^{\circ} \mathrm{F}$ endpoint and other oils equal to or greater than $401^{\circ} \mathrm{F}$ endpoint.

(s) = Less than 500 barrels per day.

$E=$ Estimated.

LRG = Liquefied Refinery Gas.

Note: Totals may not equal sum of components due to independent rounding.

Sources: - Energy Information Administration (EIA) Forms EIA-810, "Monthly Refinery Report," EIA-811, "Monthly Bulk Terminal Report," EIA-812, "Monthly Product Pipeline Report," EIA-813, "Monthly Crude Oil Report," ElA-814, "Monthly Imports Report," ElA-816, "Monthly Natural Gas Liquids Report,' ElA-817, 'Monthly Tanker and Barge Movement Report," and EIA-819M, "Monthly Oxygenate Telephone Report". - Domestic crude oil production estimates based on historical statistics from State conservation agencies and the Minerals Management Service of the U.S. Department of the interior. • Export data from the Bureau of the Census and Form ElA-810, "Monthly Refinery Report." 
Table 25. PAD District V - Year-to-Date Daily Average Supply and Disposition of Crude Oil and Petroleum Products, January-March 1995

(Thousand Barrels per Day)

\begin{tabular}{|c|c|c|c|c|c|c|c|c|c|c|}
\hline \multirow[b]{2}{*}{ Commodity } & \multicolumn{5}{|c|}{ Supply } & \multicolumn{5}{|c|}{ Disposition } \\
\hline & $\begin{array}{l}\text { Field } \\
\text { Production }\end{array}$ & $\begin{array}{c}\text { Refinery } \\
\text { Production }\end{array}$ & $\begin{array}{l}\text { Imports by } \\
\text { PAD } \\
\text { District } \\
\text { of Entry }\end{array}$ & $\begin{array}{l}\text { Unac- } \\
\text { counted } \\
\text { For } \\
\text { Crude Oil } \\
\end{array}$ & $\begin{array}{c}\text { Net } \\
\text { Receipts }\end{array}$ & $\begin{array}{c}\text { Stock } \\
\text { Change }\end{array}$ & $\begin{array}{c}\text { Crude } \\
\text { Losses }\end{array}$ & $\begin{array}{l}\text { Refinery } \\
\text { Inputs }\end{array}$ & Exports & $\begin{array}{l}\text { Products } \\
\text { Supplied }^{\text {d }}\end{array}$ \\
\hline 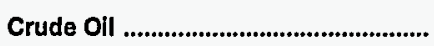 & $E_{2,531}$ & - & 267 & -7 & -310 & 25 & (s) & 2,357 & 91 & 8 \\
\hline $\begin{array}{l}\text { Natural Gas Liquids and LRGs ........... } \\
\text { Pentanes Plus ................................... } \\
\text { Liquefied Petroleum Gases ................ } \\
\text { Ethane/Ethylene ............................ } \\
\text { Propane/Propylene ........................... } \\
\text { Normal Butane/Butylene ............... } \\
\text { Isobutane/lsobutylene .................... }\end{array}$ & $\begin{array}{r}107 \\
62 \\
45 \\
(s) \\
10 \\
22 \\
13\end{array}$ & $\begin{array}{r}72 \\
- \\
72 \\
0 \\
43 \\
26 \\
3\end{array}$ & $\begin{array}{r}6 \\
0 \\
6 \\
0 \\
(s) \\
0 \\
5\end{array}$ & $\begin{array}{l}- \\
- \\
- \\
-\end{array}$ & $\begin{array}{l}0 \\
0 \\
0 \\
0 \\
0 \\
0 \\
0\end{array}$ & $\begin{array}{r}-11 \\
(s) \\
-10 \\
0 \\
-9 \\
-2 \\
1\end{array}$ & $\begin{array}{l}- \\
\overline{-} \\
\overline{-} \\
\overline{-}\end{array}$ & $\begin{array}{r}94 \\
49 \\
45 \\
0 \\
0 \\
31 \\
15\end{array}$ & $\begin{array}{r}20 \\
\text { (s) } \\
20 \\
0 \\
12 \\
7 \\
0\end{array}$ & $\begin{array}{r}82 \\
14 \\
68 \\
(s) \\
49 \\
12 \\
6\end{array}$ \\
\hline $\begin{array}{l}\text { Other Liquids ................................. } \\
\text { Other Hydrocarbons/Oxygenates ..... } \\
\text { Unfinished Olls ................................. } \\
\text { Motor Gasoline Blend. Comp. ............ } \\
\text { Avlation Gasoline Blend. Comp. ....... }\end{array}$ & $\begin{array}{r}34 \\
44 \\
-11 \\
-\end{array}$ & $\begin{array}{l}- \\
- \\
-\end{array}$ & $\begin{array}{r}38 \\
28 \\
11 \\
0 \\
0\end{array}$ & $\begin{array}{l}- \\
- \\
-\end{array}$ & $\begin{array}{l}0 \\
0 \\
0 \\
0 \\
0\end{array}$ & $\begin{array}{r}38 \\
-5 \\
34 \\
8 \\
(\mathrm{~s})\end{array}$ & $\begin{array}{l}- \\
- \\
-\end{array}$ & $\begin{array}{r}33 \\
76 \\
-24 \\
-20 \\
\text { (s) }\end{array}$ & $\begin{array}{r}1 \\
(s) \\
0 \\
1 \\
0\end{array}$ & $\begin{array}{r}\text { (s) } \\
0 \\
\text { (s) } \\
0 \\
0\end{array}$ \\
\hline 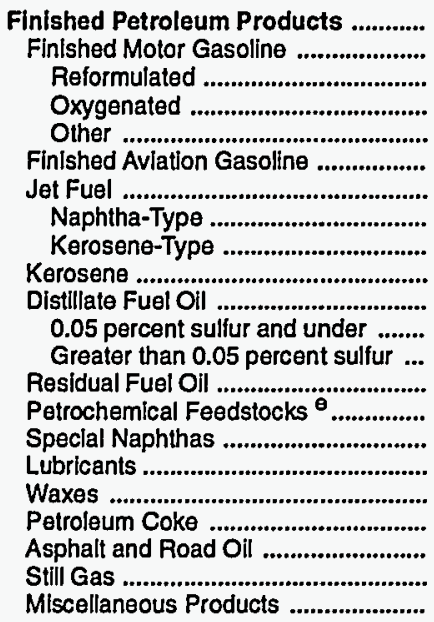 & $\begin{array}{r}17 \\
17 \\
- \\
-49 \\
- \\
- \\
- \\
- \\
- \\
- \\
- \\
- \\
- \\
- \\
-\end{array}$ & $\begin{array}{r}2,554 \\
1,172 \\
434 \\
92 \\
646 \\
3 \\
384 \\
1 \\
383 \\
3 \\
411 \\
278 \\
132 \\
247 \\
14 \\
2 \\
25 \\
2 \\
136 \\
27 \\
123 \\
7\end{array}$ & $\begin{array}{r}11 \\
1 \\
0 \\
0 \\
1 \\
(s) \\
7 \\
0 \\
7 \\
(s) \\
1 \\
0 \\
1 \\
(s) \\
1 \\
(s) \\
0 \\
(s) \\
1 \\
(s) \\
0 \\
0\end{array}$ & $\begin{array}{l}- \\
- \\
- \\
- \\
- \\
- \\
- \\
- \\
- \\
- \\
- \\
- \\
- \\
- \\
- \\
- \\
-\end{array}$ & $\begin{array}{r}88 \\
58 \\
0 \\
0 \\
58 \\
0 \\
13 \\
2 \\
11 \\
0 \\
17 \\
13 \\
5 \\
0 \\
0 \\
0 \\
0 \\
0 \\
0 \\
0 \\
0 \\
0\end{array}$ & $\begin{array}{r}-59 \\
-43 \\
-15 \\
-38 \\
10 \\
1 \\
-2 \\
(s) \\
-1 \\
(s) \\
-19 \\
-8 \\
-11 \\
5 \\
1 \\
(s) \\
-1 \\
(s) \\
-5 \\
2 \\
0 \\
2\end{array}$ & $\begin{array}{l}- \\
- \\
- \\
- \\
- \\
- \\
- \\
- \\
- \\
- \\
- \\
- \\
- \\
- \\
- \\
- \\
- \\
-\end{array}$ & $\begin{array}{l}- \\
- \\
- \\
- \\
- \\
- \\
- \\
- \\
- \\
- \\
- \\
- \\
- \\
- \\
- \\
- \\
- \\
-\end{array}$ & $\begin{array}{r}320 \\
11 \\
0 \\
(s) \\
10 \\
0 \\
11 \\
(s) \\
11 \\
(s) \\
110 \\
26 \\
84 \\
71 \\
0 \\
19 \\
4 \\
(s) \\
94 \\
1 \\
0 \\
(s)\end{array}$ & $\begin{array}{r}2,409 \\
1,280 \\
449 \\
195 \\
635 \\
2 \\
394 \\
3 \\
392 \\
3 \\
338 \\
273 \\
66 \\
171 \\
14 \\
-18 \\
23 \\
2 \\
47 \\
24 \\
123 \\
5\end{array}$ \\
\hline Total & 2,689 & 2,626 & 322 & -7 & -222 & -6 & (s) & 2,484 & 432 & 2,499 \\
\hline
\end{tabular}

Represents the PAD District in which the material entered the United States and not necessarily where the crude oil or product is processed and/or consumed.

b Unaccounted for crude oil represents the difference between the supply and disposition of crude oil.

c A negative number indicates a decrease in stocks and a positive number indicates an increase in stocks.

d Products supplied is equal to field production, plus refinery production, plus imports, plus unaccounted for crude oil, plus net receipts, minus stock change, minus crude losses, minus refinery inputs, minus exports.

- Includes naphtha less than $401^{\circ} \mathrm{F}$ endpoint and other oils equal to or greater than $401^{\circ} \mathrm{F}$ endpoint.

(s) = Less than 500 barrels per day.

$E$ = Estimated.

LRG $=$ Liquefied Refinery Gas.

Note: Totals may not equal sum of components due to independent rounding.

Sources: • Energy Information Administration (EIA) Forms EIA-810, "Monthly Refinery Report"" ElA-811, "Monthly Bulk Terminal Report" ElA-812, "Monthly Product Pipellne Report," EIA-813, "Monthly Crude Oil Report," EIA-814, "Monthly Imports Report," EIA-816, "Monthly Natural Gas Liquids Report," EIA-817, "Monthly Tanker and Barge Movement Report," and EIA-819M, "Monthly Oxygenate Telephone Report". - Domestic crude oil production estimates based on historical statistics from State conservation agencles and the Minerals Management Service of the U.S. Department of the linterior. - Export data from the Bureau of the Census and Form EIA-810, "Monthly Refinery Report." 
Table 26. Production of Crude Oil by PAD District and State (Thousand Barrels)

\begin{tabular}{|c|c|c|}
\hline \multirow{2}{*}{ PAD District and State } & \multicolumn{2}{|c|}{ January 1995} \\
\hline & Total & $\begin{array}{c}\text { Daily } \\
\text { Average }\end{array}$ \\
\hline $\begin{array}{l}\text { PAD District I } \\
\text { Florida } \\
\text { New York } \\
\text { Pennsylvania } \\
\text { Virginia . } \\
\text { West Virginia } \\
\text { Adjustment }\end{array}$ & $\begin{array}{r}E_{820} \\
E_{282} \\
E_{157} \\
1 \\
156 \\
(s)\end{array}$ & $\begin{array}{r}E_{16} \\
E_{1} \\
E_{5} \\
(s) \\
5 \\
(s)\end{array}$ \\
\hline 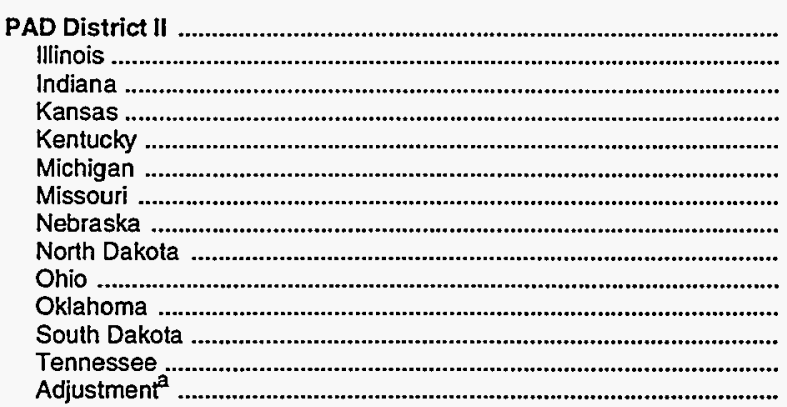 & $\begin{array}{r}17,533 \\
1,420 \\
389 \\
3,812 \\
332 \\
E_{1,000} \\
9 \\
339 \\
E_{2,350} \\
664 \\
7,378 \\
124 \\
32 \\
-316\end{array}$ & $\begin{array}{r}E_{566} \\
46 \\
13 \\
123 \\
11 \\
\text { E } 32 \\
(s) \\
11 \\
\text { E } 76 \\
\text { E } 21 \\
238 \\
4 \\
1 \\
-10\end{array}$ \\
\hline 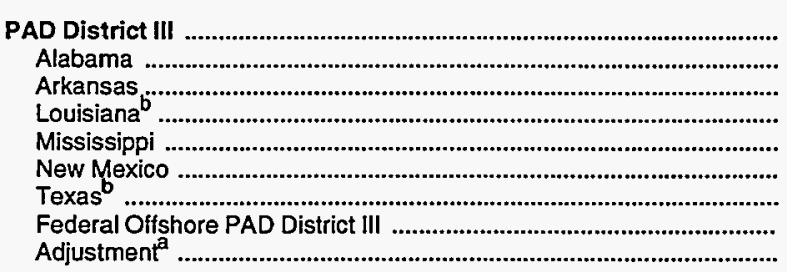 & $\begin{array}{r}E_{95,373} \\
1,612 \\
E_{164} \\
10,618 \\
1,657 \\
5,452 \\
48,568 \\
28,117 \\
-1,414\end{array}$ & $\begin{array}{r}E_{3,077} \\
52 \\
E_{25} \\
343 \\
53 \\
E_{176} \\
1,567 \\
907 \\
-46\end{array}$ \\
\hline 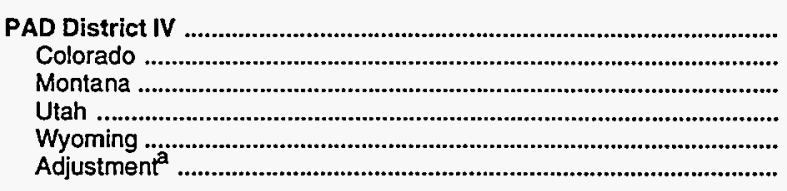 & $\begin{array}{r}E_{11,646} \\
2,204 \\
1,300 \\
1,730 \\
6,544 \\
-132\end{array}$ & $\begin{array}{r}\text { E }_{376} \\
\text { E }_{71} \\
42 \\
56 \\
211 \\
-4\end{array}$ \\
\hline $\begin{array}{l}\text { PAD District V } \\
\text { Alaska } \\
\text { South Alaska } \\
\text { North Slope ... } \\
\text { Adjustment for Alaska }{ }^{\mathrm{a}} \\
\text { Arizona } \\
\text { California } \\
\text { Fevada } \\
\text { Adjustment excluding Alaska }\end{array}$ & $\begin{array}{r}E 79,117 \\
48,819 \\
1,316 \\
47,502 \\
(s) \\
4 \\
23,639 \\
133 \\
5,926 \\
595\end{array}$ & $\begin{array}{r}E_{1,552} \\
42 \\
1,532 \\
(s) \\
(s) \\
763 \\
4 \\
191 \\
19\end{array}$ \\
\hline U.S. Total ${ }^{\mathrm{b}}$. & $E_{204,489}$ & $E_{6,596}$ \\
\hline
\end{tabular}

a These adjustments are used to reconcile the national and PAD District level sums of the State data with the independently estimated U.S. and Alaskan figures shown in the Summary Statistics portion of this issue and with the PAD District level figures published in a previous issue. Revised data at the State, PAD District, and national levels will be published without adjustments in the Petroleum Supply Annual.

$\mathrm{b}$ Includes the following current month offshore production (thousand barrels): Alaska: State - 7,997; California: State - 1,620; Louisiana: State - E1,856; Texas: State -122; U.S. Total, including Federal offishore - E45,639.

(s) = Less than 500 barrels or less than 500 barrels per day.

$E=$ Estimated.

Note: Totals may not equal sum of components due to independent rounding.

Sources: State government agencies, U.S. Department of the Interior, Minerals Management Service and the Conservation Committee of California Oil Producers. 
Table 27. Natural Gas Plant Net Production and Stocks of Petroleum Products by PAD and Refining Districts, March 1995

(Thousand Barrels)

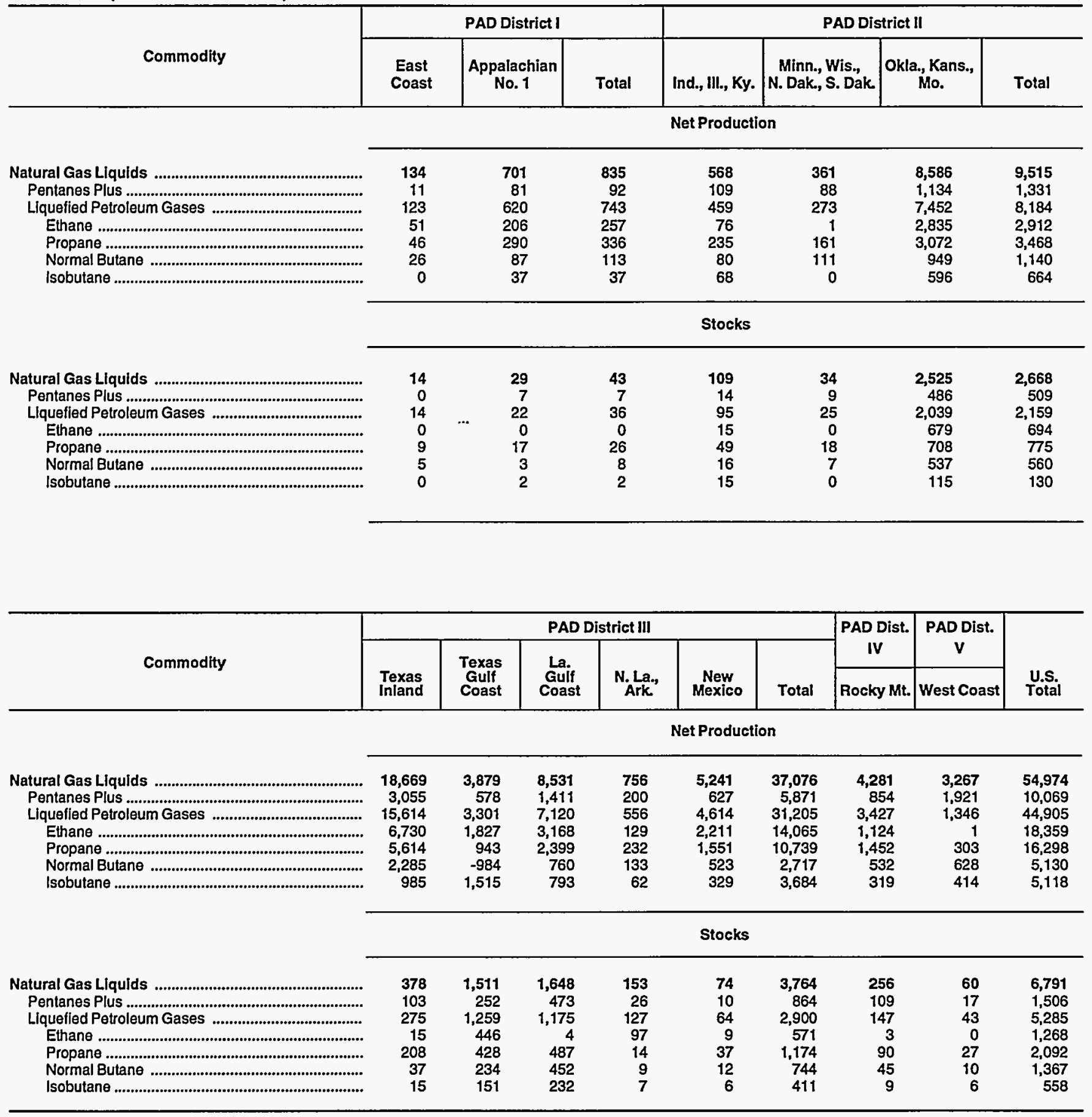

Note: Refer to Appendix A for Refining District descriptions.

Source: Energy Information Administration (EIA) Form EIA-816, "Monthly Natural Gas Liquids Report." 
Table 28. Refinery Input of Crude Oil and Petroleum Products by PAD and Refining Districts, March 1995

(Thousand Barrels, Except Where Noted)

\begin{tabular}{|c|c|c|c|c|c|c|c|}
\hline \multirow[b]{2}{*}{ Commodity } & \multicolumn{3}{|c|}{ PAD District I } & \multicolumn{4}{|c|}{ PAD District II } \\
\hline & $\begin{array}{c}\text { East } \\
\text { Coast }\end{array}$ & $\begin{array}{c}\text { Appalachian } \\
\text { No.1 }\end{array}$ & Total & Ind., III., Ky. & $\begin{array}{l}\text { Minn., Wis., } \\
\text { N. Dak,, S. Dak. }\end{array}$ & $\begin{array}{c}\text { Okla., Kans., } \\
\text { Mo. }\end{array}$ & Total \\
\hline Crude Oil & 43,981 & 2,726 & 46,707 & 60,621 & 11,473 & 17,908 & 90,002 \\
\hline $\begin{array}{l}\text { Natural Gas Liquids } \\
\text { Pentanes Plus } \\
\text { Liquefied Petroleum Gases } \\
\text { Ethane } \\
\text { Propane } \\
\text { Normal Butane } \\
\text { Isobutane }\end{array}$ & $\begin{array}{r}365 \\
0 \\
365 \\
0 \\
0 \\
238 \\
127\end{array}$ & $\begin{array}{l}0 \\
0 \\
0 \\
0 \\
0 \\
0 \\
0\end{array}$ & $\begin{array}{r}365 \\
0 \\
365 \\
0 \\
0 \\
238 \\
127\end{array}$ & $\begin{array}{r}1,997 \\
503 \\
1,494 \\
0 \\
2 \\
427 \\
1,065\end{array}$ & $\begin{array}{r}195 \\
80 \\
115 \\
0 \\
0 \\
52 \\
63\end{array}$ & $\begin{array}{r}785 \\
517 \\
268 \\
0 \\
0 \\
106 \\
162\end{array}$ & $\begin{array}{r}2,977 \\
1,100 \\
1,877 \\
0 \\
2 \\
585 \\
1,290\end{array}$ \\
\hline 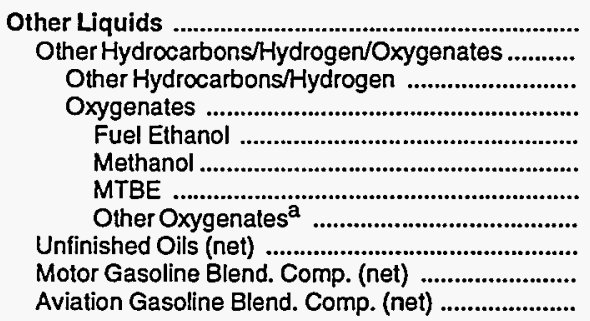 & $\begin{array}{r}5,619 \\
2,038 \\
22 \\
W \\
W \\
W \\
W \\
W \\
W \\
3,457 \\
227 \\
-103\end{array}$ & $\begin{array}{r}-43 \\
4 \\
0 \\
W \\
W \\
W \\
W \\
W \\
-61 \\
14 \\
0\end{array}$ & $\begin{array}{r}5,576 \\
2,042 \\
22 \\
2,020 \\
W \\
W \\
1,936 \\
W \\
3,396 \\
241 \\
-103\end{array}$ & $\begin{array}{r}1,845 \\
525 \\
26 \\
499 \\
W \\
W \\
W \\
W \\
931 \\
364 \\
25\end{array}$ & $\begin{array}{r}-119 \\
147 \\
0 \\
147 \\
W \\
W \\
W \\
W \\
-48 \\
-218 \\
0\end{array}$ & $\begin{array}{r}382 \\
101 \\
26 \\
75 \\
W \\
W \\
W \\
W \\
156 \\
125 \\
0\end{array}$ & $\begin{array}{r}2,108 \\
773 \\
52 \\
721 \\
448 \\
W \\
W \\
W \\
W \\
1,039 \\
271 \\
25\end{array}$ \\
\hline Total Input to Refineries & 49,965 & 2,683 & 52,648 & 64,463 & 11,549 & 19,075 & 95,087 \\
\hline 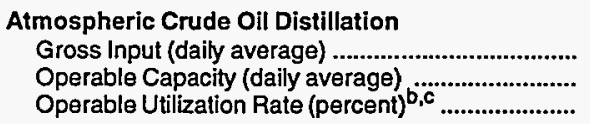 & $\begin{array}{r}1,375 \\
1,475 \\
93.3\end{array}$ & $\begin{array}{r}88 \\
97 \\
90.8\end{array}$ & $\begin{array}{r}1,464 \\
1,572 \\
93.1\end{array}$ & $\begin{array}{r}1,981 \\
2,356 \\
84.1\end{array}$ & $\begin{array}{r}370 \\
388 \\
95.3\end{array}$ & $\begin{array}{r}589 \\
703 \\
83.8\end{array}$ & $\begin{array}{r}2,940 \\
3,447 \\
85.3\end{array}$ \\
\hline 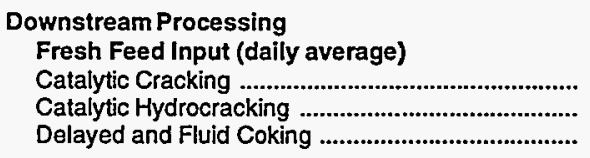 & $\begin{array}{r}591 \\
66 \\
83\end{array}$ & $\begin{array}{r}14 \\
4 \\
0\end{array}$ & $\begin{array}{r}605 \\
70 \\
83\end{array}$ & $\begin{array}{l}737 \\
100 \\
165\end{array}$ & $\begin{array}{r}75 \\
0 \\
36\end{array}$ & $\begin{array}{r}177 \\
6 \\
63\end{array}$ & $\begin{array}{l}988 \\
105 \\
263\end{array}$ \\
\hline $\begin{array}{l}\text { Crude Oil Qualities } \\
\text { Sulfur Content, Weighted Average (percent) .......... } \\
\text { API Gravity, Weighted Average (degrees) .............. }\end{array}$ & $\begin{array}{r}0.97 \\
31.38\end{array}$ & $\begin{array}{r}1.03 \\
37.19\end{array}$ & $\begin{array}{r}0.97 \\
31.73\end{array}$ & $\begin{array}{r}0.94 \\
35.02\end{array}$ & $\begin{array}{r}1.88 \\
33.34\end{array}$ & $\begin{array}{r}0.64 \\
36.23\end{array}$ & $\begin{array}{r}1.00 \\
35.06\end{array}$ \\
\hline $\begin{array}{l}\text { Operable Capacity (daily average) } \\
\text { Operating } \\
\text { Idle }\end{array}$ & $\begin{array}{r}1,475 \\
1,475 \\
0\end{array}$ & $\begin{array}{r}97 \\
97 \\
0\end{array}$ & $\begin{array}{r}1,572 \\
1,572 \\
0\end{array}$ & $\begin{array}{r}2,356 \\
2,330 \\
26\end{array}$ & $\begin{array}{r}388 \\
388 \\
0\end{array}$ & $\begin{array}{r}703 \\
698 \\
5\end{array}$ & $\begin{array}{r}3,447 \\
3,417 \\
31\end{array}$ \\
\hline 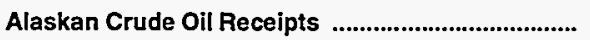 & 0 & 0 & 0 & 916 & 0 & 0 & 916 \\
\hline
\end{tabular}

See footnotes at end of table. 
Table 28. Refinery Input of Crude Oil and Petroleum Products by PAD and Refining Districts, March 1995 (Continued)

(Thousand Barrels, Except Where Noted)

\begin{tabular}{|c|c|c|c|c|c|c|c|c|c|}
\hline \multirow[b]{2}{*}{ Commodity } & \multicolumn{6}{|c|}{ PAD District Ill } & \multirow{2}{*}{\begin{tabular}{|c|}
$\begin{array}{c}\text { PAD Dist. } \\
\text { IV }\end{array}$ \\
Rocky Mt.
\end{tabular}} & \multirow{2}{*}{\begin{tabular}{|c|}
$\begin{array}{c}\text { PAD Dist. } \\
V\end{array}$ \\
West Coast
\end{tabular}} & \multirow[b]{2}{*}{$\begin{array}{l}\text { U.S. } \\
\text { Total }\end{array}$} \\
\hline & $\begin{array}{l}\text { Texas } \\
\text { Inland }\end{array}$ & $\begin{array}{l}\text { Texas } \\
\text { Gulf } \\
\text { Coast }\end{array}$ & $\begin{array}{c}\text { La. } \\
\text { Coast }\end{array}$ & $\begin{array}{c}\text { N. La., } \\
\text { Ark. }\end{array}$ & $\begin{array}{c}\text { New } \\
\text { Mexico }\end{array}$ & Total & & & \\
\hline 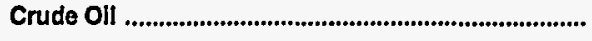 & 15,816 & 94,263 & 76,539 & 5,592 & 2,952 & 195,162 & 14,074 & 71,883 & 417,828 \\
\hline 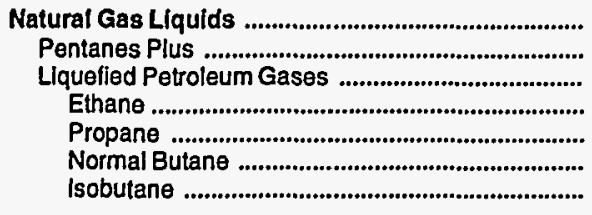 & $\begin{array}{r}1,262 \\
550 \\
712 \\
0 \\
0 \\
557 \\
155\end{array}$ & $\begin{array}{r}2,809 \\
1,359 \\
1,450 \\
0 \\
0 \\
330 \\
1,120\end{array}$ & $\begin{array}{r}2,089 \\
529 \\
1,560 \\
0 \\
0 \\
509 \\
1,051\end{array}$ & $\begin{array}{r}201 \\
159 \\
42 \\
0 \\
0 \\
0 \\
42\end{array}$ & $\begin{array}{r}231 \\
117 \\
114 \\
0 \\
0 \\
1 \\
113\end{array}$ & $\begin{array}{r}6,592 \\
2,714 \\
3,878 \\
0 \\
0 \\
1,397 \\
2,481\end{array}$ & $\begin{array}{r}352 \\
92 \\
260 \\
0 \\
0 \\
166 \\
94\end{array}$ & $\begin{array}{r}2,795 \\
1,496 \\
1,299 \\
0 \\
0 \\
807 \\
492\end{array}$ & $\begin{array}{r}13,081 \\
5,402 \\
7,679 \\
0 \\
2 \\
3,193 \\
4,484\end{array}$ \\
\hline 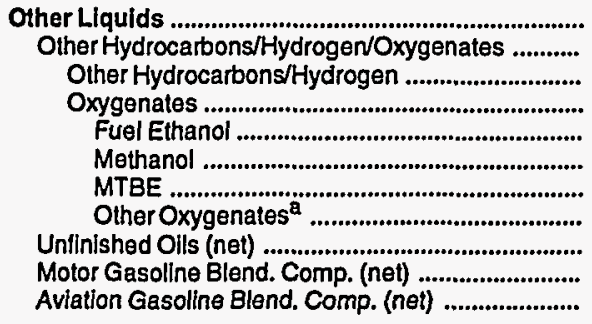 & $\begin{array}{r}-104 \\
135 \\
82 \\
53 \\
W \\
W \\
W \\
W \\
-303 \\
64 \\
0\end{array}$ & $\begin{array}{r}9,035 \\
1,807 \\
179 \\
1,628 \\
W \\
W \\
1,367 \\
W \\
7,793 \\
-565 \\
0\end{array}$ & $\begin{array}{r}1,008 \\
1,005 \\
449 \\
556 \\
W \\
W \\
W \\
W \\
612 \\
-612 \\
3\end{array}$ & $\begin{array}{r}-12 \\
0 \\
0 \\
W \\
W \\
W \\
W \\
W \\
-3 \\
-9 \\
0\end{array}$ & $\begin{array}{r}119 \\
16 \\
0 \\
W \\
W \\
W \\
W \\
W \\
58 \\
45 \\
0\end{array}$ & $\begin{array}{r}10,046 \\
2,963 \\
710 \\
2,253 \\
W \\
W \\
1,951 \\
W \\
8,157 \\
-1,077 \\
3\end{array}$ & $\begin{array}{r}-159 \\
14 \\
0 \\
14 \\
W \\
W \\
W \\
W \\
-476 \\
303 \\
0\end{array}$ & $\begin{array}{r}4,085 \\
2,189 \\
407 \\
1,782 \\
W \\
W \\
1,773 \\
W \\
1,016 \\
876 \\
4\end{array}$ & $\begin{array}{r}21,656 \\
7,981 \\
1,191 \\
6,790 \\
513 \\
17 \\
5,942 \\
318 \\
13,132 \\
614 \\
-71\end{array}$ \\
\hline Total Input to Refineries .................................... & 16,974 & 106,107 & 79,636 & 5,781 & 3,302 & 211,800 & 14,267 & 78,763 & 452,565 \\
\hline 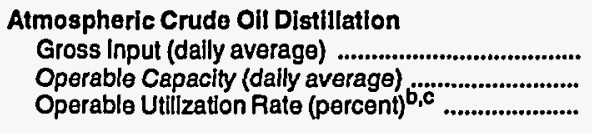 & $\begin{array}{r}516 \\
592 \\
87.1\end{array}$ & $\begin{array}{r}3,065 \\
3,412 \\
89.8\end{array}$ & $\begin{array}{r}2,496 \\
2,697 \\
92.6\end{array}$ & $\begin{array}{r}167 \\
219 \\
75.9\end{array}$ & $\begin{array}{r}95 \\
95 \\
100.7\end{array}$ & $\begin{array}{r}6,339 \\
7,015 \\
90.4\end{array}$ & $\begin{array}{r}451 \\
508 \\
88.8\end{array}$ & $\begin{array}{r}2,389 \\
2,898 \\
82.4\end{array}$ & $\begin{array}{r}13,582 \\
15,440 \\
88.0\end{array}$ \\
\hline 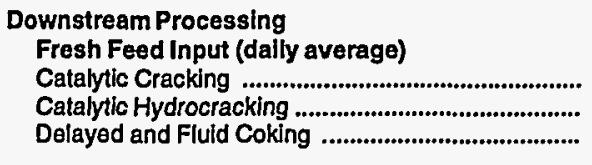 & $\begin{array}{r}160 \\
15 \\
7\end{array}$ & $\begin{array}{r}1,131 \\
166 \\
329\end{array}$ & $\begin{array}{l}816 \\
226 \\
384\end{array}$ & $\begin{array}{r}18 \\
0 \\
9\end{array}$ & $\begin{array}{r}30 \\
0 \\
0\end{array}$ & $\begin{array}{r}2,155 \\
406 \\
729\end{array}$ & $\begin{array}{r}146 \\
0 \\
36\end{array}$ & $\begin{array}{l}672 \\
387 \\
449\end{array}$ & $\begin{array}{r}4,566 \\
969 \\
1,560\end{array}$ \\
\hline $\begin{array}{l}\text { Crude Oll Qualities } \\
\text { Sulfur Content, Weighted Average (percent) ......... } \\
\text { API Gravity, Welghted Average (degrees) ............. }\end{array}$ & $\begin{array}{r}0.70 \\
38.54\end{array}$ & $\begin{array}{r}1.11 \\
31.89\end{array}$ & $\begin{array}{r}1.39 \\
30.41\end{array}$ & $\begin{array}{r}1.64 \\
31.47\end{array}$ & $\begin{array}{r}0.55 \\
39.05\end{array}$ & $\begin{array}{r}1.20 \\
31.94\end{array}$ & $\begin{array}{r}1.36 \\
34.69\end{array}$ & $\begin{array}{r}1.19 \\
25.01\end{array}$ & $\begin{array}{r}1.13 \\
31.43\end{array}$ \\
\hline 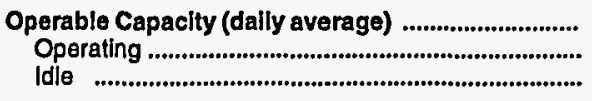 & $\begin{array}{r}592 \\
592 \\
0\end{array}$ & $\begin{array}{r}3,412 \\
3,320 \\
92\end{array}$ & $\begin{array}{r}2,697 \\
2,697 \\
0\end{array}$ & $\begin{array}{r}219 \\
207 \\
13\end{array}$ & $\begin{array}{r}95 \\
95 \\
0\end{array}$ & $\begin{array}{r}7,015 \\
6,911 \\
105\end{array}$ & $\begin{array}{r}508 \\
508 \\
0\end{array}$ & $\begin{array}{r}2,898 \\
2,856 \\
42\end{array}$ & $\begin{array}{r}15,440 \\
15,263 \\
177\end{array}$ \\
\hline 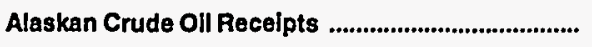 & 0 & 2,233 & 521 & $\mathbf{0}$ & $\mathbf{0}$ & 2,754 & 0 & 41,034 & 44,704 \\
\hline
\end{tabular}

a Includes ethyl tertiary butyl ether (ETBE), tertiary amyl methyl ether (TAME), tertiary butyl alcohol (TBA), and other aliphatic alcohols and ethers intended for motor gasoline blending (e.g., Isopropyl ether (IPE) or n-propanol).

Represents gross input divided by operable capacity.

c See Table H2 in the Highlights Sectlon for additional information concerning utilization rates.

$W=$ Withheld to avold disclosure of individual company data.

Note: - Totals may not equal sum of components due to independent rounding. • Refer to Appendix A for Refining District descriptions.

Source: Energy Information Administration (EIA) Form EIA-810, "Monthly Refinery Report." 
Table 29. Refinery Net Production of Finished Petroleum Products by PAD and Refining Districts, March 1995

(Thousand Barrels)

\begin{tabular}{|c|c|c|c|c|c|c|c|}
\hline \multirow[b]{2}{*}{ Commodity } & \multicolumn{3}{|c|}{ PAD District 1} & \multicolumn{4}{|c|}{ PAD District II } \\
\hline & $\begin{array}{l}\text { East } \\
\text { Coast }\end{array}$ & $\begin{array}{c}\text { Appalachian } \\
\text { No.1 }\end{array}$ & Total & Ind., III., Ky. & $\begin{array}{c}\text { Minn., Wis., } \\
\text { N. Dak., } \\
\text { S. Dak. }\end{array}$ & $\begin{array}{c}\text { Okla., Kans., } \\
\text { M.o. }\end{array}$ & Total \\
\hline 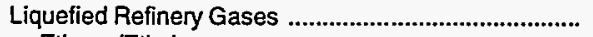 & 1,637 & -12 & 1,625 & 3,157 & 305 & 675 & 4,137 \\
\hline 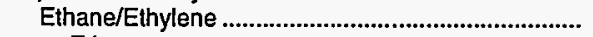 & 0 & 0 & 0 & 0 & 0 & 0 & 0 \\
\hline Ethane & W & $\mathbf{W}$ & W & $W$ & $w$ & W & $w$ \\
\hline Ethylene & W & W & W & $\ddot{W}$ & W & W & W \\
\hline 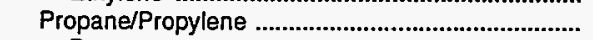 & 1,438 & 22 & 1,460 & 2,529 & 312 & 569 & 3,410 \\
\hline Propane & $W$ & W & W & $W$ & W & W & W \\
\hline 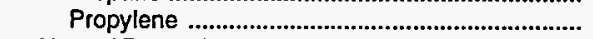 & $\ddot{W}$ & W & W & W & $\ddot{W}$ & W & W \\
\hline 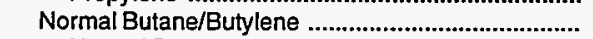 & 265 & -29 & 236 & 477 & -5 & 150 & 622 \\
\hline Normal Butane & W & W & $W$ & W & $\mathbf{W}$ & W & $\bar{W}$ \\
\hline 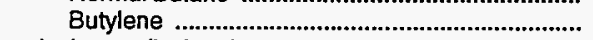 & W & w & W & W & W & W & W \\
\hline 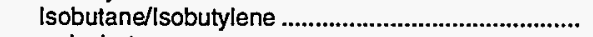 & -66 & -5 & .71 & 151 & -2 & -44 & 105 \\
\hline 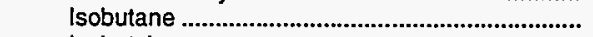 & W & $w$ & $\dot{w}$ & W & $w$ & W & W \\
\hline Isobutylene & W & $W$ & W & W & $\ddot{w}$ & W & W \\
\hline 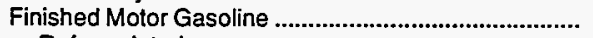 & 24,072 & 974 & 25,046 & 35,026 & 6,124 & 10,143 & 51,293 \\
\hline 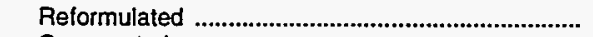 & 16,942 & 0 & 16,942 & 5,418 & 226 & 0 & 5,644 \\
\hline Oxygenated & 419 & 0 & 419 & 707 & 1,303 & 81 & 2,091 \\
\hline Other & 6,711 & 974 & 7,685 & 28,901 & 4,595 & 10,062 & 43,558 \\
\hline 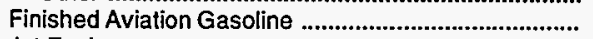 & 1 & 0 & 1 & 35 & 30 & 3 & 68 \\
\hline Jet Fuel & 1,927 & 15 & 1,942 & 3,345 & 1,026 & 895 & 5,266 \\
\hline 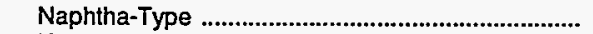 & 0 & 0 & 0 & -1 & 0 & 0 & -1 \\
\hline 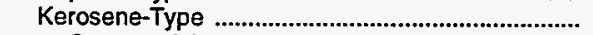 & 1,927 & 15 & 1,942 & 3,346 & 1,026 & 895 & 5,267 \\
\hline 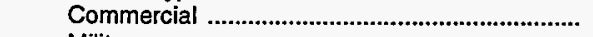 & 1,927 & 15 & 1,942 & 3,172 & 1,026 & 670 & 4,868 \\
\hline Military & 0 & 0 & 0 & 174 & 0 & 225 & 399 \\
\hline 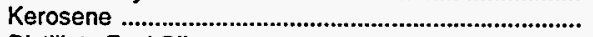 & 431 & 41 & 472 & 140 & 35 & -33 & 142 \\
\hline 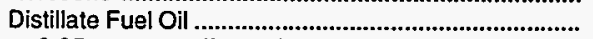 & 13,057 & 705 & 13,762 & 14,810 & 2,609 & 5,319 & 22,738 \\
\hline 0.05 percent sulfur and under & 3,486 & 532 & 4,018 & 9,230 & 492 & 3,957 & 13,679 \\
\hline 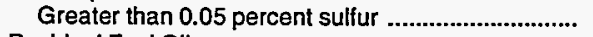 & 9,571 & $17 \overline{3}$ & 9,744 & 5,580 & 2,117 & 1,362 & 9,059 \\
\hline 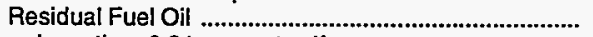 & 4,817 & 83 & 4,900 & 1,257 & 212 & 113 & 1,582 \\
\hline 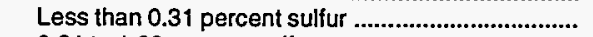 & 547 & 42 & 589 & 17 & 0 & 29 & 46 \\
\hline 0.31 to 1.00 percent sulfur ....................................... & 3,745 & 41 & 3,786 & 234 & 0 & 14 & 248 \\
\hline Greater than 1.00 percent sulfur & 525 & 0 & 525 & 1,006 & 212 & 70 & 1,288 \\
\hline Naphtha for Petrochemical Feedstock Use .................. & 243 & 0 & 243 & 504 & 0 & 75 & 579 \\
\hline Other Oils for Petrochemical Feedstock Use ............... & 14 & 0 & 14 & 409 & 0 & 53 & 462 \\
\hline Special Naphthas & 25 & 18 & 43 & 315 & 0 & 104 & 419 \\
\hline Lubricants & 284 & 262 & 546 & 460 & 0 & 212 & 672 \\
\hline 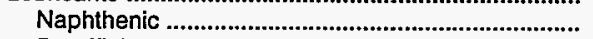 & 0 & 0 & 0 & 0 & 0 & 0 & 0 \\
\hline 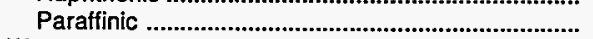 & 284 & 262 & 546 & 460 & 0 & 212 & 672 \\
\hline Waxes & 0 & 127 & 127 & 106 & 0 & 25 & 131 \\
\hline 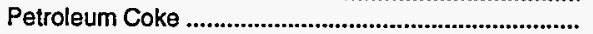 & 1,712 & 20 & 1,732 & 2,530 & 167 & 791 & 3,488 \\
\hline 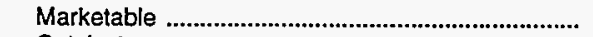 & 732 & 0 & 732 & 1,500 & 1 & 584 & 2,085 \\
\hline Catalyst & 980 & 20 & 1,000 & 1,030 & 166 & 207 & 1,403 \\
\hline 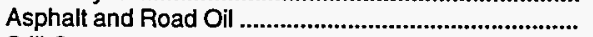 & 2,267 & 310 & 2,577 & 2,826 & 902 & 488 & 4,216 \\
\hline Still Gas & 1,686 & 121 & 1,807 & 2,582 & 386 & 807 & 3,775 \\
\hline 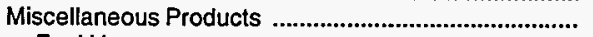 & 28 & 22 & 50 & 291 & 58 & 160 & 509 \\
\hline 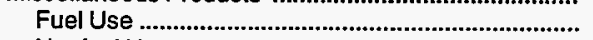 & 0 & 0 & 0 & 0 & 0 & 7 & 7 \\
\hline 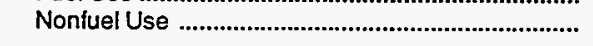 & 28 & 22 & 50 & 291 & 58 & 153 & 502 \\
\hline Total & 52,201 & 2,686 & 54,887 & 67,793 & 11,854 & 19,830 & 99,477 \\
\hline 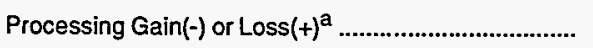 & $-2,236$ & -3 & $-2,239$ & $-3,330$ & -305 & -755 & $-4,390$ \\
\hline
\end{tabular}

See footnotes at end of table. 
Table 29. Refinery Net Production of Finished Petroleum Products by PAD and Refining Districts, March 1995 (Continued)

(Thousand Barrels)

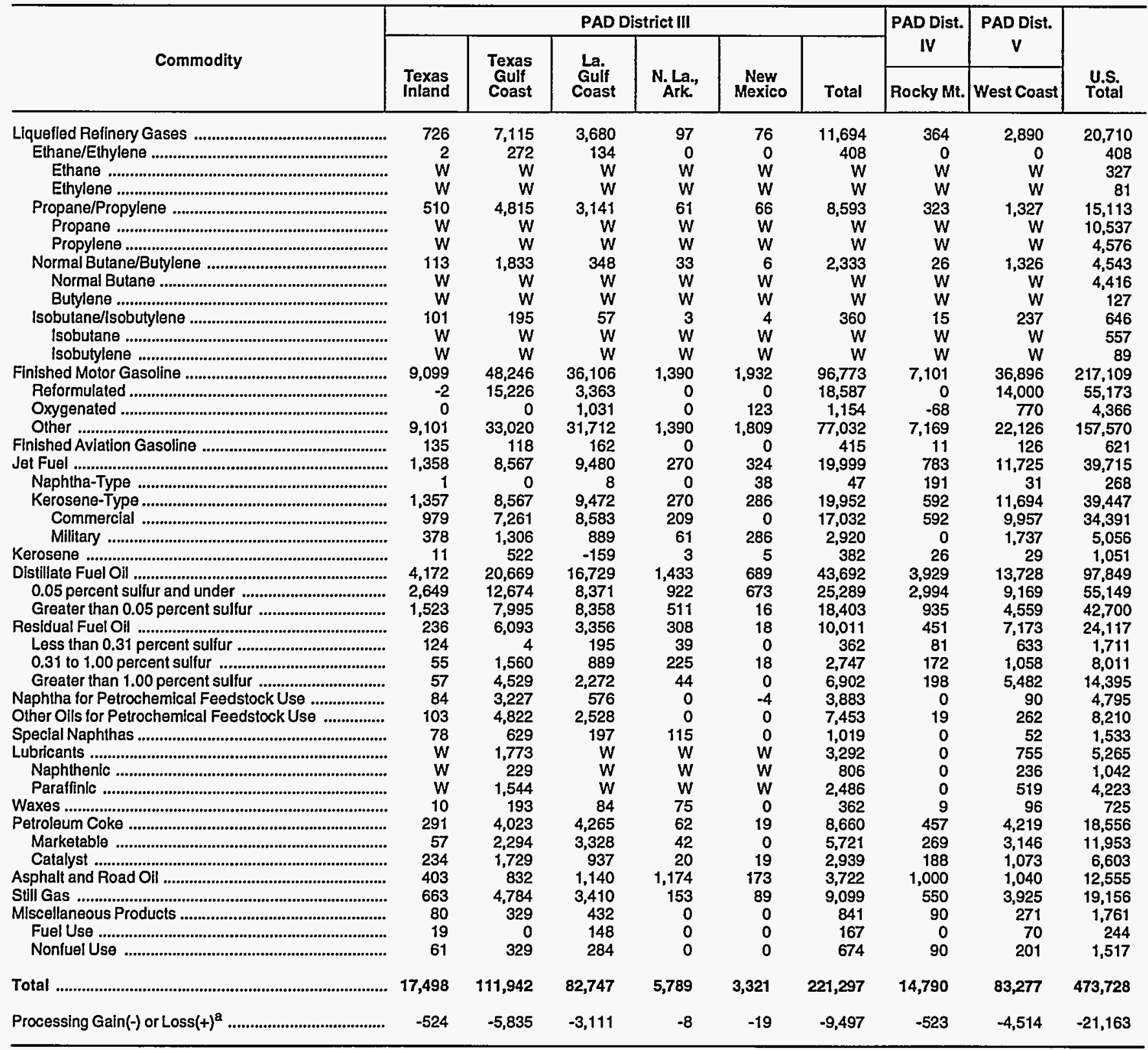

a Represents the arithmetic difference between input and production.

$W=$ Witheld to avoid disclosure of individual company data.

Note: Refer to Appendix A for Refining District descriptions.

Source: Energy Information Administration (EIA) Form EIA-810, "Monthly Refinery Report." 
Table 30. Refinery Stocks of Crude Oil and Petroleum Products by PAD and Refining Districts, March 1995

(Thousand Barrels)

\begin{tabular}{|c|c|c|c|c|c|c|c|}
\hline \multirow[b]{2}{*}{ Commodity } & \multicolumn{3}{|c|}{ PAD District I } & \multicolumn{4}{|c|}{ PAD District II } \\
\hline & $\begin{array}{l}\text { East } \\
\text { Coast }\end{array}$ & $\begin{array}{c}\text { Appalachian } \\
\text { No. } 1\end{array}$ & Total & Ind., Ill., Ky. & $\begin{array}{l}\text { Minn., Wis., } \\
\text { N. Dak., } \\
\text { S. Dak. }\end{array}$ & $\begin{array}{c}\text { Okla., Kans., } \\
\text { Mo. }\end{array}$ & Total \\
\hline 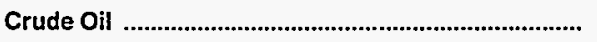 & 15,473 & 483 & 15,956 & 9,777 & 1,875 & 2,444 & 14,096 \\
\hline 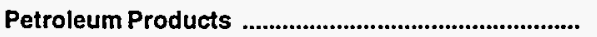 & 49,398 & 2,988 & 52,386 & 40,133 & 8,982 & 13,851 & 62,966 \\
\hline 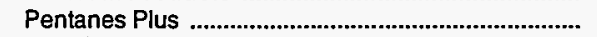 & 0 & 0 & 0 & 166 & 66 & 181 & 413 \\
\hline 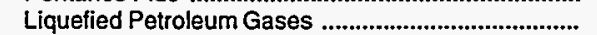 & 1,067 & 11 & 1,078 & 1,703 & 192 & 560 & 2,455 \\
\hline Ethane/Ethylene & 0 & 0 & 0 & 1 & 0 & 0 & 1 \\
\hline 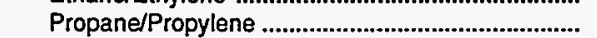 & 318 & 4 & 322 & 868 & 66 & 240 & 1,174 \\
\hline 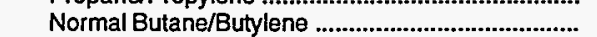 & 627 & 5 & 632 & 444 & 54 & 193 & 691 \\
\hline Isobutane/lsobutylene & 122 & 2 & 124 & 390 & 72 & 127 & 589 \\
\hline Other Hydrocarbons/Hydrogen/Oxygenates ............. & 1,780 & 20 & 1,800 & 546 & 157 & 84 & 787 \\
\hline 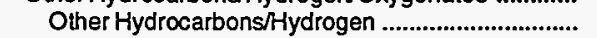 & 0 & 0 & 0 & 16 & 0 & 0 & 16 \\
\hline Oxygenates & $\mathbf{W}$ & $w$ & 1,800 & 530 & 157 & 84 & 771 \\
\hline 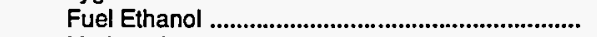 & W & W & W & W & W & W & 322 \\
\hline 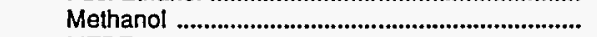 & $\ddot{W}$ & w & W & W & W & W & W \\
\hline MTBE & $\ddot{W}$ & $\ddot{w}$ & 1,655 & W & $\ddot{w}$ & w & W \\
\hline 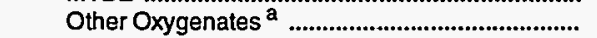 & $\ddot{W}$ & $\ddot{w}$ & W & W & $\ddot{W}$ & W & $w$ \\
\hline 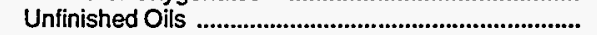 & 10,108 & 761 & 10,869 & 9,890 & 408 & 3,973 & 14,271 \\
\hline 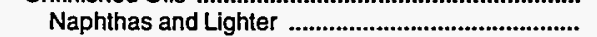 & 2,765 & 201 & 2,966 & 3,215 & 161 & 1,184 & 4,560 \\
\hline 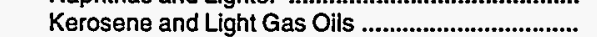 & 1,968 & 3 & 1,971 & 1,505 & 62 & 307 & 1,874 \\
\hline 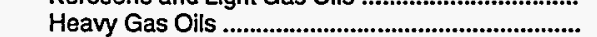 & 4,452 & 430 & 4,882 & 3,144 & 177 & 1,294 & 4,615 \\
\hline Residuum & 923 & 127 & 1,050 & 2,026 & 8 & 1,188 & 3,222 \\
\hline Motor Gasoline Blending Components ...................... & 5,665 & 92 & 5,757 & 5,936 & 1,392 & 1,282 & 8,610 \\
\hline Aviation Gasoline Blending Components .................. & 49 & 0 & 49 & 15 & 0 & 0 & 15 \\
\hline 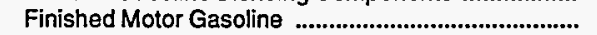 & 10,621 & 275 & 10,896 & 6,788 & 1,616 & 3,563 & 11,967 \\
\hline Reformulated & 7,189 & 0 & 7,189 & 823 & 33 & 0 & 856 \\
\hline 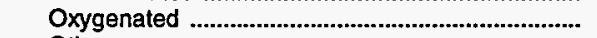 & 93 & 0 & 93 & 139 & 204 & 0 & 343 \\
\hline Other & 3,339 & 275 & 3,614 & 5,826 & 1,379 & 3,563 & 10,768 \\
\hline Finished Aviation Gasoline ........................................... & 58 & 0 & 58 & 44 & 24 & 30 & 98 \\
\hline Jet Fuel & 2,159 & 16 & 2,175 & 2,333 & 260 & 262 & 2,855 \\
\hline Naphtha-Type & 0 & 0 & 0 & 11 & 0 & 0 & 11 \\
\hline 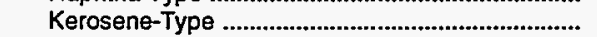 & 2,159 & 16 & 2,175 & 2,322 & 260 & 262 & 2,844 \\
\hline Kerosene & 512 & 26 & 538 & 276 & 21 & 190 & 487 \\
\hline 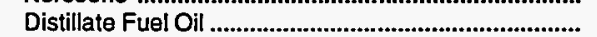 & 10,390 & 285 & 10,675 & 4,731 & 1,242 & 2,240 & 8,213 \\
\hline 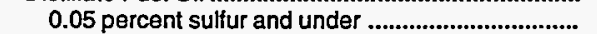 & 2,622 & 192 & 2,814 & 2,489 & 469 & 1,493 & 4,451 \\
\hline 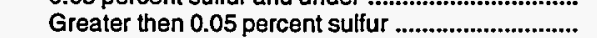 & 7,768 & 93 & 7,861 & 2,242 & 773 & 747 & 3,762 \\
\hline Residual Fuel Oil & 3,675 & 98 & 3,773 & 1,321 & 180 & 97 & 1,598 \\
\hline Less than 0.31 percent sulfur & 1,520 & $\pi$ & 1,597 & 0 & 0 & 22 & 22 \\
\hline 0.31 to 1.00 percent sulfur & 1,661 & 21 & 1,682 & 172 & 0 & 1 & 173 \\
\hline 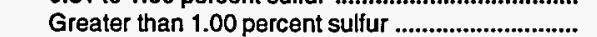 & 494 & 0 & 494 & 1,149 & 180 & 74 & 1,403 \\
\hline Naphtha for Petrochemical Feedstock Use ................ & 545 & 0 & 545 & 302 & 0 & 64 & 366 \\
\hline Other Oils for Petrochemical Feedstock Use ............. & 3 & 0 & 3 & 1 & 0 & 0 & 1 \\
\hline Special Naphthas & 3 & 35 & 38 & 143 & 0 & 48 & 191 \\
\hline 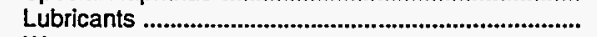 & 354 & 432 & 786 & 851 & 0 & 0 & 851 \\
\hline Waxes & 0 & 166 & 166 & 106 & 0 & 22 & 128 \\
\hline Petroleum Coke (Marketable) .................................... & 536 & 0 & 536 & 915 & 1,091 & 70 & 2,076 \\
\hline 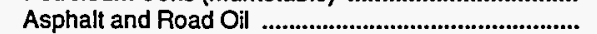 & 1,845 & 727 & 2,572 & 3,961 & 2,327 & 1,163 & 7,451 \\
\hline 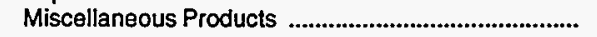 & 28 & 44 & 72 & 105 & 6 & 22 & 133 \\
\hline 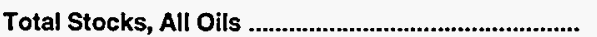 & 64,871 & 3,471 & 68,342 & 49,910 & 10,857 & 16,295 & 77,062 \\
\hline
\end{tabular}

See footnotes at end of table. 
Table 30. Refinery Stocks of Crude Oil and Petroleum Products by PAD and Refining Districts, March 1995 (Continued) (Thousand Barrels)

\begin{tabular}{|c|c|c|c|c|c|c|c|c|c|}
\hline \multirow{3}{*}{ Commodity } & \multicolumn{6}{|c|}{ PAD District III } & \multirow{3}{*}{\begin{tabular}{|c|}
$\begin{array}{c}\text { PAD Dist. } \\
\text { IV }\end{array}$ \\
Rocky Mt.
\end{tabular}} & \multirow{3}{*}{\begin{tabular}{|c|}
$\begin{array}{c}\text { PAD Dist. } \\
\text { v }\end{array}$ \\
West Coast
\end{tabular}} & \multirow[b]{3}{*}{ U.S. } \\
\hline & \multirow[b]{2}{*}{$\begin{array}{l}\text { Texas } \\
\text { Inland }\end{array}$} & \multirow{2}{*}{$\begin{array}{c}\text { Texas } \\
\text { Gulf } \\
\text { Coast }\end{array}$} & \multirow{2}{*}{ 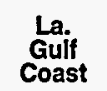 } & \multirow[b]{2}{*}{$\begin{array}{l}\text { N.La, } \\
\text { Ark. }\end{array}$} & \multirow[b]{2}{*}{$\underset{\text { Mexico }}{\text { New }}$} & \multirow[b]{2}{*}{ Total } & & & \\
\hline & & & & & & & & & \\
\hline 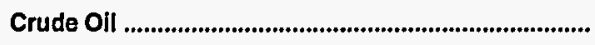 & 1,375 & 25,540 & 16,738 & 1,289 & 500 & 45,442 & 2,390 & 21,683 & 99,567 \\
\hline Petroleum Products & 10,674 & 70,267 & 44,414 & 4,209 & 1,436 & 131,000 & 13,382 & 70,276 & 330,010 \\
\hline ………………....................... & 204 & & & 11 & & 355 & & & 772 \\
\hline 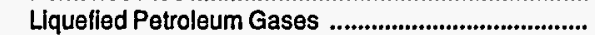 & 1,334 & 3,343 & 2,726 & 17 & 32 & 7,452 & 432 & 1,169 & 12,586 \\
\hline 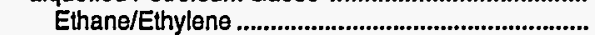 & 146 & 456 & & 0 & 0 & 602 & 0 & 0 & 603 \\
\hline 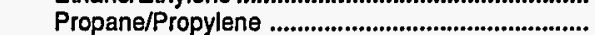 & 365 & 1,262 & 768 & 4 & 5 & 2,404 & 80 & 110 & 4,090 \\
\hline Normal Butane/Butylene ......................................... & 486 & 1,058 & 1,304 & 7 & 7 & 2,862 & 211 & 676 & 5,072 \\
\hline 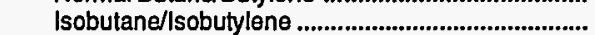 & 337 & 567 & 654 & 6 & 20 & 1,584 & 141 & 383 & 2,821 \\
\hline Other Hydrocarbons/Hydrogen/Oxygenates ............. & 154 & 1,663 & 833 & 14 & 6 & 2,670 & 129 & 3,427 & 8,813 \\
\hline Other Hydrocarbons/Hydrogen ............................... & 0 & 0 & 1 & 0 & 0 & 1 & 0 & 5 & 22 \\
\hline 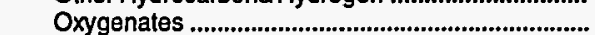 & 154 & 1,663 & 832 & $\mathbf{w}$ & $\mathbf{w}$ & 2,669 & 129 & 3,422 & 8,791 \\
\hline 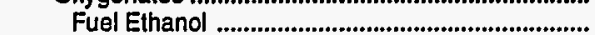 & $w$ & w & W & $\ddot{w}$ & $\ddot{w}$ & & $w$ & $w$ & 425 \\
\hline 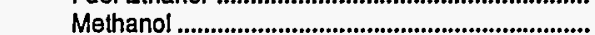 & $\ddot{w}$ & $\ddot{w}$ & $\ddot{w}$ & $\ddot{w}$ & $\ddot{w}$ & $\ddot{w}$ & $\ddot{w}$ & w & 658 \\
\hline MTBE & $w$ & 1,187 & $\underline{w}$ & $\dddot{w}$ & $w$ & 1,979 & $w$ & 3,393 & 7,516 \\
\hline Other Oxygenates a & $\ddot{w}$ & $w$ & $\ddot{w}$ & $\ddot{w}$ & $\dddot{w}$ & W & $w$ & W & 192 \\
\hline 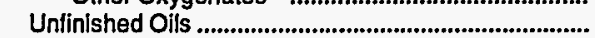 & 3,183 & 25,822 & 16,089 & 910 & 340 & 46,344 & 2,531 & 25,209 & 99,224 \\
\hline 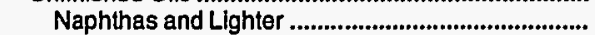 & 1,000 & 5,955 & 3,712 & 204 & 118 & 10,989 & 621 & 4,053 & 23,189 \\
\hline Kerosene and Light Gas Oils ................................... & 560 & 4,727 & 2,664 & 289 & 89 & 8,329 & 556 & 5,340 & 18,070 \\
\hline 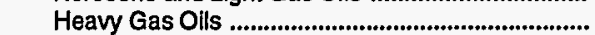 & 898 & 9,760 & 6,486 & 378 & 133 & 17,655 & 1,037 & 12,471 & 40,660 \\
\hline 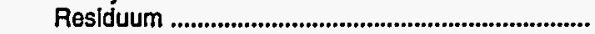 & 725 & 5,380 & 3,227 & 39 & 0 & 9,371 & 317 & 3,345 & 17,305 \\
\hline Motor Gasoline Blending Components ........................ & 1,481 & 7,200 & 5,182 & 84 & 310 & 14,257 & 2,518 & 8,154 & 39,296 \\
\hline Avlation Gasoline Blending Components ...................... & & & 23 & 0 & 0 & 23 & & & 90 \\
\hline 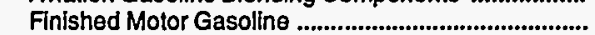 & 1,673 & 10,172 & 5,772 & 232 & 147 & 17,996 & 2,466 & 10,142 & 53,467 \\
\hline Reformulated ........................................................ & 0 & 2,889 & 396 & 0 & 0 & 3,285 & 0 & 3,189 & 14,519 \\
\hline 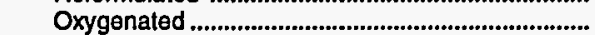 & 0 & 23 & 140 & 0 & 0 & 163 & 14 & 87 & 700 \\
\hline 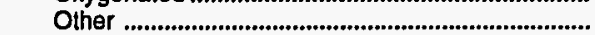 & 1,673 & 7,260 & 5,236 & 232 & 147 & 14,548 & 2,452 & 6,866 & 38,248 \\
\hline 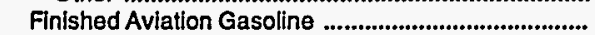 & 76 & 158 & 224 & 0 & 0 & 458 & 35 & 307 & 956 \\
\hline Jet Fuel & 474 & 3,193 & 2,254 & 121 & 84 & 6,126 & 330 & 4,345 & 15,831 \\
\hline 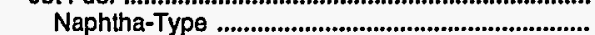 & 0 & & 175 & $\ddot{0}$ & 15 & 190 & 126 & 52 & 379 \\
\hline 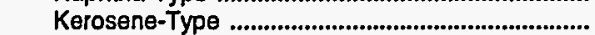 & 474 & 3,193 & 2,079 & 121 & 69 & 5,936 & 204 & 4,293 & 15,452 \\
\hline Kerosene & 27 & 283 & 171 & 5 & 18 & 504 & 88 & 45 & 1,662 \\
\hline Distllate Fuel Oil & 916 & 7,194 & 3,878 & 486 & 136 & 12,610 & 1,372 & 5,660 & 38,530 \\
\hline 0.05 percent sulfur and under ................................ & 567 & 3,133 & 1,339 & 242 & 85 & 5,366 & 1,007 & 3,815 & 17,453 \\
\hline 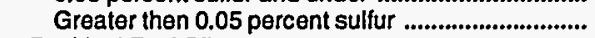 & 349 & 4,061 & 2,539 & 244 & 51 & 7,244 & 365 & 1,845 & 21,077 \\
\hline 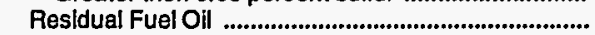 & 162 & 3,439 & 2,250 & 178 & 19 & 6,048 & 571 & 4,604 & 16,594 \\
\hline Less than 0.31 percent sulfur ...................................... & 21 & 1 & 106 & 1 & 0 & 129 & 104 & 375 & 2,227 \\
\hline 0.31 to 1.00 percent sulfur & 9 & 649 & 438 & 128 & 19 & 1,243 & 339 & 804 & 4,241 \\
\hline Greater than 1.00 percent sulfur & 132 & 2,789 & 1,706 & 49 & 0 & 4,676 & 128 & 3,425 & 10,126 \\
\hline Naphtha for Petrochemical Feedstock Use ................. & 17 & 1,472 & 491 & 8 & 5 & 1,993 & 0 & 97 & 3,001 \\
\hline Other Oils for Petrochemical Feedstock Use ............... & 57 & 1,273 & 145 & 0 & 0 & 1,475 & 0 & 233 & 1,712 \\
\hline Speclal Naphthas & 60 & 1,115 & 106 & 75 & 0 & 1,356 & 1 & 39 & 1,625 \\
\hline 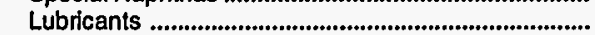 & 19 & 2,411 & 1,241 & 681 & 0 & 4,352 & 0 & 1,271 & 7,260 \\
\hline Waxes & 6 & 230 & 204 & 25 & 0 & 465 & 16 & 134 & 909 \\
\hline Petroleum Coke (Marketable) & 4 & 546 & 2,230 & 0 & 0 & 2,780 & 243 & 3,321 & 8,956 \\
\hline 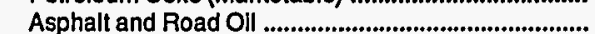 & 787 & 530 & 429 & 1,362 & 331 & 3,439 & 2,637 & 1,833 & 17,932 \\
\hline 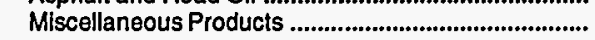 & 40 & 143 & 114 & 0 & 0 & 297 & 9 & 283 & 794 \\
\hline Total Stocks, All Oils & 12,049 & 95,807 & 61,152 & 5,498 & 1,936 & 176,442 & 15,772 & 91,959 & 429,577 \\
\hline
\end{tabular}

a Includes ethyl tertiary butyl ether (ETBE), tertiary amyl methyl ether (TAME), tertiary butyl alcohol (TBA), and other aliphatic alcohols and ethers intended for motor gasoline blending (e.g., isopropyl ether (IPE) or n-propanol).

$W=$ Withheld to avoid disclosure of individual company data.

Notes: - Stocks are reported as of the last day of the month. - Refer to Appendix A for Refining District descriptions.

Source: Energy Information Administration (EIA) Form EIA-810, "Monthly Refinery Report:" 
Table 31. Percent Refinery Yield of Petroleum Products by PAD and Refining Districts, ${ }^{a}$ March 1995

\begin{tabular}{|c|c|c|c|c|c|c|c|}
\hline \multirow[b]{2}{*}{ Commodity } & \multicolumn{3}{|c|}{ PAD District I } & \multicolumn{4}{|c|}{ PAD District II } \\
\hline & $\begin{array}{l}\text { East } \\
\text { Coast }\end{array}$ & $\begin{array}{c}\text { Appalachian } \\
\text { No.1 }\end{array}$ & Total & Ind., IIl., Ky. & $\begin{array}{l}\text { Minn., Wis., } \\
\text { N. Dak., } \\
\text { S. Dak. }\end{array}$ & $\begin{array}{c}\text { Okla., Kans., } \\
\text { Mo. }\end{array}$ & Total \\
\hline 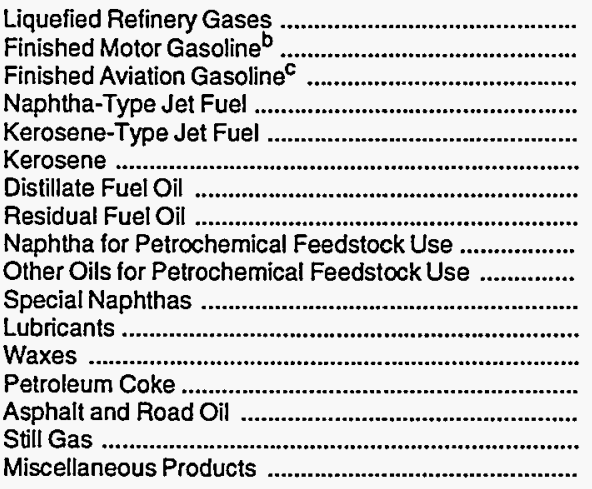 & $\begin{array}{r}3.5 \\
45.2 \\
0.2 \\
0.0 \\
4.1 \\
0.9 \\
27.5 \\
10.2 \\
0.5 \\
0.0 \\
0.1 \\
0.6 \\
0.0 \\
3.6 \\
4.8 \\
3.6 \\
0.1\end{array}$ & $\begin{array}{r}-0.5 \\
35.9 \\
0.0 \\
0.0 \\
0.6 \\
1.5 \\
26.5 \\
3.1 \\
0.0 \\
0.0 \\
0.7 \\
9.8 \\
4.8 \\
0.8 \\
11.6 \\
4.5 \\
0.8\end{array}$ & $\begin{array}{r}3.2 \\
44.7 \\
0.2 \\
0.0 \\
3.9 \\
0.9 \\
27.5 \\
9.8 \\
0.5 \\
0.0 \\
0.1 \\
1.1 \\
0.3 \\
3.5 \\
5.1 \\
3.6 \\
0.1\end{array}$ & $\begin{array}{r}5.1 \\
52.2 \\
0.0 \\
0.0 \\
5.4 \\
0.2 \\
24.1 \\
2.0 \\
0.8 \\
0.7 \\
0.5 \\
0.7 \\
0.2 \\
4.1 \\
4.6 \\
4.2 \\
0.5\end{array}$ & $\begin{array}{r}2.7 \\
52.5 \\
0.3 \\
0.0 \\
9.0 \\
0.3 \\
22.8 \\
1.9 \\
0.0 \\
0.0 \\
0.0 \\
0.0 \\
0.0 \\
1.5 \\
7.9 \\
3.4 \\
0.5\end{array}$ & $\begin{array}{r}3.7 \\
50.6 \\
0.0 \\
0.0 \\
5.0 \\
-0.2 \\
29.4 \\
0.6 \\
0.4 \\
0.3 \\
0.6 \\
1.2 \\
0.1 \\
4.4 \\
2.7 \\
4.5 \\
0.9\end{array}$ & $\begin{array}{r}4.5 \\
51.9 \\
0.0 \\
0.0 \\
5.8 \\
0.2 \\
25.0 \\
1.7 \\
0.6 \\
0.5 \\
0.5 \\
0.7 \\
0.1 \\
3.8 \\
4.6 \\
4.1 \\
0.6\end{array}$ \\
\hline Processing Gain(-) or Loss $(+)^{d}$ & -4.7 & -0.1 & -4.5 & -5.4 & -2.7 & -4.2 & -4.8 \\
\hline
\end{tabular}

\begin{tabular}{|c|c|c|c|c|c|c|c|c|c|}
\hline \multirow{3}{*}{ Commodity } & \multicolumn{6}{|c|}{ PAD District III } & \multirow{3}{*}{\begin{tabular}{|c|}
$\begin{array}{c}\text { PAD Dist. } \\
\text { IV }\end{array}$ \\
Rocky Mtt.
\end{tabular}} & \multirow{3}{*}{$\begin{array}{c}\text { PAD Dist. } \\
V\end{array}$} & \multirow[b]{3}{*}{$\begin{array}{l}\text { U.S. } \\
\text { Total }\end{array}$} \\
\hline & \multirow[b]{2}{*}{$\begin{array}{l}\text { Texas } \\
\text { Inland }\end{array}$} & \multirow{2}{*}{$\begin{array}{c}\text { Texas } \\
\text { Gulf } \\
\text { Coast }\end{array}$} & \multirow{2}{*}{$\begin{array}{c}\text { La. } \\
\text { Gulf } \\
\text { Coast }\end{array}$} & \multirow{2}{*}{$\begin{array}{c}\text { N. La., } \\
\text { Ark. }\end{array}$} & \multirow[b]{2}{*}{$\begin{array}{l}\text { New } \\
\text { Mexico }\end{array}$} & \multirow[b]{2}{*}{ Total } & & & \\
\hline & & & & & & & & & \\
\hline 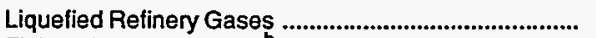 & 4.7 & 7.0 & 4.8 & 1.7 & 2.5 & 5.8 & 2.7 & 4.0 & 4.8 \\
\hline Finished Motor Gasoline ${ }^{b}$..... & 49.2 & 43.3 & 43.6 & 21.4 & 54.5 & 43.4 & 47.3 & 42.6 & 45.3 \\
\hline Finished Aviation Gasoline ${ }^{c}$. & 0.9 & 0.1 & 0.2 & 0.0 & 0.0 & 0.2 & 0.1 & 0.2 & 0.2 \\
\hline 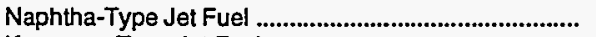 & 0.0 & 0.0 & 0.0 & 0.0 & 1.3 & 0.0 & 1.4 & 0.0 & 0.1 \\
\hline Kerosene-Type Jet Fuel .................................................. & 8.7 & 8.4 & 12.3 & 4.8 & 9.5 & 9.8 & 4.4 & 16.0 & 9.2 \\
\hline 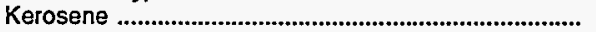 & 0.1 & 0.5 & -0.2 & 0.1 & 0.2 & 0.2 & 0.2 & 0.0 & 0.2 \\
\hline 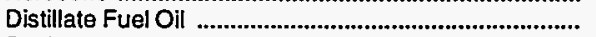 & 26.9 & 20.3 & 21.7 & 25.6 & 22.9 & 21.5 & 28.9 & 18.8 & 22.7 \\
\hline Residual Fuel Oil & 1.5 & 6.0 & 4.3 & 5.5 & 0.6 & 4.9 & 3.3 & 9.8 & 5.6 \\
\hline Naphtha for Petrochemical Feedstock Use .................. & 0.5 & 3.2 & 0.7 & 0.0 & -0.1 & 1.9 & 0.0 & 0.1 & 1.1 \\
\hline Other Oils for Petrochemical Feedstock Use ............... & 0.7 & 4.7 & 3.3 & 0.0 & 0.0 & 3.7 & 0.1 & 0.4 & 1.9 \\
\hline 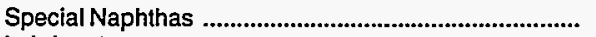 & 0.5 & 0.6 & 0.3 & 2.1 & 0.0 & 0.5 & 0.0 & 0.1 & 0.4 \\
\hline Lubricants & 0.3 & 1.7 & 1.0 & 12.7 & 0.0 & 1.6 & 0.0 & 1.0 & 1.2 \\
\hline 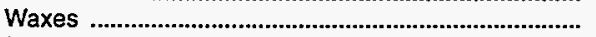 & 0.1 & 0.2 & 0.1 & 1.3 & 0.0 & 0.2 & 0.1 & 0.1 & 0.2 \\
\hline Petroleum Coke & 1.9 & 3.9 & 5.5 & 1.1 & 0.6 & 4.3 & 3.4 & 5.8 & 4.3 \\
\hline 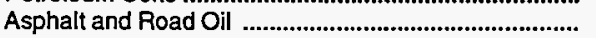 & 2.6 & 0.8 & 1.5 & 21.0 & 5.7 & 1.8 & 7.4 & 1.4 & 2.9 \\
\hline Still Gas & 4.3 & 4.7 & 4.4 & 2.7 & 3.0 & 4.5 & 4.0 & 5.4 & 4.4 \\
\hline 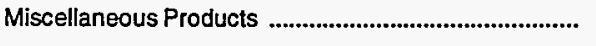 & 0.5 & 0.3 & 0.6 & 0.0 & 0.0 & 0.4 & 0.7 & 0.4 & 0.4 \\
\hline 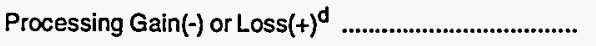 & -3.4 & -5.7 & -4.0 & -0.1 & -0.6 & -4.7 & -3.8 & -6.2 & -4.9 \\
\hline
\end{tabular}

a Based on crude oil input and net reruns of unfinished oils.

b Based on total finished motor gasoline output minus net input of motor gasoline blending components, minus input of natural gas plant liquids, other hydrocarbons and oxygenates.

Based on finished aviation gasoline output minus net input of aviation gasoline blending components.

d Represents the difference between input and production.

Notes: - Totals may not equal sum of components due to independent rounding. $\bullet$ Refer to Appendix A for Refining District descriptions.

Sources: Calculated from data on Tables 28 and 29. 
Table 32. Imports of Residual Fuel Oil by Sulfur Content and by PAD District and State of Entry, March 1995

(Thousand Barrels)

\begin{tabular}{|c|c|c|c|c|}
\hline \multirow[b]{2}{*}{ PAD District and State of Entry } & \multicolumn{4}{|c|}{ Residual Fuel Oil } \\
\hline & $\begin{array}{c}\text { Less than } \\
0.31 \% \\
\text { Sulfur }\end{array}$ & $\begin{array}{l}0.31 \text { to } \\
1.00 \% \\
\text { Sulfur }\end{array}$ & $\begin{array}{c}\text { Greater } \\
\text { than } 1.00 \% \\
\text { Sulfur }\end{array}$ & Total \\
\hline $\begin{array}{l}\text { PAD District I } \\
\text { Florida } \\
\text { Marne } \\
\text { New Hand } \\
\text { New Jersey } \\
\text { New York } \\
\text { North Carolina } \\
\text { Pennsylvanla } \\
\text { South Carolina } \\
\text { Vermont } \\
\text { Virginla }\end{array}$ & $\begin{array}{r}754 \\
0 \\
62 \\
0 \\
0 \\
203 \\
134 \\
0 \\
355 \\
0 \\
0 \\
0\end{array}$ & $\begin{array}{r}1,187 \\
0 \\
0 \\
139 \\
0 \\
319 \\
117 \\
0 \\
560 \\
51 \\
1 \\
0\end{array}$ & $\begin{array}{r}4,453 \\
623 \\
679 \\
355 \\
278 \\
423 \\
423 \\
684 \\
191 \\
61 \\
3 \\
733\end{array}$ & $\begin{array}{r}6,394 \\
623 \\
741 \\
494 \\
278 \\
945 \\
674 \\
684 \\
1,106 \\
112 \\
4 \\
733\end{array}$ \\
\hline PAD District II & $\begin{array}{l}87 \\
87\end{array}$ & $\begin{array}{l}0 \\
0\end{array}$ & $\begin{array}{l}0 \\
0\end{array}$ & $\begin{array}{l}87 \\
87\end{array}$ \\
\hline U.S. Total & 841 & 1,187 & 4,453 & 6,481 \\
\hline
\end{tabular}

Source: Energy Information Administration (EIA) Form ElA-814, "Monthly Imports Report." 
Table 33. Imports of Crude Oil and Petroleum Products by PAD District, March 1995

(Thousand Barrels)

\begin{tabular}{|c|c|c|c|c|c|c|c|}
\hline \multirow{2}{*}{ Commodity } & \multicolumn{6}{|c|}{ Petroleum Administration for Defense Districts } & \multirow[b]{2}{*}{$\begin{array}{c}\text { Daily } \\
\text { Average }\end{array}$} \\
\hline & I & II & III & IV & v & $\begin{array}{l}\text { U.S. } \\
\text { Total }\end{array}$ & \\
\hline Crude Oil ${ }^{a, b}$ & 50,319 & 34,390 & 135,039 & 3,971 & 5,952 & 229,671 & 7,409 \\
\hline $\begin{array}{l}\text { Natural Gas Liquids } \\
\text { Pentanes Plus } \\
\text { Liquefied Petroleum Gases } \\
\text { Ethane } \\
\text { Ethylene } \\
\text { Propane } \\
\text { Propylene } \\
\text { Normal Butane } \\
\text { Butylene } \\
\text { Isobutane } \\
\text { Isobutylene }\end{array}$ & $\begin{array}{r}756 \\
0 \\
756 \\
0 \\
0 \\
748 \\
0 \\
8 \\
0 \\
0 \\
0\end{array}$ & $\begin{array}{r}2,076 \\
33 \\
2,043 \\
347 \\
7 \\
1,339 \\
166 \\
134 \\
0 \\
50 \\
0\end{array}$ & $\begin{array}{r}1,208 \\
806 \\
402 \\
0 \\
0 \\
402 \\
0 \\
0 \\
0 \\
0 \\
0\end{array}$ & $\begin{array}{r}720 \\
547 \\
173 \\
0 \\
0 \\
120 \\
0 \\
49 \\
0 \\
4 \\
0\end{array}$ & $\begin{array}{r}59 \\
0 \\
59 \\
0 \\
0 \\
1 \\
0 \\
0 \\
0 \\
58 \\
0\end{array}$ & $\begin{array}{r}4,819 \\
1,386 \\
3,433 \\
347 \\
7 \\
2,610 \\
166 \\
191 \\
0 \\
112 \\
0\end{array}$ & $\begin{array}{r}155 \\
45 \\
111 \\
11 \\
(s) \\
84 \\
5 \\
6 \\
0 \\
4 \\
0\end{array}$ \\
\hline $\begin{array}{l}\text { Other Liquids } \\
\text { Other Hydrocarbons/Hydrogen/Oxygenates } \\
\text { Other Hydrocarbons/Hydrogen . } \\
\text { Oxygenates } \\
\text { Fuel Ethanol } \\
\text { MTBE } \\
\text { Other Oxygenates }{ }^{\mathrm{c}}\end{array}$ & $\begin{array}{r}4,523 \\
965 \\
0 \\
965 \\
0 \\
965 \\
0 \\
2,650 \\
0 \\
0 \\
894 \\
1,756 \\
908 \\
0\end{array}$ & $\begin{array}{l}8 \\
1 \\
1 \\
0 \\
0 \\
0 \\
0 \\
3 \\
3 \\
0 \\
0 \\
0 \\
4 \\
0\end{array}$ & $\begin{array}{r}7,581 \\
12 \\
0 \\
12 \\
0 \\
12 \\
0 \\
6,958 \\
1,597 \\
0 \\
1,692 \\
3,669 \\
611 \\
0\end{array}$ & $\begin{array}{l}0 \\
0 \\
0 \\
0 \\
0 \\
0 \\
0 \\
0 \\
0 \\
0 \\
0 \\
0 \\
0 \\
0\end{array}$ & $\begin{array}{r}1,140 \\
1,140 \\
0 \\
1,140 \\
50 \\
1,090 \\
0 \\
0 \\
0 \\
0 \\
0 \\
0 \\
0 \\
0\end{array}$ & $\begin{array}{r}13,252 \\
2,118 \\
1 \\
2,117 \\
50 \\
2,067 \\
0 \\
9,611 \\
1,600 \\
0 \\
2,586 \\
5,425 \\
1,523 \\
0\end{array}$ & $\begin{array}{r}427 \\
68 \\
(s) \\
68 \\
2 \\
67 \\
0 \\
310 \\
52 \\
0 \\
83 \\
175 \\
49 \\
0\end{array}$ \\
\hline 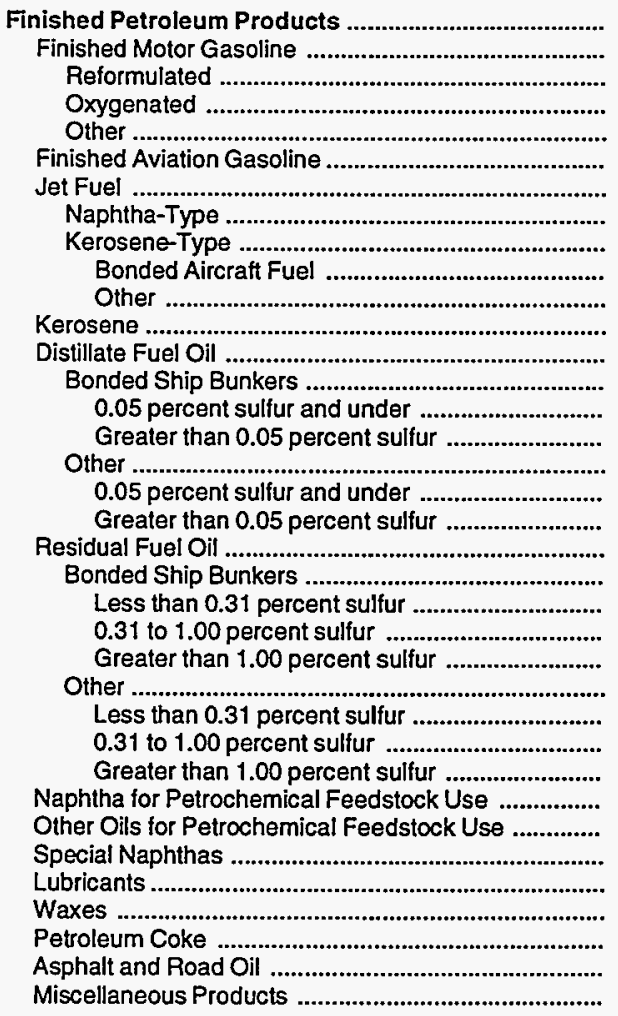 & $\begin{array}{r}26,615 \\
10,281 \\
3,976 \\
331 \\
5,974 \\
0 \\
3,034 \\
0 \\
3,034 \\
1,181 \\
1,853 \\
17 \\
5,566 \\
0 \\
0 \\
0 \\
5,566 \\
1,118 \\
4,448 \\
6,394 \\
0 \\
0 \\
0 \\
0 \\
6,394 \\
754 \\
1,187 \\
4,453 \\
69 \\
0 \\
77 \\
367 \\
24 \\
0 \\
783 \\
3\end{array}$ & $\begin{array}{r}465 \\
101 \\
0 \\
0 \\
101 \\
1 \\
0 \\
0 \\
0 \\
0 \\
0 \\
0 \\
129 \\
0 \\
0 \\
0 \\
129 \\
86 \\
43 \\
87 \\
0 \\
0 \\
0 \\
0 \\
87 \\
87 \\
0 \\
0 \\
34 \\
0 \\
45 \\
20 \\
11 \\
0 \\
34 \\
3\end{array}$ & $\begin{array}{r}4,537 \\
0 \\
0 \\
0 \\
0 \\
0 \\
20 \\
0 \\
20 \\
0 \\
20 \\
102 \\
0 \\
0 \\
0 \\
0 \\
0 \\
0 \\
0 \\
0 \\
0 \\
0 \\
0 \\
0 \\
0 \\
0 \\
0 \\
0 \\
448 \\
3,859 \\
9 \\
0 \\
2 \\
0 \\
93 \\
4\end{array}$ & $\begin{array}{r}107 \\
12 \\
0 \\
0 \\
12 \\
0 \\
0 \\
0 \\
0 \\
0 \\
0 \\
0 \\
95 \\
0 \\
0 \\
0 \\
95 \\
16 \\
79 \\
0 \\
0 \\
0 \\
0 \\
0 \\
0 \\
0 \\
0 \\
0 \\
0 \\
0 \\
0 \\
0 \\
0 \\
0 \\
0 \\
0\end{array}$ & $\begin{array}{r}166 \\
9 \\
0 \\
0 \\
9 \\
0 \\
25 \\
0 \\
25 \\
2 \\
23 \\
0 \\
27 \\
27 \\
0 \\
27 \\
0 \\
0 \\
0 \\
0 \\
0 \\
0 \\
0 \\
0 \\
0 \\
0 \\
0 \\
0 \\
59 \\
0 \\
1 \\
0 \\
5 \\
40 \\
0 \\
0\end{array}$ & $\begin{array}{r}31,890 \\
10,403 \\
3,976 \\
331 \\
6,096 \\
1 \\
3,079 \\
0 \\
3,079 \\
1,183 \\
1,896 \\
119 \\
5,817 \\
27 \\
0 \\
27 \\
5,790 \\
1,220 \\
4,570 \\
6,481 \\
0 \\
0 \\
0 \\
0 \\
6,481 \\
841 \\
1,187 \\
4,453 \\
610 \\
3,859 \\
132 \\
387 \\
42 \\
40 \\
910 \\
10\end{array}$ & $\begin{array}{r}1,029 \\
336 \\
128 \\
11 \\
197 \\
(s) \\
99 \\
0 \\
99 \\
38 \\
61 \\
4 \\
188 \\
1 \\
0 \\
1 \\
187 \\
39 \\
147 \\
209 \\
0 \\
0 \\
0 \\
0 \\
209 \\
27 \\
38 \\
144 \\
20 \\
124 \\
4 \\
12 \\
1 \\
1 \\
29 \\
(s)\end{array}$ \\
\hline Total & 82,213 & 36,939 & 148,365 & 4,798 & 7,317 & 279,632 & 9,020 \\
\hline
\end{tabular}

a Crude oil and unfinished oils are reported by the PAD District in which they are to be processed; all other products are reported by the PAD District of entry.

b Includes crude oil imported for storage in the Strategic Petroleum Reserve.

c Includes ethyl tertiary butyl ether (ETBE), tertiary amyl methyl ether (TAME), tertiary butyl alcohol (TBA), and other aliphatic alcohols and ethers intended for motor gasoline blending (e.g., isopropyl ether (IPE) or n-propanol).

(s) = Less than 500 barrels per day.

Note: Totals may not equal sum of components due to independent rounding.

Sources: Energy Information Administration (EIA) Form EIA-814, "Monthly Imports Report." 
Table 34. Year-to-Date Imports of Crude Oil and Petroleum Products by PAD District, January-March 1995

(Thousand Barrels)

\begin{tabular}{|c|c|c|c|c|c|c|c|}
\hline \multirow[b]{2}{*}{ Commodity } & \multicolumn{6}{|c|}{ Petroleum Administration for Defense Districts } & \multirow[b]{2}{*}{$\begin{array}{l}\text { Daily } \\
\text { Average }\end{array}$} \\
\hline & 1 & II & III & IV & $\mathbf{V}$ & $\begin{array}{l}\text { U.S. } \\
\text { Total }\end{array}$ & \\
\hline Crude Oll $^{\mathrm{a}, \mathrm{b}}$ & 120,432 & 98,034 & 361,396 & 11,174 & 24,031 & 615,067 & 6,834 \\
\hline 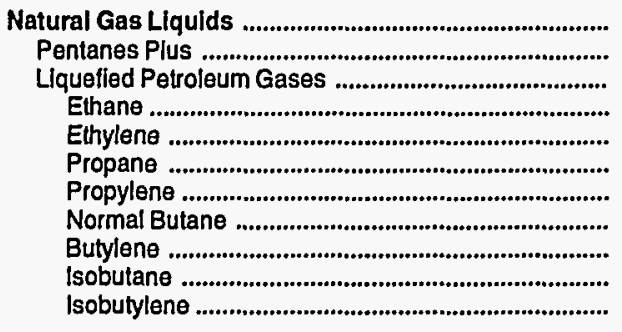 & $\begin{array}{r}2,588 \\
0 \\
2,588 \\
0 \\
0 \\
2,454 \\
0 \\
104 \\
0 \\
30 \\
0\end{array}$ & $\begin{array}{r}7,513 \\
126 \\
7,387 \\
1,008 \\
17 \\
4,941 \\
462 \\
633 \\
0 \\
326 \\
0\end{array}$ & $\begin{array}{r}3,927 \\
2,629 \\
1,298 \\
0 \\
622 \\
402 \\
0 \\
189 \\
0 \\
85 \\
0\end{array}$ & $\begin{array}{r}2,087 \\
1,343 \\
744 \\
0 \\
0 \\
485 \\
0 \\
243 \\
0 \\
16 \\
0\end{array}$ & $\begin{array}{r}500 \\
0 \\
500 \\
0 \\
0 \\
8 \\
0 \\
0 \\
0 \\
492 \\
0\end{array}$ & $\begin{array}{r}16,615 \\
4,098 \\
12,517 \\
1,008 \\
639 \\
8,290 \\
462 \\
1,169 \\
0 \\
949 \\
0\end{array}$ & $\begin{array}{r}185 \\
46 \\
139 \\
11 \\
7 \\
92 \\
5 \\
13 \\
0 \\
11 \\
0\end{array}$ \\
\hline 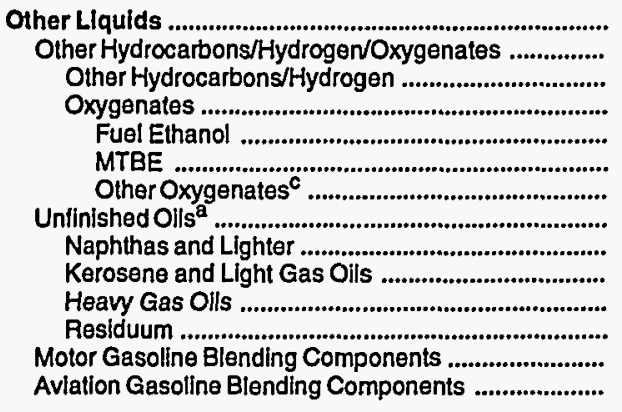 & $\begin{array}{r}14,883 \\
1,735 \\
0 \\
1,735 \\
0 \\
1,735 \\
0 \\
11,096 \\
631 \\
0 \\
5,897 \\
4,568 \\
2,052 \\
0\end{array}$ & $\begin{array}{r}14 \\
1 \\
1 \\
0 \\
0 \\
0 \\
0 \\
8 \\
8 \\
0 \\
0 \\
0 \\
5 \\
0\end{array}$ & $\begin{array}{r}18,615 \\
117 \\
0 \\
117 \\
0 \\
117 \\
0 \\
17,887 \\
4,378 \\
0 \\
4,567 \\
8,942 \\
611 \\
0\end{array}$ & $\begin{array}{l}0 \\
0 \\
0 \\
0 \\
0 \\
0 \\
0 \\
0 \\
0 \\
0 \\
0 \\
0 \\
0 \\
0\end{array}$ & $\begin{array}{r}3,442 \\
2,480 \\
0 \\
2,480 \\
90 \\
2,390 \\
0 \\
962 \\
0 \\
0 \\
79 \\
883 \\
0 \\
0\end{array}$ & $\begin{array}{r}36,954 \\
4,333 \\
1 \\
4,332 \\
90 \\
4,242 \\
0 \\
29,953 \\
5,017 \\
0 \\
10,543 \\
14,393 \\
2,668 \\
0\end{array}$ & $\begin{array}{r}411 \\
48 \\
(s) \\
48 \\
1 \\
47 \\
0 \\
333 \\
56 \\
0 \\
117 \\
160 \\
30 \\
0\end{array}$ \\
\hline 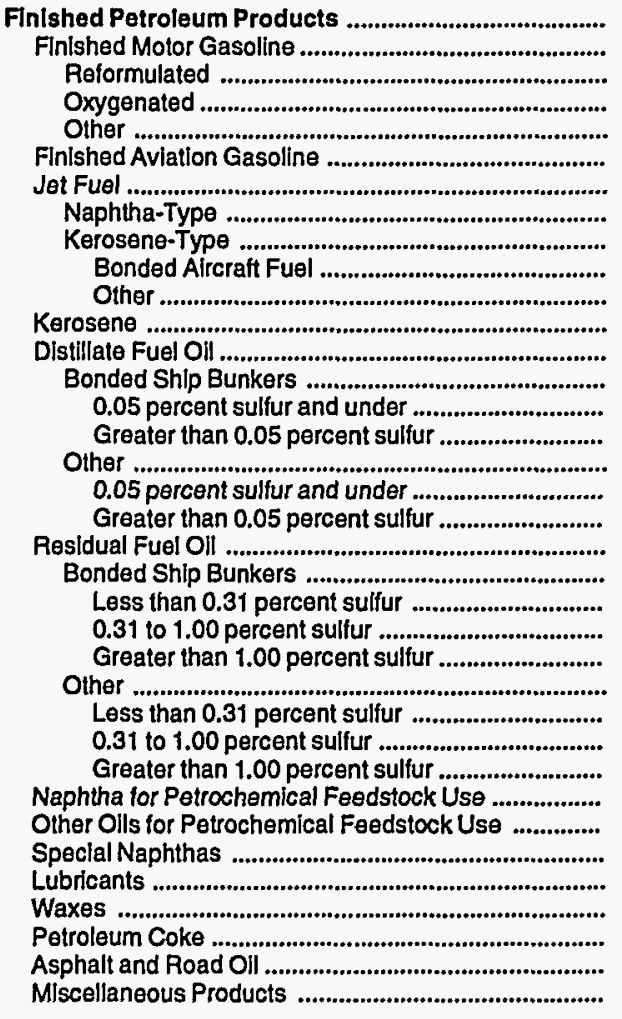 & $\begin{array}{r}72,350 \\
21,701 \\
10,274 \\
1,229 \\
10,198 \\
0 \\
8,147 \\
0 \\
8,147 \\
3,554 \\
4,593 \\
74 \\
21,316 \\
0 \\
0 \\
0 \\
21,316 \\
6,727 \\
14,589 \\
17,697 \\
0 \\
0 \\
0 \\
0 \\
17,697 \\
1,596 \\
4,434 \\
11,667 \\
125 \\
0 \\
219 \\
772 \\
85 \\
0 \\
2,205 \\
9\end{array}$ & $\begin{array}{r}1,279 \\
267 \\
0 \\
0 \\
267 \\
3 \\
0 \\
0 \\
0 \\
0 \\
0 \\
0 \\
444 \\
0 \\
0 \\
0 \\
444 \\
268 \\
176 \\
135 \\
0 \\
0 \\
0 \\
0 \\
135 \\
87 \\
0 \\
48 \\
94 \\
0 \\
217 \\
52 \\
22 \\
0 \\
34 \\
11\end{array}$ & $\begin{array}{r}16,600 \\
0 \\
0 \\
0 \\
0 \\
0 \\
202 \\
150 \\
52 \\
0 \\
52 \\
256 \\
0 \\
0 \\
0 \\
0 \\
0 \\
0 \\
0 \\
914 \\
0 \\
0 \\
0 \\
0 \\
914 \\
0 \\
0 \\
914 \\
2,072 \\
12,660 \\
99 \\
70 \\
16 \\
170 \\
128 \\
13\end{array}$ & $\begin{array}{r}383 \\
28 \\
0 \\
0 \\
28 \\
0 \\
0 \\
0 \\
0 \\
0 \\
0 \\
0 \\
355 \\
0 \\
0 \\
0 \\
355 \\
44 \\
311 \\
0 \\
0 \\
0 \\
0 \\
0 \\
0 \\
0 \\
0 \\
0 \\
0 \\
0 \\
0 \\
0 \\
0 \\
0 \\
0 \\
0\end{array}$ & $\begin{array}{r}1,008 \\
54 \\
0 \\
0 \\
54 \\
2 \\
624 \\
0 \\
624 \\
6 \\
618 \\
2 \\
92 \\
92 \\
0 \\
92 \\
0 \\
0 \\
0 \\
35 \\
0 \\
0 \\
0 \\
0 \\
35 \\
0 \\
0 \\
35 \\
59 \\
0 \\
3 \\
0 \\
17 \\
111 \\
9 \\
0\end{array}$ & $\begin{array}{r}91,620 \\
22,050 \\
10,274 \\
1,229 \\
10,547 \\
5 \\
8,973 \\
150 \\
8,823 \\
3,560 \\
5,263 \\
332 \\
22,207 \\
92 \\
0 \\
92 \\
22,115 \\
7,039 \\
15,076 \\
18,781 \\
0 \\
0 \\
0 \\
0 \\
18,781 \\
1,683 \\
4,434 \\
12,664 \\
2,350 \\
12,660 \\
538 \\
894 \\
140 \\
281 \\
2,376 \\
33\end{array}$ & $\begin{array}{r}1,018 \\
245 \\
114 \\
14 \\
117 \\
(s) \\
100 \\
2 \\
98 \\
40 \\
58 \\
4 \\
247 \\
1 \\
0 \\
1 \\
246 \\
78 \\
168 \\
209 \\
0 \\
0 \\
0 \\
0 \\
209 \\
19 \\
49 \\
141 \\
26 \\
141 \\
6 \\
10 \\
2 \\
3 \\
26 \\
(s)\end{array}$ \\
\hline Total & 210,253 & 106,840 & 400,538 & 13,644 & 28,981 & 760,256 & 8,447 \\
\hline
\end{tabular}

a Crude oil and unfinished oils are reported by the PAD District in which they are to be processed; all other products are reported by the PAD District of entry.

$b$ Includes crude oil imported for storage in the Strategic Petroleum Reserve.

c Includes ethyl tertiary butyl ether (ETBE), tertiary amyl methyl ether (TAME), tertiary butyl alcohol (TBA), and other aliphatic alcohols and ethers intended for motor gasolline blending (e.g., isopropyl ether (IPE) or n-propanoi).

(s) = Less than 500 barrels per day.

Note: Totals may not equal sum of components due to independent rounding.

Source: Energy Information Administration (EIA) Form EIA-814, "Monthly Imports Report" 
Table 35. Imports of Crude Oil and Petroleum Products into the United States by Country of Origin, ${ }^{a}$ March 1995 (Thousand Barrels)

\begin{tabular}{|c|c|c|c|c|c|c|c|c|c|c|}
\hline Country of Origin & $\begin{array}{c}\text { Crude } \\
\text { Oil }^{\mathrm{b}}\end{array}$ & $\begin{array}{c}\text { Liquefied } \\
\text { Petroleum } \\
\text { Gases } \\
\end{array}$ & $\begin{array}{c}\text { Unfinished } \\
\text { Oils }\end{array}$ & $\begin{array}{l}\text { Gasoline } \\
\text { Blending } \\
\text { Compo- } \\
\text { nents } \\
\end{array}$ & $\begin{array}{l}\text { Finished } \\
\text { Motor } \\
\text { Gasoline }\end{array}$ & Jet Fuel & $\begin{array}{c}\text { Distillate } \\
\text { Fuel Oil }\end{array}$ & $\begin{array}{c}\text { Residual } \\
\text { Fuel Oil }\end{array}$ & Kerosene & $\begin{array}{c}\text { Special } \\
\text { Naphthas }\end{array}$ \\
\hline 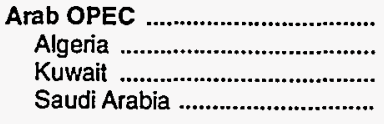 & $\begin{array}{r}52,118 \\
595 \\
7,817 \\
43,706\end{array}$ & $\begin{array}{r}402 \\
402 \\
0 \\
0\end{array}$ & $\begin{array}{r}3,628 \\
855 \\
0 \\
2,773\end{array}$ & $\begin{array}{l}0 \\
0 \\
0 \\
0\end{array}$ & $\begin{array}{l}0 \\
0 \\
0 \\
0\end{array}$ & $\begin{array}{l}0 \\
0 \\
0 \\
0\end{array}$ & $\begin{array}{l}0 \\
0 \\
0 \\
0\end{array}$ & $\begin{array}{r}650 \\
650 \\
0 \\
0\end{array}$ & $\begin{array}{l}0 \\
0 \\
0 \\
0\end{array}$ & $\begin{array}{l}0 \\
0 \\
0 \\
0\end{array}$ \\
\hline 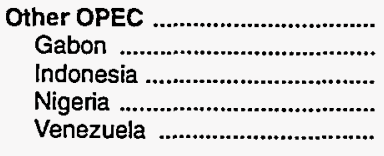 & $\begin{array}{r}64,253 \\
4,931 \\
912 \\
20,944 \\
37,466\end{array}$ & $\begin{array}{l}0 \\
0 \\
0 \\
0 \\
0\end{array}$ & $\begin{array}{r}1,821 \\
0 \\
658 \\
0 \\
1,163\end{array}$ & $\begin{array}{r}284 \\
0 \\
0 \\
0 \\
284\end{array}$ & $\begin{array}{r}1,188 \\
0 \\
0 \\
0 \\
1,188\end{array}$ & $\begin{array}{r}976 \\
0 \\
0 \\
0 \\
976\end{array}$ & $\begin{array}{r}2,225 \\
0 \\
0 \\
0 \\
2,225\end{array}$ & $\begin{array}{r}2,471 \\
0 \\
0 \\
339 \\
2,132\end{array}$ & $\begin{array}{l}0 \\
0 \\
0 \\
0 \\
0\end{array}$ & $\begin{array}{l}0 \\
0 \\
0 \\
0 \\
0\end{array}$ \\
\hline 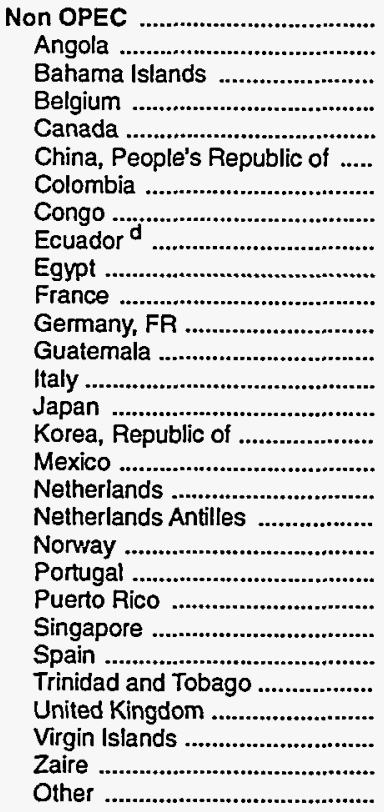 & $\begin{array}{r}113,300 \\
12,882 \\
0 \\
0 \\
27,636 \\
1,683 \\
7,380 \\
569 \\
3,212 \\
698 \\
0 \\
0 \\
206 \\
0 \\
0 \\
0 \\
29,800 \\
0 \\
0 \\
6,467 \\
0 \\
0 \\
406 \\
0 \\
2,185 \\
19,240 \\
0 \\
936 \\
0\end{array}$ & $\begin{array}{r}3,031 \\
0 \\
0 \\
0 \\
2,623 \\
0 \\
0 \\
0 \\
0 \\
0 \\
0 \\
0 \\
0 \\
0 \\
0 \\
0 \\
0 \\
0 \\
0 \\
408 \\
0 \\
0 \\
0 \\
0 \\
0 \\
0 \\
0 \\
0 \\
0\end{array}$ & $\begin{array}{r}4,162 \\
0 \\
0 \\
0 \\
237 \\
0 \\
0 \\
0 \\
0 \\
0 \\
322 \\
0 \\
0 \\
0 \\
0 \\
347 \\
0 \\
0 \\
1,221 \\
0 \\
0 \\
0 \\
642 \\
499 \\
0 \\
0 \\
894 \\
0 \\
0\end{array}$ & $\begin{array}{r}1,239 \\
0 \\
0 \\
0 \\
116 \\
0 \\
0 \\
0 \\
0 \\
0 \\
0 \\
0 \\
0 \\
0 \\
0 \\
0 \\
611 \\
247 \\
0 \\
0 \\
0 \\
0 \\
0 \\
0 \\
0 \\
0 \\
250 \\
0 \\
15\end{array}$ & $\begin{array}{r}9,215 \\
0 \\
0 \\
754 \\
2,270 \\
0 \\
0 \\
0 \\
0 \\
0 \\
0 \\
0 \\
0 \\
248 \\
0 \\
0 \\
0 \\
449 \\
0 \\
0 \\
237 \\
0 \\
0 \\
0 \\
0 \\
1,325 \\
3,845 \\
0 \\
87\end{array}$ & $\begin{array}{r}2,103 \\
0 \\
0 \\
0 \\
4 \\
0 \\
0 \\
0 \\
0 \\
0 \\
0 \\
0 \\
0 \\
0 \\
0 \\
0 \\
268 \\
0 \\
325 \\
0 \\
0 \\
0 \\
0 \\
0 \\
0 \\
0 \\
1,506 \\
0 \\
0\end{array}$ & $\begin{array}{r}3,592 \\
0 \\
0 \\
0 \\
2,298 \\
0 \\
0 \\
0 \\
0 \\
0 \\
0 \\
0 \\
0 \\
0 \\
0 \\
0 \\
0 \\
0 \\
0 \\
0 \\
0 \\
0 \\
0 \\
0 \\
0 \\
0 \\
1,294 \\
0 \\
0\end{array}$ & $\begin{array}{r}3,360 \\
355 \\
219 \\
0 \\
506 \\
0 \\
572 \\
0 \\
0 \\
0 \\
0 \\
0 \\
0 \\
0 \\
0 \\
0 \\
0 \\
0 \\
324 \\
0 \\
0 \\
0 \\
0 \\
0 \\
0 \\
0 \\
1,384 \\
0 \\
0\end{array}$ & $\begin{array}{r}119 \\
0 \\
0 \\
0 \\
17 \\
0 \\
0 \\
0 \\
0 \\
0 \\
0 \\
0 \\
0 \\
0 \\
0 \\
0 \\
102 \\
0 \\
0 \\
0 \\
0 \\
0 \\
0 \\
0 \\
0 \\
0 \\
0 \\
0 \\
0 \\
0\end{array}$ & $\begin{array}{r}132 \\
0 \\
0 \\
0 \\
123 \\
0 \\
0 \\
0 \\
0 \\
0 \\
0 \\
0 \\
0 \\
9 \\
0 \\
0 \\
0 \\
0 \\
0 \\
0 \\
0 \\
0 \\
0 \\
0 \\
0 \\
0 \\
0 \\
0 \\
0\end{array}$ \\
\hline Total & 229,671 & 3,433 & 9,611 & 1,523 & 10,403 & 3,079 & 5,817 & 6,481 & 119 & 132 \\
\hline Persian Gulf ${ }^{8}$ & 51,523 & 0 & 2,773 & 0 & $\mathbf{0}$ & 0 & $\mathbf{0}$ & 0 & 0 & 0 \\
\hline
\end{tabular}


Table 35. Imports of Crude Oil and Petroleum Products into the United States by Country of Origin, ${ }^{a}$ March 1995 (Continued)

(Thousand Barrels)

\begin{tabular}{|c|c|c|c|c|c|c|c|c|c|c|}
\hline \multirow[b]{2}{*}{ Country of Origin } & \multirow[b]{2}{*}{$\begin{array}{c}\text { Naphtha for } \\
\text { Petrochemical } \\
\text { Feedstock } \\
\text { Use }\end{array}$} & \multirow[b]{2}{*}{$\begin{array}{c}\text { Other Olls for } \\
\text { Petrochemical } \\
\text { Feedstock } \\
\text { Use }\end{array}$} & \multirow[b]{2}{*}{ Lubricants } & \multirow[b]{2}{*}{$\begin{array}{c}\text { Asphalt and } \\
\text { Road Oil }\end{array}$} & \multirow[b]{2}{*}{$\begin{array}{c}\text { Other } \\
\text { Products }\end{array}$} & \multirow[b]{2}{*}{$\begin{array}{c}\text { Total } \\
\text { Products }\end{array}$} & \multirow[b]{2}{*}{$\begin{array}{l}\text { Total } \\
\text { Crude Oil } \\
\text { and } \\
\text { Products }\end{array}$} & \multicolumn{3}{|c|}{ Daily Average } \\
\hline & & & & & & & & $\begin{array}{c}\text { Crude } \\
\text { Oil }\end{array}$ & Products & Total \\
\hline $\begin{array}{l}\text { Arab OPEC } \\
\text { Algeria } \\
\text { Kuwait } \\
\text { Saudi Arabla }\end{array}$ & $\begin{array}{l}0 \\
0 \\
0 \\
0\end{array}$ & $\begin{array}{r}3,063 \\
3,063 \\
0 \\
0\end{array}$ & $\begin{array}{l}0 \\
0 \\
0 \\
0\end{array}$ & $\begin{array}{l}0 \\
0 \\
0 \\
0\end{array}$ & $\begin{array}{r}1,625 \\
507 \\
0 \\
1,118\end{array}$ & $\begin{array}{r}9,368 \\
5,477 \\
0 \\
3,891\end{array}$ & $\begin{array}{r}61,486 \\
6,072 \\
7,817 \\
47,597\end{array}$ & $\begin{array}{r}1,681 \\
19 \\
252 \\
1,410\end{array}$ & $\begin{array}{r}302 \\
177 \\
0 \\
126\end{array}$ & $\begin{array}{r}1,983 \\
196 \\
252 \\
1,535\end{array}$ \\
\hline 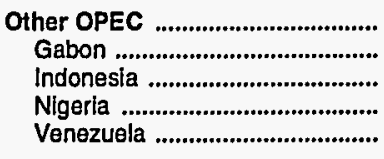 & $\begin{array}{r}277 \\
0 \\
0 \\
0 \\
277\end{array}$ & $\begin{array}{r}120 \\
0 \\
0 \\
0 \\
120\end{array}$ & $\begin{array}{l}0 \\
0 \\
0 \\
0 \\
0\end{array}$ & $\begin{array}{r}388 \\
0 \\
0 \\
0 \\
388\end{array}$ & $\begin{array}{r}261 \\
0 \\
4 \\
0 \\
257\end{array}$ & $\begin{array}{r}10,011 \\
0 \\
662 \\
339 \\
9,010\end{array}$ & $\begin{array}{r}74,264 \\
4,931 \\
1,574 \\
21,283 \\
46,476\end{array}$ & $\begin{array}{r}2,073 \\
159 \\
29 \\
676 \\
1,209\end{array}$ & $\begin{array}{r}323 \\
0 \\
21 \\
11 \\
291\end{array}$ & $\begin{array}{r}2,396 \\
159 \\
51 \\
687 \\
1,499\end{array}$ \\
\hline 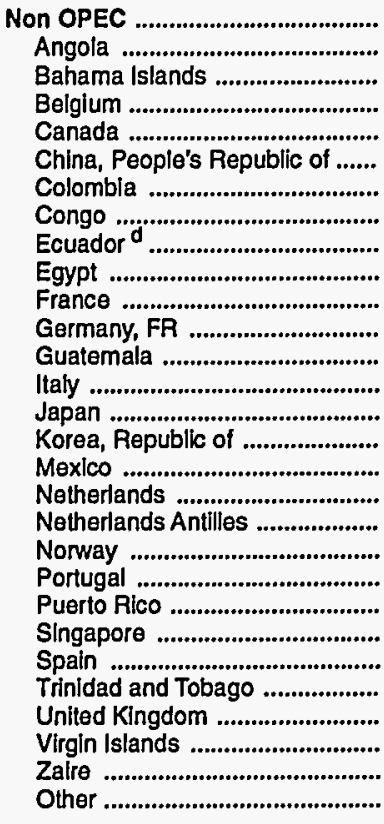 & $\begin{array}{r}333 \\
0 \\
0 \\
0 \\
98 \\
0 \\
0 \\
0 \\
0 \\
0 \\
0 \\
0 \\
0 \\
0 \\
0 \\
105 \\
0 \\
0 \\
0 \\
0 \\
0 \\
60 \\
0 \\
0 \\
0 \\
0 \\
70 \\
0 \\
0\end{array}$ & $\begin{array}{r}676 \\
0 \\
0 \\
0 \\
0 \\
0 \\
0 \\
0 \\
0 \\
0 \\
0 \\
0 \\
0 \\
0 \\
0 \\
0 \\
0 \\
0 \\
75 \\
601 \\
0 \\
0 \\
0 \\
0 \\
0 \\
0 \\
0 \\
0 \\
0\end{array}$ & $\begin{array}{r}387 \\
0 \\
0 \\
0 \\
43 \\
0 \\
0 \\
0 \\
0 \\
0 \\
0 \\
0 \\
0 \\
0 \\
0 \\
0 \\
0 \\
0 \\
0 \\
0 \\
0 \\
344 \\
0 \\
0 \\
0 \\
0 \\
0 \\
0 \\
0\end{array}$ & $\begin{array}{r}522 \\
0 \\
0 \\
0 \\
263 \\
0 \\
0 \\
0 \\
0 \\
0 \\
0 \\
0 \\
0 \\
0 \\
0 \\
0 \\
103 \\
0 \\
156 \\
0 \\
0 \\
0 \\
0 \\
0 \\
0 \\
0 \\
0 \\
0 \\
0\end{array}$ & $\begin{array}{r}1,711 \\
0 \\
0 \\
0 \\
1,153 \\
0 \\
0 \\
0 \\
0 \\
0 \\
0 \\
6 \\
0 \\
0 \\
1 \\
0 \\
304 \\
192 \\
0 \\
0 \\
0 \\
0 \\
0 \\
0 \\
0 \\
0 \\
0 \\
0 \\
55\end{array}$ & $\begin{array}{r}30,582 \\
355 \\
219 \\
754 \\
9,751 \\
0 \\
572 \\
0 \\
0 \\
0 \\
322 \\
6 \\
0 \\
257 \\
1 \\
452 \\
1,388 \\
888 \\
2,101 \\
1,009 \\
237 \\
404 \\
642 \\
499 \\
0 \\
1,325 \\
9,243 \\
0 \\
157\end{array}$ & $\begin{array}{r}143,882 \\
13,237 \\
219 \\
754 \\
37,387 \\
1,683 \\
7,952 \\
569 \\
3,212 \\
698 \\
322 \\
6 \\
206 \\
257 \\
1 \\
452 \\
31,188 \\
888 \\
2,101 \\
7,476 \\
237 \\
404 \\
1,048 \\
499 \\
2,185 \\
20,565 \\
9,243 \\
936 \\
157\end{array}$ & $\begin{array}{r}3,655 \\
416 \\
0 \\
0 \\
891 \\
54 \\
238 \\
18 \\
104 \\
23 \\
0 \\
0 \\
7 \\
0 \\
0 \\
0 \\
961 \\
0 \\
0 \\
209 \\
0 \\
0 \\
13 \\
0 \\
70 \\
621 \\
0 \\
30 \\
0\end{array}$ & $\begin{array}{r}987 \\
11 \\
7 \\
24 \\
315 \\
0 \\
18 \\
0 \\
0 \\
0 \\
10 \\
(s) \\
0 \\
8 \\
(s) \\
15 \\
45 \\
29 \\
68 \\
33 \\
8 \\
13 \\
21 \\
16 \\
0 \\
43 \\
298 \\
0 \\
5\end{array}$ & $\begin{array}{r}4,641 \\
427 \\
7 \\
24 \\
1,206 \\
54 \\
257 \\
18 \\
104 \\
23 \\
10 \\
(s) \\
7 \\
8 \\
(5) \\
15 \\
1,006 \\
29 \\
68 \\
241 \\
8 \\
13 \\
34 \\
16 \\
70 \\
663 \\
298 \\
30 \\
5\end{array}$ \\
\hline 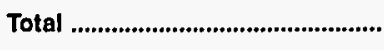 & 610 & 3,859 & 387 & 910 & 3,597 & 49,961 & 279,632 & 7,409 & 1,612 & 9,020 \\
\hline Persian Gulf ${ }^{\ominus}$ & $\mathbf{0}$ & 0 & 0 & 0 & 1,118 & 3,891 & 55,414 & 1,662 & 126 & 1,788 \\
\hline
\end{tabular}

\footnotetext{
a Crude oll and unfinished olls are reported by the PAD District in which they are to be processed; all other products are reported by the PAD District of entry.

b Includes crude oil imported for storage in the Strategic Petroleum Reserve.

c Includes avlation gasoline, aviation gasoline blending components, miscellaneous products, other hydrocarbons and oxygenates, pentanes plus, petroleum coke, and waxes.

On December 31, 1992, Ecuador withdrew as a member of OPEC. As of January 1, 1994, imports of petroleum from Ecuador appear under imports from Non-OPEC Sources.

$\theta$ Includes Bahraln, Iran, Iraq, Kuwait, Qatar, Saudi Arabla, and United Arab Emirates.

(s) = Less than 500 barrels per day.

Note: Totals may not equal sum of components due to independent rounding.

Source: Energy Information Administration (EIA) Form EIA-814, "Monthly Imports Report."
} 
Table 36. PAD District —Imports of Crude Oil and Petroleum Products by Country of Origin, ${ }^{a}$ March 1995 (Thousand Barrels)

\begin{tabular}{|c|c|c|c|c|c|c|c|c|c|c|}
\hline Country of Origin & $\begin{array}{c}\text { Crude } \\
\text { OHb }^{\mathrm{b}}\end{array}$ & $\begin{array}{l}\text { Liquefied } \\
\text { Petroleum } \\
\text { Gases } \\
\end{array}$ & $\begin{array}{c}\text { Unfinished } \\
\text { Olls }\end{array}$ & $\begin{array}{c}\text { Gasoline } \\
\text { Blending } \\
\text { Compo- } \\
\text { nents }\end{array}$ & $\begin{array}{l}\text { Finished } \\
\text { Motor } \\
\text { Gasoline }\end{array}$ & Jet Fuel & $\begin{array}{l}\text { Distillate } \\
\text { Fuel Oil }\end{array}$ & $\begin{array}{l}\text { Residual } \\
\text { Fuel Oil }\end{array}$ & Kerosene & $\begin{array}{c}\text { Special } \\
\text { Naphthas }\end{array}$ \\
\hline 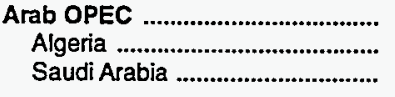 & $\begin{array}{r}7,613 \\
0 \\
7,613\end{array}$ & $\begin{array}{l}0 \\
0 \\
0\end{array}$ & $\begin{array}{r}1,114 \\
507 \\
607\end{array}$ & $\begin{array}{l}0 \\
0 \\
0\end{array}$ & $\begin{array}{l}0 \\
0 \\
0\end{array}$ & $\begin{array}{l}0 \\
0 \\
0\end{array}$ & $\begin{array}{l}0 \\
0 \\
0\end{array}$ & $\begin{array}{r}650 \\
650 \\
0\end{array}$ & $\begin{array}{l}0 \\
0 \\
0\end{array}$ & $\begin{array}{l}0 \\
0 \\
0\end{array}$ \\
\hline $\begin{array}{l}\text { Other OPEC } \\
\text { Gabon } \\
\text { Nigeria } \\
\text { Venezuela }\end{array}$ & $\begin{array}{r}19,942 \\
3,487 \\
12,151 \\
4,304\end{array}$ & $\begin{array}{l}0 \\
0 \\
0 \\
0\end{array}$ & $\begin{array}{l}0 \\
0 \\
0 \\
0\end{array}$ & $\begin{array}{r}284 \\
0 \\
0 \\
284\end{array}$ & $\begin{array}{r}1,188 \\
0 \\
0 \\
1,188\end{array}$ & $\begin{array}{r}976 \\
0 \\
0 \\
976\end{array}$ & $\begin{array}{r}2,225 \\
0 \\
0 \\
2,225\end{array}$ & $\begin{array}{r}2,471 \\
0 \\
339 \\
2,132\end{array}$ & $\begin{array}{l}0 \\
0 \\
0 \\
0\end{array}$ & $\begin{array}{l}0 \\
0 \\
0 \\
0\end{array}$ \\
\hline 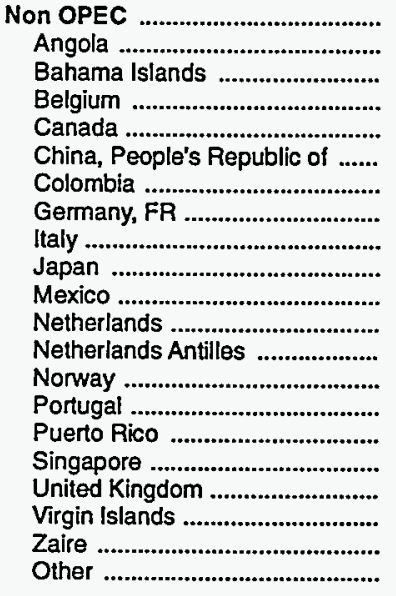 & $\begin{array}{r}22,764 \\
6,856 \\
0 \\
0 \\
1,723 \\
670 \\
500 \\
0 \\
0 \\
0 \\
2,161 \\
0 \\
0 \\
4,114 \\
0 \\
0 \\
0 \\
6,211 \\
0 \\
529 \\
0\end{array}$ & $\begin{array}{r}756 \\
0 \\
0 \\
0 \\
348 \\
0 \\
0 \\
0 \\
0 \\
0 \\
0 \\
0 \\
0 \\
408 \\
0 \\
0 \\
0 \\
0 \\
0 \\
0 \\
0\end{array}$ & $\begin{array}{r}1,536 \\
0 \\
0 \\
0 \\
0 \\
0 \\
0 \\
0 \\
0 \\
0 \\
0 \\
0 \\
0 \\
0 \\
0 \\
0 \\
642 \\
0 \\
894 \\
0 \\
0\end{array}$ & $\begin{array}{r}624 \\
0 \\
0 \\
0 \\
112 \\
0 \\
0 \\
0 \\
0 \\
0 \\
0 \\
247 \\
0 \\
0 \\
0 \\
0 \\
0 \\
0 \\
250 \\
0 \\
15\end{array}$ & $\begin{array}{r}9,093 \\
0 \\
0 \\
754 \\
2,148 \\
0 \\
0 \\
0 \\
248 \\
0 \\
0 \\
449 \\
0 \\
0 \\
237 \\
0 \\
0 \\
1,325 \\
3,845 \\
0 \\
87\end{array}$ & $\begin{array}{r}2,058 \\
0 \\
0 \\
0 \\
2 \\
0 \\
0 \\
0 \\
0 \\
0 \\
225 \\
0 \\
325 \\
0 \\
0 \\
0 \\
0 \\
0 \\
1,506 \\
0 \\
0\end{array}$ & $\begin{array}{r}3,341 \\
0 \\
0 \\
0 \\
2,047 \\
0 \\
0 \\
0 \\
0 \\
0 \\
0 \\
0 \\
0 \\
0 \\
0 \\
0 \\
0 \\
0 \\
1,294 \\
0 \\
0\end{array}$ & $\begin{array}{r}3,273 \\
355 \\
219 \\
0 \\
419 \\
0 \\
572 \\
0 \\
0 \\
0 \\
0 \\
0 \\
324 \\
0 \\
0 \\
0 \\
0 \\
0 \\
1,384 \\
0 \\
0\end{array}$ & $\begin{array}{r}17 \\
0 \\
0 \\
0 \\
17 \\
0 \\
0 \\
0 \\
0 \\
0 \\
0 \\
0 \\
0 \\
0 \\
0 \\
0 \\
0 \\
0 \\
0 \\
0 \\
0\end{array}$ & $\begin{array}{r}77 \\
0 \\
0 \\
0 \\
77 \\
0 \\
0 \\
0 \\
0 \\
0 \\
0 \\
0 \\
0 \\
0 \\
0 \\
0 \\
0 \\
0 \\
0 \\
0 \\
0\end{array}$ \\
\hline Total & 50,319 & 756 & 2,650 & 908 & 10,281 & 3,034 & 5,566 & 6,394 & 17 & $\pi$ \\
\hline Persian Gulf ${ }^{\theta}$ & 7,613 & $\mathbf{0}$ & 607 & 0 & 0 & $\mathbf{0}$ & 0 & 0 & $\mathbf{0}$ & $\mathbf{0}$ \\
\hline
\end{tabular}


Table 36. PAD District 1-Imports of Crude Oil and Petroleum Products by Country of Origin, ${ }^{a}$ March 1995 (Continued)

(Thousand Barrels)

\begin{tabular}{|c|c|c|c|c|c|c|c|c|c|c|}
\hline \multirow[b]{2}{*}{ Country of Origin } & \multirow[b]{2}{*}{$\begin{array}{c}\text { Naphtha for } \\
\text { Petrochemical } \\
\text { Feedstock } \\
\text { Use }\end{array}$} & \multirow[b]{2}{*}{$\begin{array}{c}\text { Other Oils for } \\
\text { Petrochemical } \\
\text { Feedstock } \\
\text { Use }\end{array}$} & \multirow[b]{2}{*}{ Lubricants } & \multirow[b]{2}{*}{$\begin{array}{c}\text { Asphatt and } \\
\text { Road Oil }\end{array}$} & \multirow[b]{2}{*}{$\begin{array}{c}\text { Other } \\
\text { Products }\end{array}$} & \multirow[b]{2}{*}{$\begin{array}{c}\text { Total } \\
\text { Products }\end{array}$} & \multirow[b]{2}{*}{$\begin{array}{l}\text { Tolal } \\
\text { Crude Oil } \\
\text { and } \\
\text { Products }\end{array}$} & \multicolumn{3}{|c|}{ Dally Average } \\
\hline & & & & & & & & $\begin{array}{c}\text { Crude } \\
\text { Oil } \\
\end{array}$ & Products & Total \\
\hline 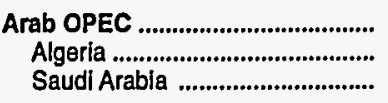 & $\begin{array}{l}0 \\
0 \\
0\end{array}$ & $\begin{array}{l}0 \\
0 \\
0\end{array}$ & $\begin{array}{l}0 \\
0 \\
0\end{array}$ & $\begin{array}{l}0 \\
0 \\
0\end{array}$ & $\begin{array}{r}645 \\
0 \\
645\end{array}$ & $\begin{array}{l}2,409 \\
1,157 \\
1,252\end{array}$ & $\begin{array}{r}10,022 \\
1,157 \\
8,865\end{array}$ & $\begin{array}{r}246 \\
0 \\
246\end{array}$ & $\begin{array}{l}78 \\
37 \\
40\end{array}$ & $\begin{array}{r}323 \\
37 \\
286\end{array}$ \\
\hline 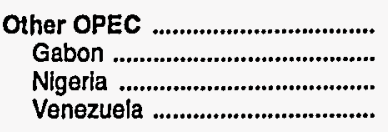 & $\begin{array}{l}0 \\
0 \\
0 \\
0\end{array}$ & $\begin{array}{l}0 \\
0 \\
0 \\
0\end{array}$ & $\begin{array}{l}0 \\
0 \\
0 \\
0\end{array}$ & $\begin{array}{r}355 \\
0 \\
0 \\
355\end{array}$ & $\begin{array}{r}128 \\
0 \\
0 \\
128\end{array}$ & $\begin{array}{r}7,627 \\
0 \\
339 \\
7,288\end{array}$ & $\begin{array}{r}27,569 \\
3,487 \\
12,490 \\
11,592\end{array}$ & $\begin{array}{l}643 \\
112 \\
392 \\
139\end{array}$ & $\begin{array}{r}246 \\
0 \\
11 \\
235\end{array}$ & $\begin{array}{r}889 \\
112 \\
403 \\
374\end{array}$ \\
\hline 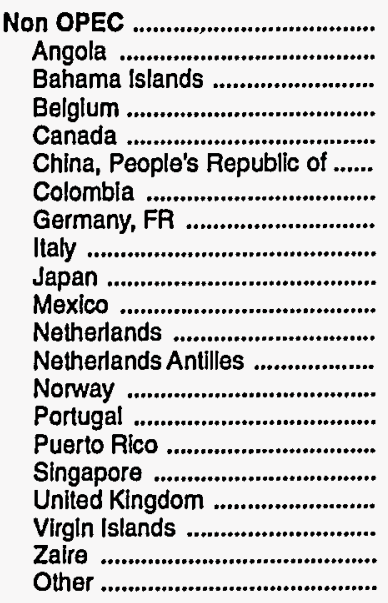 & $\begin{array}{r}69 \\
0 \\
0 \\
0 \\
9 \\
0 \\
0 \\
0 \\
0 \\
0 \\
0 \\
0 \\
0 \\
0 \\
0 \\
60 \\
0 \\
0 \\
0 \\
0 \\
0\end{array}$ & $\begin{array}{l}0 \\
0 \\
0 \\
0 \\
0 \\
0 \\
0 \\
0 \\
0 \\
0 \\
0 \\
0 \\
0 \\
0 \\
0 \\
0 \\
0 \\
0 \\
0 \\
0 \\
0\end{array}$ & $\begin{array}{r}367 \\
0 \\
0 \\
0 \\
23 \\
0 \\
0 \\
0 \\
0 \\
0 \\
0 \\
0 \\
0 \\
0 \\
0 \\
344 \\
0 \\
0 \\
0 \\
0 \\
0\end{array}$ & $\begin{array}{r}428 \\
0 \\
0 \\
0 \\
229 \\
0 \\
0 \\
0 \\
0 \\
0 \\
103 \\
0 \\
96 \\
0 \\
0 \\
0 \\
0 \\
0 \\
0 \\
0 \\
0\end{array}$ & $\begin{array}{r}219 \\
0 \\
0 \\
0 \\
17 \\
0 \\
0 \\
6 \\
0 \\
1 \\
0 \\
192 \\
0 \\
0 \\
0 \\
0 \\
0 \\
0 \\
0 \\
0 \\
3\end{array}$ & $\begin{array}{r}21,858 \\
355 \\
219 \\
754 \\
5,448 \\
0 \\
572 \\
6 \\
248 \\
1 \\
328 \\
888 \\
745 \\
408 \\
237 \\
404 \\
642 \\
1,325 \\
9,173 \\
0 \\
105\end{array}$ & $\begin{array}{r}44,622 \\
7,211 \\
219 \\
754 \\
7,171 \\
670 \\
1,072 \\
6 \\
248 \\
1 \\
2,489 \\
888 \\
745 \\
4,522 \\
237 \\
404 \\
642 \\
7,536 \\
9,173 \\
529 \\
105\end{array}$ & $\begin{array}{r}734 \\
221 \\
0 \\
0 \\
56 \\
22 \\
16 \\
0 \\
0 \\
0 \\
70 \\
0 \\
0 \\
133 \\
0 \\
0 \\
0 \\
200 \\
0 \\
17 \\
0\end{array}$ & $\begin{array}{r}705 \\
11 \\
7 \\
24 \\
176 \\
0 \\
18 \\
(s) \\
8 \\
(s) \\
11 \\
29 \\
24 \\
13 \\
8 \\
13 \\
21 \\
43 \\
296 \\
0 \\
3\end{array}$ & $\begin{array}{r}1,439 \\
233 \\
7 \\
24 \\
231 \\
22 \\
35 \\
(s) \\
8 \\
1 s) \\
80 \\
29 \\
24 \\
146 \\
8 \\
13 \\
21 \\
243 \\
296 \\
17 \\
3\end{array}$ \\
\hline Total & 69 & $\mathbf{0}$ & 367 & 783 & 992 & 31,894 & 82,213 & 1,623 & 1,029 & 2,652 \\
\hline Perslan Gulf ${ }^{\theta}$ & 0 & 0 & 0 & 0 & 645 & 1,252 & 8,865 & 246 & 40 & 286 \\
\hline
\end{tabular}

a Crude oll and unfinished oils are reported by the PAD District in which they are to be processed; all other products are reported by the PAD District of entry

b Includes crude oll imported for storage in the Strategic Petroleum Reserve.

c Includes aviation gasoline, aviation gasoline blending components, miscellaneous products, other hydrocarbons and oxygenates, pentanes plus, petroleum coke, and waxes.

On December 31, 1992, Ecuador withdrew as a member of OPEC. As of January 1, 1994, imports of petroleum from Ecuador appear under imports from Non-OPEC Sources.

- Includes Bahrain, Iran, Iraq, Kuwait, Qatar, Saudi Arabia, and United Arab Emirates.

(s) = Less than 500 barrels per day.

Note: Totals may not equal sum of components due to independent rounding.

Source: Energy Information Administration (EIA) Form EIA-814, "Monthly Imports Report." 
Table 37. PAD District II-Imports of Crude Oil and Petroleum Products by Country of Origin, ${ }^{a}$ March 1995

(Thousand Barrels)

\begin{tabular}{|c|c|c|c|c|c|c|c|c|c|c|}
\hline 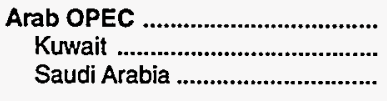 & $\begin{array}{l}2,907 \\
1,590 \\
1,317\end{array}$ & $\begin{array}{l}0 \\
0 \\
0\end{array}$ & $\begin{array}{l}0 \\
0 \\
0\end{array}$ & $\begin{array}{l}0 \\
0 \\
0\end{array}$ & $\begin{array}{l}0 \\
0 \\
0\end{array}$ & $\begin{array}{l}0 \\
0 \\
0\end{array}$ & $\begin{array}{l}0 \\
0 \\
0\end{array}$ & $\begin{array}{l}0 \\
0 \\
0\end{array}$ & $\begin{array}{l}0 \\
0 \\
0\end{array}$ & $\begin{array}{l}0 \\
0 \\
0\end{array}$ \\
\hline 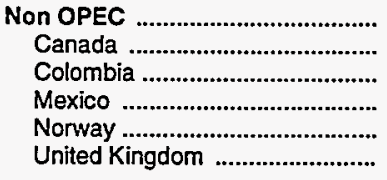 & $\begin{array}{r}24,874 \\
20,381 \\
530 \\
400 \\
574 \\
2,989\end{array}$ & $\begin{array}{r}2,043 \\
2,043 \\
0 \\
0 \\
0 \\
0\end{array}$ & $\begin{array}{l}3 \\
3 \\
0 \\
0 \\
0 \\
0\end{array}$ & $\begin{array}{l}4 \\
4 \\
0 \\
0 \\
0 \\
0\end{array}$ & $\begin{array}{r}101 \\
101 \\
0 \\
0 \\
0 \\
0\end{array}$ & $\begin{array}{l}0 \\
0 \\
0 \\
0 \\
0 \\
0\end{array}$ & $\begin{array}{r}129 \\
129 \\
0 \\
0 \\
0 \\
0\end{array}$ & $\begin{array}{r}87 \\
87 \\
0 \\
0 \\
0 \\
0\end{array}$ & $\begin{array}{l}0 \\
0 \\
0 \\
0 \\
0 \\
0\end{array}$ & $\begin{array}{r}45 \\
45 \\
0 \\
0 \\
0 \\
0\end{array}$ \\
\hline
\end{tabular}

See footnotes at end of table. 
Table 37. PAD District II-Imports of Crude Oil and Petroleum Products by Country of Origin, ${ }^{a}$ March 1995 (Continued)

(Thousand Barrels)

\begin{tabular}{|c|c|c|c|c|c|c|c|c|c|c|}
\hline \multirow[b]{2}{*}{ Country of Origin } & \multirow[b]{2}{*}{$\begin{array}{c}\text { Naphtha for } \\
\text { Petrochemical } \\
\text { Feedstock } \\
\text { Use }\end{array}$} & \multirow[b]{2}{*}{$\begin{array}{c}\text { Other Oils for } \\
\text { Petrochemical } \\
\text { Feedstock } \\
\text { Use }\end{array}$} & \multirow[b]{2}{*}{ Lubricants } & \multirow[b]{2}{*}{$\begin{array}{c}\text { Asphalt and } \\
\text { Road Oil }\end{array}$} & \multirow[b]{2}{*}{$\begin{array}{c}\text { Other } \\
\text { Products }\end{array}$} & \multirow[b]{2}{*}{$\begin{array}{c}\text { Total } \\
\text { Products }\end{array}$} & \multirow[b]{2}{*}{$\begin{array}{l}\text { Total } \\
\text { Crude Oll } \\
\text { and } \\
\text { Products }\end{array}$} & \multicolumn{3}{|c|}{ Daily Average } \\
\hline & & & & & & & & $\begin{array}{c}\text { Crude } \\
\text { Oil } \\
\end{array}$ & Products & Total \\
\hline 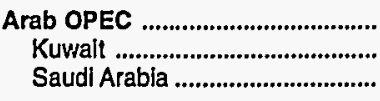 & $\begin{array}{l}0 \\
0 \\
0\end{array}$ & $\begin{array}{l}0 \\
0 \\
0\end{array}$ & $\begin{array}{l}0 \\
0 \\
0\end{array}$ & $\begin{array}{l}0 \\
0 \\
0\end{array}$ & $\begin{array}{l}0 \\
0 \\
0\end{array}$ & $\begin{array}{l}0 \\
0 \\
0\end{array}$ & $\begin{array}{l}2,907 \\
1,590 \\
1,317\end{array}$ & $\begin{array}{l}94 \\
51 \\
42\end{array}$ & $\begin{array}{l}0 \\
0 \\
0\end{array}$ & $\begin{array}{l}94 \\
51 \\
42\end{array}$ \\
\hline $\begin{array}{l}\text { Other OPEC } \\
\text { Nigeria } \\
\text { Venezuela }\end{array}$ & $\begin{array}{l}0 \\
0 \\
0\end{array}$ & $\begin{array}{l}0 \\
0 \\
0\end{array}$ & $\begin{array}{l}0 \\
0 \\
0\end{array}$ & $\begin{array}{l}0 \\
0 \\
0\end{array}$ & $\begin{array}{l}0 \\
0 \\
0\end{array}$ & $\begin{array}{l}0 \\
0 \\
0\end{array}$ & $\begin{array}{r}6,609 \\
992 \\
5,617\end{array}$ & $\begin{array}{r}213 \\
32 \\
181\end{array}$ & $\begin{array}{l}0 \\
0 \\
0\end{array}$ & $\begin{array}{r}213 \\
32 \\
181\end{array}$ \\
\hline 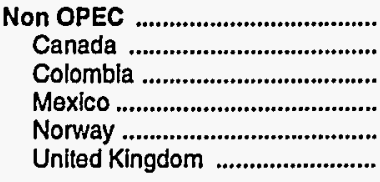 & $\begin{array}{r}34 \\
34 \\
0 \\
0 \\
0 \\
0\end{array}$ & $\begin{array}{l}0 \\
0 \\
0 \\
0 \\
0 \\
0\end{array}$ & $\begin{array}{r}20 \\
20 \\
0 \\
0 \\
0 \\
0\end{array}$ & $\begin{array}{r}34 \\
34 \\
0 \\
0 \\
0 \\
0\end{array}$ & $\begin{array}{r}49 \\
49 \\
0 \\
0 \\
0 \\
0\end{array}$ & $\begin{array}{r}2,549 \\
2,549 \\
0 \\
0 \\
0 \\
0\end{array}$ & $\begin{array}{r}27,423 \\
22,930 \\
530 \\
400 \\
574 \\
2,989\end{array}$ & $\begin{array}{r}802 \\
657 \\
17 \\
13 \\
19 \\
96\end{array}$ & $\begin{array}{r}82 \\
82 \\
0 \\
0 \\
0 \\
0\end{array}$ & $\begin{array}{r}885 \\
740 \\
17 \\
13 \\
19 \\
96\end{array}$ \\
\hline Total & 34 & 0 & 20 & 34 & 49 & 2,549 & 36,939 & 1,109 & 82 & 1,192 \\
\hline Persian Gulf ${ }^{\circ}$ & $\mathbf{0}$ & 0 & $\mathbf{0}$ & 0 & $\mathbf{0}$ & $\mathbf{0}$ & 2,907 & 94 & 0 & 94 \\
\hline
\end{tabular}

a Crude oil and unfinished oils are reported by the PAD District in which they are to be processed; all other products are reported by the PAD District of entry.

b Includes crude oil imported for storage in the Strategic Petroleum Reserve.

c Includes aviation gasoline, aviation gasoline blending components, miscellaneous products, other hydrocarbons and oxygenates, pentanes plus, petroleum coke, and waxes. Sources.

$\theta$ Includes Bahrain, Iran, Iraq, Kuwait, Qatar, Saudi Arabia, and United Arab Emirates.

(s) = Less than 500 barrels per day.

Note: Totals may not equal sum of components due to independent rounding.

Source: Energy Information Administration (EIA) Form EIA-814, "Monthly Imports Report." 
Table 38. PAD District III-Imports of Crude Oil and Petroleum Products by Country of Origin, ${ }^{a}$ March 1995 (Thousand Barrels)

\begin{tabular}{|c|c|c|c|c|c|c|c|c|c|c|}
\hline Country of Origin & $\begin{array}{c}\text { Crude } \\
\text { Oijb }\end{array}$ & $\begin{array}{c}\text { Liquefied } \\
\text { Petroleum } \\
\text { Gases }\end{array}$ & $\begin{array}{c}\text { Unfinished } \\
\text { Oils } \\
\end{array}$ & $\begin{array}{c}\text { Gasoline } \\
\text { Blending } \\
\text { Compo- } \\
\text { nents } \\
\end{array}$ & $\begin{array}{l}\text { Finished } \\
\text { Motor } \\
\text { Gasoline }\end{array}$ & Jet Fuel & $\begin{array}{c}\text { Distillate } \\
\text { Fuel Oil }\end{array}$ & $\begin{array}{l}\text { Residual } \\
\text { Fuel Oil }\end{array}$ & Kerosene & $\begin{array}{c}\text { Special } \\
\text { Naphthas }\end{array}$ \\
\hline 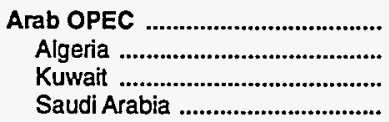 & $\begin{array}{r}40,492 \\
595 \\
5,121 \\
34,776\end{array}$ & $\begin{array}{r}402 \\
402 \\
0 \\
0\end{array}$ & $\begin{array}{r}2,514 \\
348 \\
0 \\
2,166\end{array}$ & $\begin{array}{l}0 \\
0 \\
0 \\
0\end{array}$ & $\begin{array}{l}0 \\
0 \\
0 \\
0\end{array}$ & $\begin{array}{l}0 \\
0 \\
0 \\
0\end{array}$ & $\begin{array}{l}0 \\
0 \\
0 \\
0\end{array}$ & $\begin{array}{l}0 \\
0 \\
0 \\
0\end{array}$ & $\begin{array}{l}0 \\
0 \\
0 \\
0\end{array}$ & $\begin{array}{l}0 \\
0 \\
0 \\
0\end{array}$ \\
\hline 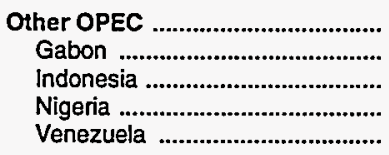 & $\begin{array}{r}36,270 \\
1,444 \\
0 \\
7,801 \\
27,025\end{array}$ & $\begin{array}{l}0 \\
0 \\
0 \\
0 \\
0\end{array}$ & $\begin{array}{r}1,821 \\
0 \\
658 \\
0 \\
1,163\end{array}$ & $\begin{array}{l}0 \\
0 \\
0 \\
0 \\
0\end{array}$ & $\begin{array}{l}0 \\
0 \\
0 \\
0 \\
0\end{array}$ & $\begin{array}{l}0 \\
0 \\
0 \\
0 \\
0\end{array}$ & $\begin{array}{l}0 \\
0 \\
0 \\
0 \\
0\end{array}$ & $\begin{array}{l}0 \\
0 \\
0 \\
0 \\
0\end{array}$ & $\begin{array}{l}0 \\
0 \\
0 \\
0 \\
0\end{array}$ & $\begin{array}{l}0 \\
0 \\
0 \\
0 \\
0\end{array}$ \\
\hline 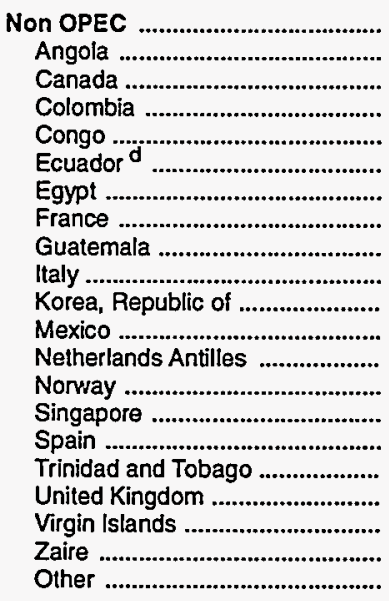 & $\begin{array}{r}58,277 \\
6,026 \\
0 \\
6,350 \\
569 \\
2,372 \\
698 \\
0 \\
206 \\
0 \\
0 \\
27,239 \\
0 \\
1,779 \\
406 \\
0 \\
2,185 \\
10,040 \\
0 \\
407 \\
0\end{array}$ & $\begin{array}{l}0 \\
0 \\
0 \\
0 \\
0 \\
0 \\
0 \\
0 \\
0 \\
0 \\
0 \\
0 \\
0 \\
0 \\
0 \\
0 \\
0 \\
0 \\
0 \\
0 \\
0\end{array}$ & $\begin{array}{r}2,623 \\
0 \\
234 \\
0 \\
0 \\
0 \\
0 \\
322 \\
0 \\
0 \\
347 \\
0 \\
1,221 \\
0 \\
0 \\
499 \\
0 \\
0 \\
0 \\
0 \\
0\end{array}$ & $\begin{array}{r}611 \\
0 \\
0 \\
0 \\
0 \\
0 \\
0 \\
0 \\
0 \\
0 \\
0 \\
611 \\
0 \\
0 \\
0 \\
0 \\
0 \\
0 \\
0 \\
0 \\
0\end{array}$ & $\begin{array}{l}0 \\
0 \\
0 \\
0 \\
0 \\
0 \\
0 \\
0 \\
0 \\
0 \\
0 \\
0 \\
0 \\
0 \\
0 \\
0 \\
0 \\
0 \\
0 \\
0 \\
0\end{array}$ & $\begin{array}{r}20 \\
0 \\
0 \\
0 \\
0 \\
0 \\
0 \\
0 \\
0 \\
0 \\
0 \\
20 \\
0 \\
0 \\
0 \\
0 \\
0 \\
0 \\
0 \\
0 \\
0\end{array}$ & $\begin{array}{l}0 \\
0 \\
0 \\
0 \\
0 \\
0 \\
0 \\
0 \\
0 \\
0 \\
0 \\
0 \\
0 \\
0 \\
0 \\
0 \\
0 \\
0 \\
0 \\
0 \\
0\end{array}$ & $\begin{array}{l}0 \\
0 \\
0 \\
0 \\
0 \\
0 \\
0 \\
0 \\
0 \\
0 \\
0 \\
0 \\
0 \\
0 \\
0 \\
0 \\
0 \\
0 \\
0 \\
0 \\
0\end{array}$ & $\begin{array}{r}102 \\
0 \\
0 \\
0 \\
0 \\
0 \\
0 \\
0 \\
0 \\
0 \\
0 \\
102 \\
0 \\
0 \\
0 \\
0 \\
0 \\
0 \\
0 \\
0 \\
0\end{array}$ & $\begin{array}{l}9 \\
0 \\
0 \\
0 \\
0 \\
0 \\
0 \\
0 \\
0 \\
9 \\
0 \\
0 \\
0 \\
0 \\
0 \\
0 \\
0 \\
0 \\
0 \\
0 \\
0\end{array}$ \\
\hline 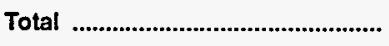 & 135,039 & 402 & 6,958 & 611 & 0 & 20 & 0 & 0 & 102 & 9 \\
\hline Persian Gulf ${ }^{e}$ & 39,897 & 0 & 2,166 & 0 & 0 & 0 & 0 & 0 & 0 & 0 \\
\hline
\end{tabular}


Table 38. PAD District III-Imports of Crude Oil and Petroleum Products by Country of Origin, ${ }^{\mathrm{a}}$ March 1995 (Continued)

(Thousand Barrels)

\begin{tabular}{|c|c|c|c|c|c|c|c|c|c|c|}
\hline \multirow[b]{2}{*}{ Country of Origin } & \multirow{2}{*}{$\begin{array}{c}\text { Naphtha for } \\
\text { Petrochemical } \\
\text { Feedstock } \\
\text { Use }\end{array}$} & \multirow[b]{2}{*}{$\begin{array}{c}\text { Other Oils for } \\
\text { Petrochemical } \\
\text { Feedstock } \\
\text { Use }\end{array}$} & \multirow[b]{2}{*}{ Lubricants } & \multirow[b]{2}{*}{$\begin{array}{c}\text { Asphatt and } \\
\text { Road Oll }\end{array}$} & \multirow[b]{2}{*}{$\begin{array}{c}\text { Other } \\
\text { Products }\end{array}$} & \multirow[b]{2}{*}{$\begin{array}{c}\text { Total } \\
\text { Products }\end{array}$} & \multirow[b]{2}{*}{$\begin{array}{l}\text { Total } \\
\text { Crude Oll } \\
\text { and } \\
\text { Products } \\
\end{array}$} & \multicolumn{3}{|c|}{ Daily Average } \\
\hline & & & & & & & & $\begin{array}{c}\text { Crude } \\
\text { Oil }\end{array}$ & Products & Total \\
\hline 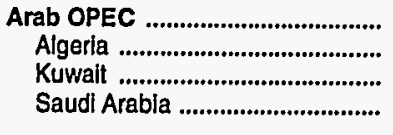 & $\begin{array}{l}0 \\
0 \\
0 \\
0\end{array}$ & $\begin{array}{r}3,063 \\
3,063 \\
0 \\
0\end{array}$ & $\begin{array}{l}0 \\
0 \\
0 \\
0\end{array}$ & $\begin{array}{l}0 \\
0 \\
0 \\
0\end{array}$ & $\begin{array}{r}519 \\
507 \\
0 \\
12\end{array}$ & $\begin{array}{r}6,498 \\
4,320 \\
0 \\
2,178\end{array}$ & $\begin{array}{r}46,990 \\
4,915 \\
5,121 \\
36,954\end{array}$ & $\begin{array}{r}1,306 \\
19 \\
165 \\
1,122\end{array}$ & $\begin{array}{r}210 \\
139 \\
0 \\
70\end{array}$ & $\begin{array}{r}1,516 \\
159 \\
165 \\
1,192\end{array}$ \\
\hline 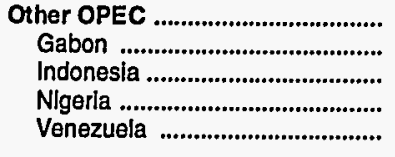 & $\begin{array}{r}277 \\
0 \\
0 \\
0 \\
277\end{array}$ & $\begin{array}{r}120 \\
0 \\
0 \\
0 \\
120\end{array}$ & $\begin{array}{l}0 \\
0 \\
0 \\
0 \\
0\end{array}$ & $\begin{array}{r}33 \\
0 \\
0 \\
0 \\
33\end{array}$ & $\begin{array}{l}4 \\
0 \\
4 \\
0 \\
0\end{array}$ & $\begin{array}{r}2,255 \\
0 \\
662 \\
0 \\
1,593\end{array}$ & $\begin{array}{r}38,525 \\
1,444 \\
662 \\
7,801 \\
28,618\end{array}$ & $\begin{array}{r}1,170 \\
47 \\
0 \\
252 \\
872\end{array}$ & $\begin{array}{r}73 \\
0 \\
21 \\
0 \\
51\end{array}$ & $\begin{array}{r}1,243 \\
47 \\
21 \\
252 \\
923\end{array}$ \\
\hline 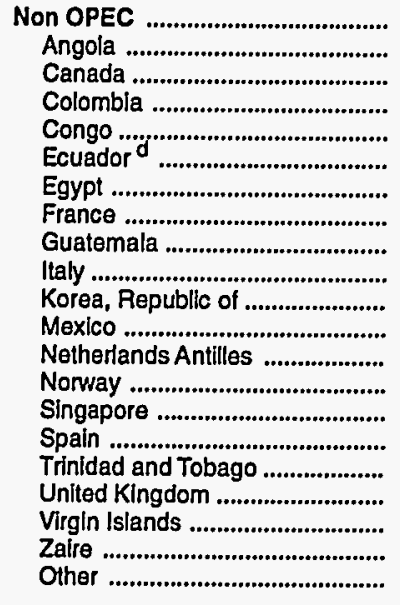 & $\begin{array}{r}171 \\
0 \\
55 \\
0 \\
0 \\
0 \\
0 \\
0 \\
0 \\
0 \\
46 \\
0 \\
0 \\
0 \\
0 \\
0 \\
0 \\
0 \\
70 \\
0 \\
0\end{array}$ & $\begin{array}{r}676 \\
0 \\
0 \\
0 \\
0 \\
0 \\
0 \\
0 \\
0 \\
0 \\
0 \\
0 \\
75 \\
601 \\
0 \\
0 \\
0 \\
0 \\
0 \\
0 \\
0\end{array}$ & $\begin{array}{l}0 \\
0 \\
0 \\
0 \\
0 \\
0 \\
0 \\
0 \\
0 \\
0 \\
0 \\
0 \\
0 \\
0 \\
0 \\
0 \\
0 \\
0 \\
0 \\
0 \\
0\end{array}$ & $\begin{array}{r}60 \\
0 \\
0 \\
0 \\
0 \\
0 \\
0 \\
0 \\
0 \\
0 \\
0 \\
0 \\
60 \\
0 \\
0 \\
0 \\
0 \\
0 \\
0 \\
0 \\
0\end{array}$ & $\begin{array}{r}301 \\
0 \\
0 \\
0 \\
0 \\
0 \\
0 \\
0 \\
0 \\
0 \\
0 \\
299 \\
0 \\
0 \\
0 \\
0 \\
0 \\
0 \\
0 \\
0 \\
2\end{array}$ & $\begin{array}{r}4,573 \\
0 \\
289 \\
0 \\
0 \\
0 \\
0 \\
322 \\
0 \\
9 \\
393 \\
1,032 \\
1,356 \\
601 \\
0 \\
499 \\
0 \\
0 \\
70 \\
0 \\
2\end{array}$ & $\begin{array}{r}62,850 \\
6,026 \\
289 \\
6,350 \\
569 \\
2,372 \\
698 \\
322 \\
206 \\
9 \\
393 \\
28,271 \\
1,356 \\
2,380 \\
406 \\
499 \\
2,185 \\
10,040 \\
70 \\
407 \\
2\end{array}$ & $\begin{array}{r}1,880 \\
194 \\
0 \\
205 \\
18 \\
77 \\
23 \\
0 \\
7 \\
0 \\
0 \\
879 \\
0 \\
57 \\
13 \\
0 \\
70 \\
324 \\
0 \\
13 \\
0\end{array}$ & $\begin{array}{r}148 \\
0 \\
9 \\
0 \\
0 \\
0 \\
0 \\
10 \\
0 \\
(s) \\
13 \\
33 \\
44 \\
19 \\
0 \\
16 \\
0 \\
0 \\
2 \\
0 \\
(\mathrm{~s})\end{array}$ & $\begin{array}{r}2,027 \\
194 \\
9 \\
205 \\
18 \\
77 \\
23 \\
10 \\
7 \\
(s) \\
13 \\
912 \\
44 \\
77 \\
13 \\
16 \\
70 \\
324 \\
2 \\
13 \\
(s)\end{array}$ \\
\hline 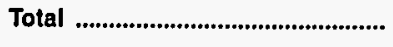 & 448 & 3,859 & 0 & 93 & 824 & 13,326 & 148,365 & 4,356 & 430 & 4,786 \\
\hline Perslan Gulf ${ }^{\circ}$................................. & 0 & 0 & 0 & 0 & 12 & 2,178 & 42,075 & 1,287 & 70 & 1,357 \\
\hline
\end{tabular}

a Crude oll and unfinished oils are reported by the PAD District in which they are to be processed; all other products are reported by the PAD District of entry.

b Includes crude oil imported for storage in the Strategic Petroleum Reserve.

c Includes aviation gasoline, avlation gasoline blending components, miscellaneous products, other hydrocarbons and oxygenates, pentanes plus, petroleum coke, and waxes. Sources.

$\theta$ Includes Bahrain, Iran, Iraq, Kuwait, Qatar, Saudi Arabia, and United Arab Emirates.

(s) = Less than 500 barrels per day.

Note: Totals may not equal sum of components due to independent rounding.

Source: Energy Information Administration (EIA) Form EIA-814, "Monthly Imports Report." 
Table 39. PAD Districts IV and V-Imports of Crude Oil and Petroleum Products by Country of Origin, ${ }^{a}$ March 1995

(Thousand Barrels)

\begin{tabular}{|c|c|c|c|c|c|c|c|c|c|c|}
\hline Country of Origin & $\begin{array}{c}\text { Crude } \\
\text { Oil }^{b}\end{array}$ & $\begin{array}{c}\text { Liquefled } \\
\text { Petroleum } \\
\text { Gases }\end{array}$ & $\begin{array}{c}\text { Unfinished } \\
\text { Oils }\end{array}$ & $\begin{array}{c}\text { Gasoline } \\
\text { Blending } \\
\text { Compo- } \\
\text { nents }\end{array}$ & $\begin{array}{l}\text { Finished } \\
\text { Motor } \\
\text { Gasoline }\end{array}$ & Jet Fuel & $\begin{array}{l}\text { Distillate } \\
\text { Fuel Oil }\end{array}$ & $\begin{array}{c}\text { Residual } \\
\text { Fuel Oil }\end{array}$ & Kerosene & $\begin{array}{c}\text { Special } \\
\text { Naphthas }\end{array}$ \\
\hline
\end{tabular}

PAD District IV

Non OPEC

Canada.

Total
3,971

3,971

3,971

$\begin{array}{ll}173 & 0 \\ 173 & 0 \\ 173 & 0\end{array}$

0
0

12
12

12

0
0
0

95
95

95

95

0
0
0

$\begin{array}{ll}0 & 0 \\ 0 & 0 \\ 0 & 0\end{array}$

PAD District $V$

\begin{tabular}{|c|c|c|c|c|c|c|c|c|c|c|}
\hline 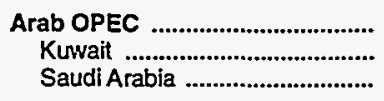 & $\begin{array}{r}1,106 \\
1,106 \\
0\end{array}$ & $\begin{array}{l}0 \\
0 \\
0\end{array}$ & $\begin{array}{l}0 \\
0 \\
0\end{array}$ & $\begin{array}{l}0 \\
0 \\
0\end{array}$ & $\begin{array}{l}0 \\
0 \\
0\end{array}$ & $\begin{array}{l}0 \\
0 \\
0\end{array}$ & $\begin{array}{l}0 \\
0 \\
0\end{array}$ & $\begin{array}{l}0 \\
0 \\
0\end{array}$ & $\begin{array}{l}0 \\
0 \\
0\end{array}$ & $\begin{array}{l}0 \\
0 \\
0\end{array}$ \\
\hline 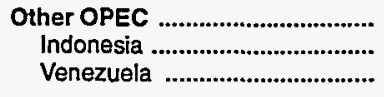 & $\begin{array}{r}1,432 \\
912 \\
520\end{array}$ & $\begin{array}{l}0 \\
0 \\
0\end{array}$ & $\begin{array}{l}0 \\
0 \\
0\end{array}$ & $\begin{array}{l}0 \\
0 \\
0\end{array}$ & $\begin{array}{l}0 \\
0 \\
0\end{array}$ & $\begin{array}{l}0 \\
0 \\
0\end{array}$ & $\begin{array}{l}0 \\
0 \\
0\end{array}$ & $\begin{array}{l}0 \\
0 \\
0\end{array}$ & $\begin{array}{l}0 \\
0 \\
0\end{array}$ & $\begin{array}{l}0 \\
0 \\
0\end{array}$ \\
\hline 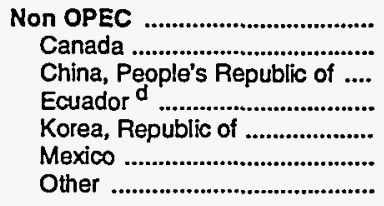 & $\begin{array}{r}3,414 \\
1,561 \\
1,013 \\
840 \\
0 \\
0 \\
0\end{array}$ & $\begin{array}{r}59 \\
59 \\
0 \\
0 \\
0 \\
0 \\
0\end{array}$ & $\begin{array}{l}0 \\
0 \\
0 \\
0 \\
0 \\
0 \\
0\end{array}$ & $\begin{array}{l}0 \\
0 \\
0 \\
0 \\
0 \\
0 \\
0\end{array}$ & $\begin{array}{l}9 \\
9 \\
0 \\
0 \\
0 \\
0 \\
0\end{array}$ & $\begin{array}{r}25 \\
2 \\
0 \\
0 \\
0 \\
23 \\
0\end{array}$ & $\begin{array}{r}27 \\
27 \\
0 \\
0 \\
0 \\
0 \\
0\end{array}$ & $\begin{array}{l}0 \\
0 \\
0 \\
0 \\
0 \\
0 \\
0\end{array}$ & $\begin{array}{l}0 \\
0 \\
0 \\
0 \\
0 \\
0 \\
0\end{array}$ & $\begin{array}{l}1 \\
1 \\
0 \\
0 \\
0 \\
0 \\
0\end{array}$ \\
\hline Total & 5,952 & 59 & $\mathbf{0}$ & 0 & 9 & 25 & 27 & 0 & 0 & 1 \\
\hline Persian Gulf ${ }^{\theta}$ & 1,106 & 0 & 0 & 0 & 0 & 0 & 0 & 0 & 0 & 0 \\
\hline
\end{tabular}


Table 39. PAD Districts IV and V-Imports of Crude Oil and Petroleum Products by Country of Origin, ${ }^{a}$ March 1995 (Continued) (Thousand Barrels)

\begin{tabular}{|c|c|c|c|c|c|c|c|c|c|c|}
\hline \multirow[b]{2}{*}{ Country of Origin } & \multirow[b]{2}{*}{$\begin{array}{c}\text { Naphtha for } \\
\text { Petrochemical } \\
\text { Feedstock } \\
\text { Use }\end{array}$} & \multirow[b]{2}{*}{$\begin{array}{c}\text { Other Oils for } \\
\text { Petrochemical } \\
\text { Feedstock } \\
\text { Use }\end{array}$} & \multirow[b]{2}{*}{ Lubricants } & \multirow[b]{2}{*}{$\begin{array}{c}\text { Asphatt and } \\
\text { Road Oll }\end{array}$} & \multirow[b]{2}{*}{$\begin{array}{c}\text { Other } \\
\text { Products }\end{array}$} & \multirow[b]{2}{*}{$\begin{array}{c}\text { Total } \\
\text { Products }\end{array}$} & \multirow[b]{2}{*}{$\begin{array}{c}\text { Total } \\
\text { Crude Oil } \\
\text { and } \\
\text { Products }\end{array}$} & \multicolumn{3}{|c|}{ Daily Average } \\
\hline & & & & & & & & $\begin{array}{c}\text { Crude } \\
\text { Oil }\end{array}$ & Products & Total \\
\hline
\end{tabular}

PAD District IV

\begin{tabular}{|c|c|c|c|c|c|c|c|c|c|c|}
\hline $\begin{array}{l}\text { Non OPEC } \\
\text { Canada } \ldots \ldots \ldots \ldots \ldots \ldots\end{array}$ & $\begin{array}{l}0 \\
0\end{array}$ & $\begin{array}{l}0 \\
0\end{array}$ & $\begin{array}{l}0 \\
0\end{array}$ & $\begin{array}{l}\mathbf{0} \\
0\end{array}$ & $\begin{array}{l}547 \\
547\end{array}$ & $\begin{array}{l}827 \\
827\end{array}$ & $\begin{array}{l}4,798 \\
4,798\end{array}$ & $\begin{array}{l}128 \\
128\end{array}$ & $\begin{array}{l}27 \\
27\end{array}$ & $\begin{array}{l}155 \\
155\end{array}$ \\
\hline 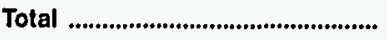 & 0 & 0 & 0 & 0 & 547 & 827 & 4,798 & 128 & 27 & 155 \\
\hline
\end{tabular}

\section{PAD District Y}

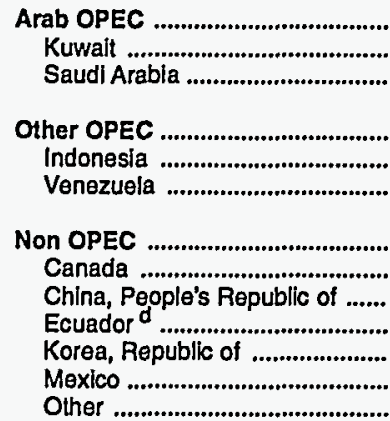

Persian Gulf ${ }^{\circ}$

0
0
0
0
0
0
59
0
0
0
59
0
0

0
0
0

0
0

0

0

0

0
0
0
0

461
0
461

0
0
0

0

0

0

\section{0
0}

0
0

0

0

129

0
129

595

595
540

0

0
0

5
50

1,185

461

46
129
129
77
638
5
59
5
1,36
461

461
0
461
129
0
129
775
638
0
0
59
28
50

1,365
461

1,567

1,106

461

36
0

1,561

912

649

4,189

2,199

1,013

840

59

28

7,317

1,567

192
36

$15 \quad 51$

a Crude oil and unfinlshed oils are reported by the PAD District in which they are to be processed; all other products are reported by the PAD District of entry.

b Includes crude oll imported tor storage in the Strategic Petroleum Reserve.

c Includes aviation gasoline, aviation gasoline blending components, miscellaneous products, other hydrocarbons and oxygenates, pentanes plus, petroleum coke, and waxes.

On December 31, 1992, Ecuador withdrew as a member of OPEC. As of January 1, 1994, imports of petroleum from Ecuador appear under imports from Non-OPEC Sources.

- Includes Bahrain, Iran, Iraq, Kuwait, Qatar, Saudi Arabla, and United Arab Emirates.

(s) = Less than 500 barrels per day.

Note: Totals may not equal sum of components due to independent rounding.

Source: Energy Information Administration (EIA) Form EIA-814, "Monthly Imports Report." 
Table 40. Year-to-Date Imports of Crude Oil and Petroleum Products into the United States by Country of Origin, $^{\text {a }}$ January-March 1995 (Thousand Barrels)

\begin{tabular}{|c|c|c|c|c|c|c|c|c|c|c|}
\hline Country of Origin & $\begin{array}{c}\text { Crude } \\
\text { Oilb }\end{array}$ & $\begin{array}{c}\text { Liquefied } \\
\text { Petroleum } \\
\text { Gases }\end{array}$ & $\begin{array}{c}\text { Unfinished } \\
\text { Oils }\end{array}$ & $\begin{array}{c}\text { Gasoline } \\
\text { Blending } \\
\text { Compo- } \\
\text { nents } \\
\end{array}$ & $\begin{array}{l}\text { Finished } \\
\text { Motor } \\
\text { Gasoline }\end{array}$ & Jet Fuel & $\begin{array}{l}\text { Distillate } \\
\text { Fuel Oil } \\
\end{array}$ & $\begin{array}{c}\text { Residual } \\
\text { Fuel Oil }\end{array}$ & Kerosene & $\begin{array}{c}\text { Special } \\
\text { Naphthas }\end{array}$ \\
\hline 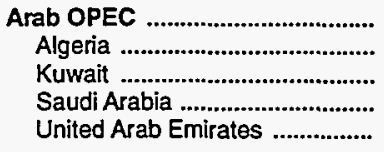 & $\begin{array}{r}138,226 \\
2,390 \\
20,608 \\
114,250 \\
978\end{array}$ & $\begin{array}{r}738 \\
738 \\
0 \\
0 \\
0\end{array}$ & $\begin{array}{r}8,423 \\
2,575 \\
906 \\
4,942 \\
0\end{array}$ & $\begin{array}{l}0 \\
0 \\
0 \\
0 \\
0\end{array}$ & $\begin{array}{l}0 \\
0 \\
0 \\
0 \\
0\end{array}$ & $\begin{array}{l}0 \\
0 \\
0 \\
0 \\
0\end{array}$ & $\begin{array}{r}940 \\
940 \\
0 \\
0 \\
0\end{array}$ & $\begin{array}{r}1,974 \\
1,974 \\
0 \\
0 \\
0\end{array}$ & $\begin{array}{l}0 \\
0 \\
0 \\
0 \\
0\end{array}$ & $\begin{array}{l}0 \\
0 \\
0 \\
0 \\
0\end{array}$ \\
\hline 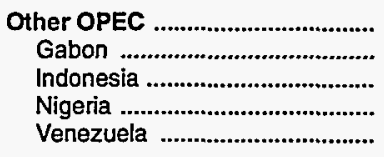 & $\begin{array}{r}173,981 \\
17,083 \\
4,533 \\
51,723 \\
100,642\end{array}$ & $\begin{array}{r}207 \\
0 \\
0 \\
0 \\
207\end{array}$ & $\begin{array}{r}6,169 \\
0 \\
1,828 \\
0 \\
4,341\end{array}$ & $\begin{array}{r}523 \\
0 \\
0 \\
0 \\
523\end{array}$ & $\begin{array}{r}3,333 \\
0 \\
0 \\
0 \\
3,333\end{array}$ & $\begin{array}{r}3,172 \\
0 \\
1 \\
0 \\
3,171\end{array}$ & $\begin{array}{r}7,175 \\
0 \\
0 \\
0 \\
7,175\end{array}$ & $\begin{array}{r}6,730 \\
0 \\
0 \\
586 \\
6,144\end{array}$ & $\begin{array}{l}0 \\
0 \\
0 \\
0 \\
0\end{array}$ & $\begin{array}{l}0 \\
0 \\
0 \\
0 \\
0\end{array}$ \\
\hline 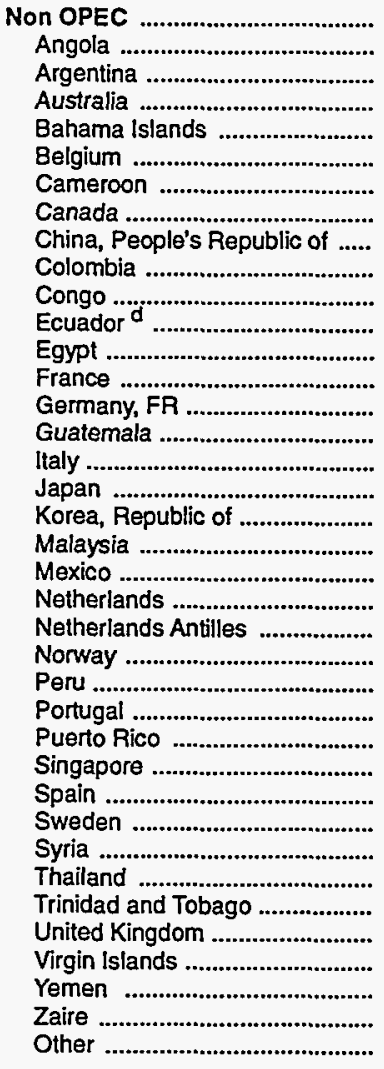 & $\begin{array}{r}302,860 \\
30,389 \\
1,578 \\
1,278 \\
0 \\
0 \\
0 \\
85,952 \\
4,171 \\
17,147 \\
569 \\
10,224 \\
2,784 \\
0 \\
0 \\
206 \\
0 \\
0 \\
0 \\
646 \\
82,838 \\
0 \\
0 \\
16,328 \\
375 \\
0 \\
0 \\
406 \\
0 \\
0 \\
0 \\
470 \\
6,670 \\
36,359 \\
0 \\
1,478 \\
1,890 \\
1,102\end{array}$ & $\begin{array}{r}11,572 \\
0 \\
0 \\
0 \\
0 \\
0 \\
0 \\
10,227 \\
0 \\
0 \\
0 \\
0 \\
0 \\
0 \\
0 \\
0 \\
64 \\
0 \\
0 \\
0 \\
625 \\
0 \\
0 \\
656 \\
0 \\
0 \\
0 \\
0 \\
0 \\
0 \\
0 \\
0 \\
0 \\
0 \\
0 \\
0 \\
0 \\
0\end{array}$ & $\begin{array}{r}15,361 \\
0 \\
0 \\
0 \\
0 \\
337 \\
315 \\
1,367 \\
0 \\
0 \\
0 \\
0 \\
0 \\
666 \\
0 \\
0 \\
0 \\
0 \\
1,810 \\
0 \\
0 \\
0 \\
3,436 \\
342 \\
0 \\
86 \\
0 \\
1,228 \\
935 \\
627 \\
753 \\
0 \\
0 \\
353 \\
2,811 \\
0 \\
0 \\
295\end{array}$ & $\begin{array}{r}2,145 \\
0 \\
0 \\
0 \\
0 \\
0 \\
0 \\
117 \\
0 \\
0 \\
0 \\
0 \\
0 \\
0 \\
0 \\
0 \\
0 \\
0 \\
0 \\
0 \\
657 \\
247 \\
0 \\
0 \\
0 \\
0 \\
0 \\
0 \\
0 \\
0 \\
0 \\
0 \\
0 \\
0 \\
577 \\
0 \\
0 \\
547\end{array}$ & $\begin{array}{r}18,717 \\
0 \\
0 \\
0 \\
0 \\
999 \\
0 \\
5,081 \\
0 \\
0 \\
0 \\
0 \\
0 \\
0 \\
0 \\
0 \\
248 \\
0 \\
0 \\
0 \\
0 \\
733 \\
0 \\
274 \\
0 \\
237 \\
0 \\
0 \\
0 \\
0 \\
0 \\
0 \\
0 \\
1,610 \\
9,448 \\
0 \\
0 \\
87\end{array}$ & $\begin{array}{r}5,801 \\
0 \\
0 \\
1 \\
0 \\
0 \\
0 \\
24 \\
0 \\
0 \\
0 \\
0 \\
0 \\
0 \\
0 \\
0 \\
0 \\
0 \\
0 \\
0 \\
321 \\
0 \\
1,784 \\
0 \\
0 \\
0 \\
0 \\
0 \\
0 \\
0 \\
0 \\
0 \\
0 \\
0 \\
3,671 \\
0 \\
0 \\
0\end{array}$ & $\begin{array}{r}14,092 \\
351 \\
0 \\
0 \\
0 \\
0 \\
0 \\
7,439 \\
0 \\
0 \\
0 \\
0 \\
0 \\
0 \\
475 \\
0 \\
0 \\
0 \\
0 \\
0 \\
0 \\
0 \\
0 \\
0 \\
0 \\
0 \\
0 \\
0 \\
0 \\
0 \\
0 \\
0 \\
0 \\
0 \\
5,827 \\
0 \\
0 \\
0\end{array}$ & $\begin{array}{r}10,077 \\
685 \\
0 \\
0 \\
643 \\
0 \\
315 \\
1,248 \\
0 \\
1,140 \\
0 \\
0 \\
0 \\
0 \\
0 \\
0 \\
0 \\
0 \\
0 \\
0 \\
305 \\
0 \\
324 \\
0 \\
35 \\
0 \\
0 \\
0 \\
0 \\
0 \\
0 \\
0 \\
0 \\
863 \\
4,519 \\
0 \\
0 \\
0\end{array}$ & $\begin{array}{r}332 \\
0 \\
0 \\
0 \\
0 \\
0 \\
0 \\
76 \\
0 \\
0 \\
0 \\
0 \\
0 \\
0 \\
0 \\
0 \\
0 \\
0 \\
0 \\
0 \\
256 \\
0 \\
0 \\
0 \\
0 \\
0 \\
0 \\
0 \\
0 \\
0 \\
0 \\
0 \\
0 \\
0 \\
0 \\
0 \\
0 \\
0\end{array}$ & $\begin{array}{r}538 \\
0 \\
0 \\
0 \\
0 \\
0 \\
0 \\
529 \\
0 \\
0 \\
0 \\
0 \\
0 \\
0 \\
0 \\
0 \\
9 \\
0 \\
0 \\
0 \\
0 \\
0 \\
0 \\
0 \\
0 \\
0 \\
0 \\
0 \\
0 \\
0 \\
0 \\
0 \\
0 \\
0 \\
0 \\
0 \\
0 \\
0\end{array}$ \\
\hline Total & 615,067 & 12,517 & 29,953 & 2,668 & 22,050 & 6,973 & 22,207 & 18,781 & 332 & 538 \\
\hline 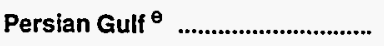 & 135,836 & 0 & 6,143 & $\mathbf{0}$ & 0 & 0 & 0 & 0 & 0 & $\mathbf{0}$ \\
\hline
\end{tabular}

See footnotes at end of table. 


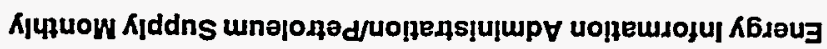

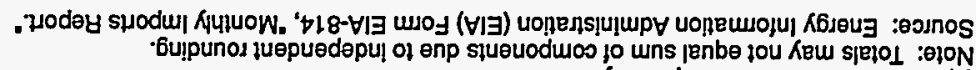
- Kep jed sjeljeq oos ueul ssej = (s)

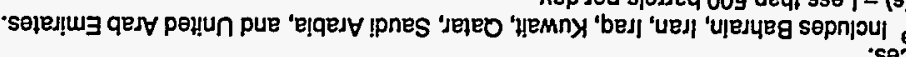

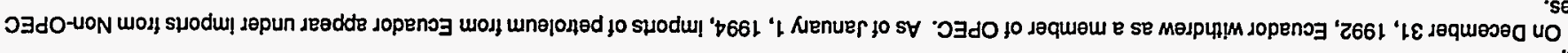
sevinos

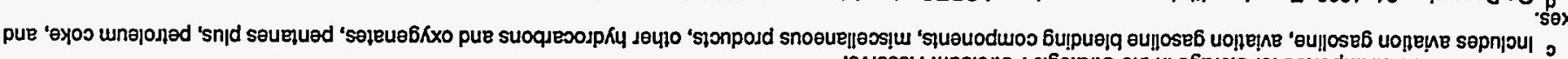

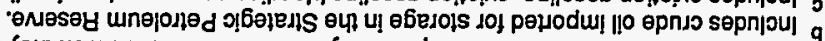

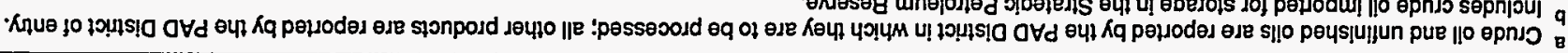

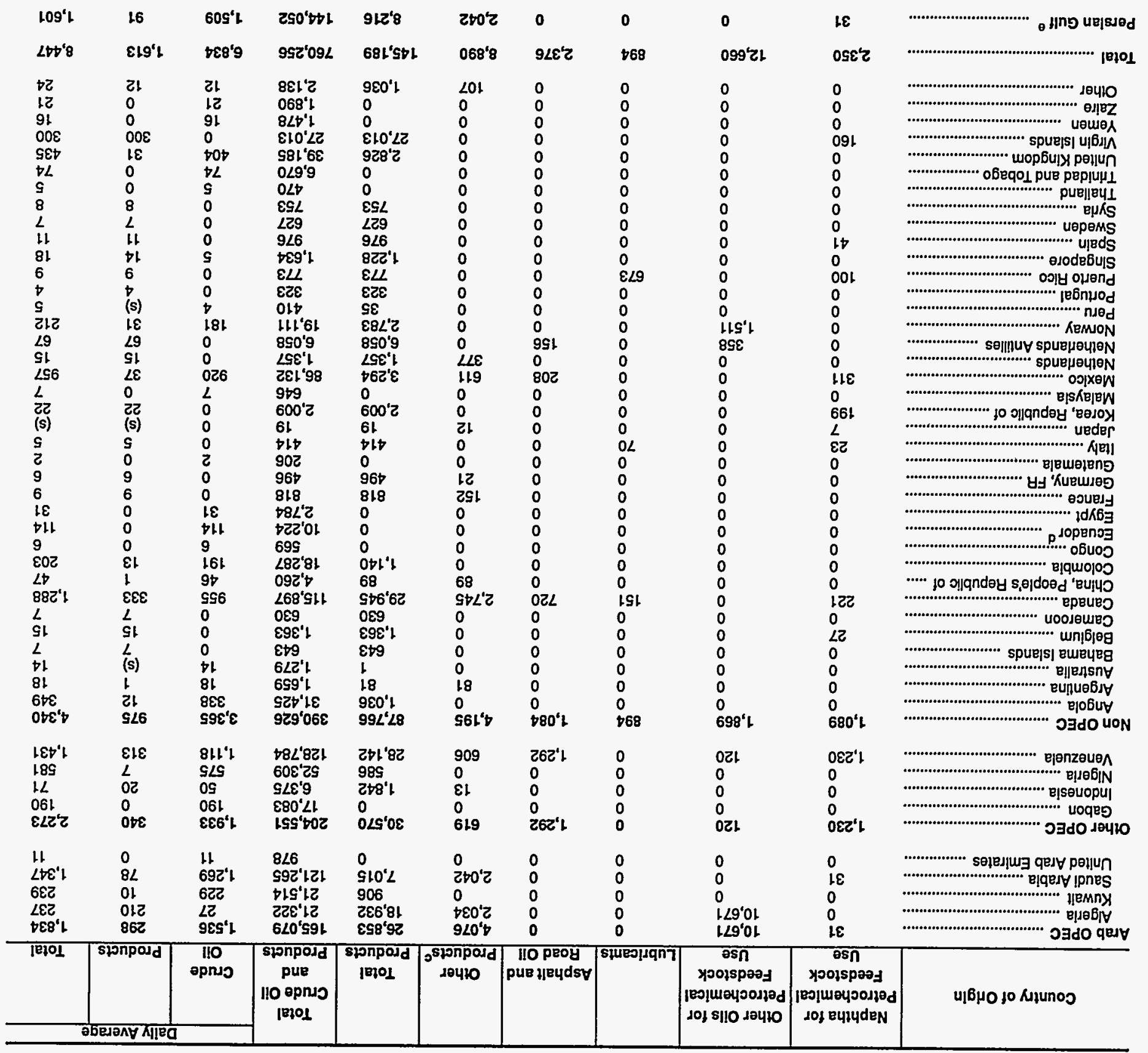


Table 41. PAD District I-Year-to-Date Imports of Crude Oil and Petroleum Products by Country of Origin, ${ }^{a}$ January-March 1995

(Thousand Barrels)

\begin{tabular}{|c|c|c|c|c|c|c|c|c|c|c|}
\hline Country of Origin & $\begin{array}{c}\text { Crude } \\
\text { Oil }^{\mathbf{b}}\end{array}$ & $\begin{array}{c}\text { Liquefied } \\
\text { Petroleum } \\
\text { Gases } \\
\end{array}$ & $\begin{array}{c}\text { Unfinished } \\
\text { Oils }\end{array}$ & $\begin{array}{l}\text { Gasoline } \\
\text { Blending } \\
\text { Compo- } \\
\text { nents }\end{array}$ & $\begin{array}{l}\text { Finished } \\
\text { Motor } \\
\text { Gasoline }\end{array}$ & Jet Fuel & $\begin{array}{l}\text { Distillate } \\
\text { Fuel Oil }\end{array}$ & $\begin{array}{l}\text { Residual } \\
\text { Fuel Oil }\end{array}$ & Kerosene & $\begin{array}{c}\text { Special } \\
\text { Naphthas }\end{array}$ \\
\hline $\begin{array}{l}\text { Arab OPEC } \\
\text { Algeria } \\
\text { Kuwait } \\
\text { Saudi Arabia }\end{array}$ & $\begin{array}{r}19,921 \\
0 \\
0 \\
19,921\end{array}$ & $\begin{array}{r}336 \\
336 \\
0 \\
0\end{array}$ & $\begin{array}{r}3,112 \\
1,896 \\
609 \\
607\end{array}$ & $\begin{array}{l}0 \\
0 \\
0 \\
0\end{array}$ & $\begin{array}{l}0 \\
0 \\
0 \\
0\end{array}$ & $\begin{array}{l}0 \\
0 \\
0 \\
0\end{array}$ & $\begin{array}{r}940 \\
940 \\
0 \\
0\end{array}$ & $\begin{array}{r}1,974 \\
1,974 \\
0 \\
0\end{array}$ & $\begin{array}{l}0 \\
0 \\
0 \\
0\end{array}$ & $\begin{array}{l}0 \\
0 \\
0 \\
0\end{array}$ \\
\hline 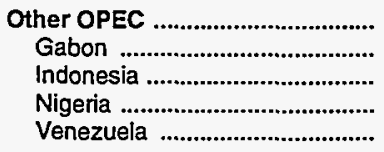 & $\begin{array}{r}45,662 \\
9,358 \\
0 \\
24,507 \\
11,797\end{array}$ & $\begin{array}{l}0 \\
0 \\
0 \\
0 \\
0\end{array}$ & $\begin{array}{r}932 \\
0 \\
722 \\
0 \\
210\end{array}$ & $\begin{array}{r}523 \\
0 \\
0 \\
0 \\
523\end{array}$ & $\begin{array}{r}3,333 \\
0 \\
0 \\
0 \\
3,333\end{array}$ & $\begin{array}{r}3,126 \\
0 \\
0 \\
0 \\
3,126\end{array}$ & $\begin{array}{r}7,175 \\
0 \\
0 \\
0 \\
7,175\end{array}$ & $\begin{array}{r}6,121 \\
0 \\
0 \\
586 \\
5,535\end{array}$ & $\begin{array}{l}0 \\
0 \\
0 \\
0 \\
0\end{array}$ & $\begin{array}{l}0 \\
0 \\
0 \\
0 \\
0\end{array}$ \\
\hline 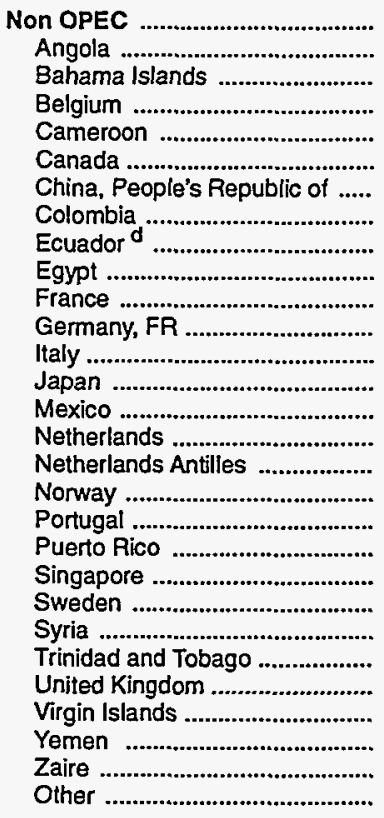 & $\begin{array}{r}54,849 \\
15,520 \\
0 \\
0 \\
0 \\
5,105 \\
1,313 \\
1,011 \\
344 \\
1,389 \\
0 \\
0 \\
0 \\
0 \\
4,492 \\
0 \\
0 \\
13,452 \\
0 \\
0 \\
0 \\
0 \\
0 \\
1,137 \\
9,700 \\
0 \\
550 \\
836 \\
0\end{array}$ & $\begin{array}{r}2,252 \\
0 \\
0 \\
0 \\
0 \\
1,596 \\
0 \\
0 \\
0 \\
0 \\
0 \\
0 \\
0 \\
0 \\
0 \\
0 \\
0 \\
656 \\
0 \\
0 \\
0 \\
0 \\
0 \\
0 \\
0 \\
0 \\
0 \\
0 \\
0\end{array}$ & $\begin{array}{r}7,052 \\
0 \\
0 \\
337 \\
315 \\
721 \\
0 \\
0 \\
0 \\
0 \\
0 \\
0 \\
0 \\
0 \\
0 \\
0 \\
720 \\
342 \\
86 \\
0 \\
642 \\
325 \\
753 \\
0 \\
0 \\
2,811 \\
0 \\
0 \\
0\end{array}$ & $\begin{array}{r}1,529 \\
0 \\
0 \\
0 \\
0 \\
112 \\
0 \\
0 \\
0 \\
0 \\
0 \\
0 \\
0 \\
0 \\
46 \\
247 \\
0 \\
0 \\
0 \\
0 \\
0 \\
0 \\
0 \\
0 \\
0 \\
577 \\
0 \\
0 \\
547\end{array}$ & $\begin{array}{r}18,368 \\
0 \\
0 \\
999 \\
0 \\
4,732 \\
0 \\
0 \\
0 \\
0 \\
0 \\
0 \\
248 \\
0 \\
0 \\
733 \\
0 \\
274 \\
237 \\
0 \\
0 \\
0 \\
0 \\
0 \\
1,610 \\
9,448 \\
0 \\
0 \\
87\end{array}$ & $\begin{array}{r}5,021 \\
0 \\
0 \\
0 \\
0 \\
18 \\
0 \\
0 \\
0 \\
0 \\
0 \\
0 \\
0 \\
0 \\
225 \\
0 \\
1,634 \\
0 \\
0 \\
0 \\
0 \\
0 \\
0 \\
0 \\
0 \\
3,144 \\
0 \\
0 \\
0\end{array}$ & $\begin{array}{r}13,201 \\
351 \\
0 \\
0 \\
0 \\
6,548 \\
0 \\
0 \\
0 \\
0 \\
0 \\
475 \\
0 \\
0 \\
0 \\
0 \\
0 \\
0 \\
0 \\
0 \\
0 \\
0 \\
0 \\
0 \\
0 \\
5,827 \\
0 \\
0 \\
0\end{array}$ & $\begin{array}{r}9,602 \\
685 \\
643 \\
0 \\
315 \\
1,113 \\
0 \\
1,140 \\
0 \\
0 \\
0 \\
0 \\
0 \\
0 \\
0 \\
0 \\
324 \\
0 \\
0 \\
0 \\
0 \\
0 \\
0 \\
0 \\
863 \\
4,519 \\
0 \\
0 \\
0\end{array}$ & $\begin{array}{r}74 \\
0 \\
0 \\
0 \\
0 \\
74 \\
0 \\
0 \\
0 \\
0 \\
0 \\
0 \\
0 \\
0 \\
0 \\
0 \\
0 \\
0 \\
0 \\
0 \\
0 \\
0 \\
0 \\
0 \\
0 \\
0 \\
0 \\
0 \\
0\end{array}$ & $\begin{array}{r}219 \\
0 \\
0 \\
0 \\
0 \\
219 \\
0 \\
0 \\
0 \\
0 \\
0 \\
0 \\
0 \\
0 \\
0 \\
0 \\
0 \\
0 \\
0 \\
0 \\
0 \\
0 \\
0 \\
0 \\
0 \\
0 \\
0 \\
0 \\
0\end{array}$ \\
\hline Total & 120,432 & 2,588 & 11,096 & 2,052 & 21,701 & 8,147 & 21,316 & 17,697 & 74 & 219 \\
\hline 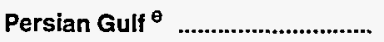 & 19,921 & 0 & 1,216 & 0 & $\mathbf{0}$ & $\mathbf{0}$ & 0 & 0 & 0 & 0 \\
\hline
\end{tabular}

See footnotes at end of table. 
Table 41. PAD District I-Year-to-Date Imports of Crude Oil and Petroleum Products by Country of Origin, ${ }^{a}$ January-March 1995 (Continued) (Thousand Barrels)

\begin{tabular}{|c|c|c|c|c|c|c|c|c|c|c|}
\hline \multirow[b]{2}{*}{ Country of Origin } & \multirow[b]{2}{*}{$\begin{array}{c}\text { Naphtha for } \\
\text { Petrochemical } \\
\text { Feedstock } \\
\text { Use } \\
\end{array}$} & \multirow[b]{2}{*}{$\begin{array}{c}\text { Other Oils for } \\
\text { Petrochemical } \\
\text { Feedstock } \\
\text { Use }\end{array}$} & \multirow[b]{2}{*}{ Lubricants } & \multirow[b]{2}{*}{$\begin{array}{c}\text { Asphalt and } \\
\text { Road Oil }\end{array}$} & \multirow[b]{2}{*}{$\begin{array}{c}\text { Other } \\
\text { Products }\end{array}$} & \multirow[b]{2}{*}{$\begin{array}{c}\text { Total } \\
\text { Products }\end{array}$} & \multirow[b]{2}{*}{$\begin{array}{c}\text { Total } \\
\text { Crude Oil } \\
\text { and } \\
\text { Products }\end{array}$} & \multicolumn{3}{|c|}{ Daily Average } \\
\hline & & & & & & & & $\begin{array}{c}\text { Crude } \\
\text { Oil }\end{array}$ & Products & Total \\
\hline $\begin{array}{l}\text { Arab OPEC } \\
\text { Algeria } \\
\text { Kuwalt } \ldots \ldots \ldots \ldots \\
\text { Saudi Arabla }\end{array}$ & $\begin{array}{l}0 \\
0 \\
0 \\
0\end{array}$ & $\begin{array}{l}0 \\
0 \\
0 \\
0\end{array}$ & $\begin{array}{l}0 \\
0 \\
0 \\
0\end{array}$ & $\begin{array}{l}0 \\
0 \\
0 \\
0\end{array}$ & $\begin{array}{r}1,183 \\
0 \\
0 \\
1,183\end{array}$ & $\begin{array}{r}7,545 \\
5,146 \\
609 \\
1,790\end{array}$ & $\begin{array}{r}27,466 \\
5,146 \\
609 \\
21,711\end{array}$ & $\begin{array}{r}221 \\
0 \\
0 \\
221\end{array}$ & $\begin{array}{r}84 \\
57 \\
7 \\
20\end{array}$ & $\begin{array}{r}305 \\
57 \\
7 \\
241\end{array}$ \\
\hline 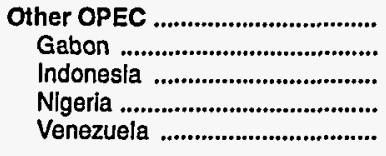 & $\begin{array}{l}0 \\
0 \\
0 \\
0 \\
0\end{array}$ & $\begin{array}{l}0 \\
0 \\
0 \\
0 \\
0\end{array}$ & $\begin{array}{l}0 \\
0 \\
0 \\
0 \\
0\end{array}$ & $\begin{array}{r}1,224 \\
0 \\
0 \\
0 \\
1,224\end{array}$ & $\begin{array}{r}128 \\
0 \\
0 \\
0 \\
128\end{array}$ & $\begin{array}{r}22,562 \\
0 \\
722 \\
586 \\
21,254\end{array}$ & $\begin{array}{r}68,224 \\
9,358 \\
722 \\
25,093 \\
33,051\end{array}$ & $\begin{array}{r}507 \\
104 \\
0 \\
272 \\
131\end{array}$ & $\begin{array}{r}251 \\
0 \\
8 \\
7 \\
236\end{array}$ & $\begin{array}{r}758 \\
104 \\
8 \\
279 \\
367\end{array}$ \\
\hline 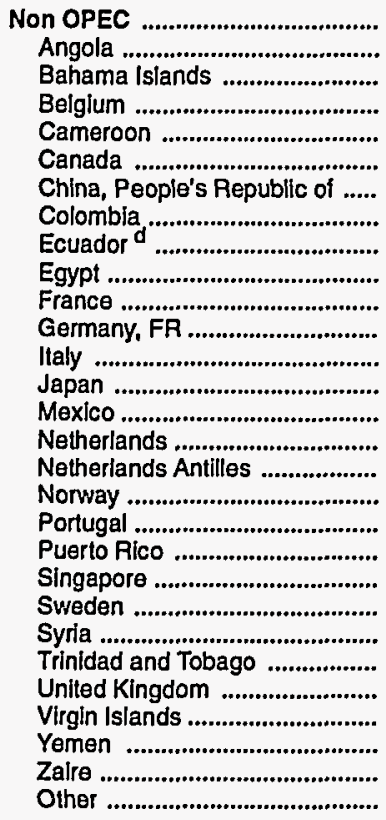 & $\begin{array}{r}125 \\
0 \\
0 \\
0 \\
0 \\
18 \\
0 \\
0 \\
0 \\
0 \\
0 \\
0 \\
0 \\
7 \\
0 \\
0 \\
0 \\
0 \\
0 \\
100 \\
0 \\
0 \\
0 \\
0 \\
0 \\
0 \\
0 \\
0 \\
0\end{array}$ & $\begin{array}{l}0 \\
0 \\
0 \\
0 \\
0 \\
0 \\
0 \\
0 \\
0 \\
0 \\
0 \\
0 \\
0 \\
0 \\
0 \\
0 \\
0 \\
0 \\
0 \\
0 \\
0 \\
0 \\
0 \\
0 \\
0 \\
0 \\
0 \\
0 \\
0\end{array}$ & $\begin{array}{r}772 \\
0 \\
0 \\
0 \\
0 \\
99 \\
0 \\
0 \\
0 \\
0 \\
0 \\
0 \\
0 \\
0 \\
0 \\
0 \\
0 \\
0 \\
0 \\
673 \\
0 \\
0 \\
0 \\
0 \\
0 \\
0 \\
0 \\
0 \\
0\end{array}$ & $\begin{array}{r}981 \\
0 \\
0 \\
0 \\
0 \\
677 \\
0 \\
0 \\
0 \\
0 \\
0 \\
0 \\
0 \\
0 \\
208 \\
0 \\
96 \\
0 \\
0 \\
0 \\
0 \\
0 \\
0 \\
0 \\
0 \\
0 \\
0 \\
0 \\
0\end{array}$ & $\begin{array}{r}518 \\
0 \\
0 \\
0 \\
0 \\
51 \\
0 \\
0 \\
0 \\
0 \\
152 \\
21 \\
0 \\
11 \\
0 \\
272 \\
0 \\
0 \\
0 \\
0 \\
0 \\
0 \\
0 \\
0 \\
0 \\
0 \\
0 \\
0 \\
11\end{array}$ & $\begin{array}{r}59,714 \\
1,036 \\
643 \\
1,336 \\
630 \\
15,978 \\
0 \\
1,140 \\
0 \\
0 \\
152 \\
496 \\
248 \\
18 \\
479 \\
1,252 \\
2,774 \\
1,272 \\
323 \\
773 \\
642 \\
325 \\
753 \\
0 \\
2,473 \\
26,326 \\
0 \\
0 \\
645\end{array}$ & $\begin{array}{r}114,563 \\
16,556 \\
643 \\
1,336 \\
630 \\
21,083 \\
1,313 \\
2,151 \\
344 \\
1,389 \\
152 \\
496 \\
248 \\
18 \\
4,971 \\
1,252 \\
2,774 \\
14,724 \\
323 \\
773 \\
642 \\
325 \\
753 \\
1,137 \\
12,173 \\
26,326 \\
550 \\
836 \\
645\end{array}$ & $\begin{array}{r}609 \\
172 \\
0 \\
0 \\
0 \\
57 \\
15 \\
11 \\
4 \\
15 \\
0 \\
0 \\
0 \\
0 \\
50 \\
0 \\
0 \\
149 \\
0 \\
0 \\
0 \\
0 \\
0 \\
13 \\
108 \\
0 \\
6 \\
9 \\
0\end{array}$ & $\begin{array}{r}663 \\
12 \\
7 \\
15 \\
7 \\
178 \\
0 \\
13 \\
0 \\
0 \\
2 \\
6 \\
3 \\
(s) \\
5 \\
14 \\
31 \\
14 \\
4 \\
9 \\
7 \\
4 \\
8 \\
0 \\
27 \\
293 \\
0 \\
0 \\
7\end{array}$ & $\begin{array}{r}1,273 \\
184 \\
7 \\
15 \\
7 \\
234 \\
15 \\
24 \\
4 \\
15 \\
2 \\
6 \\
3 \\
(s) \\
55 \\
14 \\
31 \\
164 \\
4 \\
9 \\
7 \\
4 \\
8 \\
13 \\
135 \\
293 \\
6 \\
9 \\
7\end{array}$ \\
\hline Total & 125 & 0 & 772 & 2,205 & 1,829 & 89,821 & 210,253 & 1,338 & 998 & 2,336 \\
\hline Perslan Gulf ${ }^{\theta}$............................... & $\mathbf{0}$ & 0 & 0 & 0 & 1,183 & 2,399 & 22,320 & 221 & 27 & 248 \\
\hline
\end{tabular}

\footnotetext{
a Crude oil and unfinished oils are reported by the PAD District in which they are to be processed; all other products are reported by the PAD District of entry.

b Includes crude oil imported for storage in the Strategic Petroleum Reserve. waxes.

Includes aviation gasoline, aviation gasoline blending components, miscellaneous products, other hydrocarbons and oxygenates, pentanes plus, petroleum coke, and d On December 31, 1992, Ecuador withdrew as a member of OPEC. As of January 1, 1994, imports of petroleum from Ecuador appear under imports from Non-OPEC Sources.

Includes Bahrain, Iran, Iraq, Kuwait, Qatar, Saudi Arabia, and United Arab Emirates.

(s) = Less than 500 barrels per day.

Note: Totals may not equal sum of components due to independent rounding.

Source: Energy Information Administration (EIA) Form EIA-814, "Monthly Imports Report."
} 
Table 42. PAD District II-Year-to-Date Imports of Crude Oil and Petroleum Products by Country of Origin, ${ }^{\mathrm{a}}$ January-March 1995 (Thousand Barrels)

\begin{tabular}{|c|c|c|c|c|c|c|c|c|c|c|}
\hline Country of Origin & $\begin{array}{c}\text { Crude } \\
\text { Oil }\end{array}$ & $\begin{array}{c}\begin{array}{c}\text { Liquefied } \\
\text { Petroleum } \\
\text { Gases }\end{array} \\
\end{array}$ & $\begin{array}{c}\begin{array}{c}\text { Untinished } \\
\text { Oils }\end{array} \\
\end{array}$ & $\begin{array}{c}\text { Gasoline } \\
\text { Blending } \\
\text { Compo- } \\
\text { nents }\end{array}$ & $\begin{array}{l}\text { Finished } \\
\text { Motor } \\
\text { Gasoline } \\
\end{array}$ & Jet Fuel & $\begin{array}{l}\text { Distillate } \\
\text { Fuel Oil }\end{array}$ & $\begin{array}{c}\text { Residual } \\
\text { Fuel Oil }\end{array}$ & Kerosene & $\begin{array}{c}\text { Special } \\
\text { Naphthas }\end{array}$ \\
\hline 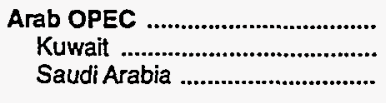 & $\begin{array}{l}7,643 \\
3,082 \\
4,561\end{array}$ & $\begin{array}{l}0 \\
0 \\
0\end{array}$ & $\begin{array}{l}0 \\
0 \\
0\end{array}$ & $\begin{array}{l}0 \\
0 \\
0\end{array}$ & $\begin{array}{l}0 \\
0 \\
0\end{array}$ & $\begin{array}{l}0 \\
0 \\
0\end{array}$ & $\begin{array}{l}0 \\
0 \\
0\end{array}$ & $\begin{array}{l}0 \\
0 \\
0\end{array}$ & $\begin{array}{l}0 \\
0 \\
0\end{array}$ & $\begin{array}{l}0 \\
0 \\
0\end{array}$ \\
\hline 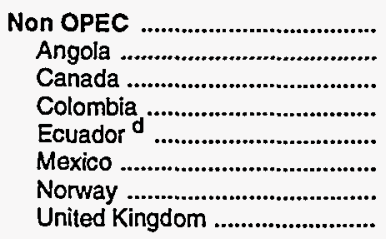 & $\begin{array}{r}73,742 \\
2,370 \\
63,563 \\
1,055 \\
353 \\
1,370 \\
574 \\
4,457\end{array}$ & $\begin{array}{r}7,387 \\
0 \\
7,387 \\
0 \\
0 \\
0 \\
0 \\
0\end{array}$ & $\begin{array}{l}8 \\
0 \\
8 \\
0 \\
0 \\
0 \\
0 \\
0\end{array}$ & $\begin{array}{l}5 \\
0 \\
5 \\
0 \\
0 \\
0 \\
0 \\
0\end{array}$ & $\begin{array}{r}267 \\
0 \\
267 \\
0 \\
0 \\
0 \\
0 \\
0\end{array}$ & $\begin{array}{l}0 \\
0 \\
0 \\
0 \\
0 \\
0 \\
0 \\
0\end{array}$ & $\begin{array}{r}444 \\
0 \\
444 \\
0 \\
0 \\
0 \\
0 \\
0\end{array}$ & $\begin{array}{r}135 \\
0 \\
135 \\
0 \\
0 \\
0 \\
0 \\
0\end{array}$ & $\begin{array}{l}0 \\
0 \\
0 \\
0 \\
0 \\
0 \\
0 \\
0\end{array}$ & $\begin{array}{r}217 \\
0 \\
217 \\
0 \\
0 \\
0 \\
0 \\
0\end{array}$ \\
\hline Persian Gulf ${ }^{\ominus}$ & 7,643 & 0 & 0 & 0 & 0 & 0 & 0 & $\mathbf{0}$ & 0 & 0 \\
\hline
\end{tabular}


Table 42. PAD District I-Year-to-Date Imports of Crude Oil and Petroleum Products by Country of Origin, ${ }^{a}$ January-March 1995 (Continued)

(Thousand Barrels)

\begin{tabular}{|c|c|c|c|c|c|c|c|c|c|c|}
\hline \multirow[b]{2}{*}{ Country of Origin } & \multirow[b]{2}{*}{$\begin{array}{c}\text { Naphtha for } \\
\text { Petrochemical } \\
\text { Feedstock } \\
\text { Use }\end{array}$} & \multirow[b]{2}{*}{$\begin{array}{c}\text { Other Oils for } \\
\text { Petrochemical } \\
\text { Feedstock } \\
\text { Use }\end{array}$} & \multirow[b]{2}{*}{ Lubricants } & \multirow[b]{2}{*}{$\begin{array}{c}\text { Asphalt and } \\
\text { Road Oll }\end{array}$} & \multirow[b]{2}{*}{$\begin{array}{c}\text { Other } \\
\text { Products }\end{array}$} & \multirow[b]{2}{*}{$\begin{array}{c}\text { Total } \\
\text { Products }\end{array}$} & \multirow[b]{2}{*}{$\begin{array}{c}\text { Total } \\
\text { Crude Oil } \\
\text { and } \\
\text { Products }\end{array}$} & \multicolumn{3}{|c|}{ Daily Average } \\
\hline & & & & & & & & $\begin{array}{c}\text { Crude } \\
\text { Oil }\end{array}$ & Products & Total \\
\hline 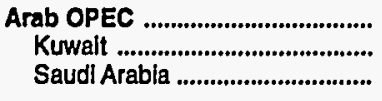 & $\begin{array}{l}0 \\
0 \\
0\end{array}$ & $\begin{array}{l}0 \\
0 \\
0\end{array}$ & $\begin{array}{l}0 \\
0 \\
0\end{array}$ & $\begin{array}{l}0 \\
0 \\
0\end{array}$ & $\begin{array}{l}0 \\
0 \\
0\end{array}$ & $\begin{array}{l}0 \\
0 \\
0\end{array}$ & $\begin{array}{l}7,643 \\
3,082 \\
4,561\end{array}$ & $\begin{array}{l}85 \\
34 \\
51\end{array}$ & $\begin{array}{l}0 \\
0 \\
0\end{array}$ & $\begin{array}{l}85 \\
34 \\
51\end{array}$ \\
\hline 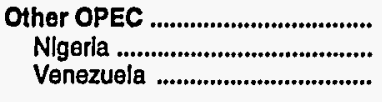 & $\begin{array}{l}0 \\
0 \\
0\end{array}$ & $\begin{array}{l}0 \\
0 \\
0\end{array}$ & $\begin{array}{l}0 \\
0 \\
0\end{array}$ & $\begin{array}{l}0 \\
0 \\
0\end{array}$ & $\begin{array}{l}0 \\
0 \\
0\end{array}$ & $\begin{array}{l}0 \\
0 \\
0\end{array}$ & $\begin{array}{r}16,649 \\
992 \\
15,657\end{array}$ & $\begin{array}{r}185 \\
11 \\
174\end{array}$ & $\begin{array}{l}0 \\
0 \\
0\end{array}$ & $\begin{array}{r}185 \\
11 \\
174\end{array}$ \\
\hline 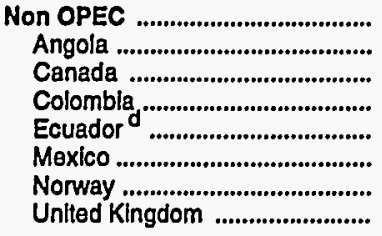 & $\begin{array}{r}94 \\
0 \\
94 \\
0 \\
0 \\
0 \\
0 \\
0\end{array}$ & $\begin{array}{l}0 \\
0 \\
0 \\
0 \\
0 \\
0 \\
0 \\
0\end{array}$ & $\begin{array}{r}52 \\
0 \\
52 \\
0 \\
0 \\
0 \\
0 \\
0\end{array}$ & $\begin{array}{r}34 \\
0 \\
34 \\
0 \\
0 \\
0 \\
0 \\
0\end{array}$ & $\begin{array}{r}163 \\
0 \\
163 \\
0 \\
0 \\
0 \\
0 \\
0\end{array}$ & $\begin{array}{r}8,806 \\
0 \\
8,806 \\
0 \\
0 \\
0 \\
0 \\
0\end{array}$ & $\begin{array}{r}82,548 \\
2,370 \\
72,369 \\
1,055 \\
353 \\
1,370 \\
574 \\
4,457\end{array}$ & $\begin{array}{r}819 \\
26 \\
706 \\
12 \\
4 \\
15 \\
15 \\
6 \\
50\end{array}$ & $\begin{array}{r}98 \\
0 \\
98 \\
0 \\
0 \\
0 \\
0 \\
0\end{array}$ & $\begin{array}{r}917 \\
26 \\
804 \\
12 \\
4 \\
15 \\
6 \\
50\end{array}$ \\
\hline 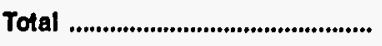 & 94 & 0 & 52 & 34 & 163 & 8,806 & 106,840 & 1,089 & 98 & 1,187 \\
\hline 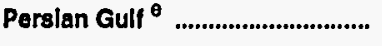 & 0 & 0 & 0 & o & 0 & 0 & 7,643 & 85 & 0 & 85 \\
\hline
\end{tabular}

\footnotetext{
a Crude oll and unfinished olls are reported by the PAD District in which they are to be processed; all other products are reported by the PAD District of entry.

b Includes crude oil Imported for storage in the Strategic Petroleum Reserve.

c Includes aviatlon gasoline, aviation gasoline blending components, miscellaneous products, other hydrocarbons and oxygenates, pentanes plus, petroleum coke, and waxes. Sources.

On December 31, 1992, Ecuador withdrew as a member of OPEC. As of January 1, 1994, imports of petroleum from Ecuador appear under imports from Non-OPEC

Includes Bahraln, Iran, Iraq, Kuwait, Qatar, Saudi Arabia, and United Arab Emirates.

(s) = Less than 500 barrels per day.

Note: Totals may not equal sum of components due to independent rounding.

Source: Energy Information Administration (EIA) Form EIA-814, "Monthly Imports Report."
}

Energy Information Administration/Petroleum Supply Monthly 
Table 43. PAD District III-Year-to-Date Imports of Crude Oil and Petroleum Products by Country of Origin, ${ }^{a}$ January-March 1995 (Thousand Barrels)

\begin{tabular}{|c|c|c|c|c|c|c|c|c|c|c|}
\hline Country of Origin & $\begin{array}{c}\text { Crude } \\
\text { Oil }^{\mathbf{b}}\end{array}$ & $\begin{array}{c}\text { Liquefied } \\
\text { Petroleum } \\
\text { Gases } \\
\end{array}$ & $\begin{array}{c}\text { Unfinished } \\
\text { Oils }\end{array}$ & $\begin{array}{l}\text { Gasoline } \\
\text { Blending } \\
\text { Compo- } \\
\text { nents } \\
\end{array}$ & $\begin{array}{l}\text { Finished } \\
\text { Motor } \\
\text { Gasoline }\end{array}$ & Jet Fuel & $\begin{array}{l}\text { Distillate } \\
\text { Fuel Oil }\end{array}$ & $\begin{array}{l}\text { Residual } \\
\text { Fuel Oil }\end{array}$ & Kerosene & $\begin{array}{c}\text { Special } \\
\text { Naphthas }\end{array}$ \\
\hline 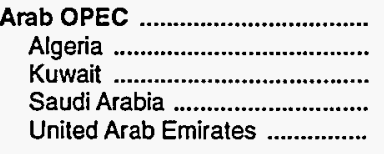 & $\begin{array}{r}106,245 \\
2,390 \\
14,123 \\
88,754 \\
978\end{array}$ & $\begin{array}{r}402 \\
402 \\
0 \\
0 \\
0\end{array}$ & $\begin{array}{r}5,014 \\
679 \\
0 \\
4,335 \\
0\end{array}$ & $\begin{array}{l}0 \\
0 \\
0 \\
0 \\
0\end{array}$ & $\begin{array}{l}0 \\
0 \\
0 \\
0 \\
0\end{array}$ & $\begin{array}{l}0 \\
0 \\
0 \\
0 \\
0\end{array}$ & $\begin{array}{l}0 \\
0 \\
0 \\
0 \\
0\end{array}$ & $\begin{array}{l}0 \\
0 \\
0 \\
0 \\
0\end{array}$ & $\begin{array}{l}0 \\
0 \\
0 \\
0 \\
0\end{array}$ & $\begin{array}{l}0 \\
0 \\
0 \\
0 \\
0\end{array}$ \\
\hline 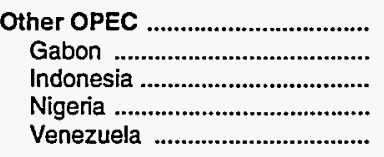 & $\begin{array}{r}105,896 \\
7,725 \\
448 \\
26,224 \\
71,499\end{array}$ & $\begin{array}{r}207 \\
0 \\
0 \\
0 \\
207\end{array}$ & $\begin{array}{r}5,237 \\
0 \\
1,106 \\
0 \\
4,131\end{array}$ & $\begin{array}{l}0 \\
0 \\
0 \\
0 \\
0\end{array}$ & $\begin{array}{l}0 \\
0 \\
0 \\
0 \\
0\end{array}$ & $\begin{array}{l}0 \\
0 \\
0 \\
0 \\
0\end{array}$ & $\begin{array}{l}0 \\
0 \\
0 \\
0 \\
0\end{array}$ & $\begin{array}{r}609 \\
0 \\
0 \\
0 \\
609\end{array}$ & $\begin{array}{l}0 \\
0 \\
0 \\
0 \\
0\end{array}$ & $\begin{array}{l}0 \\
0 \\
0 \\
0 \\
0\end{array}$ \\
\hline 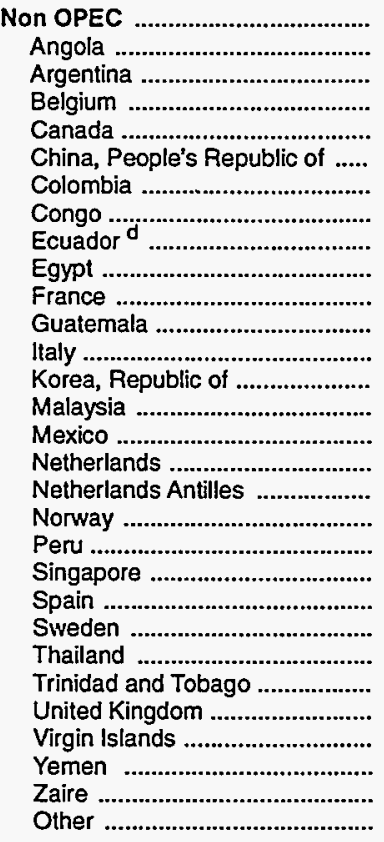 & $\begin{array}{r}149,255 \\
12,499 \\
1,578 \\
0 \\
0 \\
610 \\
15,081 \\
569 \\
6,025 \\
1,395 \\
0 \\
206 \\
0 \\
0 \\
646 \\
76,976 \\
0 \\
0 \\
2,302 \\
375 \\
406 \\
0 \\
0 \\
470 \\
5,533 \\
22,202 \\
0 \\
928 \\
1,054 \\
400\end{array}$ & $\begin{array}{r}689 \\
0 \\
0 \\
0 \\
0 \\
0 \\
0 \\
0 \\
0 \\
0 \\
0 \\
0 \\
64 \\
0 \\
0 \\
625 \\
0 \\
0 \\
0 \\
0 \\
0 \\
0 \\
0 \\
0 \\
0 \\
0 \\
0 \\
0 \\
0 \\
0 \\
0\end{array}$ & $\begin{array}{r}7,636 \\
0 \\
0 \\
0 \\
559 \\
0 \\
0 \\
0 \\
0 \\
0 \\
666 \\
0 \\
0 \\
1,810 \\
0 \\
0 \\
0 \\
2,716 \\
0 \\
0 \\
0 \\
935 \\
302 \\
0 \\
0 \\
353 \\
0 \\
0 \\
0 \\
295\end{array}$ & $\begin{array}{r}611 \\
0 \\
0 \\
0 \\
0 \\
0 \\
0 \\
0 \\
0 \\
0 \\
0 \\
0 \\
0 \\
0 \\
0 \\
611 \\
0 \\
0 \\
0 \\
0 \\
0 \\
0 \\
0 \\
0 \\
0 \\
0 \\
0 \\
0 \\
0 \\
0\end{array}$ & $\begin{array}{l}0 \\
0 \\
0 \\
0 \\
0 \\
0 \\
0 \\
0 \\
0 \\
0 \\
0 \\
0 \\
0 \\
0 \\
0 \\
0 \\
0 \\
0 \\
0 \\
0 \\
0 \\
0 \\
0 \\
0 \\
0 \\
0 \\
0 \\
0 \\
0 \\
0\end{array}$ & $\begin{array}{r}202 \\
0 \\
0 \\
0 \\
0 \\
0 \\
0 \\
0 \\
0 \\
0 \\
0 \\
0 \\
0 \\
0 \\
0 \\
52 \\
0 \\
150 \\
0 \\
0 \\
0 \\
0 \\
0 \\
0 \\
0 \\
0 \\
0 \\
0 \\
0 \\
0\end{array}$ & $\begin{array}{l}0 \\
0 \\
0 \\
0 \\
0 \\
0 \\
0 \\
0 \\
0 \\
0 \\
0 \\
0 \\
0 \\
0 \\
0 \\
0 \\
0 \\
0 \\
0 \\
0 \\
0 \\
0 \\
0 \\
0 \\
0 \\
0 \\
0 \\
0 \\
0 \\
0\end{array}$ & $\begin{array}{r}305 \\
0 \\
0 \\
0 \\
0 \\
0 \\
0 \\
0 \\
0 \\
0 \\
0 \\
0 \\
0 \\
0 \\
0 \\
305 \\
0 \\
0 \\
0 \\
0 \\
0 \\
0 \\
0 \\
0 \\
0 \\
0 \\
0 \\
0 \\
0 \\
0\end{array}$ & $\begin{array}{r}256 \\
0 \\
0 \\
0 \\
0 \\
0 \\
0 \\
0 \\
0 \\
0 \\
0 \\
0 \\
0 \\
0 \\
0 \\
256 \\
0 \\
0 \\
0 \\
0 \\
0 \\
0 \\
0 \\
0 \\
0 \\
0 \\
0 \\
0 \\
0 \\
0\end{array}$ & $\begin{array}{r}99 \\
0 \\
0 \\
0 \\
90 \\
0 \\
0 \\
0 \\
0 \\
0 \\
0 \\
0 \\
9 \\
0 \\
0 \\
0 \\
0 \\
0 \\
0 \\
0 \\
0 \\
0 \\
0 \\
0 \\
0 \\
0 \\
0 \\
0 \\
0 \\
0\end{array}$ \\
\hline Tota! & 361,396 & 1,298 & 17,887 & 611 & 0 & 202 & 0 & 914 & 256 & 99 \\
\hline Persian Gulf ${ }^{e}$ & 103,855 & 0 & 4,630 & 0 & 0 & 0 & 0 & 0 & 0 & 0 \\
\hline
\end{tabular}


Table 43. PAD District III-Year-to-Date Imports of Crude Oil and Petroleum Products by Country of Origin, ${ }^{a}$ January-March 1995 (Continued)

(Thousand Barrels)

\begin{tabular}{|c|c|c|c|c|c|c|c|c|c|c|}
\hline \multirow[b]{2}{*}{ Country of Origin } & \multirow[b]{2}{*}{$\begin{array}{c}\text { Naphtha for } \\
\text { Petrochemical } \\
\text { Feedstock } \\
\text { Use }\end{array}$} & \multirow[b]{2}{*}{$\begin{array}{c}\text { Other Oils for } \\
\text { Petrochemical } \\
\text { Feedstock } \\
\text { Use }\end{array}$} & \multirow[b]{2}{*}{ Lubricants } & \multirow[b]{2}{*}{$\begin{array}{c}\text { Asphalt and } \\
\text { Road Oil }\end{array}$} & \multirow[b]{2}{*}{$\begin{array}{c}\text { Other } \\
\text { Products }^{c}\end{array}$} & \multirow[b]{2}{*}{$\begin{array}{c}\text { Total } \\
\text { Products }\end{array}$} & \multirow[b]{2}{*}{$\begin{array}{l}\text { Total } \\
\text { Crude Oil } \\
\text { and } \\
\text { Products }\end{array}$} & \multicolumn{3}{|c|}{ Daily Average } \\
\hline & & & & & & & & $\begin{array}{c}\text { Crude } \\
\text { Oil }\end{array}$ & Products & Total \\
\hline 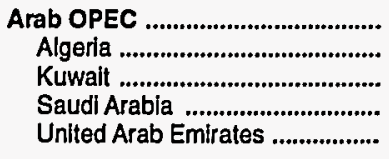 & $\begin{array}{r}31 \\
0 \\
0 \\
31 \\
0\end{array}$ & $\begin{array}{r}10,671 \\
10,671 \\
0 \\
0 \\
0\end{array}$ & $\begin{array}{l}0 \\
0 \\
0 \\
0 \\
0\end{array}$ & $\begin{array}{l}0 \\
0 \\
0 \\
0 \\
0\end{array}$ & $\begin{array}{r}2,046 \\
2,034 \\
0 \\
12 \\
0\end{array}$ & $\begin{array}{r}18,164 \\
13,786 \\
0 \\
4,378 \\
0\end{array}$ & $\begin{array}{r}124,409 \\
16,176 \\
14,123 \\
93,132 \\
978\end{array}$ & $\begin{array}{r}1,181 \\
27 \\
157 \\
986 \\
11\end{array}$ & $\begin{array}{r}202 \\
153 \\
0 \\
49 \\
0\end{array}$ & $\begin{array}{r}1,382 \\
180 \\
157 \\
1,035 \\
11\end{array}$ \\
\hline 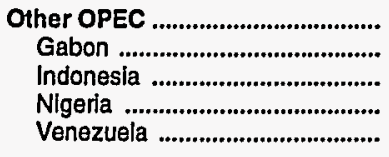 & $\begin{array}{r}1,230 \\
0 \\
0 \\
0 \\
1,230\end{array}$ & $\begin{array}{r}120 \\
0 \\
0 \\
0 \\
120\end{array}$ & $\begin{array}{l}0 \\
0 \\
0 \\
0 \\
0\end{array}$ & $\begin{array}{r}68 \\
0 \\
0 \\
0 \\
68\end{array}$ & $\begin{array}{r}13 \\
0 \\
13 \\
0 \\
0\end{array}$ & $\begin{array}{r}7,484 \\
0 \\
1,119 \\
0 \\
6,365\end{array}$ & $\begin{array}{r}113,380 \\
7,725 \\
1,567 \\
26,224 \\
77,864\end{array}$ & $\begin{array}{r}1,177 \\
86 \\
5 \\
291 \\
794\end{array}$ & $\begin{array}{r}83 \\
0 \\
12 \\
0 \\
71\end{array}$ & $\begin{array}{r}1,260 \\
86 \\
17 \\
291 \\
865\end{array}$ \\
\hline 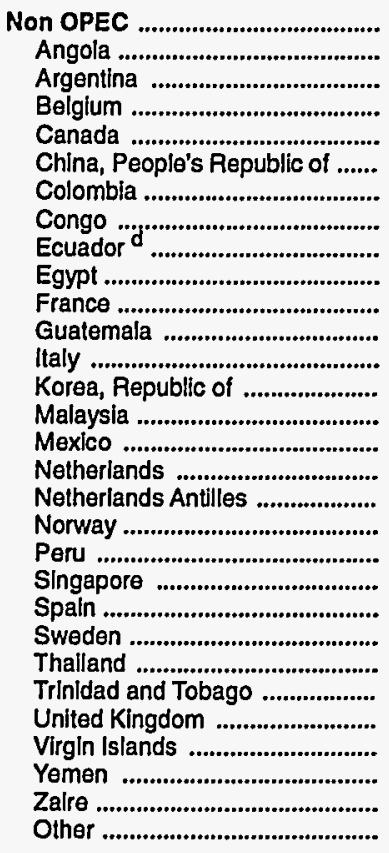 & $\begin{array}{r}811 \\
0 \\
0 \\
27 \\
109 \\
0 \\
0 \\
0 \\
0 \\
0 \\
0 \\
0 \\
23 \\
140 \\
0 \\
311 \\
0 \\
0 \\
0 \\
0 \\
0 \\
41 \\
0 \\
0 \\
0 \\
0 \\
160 \\
0 \\
0 \\
0\end{array}$ & $\begin{array}{r}1,869 \\
0 \\
0 \\
0 \\
0 \\
0 \\
0 \\
0 \\
0 \\
0 \\
0 \\
0 \\
0 \\
0 \\
0 \\
0 \\
0 \\
358 \\
1,511 \\
0 \\
0 \\
0 \\
0 \\
0 \\
0 \\
0 \\
0 \\
0 \\
0 \\
0\end{array}$ & $\begin{array}{r}70 \\
0 \\
0 \\
0 \\
0 \\
0 \\
0 \\
0 \\
0 \\
0 \\
0 \\
0 \\
70 \\
0 \\
0 \\
0 \\
0 \\
0 \\
0 \\
0 \\
0 \\
0 \\
0 \\
0 \\
0 \\
0 \\
0 \\
0 \\
0 \\
0\end{array}$ & $\begin{array}{r}60 \\
0 \\
0 \\
0 \\
0 \\
0 \\
0 \\
0 \\
0 \\
0 \\
0 \\
0 \\
0 \\
0 \\
0 \\
0 \\
0 \\
60 \\
0 \\
0 \\
0 \\
0 \\
0 \\
0 \\
0 \\
0 \\
0 \\
0 \\
0 \\
0\end{array}$ & $\begin{array}{r}886 \\
0 \\
81 \\
0 \\
10 \\
89 \\
0 \\
0 \\
0 \\
0 \\
0 \\
0 \\
0 \\
0 \\
0 \\
595 \\
105 \\
0 \\
0 \\
0 \\
0 \\
0 \\
0 \\
0 \\
0 \\
0 \\
0 \\
0 \\
0 \\
6\end{array}$ & $\begin{array}{r}13,494 \\
0 \\
81 \\
27 \\
768 \\
89 \\
0 \\
0 \\
0 \\
0 \\
666 \\
0 \\
166 \\
1,950 \\
0 \\
2,755 \\
105 \\
3,284 \\
1,511 \\
0 \\
0 \\
976 \\
302 \\
0 \\
0 \\
353 \\
160 \\
0 \\
0 \\
301\end{array}$ & $\begin{array}{r}162,749 \\
12,499 \\
1,659 \\
27 \\
768 \\
699 \\
15,081 \\
569 \\
6,025 \\
1,395 \\
666 \\
206 \\
166 \\
1,950 \\
646 \\
79,731 \\
105 \\
3,284 \\
3,813 \\
375 \\
406 \\
976 \\
302 \\
470 \\
5,533 \\
22,555 \\
160 \\
928 \\
1,054 \\
701\end{array}$ & $\begin{array}{r}1,658 \\
139 \\
18 \\
0 \\
0 \\
7 \\
168 \\
6 \\
67 \\
16 \\
0 \\
2 \\
0 \\
0 \\
7 \\
855 \\
0 \\
0 \\
26 \\
4 \\
5 \\
0 \\
0 \\
5 \\
61 \\
247 \\
0 \\
10 \\
12 \\
4\end{array}$ & $\begin{array}{r}150 \\
0 \\
1 \\
(s) \\
9 \\
1 \\
0 \\
0 \\
0 \\
0 \\
7 \\
0 \\
2 \\
22 \\
0 \\
31 \\
1 \\
36 \\
17 \\
0 \\
0 \\
11 \\
3 \\
0 \\
0 \\
4 \\
2 \\
0 \\
0 \\
3\end{array}$ & $\begin{array}{r}1,808 \\
139 \\
18 \\
(5) \\
9 \\
8 \\
168 \\
6 \\
67 \\
16 \\
7 \\
2 \\
2 \\
22 \\
7 \\
886 \\
1 \\
36 \\
42 \\
4 \\
5 \\
11 \\
3 \\
5 \\
61 \\
251 \\
2 \\
10 \\
12 \\
8\end{array}$ \\
\hline 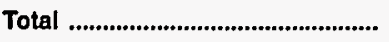 & 2,072 & 12,660 & 70 & 128 & 2,945 & 39,142 & 400,538 & 4,016 & 435 & 4,450 \\
\hline Perslan Gulf ${ }^{\theta}$ & 31 & 0 & $\mathbf{0}$ & 0 & 12 & 4,673 & 108,528 & 1,154 & 52 & 1,206 \\
\hline
\end{tabular}

\footnotetext{
Crude oll and unfinished olls are reported by the PAD District in which they are to be processed; all other products are reported by the PAD District of entry.

b Includes crude oil imported for storage in the Strategic Petroleum Reserve.

c Includes aviation gasoline, aviation gasoline blending components, miscellaneous products, other hydrocarbons and oxygenates, pentanes plus, petroleum coke, and waxes.

d On December 31, 1992, Ecuador withdrew as a member of OPEC. As of January 1, 1994, imports of petroleum from Ecuador appear under imports from Non-OPEC Sources.

- Includes Bahrain, Iran, Iraq, Kuwait, Qatar, Saudi Arabia, and United Arab Emirates.

(s) = Less than 500 barrels per day.

Note: Totals may not equal sum of components due to independent rounding.

Source: Energy Information Administration (EIA) Form EIA-814, "Monthly Imports Report."
} 
Table 44. PAD Districts IV and V-Year-to-Date Imports of Crude Oil and Petroleum Products by Country of

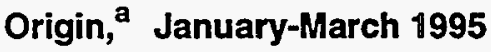

(Thousand Barrels)

\begin{tabular}{c|c|c|c|c|c|c|c|c|c|}
\hline Country of Origin & Crude & $\begin{array}{c}\text { Liquefted } \\
\text { Petroleum } \\
\text { Gases }\end{array}$ & $\begin{array}{c}\text { Unfinished } \\
\text { Oils }\end{array}$ & $\begin{array}{c}\text { Gasoline } \\
\text { Blending } \\
\text { Compo- } \\
\text { nents }\end{array}$ & $\begin{array}{c}\text { Finished } \\
\text { Motor } \\
\text { Gasoline }\end{array}$ & Jet Fuel & $\begin{array}{c}\text { Distillate } \\
\text { Fuel Oill }\end{array}$ & $\begin{array}{c}\text { Residual } \\
\text { Fuel Oil }\end{array}$ & $\begin{array}{c}\text { Special } \\
\text { Kerosene }\end{array}$ \\
\hline
\end{tabular}

PAD District IV

NON OPEC

Total

$\begin{array}{lllll}11,174 & 744 & 0 & 0 & 28 \\ 11,174 & 744 & 0 & 0 & 28 \\ 11,174 & 744 & 0 & 0 & 28\end{array}$

28
28

28
355
355

355

0

PAD District $V$

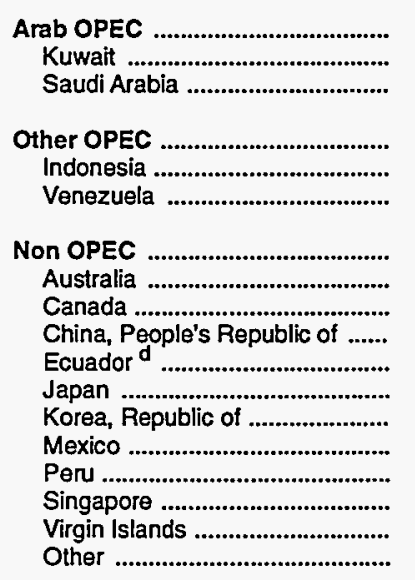

Total

Persian Gulf ${ }^{\theta}$

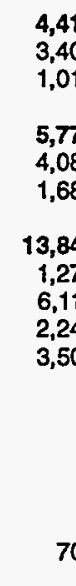

24,031

4,417

$\begin{array}{rrr}4,417 & 0 & 297 \\ 3,403 & 0 & 297 \\ 1,014 & 0 & 0 \\ 5,774 & 0 & 0 \\ 4,085 & 0 & 0 \\ 1,689 & 0 & 0 \\ 13,840 & 500 & 665 \\ 1,278 & 0 & 0 \\ 6,110 & 500 & 79 \\ 2,248 & 0 & 0 \\ 3,502 & 0 & 0 \\ 0 & 0 & 0 \\ 0 & 0 & 0 \\ 0 & 0 & 0 \\ 0 & 0 & 0 \\ 0 & 0 & 586 \\ 0 & 0 & 0 \\ 702 & 0 & 0 \\ 24,031 & 500 & 962 \\ 4,417 & 0 & 297\end{array}$

0
0
0
0
0
0
0
0
0
0
0
0
0
0
0
0
0
0

0
0
0
0
0
0
54
0
54
0
0
0
0
0
0
0
0
0

0
0
0
46
1
45
578
1
6
0
0
0
0
44
0
0
527
0
624
0

$\begin{array}{rr}0 & 0 \\ 0 & 0 \\ 0 & 0 \\ 0 & 0 \\ 0 & 0 \\ 0 & 0 \\ 92 & 35 \\ 0 & 0 \\ 92 & 0 \\ 0 & 0 \\ 0 & 0 \\ 0 & 0 \\ 0 & 0 \\ 0 & 0 \\ 0 & 35 \\ 0 & 0 \\ 0 & 0 \\ 0 & 0 \\ 92 & 35 \\ 0 & 0\end{array}$

$\begin{array}{ll}0 & 0 \\ 0 & 0 \\ 0 & 0 \\ 0 & 0 \\ 0 & 0 \\ 0 & 0 \\ 2 & 3 \\ 0 & 0 \\ 2 & 3 \\ 0 & 0 \\ 0 & 0 \\ 0 & 0 \\ 0 & 0 \\ 0 & 0 \\ 0 & 0 \\ 0 & 0 \\ 0 & 0 \\ 0 & 0 \\ 2 & 3 \\ 0 & 0\end{array}$

See footnotes at end of table. 
Table 44. PAD Districts IV and V-Year-to-Date Imports of Crude Oil and Petroleum Products by Country of Origin, ${ }^{a}$ January-March 1995 (Continued) (Thousand Barrels)

\begin{tabular}{c|c|c|c|c|c|c|c|c|}
\hline \multirow{2}{*}{ Country of Origin } & $\begin{array}{c}\text { Naphiha for } \\
\text { Petrochemical } \\
\text { Feedstock } \\
\text { Use }\end{array}$ & $\begin{array}{c}\text { Other Oils for } \\
\text { Petrochemical } \\
\text { Feedstock } \\
\text { Use }\end{array}$ & Lubricants & $\begin{array}{c}\text { Asphalt and } \\
\text { Road Oil }\end{array}$ & $\begin{array}{c}\text { Total } \\
\text { Other } \\
\text { Products }\end{array}$ & $\begin{array}{c}\text { Crude Oil } \\
\text { and } \\
\text { Products }\end{array}$ & $\begin{array}{c}\text { Total } \\
\text { Products }\end{array}$ & $\begin{array}{c}\text { Daily Average } \\
\text { Oil }\end{array}$ \\
\hline
\end{tabular}

PAD District IN

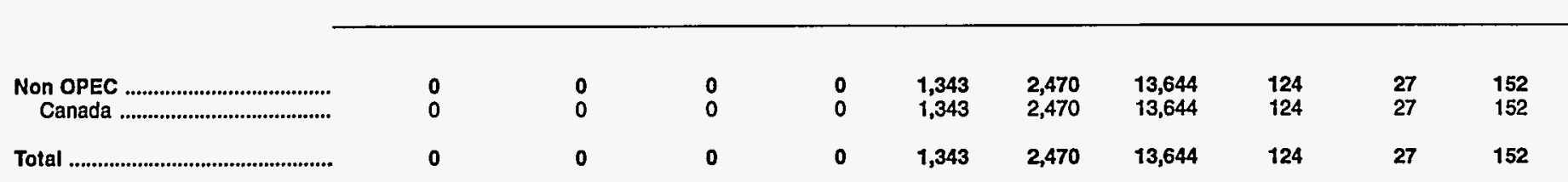

PAD District V

\begin{tabular}{|c|c|c|c|c|c|c|c|c|c|c|}
\hline 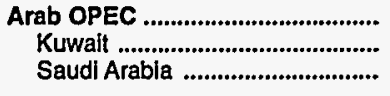 & $\begin{array}{l}0 \\
0 \\
0\end{array}$ & $\begin{array}{l}0 \\
0 \\
0\end{array}$ & $\begin{array}{l}0 \\
0 \\
0\end{array}$ & $\begin{array}{l}0 \\
0 \\
0\end{array}$ & $\begin{array}{r}847 \\
0 \\
847\end{array}$ & $\begin{array}{r}1,144 \\
297 \\
847\end{array}$ & $\begin{array}{l}5,561 \\
3,700 \\
1,861\end{array}$ & $\begin{array}{l}49 \\
38 \\
11\end{array}$ & $\begin{array}{r}13 \\
3 \\
9\end{array}$ & $\begin{array}{l}62 \\
41 \\
21\end{array}$ \\
\hline 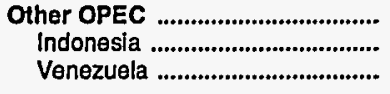 & $\begin{array}{l}0 \\
0 \\
0\end{array}$ & $\begin{array}{l}0 \\
0 \\
0\end{array}$ & $\begin{array}{l}0 \\
0 \\
0\end{array}$ & $\begin{array}{l}0 \\
0 \\
0\end{array}$ & $\begin{array}{r}478 \\
0 \\
478\end{array}$ & $\begin{array}{r}524 \\
1 \\
523\end{array}$ & $\begin{array}{l}6,298 \\
4,086 \\
2,212\end{array}$ & $\begin{array}{l}64 \\
45 \\
19\end{array}$ & $\begin{array}{r}6 \\
(s) \\
6\end{array}$ & $\begin{array}{l}70 \\
45 \\
25\end{array}$ \\
\hline 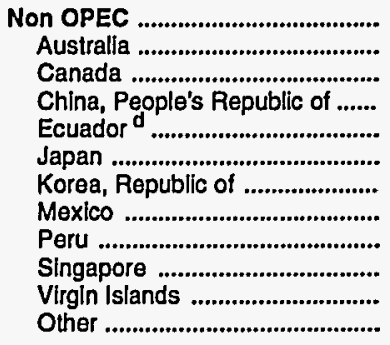 & $\begin{array}{r}59 \\
0 \\
0 \\
0 \\
0 \\
0 \\
59 \\
0 \\
0 \\
0 \\
0 \\
0\end{array}$ & $\begin{array}{l}0 \\
0 \\
0 \\
0 \\
0 \\
0 \\
0 \\
0 \\
0 \\
0 \\
0 \\
0\end{array}$ & $\begin{array}{l}0 \\
0 \\
0 \\
0 \\
0 \\
0 \\
0 \\
0 \\
0 \\
0 \\
0 \\
0\end{array}$ & $\begin{array}{l}9 \\
0 \\
9 \\
0 \\
0 \\
0 \\
0 \\
0 \\
0 \\
0 \\
0 \\
0\end{array}$ & $\begin{array}{r}1,285 \\
0 \\
1,178 \\
0 \\
0 \\
1 \\
0 \\
16 \\
0 \\
0 \\
0 \\
90\end{array}$ & $\begin{array}{r}3,282 \\
1 \\
1,923 \\
0 \\
0 \\
1 \\
59 \\
60 \\
35 \\
586 \\
527 \\
90\end{array}$ & $\begin{array}{r}17,122 \\
1,279 \\
8,033 \\
2,248 \\
3,502 \\
1 \\
59 \\
60 \\
35 \\
586 \\
527 \\
792\end{array}$ & $\begin{array}{r}154 \\
14 \\
68 \\
25 \\
39 \\
0 \\
0 \\
0 \\
0 \\
0 \\
0 \\
8\end{array}$ & $\begin{array}{r}36 \\
(s) \\
21 \\
0 \\
0 \\
(s) \\
1 \\
1 \\
(s) \\
7 \\
6 \\
1\end{array}$ & $\begin{array}{r}190 \\
14 \\
89 \\
25 \\
39 \\
(\mathrm{~s}) \\
1 \\
1 \\
(\mathrm{~s}) \\
7 \\
6 \\
9\end{array}$ \\
\hline 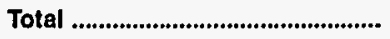 & 59 & 0 & 0 & 9 & 2,610 & 4,950 & 28,981 & 267 & $\mathbf{5 5}$ & 322 \\
\hline Persian Gulf ${ }^{\theta}$................................ & 0 & 0 & 0 & 0 & 847 & 1,144 & 5,561 & 49 & 13 & 62 \\
\hline
\end{tabular}

\footnotetext{
${ }^{a}$ Crude oil and unfinished oils are reported by the PAD District in which they are to be processed; all other products are reported by the PAD District of entry.

Includes crude oil imported for storage in the Strategic Petroleum Reserve.

c Includes aviation gasoline, avlation gasoline blending components, miscellaneous products, other hydrocarbons and oxygenates, pentanes plus, petroleum coke, and waxes.

On December 31, 1992, Ecuador withdrew as a member of OPEC. As of January 1, 1994, imports of petroleum from Ecuador appear under imports from Non-OPEC Sources.

Includes Bahrain, Iran, Iraq, Kuwait, Qatar, Saudi Arabla, and United Arab Emirates.

(s) = Less than 500 barrels per day.

Note: Totals may not equal sum of components due to independent rounding.

Source: Energy Information Administration (EIA) Form EIA-814, "Monthly Imports Report."
} 
Table 45. Exports of Crude Oil and Petroleum Products by PAD District, March 1995

(Thousand Barrels)

\begin{tabular}{|c|c|c|c|c|c|c|c|}
\hline \multirow[b]{2}{*}{ Commodity } & \multicolumn{6}{|c|}{ Petroleum Administration for Defense Districts } & \multirow[b]{2}{*}{$\begin{array}{c}\text { Daily } \\
\text { Average }\end{array}$} \\
\hline & I & II & III & IV & $\mathbf{v}$ & $\begin{array}{l}\text { U.S. } \\
\text { Total }\end{array}$ & \\
\hline Crude Oil ${ }^{\mathrm{a}}$ & 0 & 37 & 0 & 0 & 2,062 & 2,099 & 68 \\
\hline 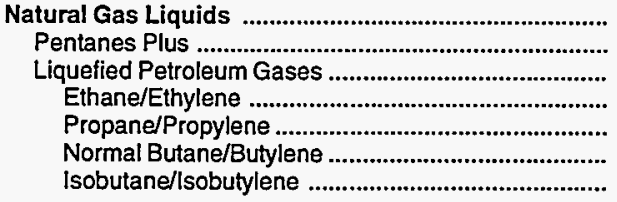 & $\begin{array}{r}23 \\
3 \\
20 \\
0 \\
19 \\
1 \\
0\end{array}$ & $\begin{array}{r}165 \\
23 \\
142 \\
0 \\
65 \\
78 \\
0\end{array}$ & $\begin{array}{r}902 \\
(s) \\
901 \\
0 \\
783 \\
118 \\
0\end{array}$ & $\begin{array}{l}1 \\
0 \\
1 \\
0 \\
0 \\
1 \\
0\end{array}$ & $\begin{array}{r}703 \\
0 \\
703 \\
0 \\
338 \\
365 \\
0\end{array}$ & $\begin{array}{r}1,794 \\
26 \\
1,768 \\
0 \\
1,204 \\
563 \\
0\end{array}$ & $\begin{array}{r}58 \\
1 \\
57 \\
0 \\
39 \\
18 \\
0\end{array}$ \\
\hline $\begin{array}{l}\text { Other Liquids } \\
\text { Other Hydrocarbons/Oxygenates } \\
\text { Motor Gasoline Blend. Comp. }\end{array}$ & $\begin{array}{r}\text { (s) } \\
\text { (s) } \\
0\end{array}$ & $\begin{array}{r}2 \\
(s) \\
2\end{array}$ & $\begin{array}{l}400 \\
233 \\
167\end{array}$ & $\begin{array}{l}0 \\
0 \\
0\end{array}$ & $\begin{array}{l}77 \\
\text { (s) } \\
76\end{array}$ & $\begin{array}{l}479 \\
234 \\
244\end{array}$ & $\begin{array}{r}15 \\
8 \\
8\end{array}$ \\
\hline $\begin{array}{l}\text { Finished Petroleum Products } \\
\text { Finished Motor Gasoline } \\
\text { Naphtha-Type Jet Fuel } \\
\text { Kerosene-Type Jet Fuel } \\
\text { Kerosene } \\
\text { Distillate Fuel Oil } \\
\text { Residual Fuel Oil } \\
\text { Special Naphthas } \\
\text { Lubricants } \\
\text { Paxes } \\
\text { Asphaleum Coke } \\
\text { Miscelland Road Oil }\end{array}$ & $\begin{array}{r}909 \\
11 \\
0 \\
(\mathrm{~s}) \\
183 \\
96 \\
1 \\
6 \\
148 \\
10 \\
442 \\
5 \\
6\end{array}$ & $\begin{array}{r}355 \\
113 \\
0 \\
0 \\
(s) \\
31 \\
57 \\
8 \\
54 \\
6 \\
65 \\
20 \\
\text { (s) }\end{array}$ & $\begin{array}{r}11,913 \\
2,601 \\
0 \\
86 \\
0 \\
2,695 \\
2,169 \\
95 \\
344 \\
43 \\
3,871 \\
8 \\
\text { (s) }\end{array}$ & $\begin{array}{r}17 \\
10 \\
0 \\
0 \\
0 \\
0 \\
0 \\
\text { (s) } \\
6 \\
0 \\
0 \\
1 \\
0\end{array}$ & $\begin{array}{r}11,818 \\
580 \\
1 \\
442 \\
1 \\
3,883 \\
2,547 \\
251 \\
157 \\
12 \\
3,929 \\
15 \\
1\end{array}$ & $\begin{array}{r}25,011 \\
3,315 \\
1 \\
528 \\
185 \\
6,706 \\
4,774 \\
360 \\
708 \\
72 \\
8,308 \\
49 \\
8\end{array}$ & $\begin{array}{r}807 \\
107 \\
(s) \\
17 \\
6 \\
216 \\
154 \\
12 \\
23 \\
2 \\
268 \\
2 \\
(s)\end{array}$ \\
\hline Total & 932 & 559 & 13,214 & 19 & 14,659 & 29,382 & 948 \\
\hline
\end{tabular}

a Crude oil exports are restricted to: (1) crude oil derived from fields under the State waters of Alaska's Cook Inlet; (2) certain domestically produced crude oil destined for Canada; and (3) shipments to U.S. territories, and California crude oil to Pacific Rim countries. On December 6, 1991, the U.S. Department of

Commerce approved a license to export 25,000 barrels per day of California heavy crude oil (less than 20 degrees API gravity) to Pacific Rim countries for one year.

$(s)=$ Less than 500 barrels or less than 500 barrels per day.

Note: Totals may not equal sum of components due to independent rounding.

Sources: Energy Information Administration (EIA) Form EIA-810, "Monthly Refinery Report" and the U.S. Bureau of the Census. 
Table 46. Year-to-Date Exports of Crude Oil and Petroleum Products by PAD District, January-March 1995

(Thousand Barrels)

\begin{tabular}{|c|c|c|c|c|c|c|c|}
\hline \multirow[b]{2}{*}{ Commodity } & \multicolumn{6}{|c|}{ Petroleum Administration for Defense Districts } & \multirow[b]{2}{*}{$\begin{array}{l}\text { Daily } \\
\text { Average }\end{array}$} \\
\hline & $\mathbf{I}$ & II & III & IV & $\mathbf{V}$ & $\begin{array}{l}\text { U.S. } \\
\text { Total }\end{array}$ & \\
\hline 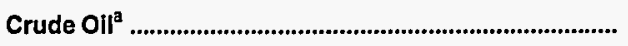 & 0 & 38 & $\mathbf{0}$ & 0 & 8,207 & 8,245 & 92 \\
\hline 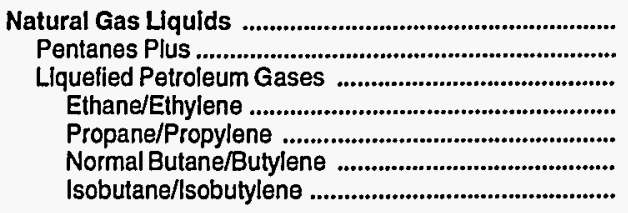 & $\begin{array}{r}92 \\
6 \\
86 \\
0 \\
83 \\
3 \\
0\end{array}$ & $\begin{array}{r}637 \\
68 \\
569 \\
0 \\
263 \\
306 \\
0\end{array}$ & $\begin{array}{r}4,741 \\
(s) \\
4,741 \\
0 \\
4,263 \\
478 \\
0\end{array}$ & $\begin{array}{l}1 \\
0 \\
1 \\
0 \\
0 \\
1 \\
0\end{array}$ & $\begin{array}{r}1,765 \\
(s) \\
1,764 \\
0 \\
1,102 \\
662 \\
0\end{array}$ & $\begin{array}{r}7,236 \\
74 \\
7,162 \\
0 \\
5,711 \\
1,451 \\
0\end{array}$ & $\begin{array}{r}80 \\
1 \\
80 \\
0 \\
63 \\
16 \\
0\end{array}$ \\
\hline 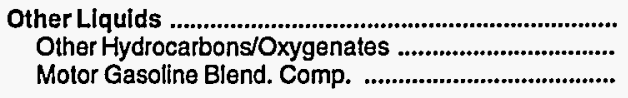 & $\begin{array}{l}5 \\
4 \\
1\end{array}$ & $\begin{array}{l}2 \\
1 \\
2\end{array}$ & $\begin{array}{r}1,243 \\
871 \\
372\end{array}$ & $\begin{array}{l}\mathbf{0} \\
\mathbf{0} \\
\mathbf{0}\end{array}$ & $\begin{array}{r}128 \\
1 \\
126\end{array}$ & $\begin{array}{r}1,377 \\
876 \\
501\end{array}$ & $\begin{array}{r}15 \\
10 \\
6\end{array}$ \\
\hline 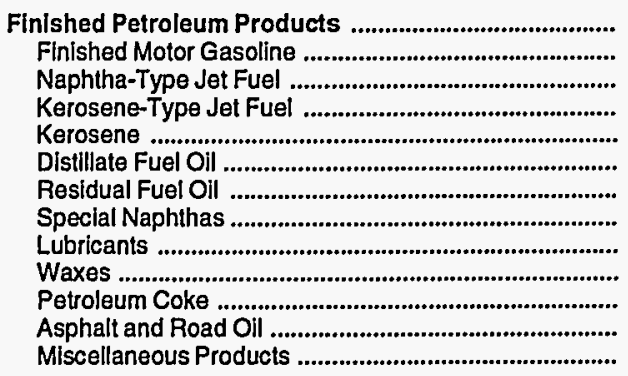 & $\begin{array}{r}2,448 \\
38 \\
1 \\
1 \\
329 \\
218 \\
682 \\
17 \\
394 \\
30 \\
706 \\
11 \\
21\end{array}$ & $\begin{array}{r}846 \\
145 \\
(\mathrm{~s}) \\
0 \\
1 \\
35 \\
181 \\
20 \\
155 \\
21 \\
194 \\
93 \\
(\mathrm{~s})\end{array}$ & $\begin{array}{r}40,409 \\
7,632 \\
296 \\
837 \\
1 \\
6,878 \\
9,653 \\
283 \\
1,295 \\
103 \\
13,364 \\
66 \\
\text { (s) }\end{array}$ & $\begin{array}{r}33 \\
16 \\
0 \\
0 \\
0 \\
0 \\
0 \\
1 \\
13 \\
0 \\
0 \\
3 \\
\text { (s) }\end{array}$ & $\begin{array}{r}28,822 \\
947 \\
1 \\
1,001 \\
3 \\
9,887 \\
6,364 \\
1,745 \\
319 \\
36 \\
8,473 \\
45 \\
3\end{array}$ & $\begin{array}{r}72,558 \\
8,777 \\
298 \\
1,839 \\
335 \\
17,017 \\
16,880 \\
2,065 \\
2,176 \\
189 \\
22,738 \\
219 \\
24\end{array}$ & $\begin{array}{r}806 \\
98 \\
3 \\
20 \\
4 \\
189 \\
188 \\
23 \\
24 \\
2 \\
253 \\
2 \\
(s)\end{array}$ \\
\hline Total & 2,544 & 1,523 & 46,393 & 34 & 38,922 & 89,416 & 994 \\
\hline
\end{tabular}

a Crude oil exports are restricted to: (1) crude oil derived from fields under the State waters of Alaska's Cook Inlet; (2) certain domestically produced crude oil destined for Canada; and (3) shipments to U.S. territories, and California crude oil to Pacific Rim countries. On December 6, 1991, the U.S. Department of Commerce approved a license to export 25,000 barrels per day of California heavy crude oil (less than 20 degrees API gravity) to Pacific Rim countries for one year.

(s) = Less than 500 barrels or less than 500 barrels per day.

Note: Totals may not equal sum of components due to independent rounding.

Sources: Energy Information Administration (EIA) Form EIA-810, "Monthly Refinery Report" and the U.S. Bureau of the Census. 
Table 47. Exports of Crude Oil and Petroleum Products by Destination, March 1995 (Thousand Barrels)

\begin{tabular}{|c|c|c|c|c|c|c|c|c|}
\hline Destination & $\begin{array}{c}\text { Crude } \\
\text { Oil }^{\mathrm{a}}\end{array}$ & $\begin{array}{c}\text { Pentanes } \\
\text { Plus }\end{array}$ & $\begin{array}{c}\text { Liquefled } \\
\text { Petroleum } \\
\text { Gases }\end{array}$ & $\begin{array}{l}\text { Finished } \\
\text { Motor } \\
\text { Gasoline }\end{array}$ & Jet Fuel & Kerosene & $\begin{array}{c}\text { Distlllate Fuel } \\
\text { Oll }\end{array}$ & $\begin{array}{l}\text { Residual } \\
\text { Fuel Oll }\end{array}$ \\
\hline 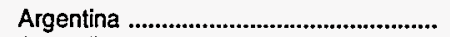 & 0 & 0 & 0 & 251 & 63 & 0 & 1 & 0 \\
\hline Australia & 0 & 0 & (s) & 274 & 0 & 0 & 31 & 0 \\
\hline 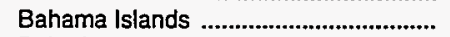 & 0 & 0 & 23 & 57 & 23 & 0 & 178 & (s) \\
\hline Bahrain & 0 & 0 & 0 & 0 & 0 & 0 & 0 & 0 \\
\hline 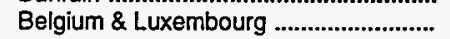 & 0 & 0 & 0 & 1 & 0 & 0 & 1 & 1 \\
\hline 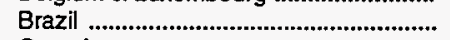 & 0 & 0 & 0 & 0 & 0 & 0 & (s) & 0 \\
\hline Canada & 37 & 23 & 198 & 250 & 147 & 1 & 296 & 159 \\
\hline Chile & 0 & 0 & 113 & 0 & 0 & 1 & 10 & 0 \\
\hline China, People's Republic of .................... & 0 & 0 & 0 & 0 & 0 & 0 & 159 & 0 \\
\hline 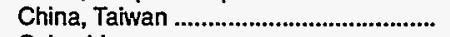 & 0 & 0 & 115 & 0 & 0 & 0 & 570 & 195 \\
\hline Colombia & 0 & 0 & 56 & 0 & 0 & 0 & 4 & 0 \\
\hline 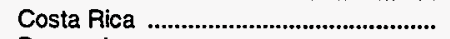 & 0 & 0 & 0 & 0 & 0 & $\mathbf{0}$ & 233 & 0 \\
\hline 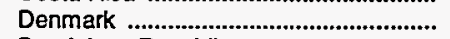 & 0 & 0 & 0 & 0 & 0 & 0 & 0 & 0 \\
\hline Dominican Republic ................................ & 0 & 1 & 0 & 82 & 0 & 0 & (s) & 0 \\
\hline 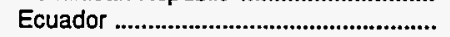 & 0 & 0 & 0 & 0 & 0 & 0 & 220 & 0 \\
\hline Egypt & 0 & 0 & 0 & 0 & 0 & 0 & 1 & 0 \\
\hline 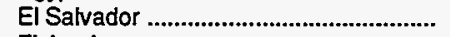 & 0 & 1 & 5 & 0 & 0 & 0 & 116 & 0 \\
\hline Finland & 0 & 0 & 0 & 0 & 0 & 0 & 0 & 0 \\
\hline France & 0 & 0 & 0 & 0 & 0 & 0 & 0 & 0 \\
\hline French Pacific Islands .............................. & 0 & 0 & 0 & 0 & 0 & 0 & 0 & 0 \\
\hline 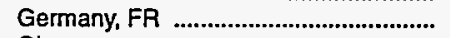 & 0 & 0 & 0 & 0 & 0 & 0 & 2 & 0 \\
\hline Ghana & 0 & 0 & 0 & 0 & 0 & 0 & 0 & 0 \\
\hline Greece & 0 & 0 & 0 & 0 & 0 & 0 & 0 & 0 \\
\hline 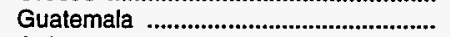 & 0 & 0 & 12 & 81 & 0 & 0 & 70 & 0 \\
\hline Guinea & 0 & 0 & 0 & 0 & 0 & 0 & (s) & 0 \\
\hline 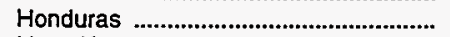 & 0 & 0 & 12 & 0 & 0 & 0 & 130 & 0 \\
\hline 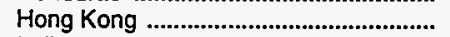 & 0 & 0 & 0 & 0 & 0 & 0 & 175 & (s) \\
\hline India & 0 & 0 & 0 & 0 & 0 & 0 & (s) & 0 \\
\hline 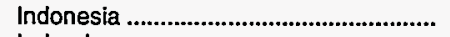 & 0 & 0 & 0 & 0 & 0 & 0 & 0 & 0 \\
\hline Ireland & 0 & 0 & 0 & 0 & 0 & 0 & 0 & 0 \\
\hline Israel & 0 & 0 & 0 & 0 & 0 & 0 & 231 & 0 \\
\hline Italy & 0 & 0 & 1 & 0 & 0 & 0 & 324 & 511 \\
\hline 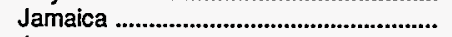 & 0 & 0 & 38 & 0 & 0 & 0 & 0 & 669 \\
\hline 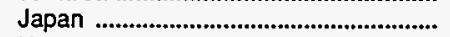 & 0 & 0 & (s) & (s) & 295 & 1 & 3 & 1 \\
\hline 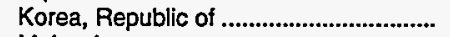 & 0 & 0 & 4 & 0 & 0 & 0 & 1,140 & 604 \\
\hline Malaysia & 0 & 0 & 0 & 0 & 0 & 0 & 0 & 0 \\
\hline Mexico & 0 & (s) & 950 & 1,814 & 0 & 1 & 23 & 710 \\
\hline 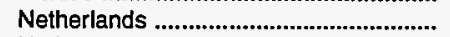 & 0 & 0 & 0 & 0 & 0 & 0 & 1 & 0 \\
\hline Netherlands Antilles ............................... & 0 & 0 & 0 & 80 & 0 & 0 & 469 & 0 \\
\hline 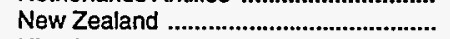 & 0 & 0 & 0 & 174 & 0 & 0 & 0 & 0 \\
\hline 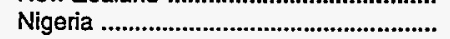 & 0 & 0 & 0 & 0 & 0 & 0 & 0 & 0 \\
\hline 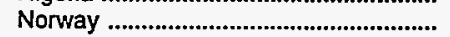 & 0 & 0 & 0 & 0 & 0 & 0 & 0 & 0 \\
\hline Panama & 0 & 0 & 0 & 0 & 0 & 0 & 270 & 449 \\
\hline Peru & 0 & 0 & 0 & 0 & 0 & 0 & (s) & 219 \\
\hline 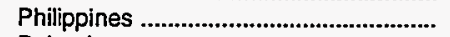 & 0 & 0 & 229 & 0 & 0 & 0 & 0 & 0 \\
\hline 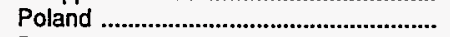 & 0 & 0 & 0 & 0 & 0 & 0 & 0 & 0 \\
\hline 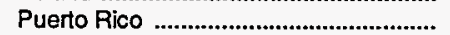 & 0 & 0 & 1 & 252 & 0 & 0 & 6 & 448 \\
\hline 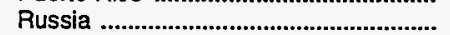 & 0 & 0 & 0 & 0 & 0 & 0 & 1 & 0 \\
\hline Saudi Arabia & 0 & 0 & 0 & 0 & 0 & 0 & (s) & 0 \\
\hline Singapore & 0 & 0 & 0 & 0 & 0 & 0 & 1,165 & 809 \\
\hline 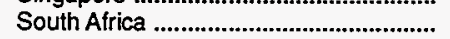 & 0 & 0 & 0 & 0 & 0 & 0 & 0 & 0 \\
\hline Spain & 0 & 0 & $(s)$ & 0 & 0 & 0 & 325 & 0 \\
\hline Suriname & 0 & 0 & 0 & 0 & 0 & 0 & 0 & 0 \\
\hline Sweden & 0 & 0 & 0 & (s) & 0 & 0 & 1 & 0 \\
\hline 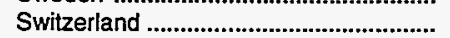 & 0 & 0 & 0 & 0 & 0 & 0 & 0 & 0 \\
\hline 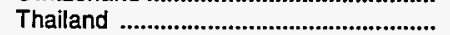 & 0 & 0 & 0 & 0 & 0 & 0 & 547 & 0 \\
\hline Trinidad and Tobago ................................. & 0 & 0 & 0 & 0 & 0 & 0 & 1 & 0 \\
\hline Turkey & 0 & 0 & 0 & 0 & 0 & 0 & 0 & 0 \\
\hline 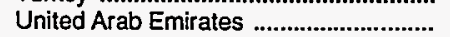 & 0 & 0 & 0 & 0 & 0 & 0 & 0 & 0 \\
\hline 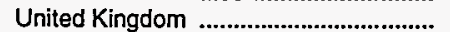 & 0 & 0 & 1 & 0 & 0 & 0 & 1 & 0 \\
\hline 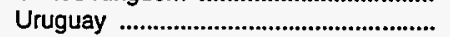 & 0 & 0 & 0 & 0 & 0 & 0 & 0 & 0 \\
\hline Venezuela & 0 & 0 & 0 & 0 & 0 & 0 & (s) & 0 \\
\hline 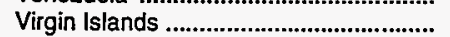 & 2,062 & 0 & 0 & 0 & 0 & 182 & 0 & 0 \\
\hline Yugoslavia & 0 & 0 & 0 & 0 & 0 & 0 & (s) & 0 \\
\hline Other & 0 & (s) & 10 & 0 & 0 & 0 & 1 & 0 \\
\hline Total & 2,099 & 26 & 1,768 & 3,315 & 528 & 185 & 6,706 & 4,774 \\
\hline
\end{tabular}


Table 47. Exports of Crude Oil and Petroleum Products by Destination, March 1995 (Continued) (Thousand Barrels)

\begin{tabular}{|c|c|c|c|c|c|c|c|c|}
\hline \multirow{2}{*}{ Destination } & \multirow[b]{2}{*}{$\begin{array}{c}\text { Special } \\
\text { Naphthas }\end{array}$} & \multirow[b]{2}{*}{ Lubricants } & \multirow[b]{2}{*}{ Waxes } & \multirow[b]{2}{*}{$\begin{array}{c}\text { Petroleum } \\
\text { Coke }\end{array}$} & \multirow{2}{*}{$\begin{array}{c}\text { Asphalt } \\
\text { and Road } \\
\text { Oil }\end{array}$} & \multirow[b]{2}{*}{$\begin{array}{c}\text { Other } \\
\text { Products }\end{array}$} & \multicolumn{2}{|c|}{ Crude Oil and Products } \\
\hline & & & & & & & Total & $\begin{array}{c}\text { Daily } \\
\text { Average }\end{array}$ \\
\hline 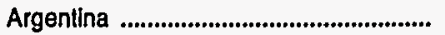 & 0 & 10 & (s) & 0 & 1 & 1 & 327 & 11 \\
\hline 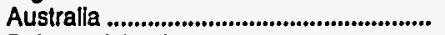 & 1 & 15 & (s) & 296 & 0 & (s) & 617 & 20 \\
\hline 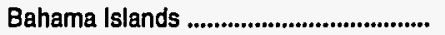 & 0 & 2 & 0 & 0 & 2 & 0 & 285 & 9 \\
\hline 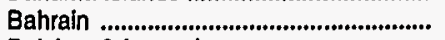 & 0 & (s) & 0 & 98 & 0 & 0 & 98 & 3 \\
\hline Belglum \& Luxembourg ............................ & 0 & 10 & (s) & 584 & 1 & (s) & 598 & 19 \\
\hline 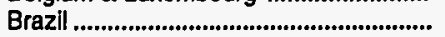 & 202 & 17 & 7 & 118 & 0 & (s) & 345 & 11 \\
\hline 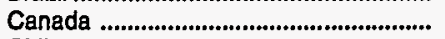 & 12 & 132 & 11 & 497 & 23 & 2 & 1,790 & 58 \\
\hline Chile & 2 & 14 & 1 & (s) & 0 & (s) & 141 & 5 \\
\hline China, People's Republic of ................... & 0 & 1 & 1 & 0 & (s) & (s) & 162 & 5 \\
\hline 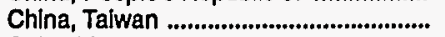 & 2 & 31 & (s) & 2 & 2 & (s) & 915 & 30 \\
\hline Colombla & $(\mathbf{s})$ & 3 & 1 & (s) & (s) & (s) & 64 & 2 \\
\hline 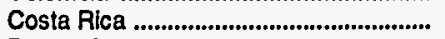 & 2 & 9 & (s) & 0 & 0 & (s) & 244 & 8 \\
\hline 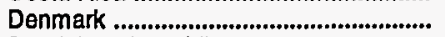 & (s) & (s) & (s) & 137 & (s) & 2 & 139 & 4 \\
\hline Dominican Republic ................................ & 0 & 4 & 1 & 0 & 0 & $\overline{0}$ & 88 & 3 \\
\hline 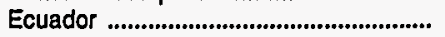 & 0 & 1 & 1 & 0 & 0 & 0 & 222 & 7 \\
\hline 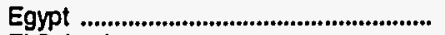 & 0 & 10 & 0 & 0 & (s) & 0 & 11 & (s) \\
\hline 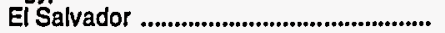 & (s) & 5 & (s) & 0 & 0 & 0 & 127 & 4 \\
\hline Finland & 0 & (s) & (s) & 0 & 0 & 0 & (s) & (s) \\
\hline France & 0 & 1 & 3 & 355 & 2 & 0 & 360 & 12 \\
\hline French Pacific Islands ............................ & 0 & (s) & 0 & 0 & 0 & 0 & (s) & (s) \\
\hline Germany, FR .......................................... & (s) & 7 & 4 & 140 & 4 & 1 & 159 & 5 \\
\hline Ghana & 0 & (s) & 0 & 48 & 0 & 0 & 48 & 2 \\
\hline 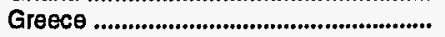 & 0 & 1 & 0 & 238 & 0 & 0 & 240 & 8 \\
\hline Guatemala .............................................. & 5 & 6 & 1 & 0 & 0 & 0 & 175 & 6 \\
\hline 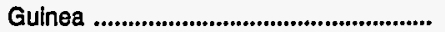 & 0 & 1 & 0 & 0 & 0 & 0 & 1 & (s) \\
\hline 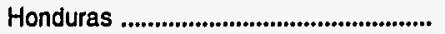 & 1 & 5 & (s) & 0 & 0 & 0 & 149 & 5 \\
\hline Hong Kong & (s) & 2 & 1 & 0 & 0 & (s) & 179 & 6 \\
\hline 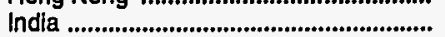 & 0 & 47 & 1 & 0 & (s) & 0 & 48 & 2 \\
\hline 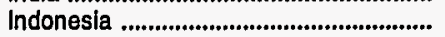 & (s) & 1 & 2 & 92 & 0 & 0 & 95 & 3 \\
\hline Ireland & 0 & (s) & (s) & 143 & 0 & (s) & 144 & 5 \\
\hline Israel & 0 & 1 & 0 & 0 & 0 & 0 & 232 & $\overline{7}$ \\
\hline Italy & (s) & (s) & 1 & 663 & 1 & (s) & 1,500 & 48 \\
\hline 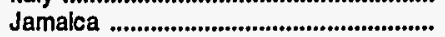 & (s) & 2 & (s) & 81 & 0 & (s) & 790 & 25 \\
\hline 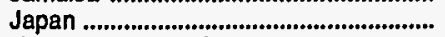 & 9 & 16 & 4 & 1,482 & 1 & (s) & 1,812 & 58 \\
\hline 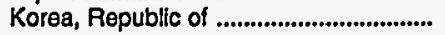 & 1 & 13 & 2 & 88 & 1 & (s) & 1,852 & 60 \\
\hline 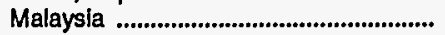 & (s) & 2 & (s) & 0 & (s) & (s) & 2 & (s) \\
\hline 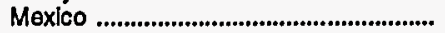 & 2 & 97 & 23 & 108 & 2 & 285 & 4,014 & 129 \\
\hline 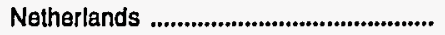 & 71 & 1 & (s) & 1,014 & 3 & (s) & 1,091 & 35 \\
\hline Netherlands Antilles ................................ & 0 & 1 & 0 & 0 & 0 & 0 & 550 & 18 \\
\hline 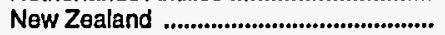 & 0 & 2 & (s) & 121 & 0 & 76 & 373 & 12 \\
\hline 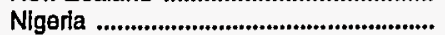 & 0 & 1 & 0 & 0 & 0 & 0 & 1 & (s) \\
\hline 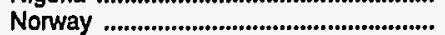 & 0 & 1 & (s) & 93 & 0 & 0 & 93 & 3 \\
\hline 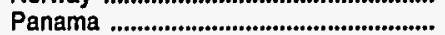 & 0 & 4 & (s) & 0 & 0 & (s) & 723 & 23 \\
\hline Peru & 0 & 30 & (s) & (s) & 0 & (s) & 250 & 8 \\
\hline & 0 & (s) & 1 & 0 & 0 & (s) & 230 & 7 \\
\hline Poland & $\mathbf{0}$ & (s) & 0 & 0 & 0 & 0 & (s) & (s) \\
\hline Puerto Rilco & 1 & 22 & 1 & 0 & 0 & (s) & 731 & 24 \\
\hline 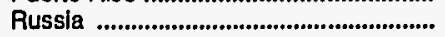 & 0 & 2 & 0 & 0 & 0 & 0 & 3 & (s) \\
\hline Saudi Arabla ............................................. & 0 & 3 & 1 & 0 & 0 & (s) & 3 & (s) \\
\hline 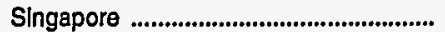 & 46 & 90 & (s) & 24 & 1 & (s) & 2,136 & 69 \\
\hline 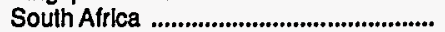 & 0 & 17 & (s) & 39 & 0 & 0 & 55 & 2 \\
\hline 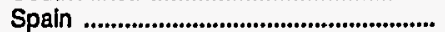 & 0 & (s) & (s) & 922 & (s) & (s) & 1,249 & 40 \\
\hline Suriname & 0 & (s) & 0 & 0 & 0 & 0 & (s) & (s) \\
\hline Sweden & 0 & 1 & (s) & 0 & 0 & 0 & 3 & (s) \\
\hline & (s) & (s) & (s) & 0 & 0 & 0 & (s) & (s) \\
\hline Thalland ..................................................... & (s) & 7 & (s) & 0 & 0 & 1 & 555 & $\begin{array}{l}\text { (3) } \\
18\end{array}$ \\
\hline TrInidad and Tobago & 0 & (s) & (s) & 0 & 0 & 0 & 1 & $\begin{array}{l}18 \\
(s)\end{array}$ \\
\hline 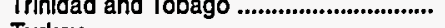 & 0 & (s) & (S) & 0 & 0 & 0 & 1 & (s) \\
\hline Turkey & (s) & 2 & (s) & 473 & 0 & 0 & 475 & 15 \\
\hline United Arab Emirates ............................... & 0 & 1 & 0 & (s) & 0 & (s) & 1 & (s) \\
\hline United Kingdom ........................................ & 0 & 2 & 1 & 58 & 1 & (s) & 64 & 2 \\
\hline Uruguay & 0 & 1 & (s) & 0 & (s) & 0 & 1 & (s) \\
\hline 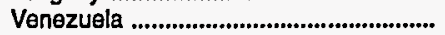 & 0 & 37 & 1 & 115 & 1 & 115 & 269 & 9 \\
\hline 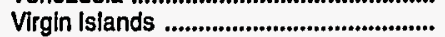 & 0 & 0 & 0 & 0 & 0 & 0 & 2,244 & 72 \\
\hline Yugoslavia .............................................. & 0 & 0 & 0 & 0 & 0 & 0 & (s) & (s) \\
\hline Other & 1 & 14 & (s) & 277 & (s) & (s) & 305 & 10 \\
\hline Total & 360 & 708 & 72 & 8,308 & 49 & 486 & 29,382 & 948 \\
\hline
\end{tabular}

a Crude oll exports are restricted to: (1) crude oll derived from fields under the State waters of Alaska's Cook Inlet; (2) certain domestically produced crude oil destined for Canada; and (3) shipments to U.S. territorles, and California crude oil to Pacilic Rim countries. On December 6, 1991, the U.S. Department of

Commerce approved a license to export 25,000 barrels per day of California heavy crude oil (less than 20 degrees API gravity) to Pacific Rim countries for one year. Includes miscellaneous products, motor gasoline blending components, and other hydrocarbons and oxygenates.

(s) = Less than 500 barrels or less than 500 barrels per day.

Note: Totals may not equal sum of components due to independent rounding.

Sources: Energy Information Administration (EIA) Form EIA-810, "Monthly Refinery Report" and the U.S. Bureau of the Census. 
Table 48. Year-to-Date Exports of Crude Oil and Petroleum Products by Destination, January-March 1995

(Thousand Barrels)

\begin{tabular}{|c|c|c|c|c|c|c|c|c|}
\hline Destination & $\begin{array}{c}\text { Crude } \\
\text { Oil }^{\mathrm{a}}\end{array}$ & $\begin{array}{c}\begin{array}{c}\text { Pentanes } \\
\text { Plus }\end{array} \\
\end{array}$ & $\begin{array}{c}\text { Liquefied } \\
\text { Petroleum } \\
\text { Gases }\end{array}$ & $\begin{array}{c}\text { Finished } \\
\text { Motor } \\
\text { Gasoline }\end{array}$ & Jet Fuel & Kerosene & $\begin{array}{c}\text { Distillate Fuel } \\
\text { Oll } \\
\end{array}$ & $\begin{array}{l}\text { Residual } \\
\text { Fuel OIl }\end{array}$ \\
\hline Argentina & 0 & 0 & 0 & 251 & 63 & 0 & 2 & 0 \\
\hline 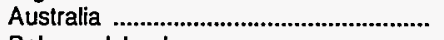 & 0 & 0 & (s) & 274 & 0 & (s) & 31 & 0 \\
\hline 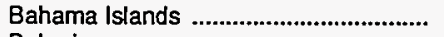 & 0 & 0 & 32 & 87 & 32 & 0 & 312 & 1 \\
\hline 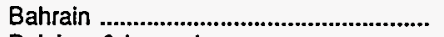 & 0 & 0 & 0 & 0 & 0 & 0 & 0 & 0 \\
\hline Belgium \& Luxembourg ............................. & 0 & 0 & 1 & 1 & 0 & 0 & 3 & 1 \\
\hline 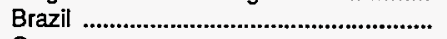 & 0 & 0 & 0 & 0 & 1 & 0 & 3 & 0 \\
\hline Cameroon & 0 & 0 & 1 & 0 & 0 & 0 & 0 & 0 \\
\hline Canada & 38 & 70 & 716 & 430 & 546 & 147 & 621 & 1,029 \\
\hline Chile & 0 & 0 & 113 & 53 & 0 & 1 & 379 & 0 \\
\hline China, People's Republic of .................... & 0 & 0 & 0 & 0 & 0 & 0 & 160 & 0 \\
\hline 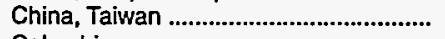 & 0 & 0 & 115 & 0 & 0 & 1 & 1,983 & 887 \\
\hline 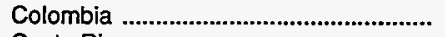 & 0 & 0 & 119 & 2 & 0 & 0 & 25 & 0 \\
\hline Costa Rica & 0 & 0 & 0 & 89 & 0 & 0 & 320 & 0 \\
\hline 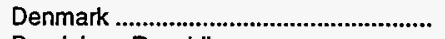 & 0 & 0 & 0 & 0 & 0 & 0 & 0 & 0 \\
\hline Dominican Republic .................................. & 0 & 3 & 86 & 172 & 0 & 0 & (s) & 49 \\
\hline 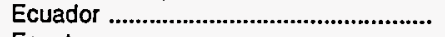 & 0 & 0 & 1,385 & 0 & 0 & 0 & 220 & 6 \\
\hline 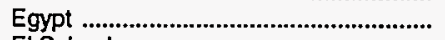 & 0 & 0 & 0 & 0 & 0 & 0 & 2 & 0 \\
\hline 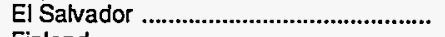 & 0 & 1 & 40 & 0 & 0 & 0 & 401 & (s) \\
\hline 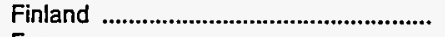 & 0 & 0 & 0 & 0 & 0 & 0 & 1 & 0 \\
\hline France & 0 & 0 & 150 & 0 & 0 & 0 & 1 & 0 \\
\hline 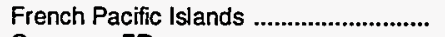 & 0 & 0 & 0 & 0 & 0 & 0 & (s) & 0 \\
\hline Germany, FR & 0 & 0 & 0 & 0 & 0 & 0 & 2 & 0 \\
\hline 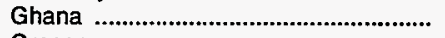 & 0 & 0 & 0 & 0 & 0 & 0 & 0 & 0 \\
\hline Greece & 0 & 0 & 0 & 0 & 0 & 0 & 0 & 0 \\
\hline Guatemala & 0 & 0 & 14 & 309 & 4 & 0 & 355 & 66 \\
\hline Guinea & 0 & 0 & 0 & 0 & (s) & 0 & (s) & 0 \\
\hline 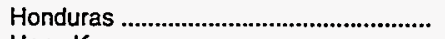 & 0 & 0 & 18 & 0 & 0 & 0 & 130 & 137 \\
\hline 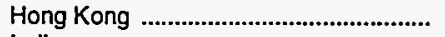 & 0 & (s) & 0 & 0 & 0 & 0 & 176 & 1 \\
\hline India & 0 & 0 & 0 & 0 & 0 & (s) & (s) & 0 \\
\hline 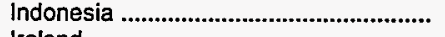 & 0 & 0 & 0 & 0 & 0 & 0 & 0 & 0 \\
\hline Ireland & 0 & 0 & 0 & 0 & 0 & 0 & 0 & 369 \\
\hline Israel & 0 & 0 & 0 & 0 & 249 & 0 & 745 & 0 \\
\hline 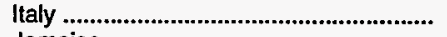 & 0 & 0 & 3 & 88 & 0 & 0 & 789 & 3,163 \\
\hline 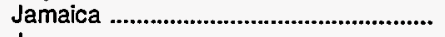 & 0 & 0 & 69 & 0 & 0 & 0 & (s) & 1,944 \\
\hline 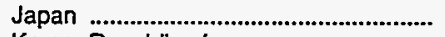 & 0 & 0 & 1 & 1 & 1,192 & 1 & 17 & 261 \\
\hline 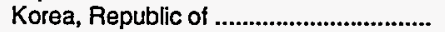 & 0 & 0 & 4 & 0 & 0 & 0 & 3,822 & 1,656 \\
\hline 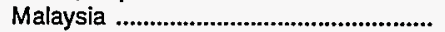 & 0 & 0 & 0 & 0 & 0 & 0 & 1 & 0 \\
\hline Mexico & 0 & (s) & 3,325 & 6,006 & (s) & 2 & 50 & 2,383 \\
\hline 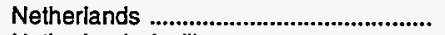 & 0 & 0 & (s) & 0 & 0 & 0 & 2 & 310 \\
\hline Netherlands Antilles ................................ & 0 & 0 & 0 & 80 & 21 & 0 & 664 & 0 \\
\hline 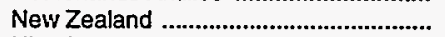 & 0 & 0 & 0 & 174 & 0 & 0 & (s) & 0 \\
\hline Nigeria & 0 & 0 & 0 & 0 & 0 & 0 & 0 & 0 \\
\hline 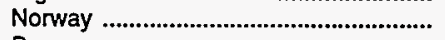 & 0 & 0 & 0 & 0 & 0 & 0 & 0 & 0 \\
\hline Panama & 0 & 0 & (s) & 231 & 0 & 0 & 955 & 1,245 \\
\hline Peru & 0 & 0 & 0 & 164 & (s) & 0 & 222 & 219 \\
\hline 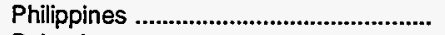 & 0 & 0 & 469 & 0 & 0 & 0 & 406 & 0 \\
\hline Poland & 0 & 0 & 0 & 0 & 0 & 0 & 0 & 0 \\
\hline 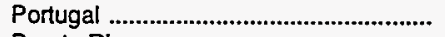 & 0 & 0 & 57 & 0 & 0 & 0 & 0 & 0 \\
\hline Puerto Rico & 0 & 0 & 4 & 362 & 23 & 0 & 93 & 449 \\
\hline Russia & 0 & 0 & 0 & 0 & 0 & 0 & 20 & 0 \\
\hline 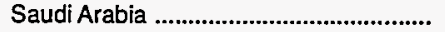 & 0 & 0 & 0 & 0 & 0 & (s) & 1 & 0 \\
\hline Singapore & 0 & (s) & 0 & 0 & 0 & 0 & 2,339 & 1,868 \\
\hline South Africa & 0 & 0 & 0 & 0 & 0 & 0 & (s) & 0 \\
\hline Spain & 0 & 0 & (s) & 0 & 0 & 0 & 640 & 0 \\
\hline 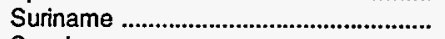 & 0 & 0 & 0 & 0 & 0 & 1 & 0 & 0 \\
\hline Sweden & 0 & 0 & 0 & (s) & 0 & 0 & 2 & 0 \\
\hline 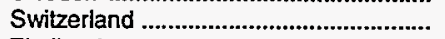 & 0 & 0 & 3 & 0 & 0 & 0 & 0 & 0 \\
\hline Thailand & 0 & 0 & (s) & 0 & 0 & 0 & 576 & 0 \\
\hline Trinidad and Tobago .................................. & 0 & 0 & 0 & 0 & 0 & 0 & 1 & 0 \\
\hline 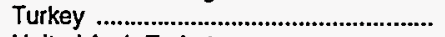 & 0 & 0 & 0 & 0 & 0 & 0 & 534 & 0 \\
\hline 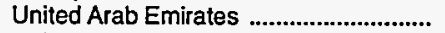 & 0 & 0 & 0 & (s) & 0 & 0 & 0 & 0 \\
\hline 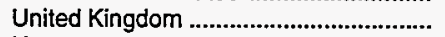 & 0 & 0 & 3 & 2 & 0 & 0 & 2 & 304 \\
\hline 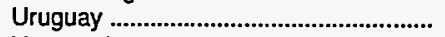 & 0 & 0 & 0 & 0 & 0 & 0 & 0 & 0 \\
\hline Venezuela & 0 & 0 & 423 & 0 & 0 & 0 & (s) & 0 \\
\hline 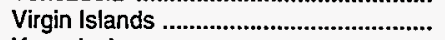 & 8,207 & 0 & 0 & 0 & 0 & 182 & 0 & 0 \\
\hline 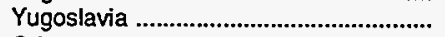 & 0 & 0 & 0 & 0 & 0 & 0 & (s) & 0 \\
\hline Other & 0 & (s) & 10 & 0 & 7 & 0 & 6 & 530 \\
\hline Total & 8,245 & 74 & 7,162 & 8,777 & 2,137 & 335 & 17,017 & 16,880 \\
\hline
\end{tabular}


Table 48. Year-to-Date Exports of Crude Oil and Petroleum Products by Destination, January-March 1995 (Continued) (Thousand Barrels)

\begin{tabular}{|c|c|c|c|c|c|c|c|c|}
\hline \multirow{2}{*}{ Destination } & \multirow[b]{2}{*}{$\begin{array}{l}\text { Special } \\
\text { Naphthas }\end{array}$} & \multirow[b]{2}{*}{ Lubricants } & \multirow[b]{2}{*}{ Waxes } & \multirow[b]{2}{*}{$\begin{array}{c}\text { Petroleum } \\
\text { Coke }\end{array}$} & \multirow{2}{*}{$\begin{array}{c}\text { Asphalt } \\
\text { and Road } \\
\text { Oil }\end{array}$} & \multirow[b]{2}{*}{$\begin{array}{c}\text { Other } \\
\text { Products }^{b}\end{array}$} & \multicolumn{2}{|c|}{ Crude Oil and Products } \\
\hline & & & & & & & Total & $\begin{array}{c}\text { Daily } \\
\text { Average }\end{array}$ \\
\hline 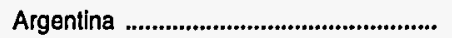 & 0 & 20 & 1 & 0 & 2 & 1 & 340 & 4 \\
\hline 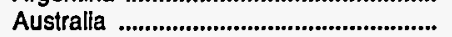 & 7 & 34 & 1 & 1,133 & 1 & (s) & 1,480 & 16 \\
\hline 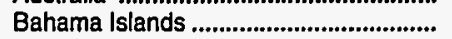 & (s) & 11 & 0 & 0 & 3 & 0 & 479 & 5 \\
\hline Bahrain ...................................................... & 0 & 1 & 0 & 294 & 0 & 0 & 294 & 3 \\
\hline Belgium \& Luxembourg .......................... & 1 & 14 & 1 & 1,355 & 2 & 1 & 1,379 & 15 \\
\hline 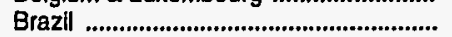 & 210 & 26 & 7 & 195 & (s) & 1 & 444 & 5 \\
\hline 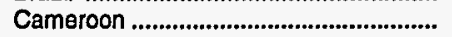 & 0 & (s) & 0 & 0 & 0 & 0 & 1 & (s) \\
\hline 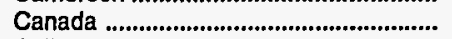 & 30 & 349 & 41 & 972 & 100 & 56 & 5,143 & 57 \\
\hline Chile & 2 & 33 & 2 & 1 & 0 & (s) & 584 & 6 \\
\hline China, People's Republic of .................. & 0 & 4 & $\overline{2}$ & 0 & 2 & (s) & 167 & 2 \\
\hline 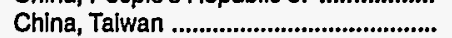 & 4 & 73 & $\overline{1}$ & 15 & 5 & (s) & 3,084 & 34 \\
\hline 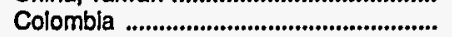 & (s) & 8 & 1 & 1 & 1 & 1 & 158 & 2 \\
\hline 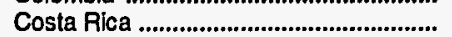 & 3 & 21 & 1 & 0 & 0 & (s) & 434 & 5 \\
\hline 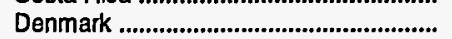 & (s) & (s) & 1 & 137 & (s) & 2 & 140 & 2 \\
\hline Dominlcan Republic ................................. & 3 & 14 & 2 & 0 & 0 & 0 & 329 & 4 \\
\hline 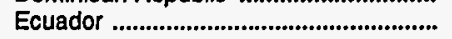 & 0 & 4 & 4 & 0 & 0 & (s) & 1,620 & 18 \\
\hline Egypt & (s) & 19 & 0 & 0 & (s) & 0 & 21 & (s) \\
\hline 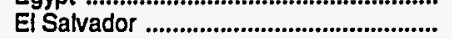 & (s) & 11 & (s) & 0 & 0 & 0 & 455 & 5 \\
\hline Finland & (s) & 2 & (s) & 0 & 0 & (s) & 3 & (s) \\
\hline France & 4 & 3 & 7 & 828 & 3 & (s) & 996 & 11 \\
\hline French Pacific Islands ............................. & 0 & 1 & 0 & 0 & 0 & 0 & 1 & (s) \\
\hline 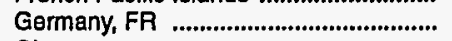 & (s) & 7 & 4 & 140 & 4 & 1 & 159 & 2 \\
\hline 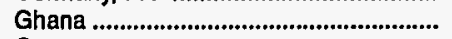 & 0 & (s) & 0 & 94 & 0 & 0 & 94 & 1 \\
\hline Greece & 0 & 3 & 0 & 484 & 0 & (s) & 487 & 5 \\
\hline Guatemala & 8 & 18 & 2 & 0 & 0 & 0 & 776 & 9 \\
\hline Guinea & 0 & 2 & 0 & 0 & 0 & 0 & 2 & (s) \\
\hline 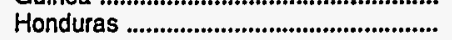 & 2 & 22 & (s) & 0 & 24 & 0 & 334 & 4 \\
\hline 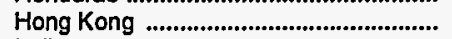 & 1 & 11 & 2 & 0 & (s) & (s) & 192 & 2 \\
\hline Indla & 0 & 91 & 2 & 0 & 3 & (s) & 96 & 1 \\
\hline 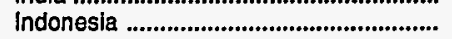 & (s) & 3 & 2 & 92 & 1 & 0 & 99 & 1 \\
\hline Ireland & 0 & (s) & $\overrightarrow{1}$ & 143 & 0 & (s) & 514 & 6 \\
\hline Israel & (s) & 6 & 0 & 320 & 0 & 0 & 1,321 & 15 \\
\hline Italy & (s) & 1 & 2 & 1,908 & 2 & (s) & 5,956 & 66 \\
\hline Jamaica .......................................................... & 4 & 5 & 1 & 81 & $\overrightarrow{0}$ & (s) & 2,105 & 23 \\
\hline Japan & 518 & 60 & 13 & 3,931 & 4 & 3 & 6,000 & 67 \\
\hline Korea, Republic of ................................... & 983 & 31 & 4 & 515 & 2 & 1 & 7,017 & 78 \\
\hline 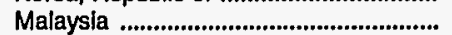 & (s) & 3 & (s) & (s) & (s) & (s) & 5 & (s) \\
\hline 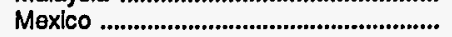 & 4 & 270 & 58 & 314 & 3 & 883 & 13,299 & 148 \\
\hline 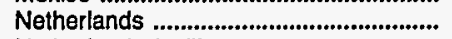 & 128 & 7 & 1 & 2,341 & 8 & 3 & 2,799 & 31 \\
\hline Netherlands Antilles ................................ & 0 & 346 & 0 & 0 & (s) & (s) & 1,111 & 12 \\
\hline 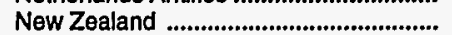 & 0 & 5 & 1 & 242 & (s) & 76 & 498 & 6 \\
\hline Nigeria & 0 & 2 & 0 & 0 & 0 & 0 & 2 & (s) \\
\hline Norway & 0 & 1 & (s) & 233 & 0 & 0 & 234 & 3 \\
\hline 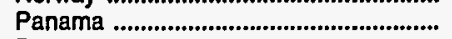 & 2 & 11 & 1 & 0 & 21 & (s) & 2,466 & 27 \\
\hline Peru & 2 & 36 & (s) & (s) & 1 & (s) & 646 & 7 \\
\hline 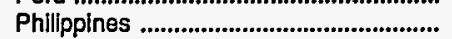 & 0 & 3 & 1 & 1 & (s) & (s) & 880 & 10 \\
\hline Poland & 0 & 2 & (s) & 6 & 0 & 0 & 8 & (s) \\
\hline 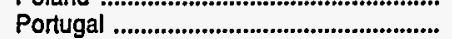 & 0 & (s) & 0 & 269 & 0 & 0 & 327 & 4 \\
\hline Puerto Rico & 96 & 258 & 3 & 0 & (s) & 2 & 1,291 & 14 \\
\hline 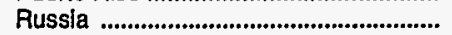 & 0 & 5 & (s) & 0 & 0 & 0 & 26 & (s) \\
\hline 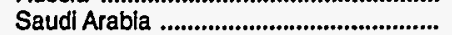 & (s) & 7 & 1 & 1 & 0 & (s) & 10 & (s) \\
\hline Singapore & 46 & 166 & 1 & 31 & 1 & 1 & 4,455 & 49 \\
\hline South Africa .............................................. & 0 & 25 & (s) & 167 & (s) & 0 & 192 & 2 \\
\hline Spaln & (s) & 1 & 1 & 3,603 & 1 & 2 & 4,248 & 47 \\
\hline Suriname ................................................... & 0 & (s) & 0 & 0 & 0 & (s) & 1 & (s) \\
\hline Sweden & 0 & 3 & 1 & 0 & 0 & (s) & 5 & (s) \\
\hline 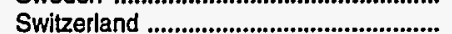 & (s) & 1 & (s) & 0 & 0 & (s) & 3 & (s) \\
\hline Thailand & (s) & 11 & 1 & (s) & 1 & 1 & 590 & (S) \\
\hline Trinidad and Tobago & (s) & 1 & (s) & 0 & (s) & 0 & 2 & (s) \\
\hline Turkey & (s) & 2 & (s) & 946 & (s) & 0 & 1.483 & $\begin{array}{l}\text { (s) } \\
16\end{array}$ \\
\hline & (s) & 18 & 0 & 150 & (s) & (s) & 169 & $\begin{array}{r}16 \\
2\end{array}$ \\
\hline 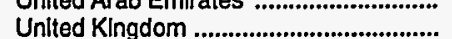 & (S) & $\begin{array}{r}10 \\
5\end{array}$ & 3 & 657 & (5) & (S) & (10) & 2 \\
\hline United Klngdom ......................................... & 2 & 5 & 3 & 657 & 8 & 2 & 987 & 11 \\
\hline 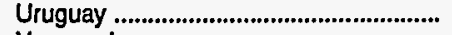 & 0 & 3 & (s) & 0 & (s) & 0 & 4 & (s) \\
\hline 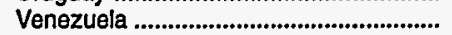 & 0 & 38 & 1 & 433 & 4 & 360 & 1,261 & 14 \\
\hline 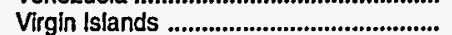 & 0 & 0 & 0 & 0 & (s) & 1 & 8,389 & 93 \\
\hline Yugoslavia & 0 & 0 & 0 & 0 & 0 & 0 & (s) & (s) \\
\hline Other & 2 & 38 & 1 & 670 & 1 & (s) & 1,266 & 14 \\
\hline 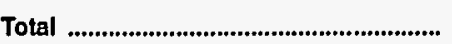 & 2,065 & 2,176 & 189 & 22,738 & 219 & 1,402 & 89,416 & 994 \\
\hline
\end{tabular}

a Crude oil exports are restricted to: (1) crude oil derived from fields under the State waters of Alaska's Cook Inlet; (2) certain domestically produced crude oil destined for Canada; and (3) shipments to U.S. territories, and California crude oil to Pacific Rim countries. On December 6, 1991, the U.S. Department of Commerce approved a license to export 25,000 barrels per day of California heavy crude oil (less than 20 degrees API gravity) to Pacific Rim countries for one year.

b Includes miscellaneous products, motor gasoline blending components, and other hydrocarbons and oxygenates.

(s) = Less than 500 barrels or less than 500 barrels per day.

Note: Totals may not equal sum of components due to independent rounding.

Sources: Energy Information Administration (EIA) Form EIA-810, "Monthly Refinery Report" and the U.S. Bureau of the Census. 
Table 49. Net Imports of Crude Oil and Petroleum Products into the United States by Country, March 1995

(Thousand Barrels per Day)

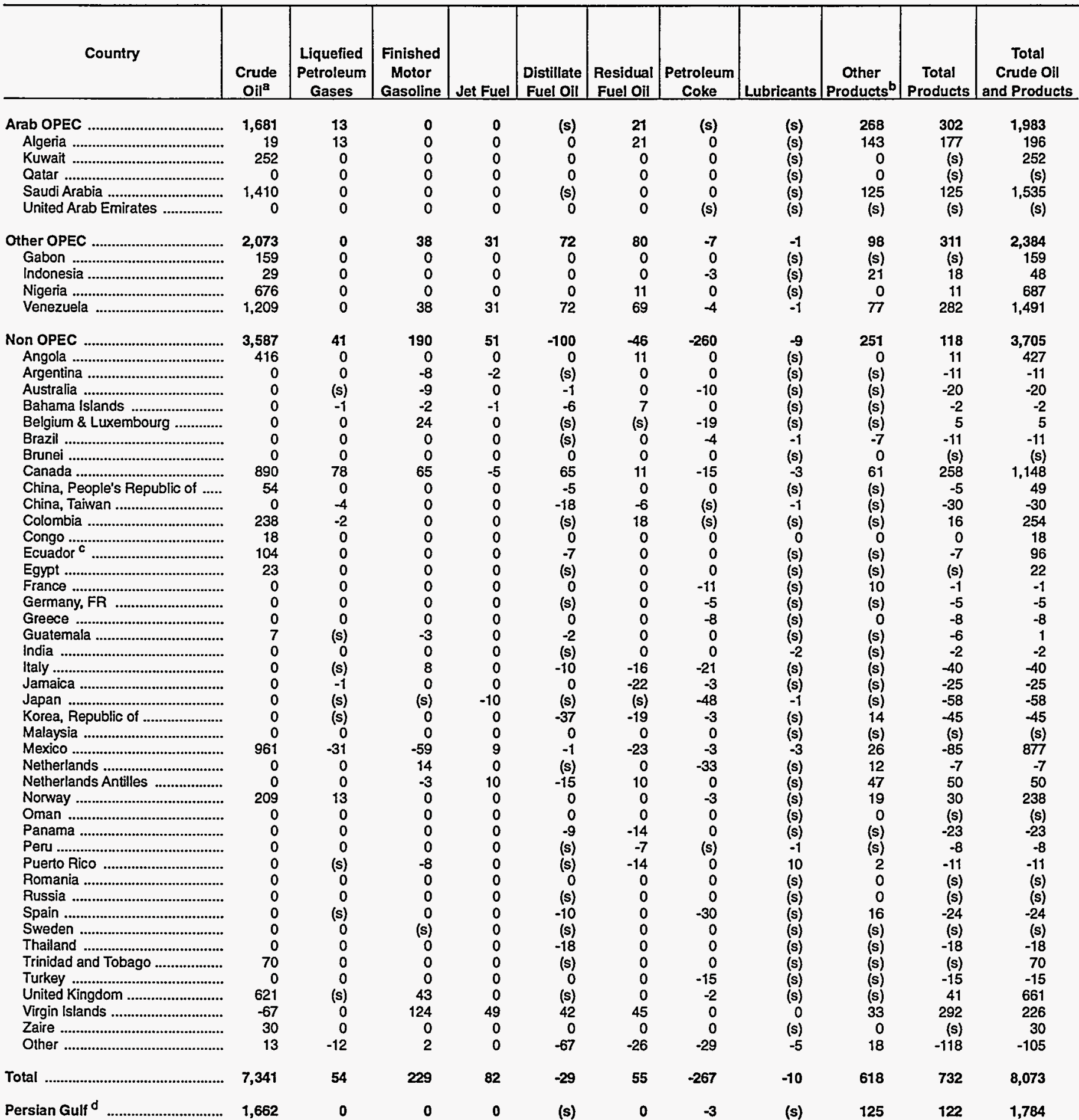

a Includes crude oil imported for storage in the Strategic Petroleum Reserve.

b Includes asphalt and road oil, aviation gasoline, aviation gasoline blending components, kerosene, miscellaneous products, motor gasoline blending components, naphtha for petrochemical feedstock use, other hydrocarbons and oxygenates, other oils for petrochemical feedstock use, pentanes plus, special naphthas, unfinished oils, and waxes.

c On December 31, 1992, Ecuador withdrew as a member of OPEC. As of January 1, 1994, imports of petroleum from Ecuador appear under imports from Non-OPEC Sources.

Includes Bahrain, Iran, Iraq, Kuwait, Qatar, Saudi Arabia, and United Arab Emirates.

(s) = Less than 500 barrels per day.

Note: Totals may not equal sum of components due to independent rounding.

Sources: Energy Information Administration (EIA) Forms EIA-810, "Monthly Refinery Report," EIA-814, "Monthly Imports Report" and the U.S. Bureau of the Census. 
Table 50. Year-to-Date Net Imports of Crude Oil and Petroleum Products into the United States by Country, January-March 1995

(Thousand Barrels per Day)

\begin{tabular}{|c|c|c|c|c|c|c|c|c|c|c|c|}
\hline Country & $\begin{array}{c}\text { Crude } \\
\text { Oil }^{\mathrm{a}}\end{array}$ & $\begin{array}{c}\text { Liquefied } \\
\text { Petroleum } \\
\text { Gases }\end{array}$ & $\begin{array}{l}\text { Finished } \\
\text { Motor } \\
\text { Gasoline }\end{array}$ & Jet Fuel & $\begin{array}{c}\text { Distillate } \\
\text { Fuel Oil }\end{array}$ & $\begin{array}{c}\text { Residual } \\
\text { Fuel OII }\end{array}$ & $\begin{array}{c}\text { Petroleum } \\
\text { Coke }\end{array}$ & Lubricants & $\begin{array}{c}\text { Other } \\
\text { Products }\end{array}$ & $\begin{array}{c}\text { Total } \\
\text { Products }\end{array}$ & $\begin{array}{c}\text { Total } \\
\text { Crude Oil } \\
\text { and Products }\end{array}$ \\
\hline 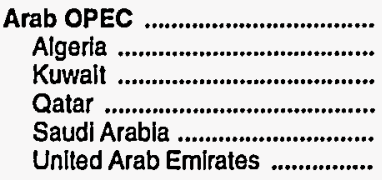 & $\begin{array}{r}1,536 \\
27 \\
229 \\
0 \\
1,269 \\
11\end{array}$ & $\begin{array}{l}8 \\
8 \\
0 \\
0 \\
0 \\
0\end{array}$ & $\begin{array}{r}\text { (s) } \\
0 \\
0 \\
0 \\
0 \\
\text { (s) }\end{array}$ & $\begin{array}{l}0 \\
0 \\
0 \\
0 \\
0 \\
0\end{array}$ & $\begin{array}{r}10 \\
10 \\
0 \\
0 \\
(s) \\
0\end{array}$ & $\begin{array}{r}22 \\
22 \\
0 \\
0 \\
0 \\
0\end{array}$ & $\begin{array}{r}-2 \\
0 \\
0 \\
0 \\
(s) \\
-2\end{array}$ & $\begin{array}{l}\text { (s) } \\
(\mathrm{s}) \\
(\mathrm{s}) \\
(\mathrm{s}) \\
\text { (s) } \\
\text { (s) }\end{array}$ & $\begin{array}{r}258 \\
170 \\
10 \\
0 \\
78 \\
(s)\end{array}$ & $\begin{array}{r}296 \\
210 \\
10 \\
(\mathrm{~s}) \\
78 \\
-2\end{array}$ & $\begin{array}{r}1,832 \\
237 \\
239 \\
(s) \\
1,347 \\
9\end{array}$ \\
\hline 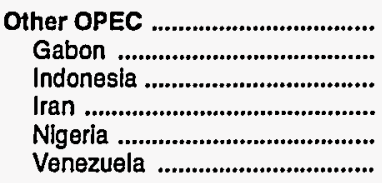 & $\begin{array}{r}1,933 \\
190 \\
50 \\
0 \\
575 \\
1,118\end{array}$ & $\begin{array}{r}-2 \\
0 \\
0 \\
0 \\
0 \\
-2\end{array}$ & $\begin{array}{r}37 \\
0 \\
0 \\
0 \\
0 \\
37\end{array}$ & $\begin{array}{r}35 \\
0 \\
(s) \\
0 \\
0 \\
35\end{array}$ & $\begin{array}{r}80 \\
0 \\
0 \\
0 \\
0 \\
80\end{array}$ & $\begin{array}{r}75 \\
0 \\
0 \\
0 \\
7 \\
68\end{array}$ & $\begin{array}{r}-6 \\
0 \\
-1 \\
0 \\
0 \\
-5\end{array}$ & $\begin{array}{l}\text { (s) } \\
\text { (s) } \\
(\mathrm{s}) \\
(\mathrm{s}) \\
(\mathrm{s}) \\
(\mathrm{s})\end{array}$ & $\begin{array}{r}106 \\
(\mathrm{~s}) \\
20 \\
0 \\
0 \\
86\end{array}$ & $\begin{array}{r}325 \\
(s) \\
19 \\
(s) \\
6 \\
299\end{array}$ & $\begin{array}{r}2,258 \\
190 \\
70 \\
(\mathrm{~s}) \\
581 \\
1,417\end{array}$ \\
\hline 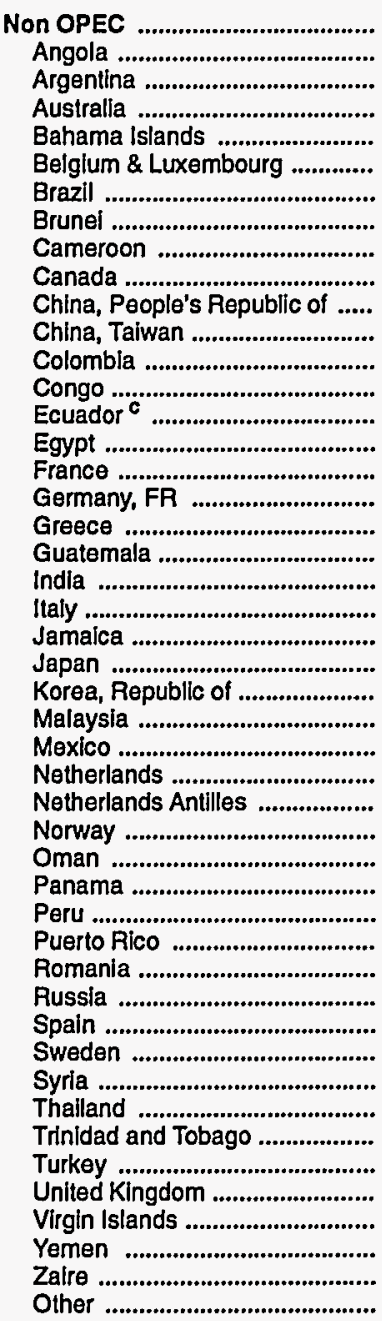 & $\begin{array}{r}3,274 \\
338 \\
18 \\
14 \\
0 \\
0 \\
0 \\
0 \\
0 \\
955 \\
46 \\
0 \\
191 \\
6 \\
114 \\
31 \\
0 \\
0 \\
0 \\
2 \\
0 \\
0 \\
0 \\
0 \\
0 \\
7 \\
720 \\
0 \\
0 \\
181 \\
0 \\
0 \\
4 \\
0 \\
0 \\
0 \\
0 \\
0 \\
0 \\
0 \\
74 \\
0 \\
-91 \\
16 \\
21 \\
17\end{array}$ & $\begin{array}{r}54 \\
0 \\
0 \\
(s) \\
(s) \\
(s) \\
0 \\
0 \\
(s) \\
106 \\
0 \\
-1 \\
-1 \\
0 \\
-15 \\
0 \\
-2 \\
0 \\
0 \\
(s) \\
0 \\
1 \\
-1 \\
(s) \\
(s) \\
0 \\
-30 \\
(s) \\
0 \\
7 \\
0 \\
(s) \\
0 \\
(s) \\
0 \\
0 \\
(s) \\
0 \\
0 \\
(s) \\
0 \\
0 \\
(s) \\
0 \\
0 \\
0 \\
-9\end{array}$ & $\begin{array}{r}110 \\
0 \\
-3 \\
-3 \\
-1 \\
11 \\
0 \\
0 \\
0 \\
52 \\
0 \\
0 \\
(s) \\
0 \\
0 \\
0 \\
0 \\
0 \\
0 \\
-3 \\
0 \\
2 \\
0 \\
(s) \\
0 \\
0 \\
-67 \\
0 \\
-1 \\
3 \\
0 \\
-3 \\
-2 \\
-4 \\
0 \\
0 \\
0 \\
0 \\
(s) \\
0 \\
0 \\
0 \\
0 \\
0\end{array}$ & $\begin{array}{r}41 \\
0 \\
-1 \\
(s) \\
(s) \\
0 \\
(s) \\
0 \\
0 \\
-6 \\
0 \\
0 \\
0 \\
0 \\
0 \\
0 \\
0 \\
0 \\
0 \\
0 \\
(s) \\
0 \\
0 \\
0 \\
-13 \\
0 \\
0 \\
0 \\
4 \\
0 \\
20 \\
0 \\
0 \\
0\end{array}$ & $\begin{array}{r}-32 \\
4 \\
(s) \\
(s) \\
-3 \\
(s) \\
(s) \\
0 \\
0 \\
76 \\
-2 \\
-22 \\
(s) \\
0 \\
-2 \\
(s) \\
(s) \\
5 \\
0 \\
-4 \\
(s) \\
-9 \\
(s) \\
(s) \\
-42 \\
(s) \\
-1 \\
(s) \\
-7 \\
0 \\
0 \\
-11 \\
-2 \\
-1 \\
0 \\
(s) \\
-7 \\
(s) \\
0 \\
-6 \\
(s) \\
-6 \\
(s) \\
65 \\
0 \\
0 \\
-49\end{array}$ & $\begin{array}{r}-76 \\
8 \\
0 \\
0 \\
7 \\
(s) \\
0 \\
0 \\
4 \\
2 \\
0 \\
-10 \\
13 \\
0 \\
(s) \\
0 \\
0 \\
0 \\
0 \\
-1 \\
0 \\
-35 \\
-22 \\
-3 \\
-18 \\
0 \\
-23 \\
-3 \\
4 \\
0 \\
0 \\
-14 \\
-2 \\
-5 \\
-6 \\
0 \\
0 \\
0 \\
0 \\
0 \\
0 \\
0 \\
6 \\
50 \\
0 \\
0 \\
-27\end{array}$ & $\begin{array}{r}-242 \\
0 \\
1 \\
-13 \\
0 \\
-15 \\
-2 \\
0 \\
0 \\
-10 \\
1 \\
(s) \\
(s) \\
0 \\
0 \\
0 \\
-9 \\
-2 \\
-5 \\
0 \\
0 \\
-21 \\
-1 \\
-44 \\
-6 \\
(s) \\
-3 \\
-26 \\
0 \\
-3 \\
0 \\
0 \\
(s) \\
0 \\
0 \\
0 \\
-40 \\
0 \\
0 \\
(s) \\
0 \\
-11 \\
-7 \\
0 \\
0 \\
0 \\
-26\end{array}$ & $\begin{array}{l}-13 \\
(s) \\
(s) \\
(s) \\
(s) \\
(s) \\
(s) \\
(s) \\
(s) \\
-2 \\
(s) \\
-1 \\
(s) \\
(s) \\
(s) \\
(s) \\
(s) \\
(s) \\
(s) \\
(s) \\
-1 \\
1 \\
(s) \\
-1 \\
(s) \\
(s) \\
3 \\
(s) \\
4 \\
(s) \\
(s) \\
(s) \\
(s) \\
5 \\
(s) \\
(s) \\
(s) \\
(s) \\
(s) \\
(s) \\
(s) \\
(s) \\
(s) \\
0 \\
0 \\
(s) \\
-4\end{array}$ & $\begin{array}{r}249 \\
(s) \\
(s) \\
(s) \\
(s) \\
4 \\
-2 \\
0 \\
4 \\
58 \\
(s) \\
(s) \\
(s) \\
(s) \\
(s) \\
(s) \\
9 \\
(s) \\
(s) \\
(s) \\
(s) \\
(s) \\
(s) \\
-6 \\
11 \\
(s) \\
12 \\
5 \\
44 \\
21 \\
(s) \\
(s) \\
(s) \\
(s) \\
0 \\
(s) \\
11 \\
7 \\
8 \\
(s) \\
(s) \\
(s) \\
4 \\
37 \\
0 \\
0 \\
23\end{array}$ & $\begin{array}{r}90 \\
12 \\
-3 \\
-16 \\
2 \\
(s) \\
-5 \\
(s) \\
7 \\
276 \\
-1 \\
-34 \\
11 \\
(s) \\
-18 \\
(s) \\
-2 \\
4 \\
-5 \\
-9 \\
-1 \\
-62 \\
-23 \\
-66 \\
-56 \\
(s) \\
-111 \\
-16 \\
55 \\
28 \\
(s) \\
-27 \\
-7 \\
-6 \\
-6 \\
(s) \\
-36 \\
7 \\
8 \\
-7 \\
(s) \\
-16 \\
20 \\
298 \\
0 \\
(s) \\
-97\end{array}$ & $\begin{array}{r}3,364 \\
349 \\
15 \\
-2 \\
2 \\
2 \\
(s) \\
-5 \\
(s) \\
7 \\
1,231 \\
45 \\
-34 \\
201 \\
6 \\
96 \\
31 \\
-2 \\
4 \\
-5 \\
-6 \\
-6 \\
-1 \\
-62 \\
-23 \\
-66 \\
-56 \\
7 \\
809 \\
-16 \\
55 \\
210 \\
210 \\
(s) \\
-27 \\
-3 \\
-6 \\
-6 \\
-6 \\
(s) \\
-36 \\
7 \\
8 \\
-1 \\
74 \\
74 \\
-16 \\
424 \\
207 \\
16 \\
21 \\
-80\end{array}$ \\
\hline Total & 6,742 & 60 & 147 & 76 & 58 & 21 & -250 & -14 & 613 & 711 & 7,454 \\
\hline 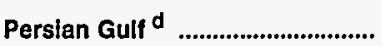 & 1,509 & 0 & (s) & 0 & (s) & 0 & -5 & (s) & 91 & 86 & 1,595 \\
\hline
\end{tabular}

a Includes crude oil imported for storage in the Strategic Petroleum Reserve.

b Includes asphalt and road oil, aviation gasoline, aviation gasoline blending components, kerosene, miscellaneous products, motor gasoline blending components, naphtha for petrochemical feedstock use, other hydrocarbons and oxygenates, other oils for petrochemical feedstock use, pentanes plus, special naphthas, unfinished olls, and waxes. Sources.

On December 31, 1992, Ecuador withdrew as a member of OPEC. As of January 1, 1994, imports of petroleum from Ecuador appear under imports from Non-OPEC

d Includes Bahrain, Iran, Iraq, Kuwait, Qatar, Saudi Arabia, and United Arab Emirates.

$(s)=$ Less than 500 barrels per day.

Note: Totals may not equal sum of components due to independent rounding.

Sources: Energy Information Administration (EIA) Forms EIA-810, "Monthly Refinery Report," EIA-814, "Monthly Imports Report" and the U.S. Bureau of the Census. 
Table 51. Stocks of Crude Oil and Petroleum Products by PAD District, March 1995

(Thousand Barrels)

\begin{tabular}{|c|c|c|c|c|c|c|}
\hline \multirow{2}{*}{ Commodity } & \multicolumn{5}{|c|}{ Petroleum Administration for Defense Districts } & \multirow[b]{2}{*}{$\begin{array}{l}\text { U.S. } \\
\text { Total }\end{array}$} \\
\hline & 1 & II & III & IV & $\mathbf{v}$ & \\
\hline 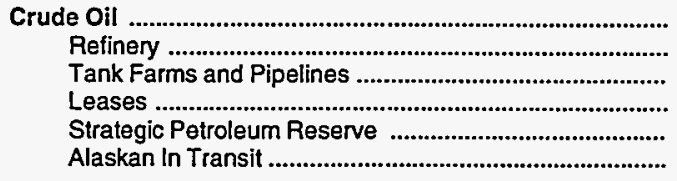 & $\begin{array}{r}16,920 \\
15,956 \\
956 \\
8 \\
0 \\
0\end{array}$ & $\begin{array}{r}73,030 \\
14,096 \\
57,871 \\
1,063 \\
0 \\
0\end{array}$ & $\begin{array}{r}745,954 \\
45,442 \\
94,485 \\
14,355 \\
591,672 \\
0\end{array}$ & $\begin{array}{r}12,844 \\
2,390 \\
9,696 \\
758 \\
0 \\
0\end{array}$ & $\begin{array}{r}80,659 \\
21,683 \\
33,915 \\
990 \\
0 \\
24,071\end{array}$ & $\begin{array}{r}929,407 \\
99,567 \\
196,923 \\
17,174 \\
591,672 \\
24,071\end{array}$ \\
\hline 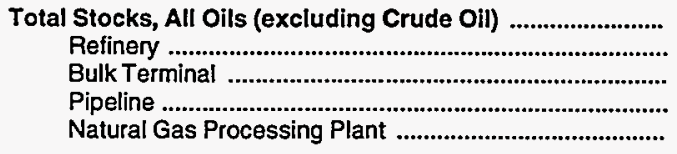 & $\begin{array}{r}158,867 \\
52,386 \\
80,068 \\
26,370 \\
43\end{array}$ & $\begin{array}{r}153,004 \\
62,966 \\
52,173 \\
35,197 \\
2,668\end{array}$ & $\begin{array}{r}244,192 \\
131,000 \\
70,034 \\
39,394 \\
3,764\end{array}$ & $\begin{array}{r}18,810 \\
13,382 \\
2,140 \\
3,032 \\
256\end{array}$ & $\begin{array}{r}94,590 \\
70,276 \\
18,336 \\
5,918 \\
60\end{array}$ & $\begin{array}{r}669,463 \\
330,010 \\
222,751 \\
109,911 \\
6,791\end{array}$ \\
\hline 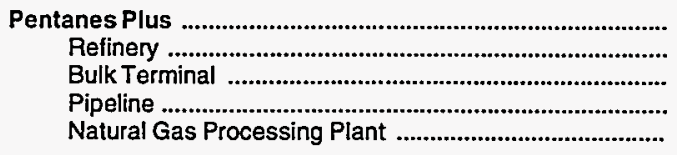 & $\begin{array}{r}20 \\
0 \\
13 \\
0 \\
7\end{array}$ & $\begin{array}{r}2,410 \\
413 \\
764 \\
724 \\
509\end{array}$ & $\begin{array}{r}4,874 \\
355 \\
2,705 \\
950 \\
864\end{array}$ & $\begin{array}{r}181 \\
4 \\
2 \\
66 \\
109\end{array}$ & $\begin{array}{r}51 \\
0 \\
34 \\
0 \\
17\end{array}$ & $\begin{array}{r}7,536 \\
772 \\
3,518 \\
1,740 \\
1,506\end{array}$ \\
\hline 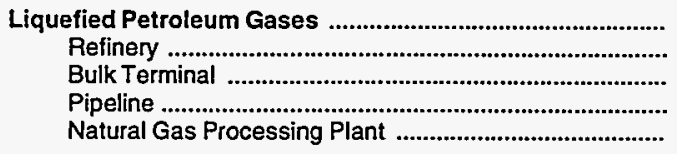 & $\begin{array}{r}2,888 \\
1,078 \\
848 \\
926 \\
36\end{array}$ & $\begin{array}{r}15,647 \\
2,455 \\
4,742 \\
6,291 \\
2,159\end{array}$ & $\begin{array}{r}53,817 \\
7,452 \\
36,288 \\
7,177 \\
2,900\end{array}$ & $\begin{array}{r}1,052 \\
432 \\
6 \\
467 \\
147\end{array}$ & $\begin{array}{r}2,089 \\
1,169 \\
877 \\
0 \\
43\end{array}$ & $\begin{array}{r}75,493 \\
12,586 \\
42,761 \\
14,861 \\
5,285\end{array}$ \\
\hline 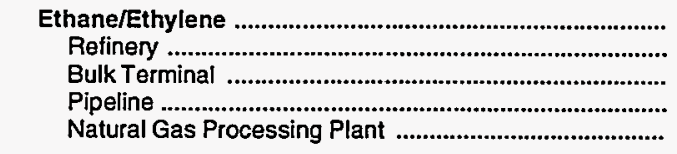 & $\begin{array}{r}13 \\
0 \\
13 \\
0 \\
0\end{array}$ & $\begin{array}{r}2,411 \\
1 \\
706 \\
1,010 \\
694\end{array}$ & $\begin{array}{r}23,769 \\
602 \\
19,596 \\
3,000 \\
571\end{array}$ & $\begin{array}{r}213 \\
0 \\
0 \\
210 \\
3\end{array}$ & $\begin{array}{l}0 \\
0 \\
0 \\
0 \\
0\end{array}$ & $\begin{array}{r}26,406 \\
603 \\
20,315 \\
4,220 \\
1,268\end{array}$ \\
\hline 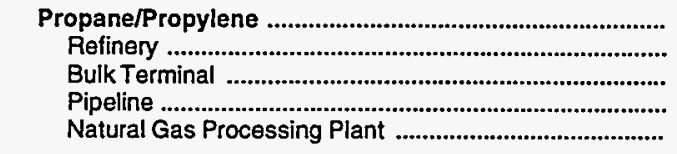 & $\begin{array}{r}2,026 \\
322 \\
826 \\
852 \\
26\end{array}$ & $\begin{array}{r}8,527 \\
1,174 \\
2,691 \\
3,887 \\
775\end{array}$ & $\begin{array}{r}14,812 \\
2,404 \\
8,512 \\
2,722 \\
1,174\end{array}$ & $\begin{array}{r}315 \\
80 \\
2 \\
143 \\
90\end{array}$ & $\begin{array}{r}370 \\
110 \\
233 \\
0 \\
27\end{array}$ & $\begin{array}{r}26,050 \\
4,090 \\
12,264 \\
7,604 \\
2,092\end{array}$ \\
\hline 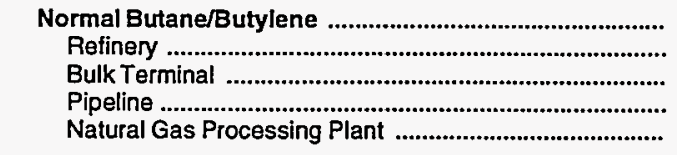 & $\begin{array}{r}649 \\
632 \\
9 \\
0 \\
8\end{array}$ & $\begin{array}{r}2,653 \\
691 \\
826 \\
576 \\
560\end{array}$ & $\begin{array}{r}10,659 \\
2,862 \\
6,174 \\
879 \\
744\end{array}$ & $\begin{array}{r}334 \\
211 \\
4 \\
74 \\
45\end{array}$ & $\begin{array}{r}1,196 \\
676 \\
510 \\
0 \\
10\end{array}$ & $\begin{array}{r}15,491 \\
5,072 \\
7,523 \\
1,529 \\
1,367\end{array}$ \\
\hline 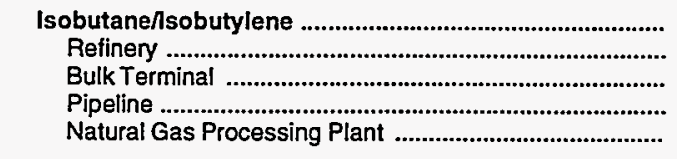 & $\begin{array}{r}200 \\
124 \\
0 \\
74 \\
2\end{array}$ & $\begin{array}{r}2,056 \\
589 \\
519 \\
818 \\
130\end{array}$ & $\begin{array}{r}4,577 \\
1,584 \\
2,006 \\
576 \\
411\end{array}$ & $\begin{array}{r}190 \\
141 \\
0 \\
40 \\
9\end{array}$ & $\begin{array}{r}523 \\
383 \\
134 \\
0 \\
6\end{array}$ & $\begin{array}{r}7,546 \\
2,821 \\
2,659 \\
1,508 \\
558\end{array}$ \\
\hline 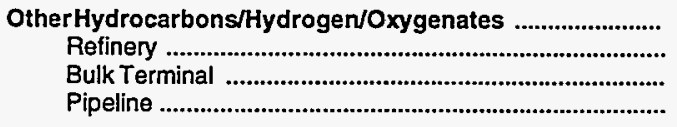 & $\begin{array}{r}2,302 \\
1,800 \\
502 \\
0\end{array}$ & $\begin{array}{r}2,533 \\
787 \\
1,560 \\
186\end{array}$ & $\begin{array}{r}5,941 \\
2,670 \\
3,157 \\
114\end{array}$ & $\begin{array}{r}250 \\
129 \\
121 \\
0\end{array}$ & $\begin{array}{r}4,292 \\
3,427 \\
264 \\
601\end{array}$ & $\begin{array}{r}15,318 \\
8,813 \\
5,604 \\
901\end{array}$ \\
\hline $\begin{array}{l}\text { Other Hydrocarbons/Hydrogen } \\
\text { Refinery }\end{array}$ & $\begin{array}{l}0 \\
0\end{array}$ & $\begin{array}{l}16 \\
16\end{array}$ & $\begin{array}{l}1 \\
1\end{array}$ & $\begin{array}{l}0 \\
0\end{array}$ & $\begin{array}{l}5 \\
5\end{array}$ & $\begin{array}{l}22 \\
22\end{array}$ \\
\hline $\begin{array}{l}\text { Fuel Ethanol } \\
\text { Refinery } \\
\text { Bulk Terminal }{ }^{\mathrm{a}} \\
\text { Pipeline }\end{array}$ & $\begin{array}{l}51 \\
W \\
W \\
W\end{array}$ & $\begin{array}{r}1,880 \\
322 \\
W \\
W\end{array}$ & $\begin{array}{l}702 \\
W \\
W \\
W\end{array}$ & $\begin{array}{l}72 \\
W \\
W \\
W\end{array}$ & $\begin{array}{r}257 \\
W \\
W\end{array}$ & $\begin{array}{r}2,962 \\
425 \\
W \\
W\end{array}$ \\
\hline 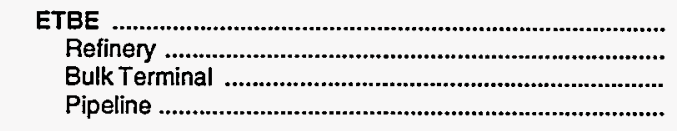 & $\begin{array}{l}W \\
W \\
W \\
W\end{array}$ & $\begin{array}{l}W \\
W \\
W \\
W\end{array}$ & $\begin{array}{l}W \\
W \\
W \\
W\end{array}$ & $\begin{array}{l}W \\
W \\
W \\
W\end{array}$ & $\begin{array}{l}w \\
w \\
w \\
w\end{array}$ & $\begin{array}{l}W \\
W \\
W \\
W\end{array}$ \\
\hline Methanol Refinery & $\begin{array}{l}\mathbf{W} \\
\mathbf{W}\end{array}$ & $\begin{array}{l}W \\
W\end{array}$ & $\begin{array}{l}W \\
W\end{array}$ & $\begin{array}{l}\mathbf{w} \\
\mathbf{W}\end{array}$ & $\begin{array}{l}\mathbf{W} \\
\mathbf{W}\end{array}$ & $\begin{array}{l}658 \\
658\end{array}$ \\
\hline
\end{tabular}

See footnotes at end of table. 
Table 51. Stocks of Crude Oil and Petroleum Products by PAD District, March 1995 (Continued) (Thousand Barrels)

\begin{tabular}{|c|c|c|c|c|c|c|}
\hline \multirow{2}{*}{ Commodity } & \multicolumn{5}{|c|}{ Petroleum Administration for Defense Districts } & \multirow{2}{*}{$\begin{array}{l}\text { U.S. } \\
\text { Total }\end{array}$} \\
\hline & $\mathbf{I}$ & II & III & IV & $\mathbf{V}$ & \\
\hline $\begin{array}{l}\text { MTBE } \\
\text { Reflnery } \\
\text { Bulk Terminal } \\
\text { Pipeline }\end{array}$ & $\begin{array}{r}2,107 \\
1,655 \\
W \\
W\end{array}$ & $\begin{array}{l}\mathbf{W} \\
\mathbf{W} \\
\mathbf{W}\end{array}$ & $\begin{array}{r}4,221 \\
1,979 \\
2,128 \\
114\end{array}$ & $\begin{array}{l}W \\
W \\
W \\
W\end{array}$ & $\begin{array}{r}4,030 \\
3,393 \\
39 \\
598\end{array}$ & $\begin{array}{r}11,143 \\
7,516 \\
2,729 \\
898\end{array}$ \\
\hline 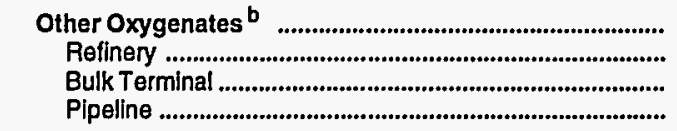 & $\begin{array}{l}\mathbf{W} \\
\mathbf{W} \\
\mathbf{W} \\
\mathbf{W}\end{array}$ & $\begin{array}{l}w \\
W \\
w \\
W\end{array}$ & $\begin{array}{l}W \\
W \\
W \\
W\end{array}$ & $\begin{array}{l}W \\
W \\
W \\
W\end{array}$ & $\begin{array}{l}W \\
W \\
W \\
W\end{array}$ & $\begin{array}{l}W \\
W \\
W \\
W\end{array}$ \\
\hline 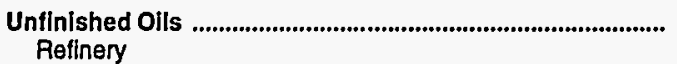 & 10,869 & 14,271 & 46,344 & 2,531 & 25,209 & 99,224 \\
\hline 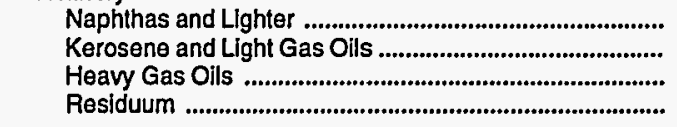 & $\begin{array}{l}2,966 \\
1,971 \\
4,882 \\
1,050\end{array}$ & $\begin{array}{l}4,560 \\
1,874 \\
4,615 \\
3,222\end{array}$ & $\begin{array}{r}10,989 \\
8,329 \\
17,655 \\
9,371\end{array}$ & $\begin{array}{r}621 \\
556 \\
1,037 \\
317\end{array}$ & $\begin{array}{r}4,053 \\
5,340 \\
12,471 \\
3,345\end{array}$ & $\begin{array}{l}23,189 \\
18,070 \\
40,660 \\
17,305\end{array}$ \\
\hline 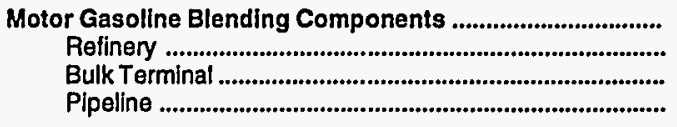 & $\begin{array}{r}6,347 \\
5,757 \\
590 \\
0\end{array}$ & $\begin{array}{r}10,157 \\
8,610 \\
561 \\
986\end{array}$ & $\begin{array}{r}15,801 \\
14,257 \\
892 \\
652\end{array}$ & $\begin{array}{r}2,518 \\
2,518 \\
0 \\
0\end{array}$ & $\begin{array}{r}8,297 \\
8,154 \\
13 \\
130\end{array}$ & $\begin{array}{r}43,120 \\
39,296 \\
2,056 \\
1,768\end{array}$ \\
\hline 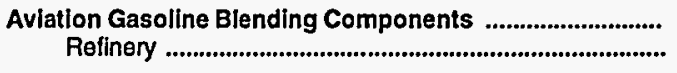 & $\begin{array}{l}49 \\
49\end{array}$ & $\begin{array}{l}15 \\
15\end{array}$ & $\begin{array}{l}23 \\
23\end{array}$ & $\begin{array}{l}0 \\
0\end{array}$ & $\begin{array}{l}3 \\
3\end{array}$ & $\begin{array}{l}90 \\
90\end{array}$ \\
\hline $\begin{array}{l}\text { Finished Motor Gasoline } \\
\text { Refinery } \\
\text { Bulk Terminal } \\
\text { Pipeline }\end{array}$ & $\begin{array}{l}50,451 \\
10,896 \\
25,734 \\
13,821\end{array}$ & $\begin{array}{l}47,103 \\
11,967 \\
19,904 \\
15,232\end{array}$ & $\begin{array}{r}45,545 \\
17,996 \\
9,400 \\
18,149\end{array}$ & $\begin{array}{r}4,757 \\
2,466 \\
845 \\
1,446\end{array}$ & $\begin{array}{r}19,879 \\
10,142 \\
7,853 \\
1,884\end{array}$ & $\begin{array}{r}167,735 \\
53,467 \\
63,736 \\
50,532\end{array}$ \\
\hline 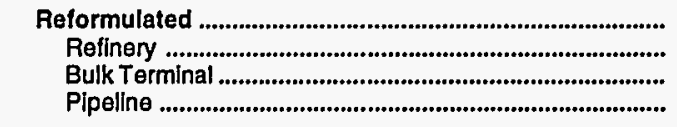 & $\begin{array}{r}23,963 \\
7,189 \\
10,945 \\
5,829\end{array}$ & $\begin{array}{r}2,574 \\
856 \\
1,293 \\
425\end{array}$ & $\begin{array}{l}7,900 \\
3,285 \\
2,030 \\
2,585\end{array}$ & $\begin{array}{l}0 \\
0 \\
0 \\
0\end{array}$ & $\begin{array}{r}5,825 \\
3,189 \\
1,807 \\
829\end{array}$ & $\begin{array}{r}40,262 \\
14,519 \\
16,075 \\
9,668\end{array}$ \\
\hline 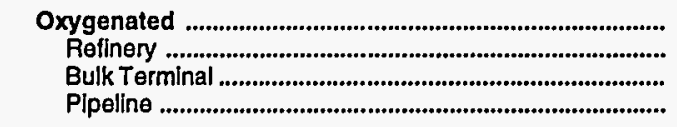 & $\begin{array}{r}349 \\
93 \\
147 \\
109\end{array}$ & $\begin{array}{r}418 \\
343 \\
75 \\
0\end{array}$ & $\begin{array}{r}396 \\
163 \\
0 \\
233\end{array}$ & $\begin{array}{r}92 \\
14 \\
78 \\
0\end{array}$ & $\begin{array}{r}127 \\
87 \\
3 \\
37\end{array}$ & $\begin{array}{r}1,382 \\
700 \\
303 \\
379\end{array}$ \\
\hline 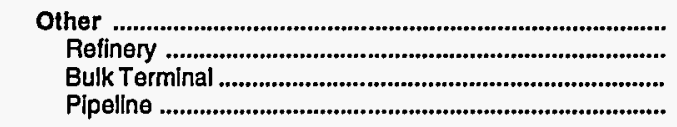 & $\begin{array}{r}26,139 \\
3,614 \\
14,642 \\
7,883\end{array}$ & $\begin{array}{l}44,111 \\
10,768 \\
18,536 \\
14,807\end{array}$ & $\begin{array}{r}37,249 \\
14,548 \\
7,370 \\
15,331\end{array}$ & $\begin{array}{r}4,665 \\
2,452 \\
767 \\
1,446\end{array}$ & $\begin{array}{r}13,927 \\
6,866 \\
6,043 \\
1,018\end{array}$ & $\begin{array}{r}126,091 \\
38,248 \\
47,358 \\
40,485\end{array}$ \\
\hline 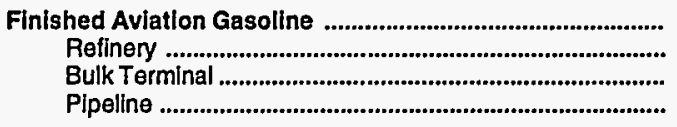 & $\begin{array}{r}694 \\
58 \\
632 \\
4\end{array}$ & $\begin{array}{r}377 \\
98 \\
247 \\
32\end{array}$ & $\begin{array}{r}505 \\
458 \\
47 \\
0\end{array}$ & $\begin{array}{r}45 \\
35 \\
10 \\
0\end{array}$ & $\begin{array}{r}532 \\
307 \\
225 \\
0\end{array}$ & $\begin{array}{r}2,153 \\
956 \\
1,161 \\
36\end{array}$ \\
\hline $\begin{array}{l}\text { Naphtha-Type Jet Fuel } \\
\text { Refinery } \\
\text { Bulk Terminal } \\
\text { Pipeline }\end{array}$ & $\begin{array}{l}0 \\
0 \\
0 \\
0\end{array}$ & $\begin{array}{r}257 \\
11 \\
66 \\
180\end{array}$ & $\begin{array}{r}190 \\
190 \\
0 \\
0\end{array}$ & $\begin{array}{r}144 \\
126 \\
0 \\
18\end{array}$ & $\begin{array}{r}323 \\
52 \\
6 \\
265\end{array}$ & $\begin{array}{r}914 \\
379 \\
72 \\
463\end{array}$ \\
\hline $\begin{array}{l}\text { Kerosene-Type Jet Fuel } \\
\text { Refinery } \\
\text { Bulk Terminal } \\
\text { Pipeline }\end{array}$ & $\begin{array}{r}10,766 \\
2,175 \\
3,930 \\
4,661\end{array}$ & $\begin{array}{l}7,196 \\
2,844 \\
2,118 \\
2,234\end{array}$ & $\begin{array}{r}11,944 \\
5,936 \\
1,720 \\
4,288\end{array}$ & $\begin{array}{l}727 \\
204 \\
285 \\
238\end{array}$ & $\begin{array}{l}7,743 \\
4,293 \\
2,173 \\
1,277\end{array}$ & $\begin{array}{l}38,376 \\
15,452 \\
10,226 \\
12,698\end{array}$ \\
\hline
\end{tabular}

See footnotes at end of table. 
Table 51. Stocks of Crude Oil and Petroleum Products by FAD District, March 1995 (Continued)

(Thousand Barrels)

\begin{tabular}{|c|c|c|c|c|c|c|}
\hline \multirow{2}{*}{ Commodity } & \multicolumn{5}{|c|}{ Petroleum Administration for Defense Districts } & \multirow{2}{*}{$\begin{array}{l}\text { U.S. } \\
\text { Total }\end{array}$} \\
\hline & $\mathbf{I}$ & ॥ & III & IV & $\mathbf{v}$ & \\
\hline 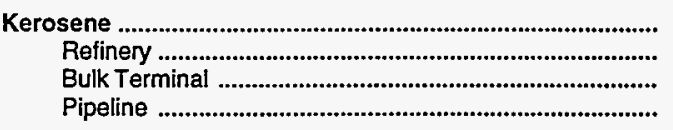 & $\begin{array}{r}4,177 \\
538 \\
3,521 \\
118\end{array}$ & $\begin{array}{r}1,325 \\
487 \\
802 \\
36\end{array}$ & $\begin{array}{r}839 \\
504 \\
297 \\
38\end{array}$ & $\begin{array}{r}103 \\
88 \\
0 \\
15\end{array}$ & $\begin{array}{r}60 \\
45 \\
10 \\
5\end{array}$ & $\begin{array}{r}6,504 \\
1,662 \\
4,630 \\
212\end{array}$ \\
\hline 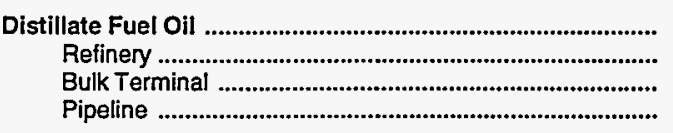 & $\begin{array}{r}45,934 \\
10,675 \\
28,419 \\
6,840\end{array}$ & $\begin{array}{r}30,480 \\
8,213 \\
12,993 \\
9,274\end{array}$ & $\begin{array}{r}25,291 \\
12,610 \\
4,707 \\
7,974\end{array}$ & $\begin{array}{r}2,748 \\
1,372 \\
600 \\
776\end{array}$ & $\begin{array}{r}11,016 \\
5,660 \\
3,793 \\
1,563\end{array}$ & $\begin{array}{r}115,469 \\
38,530 \\
50,512 \\
26,427\end{array}$ \\
\hline 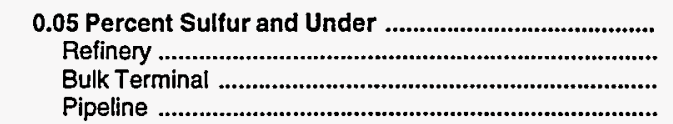 & $\begin{array}{r}15,535 \\
2,814 \\
10,591 \\
2,130\end{array}$ & $\begin{array}{r}20,825 \\
4,451 \\
9,271 \\
7,103\end{array}$ & $\begin{array}{r}13,039 \\
5,366 \\
3,113 \\
4,560\end{array}$ & $\begin{array}{r}2,238 \\
1,007 \\
548 \\
683\end{array}$ & $\begin{array}{l}7,509 \\
3,815 \\
2,681 \\
1,013\end{array}$ & $\begin{array}{l}59,146 \\
17,453 \\
26,204 \\
15,489\end{array}$ \\
\hline 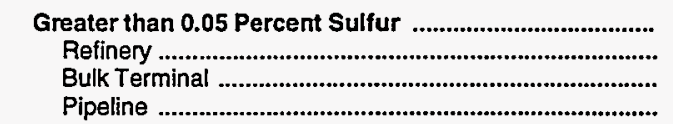 & $\begin{array}{r}30,399 \\
7,861 \\
17,828 \\
4,710\end{array}$ & $\begin{array}{l}9,655 \\
3,762 \\
3,722 \\
2,171\end{array}$ & $\begin{array}{r}12,252 \\
7,244 \\
1,594 \\
3,414\end{array}$ & $\begin{array}{r}510 \\
365 \\
52 \\
93\end{array}$ & $\begin{array}{r}3,507 \\
1,845 \\
1,112 \\
550\end{array}$ & $\begin{array}{l}56,323 \\
21,077 \\
24,308 \\
10,938\end{array}$ \\
\hline 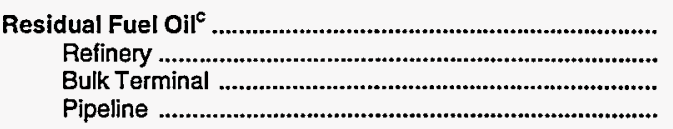 & $\begin{array}{r}14,748 \\
3,773 \\
10,975 \\
0\end{array}$ & $\begin{array}{r}2,179 \\
1,598 \\
581 \\
0\end{array}$ & $\begin{array}{r}13,286 \\
6,048 \\
7,238 \\
0\end{array}$ & $\begin{array}{r}571 \\
571 \\
0 \\
0\end{array}$ & $\begin{array}{r}6,980 \\
4,604 \\
2,183 \\
193\end{array}$ & $\begin{array}{r}37,764 \\
16,594 \\
20,977 \\
193\end{array}$ \\
\hline $\begin{array}{l}\text { Less than } 0.31 \% \text { Sulfur } \\
\text { Refinery } \\
\text { Bulk Terminal }\end{array}$ & $\begin{array}{l}3,588 \\
1,597 \\
1,991\end{array}$ & $\begin{array}{r}22 \\
22 \\
0\end{array}$ & $\begin{array}{r}1,128 \\
129 \\
999\end{array}$ & $\begin{array}{r}104 \\
104 \\
0\end{array}$ & $\begin{array}{r}447 \\
375 \\
72\end{array}$ & $\begin{array}{l}5,289 \\
2,227 \\
3,062\end{array}$ \\
\hline 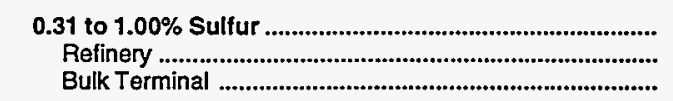 & $\begin{array}{l}5,285 \\
1,682 \\
3,603\end{array}$ & $\begin{array}{l}439 \\
173 \\
266\end{array}$ & $\begin{array}{l}3,371 \\
1,243 \\
2,128\end{array}$ & $\begin{array}{r}339 \\
339 \\
0\end{array}$ & $\begin{array}{r}1,017 \\
804 \\
213\end{array}$ & $\begin{array}{r}10,451 \\
4,241 \\
6,210\end{array}$ \\
\hline $\begin{array}{l}\text { Greater than 1.00\% Sulfur } \\
\text { Refinery } \\
\text { Bulk Terminal }\end{array}$ & $\begin{array}{r}5,875 \\
494 \\
5,381\end{array}$ & $\begin{array}{r}1,718 \\
1,403 \\
315\end{array}$ & $\begin{array}{l}8,787 \\
4,676 \\
4,111\end{array}$ & $\begin{array}{r}128 \\
128 \\
0\end{array}$ & $\begin{array}{l}5,323 \\
3,425 \\
1,898\end{array}$ & $\begin{array}{l}21,831 \\
10,126 \\
11,705\end{array}$ \\
\hline $\begin{array}{l}\text { Naphtha for Petrochemical Feedstock Use } \\
\text { Refinery }\end{array}$ & $\begin{array}{l}545 \\
545\end{array}$ & $\begin{array}{l}366 \\
366\end{array}$ & $\begin{array}{l}1,993 \\
1,993\end{array}$ & $\begin{array}{l}0 \\
0\end{array}$ & $\begin{array}{l}97 \\
97\end{array}$ & $\begin{array}{l}3,001 \\
3,001\end{array}$ \\
\hline $\begin{array}{l}\text { Other Oils for Petrochemical Feedstock Use } \\
\quad \text { Refinery }\end{array}$ & $\begin{array}{l}3 \\
3\end{array}$ & $\begin{array}{l}1 \\
1\end{array}$ & $\begin{array}{l}1,475 \\
1,475\end{array}$ & $\begin{array}{l}0 \\
0\end{array}$ & $\begin{array}{l}233 \\
233\end{array}$ & $\begin{array}{l}1,712 \\
1,712\end{array}$ \\
\hline 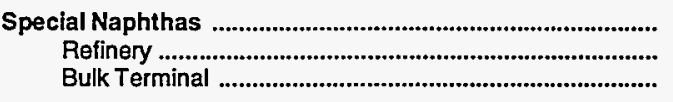 & $\begin{array}{l}83 \\
38 \\
45\end{array}$ & $\begin{array}{r}205 \\
191 \\
14\end{array}$ & $\begin{array}{r}1,732 \\
1,356 \\
376\end{array}$ & $\begin{array}{l}1 \\
1 \\
0\end{array}$ & $\begin{array}{r}39 \\
39 \\
0\end{array}$ & $\begin{array}{r}2,060 \\
1,625 \\
435\end{array}$ \\
\hline 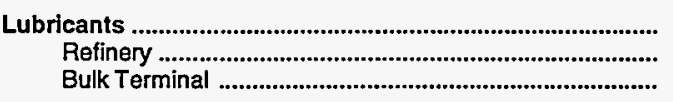 & $\begin{array}{r}2,525 \\
786 \\
1,739\end{array}$ & $\begin{array}{r}1,714 \\
851 \\
863\end{array}$ & $\begin{array}{l}5,697 \\
4,352 \\
1,345\end{array}$ & $\begin{array}{l}0 \\
0 \\
0\end{array}$ & $\begin{array}{r}1,752 \\
1,271 \\
481\end{array}$ & $\begin{array}{r}11,688 \\
7,260 \\
4,428\end{array}$ \\
\hline Waxes . & $\begin{array}{l}166 \\
166\end{array}$ & $\begin{array}{l}128 \\
128\end{array}$ & $\begin{array}{l}465 \\
465\end{array}$ & $\begin{array}{l}16 \\
16\end{array}$ & $\begin{array}{l}134 \\
134\end{array}$ & $\begin{array}{l}909 \\
909\end{array}$ \\
\hline $\begin{array}{l}\text { Petroleum Coke } \\
\text { Refinery }\end{array}$ & $\begin{array}{l}536 \\
536\end{array}$ & $\begin{array}{l}2,076 \\
2,076\end{array}$ & $\begin{array}{l}2,780 \\
2,780\end{array}$ & $\begin{array}{l}243 \\
243\end{array}$ & $\begin{array}{l}3,321 \\
3,321\end{array}$ & $\begin{array}{l}8,956 \\
8,956\end{array}$ \\
\hline 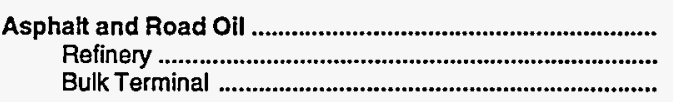 & $\begin{array}{l}5,571 \\
2,572 \\
2,999\end{array}$ & $\begin{array}{r}14,332 \\
7,451 \\
6,881\end{array}$ & $\begin{array}{r}4,268 \\
3,439 \\
829\end{array}$ & $\begin{array}{r}2,908 \\
2,637 \\
271\end{array}$ & $\begin{array}{r}2,188 \\
1,833 \\
355\end{array}$ & $\begin{array}{l}29,267 \\
17,932 \\
11,335\end{array}$ \\
\hline 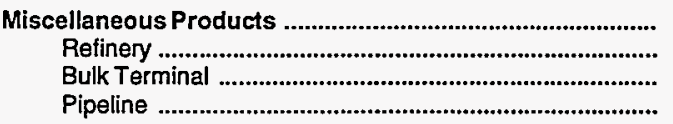 & $\begin{array}{r}193 \\
72 \\
121 \\
0\end{array}$ & $\begin{array}{r}232 \\
133 \\
77 \\
22\end{array}$ & $\begin{array}{r}1,382 \\
297 \\
1,033 \\
52\end{array}$ & $\begin{array}{r}15 \\
9 \\
0 \\
6\end{array}$ & $\begin{array}{r}352 \\
283 \\
69 \\
0\end{array}$ & $\begin{array}{r}2,174 \\
794 \\
1,300 \\
80\end{array}$ \\
\hline Total Stocks, All Oils & 175,787 & 226,034 & 990,146 & 31,654 & 175,249 & $1,598,870$ \\
\hline
\end{tabular}

a Includes stocks held by producors.

b Includes tertiary amyl methyl ether (TAME), tertiary butyl alcohol (TBA), and other aliphatic alcohols and ethers intended for motor gasoline blending (e.g., isopropyl ether (IPE) or n-propanol).

c Sulfur content not available for stocks held by pipelines.

$W=$ Withheld to avoid disciosure of individual company data.

Note: Stocks are reported as of the last day of the month.

Sources: Energy Information Administration (EIA) Forms EIA-810, "Monthly Refinery Report," ElA-811, "Monthly Bulk Terminal Report," ElA-812, "Monthly Product Pipeline Report," EIA-813, "Monthly Crude Oil Report," and EIA-816, "Monthly Natural Gas Liquids Report." 102 
Table 52. Refinery, Bulk Terminal, and Natural Gas Plant Stocks of Selected Petroleum Products by PAD District and State, March 1995

(Thousand Barrels)

\begin{tabular}{|c|c|c|c|c|c|c|c|c|c|}
\hline \multirow[b]{2}{*}{ PAD District and State } & \multicolumn{3}{|c|}{ - Motor Gasoline } & \multirow[b]{2}{*}{ Kerosene } & \multicolumn{3}{|c|}{ Distillate Fuel Oil } & \multirow[b]{2}{*}{$\begin{array}{c}\text { Residual } \\
\text { Fuel }\end{array}$} & \multirow[b]{2}{*}{$\begin{array}{c}\text { Propane/ } \\
\text { Propylene }\end{array}$} \\
\hline & Reformulated & Oxygenated & Other & & Total & $\begin{array}{c}0.05 \% \text { Sulfur } \\
\text { and Under }\end{array}$ & $\begin{array}{l}\text { Greater than } \\
0.05 \% \text { Sulfur }\end{array}$ & & \\
\hline 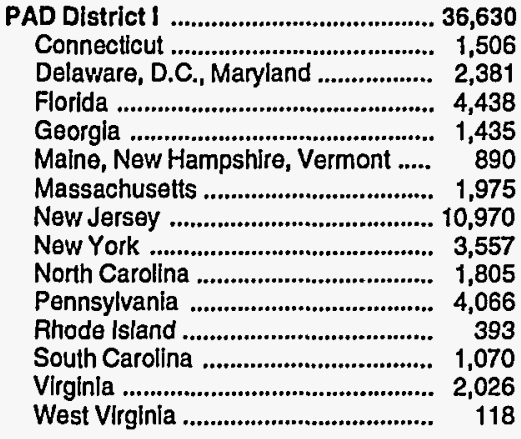 & $\begin{array}{r}18,134 \\
1,505 \\
1,952 \\
0 \\
0 \\
478 \\
1,689 \\
8,339 \\
1,441 \\
0 \\
1,221 \\
392 \\
0 \\
1,117 \\
0\end{array}$ & $\begin{array}{r}240 \\
0 \\
0 \\
0 \\
0 \\
0 \\
0 \\
95 \\
106 \\
0 \\
38 \\
0 \\
1 \\
0 \\
0\end{array}$ & $\begin{array}{r}18,256 \\
1 \\
429 \\
4,438 \\
1,435 \\
412 \\
286 \\
2,536 \\
2,010 \\
1,805 \\
2,807 \\
1 \\
1,069 \\
909 \\
118\end{array}$ & $\begin{array}{r}4,059 \\
96 \\
279 \\
142 \\
74 \\
262 \\
111 \\
936 \\
1,140 \\
304 \\
446 \\
W \\
152 \\
104 \\
W\end{array}$ & $\begin{array}{r}39,094 \\
2,927 \\
2,845 \\
1,748 \\
949 \\
1,801 \\
2,272 \\
11,331 \\
4,493 \\
1,529 \\
5,239 \\
794 \\
749 \\
2,282 \\
135\end{array}$ & $\begin{array}{r}13,405 \\
355 \\
907 \\
978 \\
616 \\
557 \\
457 \\
3,661 \\
1,373 \\
929 \\
1,863 \\
186 \\
458 \\
982 \\
83\end{array}$ & $\begin{array}{r}25,689 \\
2,572 \\
1,938 \\
770 \\
333 \\
1,244 \\
1,815 \\
7,670 \\
3,120 \\
600 \\
3,376 \\
608 \\
291 \\
1,300 \\
52\end{array}$ & $\begin{array}{r}14,748 \\
96 \\
2,050 \\
754 \\
218 \\
605 \\
980 \\
4,088 \\
2,580 \\
513 \\
1,453 \\
W \\
W \\
696 \\
W\end{array}$ & $\begin{array}{r}1,174 \\
W \\
W \\
101 \\
W \\
W \\
W \\
W \\
W \\
W \\
W \\
W \\
W \\
W \\
W\end{array}$ \\
\hline 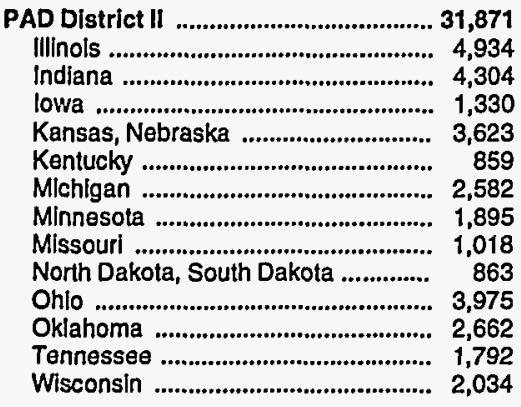 & $\begin{array}{r}2,149 \\
1,207 \\
585 \\
0 \\
0 \\
91 \\
0 \\
24 \\
0 \\
0 \\
8 \\
0 \\
0 \\
234\end{array}$ & $\begin{array}{r}418 \\
0 \\
7 \\
0 \\
0 \\
139 \\
0 \\
204 \\
0 \\
2 \\
1 \\
3 \\
62 \\
0\end{array}$ & $\begin{array}{r}29,304 \\
3,727 \\
3,712 \\
1,330 \\
3,623 \\
629 \\
2,582 \\
1,667 \\
1,018 \\
861 \\
3,966 \\
2,659 \\
1,730 \\
1,800\end{array}$ & $\begin{array}{r}1,289 \\
191 \\
201 \\
W \\
3 \\
94 \\
137 \\
W \\
W \\
W \\
332 \\
W \\
98 \\
W\end{array}$ & $\begin{array}{r}21,206 \\
3,225 \\
2,692 \\
1,358 \\
2,644 \\
886 \\
1,629 \\
1,336 \\
633 \\
937 \\
1,910 \\
1,409 \\
890 \\
1,657\end{array}$ & $\begin{array}{r}13,722 \\
1,892 \\
1,560 \\
1,151 \\
2,067 \\
456 \\
1,100 \\
940 \\
536 \\
430 \\
1,034 \\
977 \\
571 \\
1,008\end{array}$ & $\begin{array}{r}7,484 \\
1,333 \\
1,132 \\
207 \\
577 \\
430 \\
529 \\
396 \\
97 \\
507 \\
876 \\
432 \\
319 \\
649\end{array}$ & $\begin{array}{r}2,179 \\
676 \\
324 \\
W \\
26 \\
W \\
82 \\
153 \\
W \\
W \\
198 \\
224 \\
245 \\
37\end{array}$ & $\begin{array}{r}4,640 \\
268 \\
W \\
W \\
1,573 \\
W \\
912 \\
W \\
W \\
W \\
W \\
776 \\
W \\
W\end{array}$ \\
\hline 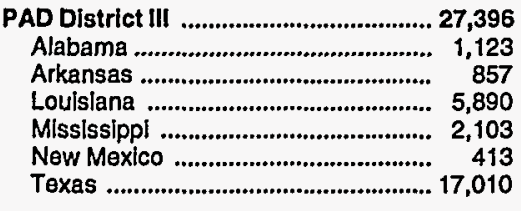 & $\begin{array}{r}5,315 \\
0 \\
0 \\
521 \\
179 \\
0 \\
4,615\end{array}$ & $\begin{array}{r}163 \\
0 \\
0 \\
140 \\
0 \\
0 \\
23\end{array}$ & $\begin{array}{r}21,918 \\
1,123 \\
857 \\
5,229 \\
1,924 \\
413 \\
12,372\end{array}$ & $\begin{array}{r}801 \\
37 \\
W \\
194 \\
17 \\
W \\
533\end{array}$ & $\begin{array}{r}17,317 \\
533 \\
493 \\
4,277 \\
1,060 \\
201 \\
10,753\end{array}$ & $\begin{array}{r}8,479 \\
346 \\
310 \\
1,746 \\
480 \\
148 \\
5,449\end{array}$ & $\begin{array}{r}8,838 \\
187 \\
183 \\
2,531 \\
580 \\
53 \\
5,304\end{array}$ & $\begin{array}{r}13,286 \\
281 \\
W \\
5,302 \\
W \\
19 \\
7,179\end{array}$ & $\begin{array}{r}12,090 \\
94 \\
W \\
2,130 \\
613 \\
W \\
9,183\end{array}$ \\
\hline 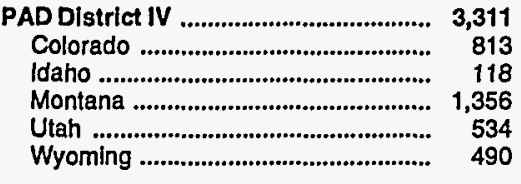 & $\begin{array}{l}0 \\
0 \\
0 \\
0 \\
0 \\
0\end{array}$ & $\begin{array}{r}92 \\
92 \\
0 \\
0 \\
0 \\
0\end{array}$ & $\begin{array}{r}3,219 \\
721 \\
118 \\
1,356 \\
534 \\
490\end{array}$ & $\begin{array}{l}88 \\
W \\
W \\
W \\
W \\
W\end{array}$ & $\begin{array}{r}1,972 \\
306 \\
211 \\
445 \\
542 \\
468\end{array}$ & $\begin{array}{r}1,555 \\
241 \\
162 \\
445 \\
305 \\
402\end{array}$ & $\begin{array}{r}417 \\
65 \\
49 \\
0 \\
237 \\
66\end{array}$ & $\begin{array}{r}571 \\
W \\
W \\
87 \\
158 \\
W\end{array}$ & $\begin{array}{r}172 \\
W \\
W \\
17 \\
72 \\
52\end{array}$ \\
\hline 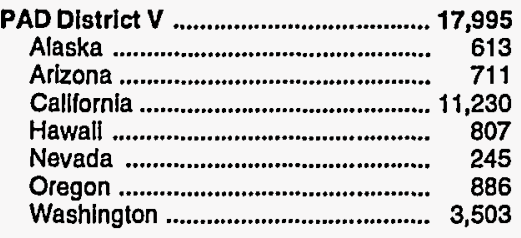 & $\begin{array}{r}4,996 \\
0 \\
0 \\
4,996 \\
0 \\
0 \\
0 \\
0\end{array}$ & $\begin{array}{r}90 \\
0 \\
84 \\
6 \\
0 \\
0 \\
0 \\
0\end{array}$ & $\begin{array}{r}12,909 \\
613 \\
627 \\
6,228 \\
807 \\
245 \\
886 \\
3,503\end{array}$ & $\begin{array}{l}\mathbf{5 5} \\
W \\
W \\
46 \\
W \\
W \\
W \\
W\end{array}$ & $\begin{array}{r}9,453 \\
721 \\
231 \\
5,662 \\
429 \\
101 \\
797 \\
1,512\end{array}$ & $\begin{array}{r}6,496 \\
238 \\
151 \\
4,643 \\
135 \\
80 \\
563 \\
686\end{array}$ & $\begin{array}{r}2,957 \\
483 \\
80 \\
1,019 \\
294 \\
21 \\
234 \\
826\end{array}$ & $\begin{array}{r}6,787 \\
W \\
W \\
4,473 \\
W \\
W \\
275 \\
768\end{array}$ & $\begin{array}{r}370 \\
W \\
W \\
124 \\
W \\
W \\
W \\
19\end{array}$ \\
\hline 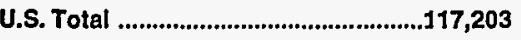 & 30,594 & 1,003 & 85,606 & 6,292 & 89,042 & 43,657 & 45,385 & 37,571 & 18,446 \\
\hline
\end{tabular}

$W=$ Withheld to avold disclosure of individual company data.

Notes: - Stocks are reported as of the last day of the month. - Totals may not equal sum of components due to independent rounding.

Sources: Energy Information Administration (EIA) Forms ElA-810, "Monthly Refinery Report," ElA-811, "Monthly Bulk Terminal Report," and EIA-816, "Monthly Natural Gas Liquids Report." 
Table 53. Movements of Crude Oil and Petroleum Products by Pipeline, Tanker, and Barge Between PAD Districts, March 1995

(Thousand Barrels)

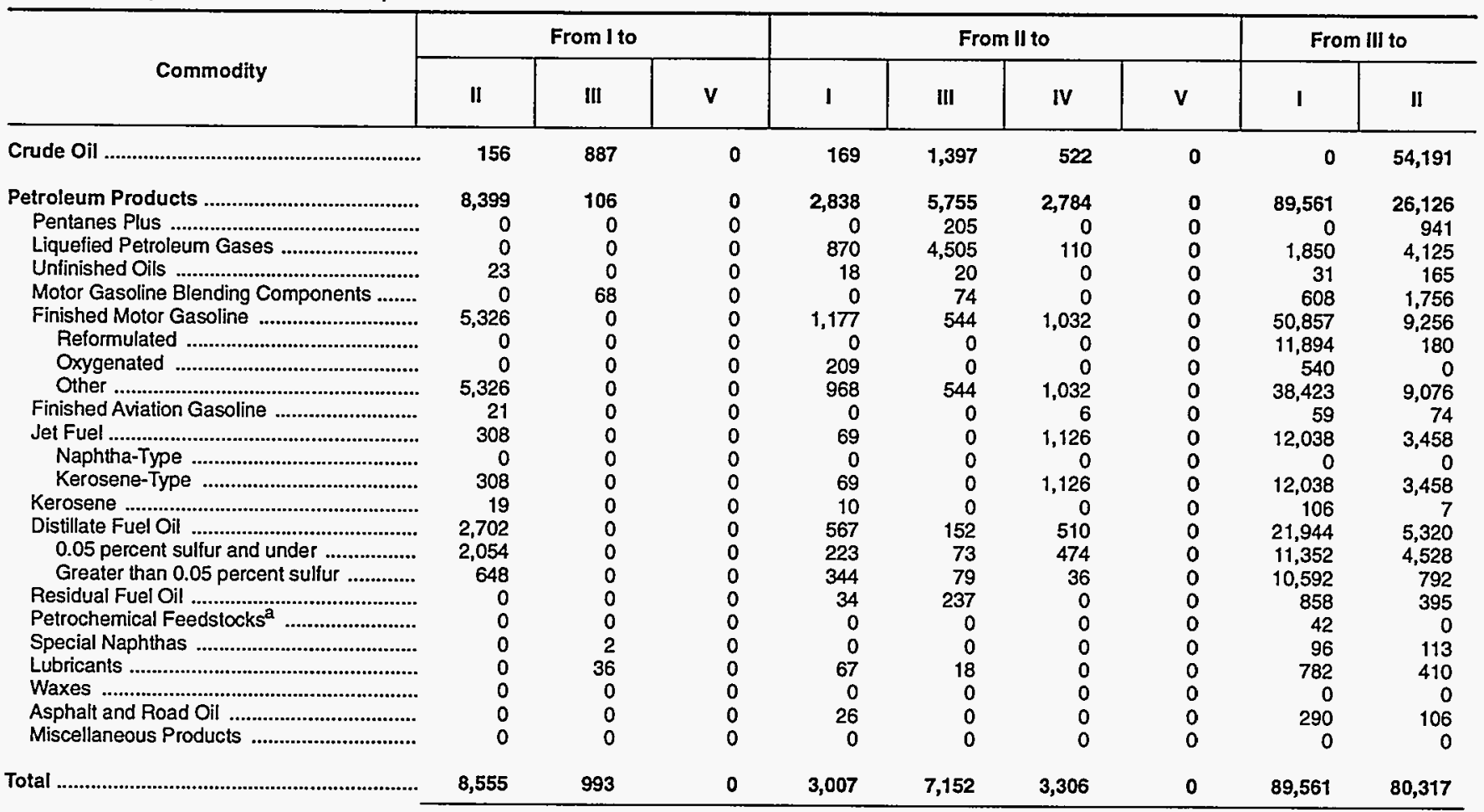

\begin{tabular}{|c|c|c|c|c|c|c|c|c|c|}
\hline \multirow[b]{2}{*}{ Commodity } & \multicolumn{2}{|c|}{ From III to } & \multicolumn{3}{|c|}{ From IV to } & \multicolumn{4}{|c|}{ From $V$ to } \\
\hline & IV & V & II & III & $\mathbf{V}$ & I & II & III & IV \\
\hline 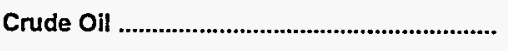 & 0 & 0 & 2,242 & 914 & 0 & $\mathbf{0}$ & 0 & 10,427 & 0 \\
\hline 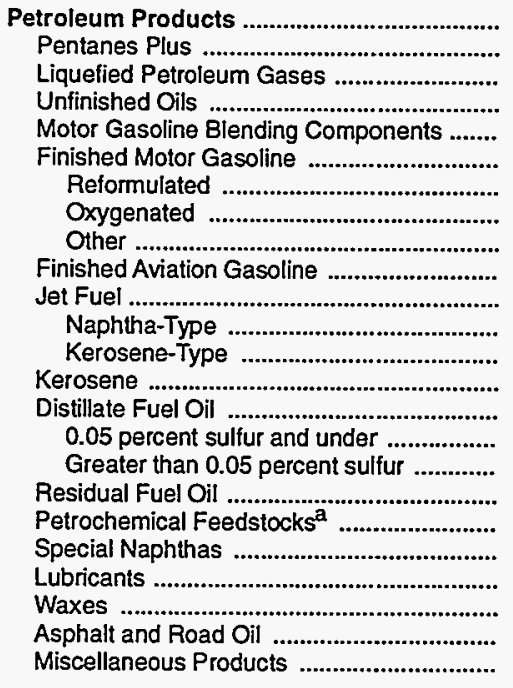 & $\begin{array}{r}353 \\
0 \\
0 \\
0 \\
0 \\
303 \\
0 \\
0 \\
303 \\
0 \\
20 \\
0 \\
20 \\
0 \\
30 \\
30 \\
0 \\
0 \\
0 \\
0 \\
0 \\
0 \\
0 \\
0\end{array}$ & $\begin{array}{r}1,661 \\
0 \\
0 \\
0 \\
0 \\
1,245 \\
0 \\
0 \\
1,245 \\
0 \\
195 \\
0 \\
195 \\
0 \\
221 \\
134 \\
87 \\
0 \\
0 \\
0 \\
0 \\
0 \\
0 \\
0\end{array}$ & $\begin{array}{r}2,439 \\
166 \\
1,365 \\
0 \\
0 \\
570 \\
0 \\
0 \\
570 \\
0 \\
68 \\
68 \\
0 \\
0 \\
270 \\
270 \\
0 \\
0 \\
0 \\
0 \\
0 \\
0 \\
0 \\
0\end{array}$ & $\begin{array}{r}1,864 \\
217 \\
1,647 \\
0 \\
0 \\
0 \\
0 \\
0 \\
0 \\
0 \\
0 \\
0 \\
0 \\
0 \\
0 \\
0 \\
0 \\
0 \\
0 \\
0 \\
0 \\
0 \\
0 \\
0\end{array}$ & $\begin{array}{r}1,217 \\
0 \\
0 \\
0 \\
0 \\
795 \\
0 \\
0 \\
795 \\
0 \\
171 \\
79 \\
92 \\
0 \\
251 \\
225 \\
26 \\
0 \\
0 \\
0 \\
0 \\
0 \\
0 \\
0\end{array}$ & $\begin{array}{r}127 \\
0 \\
0 \\
0 \\
0 \\
127 \\
0 \\
0 \\
127 \\
0 \\
0 \\
0 \\
0 \\
0 \\
0 \\
0 \\
0 \\
0 \\
0 \\
0 \\
0 \\
0 \\
0 \\
0\end{array}$ & $\begin{array}{l}0 \\
0 \\
0 \\
0 \\
0 \\
0 \\
0 \\
0 \\
0 \\
0 \\
0 \\
0 \\
0 \\
0 \\
0 \\
0 \\
0 \\
0 \\
0 \\
0 \\
0 \\
0 \\
0 \\
0\end{array}$ & $\begin{array}{r}397 \\
0 \\
0 \\
0 \\
0 \\
397 \\
0 \\
0 \\
397 \\
0 \\
0 \\
0 \\
0 \\
0 \\
0 \\
0 \\
0 \\
0 \\
0 \\
0 \\
0 \\
0 \\
0 \\
0\end{array}$ & $\begin{array}{l}0 \\
0 \\
0 \\
0 \\
0 \\
0 \\
0 \\
0 \\
0 \\
0 \\
0 \\
0 \\
0 \\
0 \\
0 \\
0 \\
0 \\
0 \\
0 \\
0 \\
0 \\
0 \\
0 \\
0\end{array}$ \\
\hline Total & 353 & 1,661 & 4,681 & 2,778 & 1,217 & 127 & 0 & 10,824 & 0 \\
\hline
\end{tabular}

a Includes naphtha less than $401^{\circ} \mathrm{F}$ endpoint and other oils equal to or greater than $401^{\circ} \mathrm{F}$ endpoint.

Sources: Energy Information Administration (EIA) Forms EIA-812, "Monthly Product Pipeline Report," EIA-813, "Monthly Crude Oil Report," and EIA-817, "Monthly Tanker and Barge Movement Report." 
Table 54. Movements of Crude Oil and Petroleum Products by Pipeline Between PAD Districts, March 1995

(Thousand Barrels)

\begin{tabular}{|c|c|c|c|c|c|c|c|}
\hline \multirow{2}{*}{ Commodity } & \multicolumn{2}{|c|}{ From I to } & \multicolumn{3}{|c|}{ From ll to } & \multicolumn{2}{|c|}{ From Ill to } \\
\hline & I! & III & $\mathbf{I}$ & III & IV & 1 & 11 \\
\hline 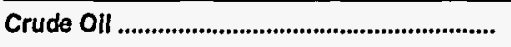 & 100 & 887 & 0 & 1,397 & 522 & 0 & 54,191 \\
\hline 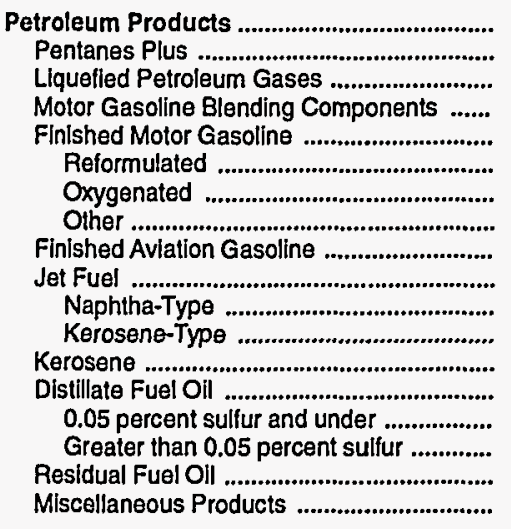 & $\begin{array}{r}8,353 \\
0 \\
0 \\
0 \\
5,303 \\
0 \\
0 \\
5,303 \\
21 \\
308 \\
0 \\
308 \\
19 \\
2,702 \\
2,054 \\
648 \\
0 \\
0\end{array}$ & $\begin{array}{l}0 \\
0 \\
0 \\
0 \\
0 \\
0 \\
0 \\
0 \\
0 \\
0 \\
0 \\
0 \\
0 \\
0 \\
0 \\
0 \\
0 \\
0\end{array}$ & $\begin{array}{r}1,371 \\
0 \\
870 \\
0 \\
476 \\
0 \\
0 \\
476 \\
0 \\
0 \\
0 \\
0 \\
0 \\
25 \\
25 \\
0 \\
0 \\
0\end{array}$ & $\begin{array}{r}5,078 \\
205 \\
4,505 \\
0 \\
309 \\
0 \\
0 \\
309 \\
0 \\
0 \\
0 \\
0 \\
0 \\
59 \\
38 \\
21 \\
0 \\
0\end{array}$ & $\begin{array}{r}2,784 \\
0 \\
110 \\
0 \\
1,032 \\
0 \\
0 \\
1,032 \\
6 \\
1,126 \\
0 \\
1,126 \\
0 \\
510 \\
474 \\
36 \\
0 \\
0\end{array}$ & $\begin{array}{r}66,654 \\
0 \\
1,659 \\
0 \\
37,949 \\
11,156 \\
0 \\
26,793 \\
21 \\
9,280 \\
0 \\
9,280 \\
106 \\
17,639 \\
9,516 \\
8,123 \\
0 \\
0\end{array}$ & $\begin{array}{r}23,069 \\
941 \\
4,125 \\
1,598 \\
8,455 \\
0 \\
0 \\
8,455 \\
67 \\
3,227 \\
0 \\
3,227 \\
0 \\
4,656 \\
4,199 \\
457 \\
0 \\
0\end{array}$ \\
\hline Total & 8,453 & 887 & 1,371 & 6,475 & 3,306 & 66,654 & 77,260 \\
\hline
\end{tabular}

\begin{tabular}{|c|c|c|c|c|c|c|c|}
\hline \multirow[b]{2}{*}{ Commodity } & \multicolumn{2}{|c|}{ From III to } & \multicolumn{3}{|c|}{ From IV to } & \multicolumn{2}{|c|}{ From V to } \\
\hline & IV & $\mathbf{V}$ & ॥ & III & $\mathbf{V}$ & III & IV \\
\hline Crude Oll & 0 & 0 & 2,242 & 914 & 0 & 7,020 & 0 \\
\hline 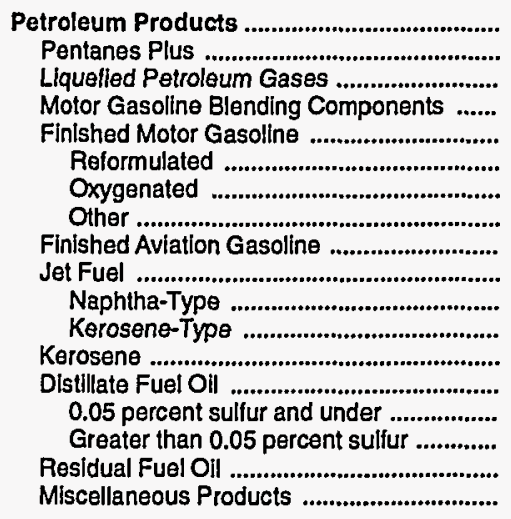 & $\begin{array}{r}353 \\
0 \\
0 \\
0 \\
303 \\
0 \\
0 \\
303 \\
0 \\
20 \\
0 \\
20 \\
0 \\
30 \\
30 \\
0 \\
0 \\
0\end{array}$ & $\begin{array}{r}1,661 \\
0 \\
0 \\
0 \\
1,245 \\
0 \\
0 \\
1,245 \\
0 \\
195 \\
0 \\
195 \\
0 \\
221 \\
134 \\
87 \\
0 \\
0\end{array}$ & $\begin{array}{r}2,439 \\
166 \\
1,365 \\
0 \\
570 \\
0 \\
0 \\
570 \\
0 \\
68 \\
68 \\
0 \\
0 \\
270 \\
270 \\
0 \\
0 \\
0\end{array}$ & $\begin{array}{r}1,864 \\
217 \\
1,647 \\
0 \\
0 \\
0 \\
0 \\
0 \\
0 \\
0 \\
0 \\
0 \\
0 \\
0 \\
0 \\
0 \\
0 \\
0\end{array}$ & $\begin{array}{r}1,217 \\
0 \\
0 \\
0 \\
795 \\
0 \\
0 \\
795 \\
0 \\
171 \\
79 \\
92 \\
0 \\
251 \\
225 \\
26 \\
0 \\
0\end{array}$ & $\begin{array}{l}0 \\
0 \\
0 \\
0 \\
0 \\
0 \\
0 \\
0 \\
0 \\
0 \\
0 \\
0 \\
0 \\
0 \\
0 \\
0 \\
0 \\
0\end{array}$ & $\begin{array}{l}0 \\
0 \\
0 \\
0 \\
0 \\
0 \\
0 \\
0 \\
0 \\
0 \\
0 \\
0 \\
0 \\
0 \\
0 \\
0 \\
0 \\
0\end{array}$ \\
\hline Total & 353 & 1,661 & 4,681 & 2,778 & 1,217 & 7,020 & 0 \\
\hline
\end{tabular}

Sources: Energy Information Administration (EIA) Forms EIA-812, "Monthly Product Pipeline Report," and EIA-813, Monthly Crude Oil Report." 
Table 55. Movements of Crude Oil and Petroleum Products by Tanker and Barge Between PAD Districts, March 1995

(Thousand Barrels)

\begin{tabular}{|c|c|c|c|c|c|c|c|c|}
\hline \multirow[b]{2}{*}{ Commodity } & \multicolumn{3}{|c|}{ From I to } & \multicolumn{3}{|c|}{ From II to } & \multicolumn{2}{|c|}{ From III to } \\
\hline & $\|$ & III & $\mathbf{V}$ & 1 & III & $\mathbf{v}$ & 1 & $\begin{array}{c}\text { New } \\
\text { Englano }\end{array}$ \\
\hline 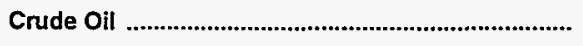 & 56 & 0 & 0 & 169 & 0 & 0 & 0 & 0 \\
\hline 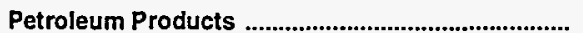 & 46 & 106 & 0 & 1,467 & 677 & $\mathbf{0}$ & 22,907 & 1,845 \\
\hline 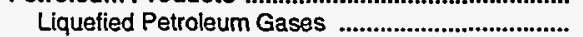 & 0 & 0 & 0 & 0 & 0 & 0 & 191 & 0 \\
\hline 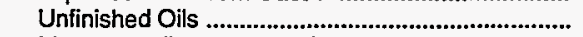 & 23 & 0 & 0 & 18 & 20 & 0 & 31 & 0 \\
\hline Motor Gasoline Blending Components .................. & 0 & 68 & 0 & 0 & 74 & 0 & 608 & 189 \\
\hline 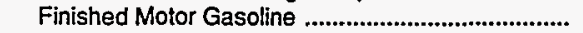 & 23 & 0 & 0 & 701 & 235 & 0 & 12,908 & 841 \\
\hline Reformulated & 0 & 0 & 0 & 0 & 0 & 0 & 738 & 494 \\
\hline 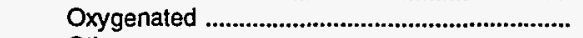 & 0 & 0 & 0 & 209 & 0 & 0 & 540 & 0 \\
\hline Other & 23 & 0 & 0 & 492 & 235 & 0 & 11,630 & 347 \\
\hline 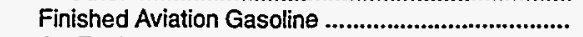 & 0 & 0 & 0 & 0 & 0 & 0 & 38 & 0 \\
\hline Jet Fuel & 0 & 0 & 0 & 69 & 0 & 0 & 2,758 & 0 \\
\hline Naphtha-Type ...................................................... & 0 & 0 & 0 & 0 & 0 & 0 & 0 & 0 \\
\hline 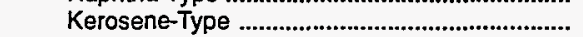 & 0 & 0 & 0 & 69 & 0 & 0 & 2,758 & 0 \\
\hline 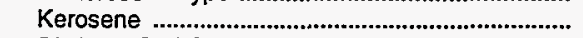 & 0 & 0 & 0 & 10 & 0 & 0 & 0 & 0 \\
\hline 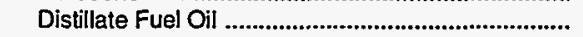 & 0 & 0 & 0 & 542 & 93 & 0 & 4,305 & 806 \\
\hline 0.05 percent sulfur and under & 0 & 0 & 0 & 198 & 35 & 0 & 1,836 & 0 \\
\hline Greater then 0.05 percent sulfur ......................... & 0 & 0 & 0 & 344 & 58 & 0 & 2,469 & 806 \\
\hline 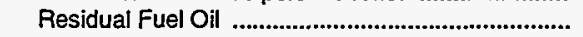 & 0 & 0 & 0 & 34 & 237 & 0 & 858 & 0 \\
\hline 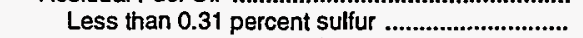 & 0 & 0 & 0 & 0 & 0 & 0 & 0 & 0 \\
\hline 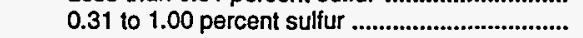 & 0 & 0 & 0 & 0 & 0 & 0 & 0 & 0 \\
\hline Greater than 1.00 percent sulfur ......................... & 0 & 0 & 0 & 34 & 237 & 0 & 858 & 0 \\
\hline 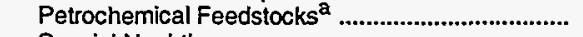 & 0 & 0 & 0 & 0 & 0 & 0 & 42 & 0 \\
\hline Special Naphthas & 0 & 2 & 0 & 0 & 0 & 0 & 96 & 0 \\
\hline 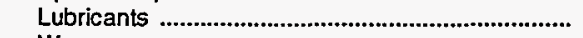 & 0 & 36 & 0 & 67 & 18 & 0 & 782 & 9 \\
\hline Waxes & 0 & 0 & 0 & 0 & 0 & 0 & 0 & 0 \\
\hline 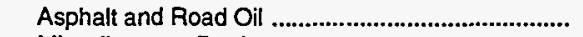 & 0 & 0 & 0 & 26 & 0 & 0 & 290 & 0 \\
\hline 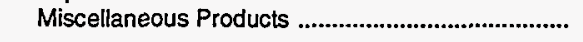 & 0 & 0 & 0 & 0 & 0 & 0 & 0 & 0 \\
\hline Total & 102 & 106 & 0 & 1,636 & 677 & 0 & 22,907 & 1,845 \\
\hline
\end{tabular}

\begin{tabular}{|c|c|c|c|c|c|c|c|}
\hline \multirow[b]{2}{*}{ Commodity } & \multicolumn{4}{|c|}{ From III to } & \multicolumn{3}{|c|}{ From V to } \\
\hline & $\begin{array}{l}\text { Central } \\
\text { Atlantic }\end{array}$ & $\begin{array}{l}\text { Lower } \\
\text { Atlantic }\end{array}$ & II & $\mathbf{v}$ & I & II & III \\
\hline 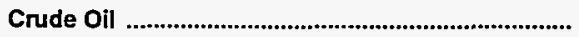 & 0 & 0 & $\mathbf{0}$ & $\mathbf{0}$ & 0 & 0 & 3,407 \\
\hline 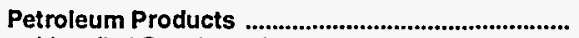 & 1,792 & 19,270 & 3,057 & 0 & 127 & 0 & 397 \\
\hline 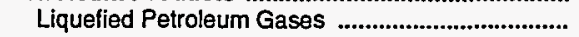 & 0 & 191 & 0 & 0 & 0 & 0 & 0 \\
\hline Unfinished Oils .......................................................... & 0 & 31 & 165 & 0 & 0 & 0 & 0 \\
\hline Motor Gasoline Blending Components .................. & 377 & 42 & 158 & 0 & 0 & 0 & 0 \\
\hline Finished Motor Gasoline ........................................ & 373 & 11,694 & 801 & 0 & 127 & 0 & 397 \\
\hline 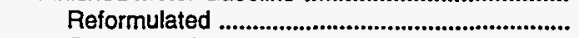 & 244 & 0 & 180 & 0 & 0 & 0 & 0 \\
\hline 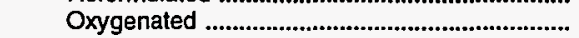 & 0 & 540 & 0 & 0 & 0 & $\mathbf{0}$ & 0 \\
\hline Other & 129 & 11,154 & 621 & 0 & 127 & 0 & 397 \\
\hline Finished Aviation Gasoline ........................................ & 5 & 33 & 7 & 0 & 0 & 0 & 0 \\
\hline Jet Fuel & 88 & 2,670 & 231 & 0 & 0 & 0 & 0 \\
\hline 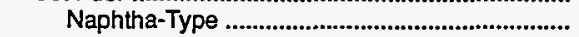 & 0 & 0 & 0 & 0 & 0 & 0 & 0 \\
\hline 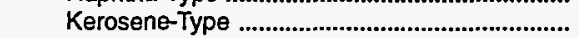 & 88 & 2,670 & 231 & 0 & 0 & 0 & 0 \\
\hline Kerosene & 0 & 0 & 7 & 0 & 0 & 0 & 0 \\
\hline 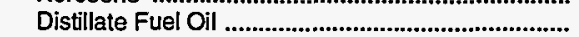 & 370 & 3,129 & 664 & 0 & 0 & 0 & 0 \\
\hline 0.05 percent sulfur and under .............................. & 29 & 1,807 & 329 & 0 & 0 & 0 & 0 \\
\hline 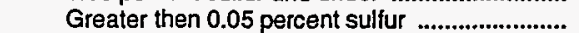 & 341 & 1,322 & 335 & 0 & 0 & 0 & 0 \\
\hline Residual Fuel Oil & 0 & 858 & 395 & 0 & 0 & 0 & 0 \\
\hline 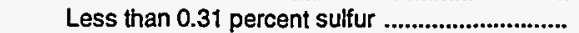 & 0 & 0 & 0 & 0 & 0 & 0 & 0 \\
\hline 0.31 to 1.00 percent sulfur ................................... & 0 & 0 & 0 & 0 & 0 & 0 & 0 \\
\hline Greater than 1.00 percent sulfur ....................... & 0 & 858 & 395 & 0 & 0 & 0 & 0 \\
\hline 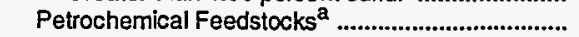 & 42 & 0 & 0 & 0 & 0 & 0 & 0 \\
\hline Special Naphthas & 0 & 96 & 113 & 0 & 0 & 0 & 0 \\
\hline 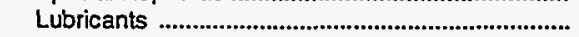 & 482 & 291 & 410 & 0 & 0 & 0 & 0 \\
\hline Waxes & 0 & 0 & 0 & 0 & 0 & 0 & 0 \\
\hline Asphalt and Road Oil & 55 & 235 & 106 & 0 & 0 & 0 & 0 \\
\hline 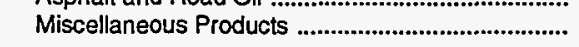 & 0 & 0 & 0 & $\mathbf{0}$ & 0 & 0 & 0 \\
\hline Total & 1,792 & 19,270 & 3,057 & 0 & 127 & 0 & 3,804 \\
\hline
\end{tabular}

a Includes naphtha less than $401^{\circ} \mathrm{F}$ endpoint and other oils equal to or greater than $401^{\circ} \mathrm{F}$ endpoint.

Source: Energy Information Administration (EIA) Form ElA-817, "Monthly Tanker and Barge Movement Report." 
Table 56. Net Movements of Crude Oil and Petroleum Products by Pipeline, Tanker, and Barge Between PAD Districts, March 1995 (Thousand Barrels)

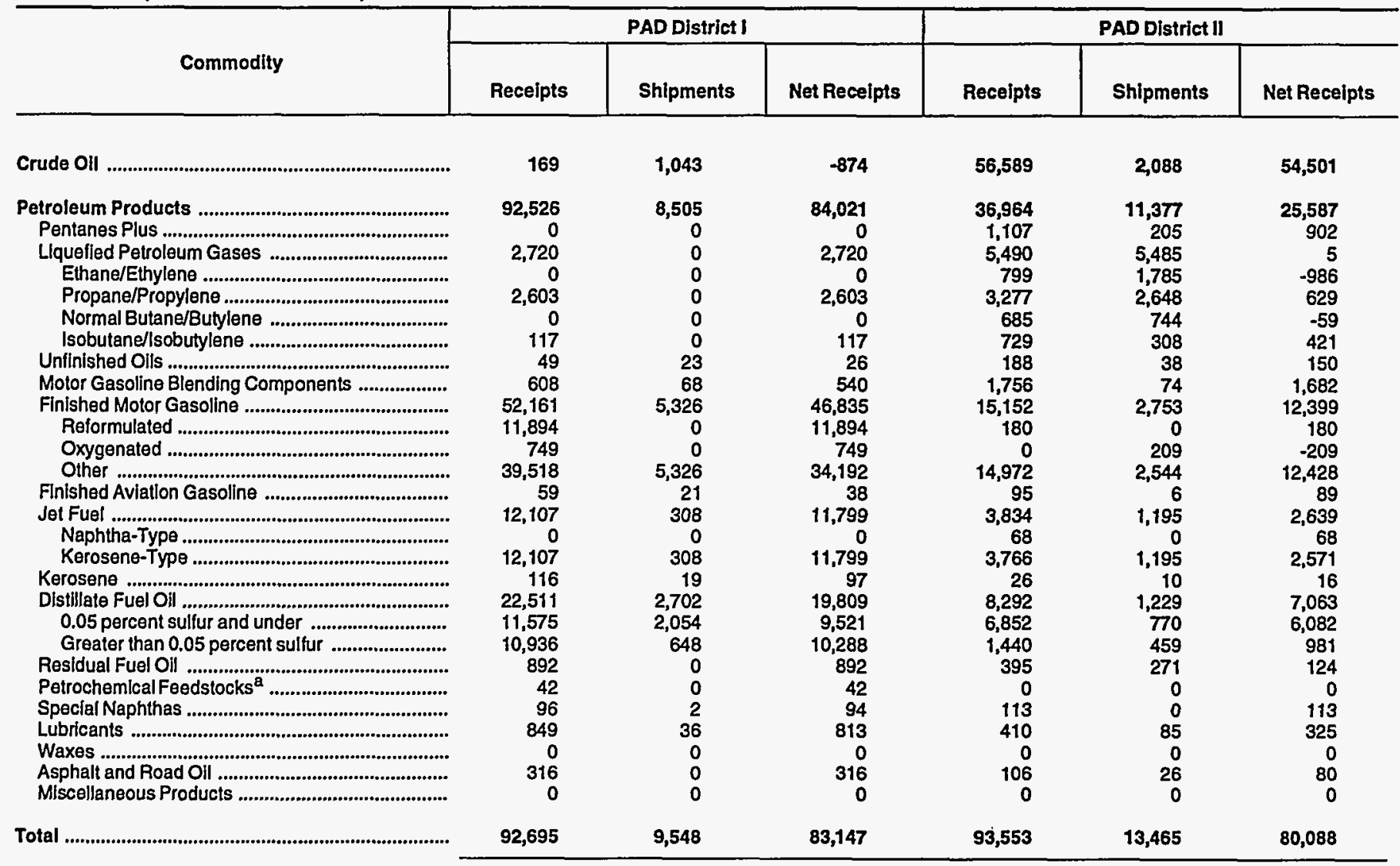

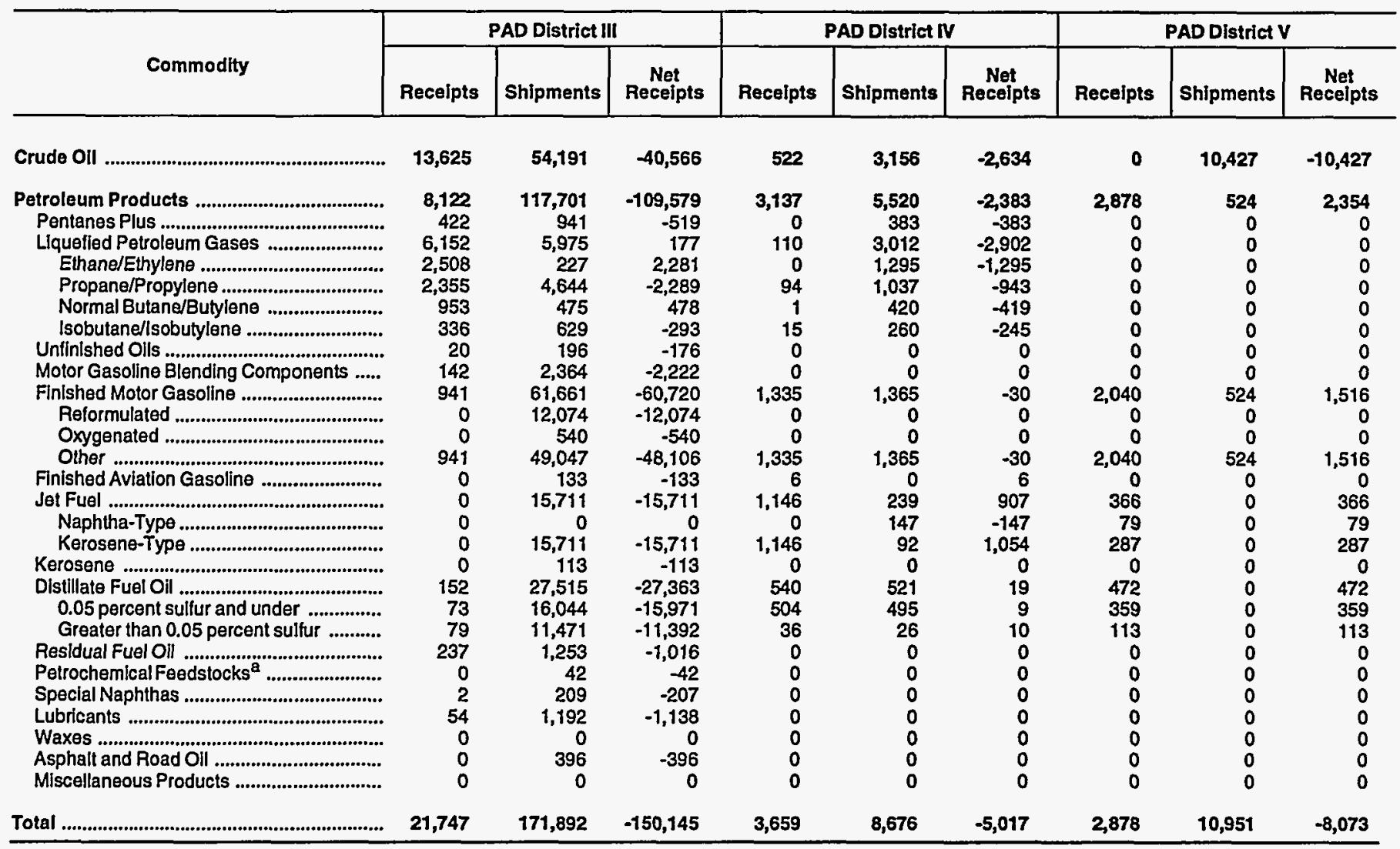

a Includes naphtha less than $401^{\circ} F$ endpoint and other oils equal to or greater than $401^{\circ} F$ endpoint

Sources: Energy Information Administration (EIA) Forms EIA-B12, "Monthly Product Pipeline Report," ElA-813, "Monthly Crude Oil Report," and EIA-817, 'Monthly Tanker and Barge Movement Report." 



\title{
Appendix A
}

\author{
District \\ Descriptions \\ and Maps
}

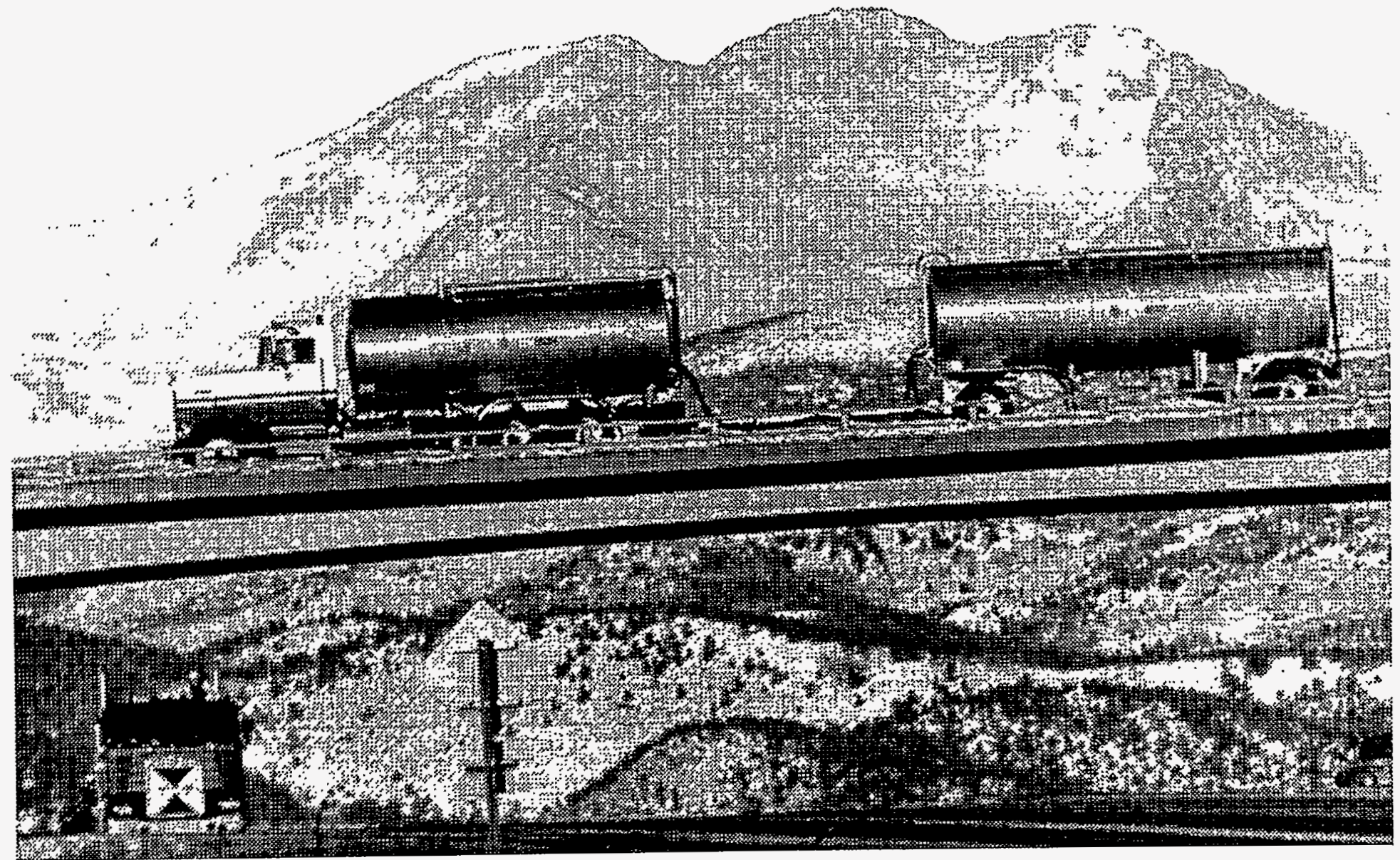

Tank trucks are used to distribute heating oil to remote areas. 



\section{District Descriptions and Maps}

The following are the Refining Districts which make up the Petroleum Administration for Defense (PAD) Districts.

\section{PAD District I}

East Coast: District of Columbia and the States of Maine, New Hampshire, Vermont, Massachusetts, Rhode Island, Connecticut, New Jersey, Delaware, Maryland, Virginia, North Carolina, South Carolina, Georgia, Florida, and the following counties of the State of New York: Cayuga, Tompkins, Chemung, and all counties east and north thereof. Also the following counties in the State of Pennsylvania: Bradford, Sullivan, Columbia, Montour, Northumberland, Dauphin, York, and all counties east thereof.

Appalachian No. 1: The State of West Virginia and those parts of the States of Pennsylvania and New York not included in the East Coast District.

\section{Sub-PAD District I}

New England: The States of Connecticut, Maine, Massachusetts, New Hampshire, Rhode Island and Vermont.

Central Atlantic: The District of Columbia and the States of Delaware, Maryland, New Jersey, New York, and Pennsylvania.

Lower Atlantic: The States of Florida, Georgia, North Carolina, South Carolina, Virginia and West Virginia.

\section{PAD District II}

Indiana-Illinois-Kentucky: The States of Indiana, I1linois, Kentucky, Tennessee, Michigan, and Ohio.

Minnesota-Wisconsin-North and South Dakota: The States of Minnesota, Wisconsin, North Dakota, and South Dakota.

Oklahoma-Kansas-Missouri: The States of Oklahoma, Kansas, Missouri, Nebraska, and Towa.

\section{PAD District III}

Texas Inland: The State of Texas except the Texas Gulf Coast District.

Texas Gulf Coast: The following counties of the State of Texas: Newton, Orange, Jefferson, Jasper, Tyler, Hardin, Liberty, Chambers, Polk, San Jacinto, Montgomery, Harris, Galveston, Waller, Fort Bend, Brazoria, Wharton, Matagorda, Jackson, Victoria, Calhoun, Refugio, Aransas, San Patricio, Nueces, Kleberg, Kenedy, Willacy, and Cameron.

Louisiana Gulf Coast: The following parishes of the State of Louisiana: Vernon, Rapides, Avoyelles, Pointe Coupee, West Feliciana, East Feliciana, Saint Helena, Tangipahoa, Washington, and all parishes south thereof. Also the following counties of the State of Mississippi: Pearl River, Stone, George, Hancock, Harrison, and Jackson. Also the following counties of the State of Alabama: Mobile and Baldwin.

North Louisiana-Arkansas: The State of Arkansas and those parts of the States of Louisiana, Mississippi, and Alabama not included in the Louisiana Gulf Coast District.

New Mexico: The State of New Mexico.

\section{PAD District IV}

Rocky Mountain: The States of Montana, Idaho, Wyoming, Utah, and Colorado.

\section{PAD District V}

West Coast: The States of Washington, Oregon, California, Nevada, Arizona, Alaska, and Hawaii. 

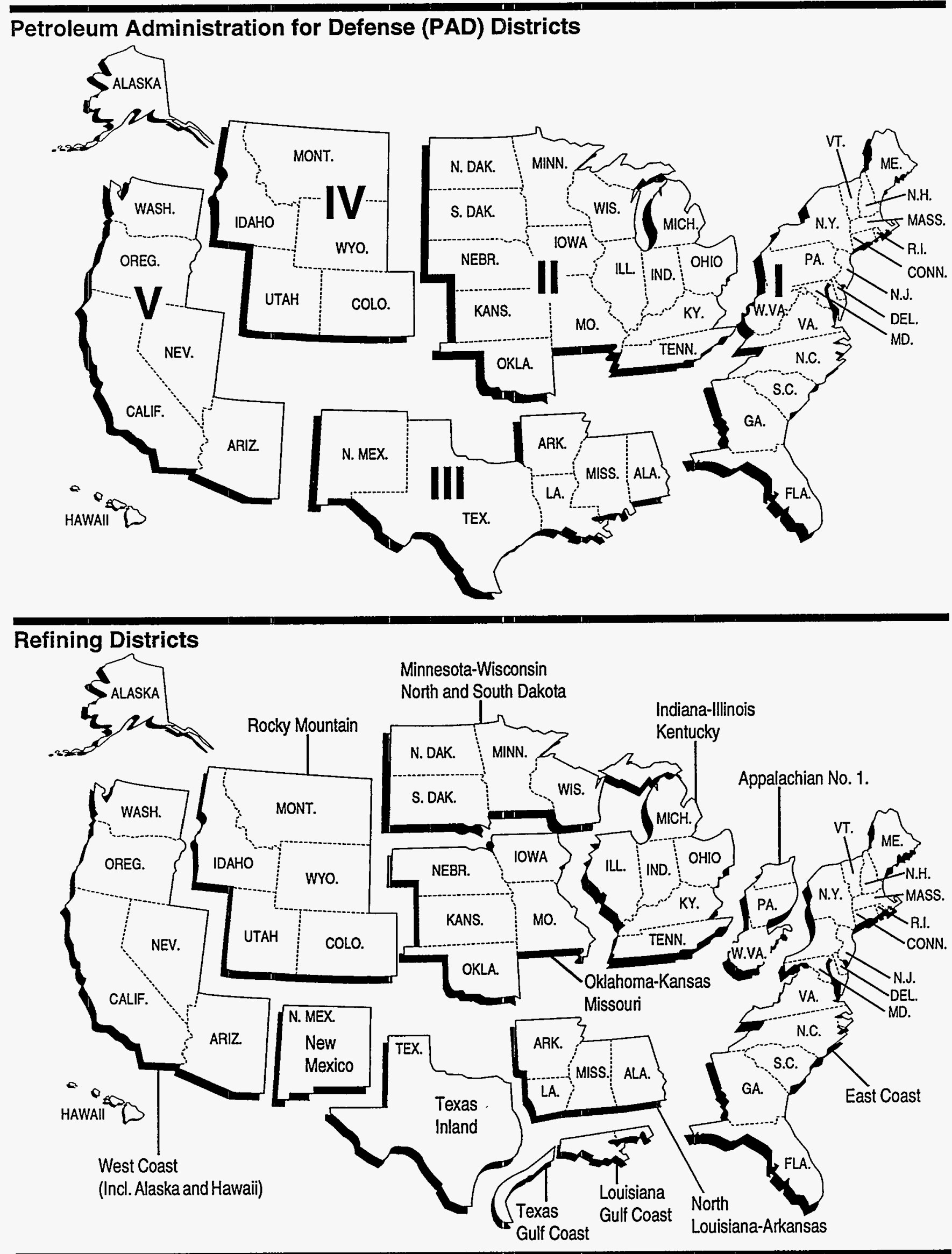


\section{Appendix B}

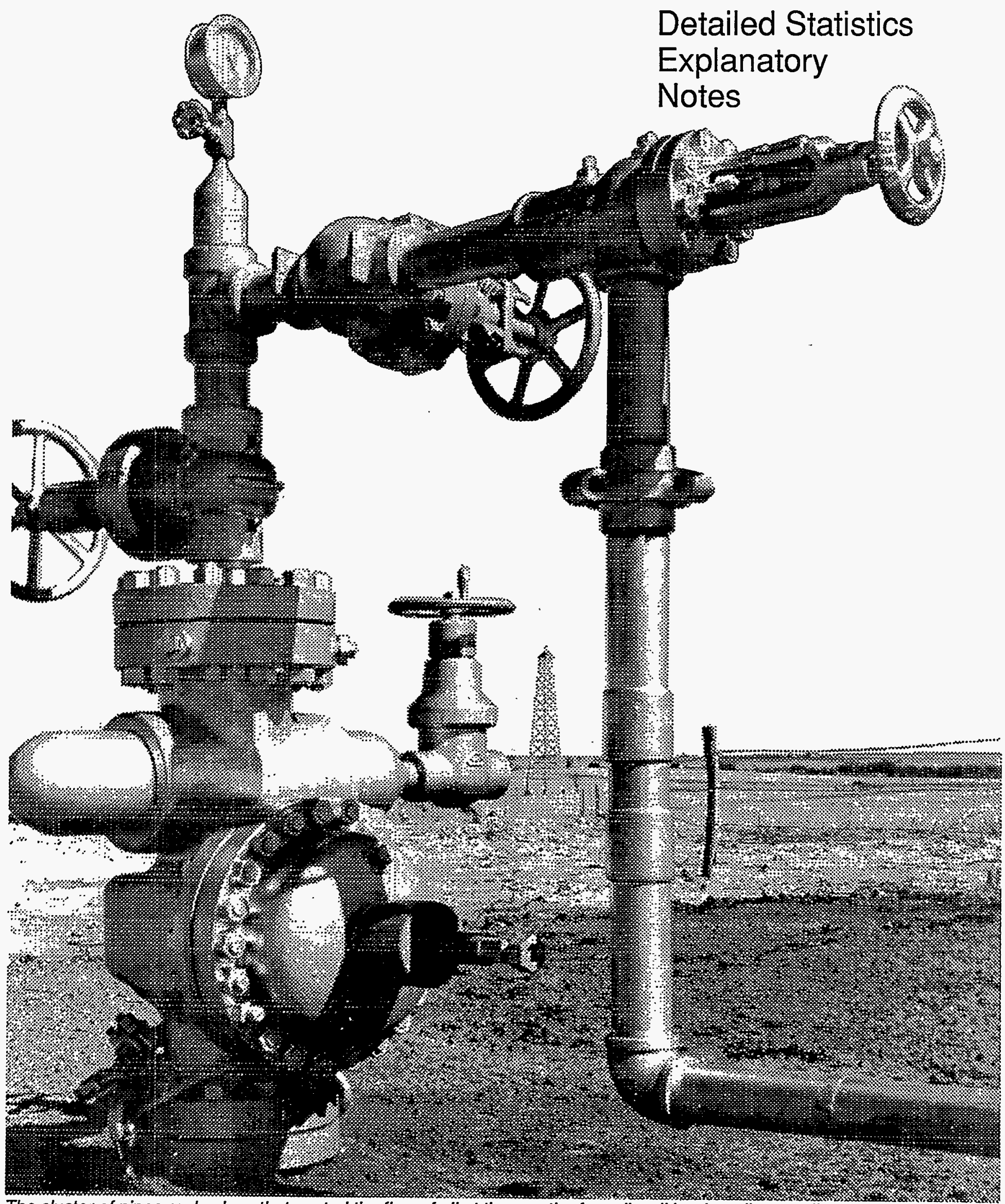

The cluster of pipes and valves that control the flow of oil at the mouth of an oil well is what oilmen call a "Christmas Tree." 



\section{Explanatory Notes}

The following Explanatory Notes are provided to assist in understanding and interpreting the data presented in the Detailed Statistics section of this publication.

- Note 1. Petroleum Supply Reporting System

- Note 2. Monthly Petroleum Supply Reporting System

- Note 3. Technical Notes for Detailed Statistics Tables

- Note 4. Domestic Crude Oil Production

- Note 5. Export Data

- Note 6. Quality Control and Data Revision

- Note 7. Frames Maintenance

- Note 8. Practical Limitations of Data Collection Efforts

- Note 9. 1994 Changes in the Petroleum Supply Monthly

\section{Note 1. Petroleum Supply Reporting System}

The Petroleum Supply Reporting System (PSRS) represents a family of data collection survey forms, data processing systems, and publication systems that have been consolidated to achieve comparability and consistency throughout. The survey forms that comprise the PSRS are listed below:

\begin{tabular}{|c|c|}
\hline $\begin{array}{l}\text { Form } \\
\text { Number }\end{array}$ & Name \\
\hline EIA-800 & "Weekly Refinery Report" \\
\hline EIA-801 & "Weekly Bulk Terminal Report" \\
\hline EIA-802 & "Weekly Product Pipeline Report" \\
\hline EIA-803 & "Weekly Crude Oil Stocks Report" \\
\hline EIA-804 & "Weekly Imports Report" \\
\hline EIA-807 & "Propane Telephone Survey" \\
\hline EIA-810 & "Monthly Refinery Report" \\
\hline EIA-811 & "Monthly Bulk Terminal Report" \\
\hline EIA-812 & "Monthly Product Pipeline Report" \\
\hline EIA-813 & "Monthly Crude Oil Report" \\
\hline EIA-814 & "Monthly Imports Report" \\
\hline EIA-816 & "Monthly Natural Gas Liquids Report" \\
\hline EIA-817 & $\begin{array}{l}\text { "Monthly Tanker and Barge Movement } \\
\text { Report" }\end{array}$ \\
\hline EIA-819. & "Monthly Oxygenate Telephone Report' \\
\hline EIA-819A & "Annual Oxygenate Capacity Report" \\
\hline EIA-820 & "Annual Refinery Report" \\
\hline
\end{tabular}

Forms EIA-800 through 804 comprise the Weekly Petroleum Supply Reporting System (WPSRS). A sample of all petroleum companies report weekly data to the Energy Information Administration (EIA) on crude oil and petroleum product stocks, refinery inputs and production, and crude oil and petroleum product imports. The sample of companies that report weekly is selected from the universe of companies that report on the comparable monthly surveys. Data collected from the WPSRS are used to develop estimates of the most current monthly quantities in the Summary Statistics section of the Petroleum Supply Monthly (PSM) and which appear in the Weekly Petroleum Status Report (WPSR).

The Form EIA-807, "Propane Telephone Survey" is used to collect data on production, stocks, and imports of propane. These data are used to monitor the supply of propane and to report to the Congress and others on supplies when requested. Data are collected from a sample of respondents reporting on the Monthly Petroleum Supply Reporting System (MPSRS) surveys. Data are collected on a weekly basis during the heating season (October through March) and published in the Winter Fuels Report. During the non-heating season (April through September) data are collected on end-of-month stocks only. These data are published in the WPSR.

Forms EIA-810 through 814,816 , and 817 comprise the MPSRS. These surveys are used to collect detailed refinery/blender and natural gas plant operations data; refinery/blender, bulk terminal, natural gas plant, and pipeline stocks data; crude oil and petroleum product imports data; and data on movements of petroleum products and crude oil between Petroleum Administration for Defense (PAD) Districts. A description of the MPSRS forms follows in Explanatory Note 2.

Data from these surveys are published in preliminary form in the PSM. They are published in final form in the Petroleum Supply Annual (PSA), Volumes 1 and 2.

Summary information on the revision error between preliminary and final data is published once a year in the PSM feature article entitled, "Timeliness and Accuracy of Petroleum Supply Data." The last article was published in the August 1993 issue and evaluated the accuracy of the data for 1992 compared with previous years.

The Form EIA-819M, "Monthly Oxygenate Telephone Report," is used to collect preliminary data on production, imports, and stocks of oxygenates by PAD District. These 
data are used to monitor the supply of oxygenates. Data are collected from a sample of respondents reporting on the MPSRS surveys and from the universe of oxygenate producers. Data are published in Appendix D of this publication and in the WPSR.

The Form EIA-819A, "Annual Oxygenate Capacity Report," is used to collect data on current and projected production capacity of oxygenates and annual production and end-of-year inventories of fuel ethanol. The results of this survey are published in the Oxygenate Capacity section of the PSA, Volume 1.

The Form EIA-820, "Annual Refinery Report," is used to collect data on refinery fuel use and consumption of steam and electricity, refinery receipts of crude oil by method of transportation, operable capacity for atmospheric crude oil distillation units and downstream units, as well as production capacity and storage capacity for petroleum products. This survey is the primary source of data in the Refinery Capacity section of the PSA Volume 1.

\section{Note 2. Monthly Petroleum Supply Reporting System}

The Monthly Petroleum Supply Reporting System (MPSRS) was implemented in January 1983 as the result of an extensive effort by the Energy Information Administration (EIA) to integrate the collection and processing of petroleum supply data that had been collected on other survey forms for many years. The collection of monthly petroleum supply statistics began as early as 1918 when the U.S. Bureau of Mines began collecting data on refinery operations, crude oil stocks and movements. The collection systems were further expanded in 1925 to include natural gas plant liquids production and storage, imports of crude oil and petroleum products and storage and movement of petroleum products in 1959, and tanker and barge movements of crude oil and petroleum products in 1964. Since their inception, each survey has undergone numerous changes, but the MPSRS was the first effort to make them all consistent and comparable. The forms that comprise the MPSRS are:

$\begin{array}{ll}\begin{array}{l}\text { Form } \\ \text { Number }\end{array} & \text { Name } \\ \text { EIA-810 } & \text { "Monthly Refinery Report" } \\ \text { EIA-811 } & \text { "Monthly Bulk Terminal Report" } \\ \text { EIA-812 } & \text { "Monthly Product Pipeline Report" } \\ \text { EIA-813 } & \text { "Monthly Crude Oil Report" } \\ \text { EIA-814 } & \text { "Monthly Imports Report" } \\ \text { EIA-816 } & \text { "Monthly Natural Gas Liquids Report" } \\ \text { EIA-817 } & \text { "Monthly Tanker and Barge Movement } \\ \text { EIA-819M } & \text { "Meport" } \\ \end{array}$

\section{Respondent Frame}

Form EIA-810, "Monthly Refinery Report" - Operators of all operating and idle petroleum refineries and blending plants located in the 50 States, the District of Columbia, Puerto Rico, the Virgin Islands, Guam and other U.S. possessions. Approximately 240 respondents report on the Form EIA-810.

Form EIA-811, "Monthly Bulk Terminal Report" - Every bulk terminal operating company located in the 50 States, the District of Columbia, Puerto Rico, the Virgin Islands, and other U.S. possessions. A bulk terminal is primarily used for storage and/or marketing of petroleum products and has a total bulk storage capacity of 50,000 barrels or more, and/or receives petroleum products by tanker, barge, or pipeline. Bulk terminal facilities associated with a product pipeline are included. In addition, the Form EIA-811 must be completed by merchant oxygenate plants that produce oxygenates. Approximately 330 respondents report on the Form EIA-811.

Form EIA-812, "Monthly Product Pipeline Report" - All product pipeline companies that carry petroleum products (including interstate, intrastate, and intracompany pipelines) in the 50 States and the District of Columbia. Approximately 80 respondents report on the Form EIA812.

Form EIA-813, "Monthly Crude Oil Report" - All companies which carry or store 1,000 barrels or more of crude oil. Included in this survey are gathering and trunk pipeline companies (including interstate, intrastate, and intracompany pipelines), crude oil producers, terminal operators, storers of crude oil (except refineries), and companies transporting Alaskan crude oil by water in the 50 States and the District of Columbia. Approximately 160 respondents report on the Form EIA-813.

Form EIA-814, "Monthly Imports Report" - All companies, including subsidiary or affiliated companies, that import crude oil or petroleum products (1) into the 50 States and the District of Columbia, (2) into Puerto Rico, the Virgin Islands and other U.S. possessions (Guam, Midway Islands, Wake Island, American Samoa, and Northern Mariana Islands), and (3) from Puerto Rico, the Virgin Islands and other U.S. possessions into the 50 States and the District of Columbia. Imports into Foreign Trade Zones located in the 50 States and the District of Columbia are considered imports into the 50 States and the District of Columbia and must be reported. A report is required only if there has been an import during the month unless the importer has been selected as part of a sample to report every month regardless of activity. Approximately 220 respondents report on the Form EIA-814.

Form EIA-816, "Monthly Natural Gas Liquids Report" Operators of all facilities that extract liquid hydrocarbons from a natural gas stream (natural gas processing plant) and/or separate a liquid hydrocarbon stream into its com- 
ponent products (fractionator). Approximately 720 respondents report on the Form EIA-816.

Form EIA-817, "Monthly Tanker and Barge Movement Report" - All companies that have custody of crude oil or petroleum products transported by tanker or barge between Petroleum Administration for Defense (PAD) Districts or between the Panama Canal and the United States. For purposes of this report, custody is defined as physical possession of crude oil or petroleum products on a company-owned tanker or barge. Also, companies which lease vessels or contract for the movement of crude oil or petroleum products on a tanker or barge between PAD Districts or between the Panama Canal and the United States are considered to have custody. Approximately 40 respondents report on the Form EIA-817.

Form EIA-819M, "Monthly Oxygenate Telephone Report" - The sample of companies that report on the EIA-819M are selected from the universe of companies that report on the MPSRS surveys and from the universe of oxygenate producers. The universe consists of (1) operators of facilities that produce (manufacture or distill) oxygenates (including MTBE plants, petrochemical plants, and refineries that produce oxygenates as part of their operations); (2) operators of petroleum refineries; (3) operators of bulk terminals, bulk stations, blending plants, and other nonrefinery facilities that store and/or blend oxygenates; and (4) importers of oxygenates (importer of record) located in or importing oxygenates into the 50 States and the District of Columbia. Approximately 100 respondents report on the Form EIA-819M.

\section{Sampling}

The sampling procedure used for the survey Form EIA$819 \mathrm{M}$ is the cut-off method and is performed using software developed by EIA's Office of Statistical Standards. In the cut-off method, companies are ranked from largest to smallest on the basis of quantities reported (oxygenate production, oxygenate stocks, and oxygenate imports) during 1993. Companies are chosen for the sample beginning with the largest and adding companies until the total sample covers approximately 90 percent of the total for each oxygenate item and supply type by geographic region (PAD Districts I through V) for which data may be published.

\section{Description of Survey Forms}

The Form EIA-810, "Monthly Refinery Report," is used to collect data on refinery input and capacity, sulfur content and API gravity of crude oil, and data on supply (beginning stocks, receipts, and production) and disposition (inputs, shipments, fuel use and losses, and ending stocks) of crude oil and refined products.

The Form EIA-811, "Monthly Bulk Terminal Report," is used to collect data on end-of-month stock levels of finished petroleum products by State in the custody of the bulk terminal company or merchant oxygenate plant regardless of ownership. Leased tankage at other facilities is excluded. All domestic and foreign stocks held at bulk terminals and in-transit thereto, except those in-transit by pipeline are included. Petroleum products in-transit by pipeline are reported by pipeline operators on Form EIA812, "Monthly Product Pipeline Report."

The Form EIA-812, "Monthly Product Pipeline Report," is used to collect data on end-of-month stock levels and movements of petroleum products transported by pipeline. Intermediate movements for pipeline systems operating in more than two PAD Districts are included.

The Form EIA-813, "Monthly Crude Oil Report," is used to collect data on end-of-month stocks of crude oil held at pipeline and tank farms (associated with the pipelines) and terminals operated by the reporting company. Also, crude oil consumed by pipelines and on leases as pump fuel, boiler fuel, etc., is reported. Data are reported on a PAD District basis.

Total Alaskan crude oil stocks in-transit by water (including stocks held at transshipment terminals between Alaska and the continental United States) to the 50 States, the District of Columbia, Puerto Rico, and the Virgin Islands are also reported by the transporting company having custody of the stocks.

Inter-PAD District movements of crude oil by pipeline are collected by the shipping and receiving PAD District. Intermediate movements for pipeline systems operating in more than two PAD Districts are not included.

The Form EIA-814, "Monthly Imports Report," is used to collect data on imports of crude oil and petroleum products (1) into the 50 States and the District of Columbia, (2) into Puerto Rico, the Virgin Islands, and other U.S. possessions (Guam, Midway Islands, Wake Island, American Samoa, and Northern Mariana Islands), and (3) from Puerto Rico, the Virgin Islands, and other U.S. possessions into the 50 States and the District of Columbia. Imports into Foreign Trade Zones located in the 50 States and the District of Columbia are considered imports into the 50 States and the District of Columbia.

The type of commodity, port of entry, country of origin, quantity (thousand barrels), sulfur percent by weight, API gravity, and name and location of the processing or storage facility are reported. Sulfur percent by weight is requested for crude oil, crude oil burned as fuel, and residual fuel oil only. API gravity is requested for crude oil only. The name and location of the processing or storage facility is requested for crude oil, unfinished oils, other hydrocarbons/hydrogen/oxygenates and blending components only.

The Form EIA-816, "Monthly Natural Gas Liquids Report," is used to collect data on the operations of natural gas processing plants and fractionators. Beginning and end-of-month stocks, receipts, inputs, production, ship- 
ments, and plant fuel use and losses during the month are collected from operators of natural gas processing plants. End-of-month stocks are collected from fractionators.

The Form EIA-817, "Monthly Tanker and Barge Movement Report," is used to collect data on the movements of crude oil and petroleum products between PAD Districts. Data are reported by shipping and receiving PAD District and sub-PAD District. Shipments to and from the Panama Canal are also included if the shipment was delivered to the Canal.

The Form EIA-819M, "Monthly Oxygenate Telephone Report," is used to collect data on production, stocks, and imports of oxygenates. Data on end-of-month stocks are reported on a custody basis regardless of ownership. Data are reported on a PAD District basis.

\section{Collection Methods}

Except for the EIA-819M, survey forms for the MPSRS can be submitted by mail, facsimile, or electronic transmission. Completed forms are required to be postmarked by the 20th calendar day following the end of the report month. Data collection for the $819 \mathrm{M}$ begins on the seventh working day of each month. Data are solicited by telephone or transmitted to the EIA by facsimile. Receipt of the reports are monitored using an automated respondent mailing list. Telephone follow-up calls are made to nonrespondents prior to the publication deadline.

\section{Response Rate}

The response rate is generally 98 to 100 percent. Chronic nonrespondents and late filing respondents are contacted in writing and reminded of their requirement to report. Companies that file late or fail to file are subject to criminal fines, civil penalties, and other sanctions as provided by Section 13(i) of the Federal Energy Administration (FEA) Act.

\section{Data Imputation}

Imputation is performed for companies that fail to file Forms EIA-810 through 813,816 , and $819 \mathrm{M}$. For such companies, previous monthly values are used for current values.

On the EIA-819M, data are aggregated for each geographic region. Estimation factors, which are derived from the previous year's data, are then applied to each cell to generate published estimates.

Data for nonrespondents on the Forms EIA-814 and 817 are not imputed because these data series, by respondent, are highly variable.

\section{Confidentiality}

The Office of Legal Counsel of the Department of Justice concluded on March 20,1991, that the Federal Energy Administration Act requires the EIA to provide companyspecific data to the Department of Justice, or to any Federal agency when requested for official use, which may include enforcement of Federal law. The information contained on this form may also be made available, upon request, to another component of the Department of Energy (DOE), to any Committee of Congress, the General Accounting Office, or other Congressional agencies authorized by law to receive such information. A court of competent jurisdiction may obtain this information in response to an order.

The information contained on Forms EIA-810 through $813,816,817$, and $819 \mathrm{M}$ are kept confidential and not disclosed to the public to the extent that they satisfy the criteria for exemption under the Freedom of Information Act (FOIA), 5 U.S.C. 552, the Department of Energy (DOE) regulations, 10 C.F.R. 1004.11, implementing the FOIA, and the Trade Secrets Act, 18 U.S.C. 1905. The information contained on Form EIA-814 are not considered confidential and historically has not been treated as such.

Upon receipt of a request for this information under the FOIA, the DOE shall make a final determination whether the information is exempt from disclosure in accordance with the procedures and criteria provided in the regulations. To assist us in this determination, respondents should demonstrate to the DOE that, for example, their information contains trade secrets or commercial or financial information whose release would be likely to cause substantial harm to their company's competitive position. A letter accompanying the submission that explains (on an element-by-element basis) the reasons why the information would be likely to cause the respondent substantial competitive harm if released to the public would aid in this determination. A new justification does not need to be provided each time information is submitted on the form, if the company has previously submitted a justification for that information and the justification has not changed. Company specific data are also provided to other DOE offices for the purpose of examining operations in the context of emergency response planning and actual emergencies.

The data collected on Forms EIA-810 through 814,816 , and 817 appear in EIA publications such as Petroleum Supply Monthly (PSM), Monthly Energy Review, Petroleum Supply Annual (PSA), and the Annual Energy Review.

Data on the breakdown between liquefied refinery gases and olefins, and lubricants is suppressed on PSM Table 29, "Refinery Net Production of Finished Petroleum Products by PAD and Refining Districts" and the corresponding 
PSA table to avoid disclosure of company identifiable data.

Statistics representing data aggregated from less than three companies or aggregated data representing 60 percent or more of a single company's data are suppressed on the PSM and corresponding PSA tables listed below. In addition, complementary suppression is performed to avoid any residual disclosure.

- Table 28, "Refinery Input of Crude Oil and Petroleum Products by PAD and Refining Districts," (inputs of oxygenates)

- Table 30, "Refinery Stocks of Crude Oil and Petroleum Products by PAD and Refining Districts," (stocks of oxygenates)

- Table 51, "Stocks of Crude Oil and Petroleum Products by PAD District," (stocks of oxygenates)

- Table 52, "Refinery, Bulk Terminal, and Natural Gas Plant Stocks of Selected Petroleum Products," (all products)

- Table D2, "Monthly Fuel Ethanol Production and Stocks by PAD Districts," and

- Table D3, "Monthly MTBE Production and Stocks by PAD Districts."

With the exception of the tables listed above, the tables in the PSM (and corresponding PSA tables) are not subject to statistical nondisclosure procedures. Thus, there may be some table cells which are based on data from only one or two respondents, or which are dominated by data from one or two large respondents. In these cases, it may be possible for a knowledgeable user of the data to make inferences about the data reported by a specific respondent.

\section{Note 3. Technical Notes for Detailed Statistics Tables}

The detailed statistics tables in the Petroleum Supply Monthly (PSM) provide complete supply and demand information for the current year. The tables are organized to locate National and Petroleum Administration for Defense (PAD) District summary data at the front followed by tables on crude oil and petroleum product production, import/export data, stocks information, and lastly, data on crude oil and petroleum product movements. To assist in the interpretation of these tables, the following technical notes are provided. Column and row headings are defined in the Glossary.

\section{Supply}

Field Production - Field production is the sum of crude oil production, natural gas plant liquids production, other liquids production, and finished petroleum products production.
Crude oil production is an estimate based on data received from State conservation agencies and the Mineral Management Service of the U.S. Department of the Interior. Refer to Explanatory Note 4 for further details.

Field production of natural gas plant liquids is reported on Form EIA-816 and published on a net basis (i.e., production minus inputs) in this column.

Other liquids field production is calculated by forcing the product supplied to be zero; thereby backing into field production.

Field production of finished petroleum products is calculated by (1) adding the amount of fuel ethanol that has been blended into finished motor gasoline, and (2) plus $(+)$ or minus $(-)$ the field production of motor gasoline blending components. Refer to Explanatory Note 8 for a further discussion of this calculation.

Negative field production of motor gasoline blending components represents an understatement for finished motor gasoline.

Negative field production of other finished motor gasoline represents an overstatement of other finished motor gasoline and an understatement of oxygenated motor gasoline.

Refinery Production - Published production of these products equal refinery production minus refinery input. Refinery production of other hydrocarbons, hydrogen and oxygenates, unfinished oils, and motor and aviation gasoline blending components appear on a net basis under refinery input. Negative refinery production will occur when the amount of a product produced during the month is less than the amount of that same product that is reprocessed (input) or reclassified to become another product during the same month.

Unaccounted for Crude Oil - This column is a balancing item for crude oil. This data element represents the difference between crude oil supply and disposition. Crude oil supply is the sum of field production and imports. Crude oil disposition is the sum of stock change, losses, refinery inputs, exports, and products supplied. A positive result indicates that refiners and exporters reported use of more crude oil than was reported to have been available to them. (This occurs, for example, when imports are undercounted due to late reporting or other problems). A negative result indicates that more crude oil was reported to have been supplied to refiners and exporters than they reported to have used.

\section{Disposition}

Stock Change - This column is calculated as the difference between the Ending Stocks column of this table and the Ending Stocks column of this table in the prior month's publication. A negative number indicates a 
decrease in stocks and a positive number indicates an increase in stocks.

Crude Losses - The volume of crude oil reported by petroleum refineries as being lost in their operations. These losses are due to spills, contamination, fires, etc., as opposed to refining processing losses or gains.

Refinery Inputs - Refinery inputs of crude oil and intermediate materials (unfinished oils, gasoline blending components, other hydrocarbons and oxygenates, liquefied petroleum gases, and pentanes plus) that are processed at refineries to produce finished petroleum products.

Crude oil inputs represents total crude oil (domestic and foreign) input to atmospheric crude oil distillation units and other refinery processing units (i.e., catalytic cracking units, cokers).

Inputs of natural gas liquids are natural gas liquids received from natural gas plants for blending and processing. Published inputs of natural gas liquids are reported on a gross basis.

Inputs of unfinished oils, motor and aviation gasoline blending components, and other hydrocarbons and oxygenates are published on a net basis (i.e., refinery input minus refinery production).

Inputs of finished petroleum products are published on a net basis (i.e., refinery production minus refinery inputs) and displayed under the refinery production column.

Exports - Exports include crude oil shipments from the 50 States to Puerto Rico, and the Virgin Islands.

Products Supplied - Products supplied is equal to field production, plus refinery production, plus imports, plus unaccounted for crude oil, (plus net receipts on a PAD District basis), minus stock change, minus crude losses, minus refinery inputs, minus exports.

Products supplied indicates those quantities of petroleum products supplied for domestic consumption. Occasionally, the result for a product is negative because total disposition of the product exceeds total supply. Negative product supplied may occur for a number of reasons: (1) product reclassification has not been reported; (2) data were misreported or reported late; (3) in the case of calculations on a PAD District basis, the figure for net receipts was inaccurate because the coverage of interdistrict movements was incomplete; and (4) products such as gasoline blending components and unfinished oils have entered the primary supply channels with their production not having been reported, e.g., streams returned to refineries from petrochemical plants.

Product supplied for crude oil is the sum of crude oil burned on leases and by pipelines as fuel. Prior to January 1983, crude oil burned on leases and by pipelines as fuel were reported as either distillate or residual fuel oil and were included in product supplied for these products.

\section{Yields}

The refinery yield of finished motor gasoline is calculated by subtracting the inputs of pentanes plus, liquefied petroleum gases, other hydrocarbons/oxygenates and motor gasoline blending components from the production of finished motor gasoline before dividing by the sum of crude oil input and unfinished oils input (net).

The refinery yield of finished aviation gasoline is calculated by subtracting the inputs of aviation gasoline blending components from the production of finished aviation gasoline before dividing by the sum of crude oil input and unfinished oils input (net).

Refinery yields for all products (except finished motor gasoline and finished aviation gasoline) are calculated by dividing the production for each product by the sum of crude oil input and unfinished oils input (net) reported in the U.S. total.

\section{Stocks}

Primary stocks of petroleum products do not include either secondary stocks held by dealers and jobbers or tertiary stocks held by consumers.

\section{Movements}

Movements of crude oil by pipeline between PAD Districts include trunk pipeline companies (interstate, intrastate, and intracompany pipelines). Intermediate movements for crude oil pipeline systems operating in more than two PAD Districts are not included.

Movements of petroleum products by pipeline between PAD Districts include trunk pipeline companies (interstate, intrastate and intracompany pipelines). Intermediate movements for product pipeline systems operating in more than two PAD Districts are included. For example, a shipment originating in PAD District 3, passing through PAD District 2 to PAD District 1 , is reported as a movement from PAD District 3 to PAD District 2 and also from PAD District 2 to PAD District 1.

Waterborne movements of crude oil and petroleum products between PAD Districts include all shipments of crude oil or petroleum products for which the transporter has custody at the time of shipment. Custody is defined as physical possession of crude oil or petroleum products on a company-owned tanker and barge.

\section{Note 4. Domestic Crude Oil Production}

The Energy Information Administration (EIA) collects monthly crude oil production data on an ongoing basis. Data on crude oil production for States are reported to the 
EIA by State government agencies. Data on crude oil production for Federal offshore areas are reported to the EIA by the Minerals Management Service of the U.S. Department of the Interior and the Conservation Committee of California Oil Producers.

Currently, all except four crude oil producing States (Michigan, New York, Ohio, and Pennsylvania) report production on a monthly basis. These four States report crude oil production on an annual basis. Estimates of monthly crude oil production for these four States are made by the EIA using data reported on Form EIA-182, "Domestic Crude Oil First Purchase Report." After the end of each calendar year, the monthly crude oil production estimates are updated using annual reports from various State agencies, the Minerals Management Service, and the Conservation Committee of California Oil Producers. The final estimate is published in the Petroleum Supply Annual (PSA).

Table 26 of this publication provides estimates of crude oil production in the latest month for which most State production data are available. There is a time lag of approximately 4 months between the end of the production month and the time when most monthly State crude oil production data become available.

In order to present more timely crude oil production estimates, the EIA prepares a weekly crude oil production estimate, which is used in the Weekly Petroleum Status Report. At the end of the production month, these weekly estimates are aggregated into an original estimate of monthly crude oil production. Approximately 45 days later, this original estimate is replaced by State-level interim estimates. The State-level interim estimates are based on: (a) data reported by the States (e.g., production data for Alaska are typically reported to the EIA before the interim estimate is made); (b) first purchase data reported on Form EIA-182, "Domestic Crude Oil First Purchase Report;" (c) exponential or hyperbolic curve fitted projections based on recent State data; or (d) constant level projections based on the average production rate during a recent time period.

Table B1 is intended to provide further insight into the EIA's estimates of monthly U.S. crude oil production. It shows: (a) how the aggregate of reported State data evolves over a period of 18 months; (b) the number of producing States that have not reported production for a given month within that period; and (c) various EIA estimates of monthly crude oil production within that period:

- The original estimate is a monthly aggregate of the weekly crude oil production estimates published in the Weekly Petroleum Status Report. This original monthly estimate is used in the Petroleum Supply Monthly (PSM) Tables S1 and S2 until replaced by the interim estimate.
- The interim estimate is used in the PSM Tables 1 through 25, and in Tables $S 1$ and $S 2$ until replaced by the final estimate.

- The initial estimate based upon first purchase data collected on the Form EIA-182 is used as an estimation tool in generating the interim estimate. The initial volume represents the best estimate available 40 days after the end of the production month and includes imputation for nonresponse and possible reporting errors. The revised volume is the best estimate available about 70 days after the production month and includes imputation as needed. A final revision is published concurrent with publication of Form EIA-182 price data in the Petroleum Marketing Annual.

- The final estimate is published in the PSA.

\section{Note 5. Export Data}

Each month the Energy Information Administration (EIA) receives magnetic tapes of aggregated export statistics from the U.S. Bureau of the Census (EM-522 and EM-594).

Census export statistics used in the Petroleum Supply Monthly reflect both government and nongovernment exports of domestic and foreign merchandise from the United States (the 50 States and the District of Columbia) to foreign countries and U.S. possessions, without regard to whether or not the exportation involves a commercial transaction. The following types of transactions are excluded from the statistics:

(1) Merchandise shipped in transit through the United States from one foreign country to another, when documented as such with U.S. Customs.

(2) Bunker fuels and other supplies and equipment for use on departing vessels, planes, or other carriers engaged in foreign trade.

\section{Source of Export Information}

The official U.S. export statistics are compiled by the U.S. Bureau of the Census. Exporters are required to file export documents with U.S. Customs officials (Customs Form 7525).

\section{Country and Area of Destination}

The country of destination is defined as the country of ultimate destination or the country where the goods are to be consumed, further processed, or manufactured, as known to the shipper at the time of exportation. If the shipper does not know the country of ultimate destination, the shipment is credited to the last country to which the shipper knows that the merchandise will be shipped in the same form as it was when exported. 
Table B1. U.S. Crude Oila Production Estimates and Reported States ${ }^{b}$ Data by Month

(Thousand Barrels per Day)

\begin{tabular}{|c|c|c|c|c|c|c|c|c|c|c|c|c|c|c|c|c|c|c|}
\hline \multirow{2}{*}{$\begin{array}{l}\text { Date of Data } \\
\text { Availability }\end{array}$} & \multicolumn{18}{|c|}{ Month of Production } \\
\hline & $11-93$ & $12-93$ & $1-94$ & $2-94$ & $3-94$ & $4-94$ & $5-94$ & $6-94$ & $7-94$ & $8-94$ & $9-94$ & $10-94$ & $11-94$ & $12-94$ & 1.95 & $2-95$ & 3-95 & $4-95$ \\
\hline & \multicolumn{18}{|c|}{ Reported State Data ${ }^{c}$} \\
\hline $1-14-94$ & 1685 & 0 & & & & & & & & & & & & & & & & \\
\hline $2-14-94$ & 3992 & 1697 & 0 & & & & & & & & & & & & & & & \\
\hline $3-14-94$ & 6276 & 3913 & 1668 & 0 & & & & & & & & & & & & & & \\
\hline $4-14-94$ & 6358 & 6316 & 3928 & 1611 & 0 & & & & & & & & & & & & & \\
\hline $5-14-94$ & 6423 & 6374 & 5224 & 3889 & 1582 & 0 & & & & & & & & & & & & \\
\hline $6-14-94$ & 6822 & 6772 & 6054 & 5170 & 3882 & 1507 & 0 & & & & & & & & & & & \\
\hline $7-14-94$ & 6822 & 6772 & 6058 & 6002 & 5957 & 3771 & 1593 & 0 & & & & & & & & & & \\
\hline $8-14-94$ & 6832 & 6785 & 6066 & 6010 & 5974 & 5845 & 3776 & 1574 & 0 & & & & & & & & & \\
\hline $9-14-94$ & 6831 & 6784 & 6066 & 6015 & 5977 & 5856 & 5926 & 3748 & 1515 & 0 & & & & & & & & \\
\hline $10-14-94$ & 6831 & 6784 & 6428 & 6023 & 5985 & 5867 & 5938 & 5020 & 3680 & 1502 & 0 & & & & & & & \\
\hline $11-14-94$ & 6828 & 6790 & 6433 & 6022 & 5986 & 5869 & 5949 & 5849 & 4950 & 3650 & 1541 & 0 & & & & & & \\
\hline $12-14-94$ & 6911 & 6790 & 6433 & 6022 & 5986 & 5868 & 5949 & 5858 & 4954 & 4954 & 3689 & 1614 & 0 & & & & & \\
\hline $1-14-95$ & 6911 & 6863 & 6422 & 6367 & 6323 & 6217 & 5949 & 5863 & 4963 & 4966 & 4996 & 3757 & 1530 & 0 & & & & \\
\hline $2-14-95$ & 6911 & 6863 & 6422 & 6367 & 6341 & 6217 & 5949 & 5865 & 4966 & 4969 & 5003 & 5068 & 3679 & 1645 & 0 & & & \\
\hline 3-14-95 & 6911 & 6863 & 6498 & 6367 & 6341 & 6217 & 5949 & 5865 & 4967 & 4968 & 5007 & 5075 & 5036 & 3839 & 1592 & 0 & & \\
\hline $4-14-95$ & 6911 & 6863 & 6544 & 6491 & 6377 & 6254 & 5985 & 5918 & 5819 & 5853 & 5919 & 5983 & 5941 & 6057 & 3626 & 1593 & 0 & \\
\hline \multirow[t]{2}{*}{ 5-14-95 } & 6911 & 6863 & 6598 & 6555 & 6522 & 6395 & 6044 & 5980 & 5877 & 5911 & 5976 & 6040 & 5999 & 6129 & 5872 & 3660 & 1540 & 0 \\
\hline & \multicolumn{18}{|c|}{ Producing States Without Reported Monthly Production ${ }^{\text {d }}$} \\
\hline \multirow[t]{3}{*}{$5-14-95$} & 0 & 0 & 3 & 3 & 3 & 3 & 5 & 5 & 5 & 5 & 5 & 5 & 5 & 5 & 10 & 19 & 27 & 33 \\
\hline & \multicolumn{18}{|c|}{ Month of Production } \\
\hline & $11-93$ & $12-93$ & $1-94$ & $2-94$ & $3-94$ & 4-94 & $5-94$ & 6-94 & $7-94$ & $8-94$ & $9-94$ & $10-94$ & $11-94$ & $12-94$ & $1-95$ & $2-95$ & 3-95 & 4-95 \\
\hline Type of & \multicolumn{18}{|c|}{ Production Estimates } \\
\hline Estimate & & & & & & & & & & & & & & & & & & \\
\hline Origina ${ }^{\theta} \ldots . . . . . .$. & 6886 & 6886 & 6870 & 6735 & 6682 & 6607 & 6685 & 6610 & 6576 & 6551 & 6580 & 6667 & 6593 & 6674 & 6616 & 6604 & 6531 & 6577 \\
\hline Interim ${ }^{\prime}$ & 6888 & 6838 & 6777 & 6745 & 6719 & 6634 & 6658 & 6567 & 6528 & 6547 & 6551 & 6578 & 6542 & 6686 & 6596 & 6703 & 6606 & \\
\hline \multicolumn{19}{|l|}{ Form EIA-182 } \\
\hline Initia! ......... & 6701 & 6534 & 6242 & 6400 & 6414 & 6228 & 6312 & 6262 & 6116 & 6261 & 6315 & 6360 & 6300 & 6467 & 6120 & 6480 & 6224 & \\
\hline Revised.... & 6730 & 6548 & 6376 & 6436 & 6384 & 6233 & 6320 & 6254 & 6225 & 6261 & 6298 & 6359 & 6290 & 6464 & 6313 & 6473 & & \\
\hline Final ${ }^{9} \ldots \ldots \ldots . . . .$. & 6912 & 6858 & & & & & & & & & & & & & & & & \\
\hline
\end{tabular}

a Includes lease condensate.

b Includes Federal offshore areas, Gulf of Mexico (PADD III) and Pacific (PADD V), as two separate reporting entities.

c Includes EIA prorated monthly production in 1993 (annual average of 67 thousand barrels per day) for four States (Michigan, New York, Ohio, and Pennsylvania) for which only annual State data are available. Includes ElA prorated monthly production in 1994 (annual average of 58 thousand barrels per day) for three states (Michigan, New York, and Ohio) for which only annual State data are available.

Michigan, New York, and Ohio are counted as having monthly reported data in 1993 after their annual reports were received. These data are first reported as of 5-14-94. Pennsylvania is counted as having monthly reported data in 1993 after its annual report was received. These data are first reported as of 8-14-94. Michigan, New York, and Ohio are counted as having monthly reported data in 1994 after their annual reports were received. These data are first reported as of 5-16-95.

Original estimates are weighted averages based on the weekly estimates published in the Weekly Petroleum Status Report.

- Interim estimates were made 44 days after the end of the production month.

9 Published in the Petroleum Supply Annual 1993, DOE/EIA 0340(93)/2. 
Note 6. Quality Control and Data Revision

\section{Quality Control}

The Energy Information Administration (EIA) monitors the supply and disposition of crude oil, petroleum products, and natural gas liquids in the United States. Through a tracking system, the EIA provides insight into the activities of primary operators and distributors in the petroleum industry. The tracking system, known as the Petroleum Supply Reporting System (PSRS), consists of production, inputs, imports, inventories, movements, and other petroleum-related data collected on weekly, month$l y$, and annual surveys.

Survey forms are periodically reviewed for completeness, meaningfulness, and clarity. Modifications are made, when needed, to maintain efficient measure of the intended data items and to track product movement accurately throughout the industry. Through this process, the EIA can maintain consistency among forms, minimize respondent burden, and eliminate ambiguity.

\section{Sampling and Nonsampling Errors}

There are two types of errors usually associated with data produced from a survey: nonsampling errors and sampling errors. Because the estimates for the monthly surveys 810 through 813,816 , and 817 are based on a complete census of the frame, there is no sampling error in the data presented. The data, however, are subject to nonsampling errors. Nonsampling errors, sometimes referred to as biases, are those which can arise from a number of sources: (1) the inability to obtain data from all companies in the frame or sample (nonresponse and the method used to account for nonresponses, (2) definitional difficulties and/or improperly worded questions which lead to different interpretations. (3) mistakes in recording or coding the data obtained from respondents, and (4) other errors of collection, response, coverage, and estimation.

Response rates on the monthly surveys are very high. In general, response rates average above 95 percent for the weekly survey and above 98 percent for monthly surveys. Whenever survey responses are not received in time to be included in published statistics, the data are imputed. Although imputing for missing data may not eliminate the total error associated with nonresponse, it can serve to reduce the error. The data reported in the previous month are used as imputed values for missing data for all surveys except the Forms EIA-814, "Monthly Imports Report," and EIA-817, "Monthly Tanker and Barge Movement Report." There is no imputation procedure for these surveys because these data series, by respondent, are highly variable.

Response error is the major factor affecting the accuracy of PSRS data. Response, or reporting error, is the dif- ference between the true value and the value reported on a survey form. Response error can occur for any number of reasons. For example, figures may be entered incorrectly when written on forms by the respondent, or errors may result from the misunderstanding of survey form instructions or definitions. Response error can also occur from the use of preliminary data when final data are not available. This can result in differences between published preliminary and final data. To help detect and minimize probable reporting errors, automated editing procedures are used to check current data for consistency with past data, as well as for internal consistency (e.g., totals equal to the sums of the parts), and to flag those data elements that fail edit criteria.

Errors can also be introduced during data processing. For example, while creating computer data files, key errors can occur in transcribing or coding the data; or information can be entered into the wrong cell. Using well designed edit criteria which examine orders of magnitude, cell position, and historical reporting patterns, many of these errors can be identified and corrected.

Monthly data are compared to weekly data on a regular basis. Discrepancies betweenly weekly and monthly data are documented and respondents are called when discrepancies are either large (usually over 300 thousand barrels) or consistent (e.g., weekly data are always lower than monthly data). In addition, a comparison of the data collected on the PSRS with other similar data series from sources outside of the Petroleum Supply Division is performed each year. The results of this data comparison are published once a year in the Petroleum Supply Monthly (PSM) feature article, "Comparison of Independent Statistics on Petroleum Supply."

Sampling errors are those errors that occur when survey estimates are based on a sample rather than being derived from a complete census of the frame. The $819 \mathrm{M}$ data, which are based on sample estimates, serve as leading indicators of the PSRS monthly data for oxygenates. To assess the accuracy of the $819 \mathrm{M}$ statistics, data are compared with the monthly aggregate data for the EIA-810, 811 , and 812 surveys. Although monthly data are still subject to error, they have been thoroughly reviewed and edited, and are considered to be the most accurate data available.

\section{Data Revision}

Resubmissions are any changes to the originally submitted data that were either requested by the EIA or initiated by the respondent. Resubmissions are compared with the original submission and processed at the time of receipt. For Forms EIA-810 through 813, 816, and 817 the Resubmission Tracking System (RTS) is run after resubmissions have been processed for the month. The RTS enables the user to study major products and data series to see how company resubmissions impact published data on a month by month basis. During the processing year, a 
summary of the effect of these resubmissions to major series is provided in Appendix C.

For the EIA-819M data, a determination is made on whether to process the resubmissions based on the magnitude of the revision. Cell entries on publication tables are marked with an " $R$ " for revised.

\section{Late Response}

Respondents who fail to respond within the prescribed time limit (25th day following the end of the report month) become nonrespondents for that particular report period and are contacted by phone to obtain the current month's data. Respondents who are chronically late (i.e., 3 consecutive months) are notified by EIA either by letter or telephone.

\section{Nonresponse}

Follow-up action is taken when a company fails to respond adequately to data requests from the EIA. Preliminary attempts to gather delinquent reports are made by phone. Noncompliance form letters are sent to those companies that have not submitted reports and have not responded to data requests by phone.

\section{Note 7. Frames Maintenance}

The Petroleum Supply Division (PSD) maintains complete lists of respondents to its monthly surveys. Each survey has a list of companies and facilities required to submit petroleum activity data. This list is known as the survey frame. Frame maintenance procedures are used to monitor the status of petroleum companies and facilities currently contained in each survey frame as well as to identify new members to be added to the frame. As a result, all known petroleum supply organizations falling within the definition of "Who Must Submit" participate in the survey.

The activities for frames maintenance are conducted on a monthly and annual basis. Monthly frames maintenance procedures focus on examining several frequently published industry periodicals that report changes in status (births, deaths, sales, and acquisitions) of petroleum facilities producing, transporting, importing, and/or storing crude oil and petroleum products. These sources are augmented by articles in newspapers, letters from respondents indicating changes in status, and information received from survey systems operated by other offices. Survey managers review these sources regularly to monitor changes in company operations and to develop lists of potential respondents. These activities assure coverage of the reporting universe and maintain accurate facility information on addresses and ownership.

Annual frames maintenance focuses on re-evaluating the "must submit" companies filing the Form EIA-814 and reviewing the sample frame for the Form EIA-819M, "Monthly Oxygenate Telephone Report."

To supplement monthly and annual frames maintenance activities and to provide more thorough coverage, the PSD periodically conducts a comprehensive frames investigation. These investigations result in the reassessment and recompilation of the complete frame for each survey. The effort also includes the evaluation of the impact of potential frame changes on the historical time series data published from these respondents. The results of this frame study are usually implemented in January to provide a full year under the same frame.

\section{Note 8. Practical Limitations of Data Collection Efforts}

\section{Crude Oil Lease Stock Adjustment}

End-of-month crude oil stocks held on leases are reported on the EIA-813, "Monthly Crude Oil Report." However, only those companies that store 1,000 barrels or more of crude oil are required to submit a report. Previous frames analysis has shown that crude oil stocks held on leases reported to the EIA are consistently lower than the lease stocks reported to individual states.

Up until 1983, monthly state government data on lease stocks were substituted for EIA data wherever possible in order to rectify the understatement of lease crude oil stocks. State data were available from three states -Texas, New Mexico, and Montana. To calculate the "lease adjustment," a comparison between EIA reported data and the state government data was made and the difference added to the ElA data for the respective states.

In 1983, the EIA modified the Form EIA-813 to eliminate state data on crude oil stocks and began collecting crude oil stock data by Petroleum Administration for Defense (PAD) District. With this change, the "lease adjustment" could no longer be calculated on a state basis and was changed to a PAD District level.

\section{Trans Alaskan Pipeline System Adjustment}

Beginning with the January 1989 data, adjustments are made to refinery inputs and product supplied of natural gas liquids (NGLs) and refinery inputs of crude oil to account for refiner misreporting. Substantial volumes of NGLs are produced at natural gas processing plants in Alaska and injected into the crude oil moving in the Trans Alaska Pipeline System (TAPS). Refiners receiving any crude oil commingled with NGLs are instructed to report the NGL portion of that stream separately from the crude oil portion. This has not been done for Alaskan crude oil because refiners are unable to identify these volumes for accounting purposes. As a result, the NGL production in Alaska has been credited directly toward product supplied and also toward product supplied from refinery production when the refiner processes the crude oil-NGL mix- 
ture. In addition, the reporting of the commingled stream as crude oil by the refiner has overstated crude oil inputs and resulted in an increase in unaccounted for crude oil equal to the volume of NGL in the crude oil.

To offset this reporting error, an adjustment is made to refinery input in all PAD Districts receiving Alaskan crude oil. The adjustment reduces the crude oil inputs and increases the NGL inputs by an equal amount. Each PAD District adjustment is a portion of the known AlaskanNGL production that is proportional to the PAD District's share of Alaskan crude oil received at all refineries in the United States. The greatest impact occurs in PAD District $V$ for butane and pentanes plus.

The reporting problem which began in 1987 grew as injections on NGLs into the TAPS increased. Data for 1988 was revised in the Petroleum Supply Annual to account for the adjustment.

\section{Finished Motor Gasoline Product Supplied Adjustment}

Beginning with the reporting of January 1993 data, adjustments were made to the product supplied series for finished motor gasoline. It was recognized that motor gasoline statistics published by the EIA through 1992 were underreported because the reporting system was not collecting all fuel ethanol and motor gasoline blending components being blended downstream from the refinery. The EIA was able to quantify these volumes and make corrective adjustments for 1992 in 1993 (refer to Table B2).

\section{Fuel Ethanol Adjustment}

Prior to 1993, an estimated 60 to 70 thousand barrels per day of fuel ethanol were added to motor gasoline to produce gasohol but were not included in the EIA finished motor gasoline production data. In 1992, the EIA attempted to collect these data from downstream fuel ethanol motor gasoline blenders but found that this effort was impractical and the results were inaccurate.

Beginning in January 1993, an estimate for the missing fuel ethanol blended into motor gasoline was calculated. This estimate was calculated as production (from the EIA819M, "Monthly Oxygenate Telephone Report"), plus imports (from the EIA-814, "Monthly Imports Report"), minus inputs at refineries (from the EIA-810, "Monthly Refinery Report"), plus or minus stock change (from the EIA-819M survey). This estimate for the amount of fuel ethanol blended into motor gasoline was added to Table 1 for Natural Gas Liquids Field Production (line 14) and in the Field Production column for finished motor gasoline in Tables 2 through 25 published in the PSM.

An estimate for the total amount of gasohol produced with the ethanol is given as 10 times the estimated fuel ethanol blended (this assumes a 10 percent ethanol blend). This amount is added to the column labeled field production of "oxygenated gasoline" and subtracted from the field production of "other" finished gasoline. The PAD District level detail was obtained by allocating the national level estimates according to the percent of gasohol sales from the U.S. Department of Transportation, Federal Highway Administration, Monthly Motor Fuel Reported by States, 1991.

\section{Motor Gasoline Blending Component Adjustment}

Prior to 1993, the EIA published a "product supplied" for motor gasoline blending components. Since these components are to be blended into finished motor gasoline, there is no actual demand for this intermediate product. The EIA corrected this series by including the quantity of "product supplied" for motor gasoline blending components with "other" finished motor gasoline. This change was accomplished in Tables 2 through 25 by adding product supplied for motor gasoline blending components to the column labeled field production of "other" motor gasoline, and subtracting it from the field production column for "motor gasoline blending components."

\section{Fuel Ethanol Stock Adjustment}

Total end-of-month stocks of fuel ethanol are underreported in the PSRS because of the inability to collect data from downstream fuel ethanol motor gasoline blenders. Total stocks of fuel ethanol are assumed to be those reported by ethanol producers on the Form EIA819M, "Monthly Oxygenate Telephone Report." The difference between the stocks reported on the EIA-819M and the stocks reported in the PSRS (from refiners, bulk terminal and pipeline operators) is added to the stocks shown for bulk terminals. If the stocks for the PSRS are higher than those reported on the EIA-819M, no adjustment is made.

\section{Note 9. 1994 Changes in the Petroleum Supply Monthly}

Effective with January 1994 data, several enhancements were made to the tables in the Petroleum Supply Monthly to reflect changes in the petroleum industry and to provide more meaningful petroleum statistics. These changes primarily affect data reported for imports, exports, and product supplied.

- On December 31, 1992, Ecuador withdrew as a member of the Organization of Petroleum Exporting Countries (OPEC). As of January 1994, imports of petroleum from Ecuador now appear under imports from NonOPEC sources. No revision was made to 1993 data. Countries have been realphabetized accordingly. This change is evident in Tables S3 and 35 through 44, 49 and 50.

- Exports data are now published for oxygenates and the sub-categories of finished motor gasoline (reformu- 
Table B2. Finished Motor Gasoline Product Supplied Adjustment, 1993 - Present (Thousand Barrels per Day)

\begin{tabular}{|c|c|c|c|c|c|c|c|c|c|c|c|c|c|}
\hline Item/Year & Jan & Feb & Mar & Apr & May & Jun & Juil & Aug & Sep & Oct & Nov & Dec & Avg \\
\hline \multicolumn{14}{|l|}{1993} \\
\hline Fuel Ethanol Adj .......... & 61 & 67 & 70 & 61 & 58 & 63 & 62 & 48 & 68 & 69 & 84 & 81 & 66 \\
\hline Motor Gas Blending .... & -59 & -61 & 15 & -32 & -3 & -5 & -19 & 54 & 79 & -72 & -72 & 48 & -10 \\
\hline Product Supplied ......... & 6,639 & 7,112 & 7,389 & 7,435 & 7,585 & 7,700 & 7,785 & 7,864 & 7,607 & 7,382 & 7,533 & 7,661 & 7,476 \\
\hline \multicolumn{14}{|l|}{1994} \\
\hline Fuel Ethanol Adj .......... & 86 & 73 & 76 & 48 & 22 & 63 & 65 & 73 & 59 & 90 & 82 & 82 & \\
\hline Motor Gas Blending .... & 35 & -11 & 17 & 58 & 33 & 76 & 90 & 97 & 82 & -18 & 55 & 104 & 52 \\
\hline Product Supplied .......... & 6,916 & 7,272 & 7,379 & 7,530 & 7,592 & 7,926 & 7,846 & 8,007 & 7,619 & 7,547 & 7,479 & 7,902 & 7,587 \\
\hline \multicolumn{14}{|l|}{1995} \\
\hline Fuel Ethanol Adj ......... & 69 & 69 & 81 & & & & & & & & & & \\
\hline Motor Gas Blending .... & 71 & 74 & 87 & & & & & & & & & & \\
\hline Product Supplied ........ & 7,157 & 7,505 & 7,780 & & & & & & & & & & \\
\hline
\end{tabular}

Note: Totals may not equal sum of components due to independent rounding.

Source: - Fuel Ethanol Adjustment - 1993, EIA, Petroleum Supply Annual, Volumes I and II: 1994 and 1995, Energy Information Administration (EIA), Petroleum Supply Monthly, Appendix D. - Motor Gasoline Blending Component Adjustment - 1993, ElA, Petroleum Supply Annual, Volumes I and II; 1994 and 1995, EIA, Petroleum Supply Monthly.

lated, oxygenated, and other) and distillate fuel oil $(0.05 \%$ sulfur and under, and greater than $0.05 \%$ sulfur).

- Product supplied is now calculated for reformulated, oxygenated, and other finished motor gasoline as well as the sulfur categories of distillate fuel oil $(0.05 \%$ sulfur and under, and greater than $0.05 \%$ sulfur). 
This section contains information on revisions to published statistics caused by resubmission of respondent survey forms. The section shows the published value in the Petroleum Supply Monthly (PSM) and the cumulative difference resulting from resubmissions for the major product series. The official published petroleum supply statistics are not changed to reflect revisions until publication of the Petroleum Supply Annual (PSA), except in cases of catastrophic error.

This section is provided as a service to analysts who need to know the latest available statistics. It should be used with caution because resubmissions are received on an irregular basis and the impact on published data can change from month to month. In some cases, the pattern of revision caused by resubmissions during the year is a poor indicator of final statistics that will be published in the PSA.

\section{Appendix C}

\author{
Impact of \\ Resubmissions \\ on Major \\ Series, 1994
}

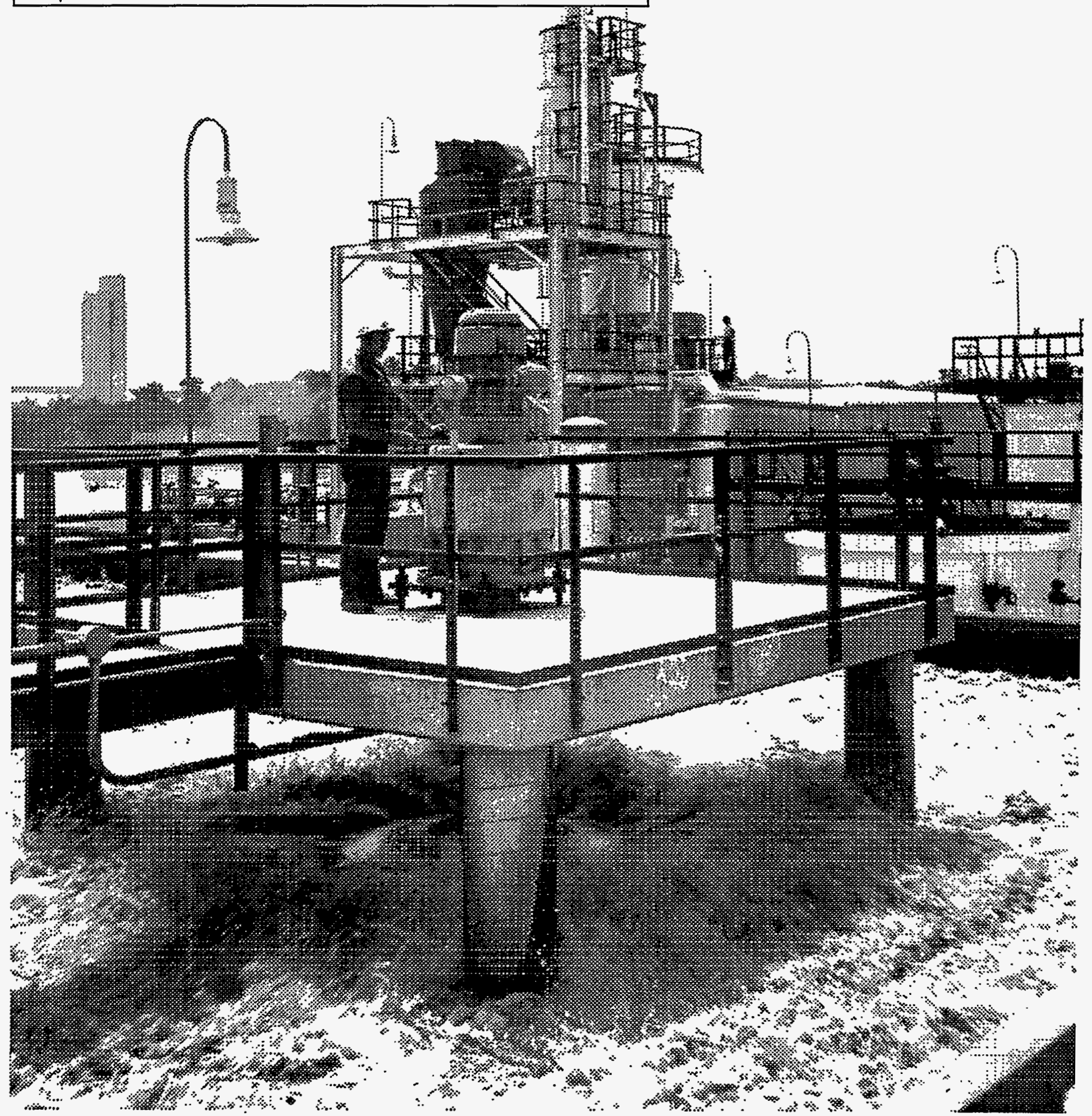

Surface aerators are used at U.S. petroleum refineries to help prevent water pollution. These aerators speed up the oxidation process by beating air into water. 
Table C1. Impact of Resubmissions on Major Series, 1995

(Thousand Barrels per Day, Except Where Noted)

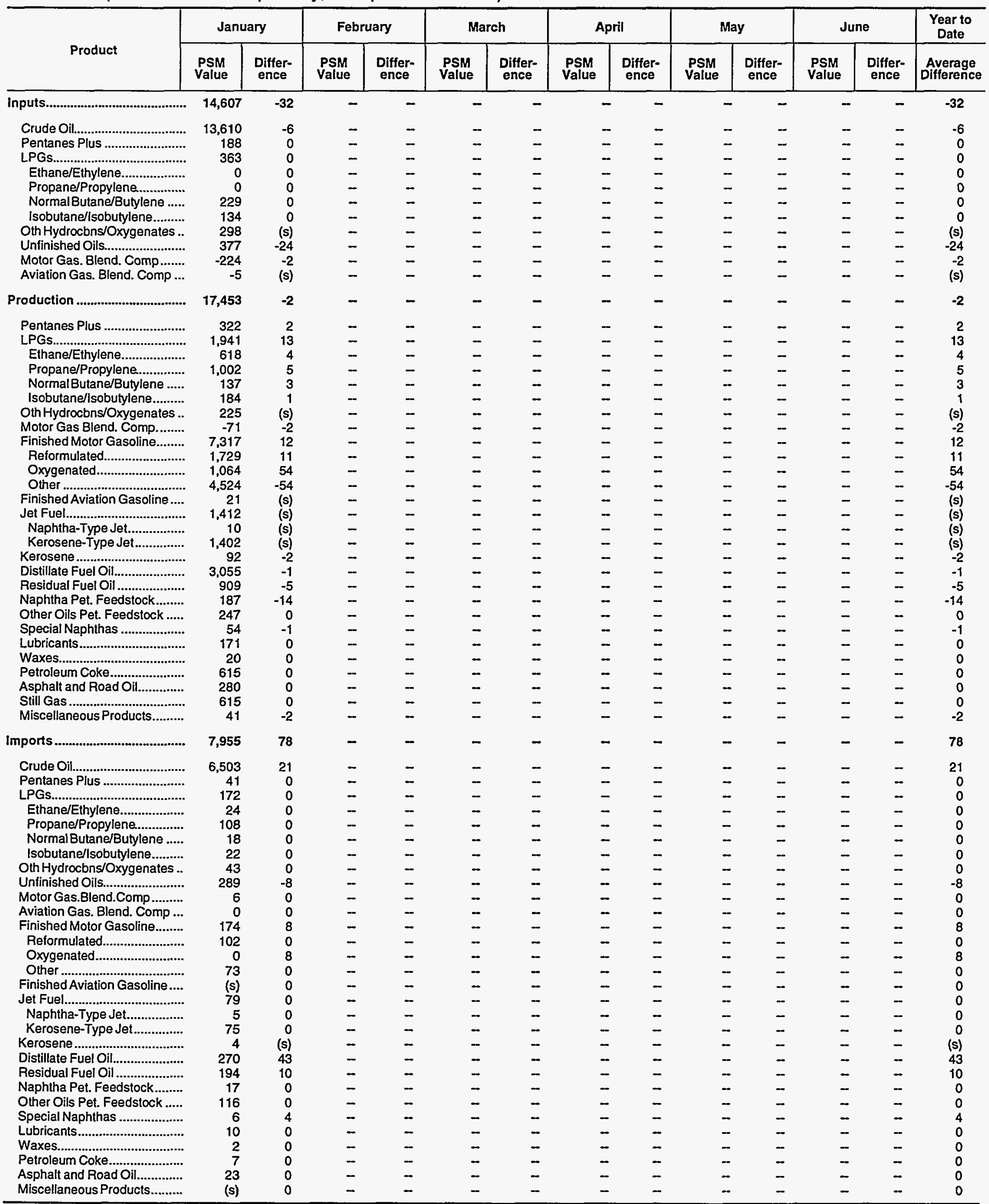

(s) = Less than 500 barrels per day.

Note: - Volumes indicate cumulative changes resulting from resubmissions received for that month as of the date of this publication. $\bullet$ Totals may not equal sum of components due to independent rounding. 
Table C1. Impact of Resubmissions on Major Series, 1995

(Thousand Barrels per Day, Except Where Noted)

\begin{tabular}{|c|c|c|c|c|c|c|c|c|c|c|c|c|c|}
\hline \multirow{2}{*}{ Product } & \multicolumn{2}{|c|}{ January } & \multicolumn{2}{|c|}{ February } & \multicolumn{2}{|c|}{ March } & \multicolumn{2}{|c|}{ April } & \multicolumn{2}{|c|}{ May } & \multicolumn{2}{|c|}{ June } & $\begin{array}{l}\text { Year to } \\
\text { Date }\end{array}$ \\
\hline & $\begin{array}{c}\text { PSM } \\
\text { Value }\end{array}$ & $\begin{array}{l}\text { Differ- } \\
\text { ence }\end{array}$ & $\begin{array}{l}\text { PSM } \\
\text { Value }\end{array}$ & $\begin{array}{c}\text { Differ- } \\
\text { ence }\end{array}$ & $\begin{array}{c}\text { PSM } \\
\text { Value }\end{array}$ & $\begin{array}{l}\text { Differ- } \\
\text { ence }\end{array}$ & $\begin{array}{c}\text { PSM } \\
\text { Value }\end{array}$ & $\begin{array}{l}\text { Differ- } \\
\text { ence }\end{array}$ & $\begin{array}{c}\text { PSM } \\
\text { Value }\end{array}$ & $\begin{array}{l}\text { Differ- } \\
\text { ence }\end{array}$ & $\begin{array}{c}\text { PSM } \\
\text { Value }\end{array}$ & $\begin{array}{l}\text { Differ- } \\
\text { ence }\end{array}$ & $\begin{array}{c}\text { Average } \\
\text { Difference }\end{array}$ \\
\hline
\end{tabular}

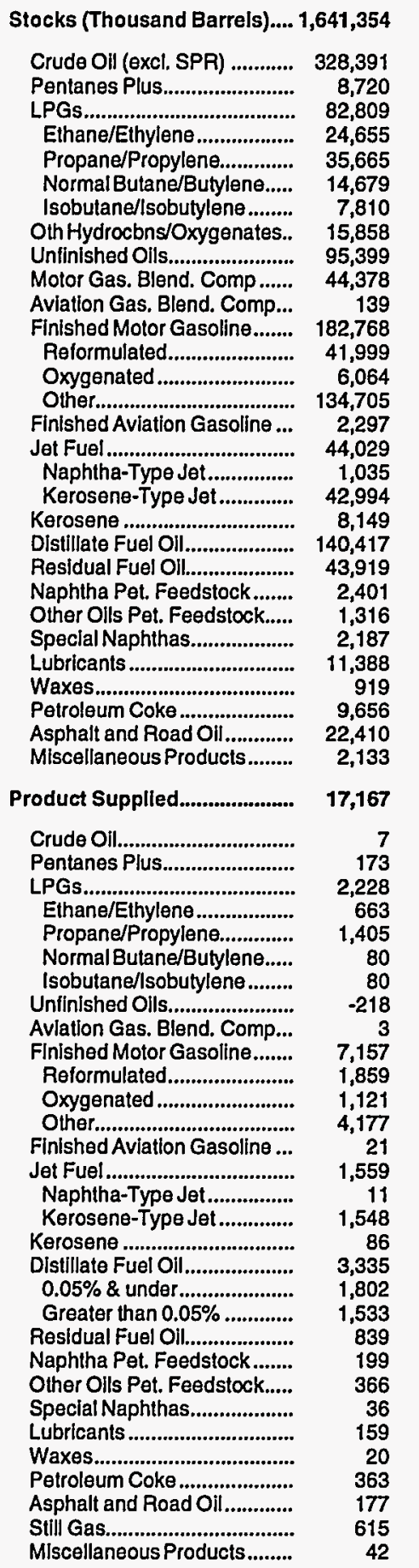

\begin{tabular}{|c|c|c|c|c|c|c|}
\hline 621 & - & - & - & - & - & - \\
\hline 302 & - & - & - & - & - & - \\
\hline 0 & - & - & - & - & - & - \\
\hline 378 & - & - & - & - & - & - \\
\hline 49 & - & - & - & - & - & - \\
\hline 59 & - & - & - & - & - & - \\
\hline 110 & - & - & - & - & - & - \\
\hline 160 & - & - & - & - & - & - \\
\hline-5 & - & - & - & - & - & - \\
\hline 12 & - & - & - & - & - & - \\
\hline 1 & - & - & - & - & - & - \\
\hline 36 & - & - & - & - & - & - \\
\hline 284 & - & - & - & - & - & - \\
\hline 11 & - & - & - & - & - & - \\
\hline-2 & - & - & - & - & - & - \\
\hline $27 \overline{5}$ & - & - & - & - & - & - \\
\hline-36 & - & - & - & - & - & - \\
\hline 8 & - & - & - & - & - & - \\
\hline-49 & - & - & - & - & - & - \\
\hline 57 & - & - & - & - & - & - \\
\hline 63 & - & - & - & - & - & - \\
\hline-170 & - & - & - & - & - & - \\
\hline-316 & - & - & - & - & - & - \\
\hline-1 & - & - & - & - & - & - \\
\hline 0 & - & - & - & - & - & - \\
\hline-63 & - & - & - & - & - & - \\
\hline 0 & - & - & - & - & - & - \\
\hline 0 & - & - & - & - & - & - \\
\hline 0 & - & - & - & - & - & - \\
\hline 238 & - & - & - & - & - & - \\
\hline-110 & $\rightarrow$ & - & - & - & - & - \\
\hline 71 & - & - & - & - & - & - \\
\hline 0 & - & - & - & - & - & - \\
\hline 2 & - & - & - & - & - & - \\
\hline (s) & - & - & - & - & - & - \\
\hline 2 & - & - & - & - & - & - \\
\hline 3 & - & - & - & - & - & - \\
\hline (s) & - & - & - & - & - & - \\
\hline-5 & - & - & - & - & - & - \\
\hline 16 & - & - & - & - & - & - \\
\hline-1 & - & - & - & - & - & - \\
\hline 11 & - & - & - & - & - & - \\
\hline 11 & - & - & - & - & - & - \\
\hline 62 & - & - & - & - & - & - \\
\hline-62 & - & - & - & - & - & - \\
\hline 1 & - & - & - & - & - & - \\
\hline-1 & - & - & - & - & - & - \\
\hline 2 & - & - & - & - & - & - \\
\hline-2 & - & - & - & - & - & - \\
\hline-5 & - & - & - & - & - & - \\
\hline 48 & - & - & - & - & - & - \\
\hline 32 & - & - & - & - & - & - \\
\hline 16 & - & - & - & - & - & - \\
\hline 15 & - & - & - & - & - & - \\
\hline-14 & - & - & - & - & - & - \\
\hline 0 & - & - & - & - & - & - \\
\hline 4 & - & - & - & - & - & - \\
\hline 0 & - & - & - & - & - & - \\
\hline 0 & - & - & - & - & - & - \\
\hline 0 & - & - & - & - & - & - \\
\hline-8 & - & - & - & - & - & - \\
\hline 0 & - & - & - & - & - & - \\
\hline 2 & - & - & - & - & - & - \\
\hline
\end{tabular}





\section{Appendix D}

\section{EIA 819M Monthly Oxygenate Telephone Report}

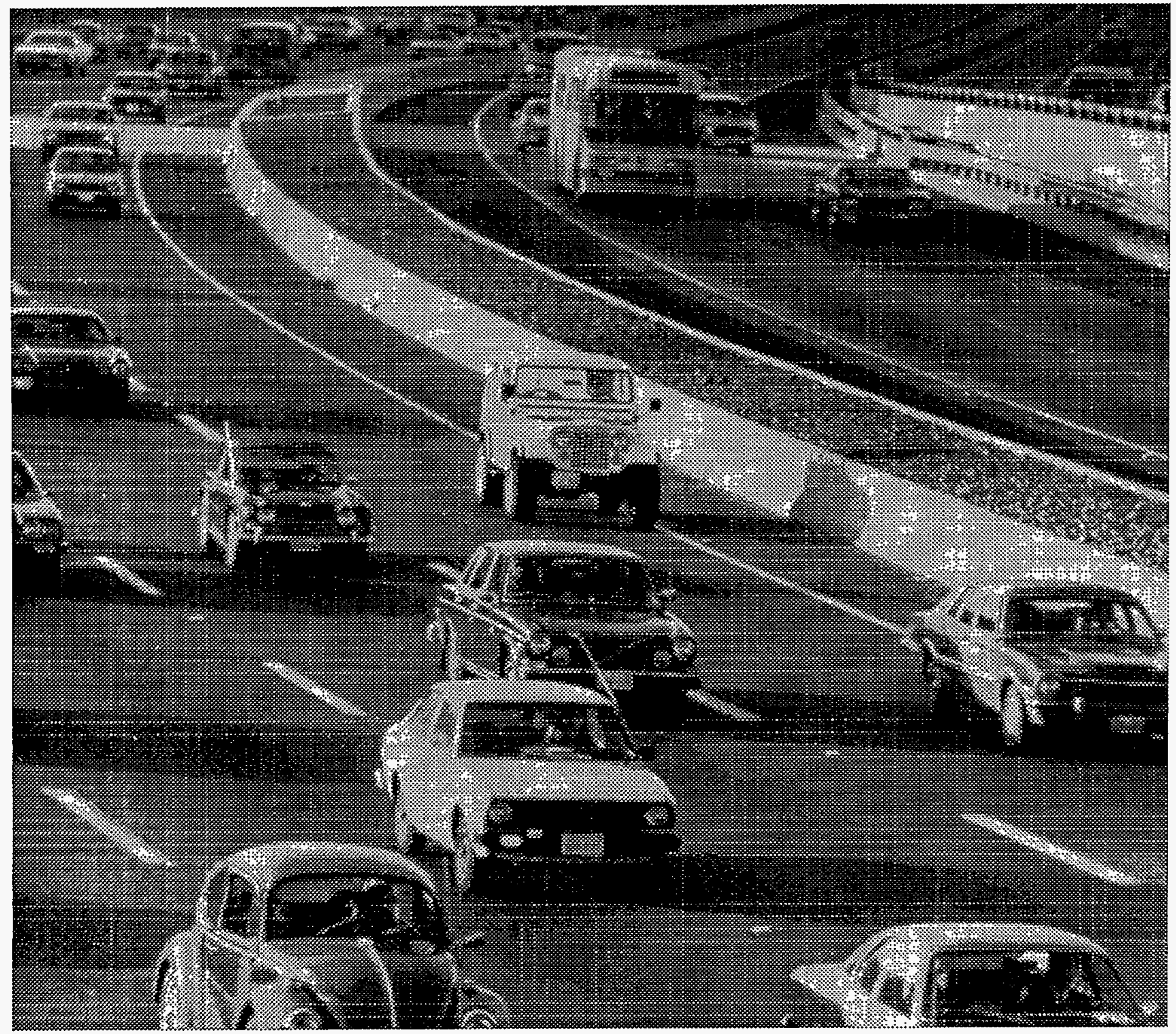

The Clean Air Act Amendments of 1990 include provisions intended to reduce toxic vehicle emissions. 



\section{EIA-819M Monthly Oxygenate Telephone Report}

The EIA-819M, "Monthly Oxygenate Telephone Report," provides production data and preliminary stock data for fuel ethanol and methyl tertiary butyl ether (MTBE) in the United States and major U.S. geographic regions. Data are collected from a sample of respondents reporting on the Monthly Petroleum Supply Reporting System surveys and from the universe of oxygenate producers. Refer to Appendix B, Explanatory Note 2 for further detail. Final data on stocks of fuel ethanol and MTBE are presented in the Detailed Statistics section. The quantity of oxygenates blended into motor gasoline previously published in this appendix is now presented in Appendix B, Table B2.

Table D1. U.S. Summary, April 1995

\begin{tabular}{|c|c|c|c|c|c|c|}
\hline \multirow[b]{2}{*}{ Products } & \multicolumn{2}{|c|}{ April 1995} & \multicolumn{2}{|c|}{ March 1995} & \multicolumn{2}{|c|}{ Year-to-Date } \\
\hline & $\begin{array}{l}\text { Thousand } \\
\text { Barrels }\end{array}$ & \begin{tabular}{|c|} 
Thousand \\
Barrels per Day
\end{tabular} & $\begin{array}{c}\text { Thousand } \\
\text { Barrels }\end{array}$ & \begin{tabular}{|c|} 
Thousand \\
Barrels per Day
\end{tabular} & $\begin{array}{c}\text { Thousand } \\
\text { Barrels }\end{array}$ & $\begin{array}{c}\text { Thousand } \\
\text { Barrels per Day }\end{array}$ \\
\hline Fuel Ethanol & & & & & & \\
\hline $\begin{array}{l}\text { Production } \\
\text { Stocks }\end{array}$ & $\begin{array}{l}2,883 \\
3,073\end{array}$ & $\begin{array}{c}96 \\
--\end{array}$ & $\begin{array}{l}2,930 \\
2,959\end{array}$ & $\begin{array}{l}95 \\
-\end{array}$ & $\begin{array}{r}11,657 \\
3,073\end{array}$ & $\begin{array}{r}97 \\
--\end{array}$ \\
\hline $\begin{array}{l}\text { MTBE } \\
\text { Production } \\
\text { Stocks }\end{array}$ & $\begin{array}{r}5,052 \\
10,264\end{array}$ & $\begin{array}{r}168 \\
--\end{array}$ & $\begin{array}{r}3,759 \\
10,586\end{array}$ & $\begin{array}{r}121 \\
--\end{array}$ & $\begin{array}{l}17,188 \\
10,264\end{array}$ & $\begin{array}{r}143 \\
\ldots\end{array}$ \\
\hline
\end{tabular}

Source: Energy Information Administration (EIA) Form EIA-819M, "Monthly Oxygenate Telephone Report." 
Table D2. Monthly Fuel Ethanol Production and Stocks by Petroleum Administration for Defense Districts (PADD)

(Thousand Barrels per Day, Except Where Noted)

\begin{tabular}{|c|c|c|c|c|c|c|c|c|c|c|c|c|}
\hline District/Year & Jan & Feb & Mar & Apr & May & Jun & Jul & Aug & Sep & Oct & Nov & Dec \\
\hline \multicolumn{13}{|l|}{ Total U.S. } \\
\hline \multicolumn{13}{|l|}{ Production } \\
\hline 1994 & 82 & 82 & 80 & 73 & 77 & 79 & 75 & 79 & 89 & 91 & 98 & 97 \\
\hline 1995 & 98 & 100 & 95 & 96 & & & & & & & & \\
\hline \multicolumn{13}{|c|}{ Stocks (thous. bbls.) } \\
\hline 1994 & 1,740 & 1,689 & 1,650 & 1,470 & 1,437 & 1,668 & 1,733 & 1,706 & 2,336 & 2,156 & 2,302 & 2,289 \\
\hline 1995 & 2,674 & 3,006 & 2,959 & 3,073 & & & & & & & & \\
\hline
\end{tabular}

\begin{tabular}{|c|c|c|c|c|c|c|c|c|c|c|c|c|}
\hline \multicolumn{13}{|c|}{ East Coast (PADD I) } \\
\hline \multicolumn{13}{|c|}{ Production } \\
\hline 1994 & $w$ & $w$ & $w$ & $W$ & $W$ & W & w & $w$ & $w$ & $w$ & $W$ & $w$ \\
\hline 1995 & W & $w$ & W & W & & & & & & & & \\
\hline \multicolumn{13}{|c|}{ Stocks (thous. bbls.) } \\
\hline 1994 & 54 & 60 & 68 & 39 & 26 & 37 & 32 & 20 & 68 & 184 & 262 & 235 \\
\hline 1995 & 65 & 390 & 51 & 87 & & & & & & & & \\
\hline
\end{tabular}

\begin{tabular}{|c|c|c|c|c|c|c|c|c|c|c|c|c|}
\hline \multicolumn{13}{|c|}{ Midwest (PADD II) } \\
\hline \multicolumn{13}{|c|}{ Production } \\
\hline 1994 & 80 & 80 & 78 & 71 & 75 & 78 & 73 & 78 & 87 & 90 & 96 & 96 \\
\hline 1995 & 96 & 98 & 93 & 94 & & & & & & & & \\
\hline \multicolumn{13}{|c|}{ Stocks (thous. bbls.) } \\
\hline 1994 & 750 & 786 & 833 & 834 & 836 & 1,000 & 979 & 940 & 1,129 & 1,152 & 1,239 & 1,188 \\
\hline 1995 & 1,460 & 1,760 & 1,880 & 2,041 & & & & & & & & \\
\hline
\end{tabular}

\begin{tabular}{|c|c|c|c|c|c|c|c|c|c|c|c|c|}
\hline \multicolumn{13}{|c|}{ Gulf Coast (PADD III) } \\
\hline \multicolumn{13}{|c|}{ Production } \\
\hline 1994 & $w$ & W & $w$ & w & W & W & $w$ & W & W & W & w & W \\
\hline 1995 & $w$ & w & $w$ & $w$ & & & & & & & & \\
\hline \multicolumn{13}{|c|}{ Stocks (thous. bbls.) } \\
\hline 1994 & 431 & 452 & 488 & 397 & 393 & 416 & 396 & 202 & 336 & 171 & 252 & 313 \\
\hline 1995 & 587 & 474 & 702 & 516 & & & & & & & & \\
\hline
\end{tabular}

\begin{tabular}{|c|c|c|c|c|c|c|c|c|c|c|c|c|}
\hline \multicolumn{13}{|c|}{ Rocky Mountain (PADD IV) } \\
\hline \multicolumn{13}{|c|}{ Production } \\
\hline 1994 & $w$ & $w$ & $W$ & $W$ & $w$ & $w$ & $W$ & $W$ & $w$ & $w$ & $w$ & $w$ \\
\hline 1995 & W & $W$ & $W$ & $W$ & & & & & & & & \\
\hline \multicolumn{13}{|c|}{ Stocks (thous. bbls.) } \\
\hline 1994 & 99 & 80 & 68 & 70 & 61 & 67 & 77 & 98 & 114 & 101 & 69 & 94 \\
\hline 1995 & 123 & 75 & 72 & 81 & & & & & & & & \\
\hline
\end{tabular}

\begin{tabular}{|c|c|c|c|c|c|c|c|c|c|c|c|c|}
\hline \multicolumn{13}{|c|}{ West Coast (PADD V) } \\
\hline \multicolumn{13}{|c|}{ Production } \\
\hline 1994 & W & W & $w$ & $W$ & $W$ & $w$ & $W$ & w & $W$ & $w$ & W & $w$ \\
\hline 1995 & W & W & W & W & & & & & & & & \\
\hline \multicolumn{13}{|c|}{ Stocks (thous. bbls.) } \\
\hline 1994 & 406 & 311 & 193 & 130 & 121 & 148 & 249 & 446 & 689 & 548 & 480 & 459 \\
\hline 1995 & 439 & 307 & 254 & 348 & & & & & & & & \\
\hline
\end{tabular}

$W=$ Withheld to avoid disclosure of individual company data.

Note: - Geographic coverage is the 50 States and the District of Columbia. • Totals may not equal sum of components due to independent rounding.

Source: Energy Information Administration (EIA) Form EIA-819M, "Monthly Oxygenate Telephone Report. * 
Table D3. Monthly Methyl Tertiary Butyl Ether (MTBE) Production and Stocks by Petroleum Administration for Defense Districts (PADD)

(Thousand Barrels per Day, Except Where Noted)

\begin{tabular}{|c|c|c|c|c|c|c|c|c|c|c|c|c|}
\hline District/Month & Jan & Feb & Mar & Apr & May & Jun & Jul & Aug & Sep & Oct & Nov & Dec \\
\hline \multicolumn{13}{|l|}{$\begin{array}{l}\text { Total U.S. } \\
\text { Production }\end{array}$} \\
\hline 1994 & 123 & 140 & 129 & 140 & 139 & 115 & 154 & 166 & 160 & 164 & 150 & 144 \\
\hline 1995 & 149 & 135 & 121 & 168 & & & & & & & & \\
\hline \multicolumn{13}{|c|}{ Stocks (thous. bbls.) } \\
\hline 1994 & 8,226 & 8,444 & 10,619 & 11,547 & 12,008 & 14,004 & 17,414 & 19,786 & 19,703 & 19,400 & 15,998 & 12,176 \\
\hline 1995 & 11,406 & 11,047 & 10,586 & 10,264 & & & & & & & & \\
\hline \multicolumn{13}{|c|}{$\overline{\text { East Coast (PADD I) }}$} \\
\hline \multicolumn{13}{|l|}{ Production } \\
\hline 1994 & W & $w$ & $W$ & $w$ & $W$ & W & W & w & W & $w$ & W & W \\
\hline 1995 & $w$ & $w$ & $W$ & $w$ & & & & & & & & \\
\hline \multicolumn{13}{|c|}{ Stocks (thous. bbls.) } \\
\hline 1994 & 836 & 1,048 & 1,356 & 1,450 & 1,408 & 1,707 & 2,490 & 2,720 & 3,168 & 4,403 & 3,460 & 2,973 \\
\hline 1995 & 2,617 & 2,132 & 1,951 & 1,335 & & & & & & & & \\
\hline \multicolumn{13}{|c|}{ Midwest (PADD II) } \\
\hline \multicolumn{13}{|l|}{ Production } \\
\hline 1994 & $w$ & W & $W$ & $W$ & W & W & $w$ & W & W & W & W & W \\
\hline 1995 & $w$ & $w$ & $w$ & $w$ & & & & & & & & \\
\hline \multicolumn{13}{|c|}{ Stocks (thous. bbls.) } \\
\hline 1994 & $w$ & $w$ & w & $w$ & $w$ & W & $w$ & $w$ & W & W & W & w \\
\hline 1995 & $w$ & W & W & W & & & & & & & & \\
\hline \multicolumn{13}{|c|}{ Gulf Coast (PADD III) } \\
\hline \multicolumn{13}{|l|}{ Production } \\
\hline 1994 & 108 & 122 & 115 & 124 & 124 & 97 & 135 & 147 & 141 & 145 & 135 & 125 \\
\hline 1995 & 132 & 118 & 103 & 148 & & & & & & & & \\
\hline \multicolumn{13}{|c|}{ Stocks (thous. bbls.) } \\
\hline 1994 & 4,057 & 4,756 & 5,375 & 5,768 & 6,011 & 6,823 & 9,247 & 10,130 & 10,018 & 9,052 & 7,149 & 4,605 \\
\hline 1995 & 4,716 & 4,375 & 3,933 & 3,599 & & & & & & & & \\
\hline \multicolumn{13}{|c|}{ Rocky Mountain (PADD IV) } \\
\hline \multicolumn{13}{|l|}{ Production } \\
\hline 1994 & $W$ & W & $w$ & $W$ & $w$ & W & W & W & $w$ & W & W & W \\
\hline 1995 & $w$ & W & $w$ & $W$ & & & & & & & & \\
\hline \multicolumn{13}{|c|}{ Stocks (thous. bbls.) } \\
\hline 1994 & $W$ & W & W & $w$ & $w$ & W & $w$ & W & $w$ & $w$ & W & W \\
\hline 1995 & W & W & $w$ & $w$ & & & & & & & & \\
\hline \multicolumn{13}{|c|}{$\overline{\text { West Coast (PADD V) }}$} \\
\hline \multicolumn{13}{|l|}{ Production } \\
\hline 1994 & W & $w$ & $w$ & $w$ & W & W & $W$ & W & w & $w$ & $w$ & W \\
\hline 1995 & W & $w$ & W & $W$ & & & & & & & & \\
\hline \multicolumn{13}{|c|}{ Stocks (thous. bbls.) } \\
\hline 1994 & 2,909 & 2,186 & 3,405 & 3,922 & 4,275 & 5,176 & 5,306 & 6,402 & 5,983 & 5,262 & 4,871 & 4,170 \\
\hline 1995 & 3,614 & 3,950 & 4,055 & 4,810 & & & & & & & & \\
\hline
\end{tabular}

$W=$ Withheld to avoid disclosure of individual company data.

Note: Geographic coverage is the 50 States and the District of Columbia. $\bullet$ Totals may not equal sum of components due to independent rounding.

Source: Energy Information Administration (EIA) Form EIA-819M, "Monthly Oxygenate Telephone Report. " 
Table D4. Monthly Methyl Tertiary Butyl Ether (MTBE) Production by Merchant and Captive Plants (Thousand Barrels per Day, Except Where Noted)

\begin{tabular}{|c|c|c|c|c|c|c|c|c|c|c|c|c|}
\hline Year & Jan & Feb & Mar & Apr & May & Jun & Jul & Aug & Sep & Oct & Nov & Dec \\
\hline \multicolumn{13}{|l|}{ Total U.S. } \\
\hline 1992 & 98 & 94 & 89 & 79 & 90 & 90 & 101 & 91 & 104 & 118 & 128 & 125 \\
\hline 1993 & 115 & 114 & 112 & 138 & 132 & 126 & 155 & 142 & 157 & 146 & 148 & 144 \\
\hline 1994 & 123 & 140 & 129 & 140 & 139 & 115 & 154 & 166 & 160 & 164 & 150 & 144 \\
\hline 1995 & 149 & 135 & 121 & 168 & & & & & & & & \\
\hline \multicolumn{13}{|c|}{ Merchant Plants } \\
\hline 1992 & 65 & 62 & 58 & 48 & 55 & 53 & 63 & 53 & 61 & 76 & 81 & 77 \\
\hline 1993 & 63 & 66 & 66 & 87 & 75 & 70 & 89 & 79 & 87 & 78 & 81 & 75 \\
\hline 1994 & 63 & 76 & 66 & 73 & 72 & 50 & 73 & 78 & 78 & 72 & 81 & 67 \\
\hline 1995 & 68 & 45 & 45 & 62 & & & & & & & & \\
\hline \multicolumn{13}{|c|}{ Captive Plants } \\
\hline 1992 & 33 & 32 & 31 & 31 & 35 & 37 & 38 & 38 & 43 & 42 & 47 & 48 \\
\hline 1993 & 52 & 48 & 46 & 51 & 57 & 56 & 66 & 63 & 70 & 68 & 67 & 69 \\
\hline 1994 & 60 & 64 & 63 & 67 & 67 & 65 & 81 & 88 & 82 & 92 & 69 & 77 \\
\hline 1995 & 81 & 90 & 77 & 107 & & & & & & & & \\
\hline
\end{tabular}

Note: - Geographic coverage is the 50 States and the District of Columbia. - Totals may not equal sum of components due to independent rounding. Source: Energy Information Administration (EIA) Form EIA-819M, "Monthly Oxygenate Telephone Report. " 


\section{Glossary}

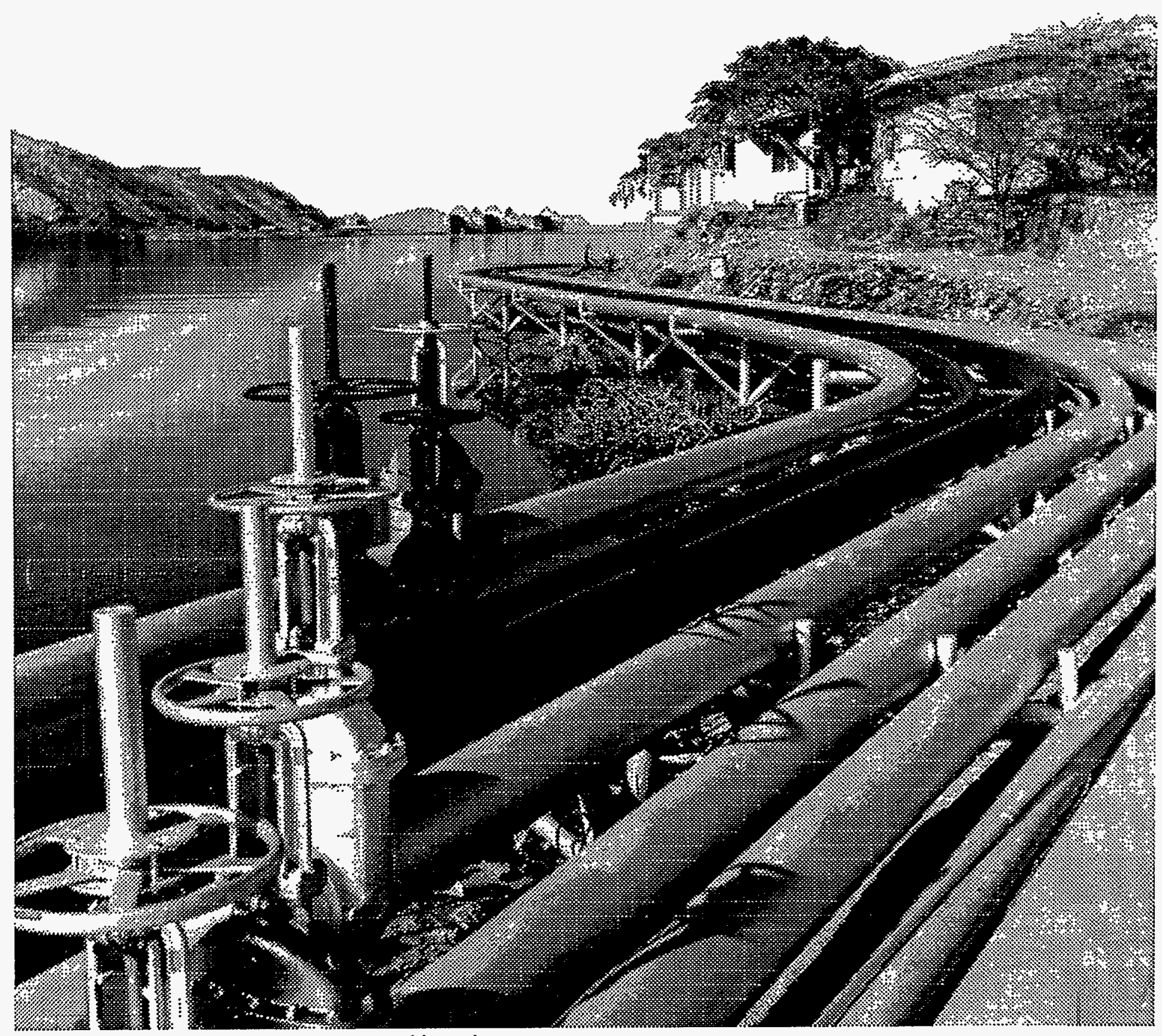

Pipelines carry natural gas across geographic regions. 


\section{Definitions of Petroleum Products and Other Terms}

Alcohol. The family name of a group of organic chemical compounds composed of carbon, hydrogen, and oxygen. The series of molecules vary in chain length and are composed of a hydrocarbon plus a hydroxyl group; $\mathrm{CH}_{3}-\left(\mathrm{CH}_{2}\right)$ n-OH (e.g., methanol, ethanol, and tertiary butyl alcohol).

Alkylate. The product of an alkylation reaction. It usually refers to the high octane product from alkylation units. This alkylate is used in blending high octane gasoline.

Alkylation. A refining process for chemically combining isobutane with olefin hydrocarbons (e.g., propylene, butylene) through the control of temperature and pressure in the presence of an acid catalyst, usually sulfuric acid or hydrofluoric acid. The product, alkylate, an isoparaffin, has high octane value and is blended with motor and aviation gasoline to improve the antiknock value of the fuel.

API Gravity. An arbitrary scale expressing the gravity or density of liquid petroleum products. The measuring scale is calibrated in terms of degrees API; it is calculated as follows:

$$
\text { Degrees API }=\frac{141.5}{s p . g r .60^{\circ} \mathrm{F} / 60^{\circ} \mathrm{F}}-131.5
$$

Aromatics. Hydrocarbons characterized by unsaturated ring structures of carbon atoms. Commercial petroleum aromatics are benzene, toluene, and xylene (BTX).

Asphalt. A dark-brown-to-black cement-like material containing bitumens as the predominant constituent obtained by petroleum processing. The definition includes crude asphalt as well as the following finished products: cements, fluxes, the asphalt content of emulsions (exclusive of water), and petroleum distillates blended with asphalt to make cutback asphaits. The conversion factor for asphalt is $\mathbf{5 . 5}$ barrels per short ton.

ASTM. The acronym for the American Society for Testing and Materials.

Atmospheric Crude Oil Distillation. The refining process of separating crude oil components at atmospheric pressure by heating to temperatures of about $600^{\circ}$ to $750^{\circ} \mathrm{F}$ (depending on the nature of the crude oil and desired products) and subsequent condensing of the fractions by cooling.
Aviation Gasoline (Finished). All special grades of gasoline for use in aviation reciprocating engines, as given in ASTM Specification D910 and Military Specification MIL-G-5572. Excludes blending components which will be used in blending or compounding into finished aviation gasoline.

Aviation Gasoline Blending Components. Naphthas which will be used for blending or compounding into finished aviation gasoline (e.g., straight-run gasoline, alkylate, reformate, benzene, toluene, and xylene). Excludes oxygenates (alcohols, ethers), butane, and pentanes plus. Oxygenates are reported as other hydrocarbons, hydrogen, and oxygenates.

Barrel. A volumetric unit of measure for crude oil and petroleum products equivalent to 42 U.S. gallons. This measure is used in most statistical reports. Factors for converting petroleum coke, asphalt, still gas and wax to barrels are given in the definitions of these products.

Barrels Per Calendar Day. The maximum number of barrels of input that can be processed during a 24-hour period after making allowances for the following limitations:

the capability of downstream facilities to absorb the output of crude oil processing facilities of a given refinery. No reduction is made when a planned distribution of intermediate streams through other than downstream facilities is part of a refinery's normal operation;

the types and grades of inputs to be processed;

the types and grades of products expected to be manufactured;

the environmental constraints associated with refinery operations;

the reduction of capacity for scheduled downtime such as routine inspection, mechanical problems, maintenance, repairs, and turnaround; and

the reduction of capacity for unscheduled downtime such as mechanical problems, repairs, and slowdowns.

Barrels Per Stream Day. The amount a unit can process running at full capacity under optimal crude oil and product slate conditions.

Shaded areas in the definitions represent changes introduced in October 1994 
Benzene $\left(\mathrm{C}_{6} \mathrm{H}_{6}\right)$. An aromatic hydrocarbon present in small proportion in some crude oils and made commercially from petroleum by the catalytic reforming of naphthenes in petroleum naphtha. Also made from coal in the manufacture of coke. Used as a solvent, in manufacturing detergents, synthetic fibers, and petrochemicals and as a component of high-octane gasoline.

Blending Components. See Motor or Aviation Gasoline Blending Components.

Blending Plant. A facility which has no refining capability but is either capable of producing finished motor gasoline through mechanical blending or blends oxygenates with motor gasoline.

Bonded Petroleum Imports. Petroleum imported and entered into Customs bonded storage. These imports are not included in the import statistics until they are: (1) withdrawn from storage free of duty for use as fuel for vessels and aircraft engaged in international trade; or (2) withdrawn from storage with duty paid for domestic use.

BTX. The acronym for the commercial petroleum aromatics benzene, toluene, and xylene. See individual categories for definitions.

Bulk Station. A facility used primarily for the storage and/or marketing of petroleum products which has a total bulk storage capacity of less than 50,000 barrels and receives its petroleum products by tank car or truck.

Bulk Terminal. A facility used primarily for the storage and/or marketing of petroleum products which has a total bulk storage capacity of 50,000 barrels or more and/or receives petroleum products by tanker, barge, or pipeline.

Butane $\left(\mathrm{C}_{4} \mathrm{H}_{10}\right)$. A normally gaseous straight-chain or branch-chain hydrocarbon extracted from natural gas or refinery gas streams. It includes isobutane and normal butane and is designated in ASTM Specification D1835 and Gas Processors Association Specifications for commercial butane.

Isobutane $\left(\mathrm{C}_{4} \mathrm{H}_{10}\right)$. A normally gaseous branch-chain hydrocarbon. It is a colorless paraffinic gas that boils at a temperature of $10.9^{\circ} \mathrm{F}$. It is extracted from natural gas or refinery gas streams.

Normal Butane ( $\left.\mathrm{C}_{4} \mathrm{H}_{10}\right)$. A normally gaseous straight-chain hydrocarbon. It is a colorless paraffinic gas that boils at a temperature of $31.1^{\circ} \mathrm{F}$. It is extracted from natural gas or refinery gas streams.

Butylene $\left(\mathrm{C}_{4} \mathrm{H}_{8}\right)$. An olefinic hydrocarbon recovered from refinery processes.
Captive Refinery Oxygenate Plants. Oxygenate production facilities located within or adjacent to a refinery complex.

Catalytic Cracking. The refining process of breaking down the larger, heavier, and more complex hydrocarbon molecules into simpler and lighter molecules. Catalytic cracking is accomplished by the use of a catalytic agent and is an effective process for increasing the yield of gasoline from crude oil. Catalytic cracking processes fresh feeds and recycled feeds.

Fresh Feeds. Crude oil or petroleum distillates which are being fed to processing units for the first time.

Recycled Feeds. Feeds that are continuously fed back for additional processing.

Catalytic Hydrocracking. A refining process that uses hydrogen and catalysts with relatively low temperatures and high pressures for converting middle boiling or residual material to high-octane gasoline, reformer charge stock, jet fuel, and/or high grade fuel oil. The process uses one or more catalysts, depending upon product output, and can handle high sulfur feedstocks without prior desulfurization.

Catalytic Hydrotreating. A refining process for treating petroleum fractions from atmospheric or vacuum distillation units (e.g., naphthas, middle distillates, reformer feeds, residual fuel oil, and heavy gas oil) and other petroleum (e.g., cat cracked naphtha, coker naphtha, gas oil, etc.) in the presence of catalysts and substantial quantities of hydrogen. Hydrotreating includes desulfurization, removal of substances (e.g., nitrogen compounds) that deactivate catalysts, conversion of olefins to paraffins to reduce gum formation in gasoline, and other processes to upgrade the quality of the fractions.

Catalytic Reforming. A refining process using controlled heat and pressure with catalysts to rearrange certain hydrocarbon molecules, thereby converting paraffinic and naphthenic type hydrocarbons (e.g., low-octane gasoline boiling range fractions) into petrochemical feedstocks and higher octane stocks suitable for blending into finished gasoline. Catalytic reforming is reported in two categories. They are:

Low Pressure. A processing unit operating at less than 225 pounds per square inch gauge (PSIG) measured at the outlet separator.

High Pressure. A processing unit operating at either equal to or greater than 225 pounds per square inch gauge (PSIG) measured at the outlet separator. 
Charge Capacity. The input (feed) capacity of the refinery processing facilities.

Coal. A black or brownish-black solid combustible substance formed by the partial decomposition of vegetable matter without access to air. The rank of coal, which includes anthracite, bituminous coal, subbituminous coal, and lignite, is based on fixed carbon, volatile matter, and heating value. Coal rank indicates the progressive alteration, or coalification, from lignite to anthracite. Lignite contains approximately 9 to 17 million BTU per ton. The heat contents of subbituminous and bituminous coal range from 16 to 24 million BTU per ton, and from 19 to 30 million BTU per ton, respectively. Anthracite contains approximately 22 to 28 million BTU per ton.

\section{Commercial Kerosene-Type Jet Fuel. See} Kerosene-Type Jet Fuel.

Crude Oil (Including Lease Condensate). A mixture of hydrocarbons that exists in liquid phase in underground reservoirs and remains liquid at atmospheric pressure after passing through surface-separating facilities. Included are lease condensate and liquid hydrocarbons produced from tar sands, gilsonite, and oil shale. Drip gases are also included, but topped crude oil (residual oil) and other unfinished oils are excluded. Liquids produced at natural gas processing plants and mixed with crude oil are likewise excluded where identifiable. Crude oil is considered as either domestic or foreign, according to the following:

Domestic. Crude oil produced in the United States or from its "outer continental shelf" as defined in 43 USC 1331.

Foreign. Crude oil produced outside the United States. Imported Athabasca hydrocarbons are included.

Crude Oil, Refinery Receipts. Receipts of domestic and foreign crude oil at a refinery. Includes all crude oil in transit except crude oil in transit by pipeline. Foreign crude oil is reported as a receipt only after entry through customs. Crude oil of foreign origin held in bonded storage is excluded.

Crude Oil Losses. Represents the volume of crude oil reported by petroleum refineries as being lost in their operations. These losses are due to spills, contamination, fires, etc. as opposed to refinery processing losses.

Crude Oil Production. The volume of crude oil produced from oil reservoirs during given periods of time. The amount of such production for a given period is measured as volumes delivered from lease storage tanks (i.e., the point of custody transfer) to pipelines, trucks, or other media for transport to refineries or terminals with adjustments for (1) net differences between opening and closing lease inventories, and (2) basic sediment and water (BS\&W).

Crude Oil Qualities. Refers to two properties of crude oil, the sulfur content and API gravity, which affect processing complexity and product characteristics.

Delayed Coking. A process by which heavier crude oil fractions can be thermally decomposed under conditions of elevated temperatures and pressure to produce a mixture of lighter oils and petroleum coke. The light oils can be processed further in other refinery units to meet product specifications. The coke can be used either as a fuel or in other applications such as the manufacturing of steel or aluminum.

Disposition. The components of petroleum disposition are stock change, crude oil losses, refinery inputs, exports, and products supplied for domestic consumption.

Distillate Fuel Oil. A general classification for one of the petroleum fractions produced in conventional distillation operations. It is used primarily for space heating, on-and-off-highway diesel engine fuel (including railroad engine fuel and fuel for agricultural machinery), and electric power generation. Included are products known as No. 1, No. 2, and No. 4 fuel oils; No. 1, No. 2, and No. 4 diesel fuels. Distillate fuel oil is reported in the following sulfur categories: $0.05 \%$ sulfur and under and Greater than $0.05 \%$ sulfur.

No. 1 Distillate. A petroleum distillate which meets the specifications for No. 1 heating or fuel oil as defined in ASTM D 396 and/or the specifications for No. 1 diesel fuel as defined in ASTM Specification D 975 with distillation temperatures of $420^{\circ} \mathrm{F}$ at the 10-percent recovery point and $550^{\circ} \mathrm{F}$ at the 90-percent recovery point, and kinematic viscosities between 1.4 and 2.2 centistokes at $100^{\circ} \mathrm{F}$.

No. 2 Distillate. A petroleum distillate which meets the specifications for No. 2 heating or fuel oil as defined in ASTM D 396 and/or the specifications for No. 2 diesel fuel as defined in ASTM Specification D 975 with distillation temperatures of $540^{\circ}$ and $640^{\circ} \mathrm{F}$ at the 90-percent recovery point, and kinematic viscosities between 2.0 and 4.3 centistokes at $100^{\circ} \mathrm{F}$.

No. 4 Fuel Oil. A fuel oil for commercial burner installations not equipped with preheating facilities. It is used extensively in industrial plants. This grade is a blend of distillate fuel oil and residual fuel oil stocks that conforms to ASTM Specification D396 or Federal Specification VV-F-815C; with minimum and maximum kinematic viscosities between 5.8 and 26.4 centistokes at 
$100^{\circ} \mathrm{F}$. Also included is No. 4-D, a fuel oil for low and medium-speed diesel engines that conforms to ASTM Specification D975.

Electricity (Purchased). Electricity purchased for refinery operations that is not produced within the refinery complex.

Ending Stocks. Primary stocks of crude oil and petroleum products held in storage as of 12 midnight on the last day of the month. Primary stocks include crude oil or petroleum products held in storage at (or in) leases, refineries, natural gas processing plants, pipelines, tank farms, and bulk terminals that can store at least 50,000 barrels of petroleum products or that can receive petroleum products by tanker, barge, or pipeline. Crude oil that is in-transit by water from Alaska, or that is stored on Federal leases or in the Strategic Petroleum Reserve is included. Primary Stocks exclude stocks of foreign origin that are held in bonded warehouse storage.

ETBE (Ethyl tertiary butyl ether) $\left(\mathrm{CH}_{3}\right)_{3} \mathrm{COC}_{2} \mathrm{H}_{5}$. An oxygenate blend stock formed by the catalytic etherification of isobutylene with ethanol.

Ethane $\left(\mathrm{C}_{2} \mathrm{H}_{6}\right)$. A normally gaseous straight-chain hydrocarbon. It is a colorless paraffinic gas that boils at a temperature of $-127.48^{\circ} \mathrm{F}$. It is extracted from natural gas and refinery gas streams.

Ether. A generic term applied to a group of organic chemical compounds composed of carbon, hydrogen, and oxygen, characterized by an oxygen atom attached to two carbon atoms (e.g., methyl tertiary butyl ether).

Ethylene $\left(\mathrm{C}_{2} \mathrm{H}_{4}\right)$. An olefinic hydrocarbon recovered from refinery processes or petrochemical processes.

Exports. Shipments of crude oil and petroleum products from the 50 States and the District of Columbia to foreign countries, Puerto Rico, the Virgin Islands, and other U.S. possessions and territories.

Field Production. Represents crude oil production on leases, natural gas liquids production at natural gas processing plants, new supply of other hydrocarbons/ oxygenates and motor gasoline blending components, and fuel ethanol blended into finished motor gasoline.

Flexicoking. A thermal cracking process which converts heavy hydrocarbons such as crude oil, tar sands bitumen, and distillation residues into light hydrocarbons. Feedstocks can be any pumpable hydrocarbons including those containing high concentrations of sulfur and metals.

Fluid Coking. A thermal cracking process utilizing the fluidized-solids technique to remove carbon (coke) for continuous conversion of heavy, low-grade oils into lighter products.

Fresh Feed Input. Represents input of material (crude oil, unfinished oils, natural gas liquids, other hydrocarbons and oxygenates or finished products) to processing units at a refinery that is being processed (input) into a particular unit for the first time.

Examples:

(1) Unfinished oils coming out of a crude oil distillation unit which are input into a catalytic cracking unit are considered fresh feed to the catalytic cracking unit.

(2) Unfinished oils coming out of a catalytic cracking unit being looped back into the same catalytic cracking unit to be reprocessed are not considered fresh feed.

Fuel Ethanol $\left(\mathrm{C}_{2} \mathrm{H}_{5} \mathrm{OH}\right)$. An anhydrous denatured aliphatic alcohol intended for gasoline blending as described in Oxygenates definition.

Fuels Solvent Deasphalting. A refining process for removing asphalt compounds from petroleum fractions, such as reduced crude oil. The recovered stream from this process is used to produce fuel products.

Gas Oil. A liquid petroleum distillate having a viscosity intermediate between that of kerosene and lubricating oil. It derives its name from having originally been used in the manufacture of illuminating gas. It is now used to produce distillate fuel oils and gasoline.

Gasohol. A blend of finished motor gasoline and alcohol (generally ethanol but sometimes methanol), limited to 10 percent by volume of alcohol.

Gasoline Blending Components. Naphthas which will be used for blending or compounding into finished aviation or motor gasoline (e.g., straight-run gasoline, alkylate, reformate, benzene, toluene, and xylene). Excludes oxygenates (alcohols, ethers), butane, and pentanes plus.

Gross Input to Atmospheric Crude Oil Distillation Units. Total input to atmospheric crude oil distillation units. Includes all crude oil, lease condensate, natural gas plant liquids, unfinished oils, liquefied refinery gases, slop oils, and other liquid hydrocarbons produced from tar sands, gilsonite, and oil shale.

Heavy Gas Oil. Petroleum distillates with an approximate boiling range from $651^{\circ}$ to $1000^{\circ} \mathrm{F}$. 
Hydrogen. The lightest of all gases, occurring chiefly in combination with oxygen in water; exists also in acids, bases, alcohols, petroleum, and other hydrocarbons.

Idle Capacity. The component of operable capacity that is not in operation and not under active repair, but capable of being placed in operation within 30 days; and capacity not in operation but under active repair that can be completed within 90 days.

Imported Crude Oil Burned As Fuel. The amount of foreign crude oil burned as a fuel oil, usually as residual fuel oil, without being processed as such. Imported crude oil burned as fuel includes lease condensate and liquid hydrocarbons produced from tar sands, gilsonite, and oil shale.

Imports. Receipts of crude oil and petroleum products into the 50 States and the District of Columbia from foreign countries, Puerto Rico, the Virgin Islands, and other U.S. possessions and territories.

Isobutane. See Butane.

Isobutylene $\left(\mathrm{C}_{4} \mathrm{H}_{8}\right)$. An olefinic hydrocarbon recovered from refinery processes or petrochemical processes.

Isohexane $\left(\mathrm{C}_{6} \mathrm{H}_{14}\right)$. A saturated branch-chain hydrocarbon. It is a colorless liquid that boils at a temperature of $156.2^{\circ} \mathrm{F}$.

Isomerization. A refining process which alters the fundamental arrangement of atoms in the molecule without adding or removing anything from the original material. Used to convert normal butane into isobutane $\left(\mathrm{C}_{4}\right)$, an alkylation process feedstock, and normal pentane and hexane into isopentane $\left(\mathrm{C}_{5}\right)$ and isohexane $\left(\mathrm{C}_{6}\right)$, high-octane gasoline components.

\section{Isopentane. See Natural Gasoline and Isopentane.}

Kerosene. A petroleum distillate that has a maximum distillation temperature of $401^{\circ} \mathrm{F}$ at the 10-percent recovery point, a final boiling point of $572^{\circ} \mathrm{F}$, and a minimum flash point of $100^{\circ} \mathrm{F}$. Included are the two grades designated in ASTM D3699: No. 1-K and No. $2-K$, and all grades of kerosene called range or stove oil. Kerosene is used in space heaters, cook stoves, and water heaters and is suitable for use as an illuminant when burned in wick lamps.

Kerosene-Type Jet Fuel. A quality kerosene product with a maximum distillation temperature of $400^{\circ} \mathrm{F}$ at the 10-percent recovery point and a final maximum boiling point of $572^{\circ} \mathrm{F}$. The fuel is designated in ASTM Specification D1655 and Military Specification MIL-T-5624L (Grades JP-5 and JP-8). A relatively low-freezing point distillate of the kerosene type used primarily for turbojet and turboprop aircraft engines.

Commercial. Kerosene-type jet fuel intended for commercial use.

Military. Kerosene-type jet fuel intended for military use.

Lease Condensate. A natural gas liquid recovered from gas well gas (associated and non-associated) in lease separators or natural gas field facilities. Lease condensate consists primarily of pentanes and heavier hydrocarbons.

Light Gas Oils. Liquid petroleum distillates heavier than naphtha, with an approximate boiling range from $401^{\circ} \mathrm{F}$ to $650^{\circ} \mathrm{F}$.

Liquefied Petroleum Gases (LPG). Ethane, ethylene, propane, propylene, normal butane, butylene, isobutane, and isobutylene produced at refineries or natural gas processing plants, including plants that fractionate raw natural gas plant liquids.

Liquefied Refinery Gases (LRG). Liquefied petroleum gases fractionated from refinery or still gases. Through compression and/or refrigeration, they are retained in the liquid state. The reported categories are ethane/ethylene, propane/propylene, normal butane/butylene, and isobutane/isobutylene. Excludes still gas.

Lubricants. A substance used to reduce friction between bearing surfaces or as process materials either incorporated into other materials used as processing aids in the manufacturing of other products, or as carriers of other materials. Petroleum lubricants may be produced either from distillates or residues. Other substances may be added to impart or improve certain required properties. Do not include byproducts of lubricating oil refining such as aromatic extracts derived from solvent extraction or tars derived from deasphalting. "Lubricants" includes all grades of lubricating oils from spindle oil to cylinder oil and those used in greases. Reporting categories include:

Paraffinic. Includes all grades of bright stock and neutrals with a Viscosity Index $>75$.

Naphthenic. Includes all lubricating oil base stocks with a Viscosity Index $<75$.

Note: The criterion for categorizing the lubricants is based solely on the Viscosity Index of the stocks and is independent of crude sources and type of processing used to produce the oils. 
Exceptions: Lubricating oil base stocks that have been historically classified as naphthenic or paraffinic by a refiner may continue to be so categorized irrespective of the Viscosity Index criterion.

Example:

(1) Unextracted paraffinic oils that would not meet the Viscosity Index test.

Merchant Oxygenate Plants. Oxygenate production facilities that are not associated with a petroleum refinery. Production from these facilities is sold under contract or on the spot market to refiners or other gasoline blenders.

Methanol $\left(\mathrm{CH}_{3} \mathrm{OH}\right)$. A light, volatile alcohol intended for gasoline blending as described in Oxygenate definition.

Middle Distillates. A general classification of refined petroleum products that includes distillate fuel oil and kerosene.

Military Kerosene-Type Jet Fuel. See Kerosene-Type Jet Fuel.

Miscellaneous Products. Includes all finished products not classified elsewhere (e.g., petrolatum, lube refining byproducts (aromatic extracts and tars), absorption oils, ram-jet fuel, petroleum rocket fuels, synthetic natural gas feedstocks, and specialty oils).

Motor Gasoline (Finished). A complex mixture of relatively volatile hydrocarbons, with or without small quantities of additives, that has been blended to form a fuel suitable for use in spark-ignition engines. Motor gasoline, as given in ASTM Specification D-4814 or Federal Specification VV-G-1690B, includes a range in distillation temperatures from 122 degrees to 158 degrees $\mathrm{F}$ at the 10-percent recovery point and from 365 degrees to 374 degrees $\mathrm{F}$ at the 90 -percent recovery point. "Motor gasoline" includes reformulated gasoline, oxygenated gasoline, and other finished gasoline. Blendstock is excluded until blending has been completed.

Reformulated Gasoline. Gasoline formulated for use in motor vehicles, the composition and properties of which meet the requirements of the reformulated gasoline regulations promulgated by the U.S. Environmental Protection Agency under Section $211 \mathrm{~K}$ of the Clean Air Act. Inclüdes oxygenated fuels program reformulated gâsoline (OPRG) Exclùdes reformulated gasoline blendstock for oxygenate blending $(\mathbf{R B O B})$

Oxygenated Gasoline. Gasoline formulated for use in motor vehicles that has an oxygen content of 1.8 percent or higher, by weight. Includes gasohol. Excludes reformulated gasoline, oxygenated fuels program reformulated gasoline (OPRG) and reformulated gasoline blendstock for oxygenate blending (RBOB).

Other Finished or Conventional Gasoline. Motor gasoline not included in the oxygenated or reformulated gasoline categories. Excludes reformulated gasoline Blendstock for oxygenate blending (RBOB):

Motor Gasoline Blending. Mechanical mixing of motor gasoline blending components and oxygenates to produce finished motor gasoline. Mechanical mixing of finished motor gasoline with motor gasoline blending components or oxygenates which results in increased volumes of finished motor gasoline, and/or changes in the classification of finished motor gasoline (e.g., other finished motor gasoline mixed with MTBE to produce? oxygenated notor gasoline), is considered motor gasoline blending.

Motor Gasoline Blending Components. Naphthas which will be used for blending or compounding into finished motor gasoline (e.g., straight-run gasoline, alkylate, reformate, benzene, toluene, xylene) and includes reformulated gasoline blendstock for oxygenate blending (RBOB ), Excludes oxygenates (alcohols, ethers), butane, and pentanes plus. Oxygenates are reported as individual components and included in the total for other hydrocarbons, hydrogens, and oxygenates.

MTBE (Methyl tertiary butyl ether) $\left(\mathrm{CH}_{3}\right)_{3} \mathrm{COCH}_{3}$. An ether intended for gasoline blending as described in Oxygenate definition.

Naphtha. A generic term applied to a petroleum fraction with an approximate boiling range between $122^{\circ}$ and $400^{\circ}$ F.

Naphtha Less Than $401^{\circ}$ F. See Petrochemical Feedstocks.

Naphtha-Type Jet Fuel. A fuel in the heavy naphtha boiling range. ASTM Specification D1655 specifies for this fuel maximum distillation temperatures of $290^{\circ} \mathrm{F}$ at the 20-percent recovery point and $470^{\circ} \mathrm{F}$ at the 90 -percent point, meeting Military Specification MIL-T-5624L (Grade JP-4). JP-4 is used for turbojet and turboprop aircraft engines, primarily by the military. Excludes ram-jet and petroleum rocket fuels.

Natural Gas. A mixture of hydrocarbons and small quantities of various nonhydrocarbons existing in the gaseous phase or in solution with crude oil in underground reservoirs. 
Natural Gas Field Facility. A field facility designed to process natural gas produced from more than one lease for the purpose of recovering condensate from a stream of natural gas; however, some field facilities are designed to recover propane, normal butane, pentanes plus, etc., and to control the quality of natural gas to be marketed.

Natural Gas Plant Liquids. Natural gas liquids recovered from natural gas in gas processing plants, and in some situations, from natural gas field facilities. Natural gas liquids extracted by fractionators are also included. These liquids are defined according to the published specifications of the Gas Processors Association and the American Society for Testing and Materials and are classified as follows: ethane, propane, normal butane, isobutane, and pentanes plus.

Natural Gas Processing Plant. A facility designed (1) to achieve the recovery of natural gas liquids from the stream of natural gas which may or may not have been processed through lease separators and field facilities, and (2) to control the quality of the natural gas to be marketed. Cycling plants are classified as gas processing plants.

Natural Gasoline and Isopentane. A mixture of hydrocarbons, mostly pentanes and heavier, extracted from natural gas, that meets vapor pressure, end-point, and other specifications for natural gasoline set by the Gas Processors Association. Includes isopentane which is a saturated branch-chain hydrocarbon, $\left(\mathrm{C}_{5} \mathrm{H}_{12}\right)$, obtained by fractionation of natural gasoline or isomerization of normal pentane.

Net Receipts. The difference between total movements into and total movements out of each PAD District by pipeline, tanker, and barge.

Normal Butane. See Butane.

OPEC. The acronym for the Organization of Petroleum Exporting Countries, that have organized for the purpose of negotiating with oil companies on matters of oil production, prices and future concession rights. Current members are Algeria, Gabon, Indonesia, Iran, Iraq, Kuwait, Libya, Nigeria, Qatar, Saudi Arabia, United Arab Emirates, and Venezuela. The Neutral Zone between Kuwait and Saudi Arabia is considered part of OPEC.

OPRG, "Oxygenated fuels Program Reformulated Gasoline" is reformulated gasoline which is intended for use in an oxygenated fuels program control area during an oxygenated fuels program control period.

Operable Capacity. The amount of capacity that, at the beginning of the period, is in operation; not in operation and not under active repair, but capable of being placed in operation within 30 days; or not in operation but under active repair that can be completed within 90 days. Operable capacity is the sum of the operating and idle capacity and is measured in barrels per calendar day or barrels per stream day.

Operating Capacity. The component of operable capacity that is in operation at the beginning of the period.

Operable Utilization Rate. Represents the utilization of the atmospheric crude oil distillation units. The rate is calculated by dividing the gross input to these units by the operable refining capacity of the units.

Operating Utilization Rate. Represents the utilization of the atmospheric crude oil distillation units. The rate is calculated by dividing the gross input to these units by the operating refining capacity of the units.

\section{Other Finished. See Motor Gasoline (Finished).}

Other Hydrocarbons. Materials received by a refinery and consumed as a raw material. Includes hydrogen, coal tar derivatives, gilsonite, and natural gas received by the refinery for reforming into hydrogen. Natural gas to be used as fuel is excluded.

Other Oils Equal To or Greater Than $401^{\circ} \mathrm{F}$. See Petrochemical Feedstocks.

Other Oxygenates. Other aliphatic alcohols and aliphatic ethers intended for motor gasoline blending (e.g., isopropyl ether (IPE) or n-propanol).

Oxygenated Gasoline. See Motor Gasoline (Finished).

Oxygenates. Any substance which, when added to gasoline, increases the amount of oxygen in that gasoline blend. Through a series of waivers and interpretive rules, the Environmental Protection Agency (EPA) has determined the allowable limits for oxygenates in unleaded gasoline. The "Substantially Similar" Interpretive Rules (56 FR (February 11, 1991)) allows blends of aliphatic alcohols other than methanol and aliphatic ethers, provided the oxygen content does not exceed 2.7 percent by weight. The "Substantially Similar" Interpretive Rules also provides for blends of methanol up to 0.3 percent by volume exclusive of other oxygenates, and butanol or alcohols of a higher molecular weight up to 2.75 percent by weight. Individual waivers pertaining to the use of oxygenates in unleaded gasoline have been issued by the EPA. They include:

Fuel Ethanol. Blends of up to 10 percent by volume anhydrous ethanol (200 proof) (commonly referred to as the "gasohol waiver"). 
Methanol. Blends of methanol and gasoline-grade tertiary butyl alcohol (GTBA) such that the total oxygen content does not exceed 3.5 percent by weight and the ratio of methanol to GTBA is less than or equal to 1 . It is also specified that this blended fuel must meet ASTM volatility specifications (commonly referred to as the "ARCO" waiver).

Blends of up to 5.0 percent by volume methanol with a minimum of 2.5 percent by volume cosolvent alcohols having a carbon number of 4 or less (i.e., ethanol, propanol, butanol, and/or GTBA). The total oxygen must not exceed 3.7 percent by weight, and the blend must meet ASTM volatility specifications as well as phase separation and alcohol purity specifications (commonly referred to as the "DuPont" waiver).

MTBE (Methyl tertiary butyl ether). Blends up to 15.0 percent by volume MTBE which must meet the ASTM D4814 specifications. Blenders must take precautions that the blends are not used as base gasolines for other oxygenated blends (commonly referred to as the "Sun" waiver).

Pentanes Plus. A mixture of hydrocarbons, mostly pentanes and heavier, extracted from natural gas. Includes isopentane, natural gasoline, and plant condensate.

Persian Gulf. The countries that comprise the Persian Gulf are: Bahrain, Iran, Iraq, Kuwait, Qatar, Saudi Arabia, and the United Arab Emirates.

Petrochemical Feedstocks. Chemical feedstocks derived from petroleum principally for the manufacture of chemicals, synthetic rubber, and a variety of plastics. The categories reported are "Naphtha Less Than $401^{\circ} \mathrm{F}$ " and "Other Oils Equal To or Greater Than $401^{\circ}$ F."

Naphtha Less Than $401^{\circ} \mathrm{F}$. A naphtha with a boiling range of less than $401^{\circ} \mathrm{F}$ that is intended for use as a petrochemical feedstock.

Other Oils Equal To or Greater Than $401^{\circ} \mathrm{F}$. Oils with a boiling range equal to or greater than $401^{\circ} \mathrm{F}$ that are intended for use as a petrochemical feedstock.

Petroleum Administration for Defense (PAD) Districts. Geographic aggregations of the 50 States and the District of Columbia into five districts by the Petroleum Administration for Defense in 1950. These districts were originally defined during World War II for purposes of administering oil allocation.

Petroleum Coke. A residue, the final product of the condensation process in cracking. This product is reported as marketable coke or catalyst coke. The conversion factor is 5 barrels per short ton.

Marketable Coke. Those grades of coke produced in delayed or fluid cokers which may be recovered as relatively pure carbon. This "green" coke may be sold as is or further purified by calcining.

Catalyst Coke. In many catalytic operations (e.g., catalytic cracking) carbon is deposited on the catalyst, thus deactivating the catalyst. The catalyst is reactivated by burning off the carbon, which is used as a fuel in the refining process. This carbon or coke is not recoverable in a concentrated form.

Petroleum Products. Petroleum products are obtained from the processing of crude oil (including lease condensate), natural gas, and other hydrocarbon compounds. Petroleum products include unfinished oils, liquefied petroleum gases, pentanes plus, aviation gasoline, motor gasoline, naphtha-type jet fuel, kerosene-type jet fuel, kerosene, distillate fuel oil, residual fuel oil, petrochemical feedstocks, special naphthas, lubricants, waxes, petroleum coke, asphalt, road oil, still gas, and miscellaneous products.

Pipeline (Petroleum). Crude oil and product pipelines used to transport crude oil and petroleum products respectively, (including interstate, intrastate, and intracompany pipelines) within the 50 States and the District of Columbia.

Plant Condensate. One of the natural gas liquids, mostly pentanes and heavier hydrocarbons, recovered and separated as liquids at gas inlet separators or scrubbers in processing plants.

Processing Gain. The volumetric amount by which total output is greater than input for a given period of time. This difference is due to the processing of crude oil into products which, in total, have a lower specific gravity than the crude oil processed.

Processing Loss. The volumetric amount by which total refinery output is less than input for a given period of time. This difference is due to the processing of crude oil into products which, in total, have a higher specific gravity than the crude oil processed.

Product Supplied, Crude Oil. Crude oil burned on leases and by pipelines as fuel.

Production Capacity. The maximum amount of product that can be produced from processing facilities.

Products Supplied. Approximately represents consumption of petroleum products because it measures 


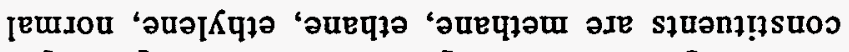

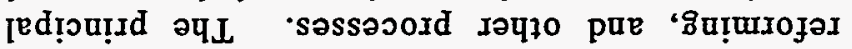

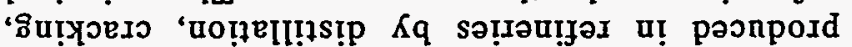

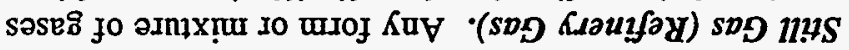

'xəृุduos

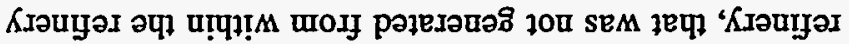

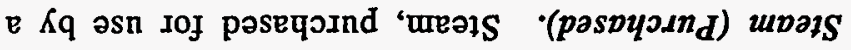

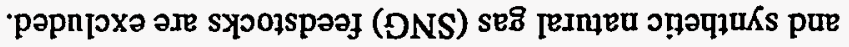

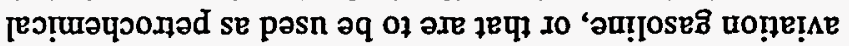

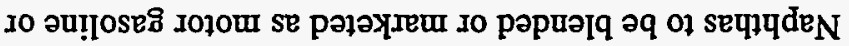

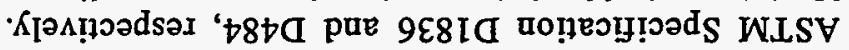

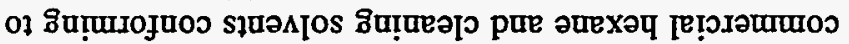
II

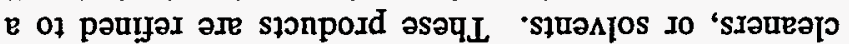

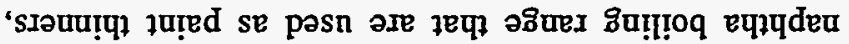

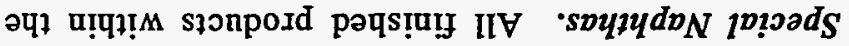

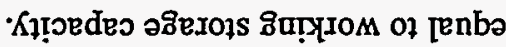

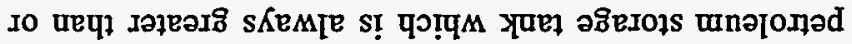

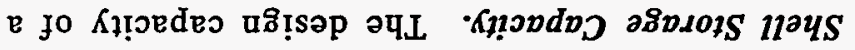

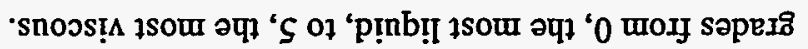

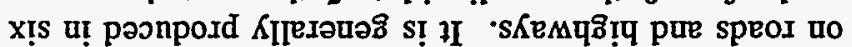

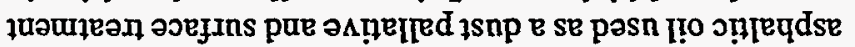

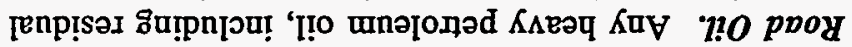

अ. .000I पर्प

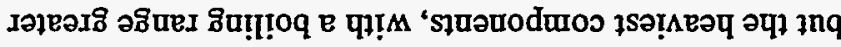

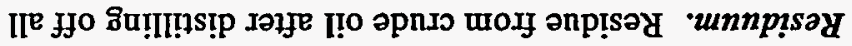

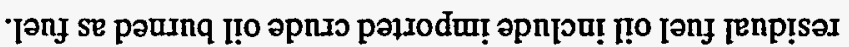

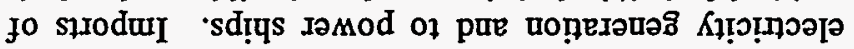

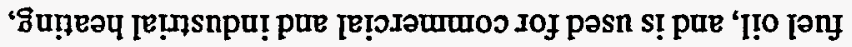

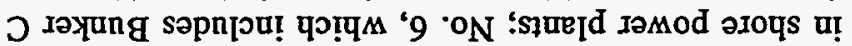

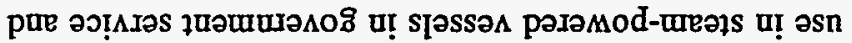

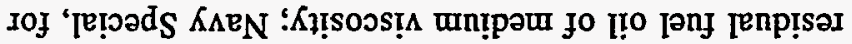

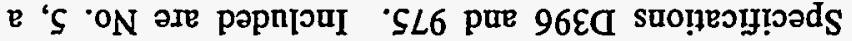

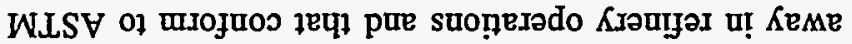

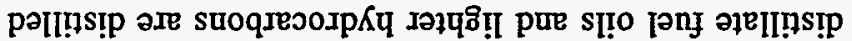

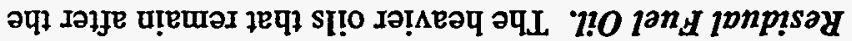

\section{วน!IOSE⿹ J010W ว2S}

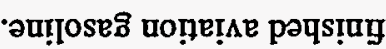

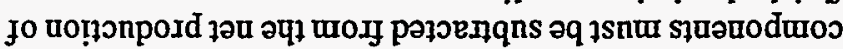

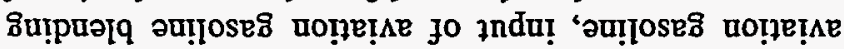

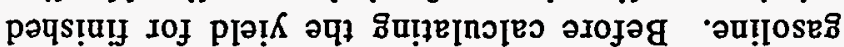

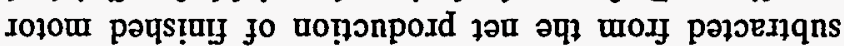
әq 7snu słuəuoduros su!puəIq әแ!

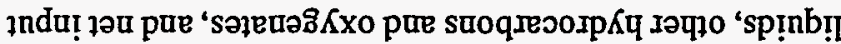

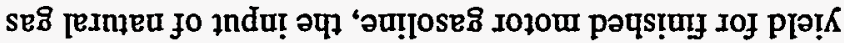

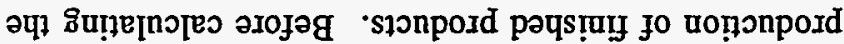

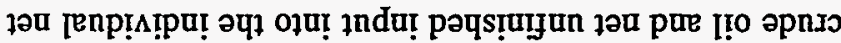

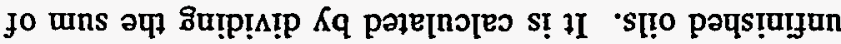

fo łndu! zau pue I!o วpnso jo łndu! worł paonposd

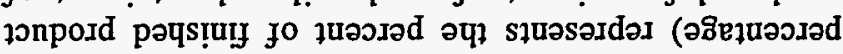

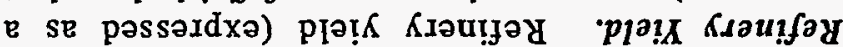

วndu! KIəuyər səpun s!̣seq jəu

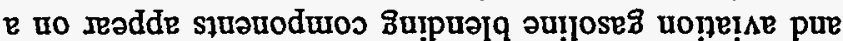

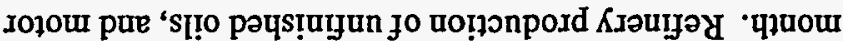

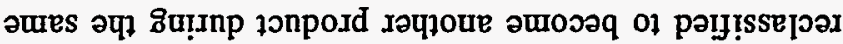

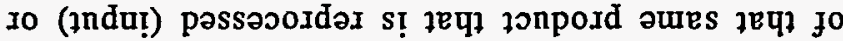

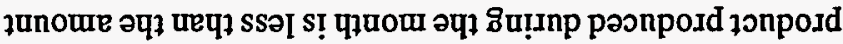

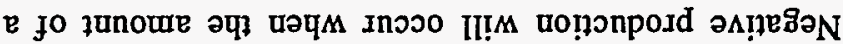

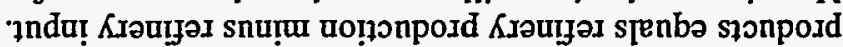

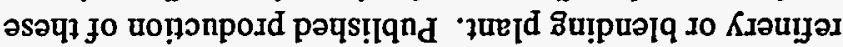

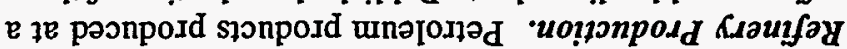

'słonpord unค[oगəd

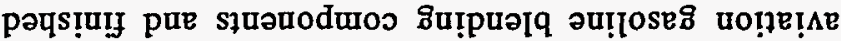

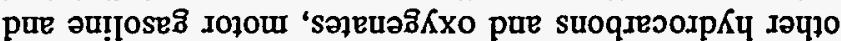

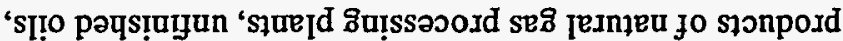

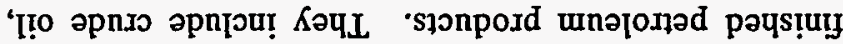

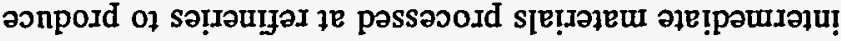

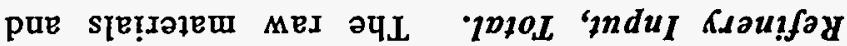

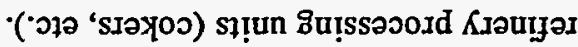
Iәq10 pue sł! un uo!̣e

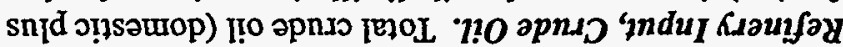

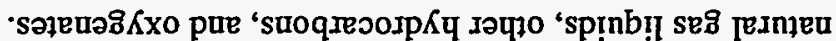

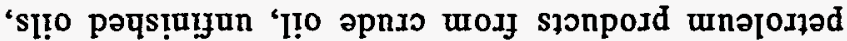

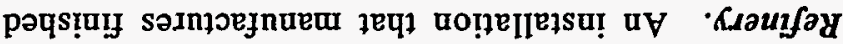

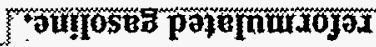

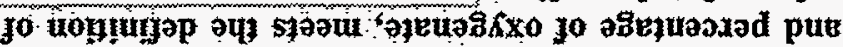

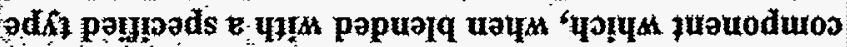

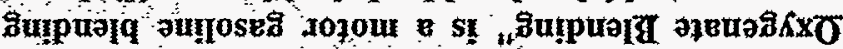

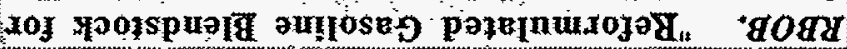

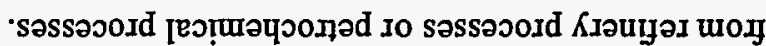

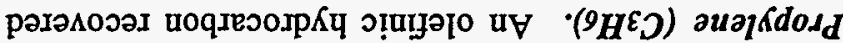

•auedord $\varsigma$ - $₫ H$ pue auedosd

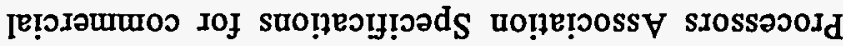
SED pue ऽE8I słonpord IIE sapnjou! II 'sureajis se8 Kxau!jad do

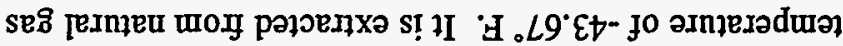

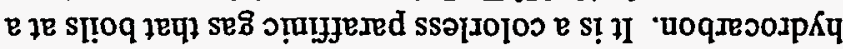

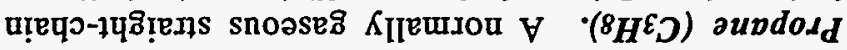

'słrodxa snutur 'sindu!

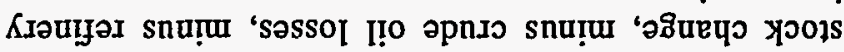

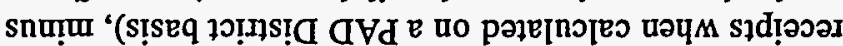

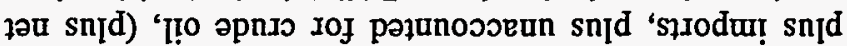

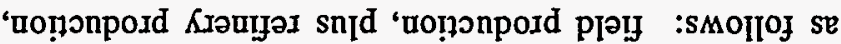
pə⿱一𫝀)

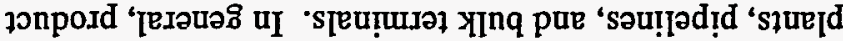

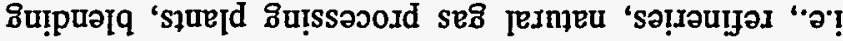

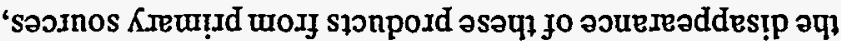


butane, butylene, propane, propylene, etc. Still gas is used as a refinery fuel and a petrochemical feedstock. The conversion factor is 6 million BTU's per fuel oil equivalent barrel.

Stock Change. The difference between stocks at the beginning of the month and stocks at the end of the month.

A negative number indicates a decrease in stocks and a positive number indicates an increase in stocks.

Strategic Petroleum Reserve (SPR). Petroleum stocks maintained by the Federal Government for use during periods of major supply interruption.

Sulfur. A yellowish nonmetallic element, sometimes known as "brimstone".

Supply. The components of petroleum supply are field production, refinery production, imports, and net receipts when calculated on a PAD District basis.

TAME (Tertiary amyl methyl ether) $\left(\mathrm{CH}_{3}\right)_{2}\left(\mathrm{C}_{2} \mathrm{H}_{5}\right) \mathrm{COCH}_{3}$. An oxygenate blend stock formed by the catalytic etherification of isoamylene with methanol.

Tank Farm. An installation used by gathering and trunk pipeline companies, crude oil producers, and terminal operators (except refineries) to store crude oil.

Tanker and Barge. Vessels that transport crude oil or petroleum products. Data are reported for movements between PAD Districts; from a PAD District to the Panama Canal; or from the Panama Canal to a PAD District.

TBA (Tertiary butyl alcohol) $\left(\mathrm{CH}_{3}\right)_{3} \mathrm{COH}$. An alcohol primarily used as a chemical feedstock, a solvent or feedstock for isobutylene production for MTBE; produced as a co-product of propylene oxide production or by direct hydration of isobutylene.

Thermal Cracking. A refining process in which heat and pressure are used to break down, rearrange, or combine hydrocarbon molecules. Thermal cracking includes gas oil, visbreaking, fluid coking, delayed coking, and other thermal cracking processes (e.g., flexicoking). See individual categories for definition.

Toluene $\left(\mathrm{C}_{6} \mathrm{H}_{5} \mathrm{CH}_{3}\right)$. Colorless liquid of the aromatic group of petroleum hydrocarbons, made by the catalytic reforming of petroleum naphthas containing methyl cyclohexane. A high-octane gasoline-blending agent, solvent, and chemical intermediate, base for TNT.

Unaccounted for Crude Oil. Represents the arithmetic difference between the calculated supply and the calculated disposition of crude oil. The calculated supply is the sum of crude oil production plus imports minus changes in crude oil stocks. The calculated disposition of crude oil is the sum of crude oil input to refineries, crude oil exports, crude oil burned as fuel, and crude oil losses.

Unfinished Oils. Includes all oils requiring further processing, except those requiring only mechanical blending. Includes naphthas and lighter oils, kerosene and light gas oils, heavy gas oils, and residuum. See individual categories for definition.

Unfractionated Streams. Mixtures of unsegregated natural gas liquid components excluding those in plant condensate. This product is extracted from natural gas.

United States. The United States is defined as the 50 States and the District of Columbia.

Vacuum Distillation. Distillation under reduced pressure (less the atmospheric) which lowers the boiling temperature of the liquid being distilled. This technique with its relatively low temperatures prevents cracking or decomposition of the charge stock.

Visbreaking. A thermal cracking process in which heavy atmospheric or vacuum-still bottoms are cracked at moderate temperatures to increase production of distillate products and reduce viscosity of the distillation residues.

Wax. A solid or semi-solid material derived from petroleum distillates or residues by such treatments as chilling, precipitating with a solvent, or de-oiling. It is light-colored, more-or-less translucent crystalline mass, slightly greasy to the touch, consisting of a mixture of solid hydrocarbons in which the paraffin series predominates. Includes all marketable wax whether crude scale or fully refined. The three grades included are microcrystalline, crystalline-fully refined, and crystalline-other. The conversion factor is 280 pounds per 42 U.S. gallons per barrel.

Microcrystalline Wax. Wax extracted from certain petroleum residues having a finer and less apparent crystalline structure than paraffin wax and having the following physical characteristics: penetration at $77^{\circ} \mathrm{F}$ (D1321)-60 maximum; viscosity at $210^{\circ} \mathrm{F}$ in Saybolt Universal Seconds (SUS); (D88)-60 SUS (10.22 centistokes) minimum to 150 SUS (31.8 centistokes) maximum; oil content (D721)-5 percent minimum.

Crystalline-Fully Refined Wax. A light-colored paraffin wax having the following characteristics: viscosity at $210^{\circ} \mathrm{F}$ (D88)-59.9 SUS (10.18 centistokes) maximum; oil content (D721)-0.5 percent maximum; other +20 color, Saybolt minimum. 
Crystalline-Other Wax. A paraffin wax having the following characteristics: viscosity at $210^{\circ} \mathrm{F}$ (D88)-59.9 SUS (10.18 centistokes) maximum; oil content (D721)-0.51 percent minimum to 15 percent maximum.

Working Storage Capacity. The difference in volume between the maximum safe fill capacity and the quantity below which pump suction is ineffective (bottoms).
Xylene $\left(\mathrm{C}_{6} \mathrm{H}_{4}\left(\mathrm{CH}_{3}\right)_{2}\right)$. Colorless liquid of the aromatic group of hydrocarbons made the catalytic reforming of certain naphthenic petroleum fractions. Used as high-octane motor and aviation gasoline blending agents, solvents, chemical intermediates. Isomers are metaxylene, orthoxylene, paraxylene. 